NASA/TP-2010-216845

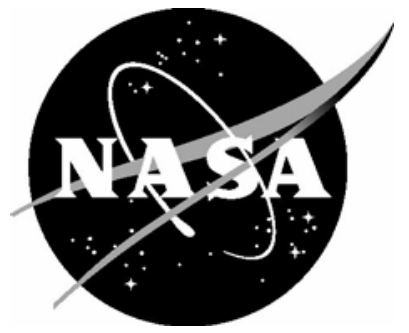

\title{
Hazard Detection Analysis for a Forward-Looking Interferometer
}

Leanne West, Gary Gimmestad, and Ralph Herkert

Georgia Tech Research Institute, Atlanta, Georgia

William L. Smith and Stanislav Kireev

Hampton University, Hampton, Virginia

Philip R. Schaffner and Taumi S. Daniels

NASA Langley Research Center, Hampton, Virginia

Larry B. Cornman, Robert Sharman, and Andrew Weekley

University Corporation for Atmospheric Research, Boulder, Colorado

Glen Perram, Kevin Gross, and Greg Smith

Air Force Institute of Technology, Dayton, Ohio

Wayne Feltz, Joe Taylor, and Erik Olson

University of Wisconsin-Madison, Madison, Wisconsin 


\section{NASA STI Program ... in Profile}

Since its founding, NASA has been dedicated to the advancement of aeronautics and space science. The NASA scientific and technical information (STI) program plays a key part in helping NASA maintain this important role.

The NASA STI program operates under the auspices of the Agency Chief Information Officer. It collects, organizes, provides for archiving, and disseminates NASA's STI. The NASA STI program provides access to the NASA Aeronautics and Space Database and its public interface, the NASA Technical Report Server, thus providing one of the largest collections of aeronautical and space science STI in the world. Results are published in both non-NASA channels and by NASA in the NASA STI Report Series, which includes the following report types:

- TECHNICAL PUBLICATION. Reports of completed research or a major significant phase of research that present the results of NASA programs and include extensive data or theoretical analysis. Includes compilations of significant scientific and technical data and information deemed to be of continuing reference value. NASA counterpart of peerreviewed formal professional papers, but having less stringent limitations on manuscript length and extent of graphic presentations.

- TECHNICAL MEMORANDUM. Scientific and technical findings that are preliminary or of specialized interest, e.g., quick release reports, working papers, and bibliographies that contain minimal annotation. Does not contain extensive analysis.

- CONTRACTOR REPORT. Scientific and technical findings by NASA-sponsored contractors and grantees.
- CONFERENCE PUBLICATION. Collected papers from scientific and technical conferences, symposia, seminars, or other meetings sponsored or co-sponsored by NASA.

- SPECIAL PUBLICATION. Scientific, technical, or historical information from NASA programs, projects, and missions, often concerned with subjects having substantial public interest.

- TECHNICAL TRANSLATION. Englishlanguage translations of foreign scientific and technical material pertinent to NASA's mission.

Specialized services also include creating custom thesauri, building customized databases, and organizing and publishing research results.

For more information about the NASA STI program, see the following:

- Access the NASA STI program home page at http://www.sti.nasa.gov

- E-mail your question via the Internet to help@sti.nasa.gov

- Fax your question to the NASA STI Help Desk at $443-757-5803$

- Phone the NASA STI Help Desk at 443-757-5802

- Write to:

NASA STI Help Desk NASA Center for AeroSpace Information 7115 Standard Drive Hanover, MD 21076-1320 
NASA/TP-2010-216845

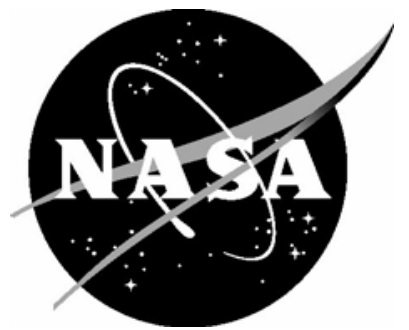

\section{Hazard Detection Analysis for a Forward-Looking Interferometer}

Leanne West, Gary Gimmestad, and Ralph Herkert

Georgia Tech Research Institute, Atlanta, Georgia

William L. Smith and Stanislav Kireev

Hampton University, Hampton, Virginia

Philip R. Schaffner and Taumi S. Daniels

NASA Langley Research Center, Hampton, Virginia

Larry B. Cornman, Robert Sharman, and Andrew Weekley

University Corporation for Atmospheric Research, Boulder, Colorado

Glen Perram, Kevin Gross, and Greg Smith

Air Force Institute of Technology, Dayton, Ohio

Wayne Feltz, Joe Taylor, and Erik Olson

University of Wisconsin-Madison, Madison, Wisconsin

National Aeronautics and

Space Administration 
Trade names and trademarks are used in this report for identification only. Their usage does not constitute an official endorsement, either expressed or implied, by the National Aeronautics and Space Administration.

Available from:

NASA Center for AeroSpace Information

7115 Standard Drive

Hanover, MD 21076-1320

443-757-5802 


\section{Table of Contents}

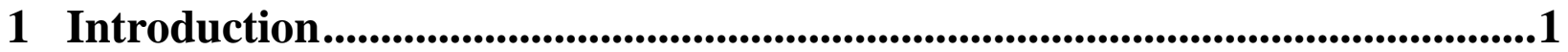

2 Theoretical and Simulation Studies .........................................................2

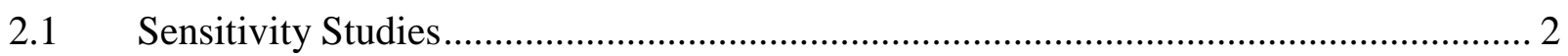

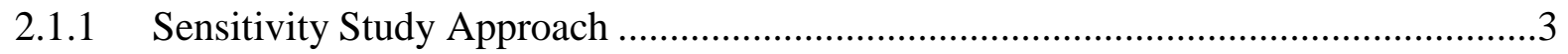

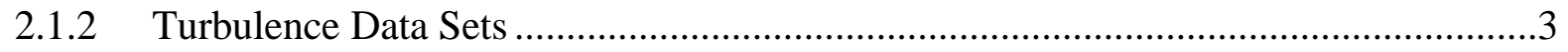

2.1.2.1 Idealized turbulence simulations .................................................................... 4

2.1.2.2 High-rate recorded data .......................................................................... 4

2.1.2.2.1 Research Aircraft Data ............................................................................ 4

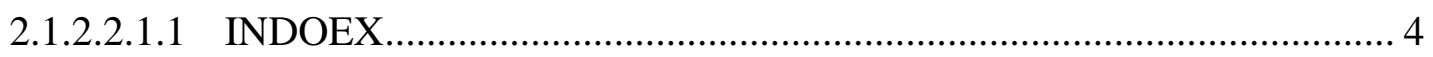

2.1.2.2.1.2 T-REX cases.............................................................................. 4

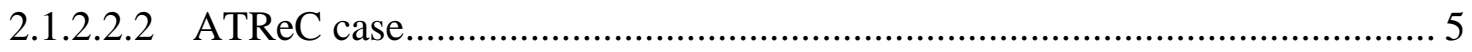

2.1.2.2.3 Commercial aircraft Flight Data Recorder (FDR) ..................................... 5

2.1.2.2.3.1 Convective case over southeastern Iowa, 22 Oct 2004 …….................. 5

2.1.2.2.3.2 Convective case over northwestern TN, 22 July $2002 \ldots \ldots \ldots \ldots \ldots \ldots \ldots \ldots \ldots . . .5$

2.1.2.3 Numerical simulation output ..................................................................... 6

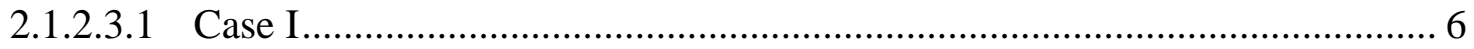

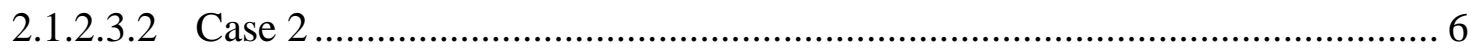

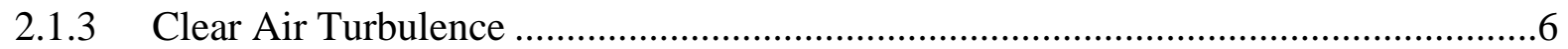

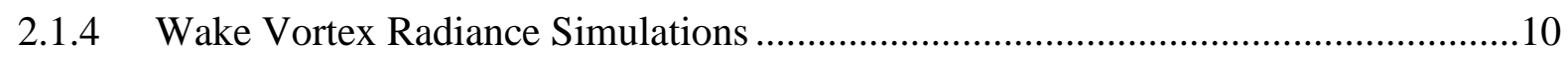

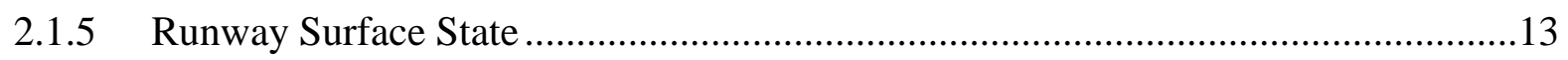

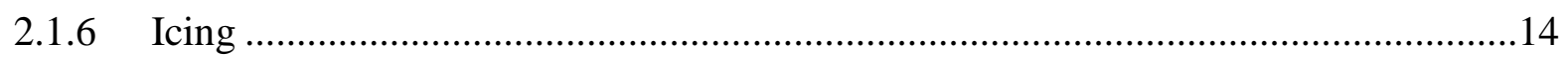

2.1.7 Lee Wave Turbulence FLI Detection Study .........................................................15

2.1.7.1 FLI Radiative Transfer Model Simulations ………………………………….. 17

2.1.7.2 FLI Multi-spectral Imagery …………………...................................... 22

2.1.7.3 Empirical Orthogonal Function Analysis......................................................... 24

2.2 Correlation of Atmospheric Parameters with Radiometric Signals ............................... 31

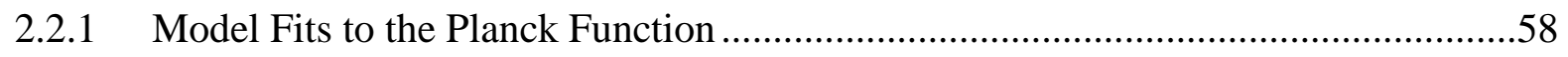

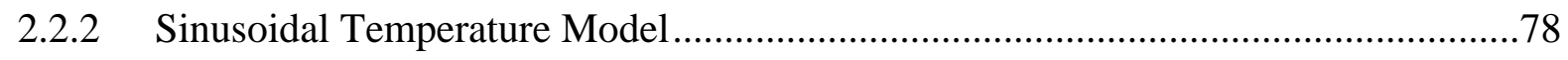

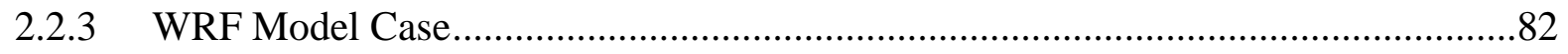

2.2.4 von Karman Simulation Cases ............................................................................... 


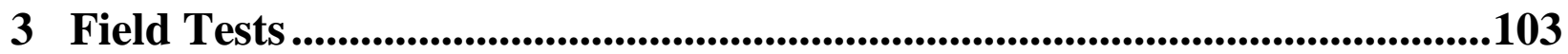

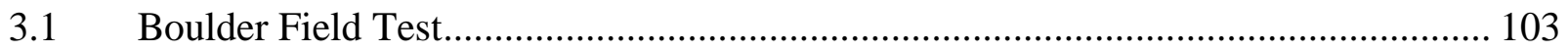

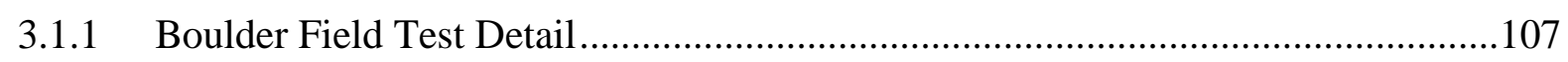

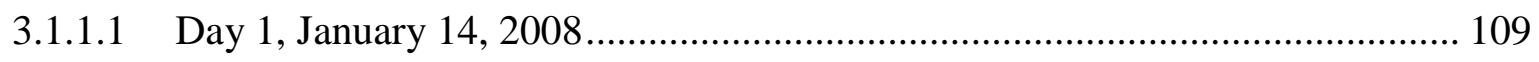

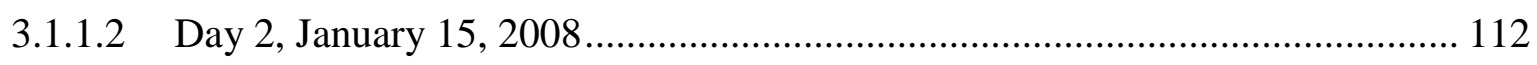

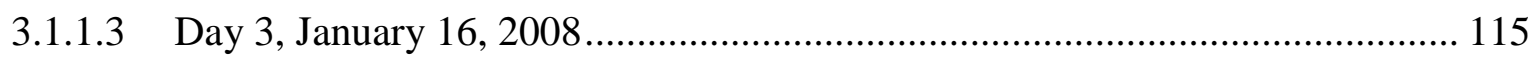

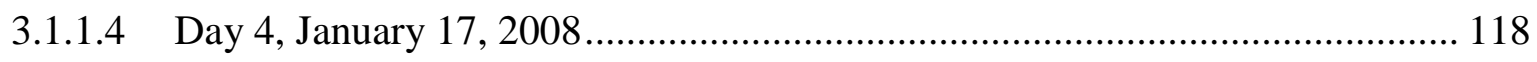

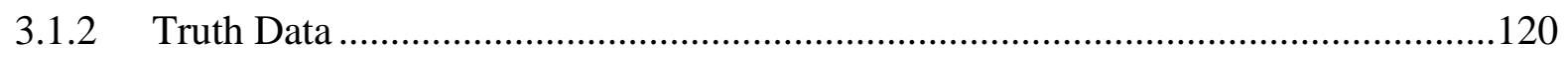

3.1.3 Boulder Field Test Summary …………………..........................................121

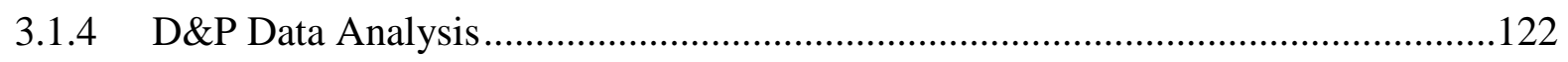

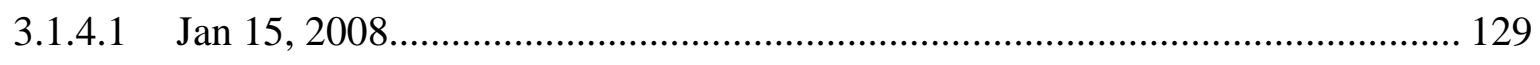

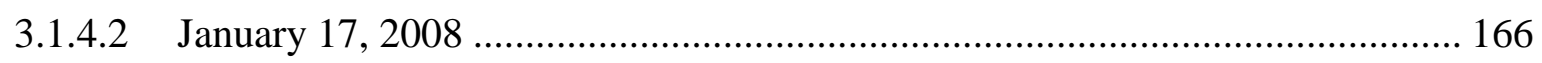

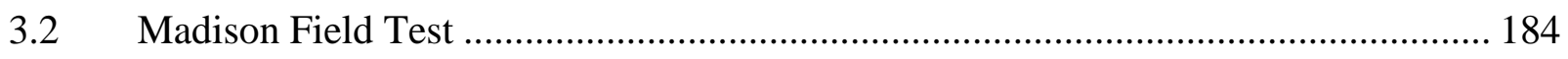

3.2.1 Telops MWIR FIRST Data Analysis …………………………………….......189

3.2.1.1 Signal Processing for Analysis of MWIR FIRST WAVEx Data ..................... 192

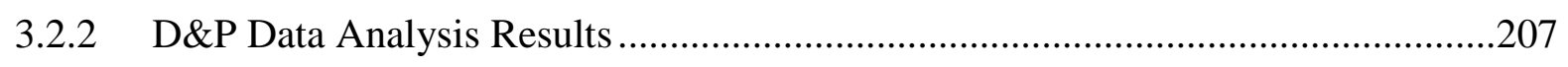

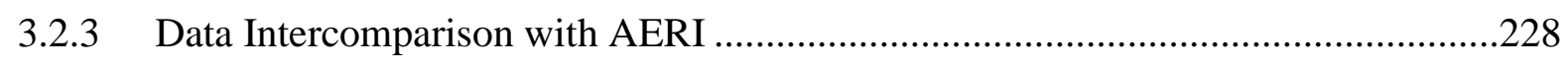

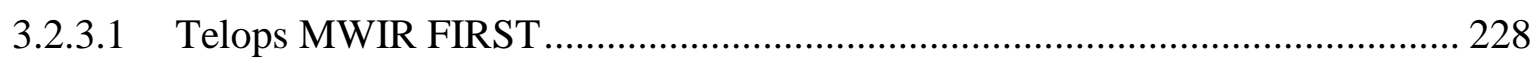

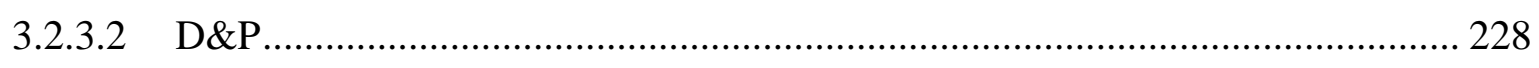

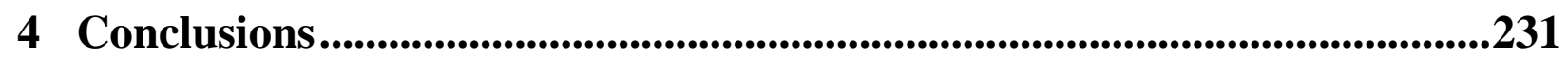

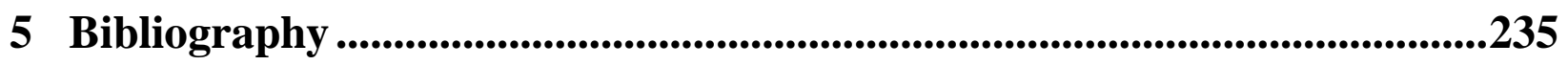

Appendix A: Publications and Presentations ...................................................236

Appendix B: WAVEx Auxiliary Data .............................................................................237 


\section{Introduction}

This Final Report on the feasibility of airborne detection of aviation hazards under Task NNX07AN17A was prepared by personnel at the Georgia Tech Research Institute (GTRI), Hampton University (HU), and the University Corporation for Atmospheric Research (UCAR), with input from other team members from the University of Wisconsin-Madison (UW) and the Air Force Institute of Technology (AFIT). Work under this project specifically addresses the use of a forward-looking interferometer as an in-flight hazard sensor.

The Forward-Looking Interferometer (FLI) is a new instrument concept for obtaining the measurements required to alert flight crews to potential weather hazards to safe flight. To meet the needs of the commercial fleet, such a sensor should address multiple hazards to warrant the costs of development, certification, installation, training, and maintenance. The FLI concept is based on high-resolution Infrared Fourier Transform Spectrometry (FTS) technologies that have been developed for satellite remote sensing. These technologies have also been applied to the detection of aerosols and gases for other purposes. The FLI concept is being evaluated for its potential to address multiple hazards including clear air turbulence (CAT), volcanic ash, wake vortices, low slant range visibility, dry wind shear, and icing during all phases of flight (takeoff, cruise, and landing).

Several project team members previously investigated the physical basis for radiometric detection of the six hazards listed above (Gimmestad 2001, West 2007). The ability of the FLI to provide estimates of the range to hazards was also investigated, with the result that both detection and ranging are enabled by the high spectral resolution provided by the FTS. A sufficient infrared spectral signature appears to be associated with the hazards to enable detection and/or mitigation of all of them, although detection of CAT at relevant ranges may also require that the FLI have high temperature resolution (low noise). The combination of high spectral and temperature resolutions in an imaging instrument is expected to enable sophisticated algorithms with high detection rates and low false alarm rates. The FLI will also function as an infrared imager, providing a real-time video display with night vision capability and enhanced vision in obscured conditions.

The research accomplished in this second phase of the FLI project was in three major areas: further sensitivity studies to better understand the potential capabilities and requirements for an airborne FLI instrument, field measurements that were conducted in an effort to provide empirical demonstrations of radiometric hazard detection, and theoretical work to support the development of algorithms to determine the severity of detected hazards.

The sensitivity studies employed the EOF regression technique, primarily for CAT. The EOF technique requires a large number of data sets, and three types of data sets were used: idealized simulations based on a von Kármán representation of the atmospheric turbulence spectrum; measured data from commercial aircraft flight data recorders and from research aircraft; and four-dimensional simulations of actual severe turbulence encounters. The data sets were used to simulate the radiance spectra that would be observed by an airborne FLI instrument for different aircraft altitudes and positions, over a wide range of azimuth and elevation observing angles.

The ability of the FLI to detect ice on runways was also investigated. In the 8-12 micron region, asphalt, concrete, water, ice, and snow all have different spectral signatures, which should enable FLI algorithms to determine the runway condition, and hence estimate the coefficient of friction, 
during an aircraft approach. This information would tell the pilot whether a safe landing is possible, and if not, it would enable the decision to divert to another airport.

Theoretical calculations were also performed to determine the size of the radiance signals associated with wake vortices. The vortex dimensions, temperature differences, and concentrations of entrained exhaust gasses all depend on the aircraft size, configuration, weight, and flight conditions in a complicated way, so the calculations were performed over ranges of vortex parameters. The calculations were initially done for the 3 to 5 micron (MWIR) wavelength region, the same region covered by the FLI that was used in the field measurements. For reasonable estimates of vortex size, core temperature, and gas concentration, the calculated vortex brightness temperature signatures were fairly small, on the order of $0.1 \mathrm{~K}$. The calculations were also performed for the long wave infrared (LWIR) region with the same result, i.e. signatures on the order of $0.1 \mathrm{~K}$.

Two field measurement campaigns were conducted in an effort to provide empirical demonstrations of radiometric hazard detection. In order to minimize costs, both campaigns were ground-based rather than airborne. The disadvantages of being on the ground looking upward along a slant path were that the simulations performed in the first phase of the project were for the airborne, horizontal-viewing scenario; detailed truth data were unavailable; and viewing angles tended to decrease the radiometric hazard signatures.

The goal of the Boulder measurements was to detect turbulence associated with mountain lee waves by looking for disturbances in long time series of radiance spectra. Although detailed truth data along the radiometer's line of site was not measurable, an NCAR turbulence forecast product was available, along with pilot reports and automated reports from some commercial aircraft.

The goal of the field measurement campaign in Madison, WI was to detect aircraft wake vortices by radiometry, preferably imaging radiometry. During the week of 1-7 June 2008, a suite of instruments was set up directly in line with the main runway of the Dane County Regional Airport. The instruments included the Telops MWIR FIRST ${ }^{\mathrm{TM}}$, the D\&P radiometer, a Bomem MR $-154^{\mathrm{TM}}$ radiometer, and the University of Wisconsin-Madison's AERIbago, which housed the precision-calibrated AERI radiometer, as well as a weather station. The MWIR FIRST and the Bomem were aimed along the path of landing/departing aircraft at a fixed angle of $\sim 20$ degrees above the horizon, while the D\&P and AERI were pointed vertically.

\section{Theoretical and Simulation Studies}

\subsection{Sensitivity Studies}

Theoretical simulation studies were conducted to develop and demonstrate the EOF regression hazard retrieval approach that is discussed extensively in Section 3.3 of the feasibility study Final Report. (West et. al. 2007) In the feasibility study, the EOF regression technique was applied to the enhancement of slant range visibility. In this project, the EOF regression technique was applied to clear air turbulence, wake vortices, Lee wave turbulence, icing, and volcanic ash. Turbulence data sets were provided by UCAR. 


\subsubsection{Sensitivity Study Approach}

In order to generate the large number of atmospheric conditions required to employ EOF regression analysis, the physical variables regulating the hazard radiometric signal (e.g., temperature and moisture variance) were varied through the use of large, four-dimensional mesoscale forecast model simulated turbulence data sets. In this manner, many atmospheric and aviation turbulence measurement observation conditions can be simulated. These simulations produce stable statistical covariance matrices used for calculating the radiance spectra EOFs, as well as the statistical regression equations for predicting the turbulence from EOF amplitudes associated with "observed" radiance spectra.

In order to maximize the skill of the CAT EOF prediction equations, separate radiance EOFs and regression coefficients relating the EOF amplitudes (i.e., PC scores) to the hazard (e.g., turbulence intensity, distance of turbulent region from the aircraft) are computed for different aircraft altitudes and aircraft positions within the forecast model data field, over the full range of azimuth and elevation angles observed within the field of regard of the simulated FLI large area format focal plane detector array. Two computational steps are necessary for this retrieval process. The first step is the specification of the EOF amplitudes by convolution of the transpose of the matrix of EOF elements with the vector of spectral radiance observations for each detector element. The second step is the convolution of the resulting EOF amplitudes with the prediction coefficients relating CAT variables (e.g., intensity and distance from the aircraft) to the EOF amplitudes. In actual practice, these two processes can be combined into a single computational step.

The various EOFs and prediction equations described above were applied to the same fourdimensional ( $\mathrm{x}, \mathrm{y}, \mathrm{d}$, and $\mathrm{t}$ ) data set used to derive the EOFs and retrieval coefficients, but for independent sets of aircraft positions and viewing conditions, as a test of the sensitivity and potential accuracy of the technique. A method of utilizing the four dimensional turbulence indicators (i.e., temperature variance, water vapor variance, vertical motion, horizontal shear, horizontal wind vertical shear, etc.) produced from the simulations will be analyzed to define objective criteria, which can be used to minimize false alarms. An estimate of the false alarm percentage resulting from the application of the technique to actual imaging FLI in-flight data has been made.

\subsubsection{Turbulence Data Sets}

The data sets provided by NCAR for test and evaluation of the FLI algorithm consisted of three types:

1. Idealized turbulence simulations, based on a von Kármán representation of the atmospheric turbulence spectrum.

2. High-rate recorded data from aircraft encounters of turbulence. These data are from an archive of commercial aircraft flight data recordings (FDR) and high-rate data taken by research aircraft during various field campaigns.

3. Multi-nested 3D plus time high-resolution numerical simulation output from modeled after actual severe turbulence encounters. 


\subsubsection{Idealized turbulence simulations}

A first data set was provided from idealized high resolution numerical simulations of von Kármán temperature turbulence. In spite of some limitations, the von Kármán turbulence spectrum has been shown to provide a reasonable approximation for localized turbulence events at mid to upper levels (Murrow et al. 1982, Murrow 1987). One-dimensional homogeneous and isotropic turbulence fields can be generated for a wide range of turbulent conditions by constructing many realizations of a von Kármán temperature field. Each realization is generated with a spectral technique that produces the exact spatial covariance defined by the parameters of a von Kármán model (Frehlich 1997), i.e., the random temperature is produced by generating statistically independent zero mean Gaussian random numbers for the real and imaginary parts of the Fourier coefficients. The variance of each Fourier coefficient is chosen to produce the desired spatial correlation function. The spatial realizations are calculated from the random Fourier coefficients using the Fast Fourier Transform (FFT). Each simulation produces two (onedimensional) turbulence fields - one corresponding to the real part and the other to the imaginary part of the FFT. One simulation output (with to two realizations) was provided. Each realization contained 1-D temperature fluctuations in the longitudinal direction with a spacing of $1 \mathrm{~m}$. This was produced using an outer scale of $2000 \mathrm{~m}$ and a standard deviation of $1 \mathrm{~K}$, which gives a peak-to-peak variation of 4-5 K. The output could be scaled up or down to correspond to different turbulence intensities. These realizations of temperature were scaled to typical temperature signals for Kelvin-Helmholtz instabilities observed in research aircraft measurements (Whiteway et al. 2004) and located at various distances in front of the aircraft.

\subsubsection{High-rate recorded data}

\subsection{Research Aircraft Data}

Since a von Kármán field does not provide a realistic connection between the temperature and velocity fields, examples of traces of spatial variability derived from the NCAR research aircraft were collected and were provided to GTRI/HU for evaluation. The test cases are described below.

\subsection{INDOEX}

The NCAR C-130 research aircraft deployed as part of the INDOEX (Indian Ocean Experiment) campaign in the tropics during 1999 contained a turbulent event at an altitude of $4.8 \mathrm{~km}$. The spectrum of this event showed a classical $\mathrm{k}^{-5 / 3}$ spectrum produced from about $\mathrm{k}=0.003-0.02 \mathrm{~m}^{-1}$ with an EDR value of approximately $0.30 \mathrm{~m}^{2 / 3} \mathrm{~s}^{-1}$, which would put the turbulence experience in roughly the moderate intensity category. The temperature and water vapor density have some correlated features to the enhanced vertical velocity and also to some features that are not correlated. This may be related to the conditions in the tropics.

\subsection{T-REX cases}

The new NCAR HIAPER G5 aircraft collected considerable data over the Rocky Mountains and the Sierra-Nevada Mountains in March and April of 2006 in support of the Terrain Induced Rotor Experiment (T-REX). This data was collected at a higher altitude than the INDOEX data. Several of the ferry legs from the RAF facility to Northern California exhibited relatively large temperature fluctuations that should produce large signatures in the FLI analyses. However, the

vertical velocity spectrum in wavenumber region $\mathrm{k}=0.0025-0.01 \mathrm{~m}^{-1}$ has only modest values of 
the eddy dissipation rate ${ }^{1 / 3}$ (EDR) were observed, reinforcing our lack of understanding of the thermal turbulence - velocity turbulence connection. Two cases were provided, corresponding to flights 3 on 9 Mar 2006 and 17 on 24 Mar 2006. Both data sets included only data extracted for the portions of the flights over the Colorado Rockies. They are mountain wave turbulence generated.

\subsection{ATReC case}

During the Atlantic THORPEX Regional Campaign (ATReC) the NOAA G4 weather reconnaissance aircraft experienced severe clear-air turbulence on 6 Dec 2003 while above a region of active moist convection, to the east of a surface low pressure system off the north-east coast of the United States. This case has been analyzed in detail and numerical simulations of the event have been performed (Lane et al. 2005). The in-situ measurements from the G4 were taken at $1 \mathrm{~Hz}$ time resolution. Shortly after $2000 \mathrm{UTC}$, the aircraft measured strong fluctuations in all three velocity components, especially vertical velocity, temperature, true air speed, and relative humidity. These turbulent fluctuations occurred at approximately $13.5 \mathrm{~km}$ altitude. Of note is the relative humidity which attains a maximum value of approximately $40 \%$. Therefore, this turbulence did occur in clear air, but its existence is probably related to the intense convection over the warm Gulf Stream ocean surface.

\subsection{Commercial aircraft Flight Data Recorder (FDR)}

Analysis of the FDR information from 7 turbulent events produced a couple of interesting cases. Unfortunately, in all cases, humidity information is not recorded, and for several of the cases temperature information was not recorded either. The three datasets that seem to have at least temperature information in them are summarized below.

\subsection{Convective case over southeastern Iowa, 22 Oct 2004}

A commercial aircraft encountered what was reported as severe turbulence over southeastern Iowa on 22 Oct. 2004 at FL370 at about 1636 UTC. A time series of the recorded vertical acceleration, total air temperature (TAT) and derived static air temperature (SAT) was provided. The vertical acceleration is measured at $8 \mathrm{~Hz}$ on the recorder and showed a large swing from a maximum of $1.80 \mathrm{~g}$ to a minimum of $0.18 \mathrm{~g}$ in just a few seconds. The TAT is recorded at $1 \mathrm{~Hz}$ and showed a sharp increase at about the time of the event, followed by a more gradual decrease and then followed by a gradual recovery. But the TAT includes the effects of adiabatic compression in the temperature probe. It is related to the SAT or ambient temperature through

the relation SAT=TAT/ $\left(1+\mathrm{CM}^{2}\right)$, where $\mathrm{M}$ is the aircraft Mach number and $\mathrm{C}$ is a sensor specific constant. For the cruise Mach number, SAT/TAT 0.5.

\subsection{Convective case over northwestern TN, 22 July 2002}

A commercial B757 aircraft encountered what was reported as severe turbulence over northwestern Tennessee on 22 July 2002 at 1848 UTC at about 35,000 ft. while climbing to a destination cruise altitude of FL390. The vertical acceleration was recorded at $8 \mathrm{~Hz}$ and showed a large swing from a maximum of $1.74 \mathrm{~g}$ to a minimum of $-0.37 \mathrm{~g}$ in just a few seconds. The TAT is recorded at $1 \mathrm{~Hz}$. Unfortunately, SAT was not recorded, so a conversion factor could not be applied to derive a high rate SAT during the event. However, the TAT did show a gradual decrease, as expected during a climb, prior to the event, with oscillations coincident with the 
event. Thus in this case there seems to be a temperature signature during the time of the event, but it's difficult to know how much of this may be due to Mach variations. Note though that this event occurred only over a very small time/space interval and is superposed on a larger more gradual temperature trend, making FLI detection of the event challenging.

\subsubsection{Numerical simulation output}

The 3D model output for two cases was analyzed. This included 3D wind fields, temperature, humidity, density, pressure, and altitude, as well as terrain information. Each case was documented with an in-depth description of the conditions surrounding the encounter and an analysis of the genesis of the event. Both cases used the Clark-Hall mesoscale model (Clark 1977, Clark et al. 1996).

\subsection{Case I}

This event was based on a high-resolution simulation of an extreme turbulence encounter over Evergreen, Colorado in 1992. The encounter and the results of the simulation were documented in Clark et al. 2000. This was a multi-nested simulation with 5 nests (see Fig. 11 in the Clark et al. 2000 paper). The archived output from the simulation was in netcdf format. All 5 nests were provided at four model times 15 min apart.

\subsection{Case 2}

This event was based on the results of a high-resolution simulation of a commercial aircraft severe mountain wave turbulence encounter over northern Colorado on 15 March 2006. This event was brought to our attention by the airline. One flight attendant was injured and the flight had to be diverted. The simulation of this event also used the Clark-Hall model with three nested domains, the inner domain resolution being $1 \mathrm{~km}$. The three grids were at simulation times $3 \mathrm{~min}$ apart.

\subsubsection{Clear Air Turbulence}

HU obtained from AER (Atmospheric Environmental Research) a new version of the Line-byLine Radiative Transfer Model (LBLRTM) that is used for FLI simulations. The new code has an improved carbon dioxide line-coupling approach and some improvements in the absorption line shape specification. The new LBLRTM has undergone extensive testing during the first six months of this investigation. In particular, the new LBLRTM has been validated experimentally using radiosondes and simultaneous radiance spectra observed from the MetOP satellite IASI (Infrared Atmospheric Sounding Interferometer) measurements.

HU has developed a new, fast 3-D (horizontal, vertical, and wavenumber) radiative transfer model to enable the rapid retrieval of atmospheric parameters from interferometric radiance measurements, such as will be obtained with the FLI. This model may enable turbulent fluctuations of temperature and water vapor to be retrieved from physical radiative transfer principles, rather than through the use of statistical Principle Component (PC) functions, which requires an extensive statistical training database. The achievement of an adequate statistical data base for the implementation of PC regression retrieval techniques for turbulence detection is a major technical issue in the current effort.

HU (a) provided UCAR with the FLI radiance spectra, as a function of distance from the turbulent region, for several von Kármán cases, (b) developed a new 3-D forward radiative 
transfer model for simulating large area format 3-D imaging FLI measurements and has performed testing of this model using the UW forecast model generated turbulence atmospheric data set, and (c) developed a Principal Component Analysis (PCA) statistical regression turbulence retrieval approach using simulated 4-D FLI radiance spectra.

FLI spectra have been simulated for two cases of von Kármán turbulent perturbations of temperature along the line of sight. The goal was to study the variability in spectra depending on the distance to the turbulent event. Von Kármán turbulent temperature perturbations, provided by UCAR, have $20 \mathrm{~km}$ length with initial step of $1 \mathrm{~m}$. To optimize calculations, and to take into account the real spatial resolution of FLI, these realizations have been averaged so that their spatial resolution is degraded to $100 \mathrm{~m}$. Summary data sets prepared for UCAR:

1. Two cases of von Kármán temperature perturbations (each $20 \mathrm{~km}$ long with increment of $100 \mathrm{~m})$

2. Three aircraft altitudes: $1.5,4.5$, and $9.5 \mathrm{~km}$;

3. Distance to event from $0 \mathrm{~km}$ to $60 \mathrm{~km}$ with increment $0.2 \mathrm{~km}$;

4. Wavenumber range $\sim 650-1650 \mathrm{~cm}^{-1}$ with increment $\sim 0.5 \mathrm{~cm}-1$.

The total number of spectra that were computed is 1809 (i.e. 2 cases $\mathrm{x} 3$ altitudes $\mathrm{x} 301$ distances from aircraft + the unperturbed spectra at the 3 different altitudes for the reference atmospheric condition).

Figure 1 is an example showing three different spectral radiance deviations from a non-turbulent radiance spectrum for FLI measurements simulated from Von Kármán case 1, for an aircraft altitude of $9.5 \mathrm{~km}$. The three spectra correspond to FLI sensor distances from the turbulence of 0 $\mathrm{km}, 1 \mathrm{~km}$, and $2 \mathrm{~km}$. One can see significant spectral radiance turbulence signal in the 650-700 $\mathrm{cm}^{-1}$ opaque region when the aircraft is just about to encounter the turbulent region (i.e., a distance of $0 \mathrm{~km}$.). This strong radiance signal diminishes rapidly with distance from the turbulence. However, in more transparent spectral regions (e.g., $700-800 \mathrm{~cm}^{-1}$, and 1000-1100 $\mathrm{cm}^{-1}$ ), the turbulent radiance signal can be seen in the FLI measurements made at some distance from the turbulent region. Thus, the distance of the turbulence from the aircraft (i.e., the warning time) should be predictable from the spectral dependence of the turbulent radiance signal observed with the FLI. 


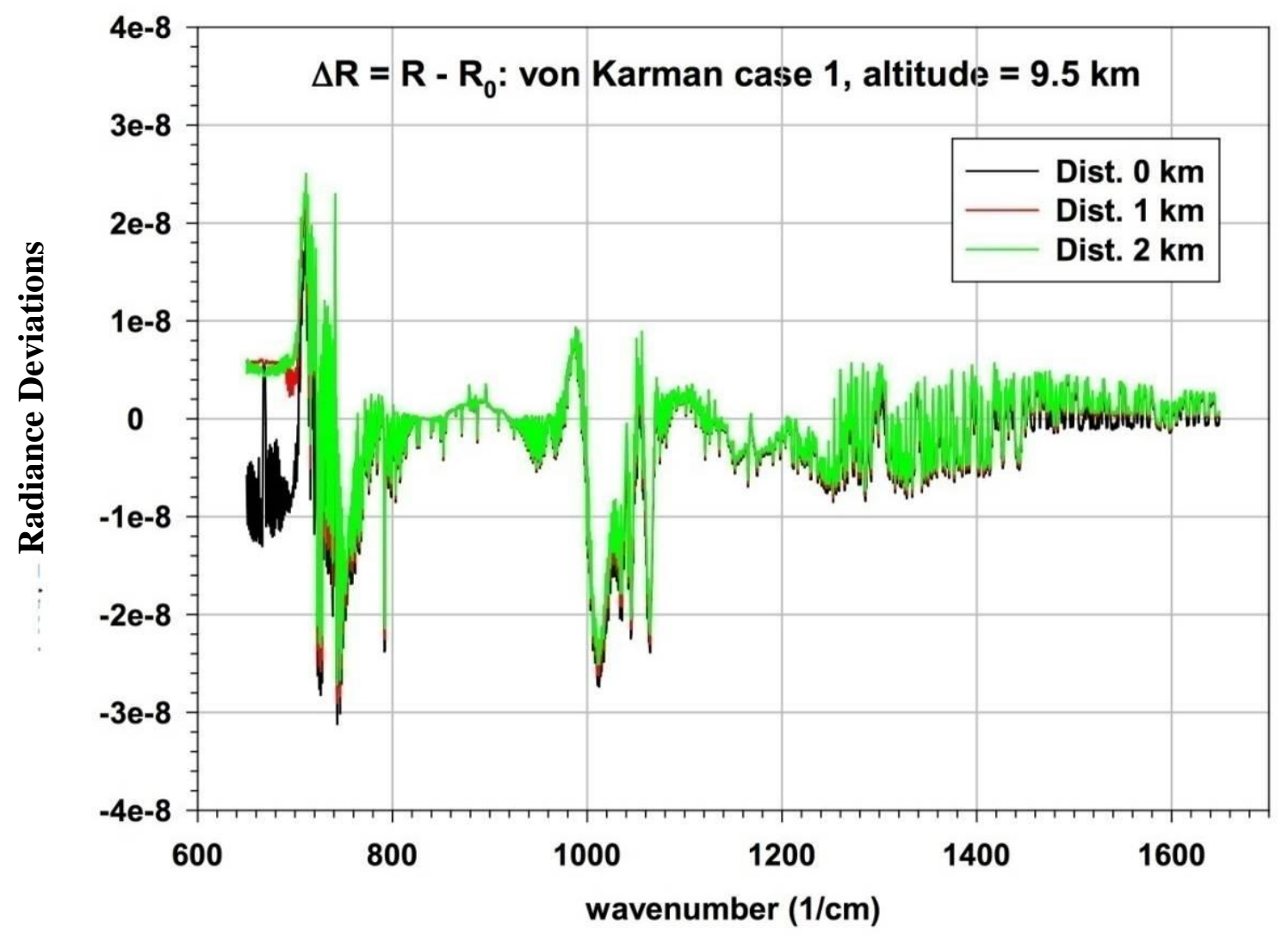

Figure 1. Spectral radiance deviations from a non-turbulent radiance spectrum.

The atmospheric fields were simulated using version 2.2 of the WRF model. The simulation was initialized at 00 UTC on 06 March 2004 with $1^{\circ}$ GFS data and then run for 24 hours. The simulation contains 2 domains with $5-\mathrm{km}$ and $1-\mathrm{km}$ horizontal grid spacing, respectively. The simulation employed the following parameterization schemes: (a) Thompson microphysics, (b) Eta planetary boundary layer, (c) RRTM/Dudhia radiation, (e) NOAH land-surface model, and (f) Explicit convection (i.e. no cumulus parameterization).

Figure 2 shows a comparison of $700 \mathrm{mb}$ vertical velocity and water vapor mixing ratio at 1800 UTC on March 6, 2004, illustrating the lee wave turbulence generated by the Rocky Mountains. One can see a very strong correspondence between the vertical velocity and water vapor mixing ratio fields. Since the radiance within the $1200-1600 \mathrm{~cm}^{-1}$ region of the spectrum is strongly dependent on the water vapor mixing ratio profile along the viewed path, a strong sensitivity of the FLI radiance spectra to these atmospheric turbulence fluctuations is expected. 
$700 \mathrm{mb}$ Vertical Velocity (cm/s) 1800 UTC 03/06/04

$700 \mathrm{mb}$ Mixing Ratio 1800 UTC 03/06/04
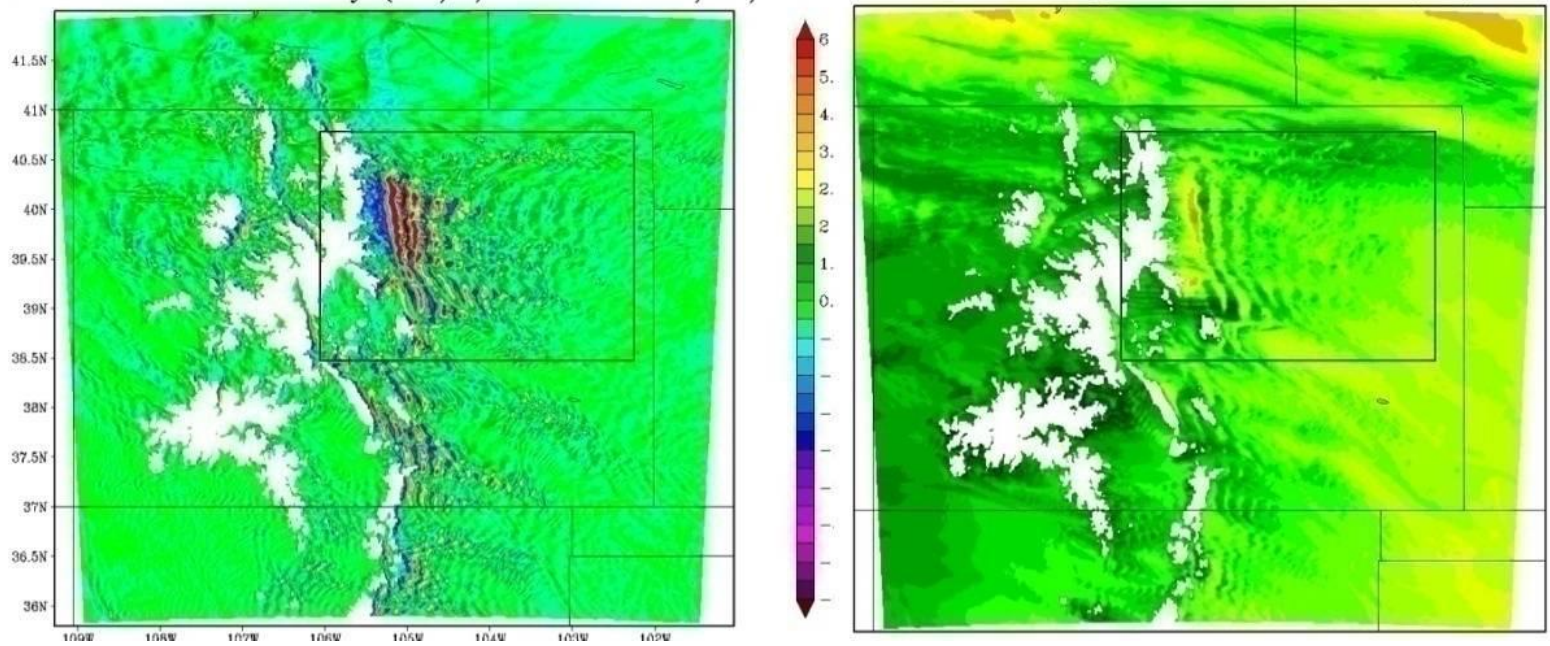

Figure 2. Comparison of $700 \mathrm{mb}$ vertical velocity and water vapor mixing ratio.

The atmospheric fields for this case have been accessed and were being used to conduct FLI radiance simulations and to test the new turbulence EOF (Empirical Orthogonal Functions) regression retrieval procedures discussed in the next paragraph.

A retrieval model based on PCA was formulated and tested using real interferometer data provided by the Infrared Atmospheric Sounding Interferometer (IASI) instrument flying on the European MetOp satellite. (The IASI instrument provides radiance data similar to the NAST-I aircraft instrument). The new formulation links temperature and water vapor through a single atmospheric state vector which enables the correlation between temperature and moisture to be exploited through a single EOF matrix for both constituents. For FLI retrievals, the formulation expands the atmospheric state vector to include vertical velocity as well, exploiting the correlation between vertical velocities with mixing ratio, as depicted in Figure 2. Turbulence warnings are derived from three dimensional $(\mathrm{x}, \mathrm{y}, \mathrm{t})$ vertical velocity retrievals obtained via the EOF regression retrieval technique using the FLI imaging spectrometer radiance observations. This methodology is being tested using the four dimensional Colorado turbulence simulation discussed above.

The physical parameter retrieval methodology for performing turbulence and other aviation hazard detection from FLI spectra was tested using real, as opposed to simulated, MetOp IASI radiance spectra, which are observed with a spectral resolution of $0.5 \mathrm{~cm}^{-1}$ throughout the spectral range from $650-2750 \mathrm{~cm}^{-1}$. Although the profile results appeared to be generally satisfactory, there was a discrepancy of the temperature profile retrieval with a validating balloon measurement (i.e., a coincident radiosonde) in the upper troposphere that indicates that the $\mathrm{CO}_{2}$ mixing ratio used for LBLRTM needs to be increased in order to get agreement between observed and calculated radiance. This increase is believed to be consistent with the annual increases in carbon dioxide measured by surface stations (e.g., the Mauna Loa Observatory, Hawaii).

Figure 3 shows a comparison between radiance spectra computed using the original LBLRTM $\mathrm{CO}_{2}$ mixing ratio (360 ppm) versus that using the 2007 annual value (385 ppm) measured at the Mauna Loa Observatory. The large reduction of the discrepancy between observed and 
calculated radiance in the $700-750 \mathrm{~cm}^{-1} \mathrm{CO}_{2}$ absorption band is obvious from the comparison shown.

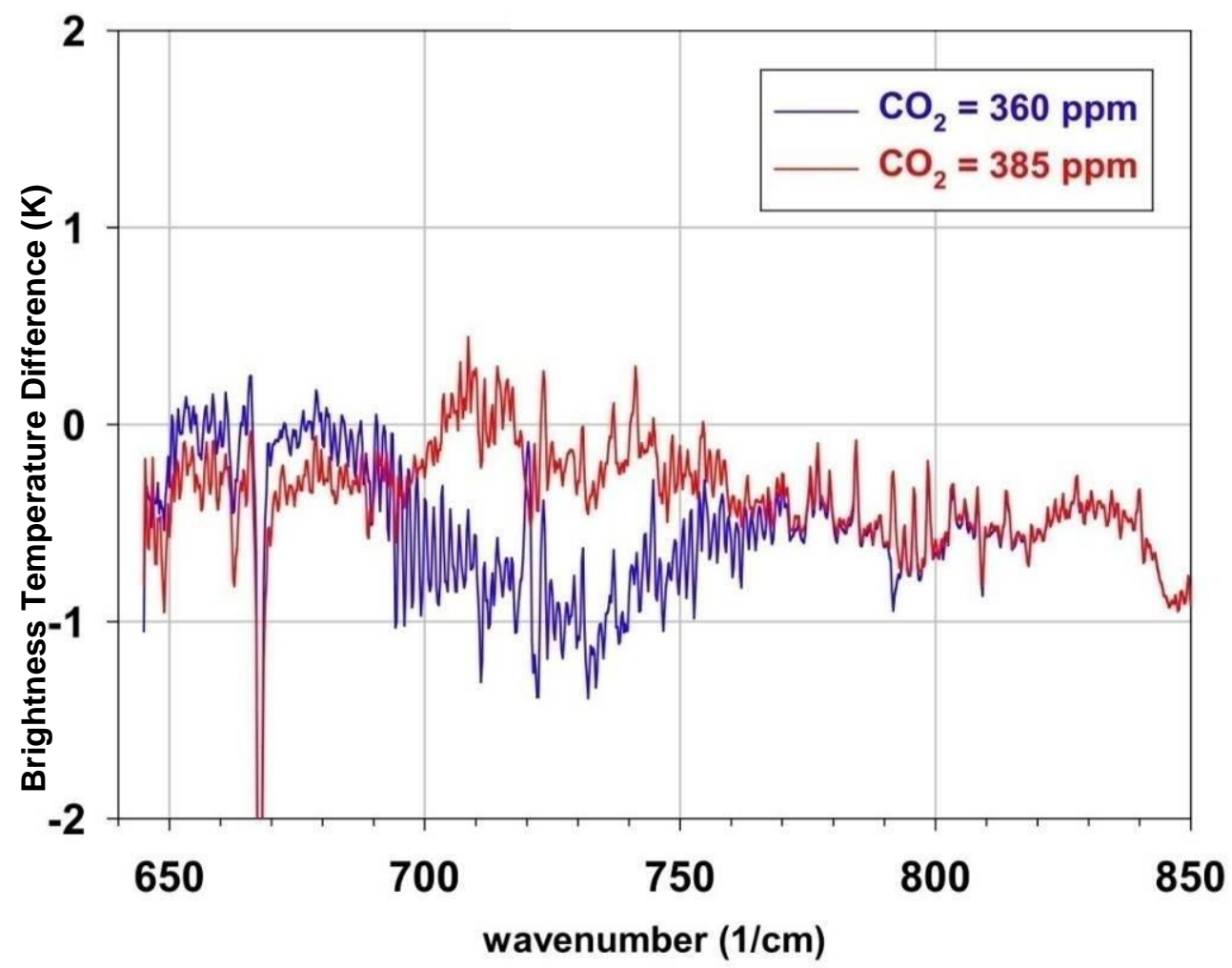

Figure 3. Comparison between computed and measured radiance spectra.

\subsubsection{Wake Vortex Radiance Simulations}

Theoretical calculations were performed to determine the size of the radiance signal associated with wake vortices. The effects of temperature differentials, produced by expansion of the wake vortex, and enhancement of ambient air mixing ratios, produced by entrainment of the engine's exhaust gases, were considered.

The calculations were performed for a viewing angle: of 20 degrees above the horizon, the pointing angle of the MWIR FIRST instrument. The width of the vortex along the instruments lime of site was varied, being $3 \mathrm{~m}, 6 \mathrm{~m}, 12 \mathrm{~m}$, and $24 \mathrm{~m}$, which provides a height cross section of about $1 \mathrm{~m}, 2 \mathrm{~m}, 4 \mathrm{~m}$, and $8 \mathrm{~m}$, respectively. Calculations were performed for different distances of $500 / 300 / 100 \mathrm{~m}$ (along the line of view) from the instrument to the vortex, which provides an altitude of the vortex of approximately 171/103/34 m, respectively. Wave Vortex core temperature perturbations from the environment of $-10 /-5 /-1 \mathrm{~K}$ were assumed. The wavenumber resolution of the radiance spectra calculated was $0.25 \mathrm{~cm}^{-1}$. A moderate Norton- Beer apodization was applied to the line-by-line radiative transfer model simulated interferograms before Fourier transformation to the resulting radiance spectra.

Figure 4 shows a few example longwave and shortwave background radiance spectra and vortex signature (i.e., the difference between vortex core radiant brightness temperature and background brightness temperature) spectra computed for different distances between the instrument and the vortex core and for different vortex core temperature perturbations from the background 
atmospheric temperature. As can be seen, the vortex brightness temperature signal is expected to be quite small $(<0.2 \mathrm{~K})$.

The vortex brightness temperature signal dependence on the mixing ratio enhancement produced by the entrained gases is shown in Figure 5. Here, the vortex brightness temperature signals were computed for a vortex distance from the instrument of $500 \mathrm{~m}$, and a vortex temperature perturbation of $-5 \mathrm{~K}$ from the environment. Entrained $\mathrm{CO}_{2}, \mathrm{~N}_{2} \mathrm{O}$, and $\mathrm{CO}$ exhaust gas vortex air mixing ratio enhancements of 10 times and 100 times the environmental atmospheric mixing ratio (i.e., factor $=1$ ) is assumed. As can be seen the brightness temperature enhancement is nearly linearly related the entrained gas mixing ratio enhancement. Since the degree to which exhaust gases become entrained into the environmental air forming the vortex depends on the type of aircraft and the distance of the engines from the wing tip where the vortex is formed, it is difficult to predict exactly how much of the exhaust gas will be entrained into the vortex.

Finally, Figure 6 shows the dependence of the vortex brightness temperature signal on the thickness of the vortex. Here the vortex brightness temperature signals were computed for a vortex distance from the instrument of $500 \mathrm{~m}$, a vortex temperature perturbation of $-5 \mathrm{~K}$ from the environment, and a $\mathrm{CO}_{2}, \mathrm{~N}_{2} \mathrm{O}$, and $\mathrm{CO}$ exhaust gas vortex air enhancements of 10 times the environmental atmospheric mixing ratio. The vortex signals were computed along viewed path vortex thicknesses of $3,6,12$, and $24 \mathrm{~m}$. As can be seen, the vortex brightness temperature signal appears to increase linearly with the vortex thickness.

In summary, it is apparent from these calculations that a very high measurement signal to noise ratio is needed to detect wake vortices. 


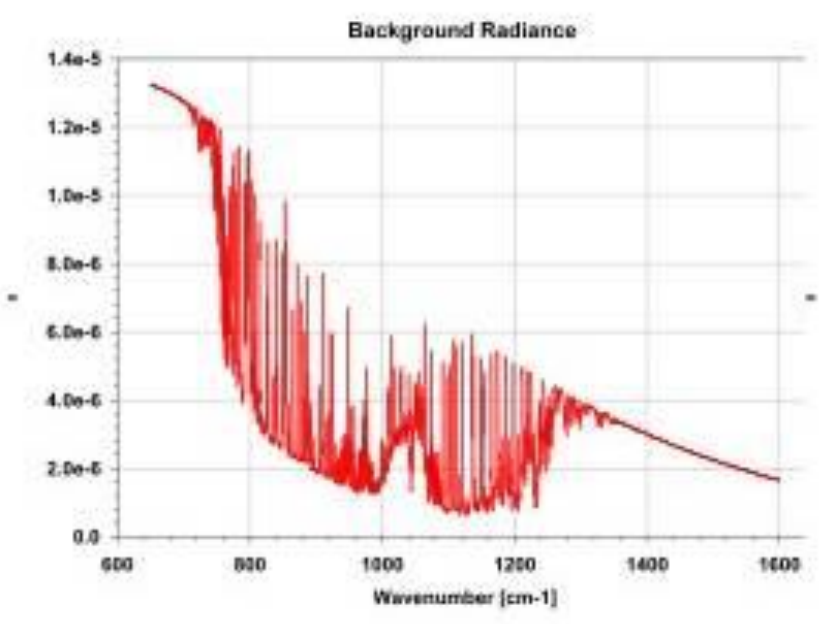

seT, Constant Voctex of $=-10 \mathrm{~K}$
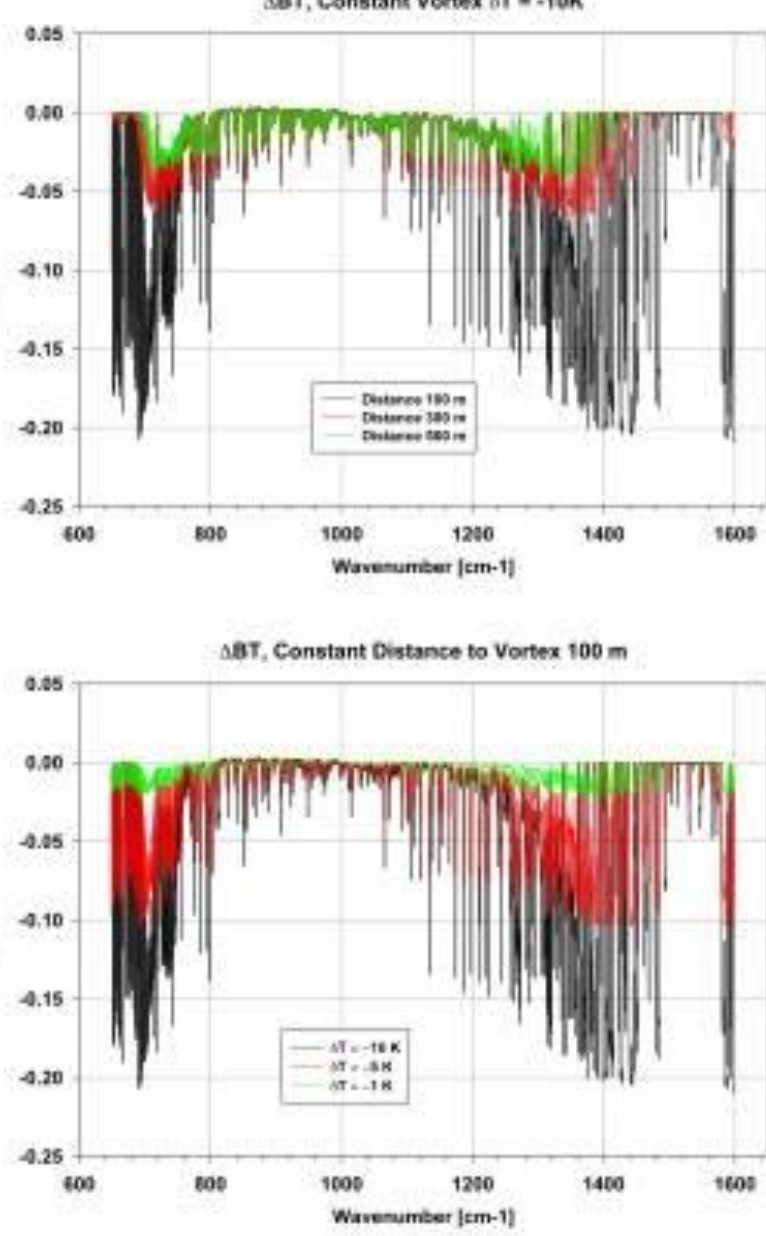
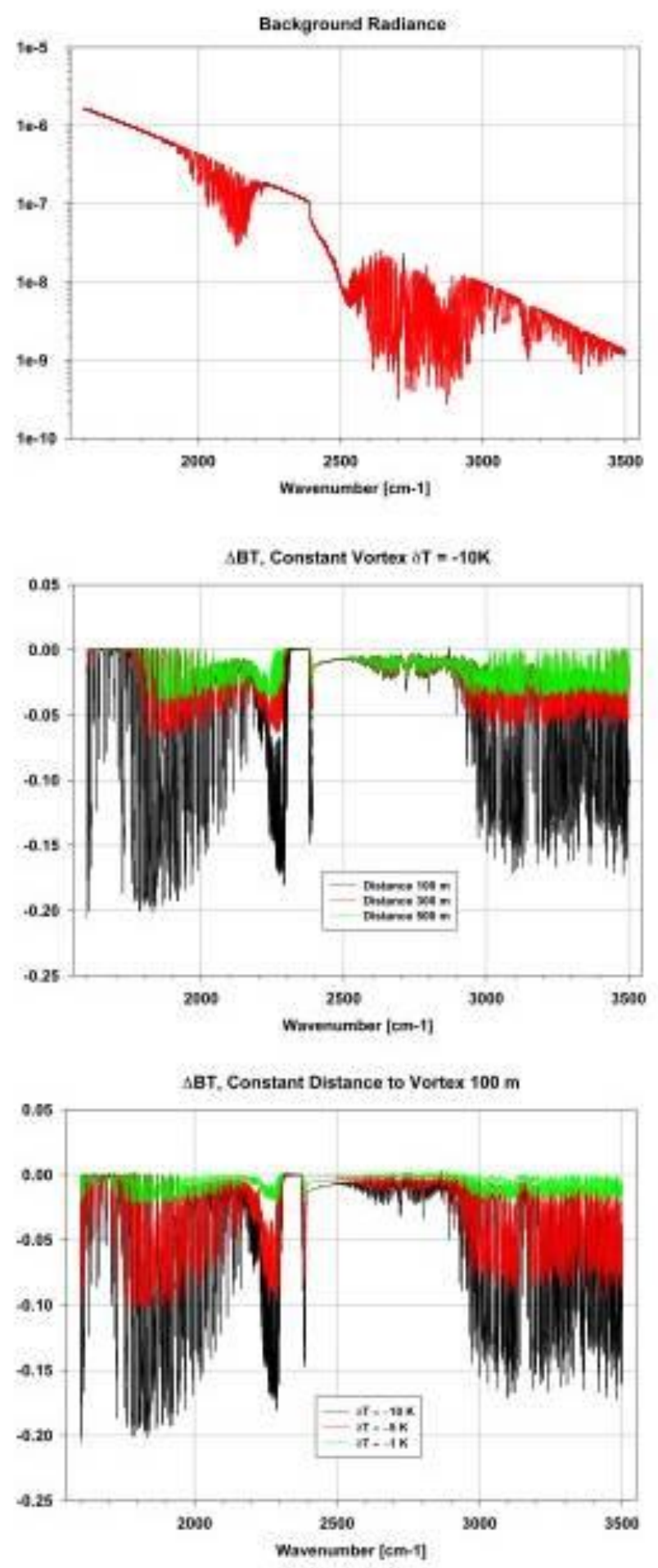

Figure 4. Radiance spectra and vortex signature. 

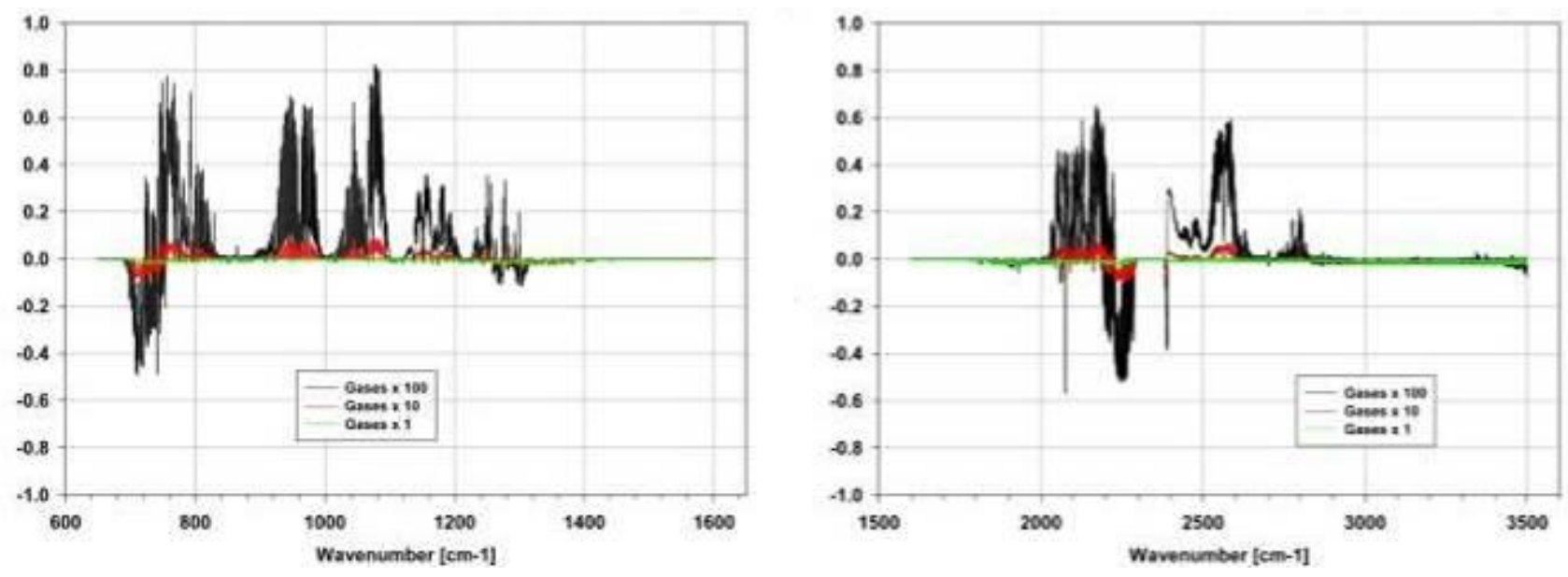

Figure 5. Vortex brightness temperature with ambient gas concentrations.
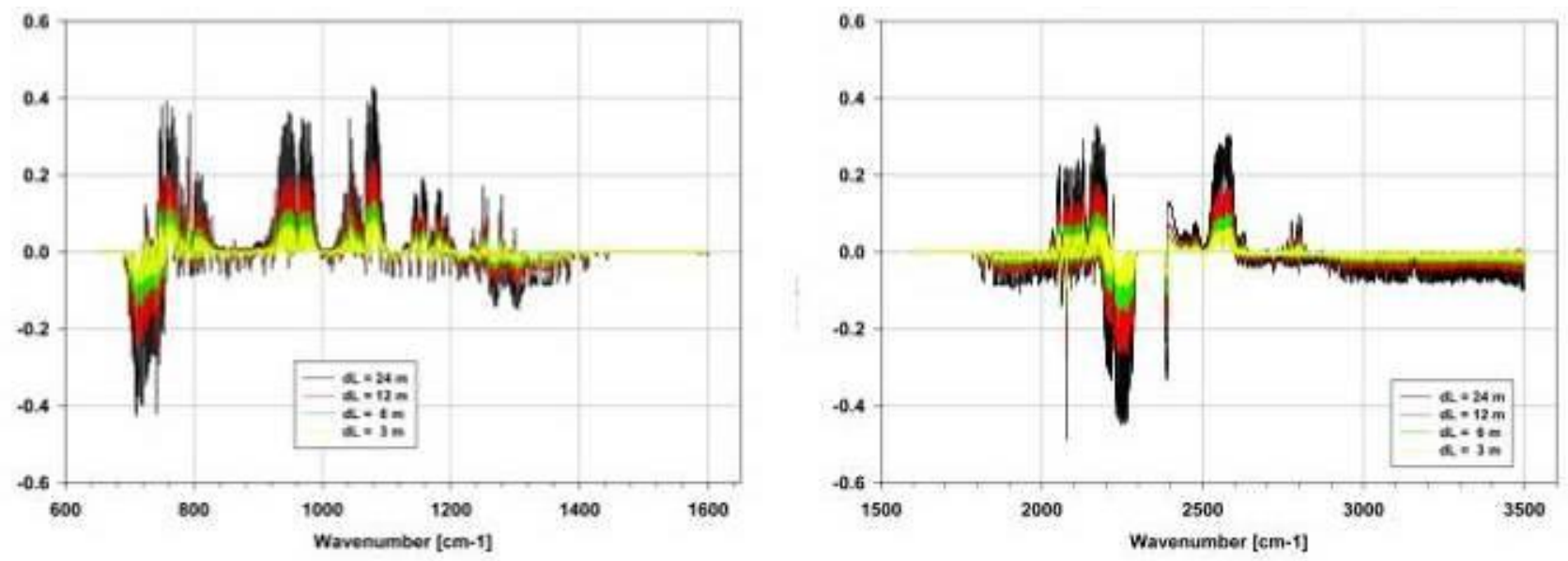

Figure 6. Vortex brightness temperature with 10x enhanced gases.

\subsubsection{Runway Surface State}

As part of a separate but related study to detect runway friction conditions from the cockpit of an aircraft just prior to landing, the physical basis for the detection of runway friction conditions using a FLI was defined. Two separate accidents caused by poor runway friction induced by heavy rain occurred on July 17, 2007 at Sao Paulo Brazil and on September 16, 2007, at Phuket, Thailand. Poor runway friction caused by ice, snow, and frost have also been responsible for hundreds of accidents throughout the history of commercial aviation.

For a FLI viewing a runway from an aircraft close to the runway surface, the radiance observed across the 8-13 $\mu \mathrm{m}$ (i.e., 770 - $1250 \mathrm{~cm}^{-1}$ ) "window" wavelength portion of the spectrum, where clear-air atmospheric transmittance is close to unity, will be related to the runway emissivity through the relation

$$
\mathrm{R}(\theta)=\varepsilon_{\mathrm{s}} \mathrm{B}(\mathrm{T})
$$


Where, according to equation (1), the spectral character of the FLI runway radiance signal will be dominated by the spectral character of runway surface emissivity, $\varepsilon_{s}$. In equation (1), B(T) is a slowly varying monotonic function of spectral wavelength, and $\theta$ is the depression angle (below the horizon) of the line of sight from the instrument to a point on the runway.

Figure 7 shows the emissivity of various types of runway surfaces in which the runway surface is either dry or saturated with pure water, snow, or ice. The runway spectral radiance distribution formed by a mixture of these surface conditions will be a linear combination of the spectral radiance distributions from each type of constituent forming the mixture. The weights are the fractions of runway cover by each constituent.

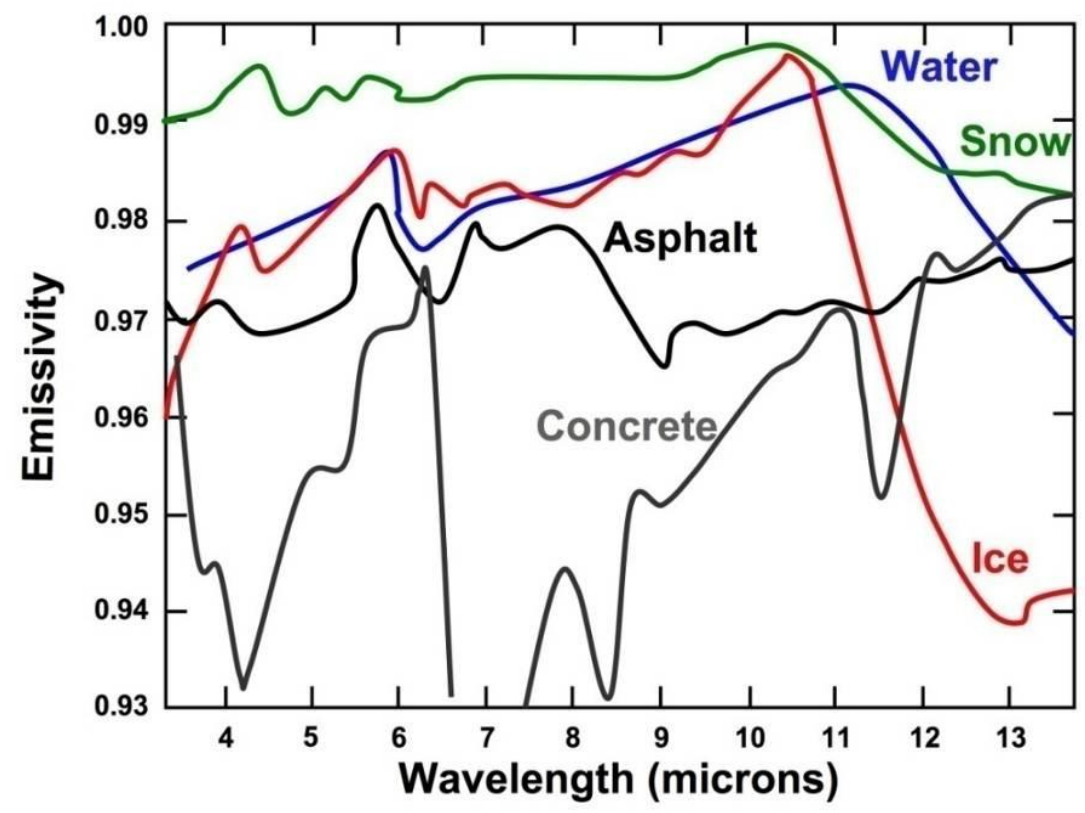

Figure 7. The spectral distribution of emissivity of various types of runway surfaces.

The unique spectral character of the radiance emission by each of these runway surface types, together with the knowledge that any particular runway condition is characterized by a linear combination of these spectral distributions, enables determination of the Coefficient of Friction (COF) for any viewed spot under clear line of sight conditions. Given the spatial distribution of the COF along the entire length of the runway would enable the stopping distance for any particular aircraft to be determined as a function of the touchdown position along the runway. This information would tell the pilot prior to touchdown whether a safe landing is possible, and if not, it would enable the decision to divert to another runway or airport.

\subsubsection{Icing}

Sensitivity studies of icing were to be conducted during this program. However, the icing work was to be performed with the Madison Telops data, which was originally planned to be conducted during winter when super cooled liquid water clouds would have been visible. When the experiment was delayed due to the delay in delivery of the Telops MWIR FIRST, the 
possibility of obtaining the required data was negated. Instead, the research team focused on the Boulder lee wave turbulence data set.

\subsubsection{Lee Wave Turbulence FLI Detection Study}

Lee wave turbulence is produced in conjunction with lee waves, which are gravity waves that occur when stable air flows over a mountain barrier. Lee wave turbulence is responsible for some of the most violent turbulence encountered by aircraft away from thunderstorms. The intensity of lee wave turbulence depends on the wind speed near the mountain peaks. The upper layer of a lee wave system (within 5,000 ft. of the tropopause), where smooth wave flow usually dominates, is where microscale turbulence occasionally occurs. Near the tropopause, winds reach maximum speeds with vertical shears above and below. Lee wave activity strengthens the shear, which promotes the development of shearing-gravity waves, especially near stable layers. Mountain wave turbulence, like Clear Air Turbulence (CAT), is more favorable when a jet stream is present over a mountainous area. The lower layer of the lee wave system, extending from ground to just above the mountaintop, is characterized by turbulence due to strong winds and rotors (mesoscale circulation around a horizontal axis located parallel to a mountain ridge on the downwind side of the mountain). Widespread turbulence is produced in this region by strong winds and wind shear. Typically, the worst turbulence occurs along the lee slopes of the mountain. The greatest lee wave turbulence occurs in the lower turbulent zone in rotor circulations, which are found under the lee wave crests.

Figure 8 shows mountain lee waves in satellite moisture channel imagery at several different spatial resolutions; $8 \mathrm{~km}, 4 \mathrm{~km}$, and $1 \mathrm{~km}$. As can be seen, the mountain lee waves are relatively large scale, being generally greater than $1 \mathrm{~km}$ in wavelength, and therefore should be visible with a FLI system. Because of the severity of the lee wave turbulence as an aviation hazard, it is important to be able determine whether an airborne mounted FLI system could be used to detect this turbulence with a warning time useful for aircraft avoidance, or at least in time to secure the passengers before the aircraft encounters it. Since there is not yet an airborne FLI instrument to experimentally determine this capability, it is necessary to simulate FLI radiance data using atmospheric data created by realistic high-resolution numerical weather prediction model. 


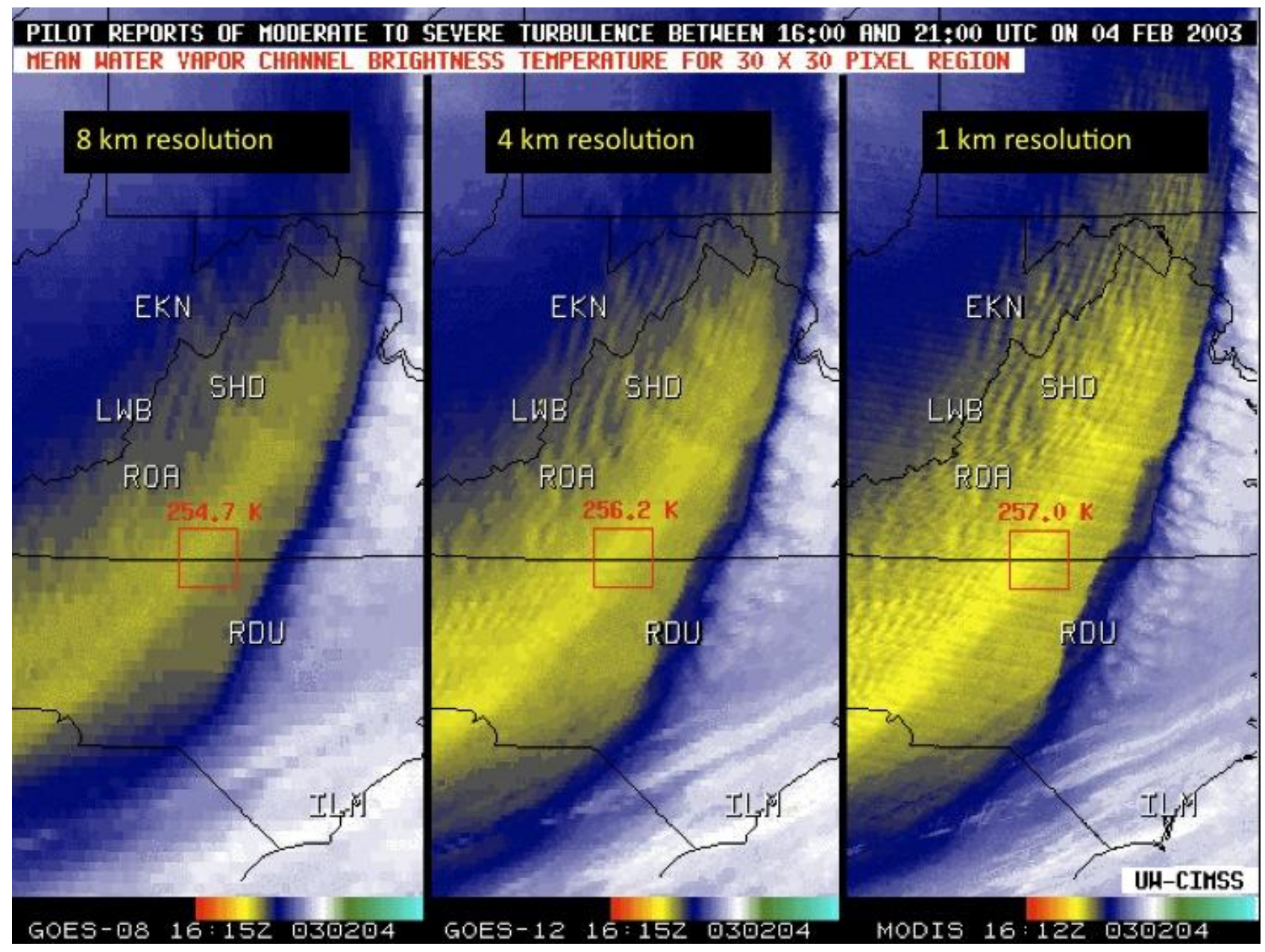

Figure 8. Mountain Lee waves seen in satellite moisture radiance imagery.

The Cooperative Institute of Meteorological Satellite Studies (CIMSS) at the University of Wisconsin produced the atmospheric data needed for the FLI simulation for a 06 March 2004 Colorado Rocky Mountain lee wave turbulence occurrence. The atmospheric fields needed were simulated using version 2.2 of the WRF model. The simulation was initialized at 00 UTC on 06 March 2004 with $1^{\circ}$ GFS data and then run for 24 hours. The simulation contained a 1-km horizontal grid spacing suitable for simulating FLI radiance spectra via radiative transfer calculation. The simulation employed the following atmospheric process parameterization schemes: (a) Thompson microphysics, (b) Eta planetary boundary layer, (c) RRTM/Dudhia radiation, (e) NOAH land-surface model, and (f) Explicit convection (i.e. no cumulus parameterization).

Figure 9 shows the mixing ratio and the vertical velocity variations associated with the lee wave turbulence generated by the Rocky Mountains at 1800 UTC on March 6, 2004. A very strong correspondence between the vertical velocity and water vapor mixing ratio fields is apparent. Since the radiance within the $1200-1600 \mathrm{~cm}^{-1}$ region of the spectrum is strongly dependent on the water vapor mixing ratio profile along the viewed path, a strong sensitivity of the FLI radiance spectra to these atmospheric turbulence fluctuations is expected. 
$700 \mathrm{mb}$ Vertical Velocity $(\mathrm{cm} / \mathrm{s}) 1800$ UTC 03/06/04
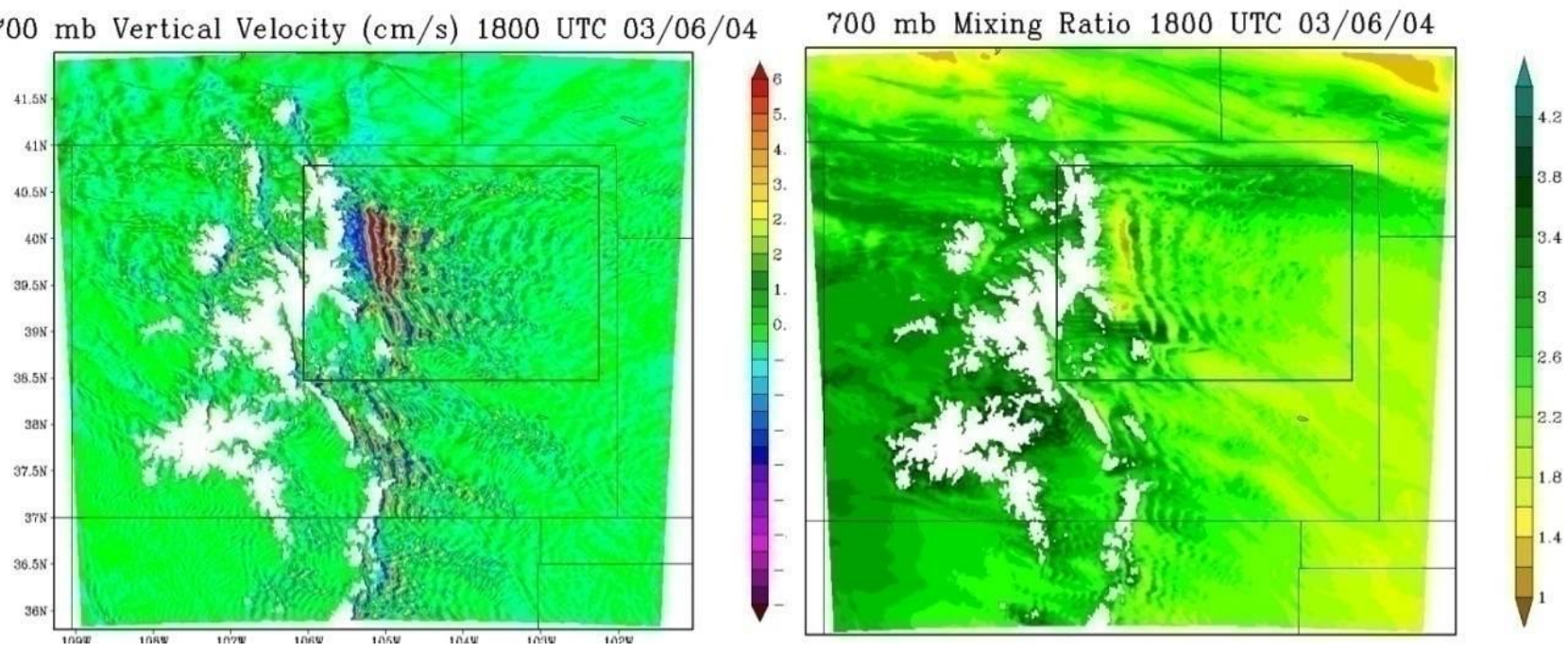

Figure 9. Comparison of $700 \mathrm{mb}$ vertical velocity and water vapor mixing ratio.

The atmospheric fields generated for this case have been used to conduct FLI radiance measurement simulations and to test a new turbulence EOF (Empirical Orthogonal Functions) regression retrieval procedure, to be described below.

\subsubsection{FLI Radiative Transfer Model Simulations}

The procedure for testing the FLI aviation lee wave turbulence hazard retrieval algorithm, using the UW model simulation of mountain lee wave turbulence, is defined as follows. For the initial radiative transfer calculations, it was assumed that the FLI instrument is an imaging interferometer with a square Field of Regard (FOR) of $250 \mathrm{mrad}$ in azimuth by $250 \mathrm{mrad}$ in elevation, with instantaneous spatial resolution of $1 \mathrm{mrad}$ (i.e., a focal plane size of $250 \times 250=$ 62,500 detector elements), an unapodized spectral resolution of $0.5 \mathrm{~cm}^{-1}$, and a spectral range from $650-1650 \mathrm{~cm}^{-1}$. Simulations were performed initially for an aircraft altitude of $8 \mathrm{~km}$ $(\sim 350 \mathrm{mb})$, the altitude where severe lee wave turbulence is observed from the model output. Simulations were performed for various distances from the turbulent region (i.e., the eastern slope of the Rocky Mountains), and for various angles of observation relative to the wave pattern established by the mountain geometry; that is, the FLI measurements were simulated for an aircraft approaching the turbulence region from a wide range of flight headings, including north $\left(360^{\circ}\right)$, northeast $\left(45^{\circ}\right)$, east $\left(90^{\circ}\right)$, southeast $\left(135^{\circ}\right)$, south $\left(180^{\circ}\right)$, southwest $\left(235^{\circ}\right)$, west $\left(270^{\circ}\right)$, and northwest $\left(315^{\circ}\right)$ directions. Radiance EOFs were produced from two-dimensional radiance spectra obtained for various distances from the turbulence region by combining the spectra simulated over the wide variety of approach angles and a sampling by 25 (a 5 x 5 representative sample) detector elements forming the FLI detector array.

Once all the FLI radiances were simulated as described above, we evaluated the correlations of the PC amplitudes with the magnitude of the turbulence ahead of the aircraft (i.e., applying the EOF regression approach for the retrieval of the distance of the turbulence from the aircraft. As will be shown, the actual spatial distribution of the turbulence is evident in the display of the spatial distribution of the radiances, or EOF amplitudes. The turbulence has a different spectral signature dependent upon the aircrafts distance from the turbulent region (i.e., if the turbulence is 
close, strong absorption line signals are sensitive to it, whereas, if the turbulence is far from the turbulence, only weak absorption regions are influenced by the turbulent fluctuations of temperature and mixing ratio of the absorbing constituents).

In principle, it should be possible to perform an EOF regression retrieval of the turbulence amplitude based on correlation of atmospheric variable turbulence indicators (e.g., vertical velocity horizontal shear, temperature deviations from the mean, absorbing gas mixing ratio deviations from the mean, etc. and their EOF representations) and the radiance EOF amplitudes (i.e., coefficients of the EOF expansion) associated with the "observed" radiance spectrum observations. Spatial variations of the radiance EOF amplitudes (predicted temperature, absorbing gas, and vertical velocity EOF amplitudes) across the detector array should also be useful indicators of the existence of turbulence and its severity. As will be shown, the distance to the turbulent region can be diagnosed from the EOF amplitudes associated with the radiance spectra observed for a wide range of aircraft-to-turbulence-region distances.

Figure 10 shows the results of model simulations of FLI radiance spectra for the 6 March 2004 turbulence simulation observed at five different elevation angles, for a local azimuth angle of zero (i.e., the forward looking straight and level direction) and for five different azimuth directions for each of the five elevation angles considered. The azimuth angles of view are overlaid on a horizontal cross-section of vertical velocity near the assumed $8 \mathrm{~km}$ level of the FLI located at $39.5 \mathrm{~N}$ and $103 \mathrm{~W}$, at $18 \mathrm{UTC}$. The angles correspond to those shown in Figure 11. The top left plot is the mean radiance averaged over all azimuth angle $\alpha$ elements for the given elevation angle $\beta$. Continuing down the left-hand side and the right-hand column are plots of radiance deviation from the mean. As stated earlier, these initial calculations assumed a square detector array with a $250 \times 250$ mrad field of regard. The radiance spectra shown in Figure 10 correspond to the deviation of the radiance observed for each of twenty detector elements (i.e., the $5 \times 5$ sub-sampling of the $250 \times 250$ detector elements) considered (i.e., the central, half angle, and full angle elements) and the average radiance value observed for each elevation angle. The averaging of the radiance observed over all azimuth directions averages out all the turbulence signals, to produce a turbulence-free signal reference. Thus, the deviation signals shown in Figure 10 represent turbulent fluctuations of radiance for this atmospheric condition and assumed FLI viewing scenario. 

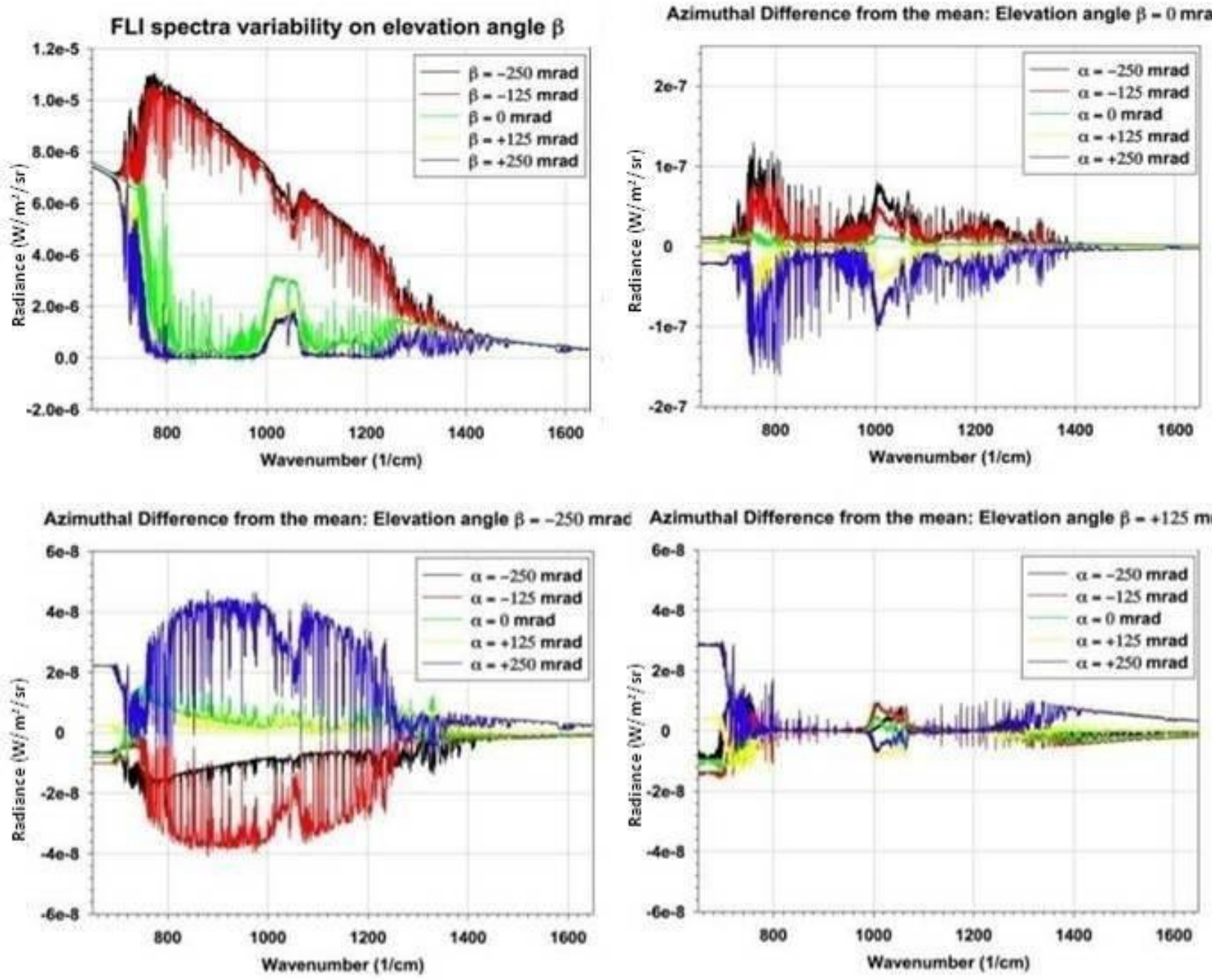

Azimuthal Difference from the mean: Elevation angle $\beta=-125$ mrad
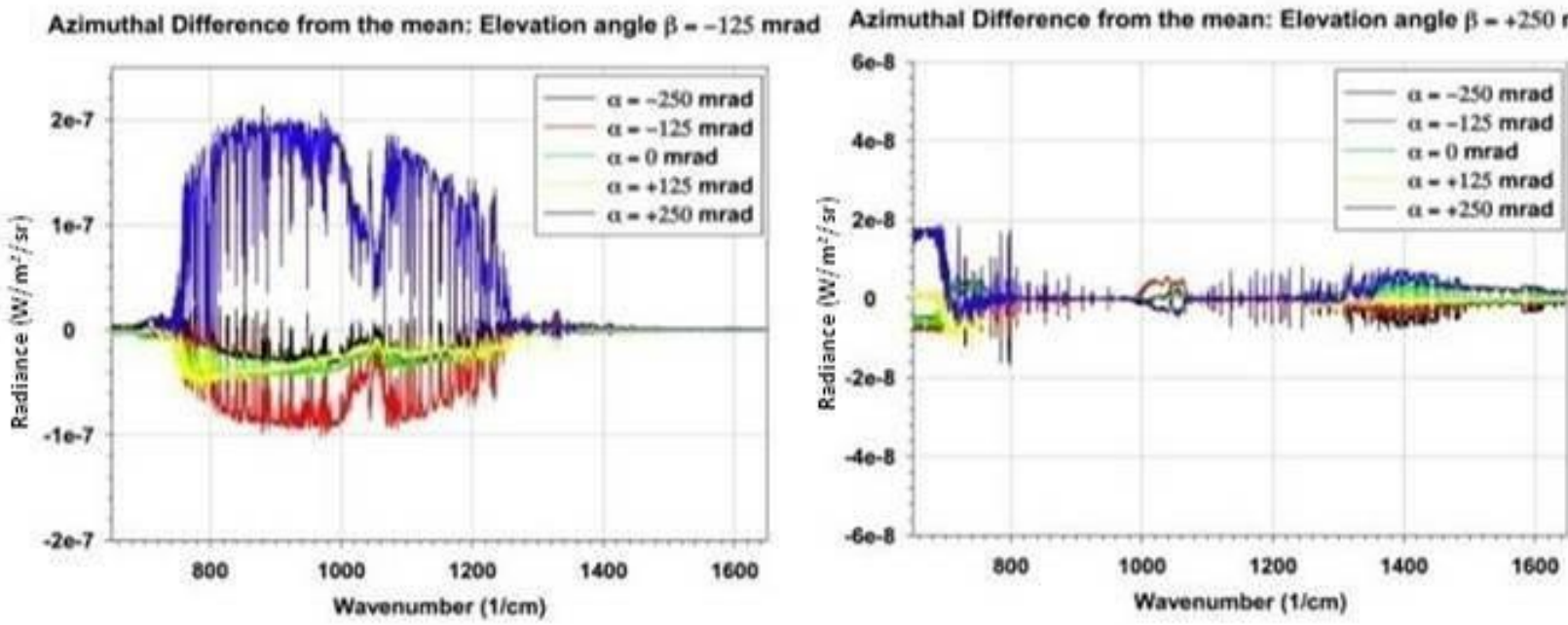

Figure 10. The radiance spectra computed for various azimuth and elevation angles. 
$400 \mathrm{mb}$ Vertical Velocity (m/s) 1800 UTC $03 / 06 / 04$
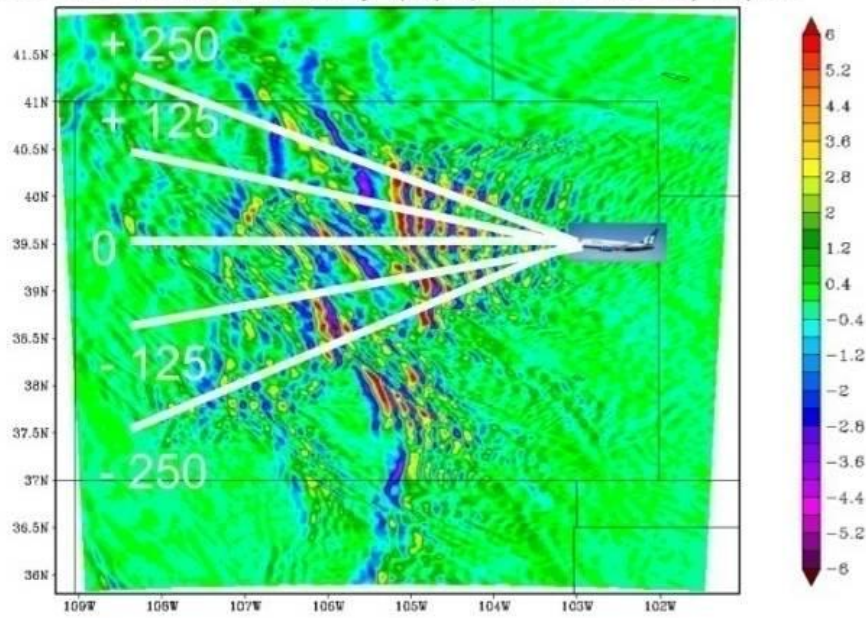

W-Q Cross-Section 39.5 N 1800 UTC 03/06/04

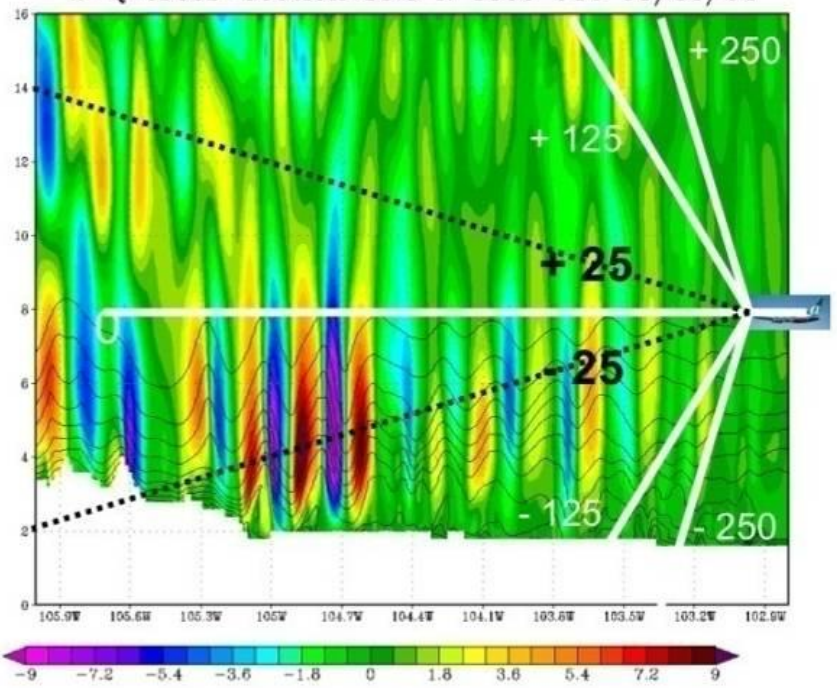

Figure 11. Viewing geometry for the radiance calculations shown in Figure 10.

It is clearly shown by these simulations that the elevation angle range of the FLI imaging array should be restricted to about $50 \mathrm{mrad}(+/-25 \mathrm{mrad})$, in order to optimize the FLI turbulence detection capability. Elevation angles larger than these become dominated by ground surface and upper atmosphere and space background radiance signals. As a result, the elevation angle range of the FLI imaging array was restricted to $50 \mathrm{mrad}(+/-25 \mathrm{mrad})$ for the conduct of the turbulence detection and retrieval studies performed during the latter part of this project and described below.

Thus, for the FLI radiance simulations whose results follow, the FLI instrument field of regard was considered to be $250 \times 50 \mathrm{mrad}$, with each detector element resolving a 1-mrad field of view (i.e., a 12,500 detector element imaging focal plane array). Radiance measurement simulations were then performed for a small, but representative, 125-detector element sampling (5 elevations x 25 azimuth angular positions) of the 12,500-element detector array. The FLI observations were performed for 8 viewing directions (as shown in Figure 12), 10 different distances to the "turbulence center" of $39.5^{\circ} \mathrm{N}, 105^{\circ} \mathrm{W}$ (as shown in Figure 13). There are twenty-five different detector positions simulated for each viewing direction and aircraft distance from the center of turbulence. As a result, the FLI imaging spectrometer ray trace radiative transfer model produced 10,000 radiance spectra to approximate what would be observed with a FLI imaging spectrometer. The spectral range considered was $750-1250 \mathrm{~cm}^{-1}$ with a spectral resolution of 0.5 $\mathrm{cm}^{-1}$ unapodized. 


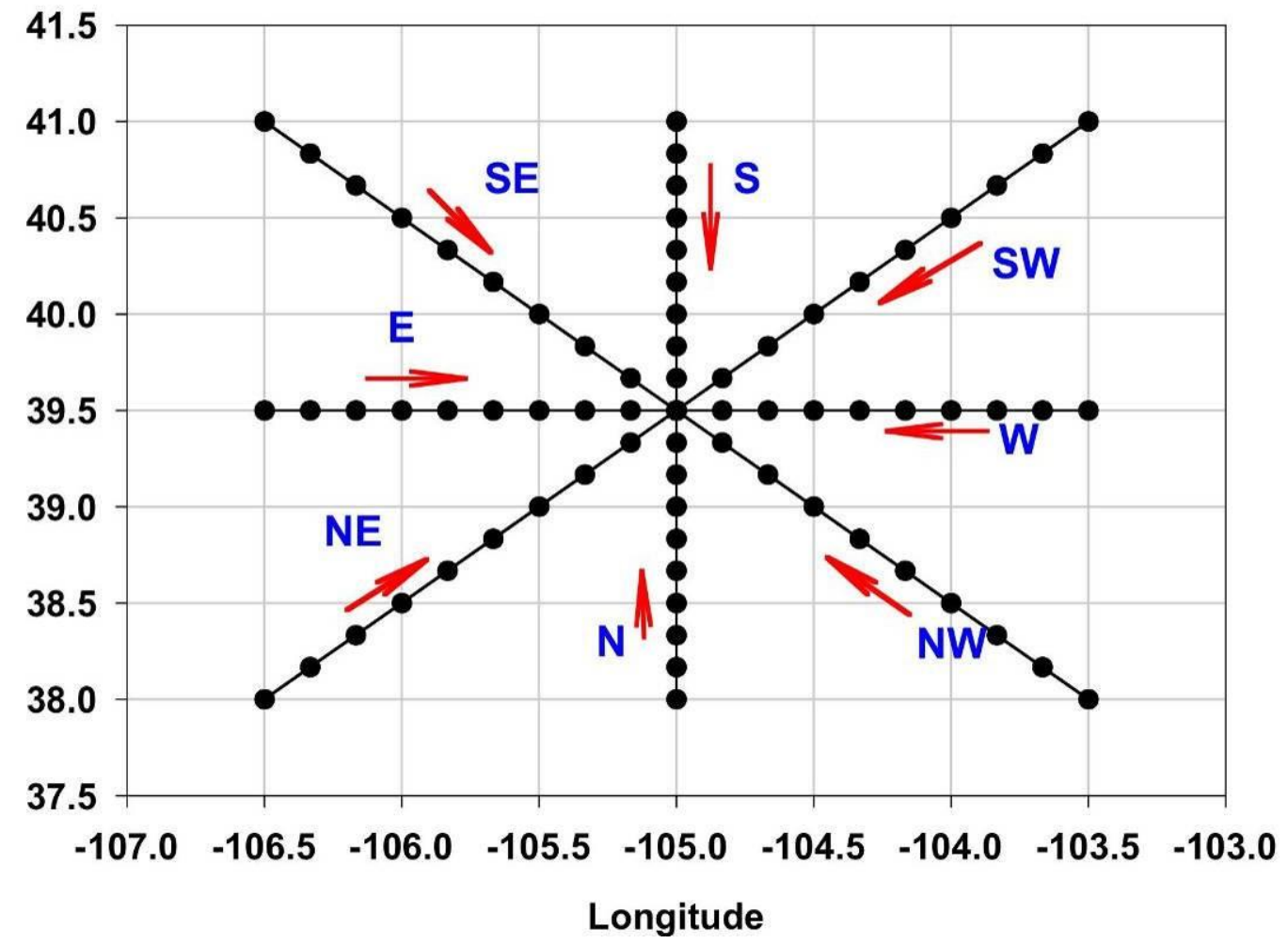

Figure 12. Viewing directions for the FLI measurement simulation

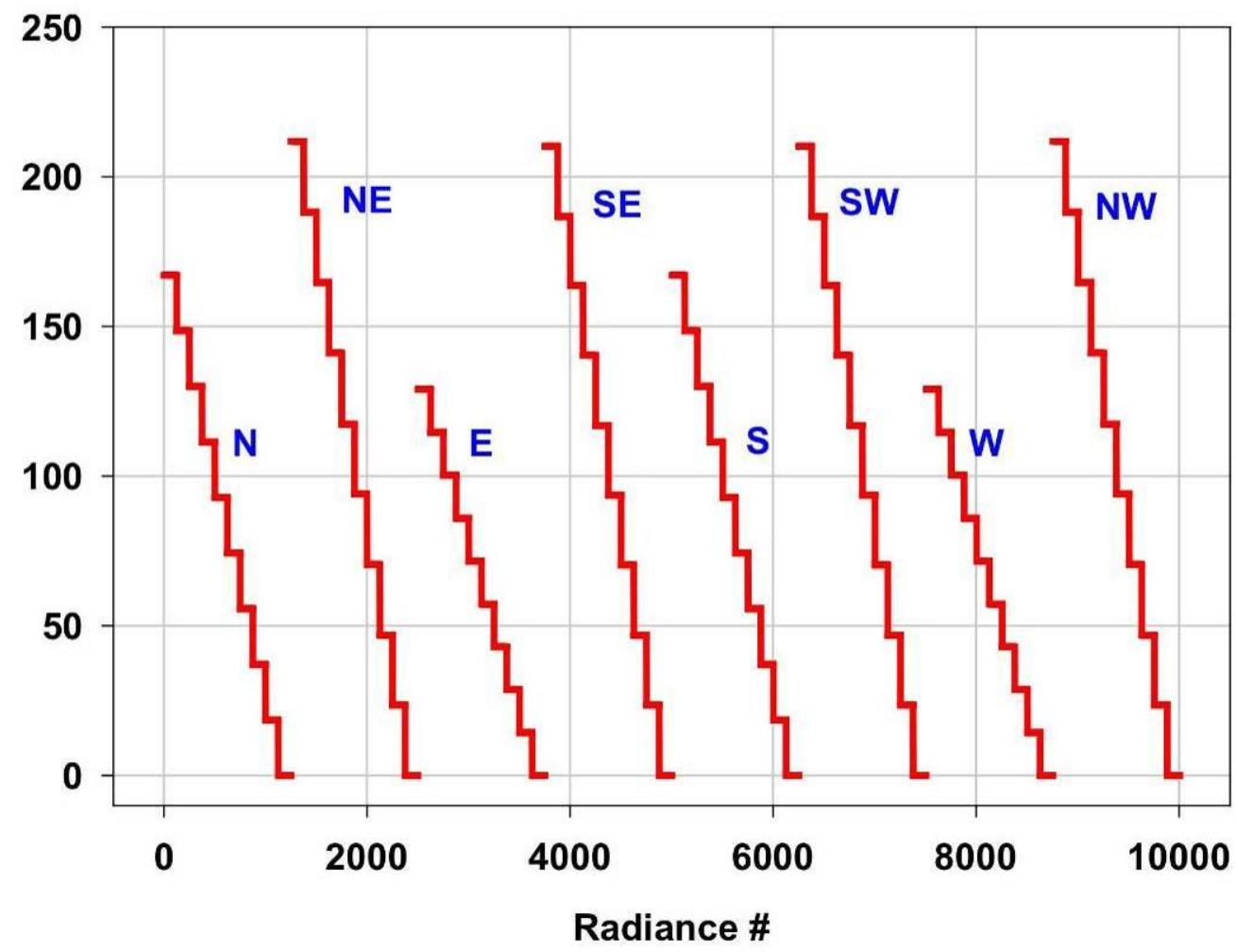

Figure 13. Distance from turbulence for each viewing directions. 


\subsubsection{FLI Multi-spectral Imagery}

FLI multi-spectral imagery was produced from the FLI simulated radiance spectra. Figure 14 shows brightness temperature imagery, for various spectral channels. The aircraft was at a distance of about $150 \mathrm{~km}$ to the center of turbulence. This information could be processed to produce a cockpit display in a commercial airliner. The figure includes three sets of viewing scenarios. The upper panels of each scenario show imagery of the absolute brightness temperature values observed by the detector array. These absolute brightness temperature images, shown in the upper panels, do not reveal the turbulence because the turbulent brightness temperature signal is very small relative to the strong variation of brightness temperature with elevation angle. As shown in the lower panels of each scenario, a display was generated showing the brightness temperature deviation from its azimuth mean value. This enables delineation of the turbulence signal imbedded within the brightness temperature measurements, for each elevation angle subtended by the imaging array. This difference processing eliminates the strong dependence of the brightness temperature features on elevation angle, therefore enhancing the small-scale variations of the brightness temperature signal. As can be seen, the turbulent signal is now very evident in the brightness temperature imagery. 
Westward Viewing Brightness Temperature $800.8 \mathrm{~cm}^{-1}$

$1219.2 \mathrm{~cm}^{-1}$
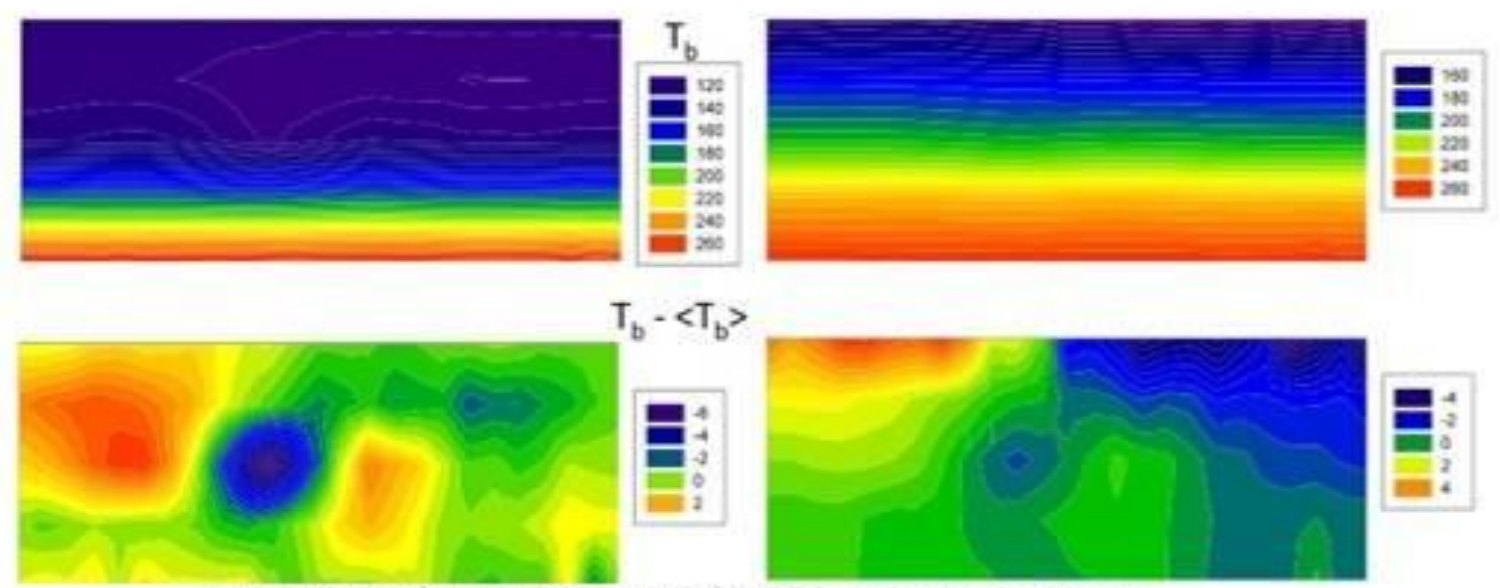

Westward Viewing Brightness Temperature $800.3 \mathrm{~cm}^{-1}$ $805.8 \mathrm{~cm}^{-1}$

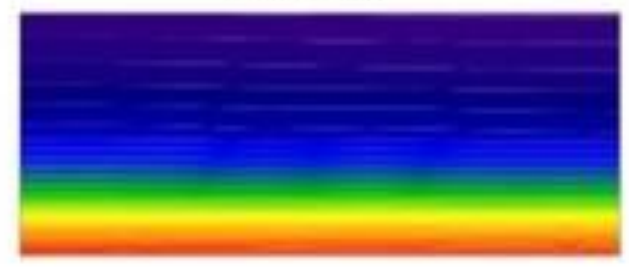
$T_{b}$
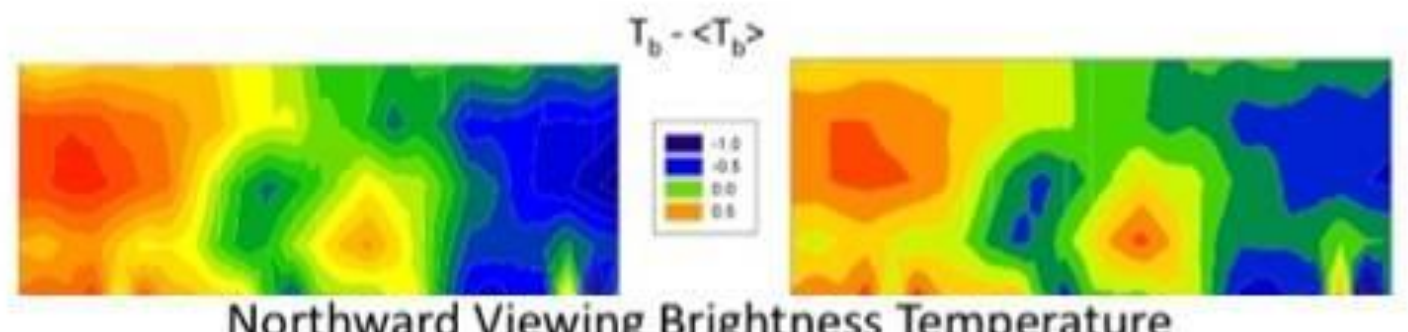

Northward Viewing Brightness Temperature
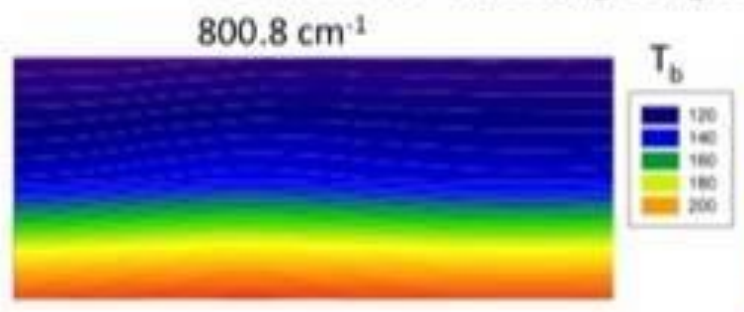

$1219.2 \mathrm{~cm}^{-1}$
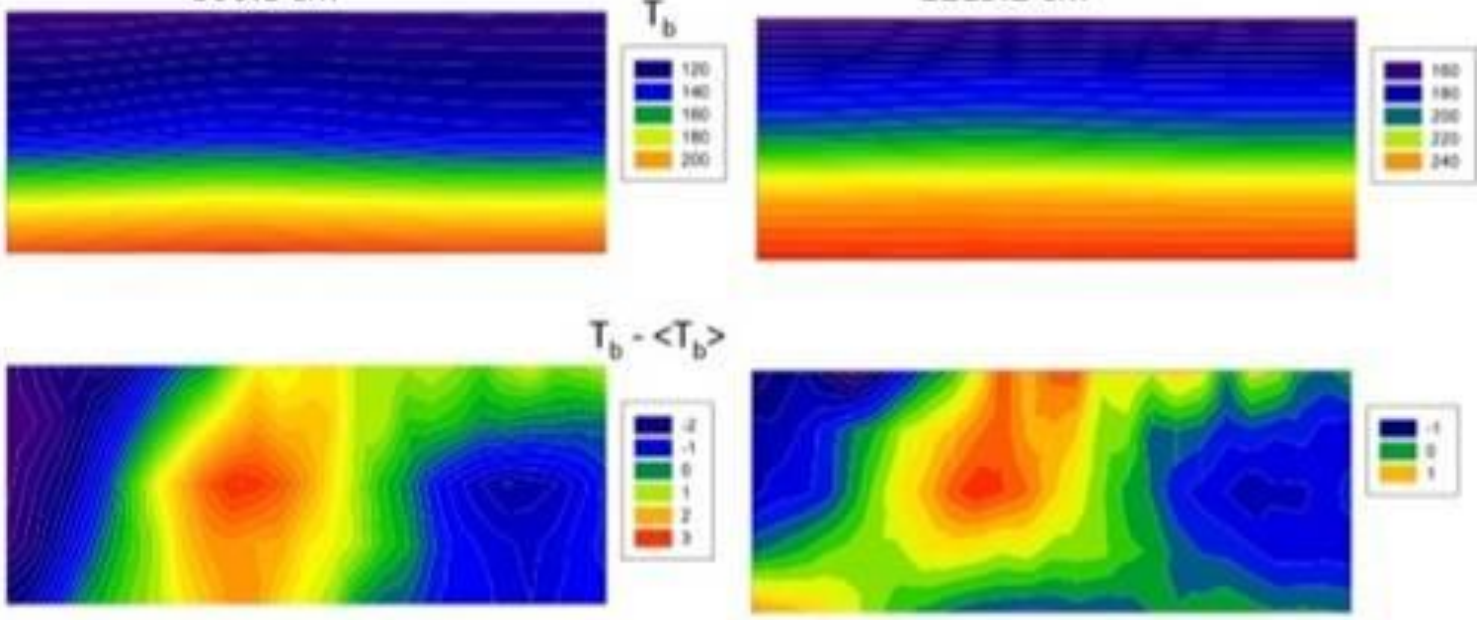

Figure 14. FLI imaging spectrometer brightness temperature imagery. 


\subsubsection{Empirical Orthogonal Function Analysis}

Empirical orthogonal functions were calculated for the 10,000 spectra without regard to viewing angle, and the direction and distance to the turbulence. Figure 15 shows the mean and standard deviation of the 10,000 radiance spectra while Figure 16 displays the eigenvalues (i.e., residual error variance) as a function of the EOF number used to represent these 10,000 different spectra. As can be seen from Figure 15, the highest degree of radiance variance is in the window regions of the spectra, where the turbulence signal is expected to be maximized. Figure 16 shows eigenvalues as a function of EOF number for all 10000 EOFs (left hand side) and for the first 100 EOFs (right hand side). As shown in the plots, the first 200 eigenvalues decrease relatively rapidly with a very slow, but consistent, decrease in EOF value beyond this point. It is believed that a major amount of the variance, as well as the rapid decrease in eigenvalues with EOF number, is due to the variation produced by the known measurement elevation angle, rather than due to the atmospheric signal. Thus, in order to use the coefficients of EOFs as predictors of turbulence, it was important to classify the data with respect to elevation angle in order to optimize the sensitivity of the EOF coefficients to the unknown turbulence parameters.

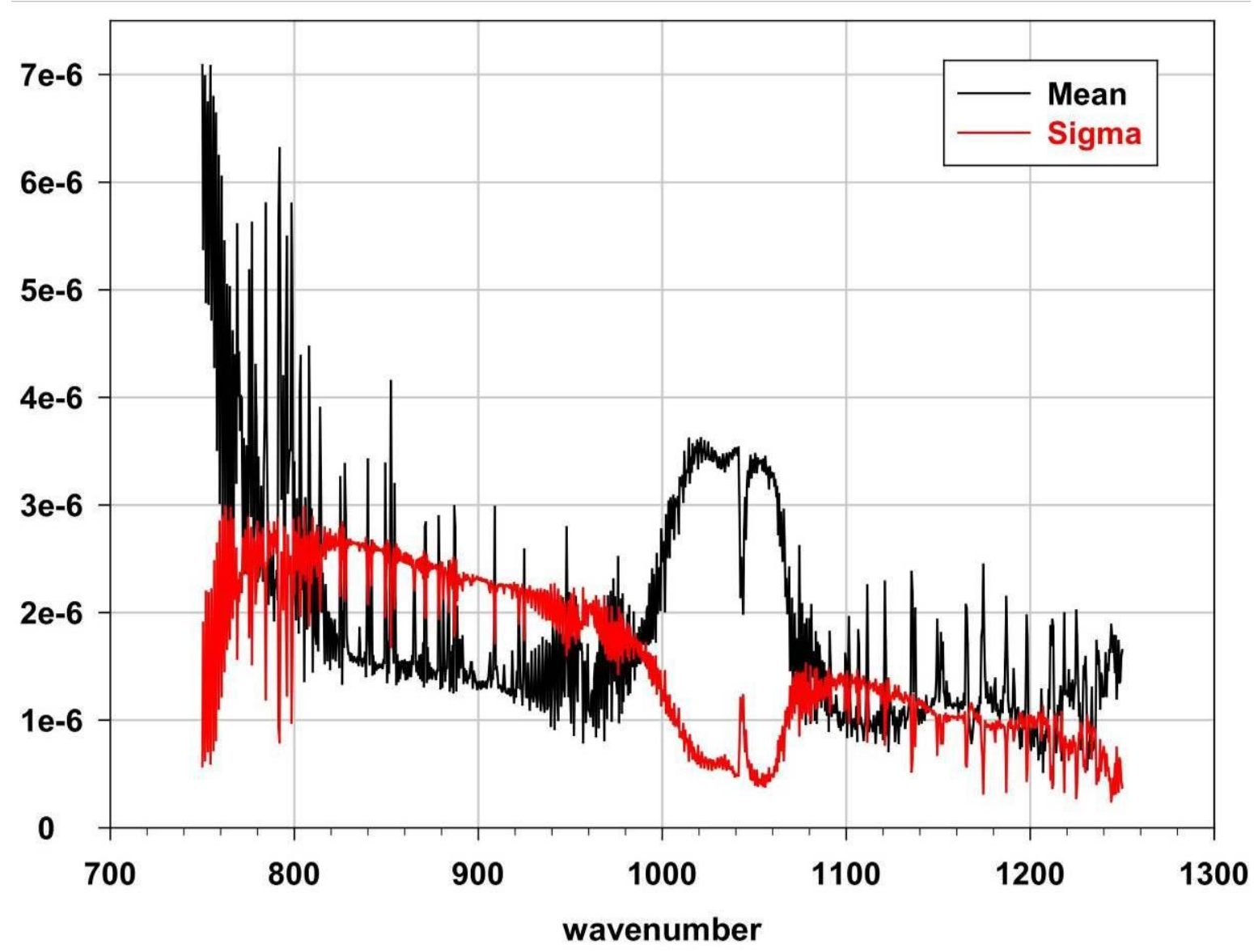

Figure 15. Mean and standard deviation of the 10,000 simulated FLI radiance spectra 

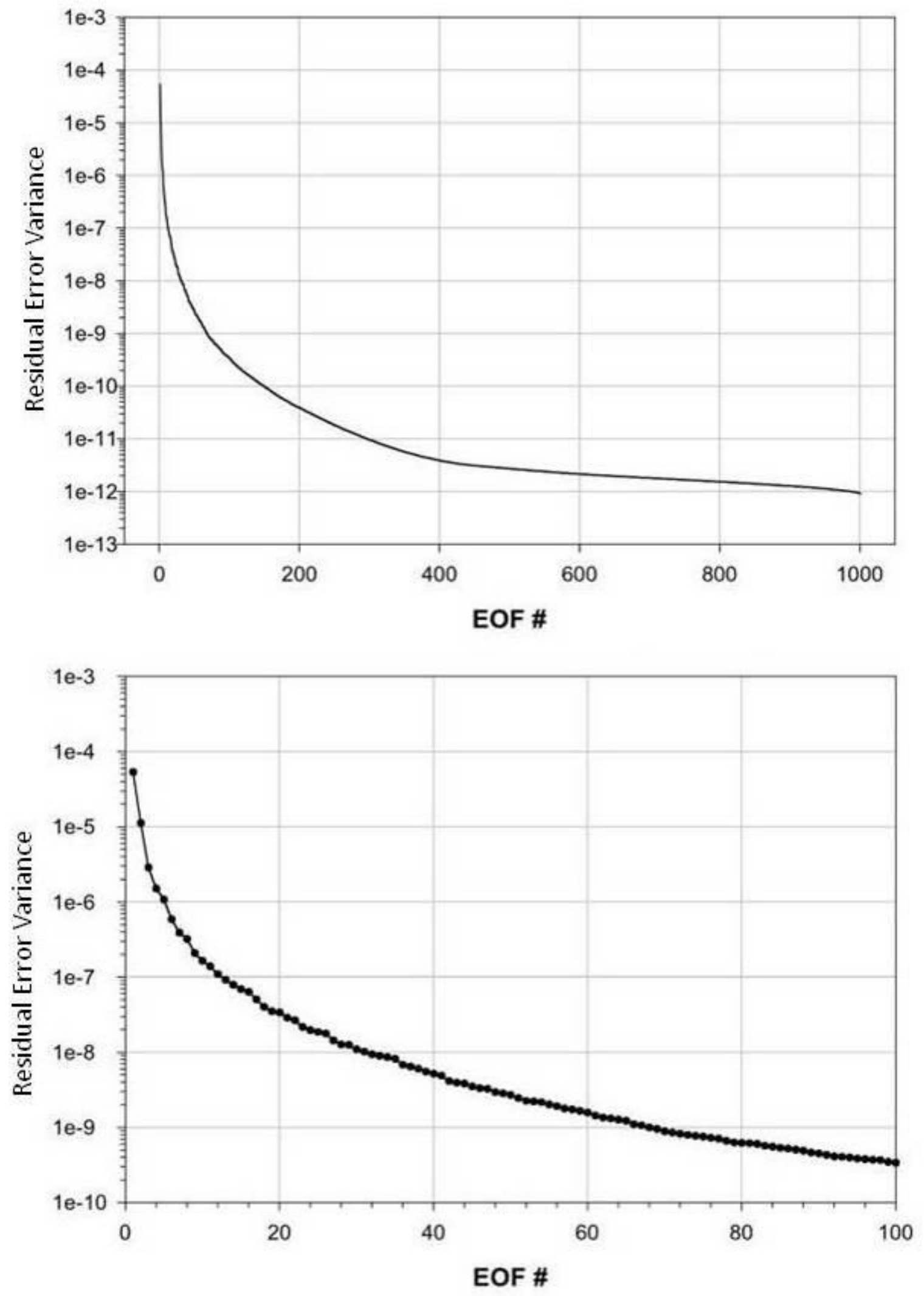

Figure 16. Eigenvalues as a function of EOF number. 
Thus, the following procedure was adopted to produce an EOF coefficient turbulence predictor model:

(a) Compute separate EOFs for each of the five different elevation angles. All the azimuth angles are grouped together but separate EOFs were computed for each different elevation angle of measurement.

(b) Further classify the spectra (to produce separate EOFS) for separate directions of the turbulence wave orientation.

Thus, the final result of performing three classifications ( $a$ and $b$ ) yields 40 different EOF classes for a given aircraft altitude (i.e., 5 elevations x 8 directions).

In summary, analyses of the empirical orthogonal functions have been produced for FLI observations from an aircraft at an 8-km aircraft altitude. The spectral range considered was $750-1250 \mathrm{~cm}^{-1}$, with a spectral resolution of $0.5 \mathrm{~cm}^{-1}$, unapodized. EOFs for 9,000 spectra associated with eight (8) viewing directions, ten (10) different distances to the "turbulence center"(i.e., $39.5^{\circ} \mathrm{N}, 105^{\circ} \mathrm{W}$ ) and five (5) elevations $x$ twenty-five (25) azimuth angular positions of the detector array were produced from the WARF simulated atmospheric pressure , temperature, and moisture data.

Figure 17 shows on the top plot the mean and on the bottom plot the standard deviation of the radiance of simulated FLI observations corresponding to observations from different distances to the turbulence. The eigenvalues for different elevation angles, corresponding to the unexplained variance, as a function of the number of eigenvectors used to represent the spectral radiance data set is shown in Figure 18. As can be seen by comparing Figure 18 with Figure 16, there is an order of magnitude, or more, reduction in the unexplained variance by classifying the EOFs with respect to viewing angle (see for example, the case where 150 EOFs are to be used for the representation). 

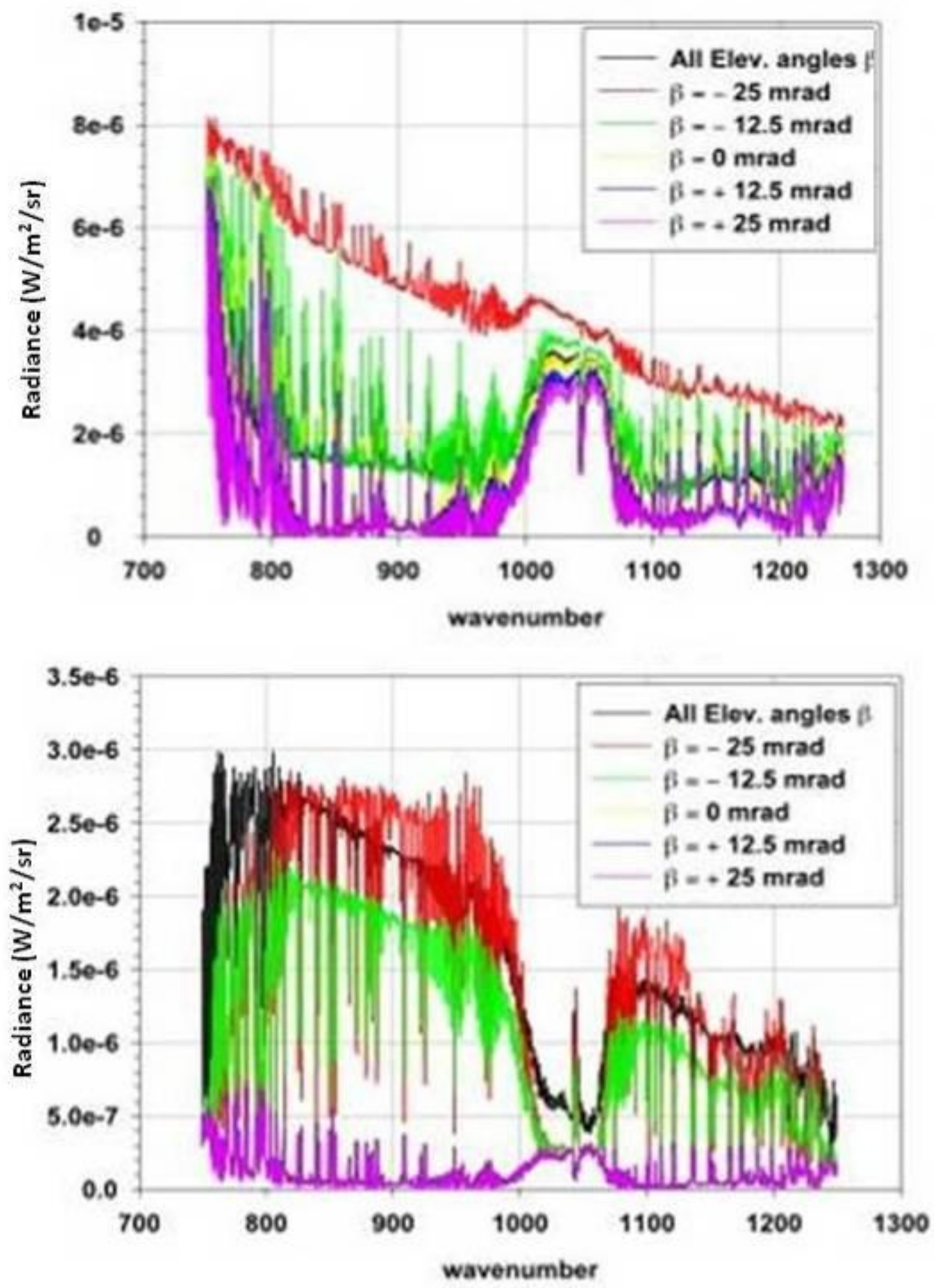

Figure 17. Mean and standard deviations of FLI radiance spectra. 


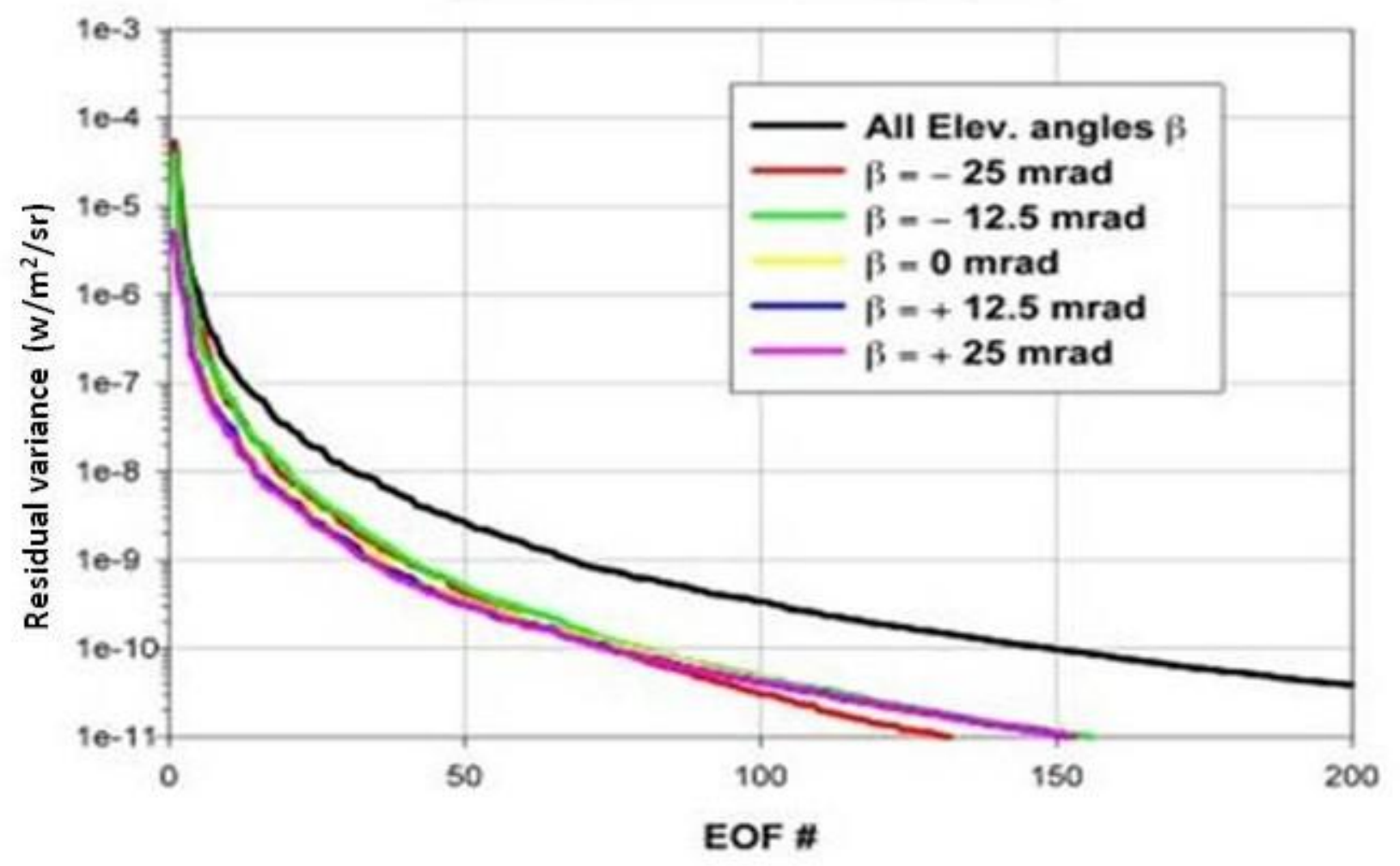

Figure 18. Residual variance of the radiance spectra.

In summary, an algorithm was developed for using the WRF model simulated FLI radiances to predict the aircraft distance to center of lee wave turbulence. The algorithm relates the distance to the center of turbulence to "observed" local variance of the EOF coefficients across a portion of the detector array. The local variance of the EOF amplitudes (i.e., sum of the square of the EOF amplitude deviations from the mean) was computed for different combinations of detector elements forming the $5 \times 25$ array (125 different geographical footprints). A regression equation was produced to predict the distance of the aircraft from the center of turbulence from a linear combination of the observed PC coefficient (EOF amplitude) variances. The regression equation was produced using the spectra for each of the eight aircraft flight directions.

Figure 19 shows the fractional explained variance (i.e., relative where $1.0=100 \%$ ) of Principal Component score (i.e., EOF coefficient) variation and distance from the aircraft as a function of PC (i.e., EOF) number. It can be seen that the best-correlated Principal Component number (i.e., EOF number) varies with the elevation angles of the detector elements used to compute the variance for the detection. The largest explained variances seem to be obtained when only the detector elements that see the turbulence (Elevation -1 and 0 ) are considered. 


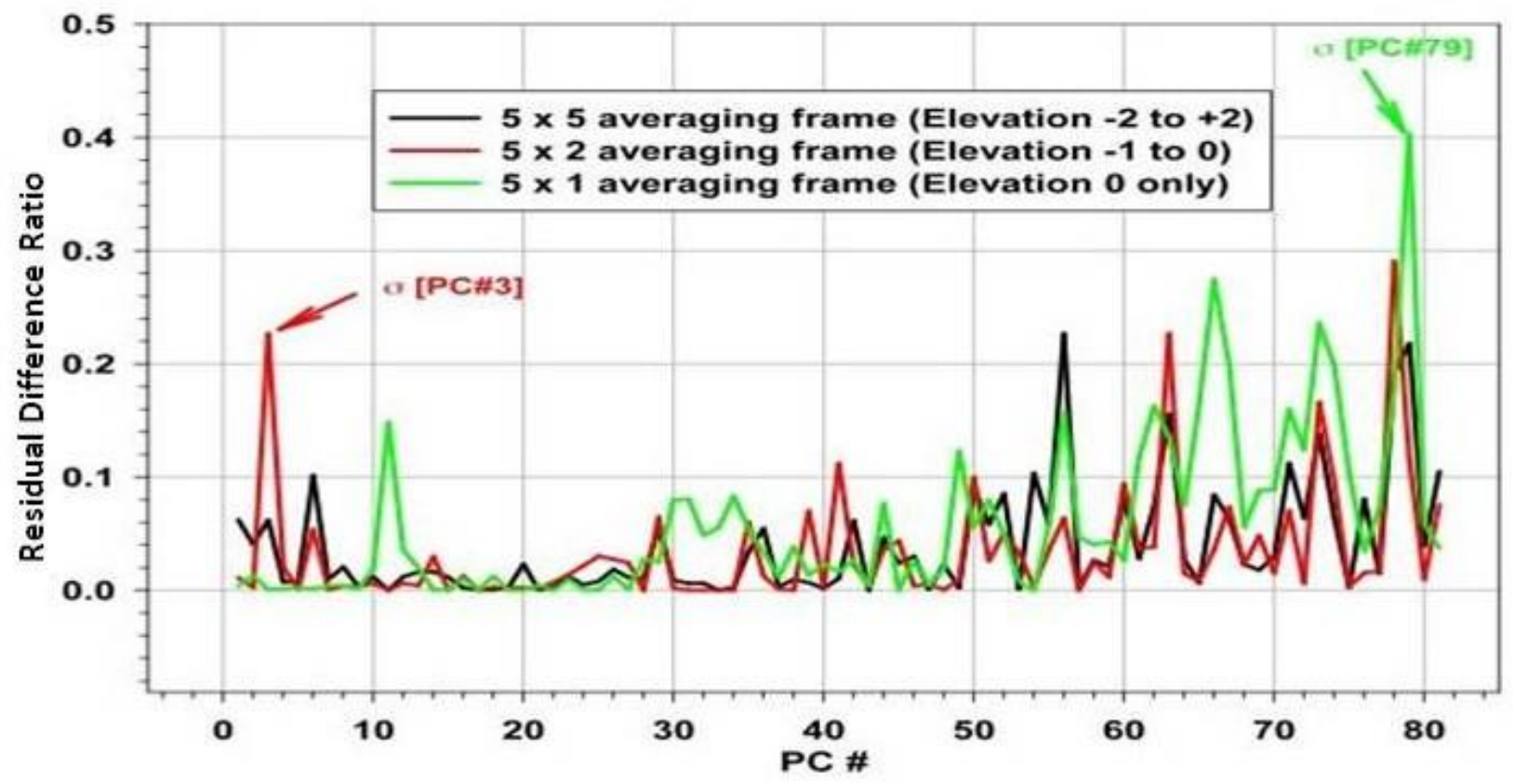

Figure 19. Fractional explained variance of distance to turbulence.

Further analyses of the simulated FLI results indicated that the correlations between the local variance of the radiances observed in individual spectral channels was more highly correlated with the distance from the turbulence center than was the correlation with individual PC scores. Figure 20 below shows the correlation between the relative radiance (i.e., deviation from azimuth mean) and the distance from the center of turbulence for $125 \times 2$ (elevation angles 0 and-1) portion of the imaging frame of FLI radiance data. As can be seen, there are both highly positive and highly negative correlations between the distance to turbulence and the local variance of relative radiance for specific spectral channels, depending on the direction of flight. This reversal in correlation sign must be related to the orientation of the lee wave train, relative to radiance image scene, which varies with flight direction. As a consequence of this result it was decided to produce a new linear regression algorithm that related the distance to turbulence to two different predictors, the sum of local variances of relative radiance for the highly positively correlated channels and the sum of local variances of relative radiance for the highly negatively correlated channels.

Figure 21 shows the result of the application of this regression in terms of a plot showing the relationship between the observed (true) and predicted distance from the turbulence associated with regression equations generated using the spectral averages of relative radiance variance for the highly positively and highly negatively correlated radiance channels as predictors. It can be seen, that there is excellent correspondence between the observed and predicted distance to turbulence values. However, as shown in Figure 20, these relationships are highly dependent on the direction of flight relative to the turbulence; thus, there is no universal relationship for all possible flight directions. Fortunately, in the case of Lee wave turbulence, the relative direction of flight is known from the aircraft heading and the known geographical orientation of the mountains. 


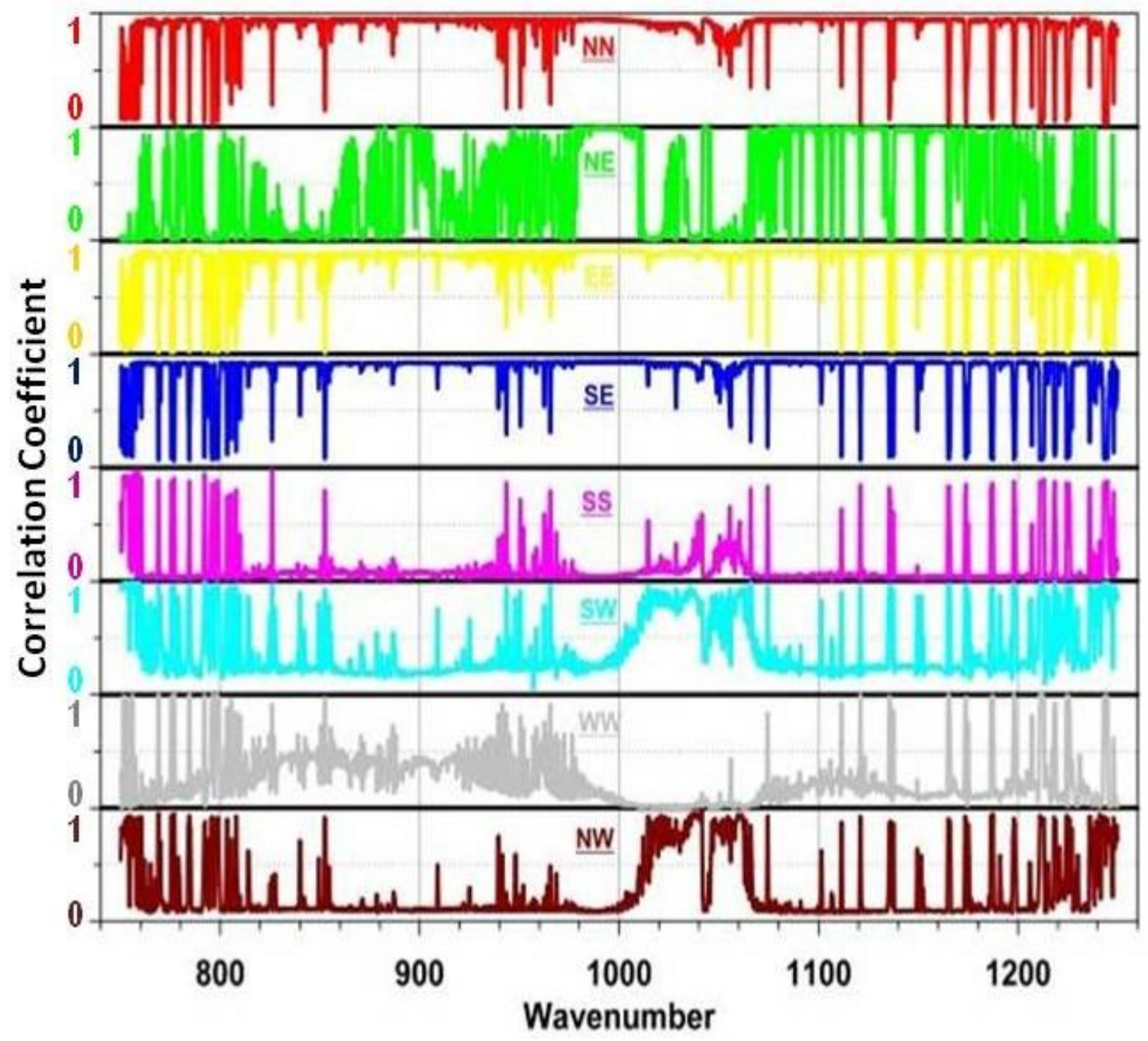

Figure 20. Correlation between the local variance and distance. 


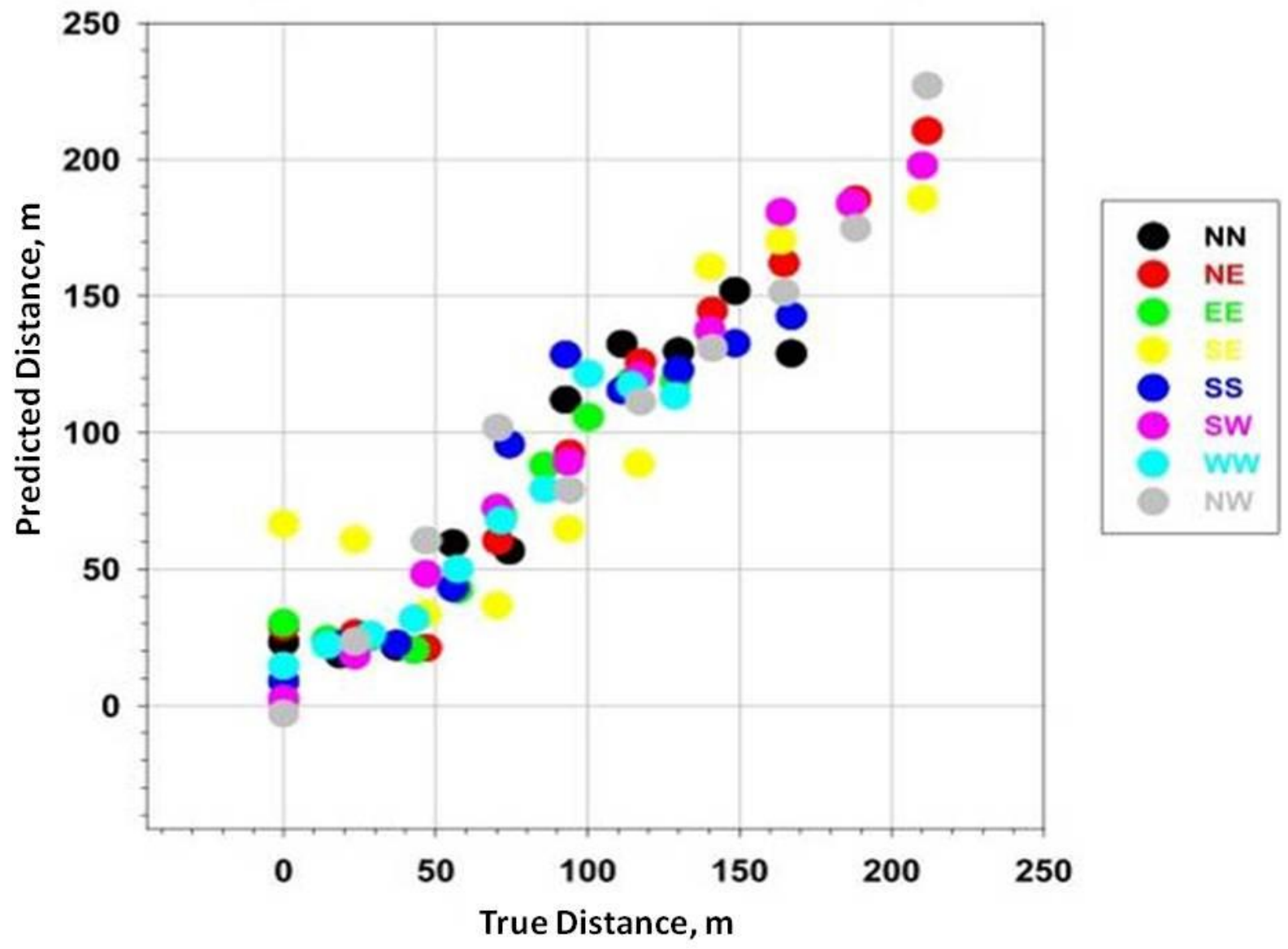

Figure 21. Mountain lee turbulence comparison.

In summary, it is concluded that an aircraft-mounted FLI could be used to detect lee wave turbulence when the aircraft is at a considerable distance from the aircraft. The distance from the aircraft is highly correlated with the local variance of the radiance for certain individual spectral channels, and the averages of the highly positively correlated and the highly negatively correlated radiances can be used to predict the distance to the turbulence center, given the direction of flight relative to the lee wave front. The use of the FLI brightness temperature imagery to quantify the severity of the turbulence detected remains to be investigated.

\subsection{Correlation of Atmospheric Parameters with Radiometric Signals}

In this section we discuss the theoretical aspects regarding the detection of turbulence with a forward-looking passive IR interferometer. In a previous work, a development was given that had some subtle errors, as well as analyzing a more complicated problem (West 2008). That problem was to compute the correlation function of the radiance field as a function of aircraft motion. In that analysis, it is assumed that all of the pertinent quantities (temperature and absorption coefficient), contained a mean and a fluctuating part. Furthermore, assuming that the absorption coefficient and the temperature field were correlated created a rather intractable problem. In this work, a simpler model is assumed, and this allows us to calculate the radiance correlation function in terms of the temperature correlation function. The assumptions in this model include: constant absorption coefficient in space; no correlation between the absorption 
coefficient and the temperature field; a linear approximation to the Planck function; and a known form for the temperature correlation function.

Following on the methods used in airborne radar turbulence detection algorithms, the concept is to derive equations that relate the statistics of the atmospheric turbulence (e.g., temperature field) to those of the sensor measurements (e.g., the radiances). Two approaches can be considered: (1) the turbulent temperature field is homogeneous and extends along the whole measurement path, and (2) the temperature field is laminar, excepting for a "patch" of turbulence at a certain distance from the sensor. In this work, the first method it utilized.

Start with the one-dimensional Radiative Transfer Equation (RTE) for a gas in local thermal equilibrium (LTE), with negligible scattering, and where the measurement is made along the direction $x$ :

$$
\frac{d I_{v}(x)}{d x}=\alpha_{v}(x)\left[I_{v}(x)-B_{v}(x)\right]
$$

The subscript $v$ is to indicate the wavenumber dependence on these quantities. These subscripts will be dropped, excepting for where their explicit functionality is important. $I(x)$ is the radiance at the position $x, B_{v}(T(x))$ is the Planck function as a function of the temperature at the position $x$, and $\alpha_{v}(x)$ is the absorption coefficient.

We are using a different coordinate system than that which is standard in the literature; hence, a few explanatory words are required. In the literature, it is assumed that the coordinate system is positive from the source to the receiving point, call this coordinate $s$. If we visualize this with the source to the right and the receiving point to the left, as the radiation propagates through a cylinder of width $d s$, the radiance at the point $s$ is decreased by absorption going through the cylinder. (Recall that we are assuming negligible scattering.) That is, from the point $s$, we are adding a cylinder that goes from $s$ to $s+d s$. Thus the radiance decreases such that $d I(s)=-\alpha(s) I(s) d s$. Now consider the emission of radiance from the cylinder. In LTE, this increase in radiance is given by, $d I(s)=+\alpha(s) B(s) d s$, and hence the RTE is given in the standard form in the literature.

$$
\frac{d I(s)}{d s}=\alpha(s)[B(s)-I(s)]
$$

Note that the sign is reversed from Eq. (1). In our case, the source and receiver are in the same locations, but we choose a coordinate system where the positive sense goes from receiver to transmitter, $d x=-d s$. The reason for this choice is that we are considering an aircraft moving towards the source, and hence want the coordinate system to be positive in that sense. In this case, we are removing a cylinder as we move from $x$ to $x+d x$, and hence there is less radiation from emission and more from the lessened amount of absorption - thus the change in signs.

Eq. (1) is a linear first order differential equation of the form, 


$$
\frac{d y}{d x}+a(x) y(x)=b(x)
$$

With initial condition $y=y\left(x_{0}\right)$. This equation has the solution,

$$
y(x)=\exp \left[-\int_{x_{0}}^{x} a\left(x^{\prime}\right) d x^{\prime}\right]\left\{y\left(x_{0}\right)+\int_{x_{0}}^{x} b\left(x^{\prime}\right) \exp -\left[\int_{x_{0}}^{x^{\prime}} a\left(x^{\prime \prime}\right) d x^{\prime \prime}\right] d x^{\prime}\right\}
$$

Note that in this form, $x_{0} \leq x^{\prime} \leq x$. For our problem, however, we would like to have $x_{0}$ be at the source, in which case, $x \leq x^{\prime} \leq x_{0}$. In this case, we must change the sign of the integrals, e.g.,

$$
\int_{x_{0}}^{x} a\left(x^{\prime}\right) d x^{\prime} \rightarrow-\int_{x}^{x_{0}} a\left(x^{\prime}\right) d x^{\prime}
$$

Thus, Eq. (3) becomes,

$$
y(x)=\exp \left[\int_{x}^{x_{0}} a\left(x^{\prime}\right) d x^{\prime}\right]\left\{y\left(x_{0}\right)-\int_{x}^{x_{0}} b\left(x^{\prime}\right) \exp \left[\int_{x^{\prime}}^{x_{0}} a\left(x^{\prime \prime}\right) d x^{\prime \prime}\right] d x^{\prime}\right\}
$$

Referring to Eq.(1), we let $y(x) \rightarrow I(x), a(x) \rightarrow-\alpha(x), b(x) \rightarrow-\alpha(x) B(x), \quad x_{0} \rightarrow L$, and $y\left(x_{0}\right) \rightarrow I(L)$. Eq. (3) becomes,

$$
I(x)=\exp \left[-\int_{x}^{L} \alpha\left(x^{\prime}\right) d x^{\prime}\right]\left\{I(L)+\int_{x}^{L} \alpha\left(x^{\prime}\right) B\left(x^{\prime}\right) \exp \left[\int_{x^{\prime}}^{L} \alpha\left(x^{\prime \prime}\right) d x^{\prime \prime}\right] d x^{\prime}\right\}
$$

Consider, $\int_{x^{\prime}}^{L} \alpha\left(x^{\prime \prime}\right) d x^{\prime \prime}$. Since $x \leq x^{\prime} \leq L, \int_{x^{\prime}}^{L} \alpha\left(x^{\prime \prime}\right) d x^{\prime \prime}=\int_{x}^{L} \alpha\left(x^{\prime \prime}\right) d x^{\prime \prime}-\int_{x}^{x^{\prime}} \alpha\left(x^{\prime \prime}\right) d x^{\prime \prime}$. Since the first integral on the RHS is independent of $x^{\prime}$, it can be taken outside the integral over $x^{\prime}$. This gives,

$$
I(x)=\exp \left[-\int_{x}^{L} \alpha\left(x^{\prime}\right) d x^{\prime}\right] I(L)+\int_{x}^{L} \alpha\left(x^{\prime}\right) B\left(x^{\prime}\right) \exp \left[-\int_{x}^{x^{\prime}} \alpha\left(x^{\prime \prime}\right) d x^{\prime \prime}\right] d x^{\prime}
$$

This the full form of the RTE, which, excepting for the use of a different coordinate system is a standard result in the literature. Next, consider the radiance at a point $x+\rho$, i.e., the aircraft has moved a positive distance $\rho$ towards the source. 


$$
I(x+\rho)=\exp \left[-\int_{x+\rho}^{L} \alpha\left(x^{\prime}\right) d x^{\prime}\right] I(L)+\int_{x+\rho}^{L} \alpha\left(x^{\prime}\right) B\left(x^{\prime}\right) \exp \left[-\int_{x+\rho}^{x^{\prime}} \alpha\left(x^{\prime \prime}\right) d x^{\prime \prime}\right] d x^{\prime}
$$

The correlation function of the radiances is given by,

$$
R_{I}(x, \rho)=\langle I(x) I(x+\rho)\rangle
$$

As pointed out in the earlier work, this is a very intractable problem in general. However, with the simplifications mentioned above, we can develop an equation of the correlation function of the radiances that is tractable. The accuracy of the results given these assumptions is beyond the scope of this work.

First consider constancy of the absorption coefficient in space. This allows Eq. (4) to be written as,

$$
I(x)=I(L) \mathrm{e}^{-\alpha(L-x)}+\alpha e^{\alpha x} \int_{x}^{L} B\left(x^{\prime}\right) e^{-\alpha x^{\prime}} d x^{\prime}
$$

If we further assume that $I(L)=0$, i.e., that there are no fluctuating sources for $x>L$. Any radiation from a laminar source is assumed to have been removed, hence the assumption is valid. So, we are left with a fairly simple equation,

$$
I(x)=\alpha e^{\alpha x} \int_{x}^{L} B\left(x^{\prime}\right) e^{-\alpha x^{\prime}} d x^{\prime}
$$

Next, we compute the similar form for Eq. (5), multiply and take the expected value; hence,

$$
R_{I}(x, \rho)=\langle I(x) I(x+\rho)\rangle=\alpha^{2} e^{\alpha(2 x+\rho)} \int_{x}^{L} \int_{x+\rho}^{L}\left\langle B\left(x^{\prime}\right) B\left(x^{\prime \prime}\right)\right\rangle e^{-\alpha\left(x^{\prime}+x^{\prime \prime}\right)} d x^{\prime \prime} d x^{\prime}
$$

Where we have used the fact that the Planck function (e.g., the temperature field) is uncorrelated with the constant absorption coefficient. Next, we choose a linear model for the Planck function, 


$$
B_{v}(T(x))=\frac{k_{1}}{k_{2}} v^{2} T(x)
$$

(See the following section.) Plugging this into Eq. (9) gives,

$$
R_{I}(x, \rho)=\alpha^{2} \mathrm{C}_{\nu}^{2} e^{\alpha(2 x+\rho)} \int_{x}^{L} \int_{x+\rho}^{L}\left\langle T\left(x^{\prime}\right) T\left(x^{\prime \prime}\right)\right\rangle e^{-\alpha\left(x^{\prime}+x^{\prime \prime}\right)} d x^{\prime \prime} d x^{\prime}
$$

where $\mathrm{C}_{v}^{2}=\left(\frac{k_{1}}{k_{2}} v^{2}\right)^{2}$. If it is assumed that the temperature fluctuations are homogeneous, then $R_{T}\left(x^{\prime}, x^{\prime \prime}\right)=\left\langle T\left(x^{\prime}\right) T\left(x^{\prime \prime}\right)\right\rangle=R_{T}\left(x^{\prime \prime}-x^{\prime}\right)$. It can be seen that Eq. (11) is a function of $\eta=x^{\prime \prime}-x^{\prime}$ and $\mu=x^{\prime \prime}+x^{\prime}$. This leads us naturally to this change of variables in the double integral. From Calculus, we know that the change of variable formula is given by,

$$
\iint f\left(x^{\prime}, x^{\prime \prime}\right) d x^{\prime \prime} d x^{\prime}=\iint f(\eta, \mu)|J(\eta, \mu)| d \mu d \eta
$$

Where $f(\eta, \mu)=R_{T}(\eta) e^{-\alpha \mu}$, and the Jacobian of the transformation is given by,

$$
J(\eta, \mu)=\left|\begin{array}{ll}
\frac{\partial x^{\prime}}{\partial \eta} & \frac{\partial x^{\prime}}{\partial \mu} \\
\frac{\partial x^{\prime \prime}}{\partial \eta} & \frac{\partial x^{\prime \prime}}{\partial \mu}
\end{array}\right|=\left|\begin{array}{cc}
-1 / 2 & 1 / 2 \\
1 / 2 & 1 / 2
\end{array}\right|=-\frac{1}{2}
$$

and hence, $|J(\eta, \mu)|=1 / 2$. Notice that this transformation has separated the integrand into two independent parts, and also where the integral over $\mu$ will be especially easy. Special care must be taken in determining the new limits of integration. In the left side of Figure 22, the $\left(x^{\prime}, x^{\prime \prime}\right)$ space shows the integration region as a rectangle. 


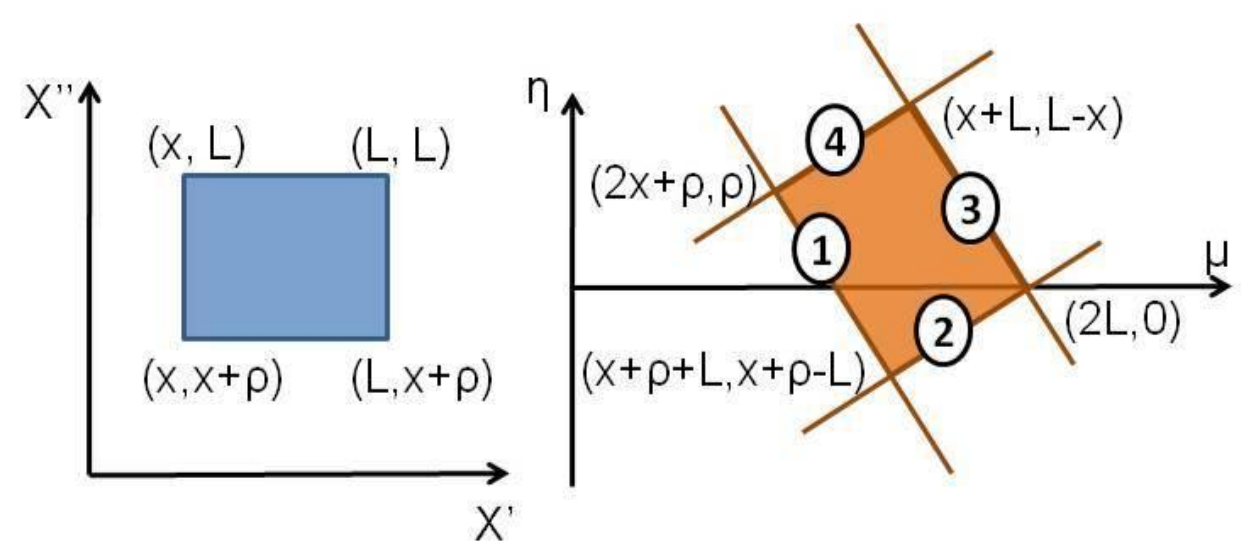

Figure 22. Comparison of regions for integration.

Whereas, the right side of Figure 22 shows the $(\mu, \eta)$ space with the region of integration as a parallelogram and the mapping of the corners in $\left(x^{\prime}, x^{\prime \prime}\right)$ space to those in the $(\mu, \eta)$ space is given by, $\left(x^{\prime}, x^{\prime \prime}\right) \rightarrow(\mu, \eta)=\left(x^{\prime \prime}+x^{\prime}, x^{\prime \prime}-x^{\prime}\right)$. Assume that for $\mu: 2 x+\rho<x+L \leq x+\rho+L \leq 2 L$, and for $\eta: x+\rho-L<0 \leq \rho<L-x$. Note that this region is bounded by the four numbered lines. Holding $\eta$ fixed, and integrating over $\mu$, there are three distinct regions: (1) $x+\rho-L<\eta<0$, (2) $0 \leq \eta \leq \rho$, and (3) $\rho<\eta<L-x$. In region $1, \mu$ varies between line 1 and line 2 (increasing $\mu$ ); in region 2, $\mu$ varies between lines 1 and 3; and finally, in region 3, $\mu$ varies between lines 4 and 3 . The equations of the four lines must be computed first and then solved for $\mu$ to get the new integration limits. The two-point equation for a line is given by,

$$
\eta=\frac{\eta_{2}-\eta_{1}}{\mu_{2}-\mu_{1}}\left(\mu-\mu_{1}\right)+\eta_{1}
$$

For line $1, \mu=2 x+2 \rho-\eta$; for line $2, \mu=\eta+2 L$; for line $3, \mu=2 L-\eta$; and for line 4 , $\mu=\eta+2 x$.

Returning to Eq. (12), we have the following integrals over the three regions. For region 1, $x+\rho-L<\eta<0$,

$$
\int_{\rho+x-L}^{0} \int_{2 x+2 \rho-\eta}^{\eta+2 L} R_{T}(\eta) e^{-\alpha \mu} d \mu d \eta
$$

The integral over $\mu$ is,

$$
\int_{2 x+2 \rho-\eta}^{\eta+2 L} e^{-\alpha \mu} d \mu=\frac{-1}{\alpha}\left[e^{-\alpha(2 L+\eta)}-e^{-\alpha(2 x+2 \rho-\eta)}\right]=\frac{-1}{\alpha}\left[e^{-\alpha 2 L} e^{-\alpha \eta}-e^{-\alpha(2 x+2 \rho)} e^{\alpha \eta}\right]
$$


For region $2,0 \leq \eta \leq \rho$,

$$
\int_{0}^{\rho} \int_{2 x+2 \rho-\eta}^{2 L-\eta} R_{T}(\eta) e^{-\alpha \mu} d \mu d \eta
$$

The integral over $\mu$ is,

$$
\int_{2 x+2 \rho-\eta}^{2 L-\eta} e^{-\alpha \mu} d \mu=\frac{-1}{\alpha}\left[e^{-\alpha(2 L-\eta)}-e^{-\alpha(2 x+2 \rho-\eta)}\right]=\frac{-1}{\alpha}\left[e^{-\alpha 2 L} e^{\alpha \eta}-e^{-\alpha(2 x+2 \rho)} e^{\alpha \eta}\right]
$$

And for region 3, $\rho<\eta<L-x$,

$$
\int_{\rho}^{L-x} \int_{\eta+2 x}^{2 L-\eta} R_{T}(\eta) e^{-\alpha \mu} d \mu d \eta
$$

The integral over $\mu$ is,

$$
\int_{\eta+2 x}^{2 L-\eta} e^{-\alpha \mu} d \mu=\frac{-1}{\alpha}\left[e^{-\alpha(2 L-\eta)}-e^{-\alpha(\eta+2 x)}\right]=\frac{-1}{\alpha}\left[e^{-\alpha 2 L} e^{\alpha \eta}-e^{-\alpha 2 x} e^{-\alpha \eta}\right]
$$

Combining all of these results with Eq. (11), gives:

$$
\begin{aligned}
R_{I}(x, \rho)=\frac{-\alpha}{2} \mathrm{C}_{v}^{2} e^{\alpha(2 x+\rho)} & \left\{\left[e^{-\alpha 2 L} \int_{\rho+x-L}^{0} R_{T}(\eta) e^{-\alpha \eta} d \eta-e^{-\alpha(2 x+2 \rho)} \int_{\rho+x-L}^{0} R_{T}(\eta) e^{\alpha \eta} d \eta\right]\right. \\
& +\left[e^{-\alpha 2 L} \int_{0}^{\rho} R_{T}(\eta) e^{\alpha \eta} d \eta-e^{-\alpha(2 x+2 \rho)} \int_{0}^{\rho} R_{T}(\eta) e^{\alpha \eta} d \eta\right] \\
& \left.+\left[e^{-\alpha 2 L} \int_{\rho}^{L-x} R_{T}(\eta) e^{\alpha \eta} d \eta-e^{-\alpha 2 x} \int_{\rho}^{L-x} R_{T}(\eta) e^{-\alpha \eta} d \eta\right]\right\}
\end{aligned}
$$


Multiplying through by $e^{\alpha(2 x+\rho)}$ gives,

$$
\begin{aligned}
R_{I}(x, \rho)=\frac{-\alpha}{2} \mathrm{C}_{v}^{2}\left\{\left[e^{-\alpha(2 L-2 x-\rho)} \int_{\rho+x-L}^{0} R_{T}(\eta) e^{-\alpha \eta} d \eta-e^{-\alpha \rho} \int_{\rho+x-L}^{0} R_{T}(\eta) e^{\alpha \eta} d \eta\right]\right. \\
+\left[e^{-\alpha(2 L-2 x-\rho)} \int_{0}^{\rho} R_{T}(\eta) e^{\alpha \eta} d \eta-e^{-\alpha \rho} \int_{0}^{\rho} R_{T}(\eta) e^{\alpha \eta} d \eta\right] \\
\left.+\left[e^{-\alpha(2 L-2 x-\rho)} \int_{\rho}^{L-x} R_{T}(\eta) e^{\alpha \eta} d \eta-e^{\alpha \rho} \int_{\rho}^{L-x} R_{T}(\eta) e^{-\alpha \eta} d \eta\right]\right\}
\end{aligned}
$$

For the first term in brackets, we would like to have the integral over positive values of $\eta$. This is because, as it will be seen, the form of the temperature correlation function is not defined for values along the negative real axis. In order to make this so, we change variables from $\eta$ to $-\omega$, which implies that $d \eta=-d \omega$, and the lower limit becomes $L-\rho-x$. Thus,

$$
\int_{\rho+x-L}^{0} R_{T}(\eta) e^{\alpha \eta} d \eta \rightarrow-\int_{L-\rho-x}^{0} R_{T}(\omega) e^{\alpha \omega} d \omega=\int_{0}^{L-\rho-x} R_{T}(\omega) e^{\alpha \omega} d \omega
$$

and similarly for the $e^{-\alpha \eta}$ integral. Further, we note that since,

$$
\int_{0}^{L-x}=\int_{0}^{\rho}+\int_{\rho}^{L-x}
$$

the first integrals in the second and third brackets can be combined. The final result is then,

$$
\begin{gathered}
R_{I}(x, \rho)=\frac{-\alpha}{2} C_{v}^{2}\left\{e^{-\alpha(2 L-2 x-\rho)} \int_{0}^{L-x-\rho} R_{T}(\omega) e^{-\alpha \omega} d \omega-e^{-\alpha \rho} \int_{0}^{L-x-\rho} R_{T}(\omega) e^{\alpha \omega} d \omega\right. \\
+e^{-\alpha(2 L-2 x-\rho)} \int_{0}^{L-x} R_{T}(\eta) e^{\alpha \eta} d \eta-e^{-\alpha \rho} \int_{0}^{\rho} R_{T}(\eta) e^{\alpha \eta} d \eta \\
\left.-e^{\alpha \rho} \int_{\rho}^{L-x} R_{T}(\eta) e^{-\alpha \eta} d \eta\right\}
\end{gathered}
$$


It can be seen that each of the integrals has one of two integrand types: $R_{T}(\eta) e^{ \pm \alpha \eta}$. Next, we must choose a functional form of the temperature correlation function. A commonly used form is a von Karman one:

$$
R_{T}(\eta)=\sigma_{T}^{2} \frac{2^{2 / 3}}{\Gamma(1 / 3)}\left(k_{0} \eta\right)^{1 / 3} K_{1 / 3}\left(k_{0} \eta\right)
$$

where, $\sigma_{T}^{2}$ is the variance of the temperature turbulence; $\Gamma$ is the gamma function; $K_{1 / 3}$ is a modified Bessel function of the second kind, with order 1/3; and $k_{0}=\frac{\sqrt{\pi} \Gamma(5 / 6)}{\Gamma(1 / 3) L_{T}}$, where $L_{T}=\frac{1}{\sigma_{T}^{2}} \int_{0}^{\infty} R(\eta) d \eta$ is the integral length scale. It was mentioned above that we wanted the integrals to be over positive values of $\eta$. The reason is this Bessel function is defined everywhere in the complex plane, excepting for the negative real axis. It can be seen that the integrals in Eq. (21), will be of the form:

$$
\sigma_{T}^{2} \frac{2^{2 / 3}}{\Gamma(1 / 3)} \int_{a}^{b}\left(k_{0} \eta\right)^{1 / 3} K_{1 / 3}\left(k_{0} \eta\right) e^{ \pm \alpha \eta}
$$

These integrals cannot be calculated in closed form (excepting for $a=0, b=\infty$ ). So there are two options: numerical integration, or approximate integrals. The first method is fairly efficient numerically, as all the functions are well behaved. The second method, however allows for analytical analysis. We shall pursue both options. The numerical approach is straightforward, so we will only discuss the approximation methodology.

Consider the full integrands, Eq. (23), for the positive and negative exponentials. These are shown in Figure 23, along with the von Karman correlation function and the positive and negative exponential functions (with $\alpha=10^{-5} \mathrm{~m}^{-1}, L_{T}=1000 \mathrm{~m}$, and $\sigma_{T}^{2}=1$. In the figure, the full integrand with negative exponential is orange, the full integrand with positive exponential is black, the Von Karman function is magenta, and positive and negative exponentials are red and blue, respectively. It can be seen that the exponential functions - obviously due to the small value for $\alpha$ - have very little effect on the overall values. Figure 24 shows a sub-region of the first plot, from 500-1500 m. Figure 25 and Figure 26 are the same as the previous two figures, excepting for the turbulence integral length scale is now $2000 \mathrm{~m}$. Figure 27 illustrates the dramatic effect in changing $\alpha$ from $10^{-5}$ to $10^{-3}$. Note that the plot region has changed, to illustrate the pertinent quantities. Referring to Eq. (21), however, it can be seen that the integrands that have positive (negative) exponential functions have oppositely-signed exponential multiplying factors. 


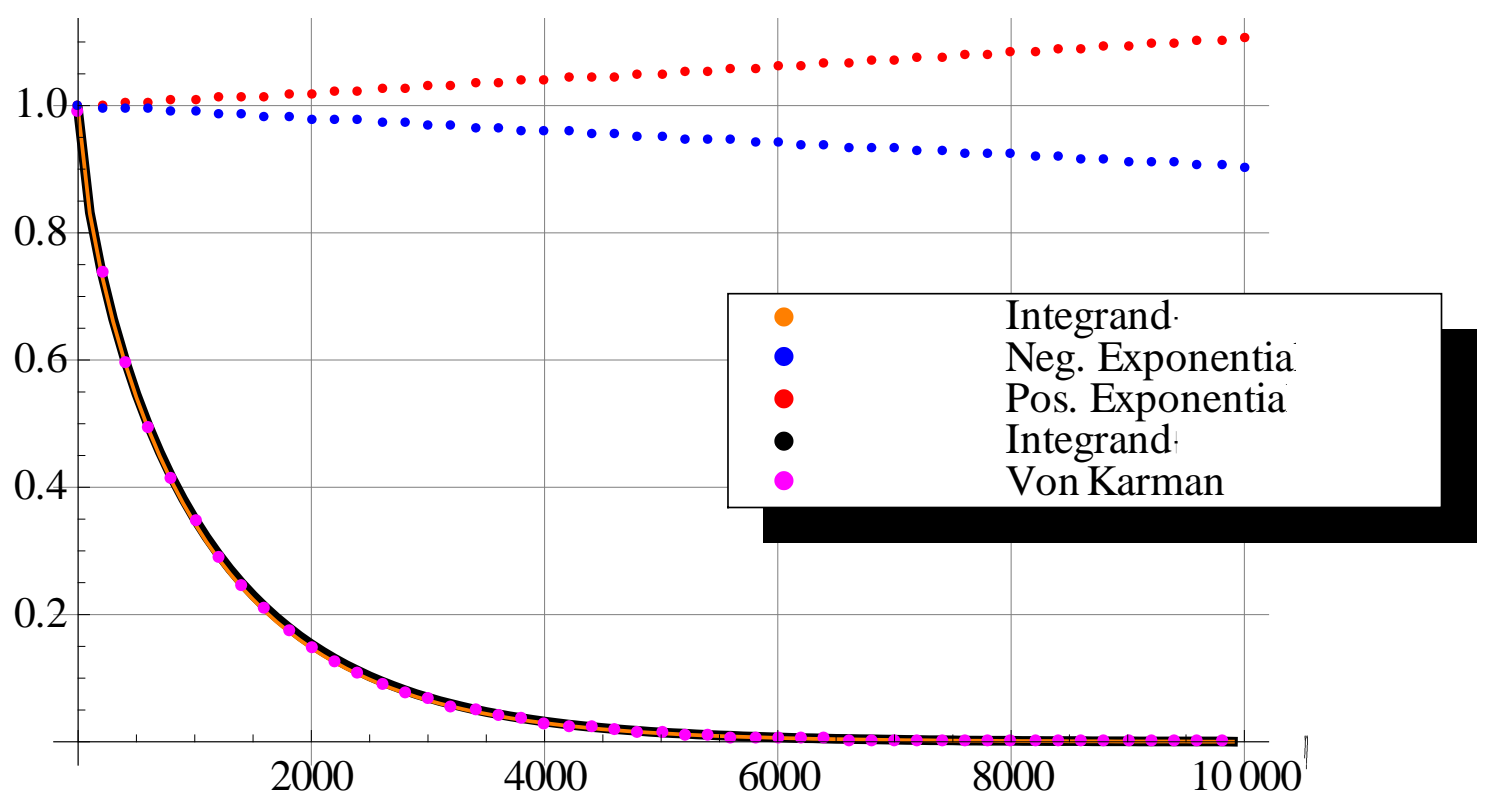

Figure 23. Comparison of Integrands.

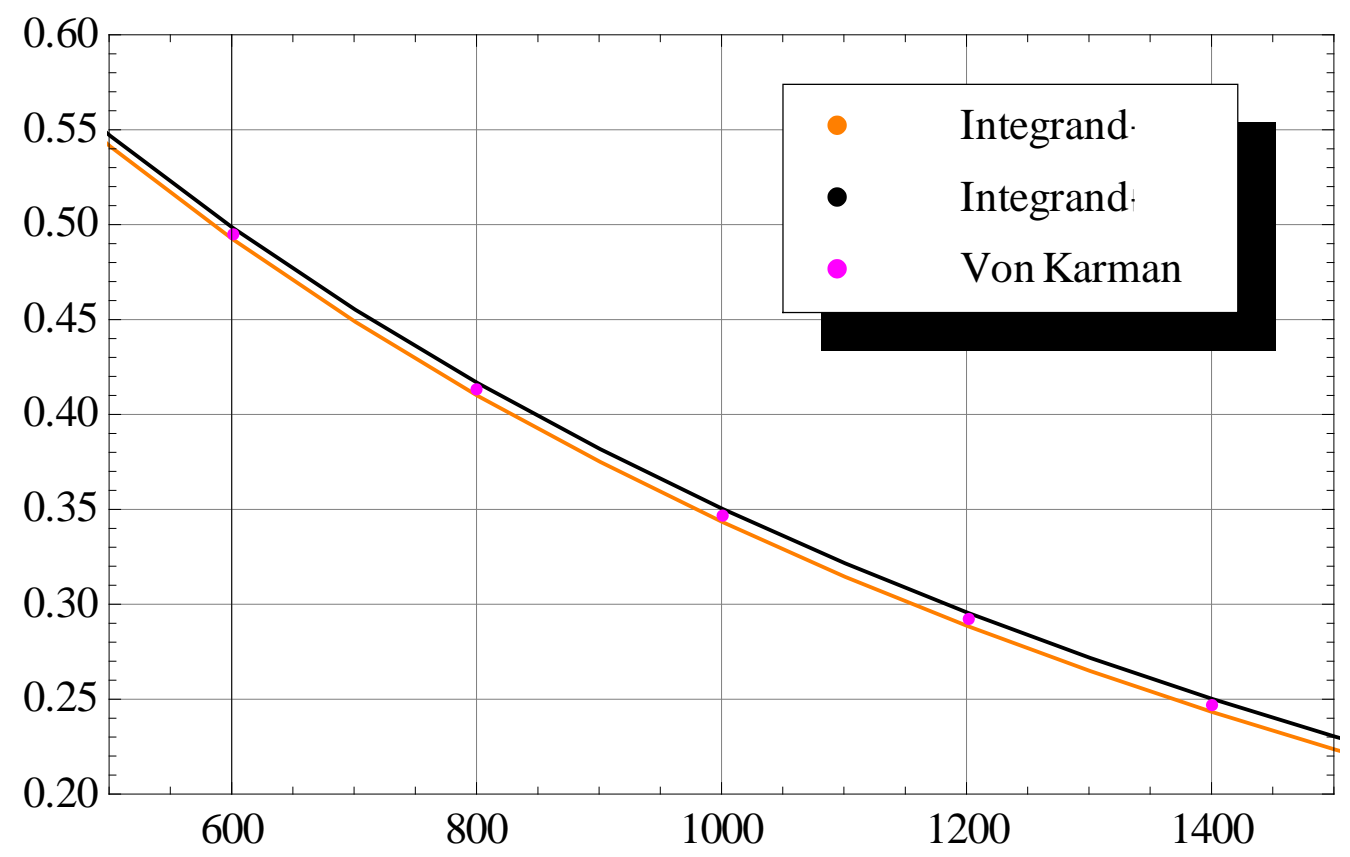

Figure 24. Detail of previous figure, over a smaller range of $\eta$ 's. 


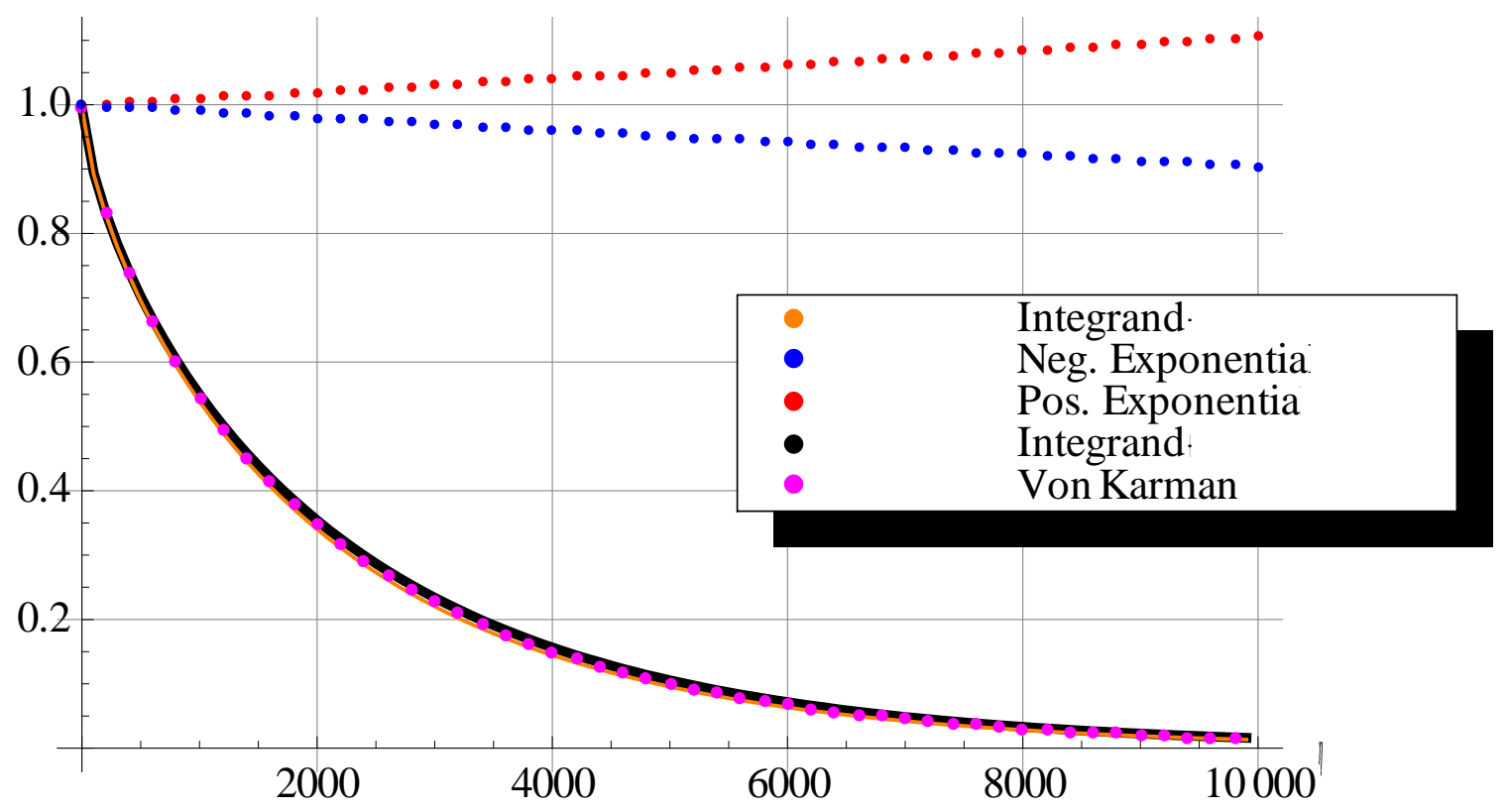

Figure 25. Comparison of Integrands, excepting for $L_{T}=2000 \mathrm{~m}$.

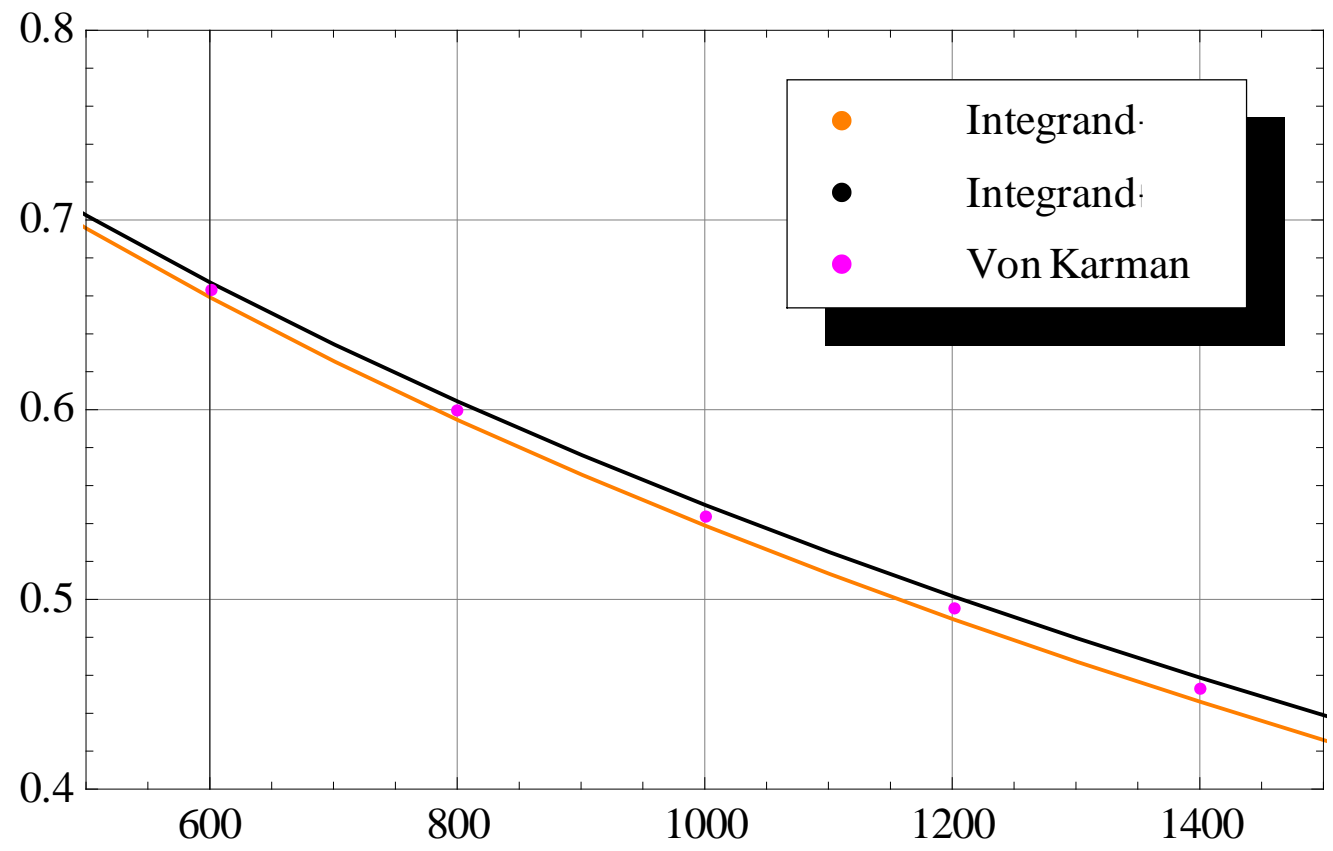

Figure 26. Detail of previous figure, excepting for $L_{T}=2000 \mathrm{~m}$. 


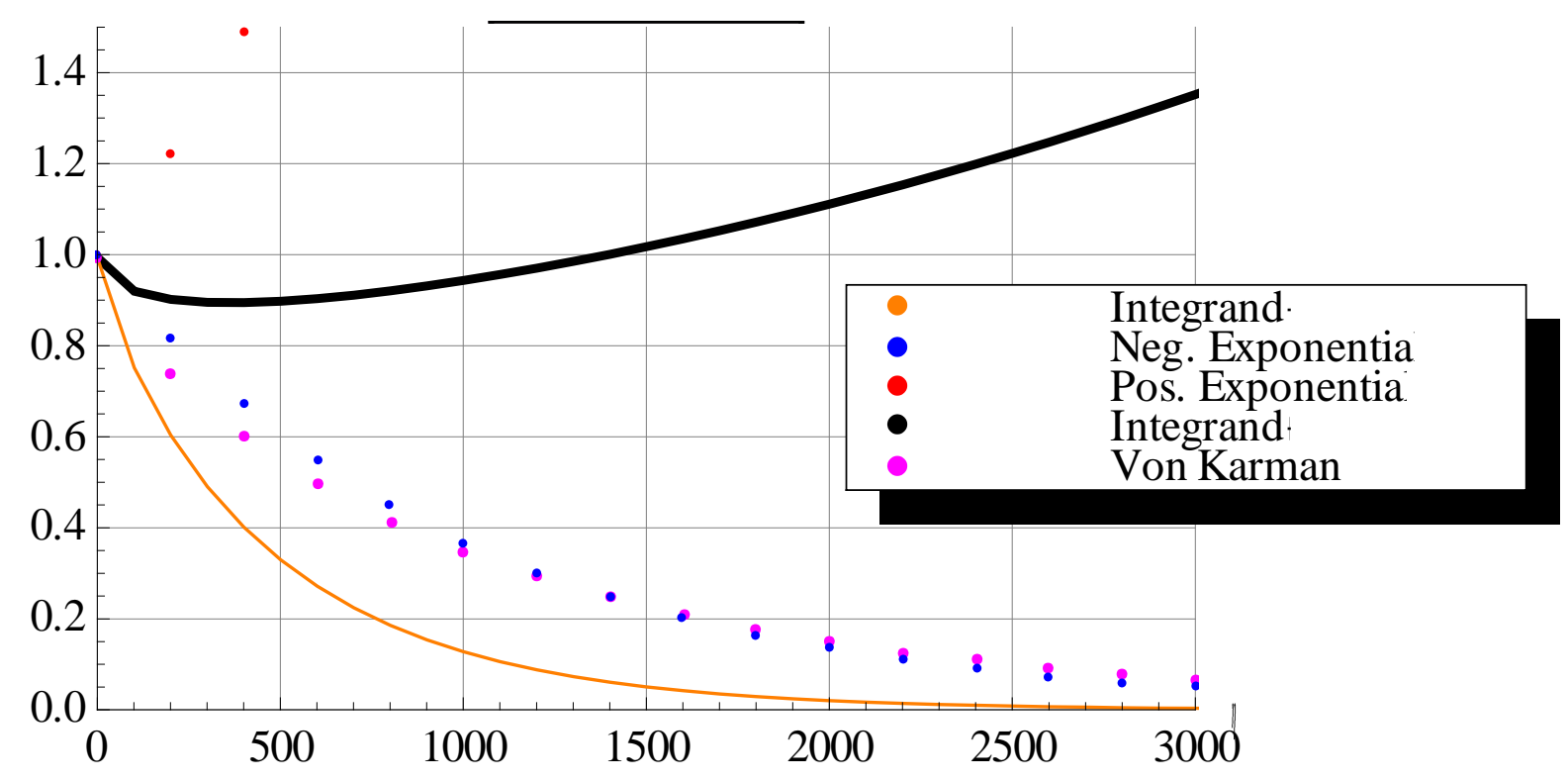

Figure 27. Comparison of Integrands, excepting that $\alpha=10^{-3}$.

Returning to the integral approximation, we first look at the small- $\eta$ range, and look for reasonably accurate power series approximations. These approximations - while potentially messy - can then be easily integrated. We will look at a $3^{\text {rd }}$ and a $5^{\text {th }}$ order expansions around zero. Using Mathematica, the $3^{\text {rd }}$ order series expansion for the integrand with the positive exponential is found to be,

$$
\begin{aligned}
& \frac{\sqrt[3]{\pi} \sigma \mathrm{T}^{2} \Gamma\left(-\frac{1}{3}\right)\left(\frac{1}{2} \Gamma\left(\frac{5}{6}\right)\right)^{2 / 3}\left(8 \mathrm{LT}^{2} \Gamma\left(\frac{1}{3}\right)^{2} \alpha^{2}+3 \pi \Gamma\left(\frac{5}{6}\right)^{2}\right) \eta^{8 / 3}}{16 \mathrm{LT}^{8 / 3} \Gamma\left(\frac{1}{3}\right)^{11 / 3}}+\frac{1}{8} \sigma \mathrm{T}^{2}\left(4 \alpha^{2}+\frac{3 \pi \Gamma\left(\frac{5}{6}\right)^{2}}{\mathrm{LT}^{2} \Gamma\left(\frac{1}{3}\right)^{2}}\right) \eta^{2}+ \\
& \frac{\sqrt[3]{\pi} \alpha \sigma \mathrm{T}^{2} \Gamma\left(-\frac{1}{3}\right)\left(\frac{1}{2} \Gamma\left(\frac{5}{6}\right)\right)^{2 / 3} \eta^{5 / 3}}{\mathrm{LT}^{2 / 3} \Gamma\left(\frac{1}{3}\right)^{5 / 3}}+\alpha \sigma \mathrm{T}^{2} \eta+\frac{\sqrt[3]{\pi} \sigma \mathrm{T}^{2} \Gamma\left(-\frac{1}{3}\right)\left(\frac{1}{2} \Gamma\left(\frac{5}{6}\right)\right)^{2 / 3} \eta^{2 / 3}}{\mathrm{LT}^{2 / 3} \Gamma\left(\frac{1}{3}\right)^{5 / 3}}+\sigma \mathrm{T}^{2}
\end{aligned}
$$

and the $5^{\text {th }}$ order expansion is, 


$$
\begin{aligned}
& \frac{\sqrt[3]{\pi} \sigma \mathrm{T}^{2} \Gamma\left(-\frac{1}{3}\right)\left(\frac{1}{2} \Gamma\left(\frac{5}{6}\right)\right)^{2 / 3}\left(112 \mathrm{LT}^{4} \Gamma\left(\frac{1}{3}\right)^{4} \alpha^{4}+252 \mathrm{LT}^{2} \pi \Gamma\left(\frac{1}{3}\right)^{2} \Gamma\left(\frac{5}{6}\right)^{2} \alpha^{2}+27 \pi^{2} \Gamma\left(\frac{5}{6}\right)^{4}\right) \eta^{14 / 3}}{2688 \mathrm{LT}^{14 / 3} \Gamma\left(\frac{1}{3}\right)^{17 / 3}}+ \\
& \frac{1}{960} \sigma \mathrm{T}^{2}\left(40 \alpha^{4}+\frac{180 \pi \Gamma\left(\frac{5}{6}\right)^{2} \alpha^{2}}{\mathrm{LT}^{2} \Gamma\left(\frac{1}{3}\right)^{2}}+\frac{27 \pi^{2} \Gamma\left(\frac{5}{6}\right)^{4}}{\mathrm{LT}^{4} \Gamma\left(\frac{1}{3}\right)^{4}}\right) \eta^{4}+ \\
& \frac{\sqrt[3]{\pi} \alpha \sigma \mathrm{T}^{2} \Gamma\left(-\frac{1}{3}\right)\left(\frac{1}{2} \Gamma\left(\frac{5}{6}\right)\right)^{2 / 3}\left(8 \mathrm{LT}^{2} \Gamma\left(\frac{1}{3}\right)^{2} \alpha^{2}+9 \pi \Gamma\left(\frac{5}{6}\right)^{2}\right) \eta^{11 / 3}}{48 \mathrm{LT}^{8 / 3} \Gamma\left(\frac{1}{3}\right)^{11 / 3}}+\frac{1}{24} \alpha \sigma \mathrm{T}^{2}\left(4 \alpha^{2}+\frac{9 \pi \Gamma\left(\frac{5}{6}\right)^{2}}{\mathrm{LT}^{2} \Gamma\left(\frac{1}{3}\right)^{2}}\right) \eta^{3}+ \\
& \frac{\sqrt[3]{\pi} \sigma \mathrm{T}^{2} \Gamma\left(-\frac{1}{3}\right)\left(\frac{1}{2} \Gamma\left(\frac{5}{6}\right)\right)^{2 / 3}\left(8 \mathrm{LT}^{2} \Gamma\left(\frac{1}{3}\right)^{2} \alpha^{2}+3 \pi \Gamma\left(\frac{5}{6}\right)^{2}\right) \eta^{8 / 3}}{16 \mathrm{LT}^{8 / 3} \Gamma\left(\frac{1}{3}\right)^{11 / 3}}+\frac{1}{8} \sigma \mathrm{T}^{2}\left(4 \alpha^{2}+\frac{3 \pi \Gamma\left(\frac{5}{6}\right)^{2}}{\mathrm{LT}^{2} \Gamma\left(\frac{1}{3}\right)^{2}}\right) \eta^{2}+ \\
& \frac{\sqrt[3]{\pi} \alpha \sigma \mathrm{T}^{2} \Gamma\left(-\frac{1}{3}\right)\left(\frac{1}{2} \Gamma\left(\frac{5}{6}\right)\right)^{2 / 3} \eta^{5 / 3}}{\mathrm{LT}^{2 / 3} \Gamma\left(\frac{1}{3}\right)^{5 / 3}}+\alpha \sigma \mathrm{T}^{2} \eta+\frac{\sqrt[3]{\pi} \sigma \mathrm{T}^{2} \Gamma\left(-\frac{1}{3}\right)\left(\frac{1}{2} \Gamma\left(\frac{5}{6}\right)\right)^{2 / 3} \eta^{2 / 3}}{\mathrm{LT}^{2 / 3} \Gamma\left(\frac{1}{3}\right)^{5 / 3}}+\sigma \mathrm{T}^{2}
\end{aligned}
$$

The analogous expansions for the integrands with negative exponentials are given by,

$$
\begin{aligned}
& \frac{\sqrt[3]{\pi} \sigma \mathrm{T}^{2} \Gamma\left(-\frac{1}{3}\right)\left(\frac{1}{2} \Gamma\left(\frac{5}{6}\right)\right)^{2 / 3}\left(8 \mathrm{LT}^{2} \Gamma\left(\frac{1}{3}\right)^{2} \alpha^{2}+3 \pi \Gamma\left(\frac{5}{6}\right)^{2}\right) \eta^{8 / 3}}{16 \mathrm{LT}^{8 / 3} \Gamma\left(\frac{1}{3}\right)^{11 / 3}}+\frac{1}{8} \sigma \mathrm{T}^{2}\left(4 \alpha^{2}+\frac{3 \pi \Gamma\left(\frac{5}{6}\right)^{2}}{\mathrm{LT}^{2} \Gamma\left(\frac{1}{3}\right)^{2}}\right) \eta^{2}- \\
& \frac{\sqrt[3]{\pi} \alpha \sigma \mathrm{T}^{2} \Gamma\left(-\frac{1}{3}\right)\left(\frac{1}{2} \Gamma\left(\frac{5}{6}\right)\right)^{2 / 3} \eta^{5 / 3}}{\mathrm{LT}^{2 / 3} \Gamma\left(\frac{1}{3}\right)^{5 / 3}}-\alpha \sigma \mathrm{T}^{2} \eta+\frac{\sqrt[3]{\pi} \sigma \mathrm{T}^{2} \Gamma\left(-\frac{1}{3}\right)\left(\frac{1}{2} \Gamma\left(\frac{5}{6}\right)\right)^{2 / 3} \eta^{2 / 3}}{\mathrm{LT}^{2 / 3} \Gamma\left(\frac{1}{3}\right)^{5 / 3}}+\sigma \mathrm{T}^{2}
\end{aligned}
$$

and for the $5^{\text {th }}$ order expansion, 


$$
\begin{aligned}
& \frac{\sqrt[3]{\pi} \sigma \mathrm{T}^{2} \Gamma\left(-\frac{1}{3}\right)\left(\frac{1}{2} \Gamma\left(\frac{5}{6}\right)\right)^{2 / 3}\left(112 \mathrm{LT}^{4} \Gamma\left(\frac{1}{3}\right)^{4} \alpha^{4}+252 \mathrm{LT}^{2} \pi \Gamma\left(\frac{1}{3}\right)^{2} \Gamma\left(\frac{5}{6}\right)^{2} \alpha^{2}+27 \pi^{2} \Gamma\left(\frac{5}{6}\right)^{4}\right) \eta^{14 / 3}}{2688 \mathrm{LT}^{14 / 3} \Gamma\left(\frac{1}{3}\right)^{17 / 3}}+ \\
& \frac{1}{960} \sigma \mathrm{T}^{2}\left(40 \alpha^{4}+\frac{180 \pi \Gamma\left(\frac{5}{6}\right)^{2} \alpha^{2}}{\mathrm{LT}^{2} \Gamma\left(\frac{1}{3}\right)^{2}}+\frac{27 \pi^{2} \Gamma\left(\frac{5}{6}\right)^{4}}{\mathrm{LT}^{4} \Gamma\left(\frac{1}{3}\right)^{4}}\right) \eta^{4}- \\
& \frac{\sqrt[3]{\pi} \alpha \sigma \mathrm{T}^{2} \Gamma\left(-\frac{1}{3}\right)\left(\frac{1}{2} \Gamma\left(\frac{5}{6}\right)\right)^{2 / 3}\left(8 \mathrm{LT}^{2} \Gamma\left(\frac{1}{3}\right)^{2} \alpha^{2}+9 \pi \Gamma\left(\frac{5}{6}\right)^{2}\right) \eta^{11 / 3}}{48 \mathrm{LT}^{8 / 3} \Gamma\left(\frac{1}{3}\right)^{11 / 3}}+\frac{1}{24} \alpha \sigma \mathrm{T}^{2}\left(-4 \alpha^{2}-\frac{9 \pi \Gamma\left(\frac{5}{6}\right)^{2}}{\mathrm{LT}^{2} \Gamma\left(\frac{1}{3}\right)^{2}}\right) \eta^{3}+ \\
& \frac{\sqrt[3]{\pi} \sigma \mathrm{T}^{2} \Gamma\left(-\frac{1}{3}\right)\left(\frac{1}{2} \Gamma\left(\frac{5}{6}\right)\right)^{2 / 3}\left(8 \mathrm{LT}^{2} \Gamma\left(\frac{1}{3}\right)^{2} \alpha^{2}+3 \pi \Gamma\left(\frac{5}{6}\right)^{2}\right) \eta^{8 / 3}}{16 \mathrm{LT}^{8 / 3} \Gamma\left(\frac{1}{3}\right)^{11 / 3}}+\frac{1}{8} \sigma \mathrm{T}^{2}\left(4 \alpha^{2}+\frac{3 \pi \Gamma\left(\frac{5}{6}\right)^{2}}{\mathrm{LT}^{2} \Gamma\left(\frac{1}{3}\right)^{2}}\right) \eta^{2}- \\
& \frac{\sqrt[3]{\pi} \alpha \sigma \mathrm{T}^{2} \Gamma\left(-\frac{1}{3}\right)\left(\frac{1}{2} \Gamma\left(\frac{5}{6}\right)\right)^{2 / 3} \eta^{5 / 3}}{\mathrm{LT}^{2 / 3} \Gamma\left(\frac{1}{3}\right)^{5 / 3}}-\alpha \sigma \mathrm{T}^{2} \eta+\frac{\sqrt[3]{\pi} \sigma \mathrm{T}^{2} \Gamma\left(-\frac{1}{3}\right)\left(\frac{1}{2} \Gamma\left(\frac{5}{6}\right)\right)^{2 / 3} \eta^{2 / 3}}{\mathrm{LT}^{2 / 3} \Gamma\left(\frac{1}{3}\right)^{5 / 3}}+\sigma \mathrm{T}^{2}
\end{aligned}
$$

It is possible that these expressions can be further simplified.

Next, consider the large- $\eta$ expansions. In this case, we perform an $3^{\text {rd }}$ and $4^{\text {th }}$ order expansion around $\eta \rightarrow \infty$ for the positive and negative exponential cases, respectively. The $3^{\text {rd }}$ and $4^{\text {th }}$ order expansions for the positive exponential functions are,

$$
e^{\eta\left(\alpha-\frac{\sqrt{\pi} \Gamma\left(\frac{5}{6}\right)}{\operatorname{LT} \Gamma\left(\frac{1}{3}\right)}\right)}\left(\frac{385 \sigma \mathrm{T}^{2} \Gamma\left(\frac{1}{3}\right)^{7 / 6} \mathrm{LT}^{13 / 6}}{51842^{5 / 6} \pi^{7 / 12} \eta^{13 / 6} \Gamma\left(\frac{5}{6}\right)^{13 / 6}}-\frac{5 \sigma \mathrm{T}^{2} \sqrt[6]{\Gamma\left(\frac{1}{3}\right)} \mathrm{LT}^{7 / 6}}{362^{5 / 6} \sqrt[12]{\pi} \eta^{7 / 6} \Gamma\left(\frac{5}{6}\right)^{7 / 6}}+\frac{\pi^{5 / 12} \sigma \mathrm{T}^{2} \sqrt[6]{\frac{2}{\Gamma\left(\frac{5}{6}\right)}} \sqrt[6]{\mathrm{LT}}}{\sqrt[6]{\eta} \Gamma\left(\frac{1}{3}\right)^{5 / 6}}\right)
$$

and, 


$$
\begin{aligned}
& e^{\eta\left(\alpha-\frac{\sqrt{\pi} \Gamma\left(\frac{5}{6}\right)}{\operatorname{LT} \Gamma\left(\frac{1}{3}\right)}\right)}\left(-\frac{85085 \sigma \mathrm{T}^{2} \Gamma\left(\frac{1}{3}\right)^{13 / 6} \mathrm{LT}^{19 / 6}}{11197442^{5 / 6} \pi^{13 / 12} \eta^{19 / 6} \Gamma\left(\frac{5}{6}\right)^{19 / 6}}+\right. \\
& \\
& \left.\quad \frac{385 \sigma \mathrm{T}^{2} \Gamma\left(\frac{1}{3}\right)^{7 / 6} \mathrm{LT}^{13 / 6}}{51842^{5 / 6} \pi^{7 / 12} \eta^{13 / 6} \Gamma\left(\frac{5}{6}\right)^{13 / 6}}-\frac{5 \sigma \mathrm{T}^{2} \sqrt[6]{\Gamma\left(\frac{1}{3}\right)} \mathrm{LT}^{7 / 6}}{362^{5 / 6} \sqrt[12]{\pi} \eta^{7 / 6} \Gamma\left(\frac{5}{6}\right)^{7 / 6}}+\frac{\pi^{5 / 12} \sigma \mathrm{T}^{2} \sqrt[6]{\frac{2}{\Gamma\left(\frac{5}{6}\right)}} \sqrt[6]{\mathrm{LT}}}{\sqrt[6]{\eta} \Gamma\left(\frac{1}{3}\right)^{5 / 6}}\right)
\end{aligned}
$$

For the negative exponentials we have,

$$
e^{-\alpha \eta-\frac{\sqrt{\pi} \Gamma\left(\frac{5}{6}\right) \eta}{\operatorname{LT} \Gamma\left(\frac{1}{3}\right)}}\left(\frac{385 \sigma \mathrm{T}^{2} \Gamma\left(\frac{1}{3}\right)^{7 / 6} \mathrm{LT}^{13 / 6} \sqrt[5]{5}}{51842^{5 / 6} \pi^{7 / 12} \eta^{13 / 6} \Gamma\left(\frac{5}{6}\right)^{13 / 6}}-\frac{5 \sigma \mathrm{T}^{2} \sqrt[6]{\Gamma\left(\frac{1}{3}\right)} \mathrm{LT}^{7 / 6}}{362^{5 / 6} \sqrt[12]{\pi} \eta^{7 / 6} \Gamma\left(\frac{5}{6}\right)^{7 / 6}}+\frac{\pi^{5 / 12} \sigma \mathrm{T}^{2} \sqrt[6]{\frac{2}{\Gamma\left(\frac{5}{6}\right)}} \sqrt[6]{\mathrm{LT}}}{\sqrt[6]{\eta} \Gamma\left(\frac{1}{3}\right)^{5 / 6}}\right)
$$

and,

$$
e^{-\alpha \eta-\frac{\sqrt{\pi} \Gamma\left(\frac{5}{6}\right) \eta}{\operatorname{LT} \Gamma\left(\frac{1}{3}\right)}}\left(-\frac{85085 \sigma \mathrm{T}^{2} \Gamma\left(\frac{1}{3}\right)^{13 / 6} \mathrm{LT}^{19 / 6}}{11197442^{5 / 6} \pi^{13 / 12} \eta^{19 / 6} \Gamma\left(\frac{5}{6}\right)^{19 / 6}}+\right.
$$

$$
\left.\frac{385 \sigma \mathrm{T}^{2} \Gamma\left(\frac{1}{3}\right)^{7 / 6} \mathrm{LT}^{13 / 6}}{51842^{5 / 6} \pi^{7 / 12} \eta^{13 / 6} \Gamma\left(\frac{5}{6}\right)^{13 / 6}}-\frac{5 \sigma \mathrm{T}^{2} \sqrt[6]{\Gamma\left(\frac{1}{3}\right)} \mathrm{LT}^{7 / 6}}{362^{5 / 6} \sqrt[12]{\pi} \eta^{7 / 6} \Gamma\left(\frac{5}{6}\right)^{7 / 6}}+\frac{\pi^{5 / 12} \sigma \mathrm{T}^{2} \sqrt[6]{\frac{2}{\Gamma\left(\frac{5}{6}\right)} \sqrt[6]{\mathrm{LT}}}}{\sqrt[6]{\eta} \Gamma\left(\frac{1}{3}\right)^{5 / 6}}\right)
$$

Note that while the high- $\eta$ approximations are simpler in form, they will be slightly more difficult to integrate due to the combined exponential and power terms (i.e., integration by parts will be required.) 
The following figures illustrate the performance of the small- $\eta$ integrand approximations for both positive and negative exponentials, varying the integral length scale, and varying the absorption coefficient. (Note: In order to enhance pertinent features, different scales are often used for different plots.) Figure 28 is a plot of full, 3rd and 5th order, small- approximations to the integrand with the positive exponential. The figure shows the improved performance of the $5^{\text {th }}$ order approximation to the positive exponential $\left(L_{T}=1000 \mathrm{~m}\right.$ and $\alpha=10^{-5} \mathrm{~m}^{-1}$ ). By improved, it is meant that the $5^{\text {th }}$ order approximation is closer to the full integrand for larger values of $\eta$. This is important, as we want as large a range for the low- $\eta$ approximation in order to have as much functional overlap with the high- $\eta$ approximations, such that the combination can then hopefully cover the entire range of $\eta$-values for a given integral's limits. Figure 29 shows that for $L_{T}=2000 \mathrm{~m}$, both the approximations perform well. This is to be expected, as the larger length scales means that the von Karman correlation does not decay as fast as with smaller length scales. Figure 30 has the same length scale as with the data shown in Figure 28, however the absorption coefficient is two orders of magnitude larger $\left(10^{-3}\right)$. It can be seen that this change has a dramatic effect on the accuracy of the approximations. This is not too surprising, as the larger absorption coefficient means that the exponential function has a stronger influence, and the $3^{\text {rd }}$ and $5^{\text {th }}$ order power series approximations around $\eta=0$ do not give a good approximation to the exponential function. The same pattern as with the positive exponential case occurs for the negative exponential cases, as can be seen from Figure 31 - Figure 33.

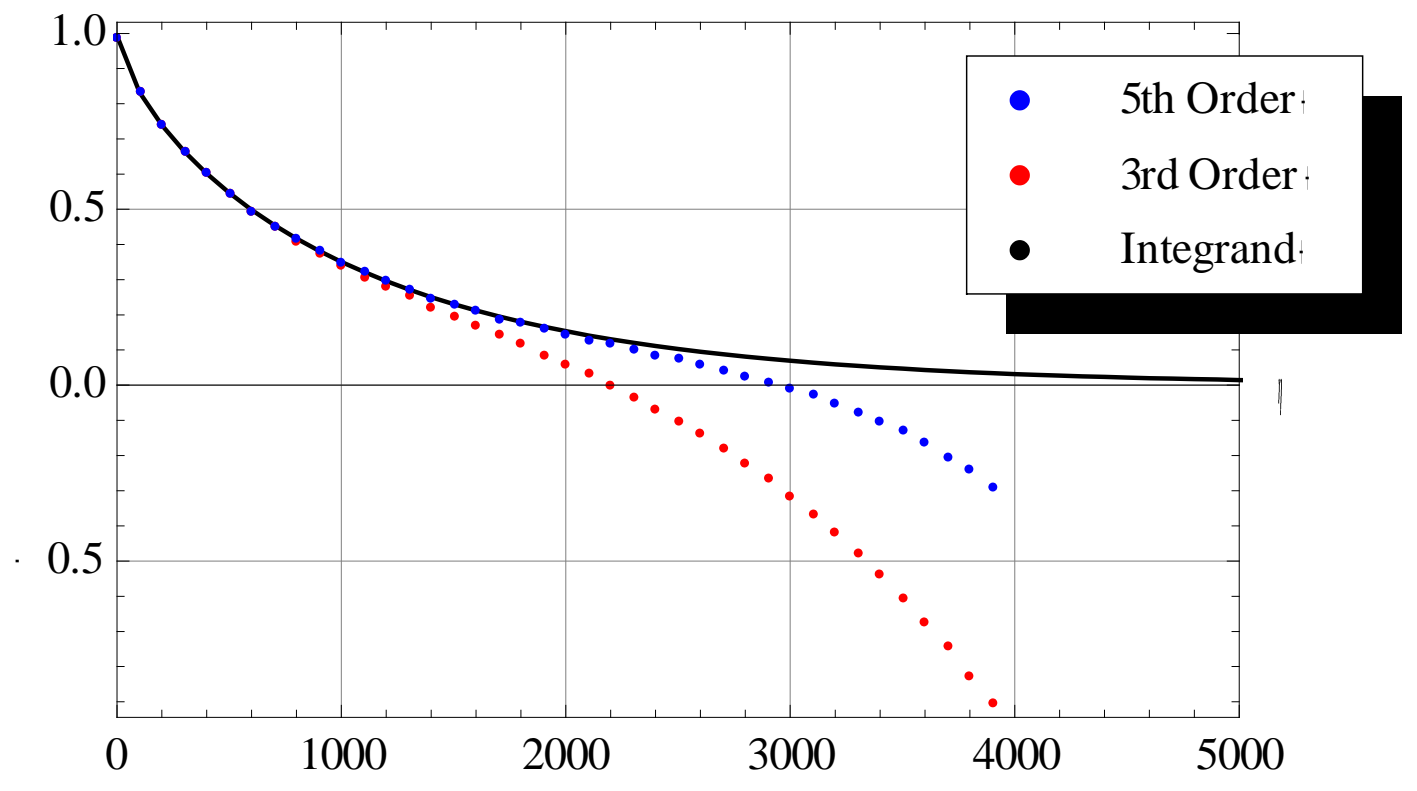

Figure 28. Integrand with the positive exponential and $L_{T}=1000 \mathrm{~m}$. 


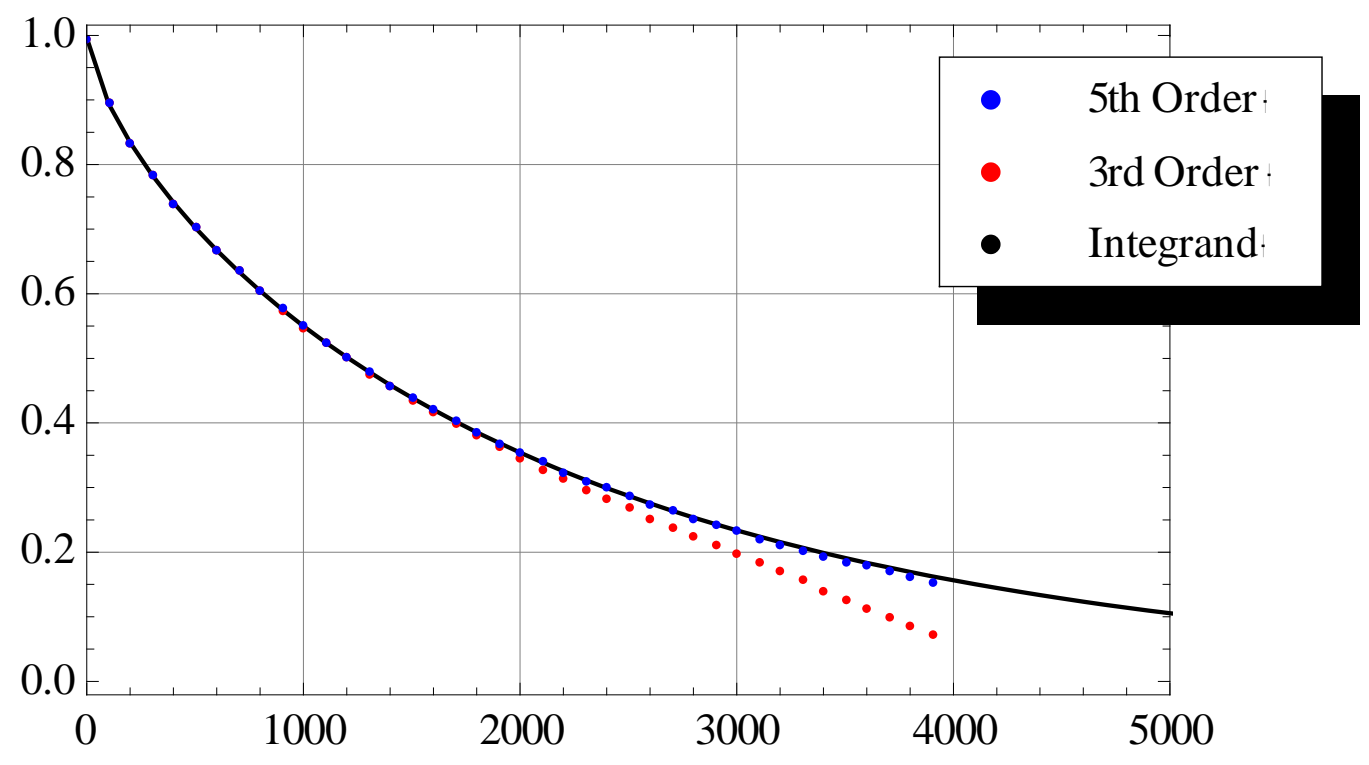

Figure 29. Same as Figure 28, but with $L_{T}=2000 \mathrm{~m}$.

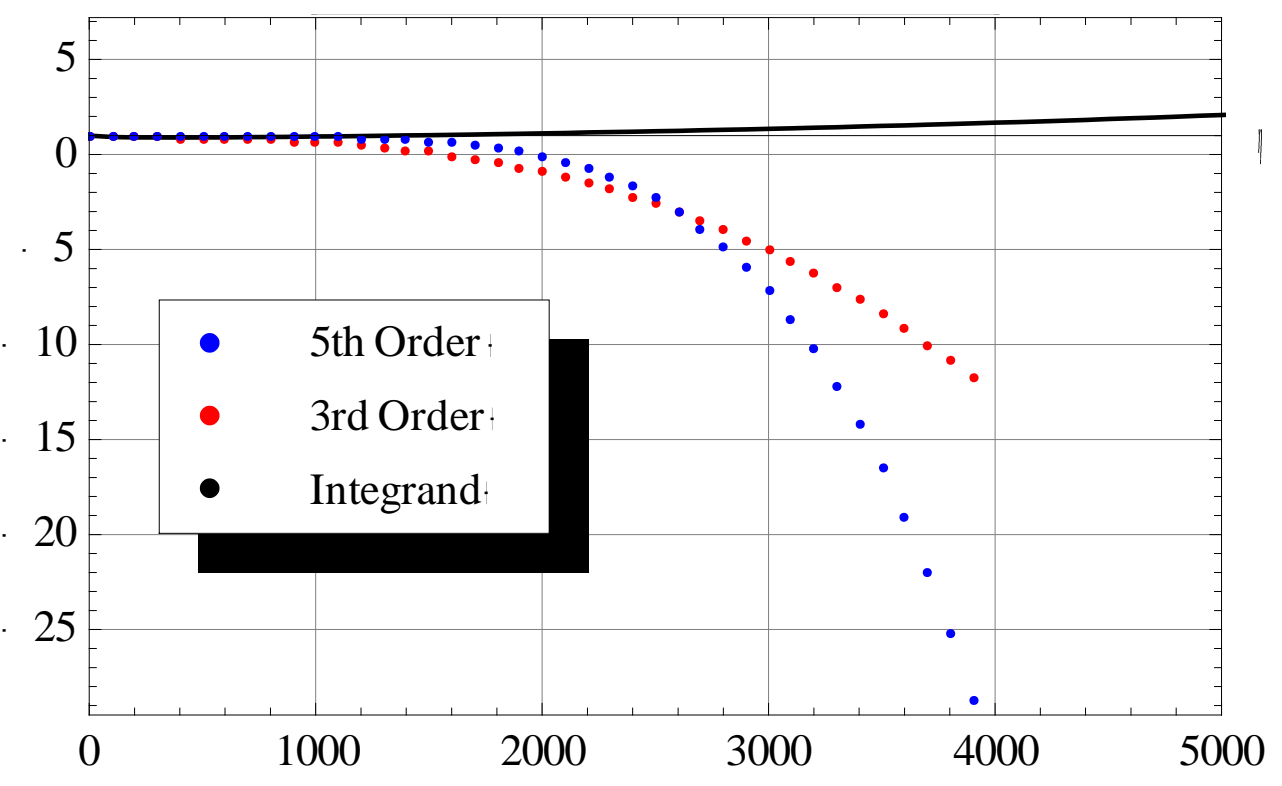

Figure 30. Same as Figure 28, but with $\alpha=10^{-3}$. 


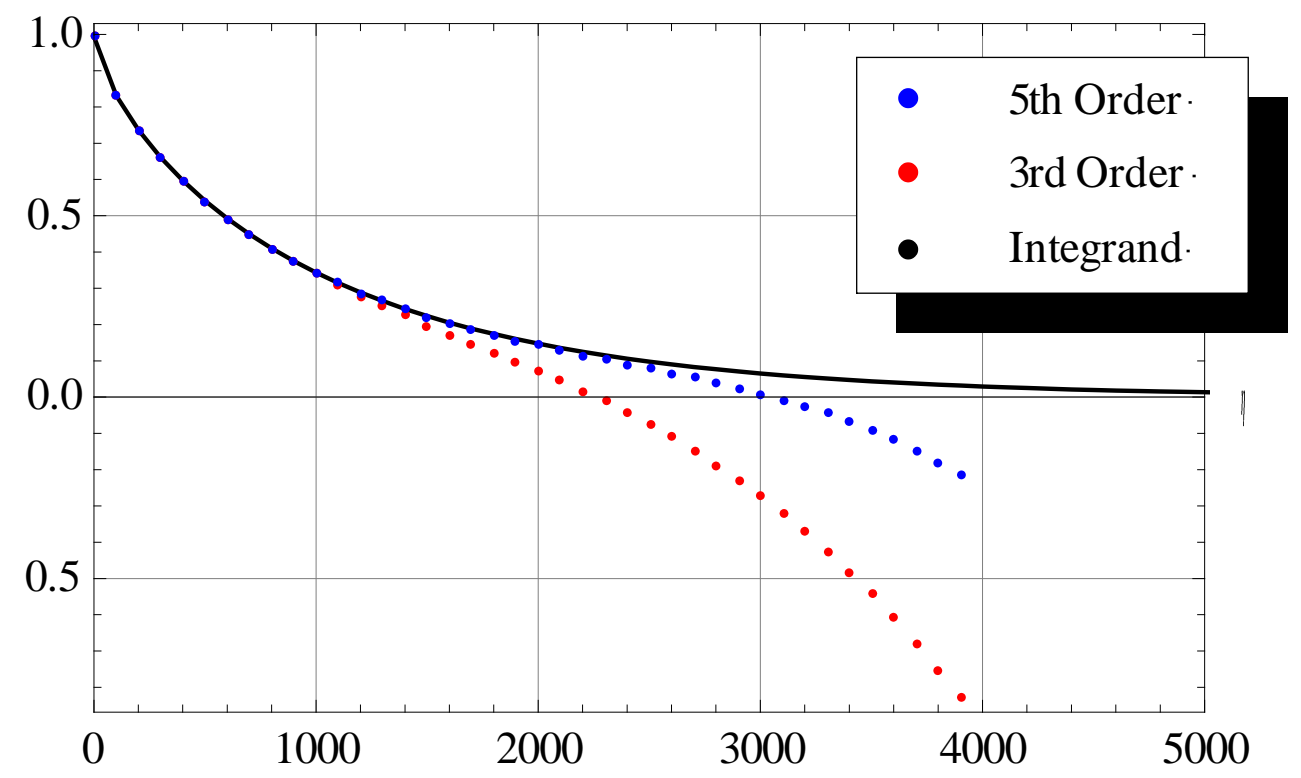

Figure 31. Same as Figure 28, but with negative exponential.

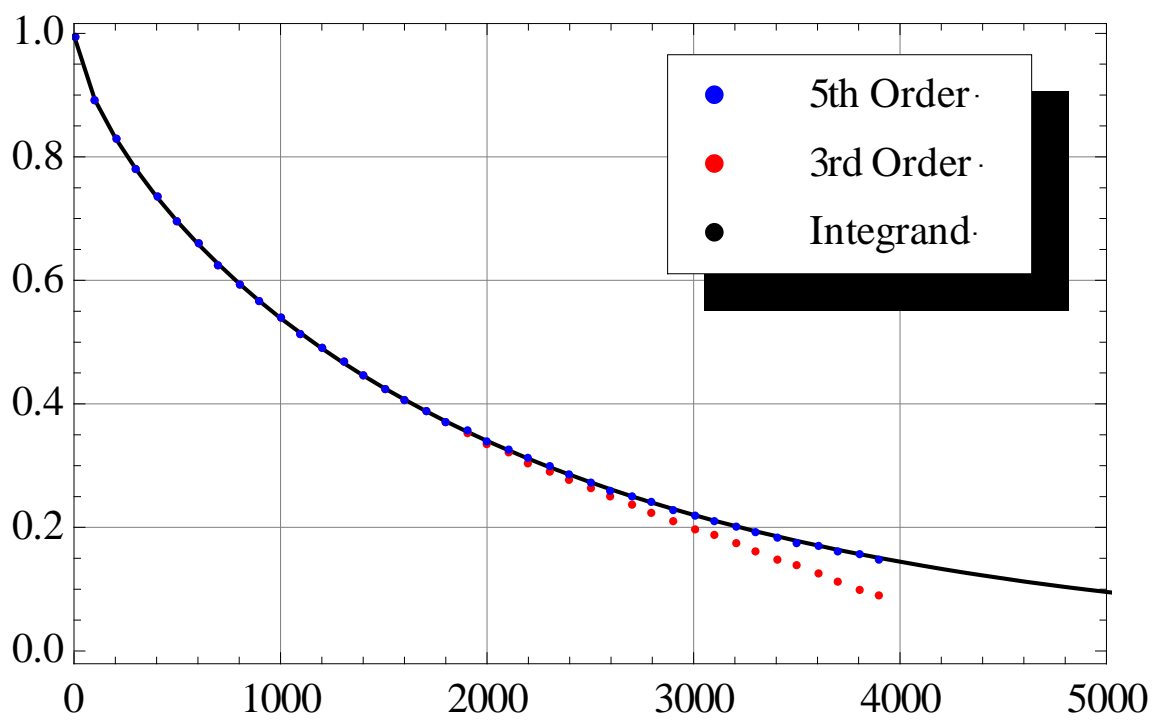

Figure 32. Same as Figure 29, but with negative exponential. 


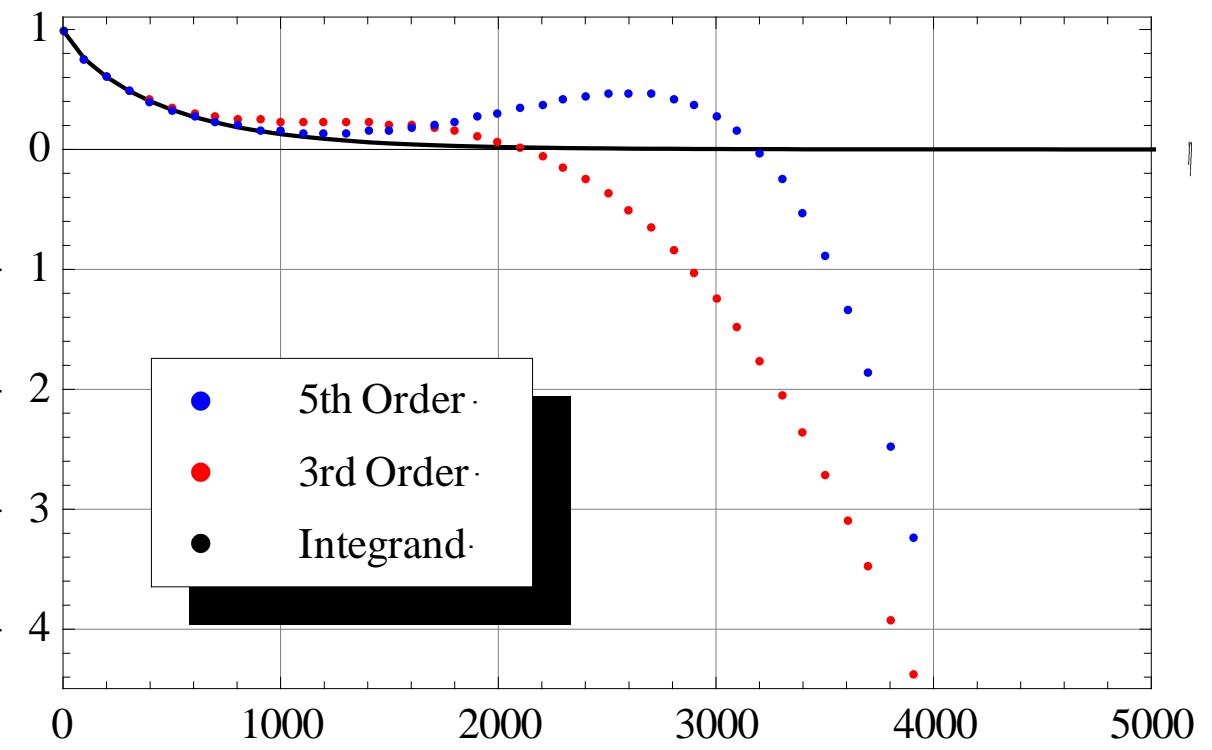

Figure 33. Same as Figure 30, but with negative exponential.

Next, consider the large- $\eta$ approximation regime. Figure 34 - Figure 40 illustrate the fits of the $5^{\text {th }}$-order, small- $\eta$ approximation, as well as the large- $\eta 3^{\text {rd }}$ and $4^{\text {th }}$ order approximations, to the negative and positive exponential integrands. The same type of variations in $L_{T}$ and $\alpha$ are used as above. From Figure 34 it can be seen that the large- $\eta$ approximations do a very good job of matching the full integrand for the positive exponential case. There is a reasonable overlap between these approximations and the $5^{\text {th }}$-order, small- $\eta$ approximation - indicating that in this case $\left(L_{T}=1000 \mathrm{~m}\right.$ and $\alpha=10^{-5} \mathrm{~m}^{-1}$ ) the combination of small- and large- $\eta$ approximations cover the entire range of $\eta$ values. Figure 35 illustrates the $L_{T}=2000 m$ case. Here, as expected the small- $\eta$ approximation is accurate over a much larger range, whereas the large- $\eta$ approximations perform somewhat more poorly in the smaller- $\eta$ range. Nevertheless, due to the large overlap region, this case is very well handled by the combination. Also shown in this figure, as well as in subsequent ones, the average of the $3^{\text {rd }}$ and $4^{\text {th }}$ order, large- $\eta$ approximations is shown. Due to the odd-even nature of these approximations, they start diverging from each other at smaller- $\eta$ values. The average of the two allows for a somewhat better approximation at these smaller values. Figure 36 shows the effect of increasing the absorption coefficient from $10^{-5}$ to $10^{-3}$. It can be seen that the overlap region is greatly reduced in this case. Figure 37 , a detail view of Figure 36, reinforces this condition. This indicates that a better approximation scheme is required to adequately handle this case. Perhaps the average of $4^{\text {th }}$ and $5^{\text {th }}$ order, small$\eta$ approximations could work. Further analysis would be needed. Figure 38 through Figure 40 are the negative exponential integrand analogues to those figures presented above. The behaviors over the different parameter settings are quite similar to those with the positive exponential integrand. 


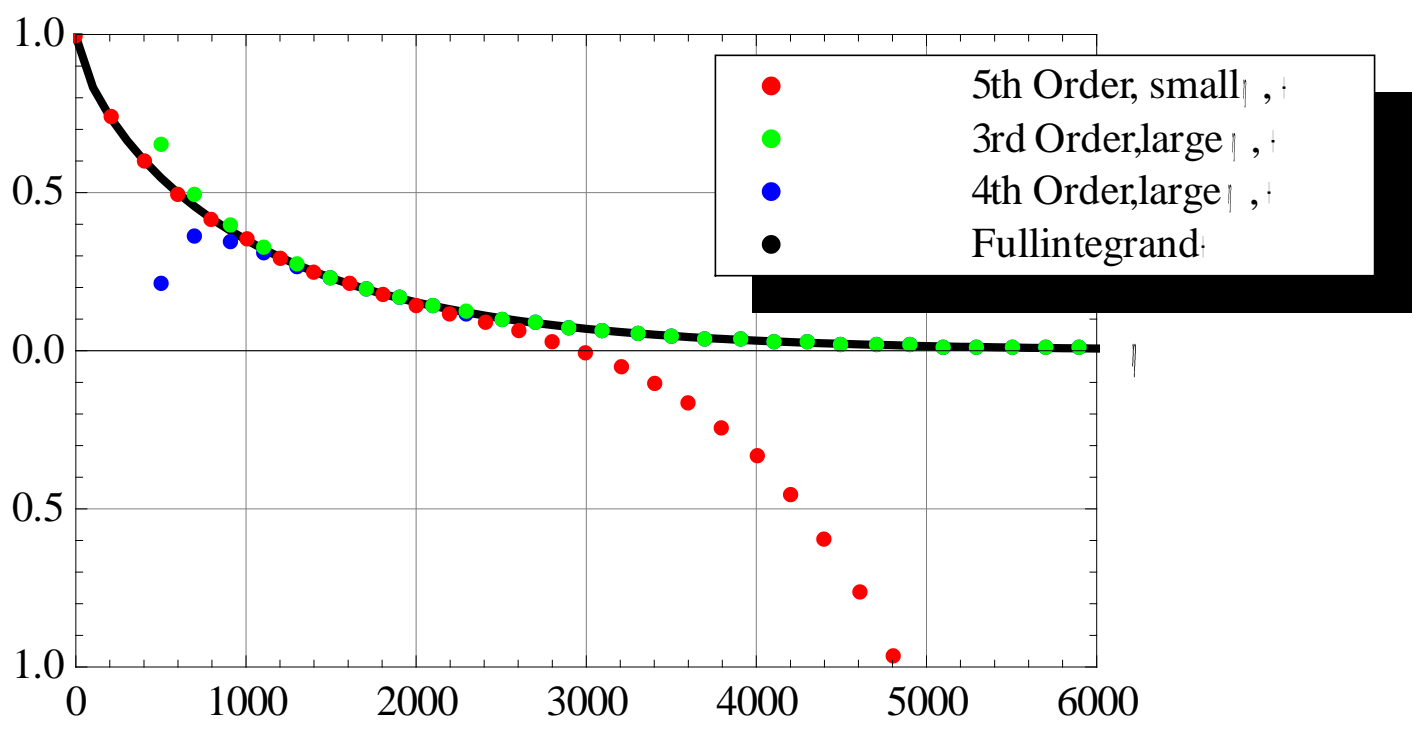

Figure 34. Large- $\eta$ approximations to the integrand, $L_{T}=1000 \mathrm{~m}$.

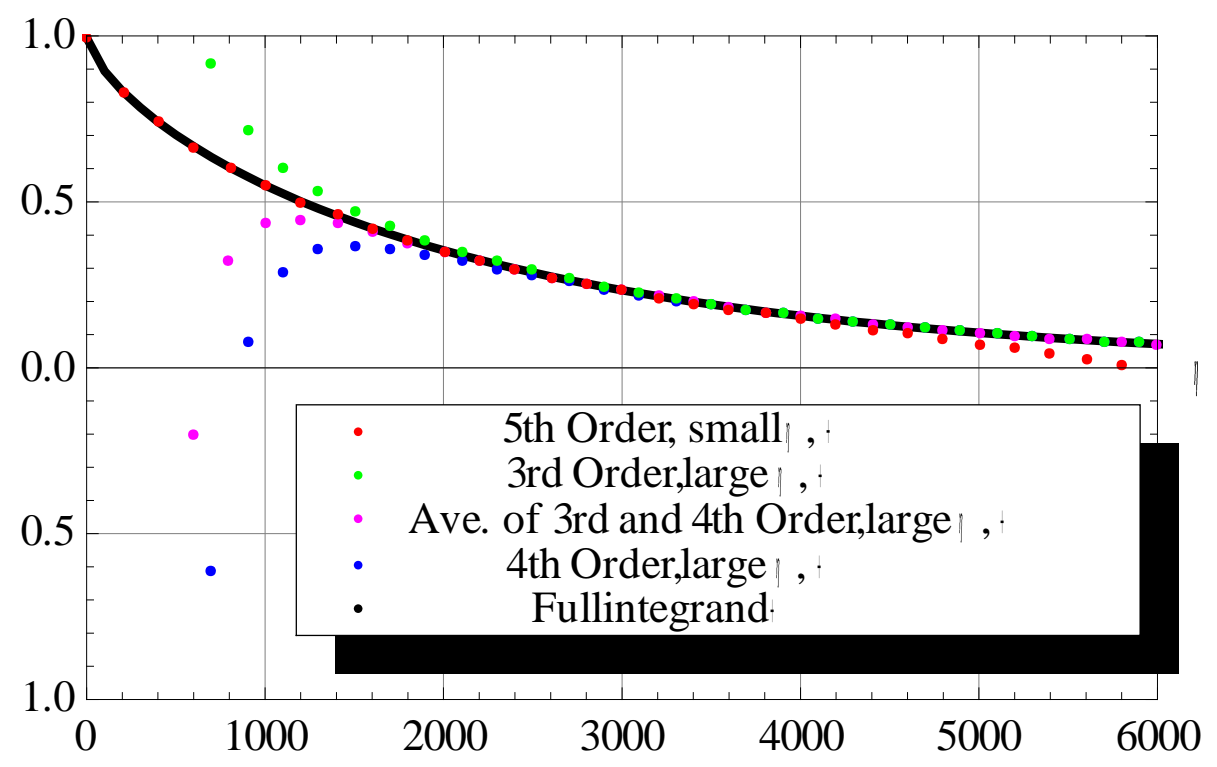

Figure 35. Same as for Figure 34, but with $L_{T}=2000 \mathrm{~m}$. 


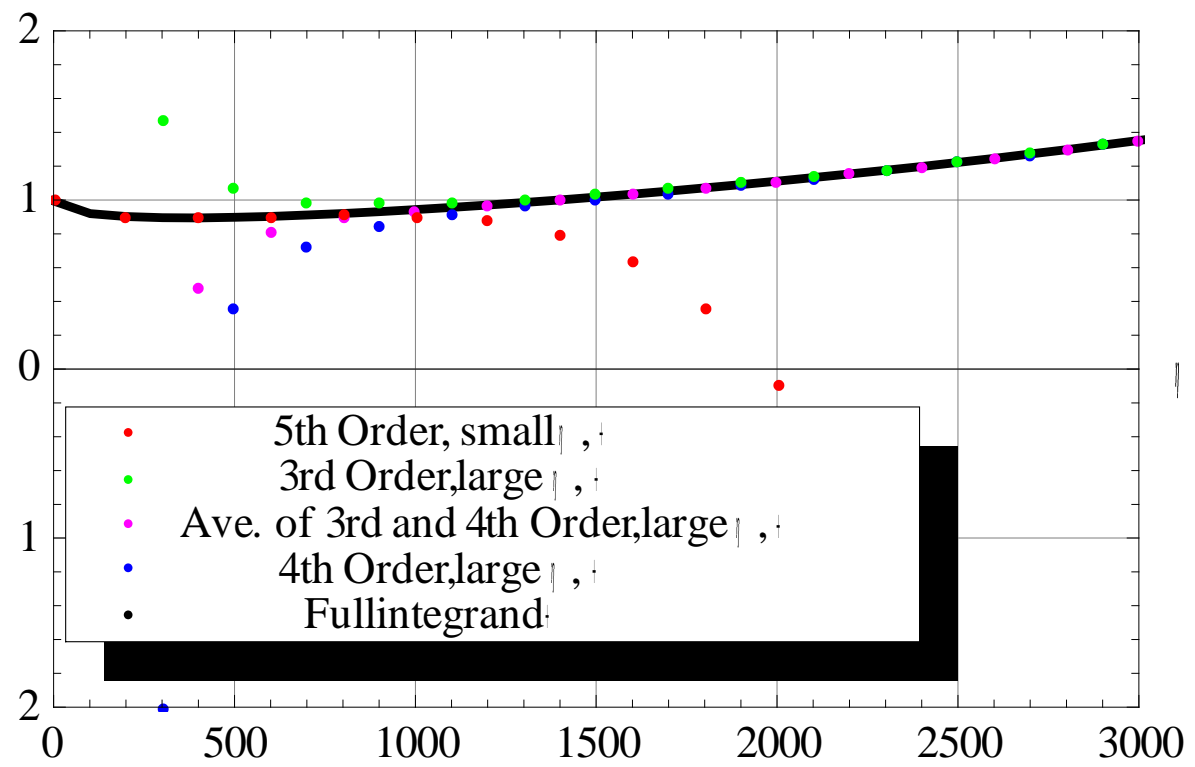

Figure 36. Same as for Figure 34, but with $\alpha=10^{-3} \mathrm{~m}^{-1}$.

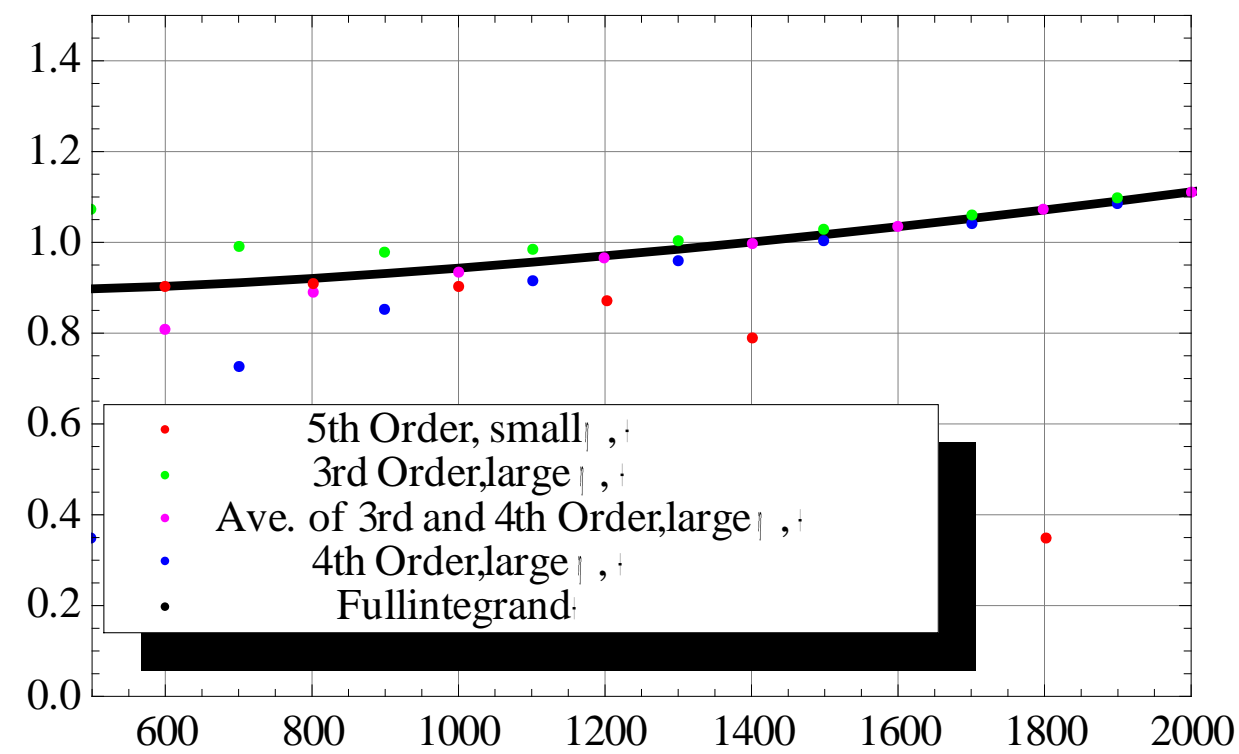

Figure 37. Detail of Figure 36 for $500 \leq \eta \leq 2000 m$. 


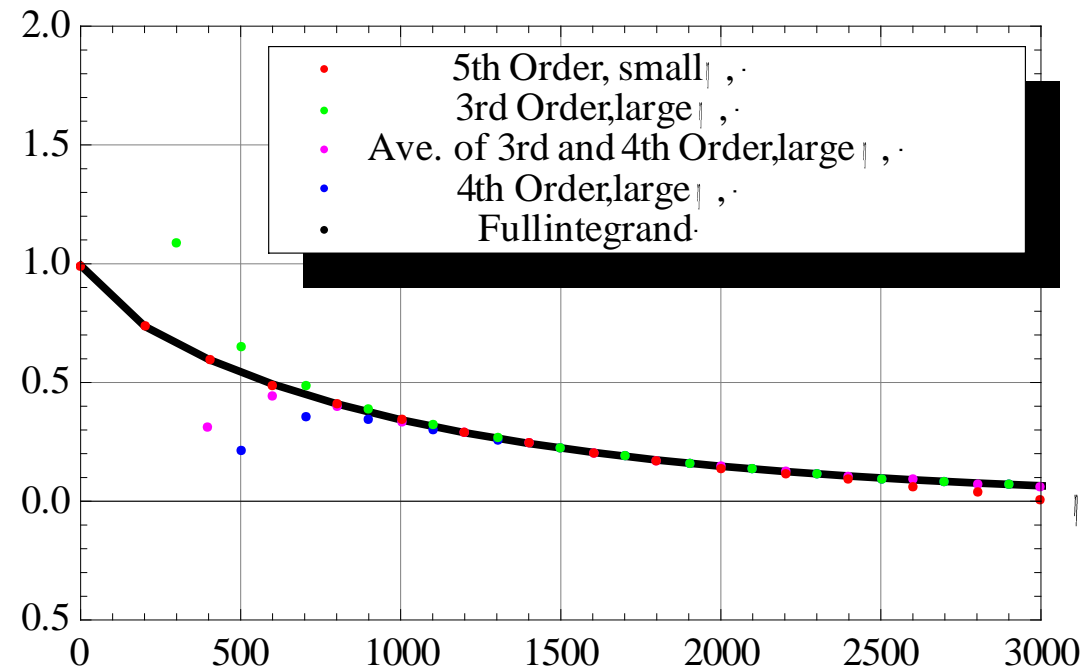

Figure 38. Large- $\eta$ approximations to the integrand, $L_{T}=1000 \mathrm{~m}$.

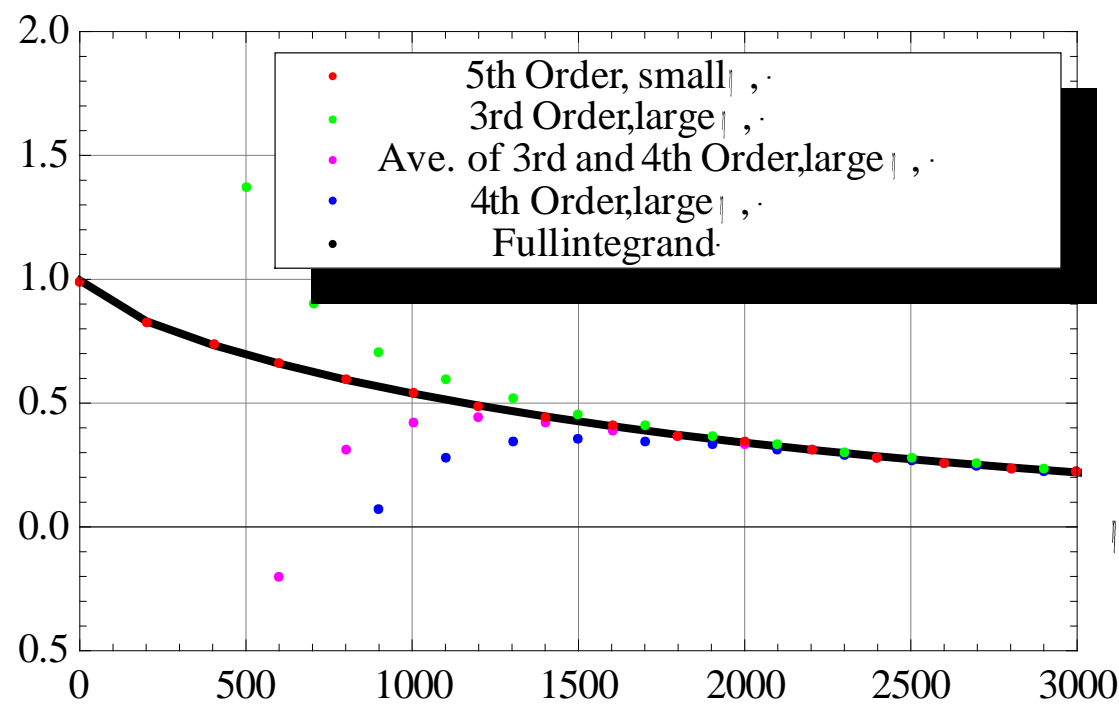

Figure 39. Same as Figure 38, but with $L_{T}=2000 \mathrm{~m}$. 


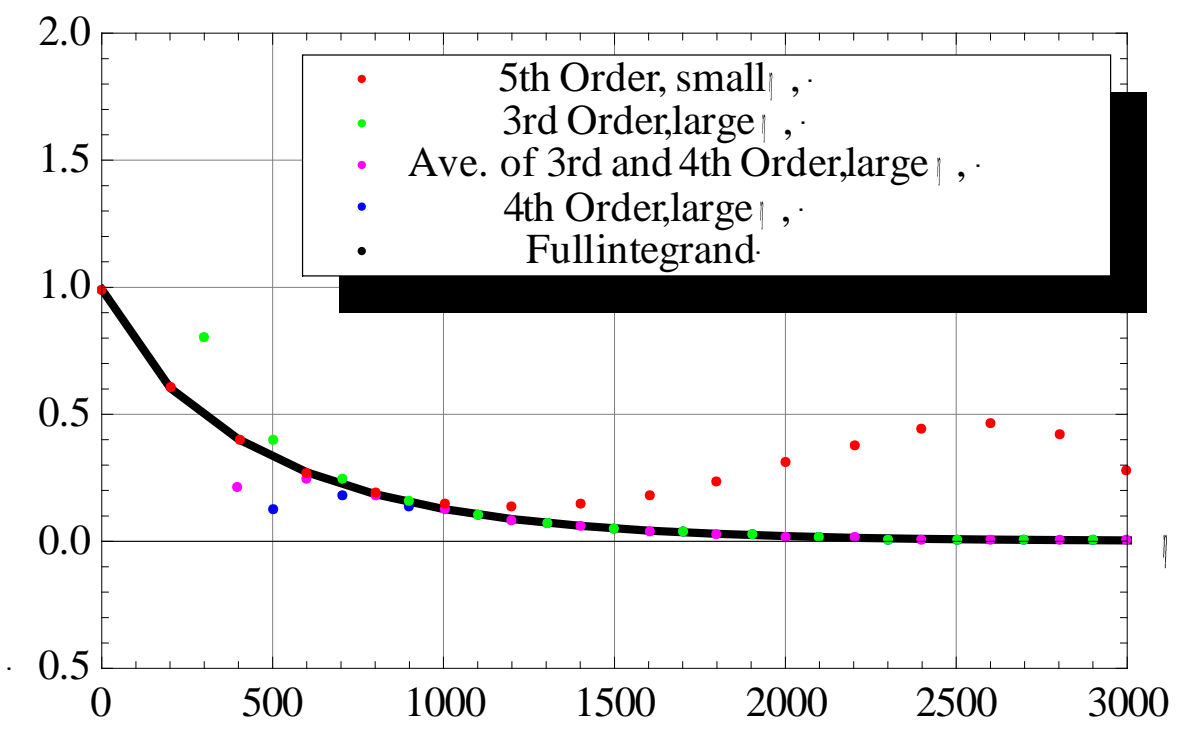

Figure 40. Same as Figure 38, but with $\alpha=10^{-3} \mathrm{~m}^{-1}$.

Next we turn to the analysis of the radiance correlation function, Eq. (21). Figure 41 is a plot of Radiance correlation function varying $\sigma_{T}$ - for $L_{T}=1000 \mathrm{~m}, x=0, L=10 \mathrm{~km}$, and $v=704 \mathrm{~cm}^{-1}$. The plot shows the dependency of the radiance correlation function on the turbulence intensity. It can be seen that the correlation function is clearly sensitive to this parameter. This is not unexpected since each of the terms in Eq. (21) (via Eq. (22)) has $\sigma_{T}^{2}$ as a multiplier. Nevertheless, this is a key fact - since if there was no clear sensitivity to the turbulence intensity, this approach would be fruitless. The next two plots illustrate a weaker dependency on the total turbulence path length. This also is an a fortiori fact, since the path length through the turbulence will never be known a priori. Figure 44 and Figure 45 indicate that the radiance correlation function is significantly dependent on the turbulence length scale. Nevertheless, the same general pattern occurs: larger turbulence intensity gives a larger correlation function value. Figure 46 continues with the pattern seen above - the absorption coefficient has a very significant effect. Note that this figure is plotted in log space, due to the large dynamic range.

The next set of plots involves the radiance correlation coefficient, that is, the correlation function divided by its value at zero lag $(\rho=0)$. As expected, the variation due to the turbulence intensity is gone (same multiplier in numerator and denominator). Figure 48 shows that there is still a small effect on the correlation coefficient due to the turbulence length scale. Note how this effect is opposite to the effect on the correlation function itself. As the length scale was increased the correlation function increased, so when normalizing by the value at zero lag, the correlation coefficient will get smaller. Figure 49 again shows the dramatic effect due to large changes in the absorption coefficient, and Figure 50 shows the effect due to the turbulence path length. The von Karman correlation function (with $\sigma_{T}^{2}=1$ ) is shown for reference. It is clear that the radiance, being a path-weighted integrated value, increases the correlation over what one might measure with a temperature probe on the aircraft. 


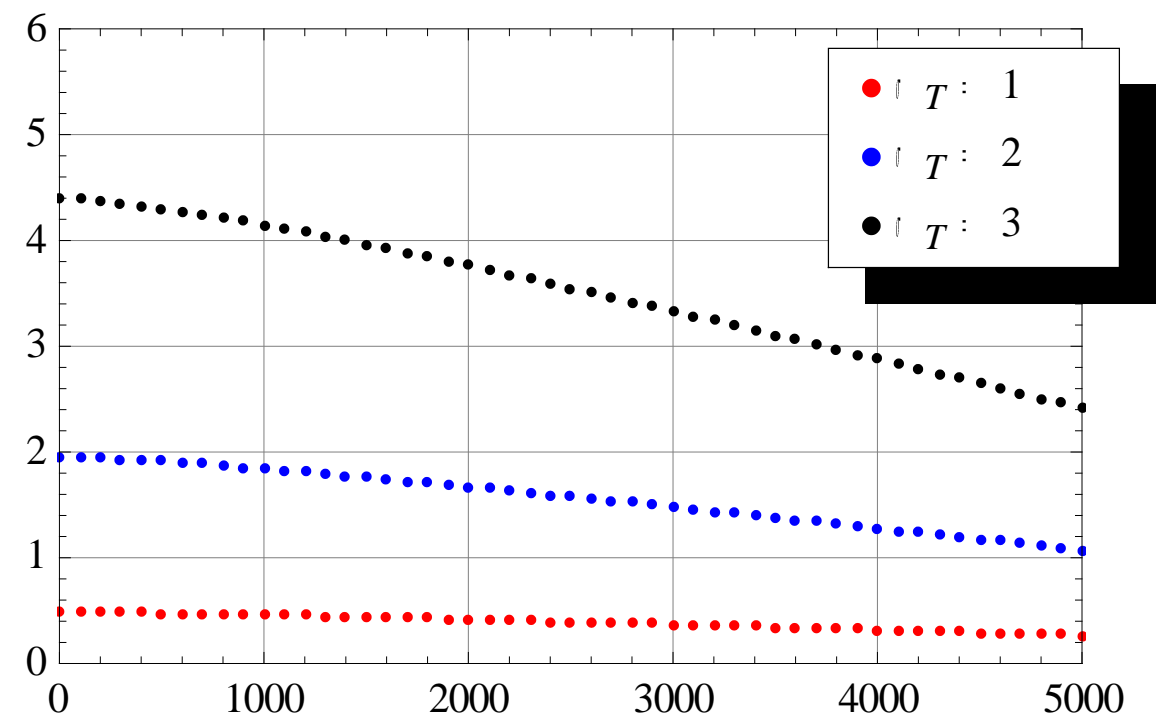

Figure 41. Radiance correlation function, varying $\sigma_{T}$.

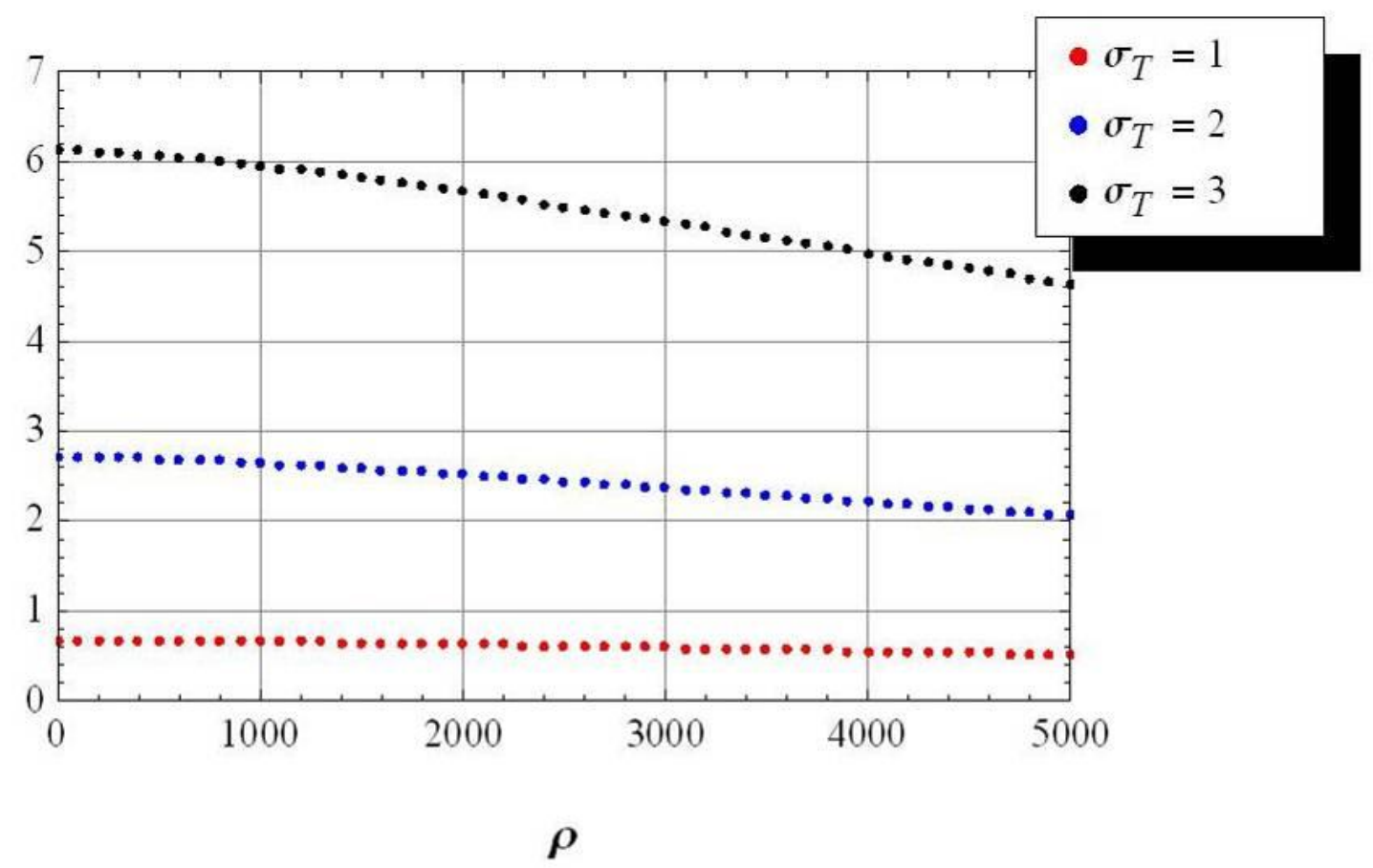

Figure 42. Same as Figure 41, excepting for $L=20 \mathrm{~km}$. 


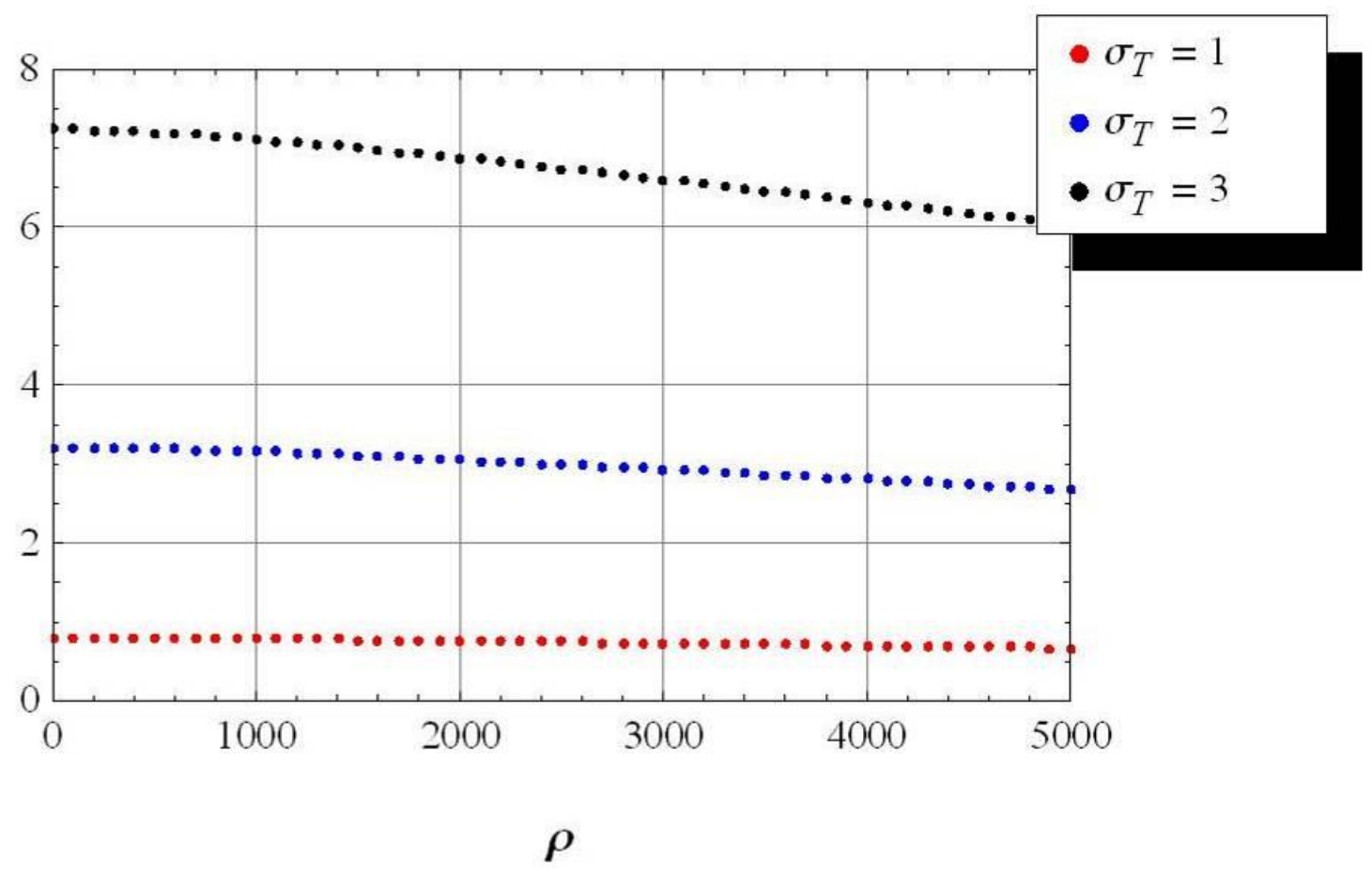

Figure 43. Same as Figure 41, excepting for $L=100 \mathrm{~km}$.

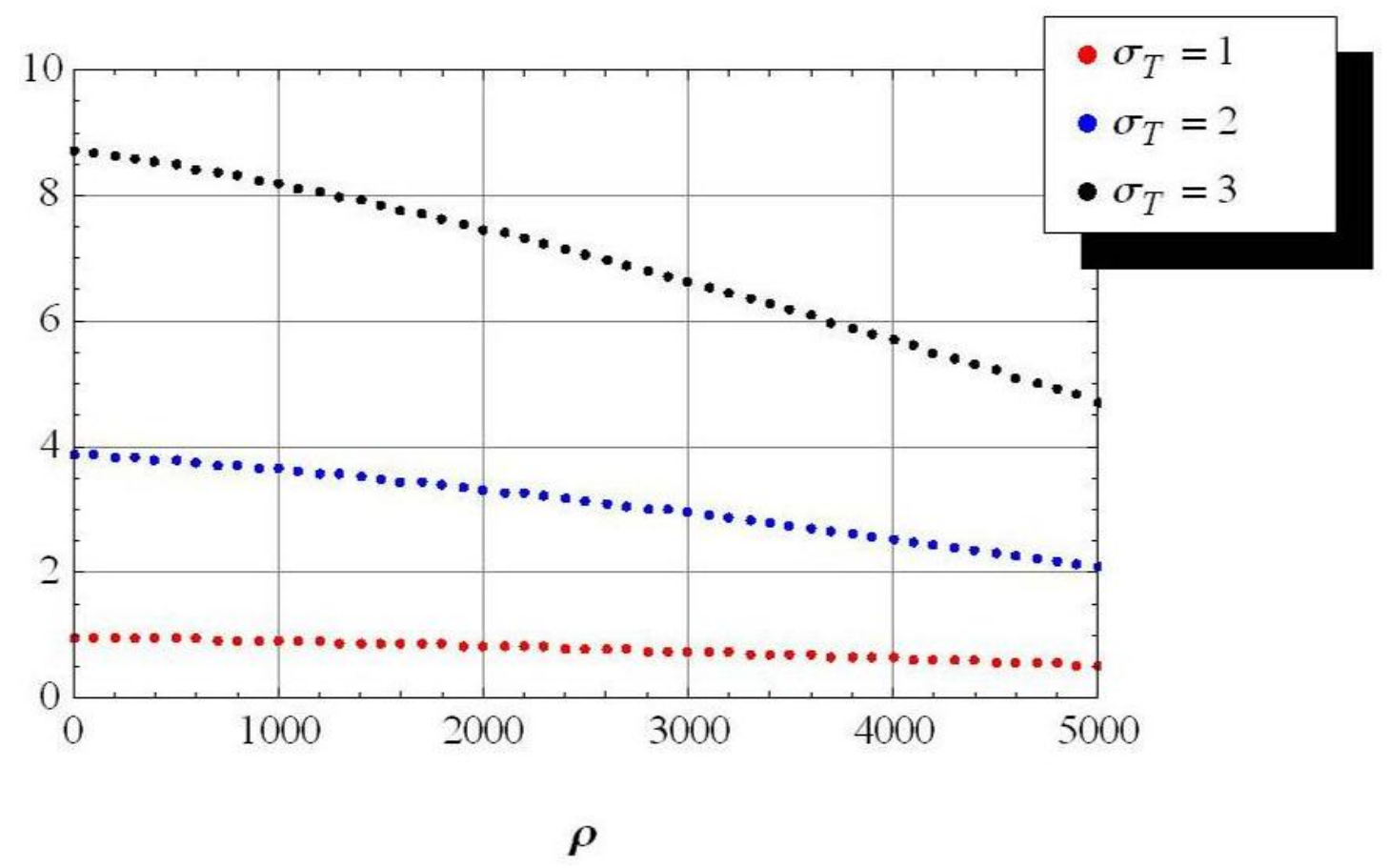

Figure 44. Same as Figure 41, excepting $L_{T}=2000 \mathrm{~m}$. 


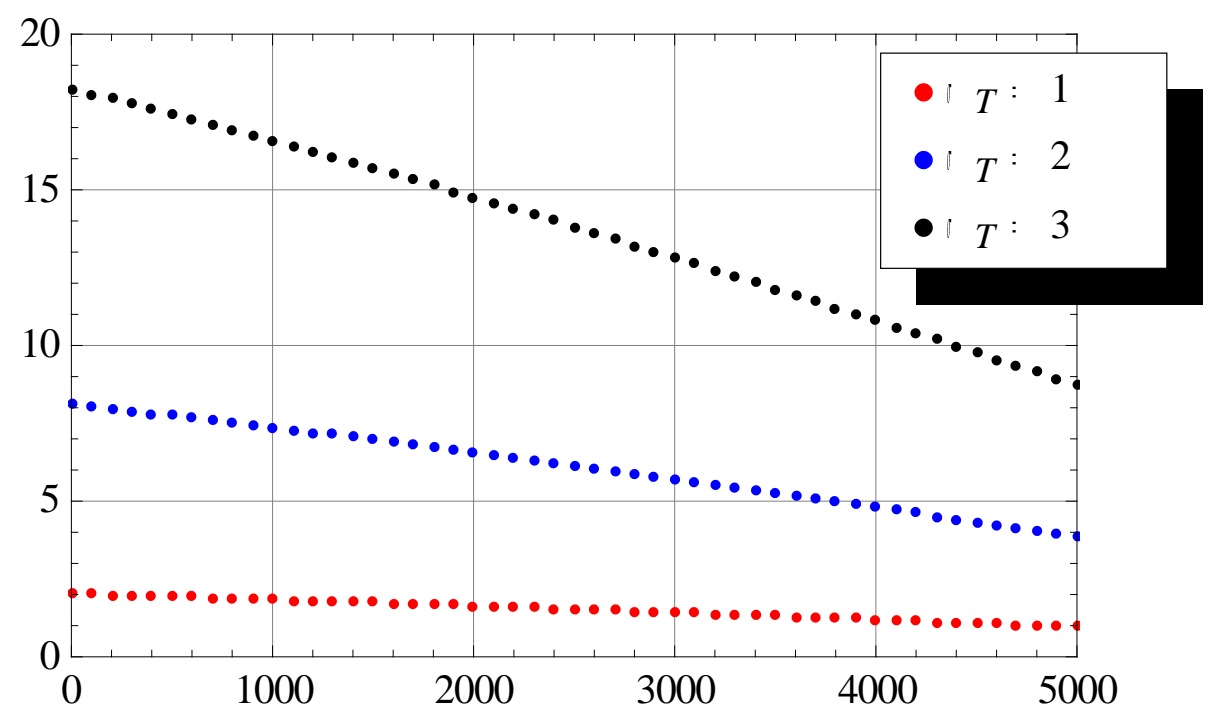

Figure 45. Same as Figure 41, but with $L_{T}=5000 \mathrm{~m}$.

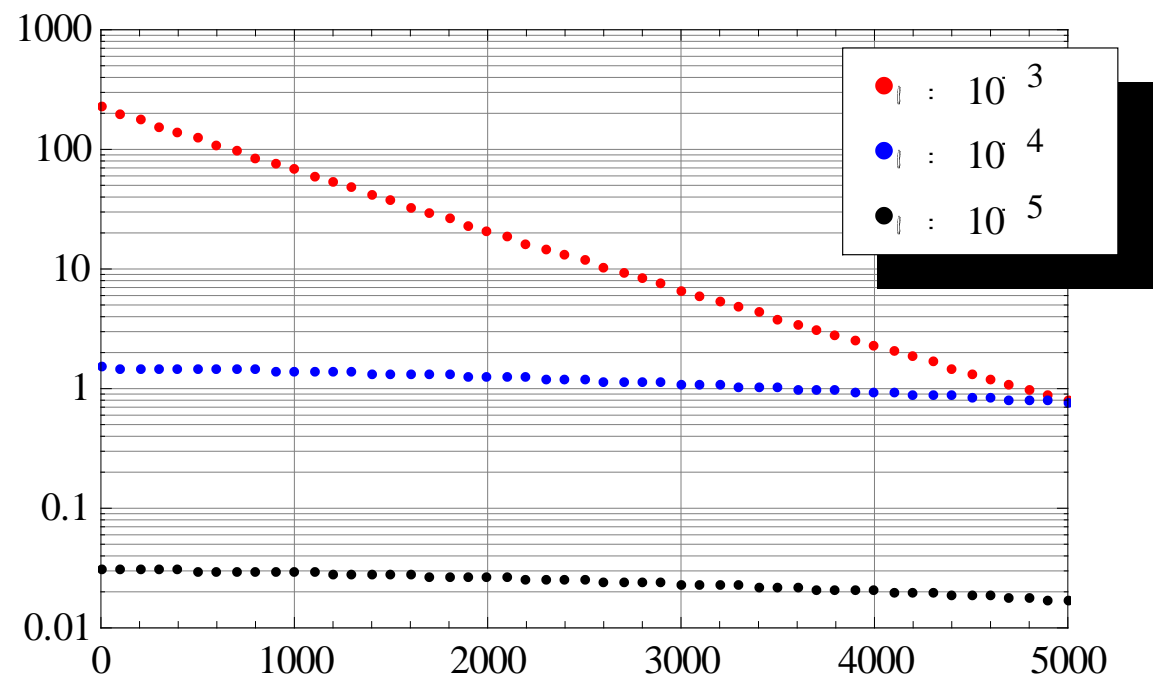

Figure 46. Same as Figure 41, excepting that $\alpha=10^{-3}, 10^{-4}, 10^{-5}$. 


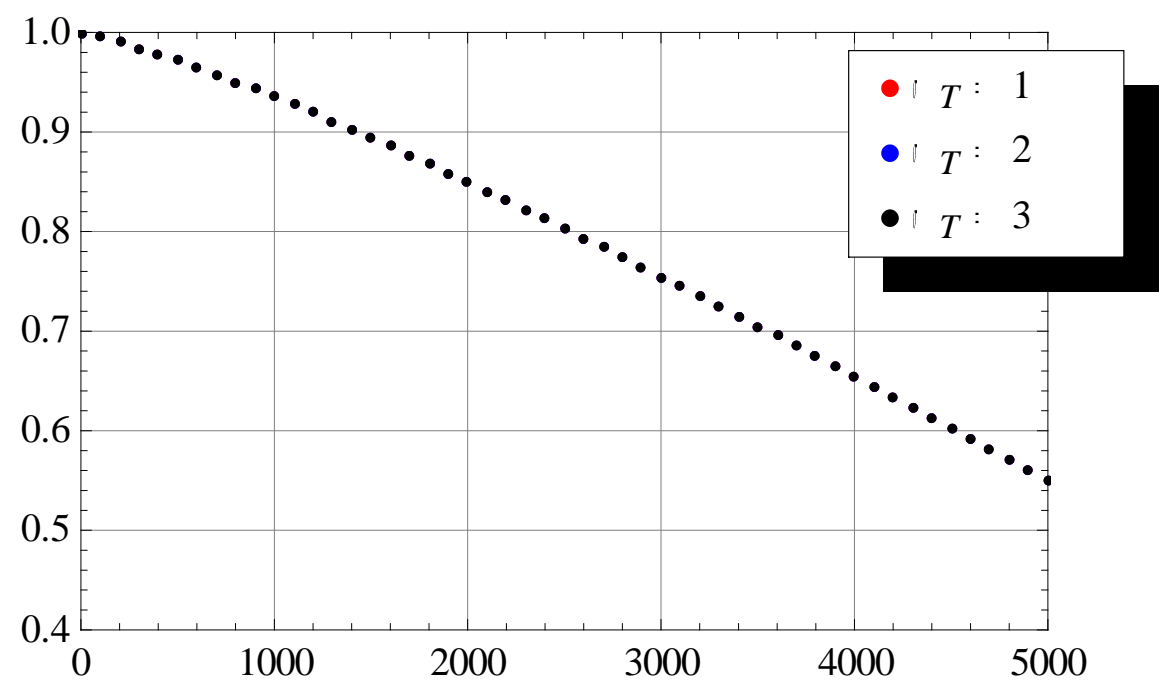

Figure 47. Correlation coefficient using same parameters as Figure 41.

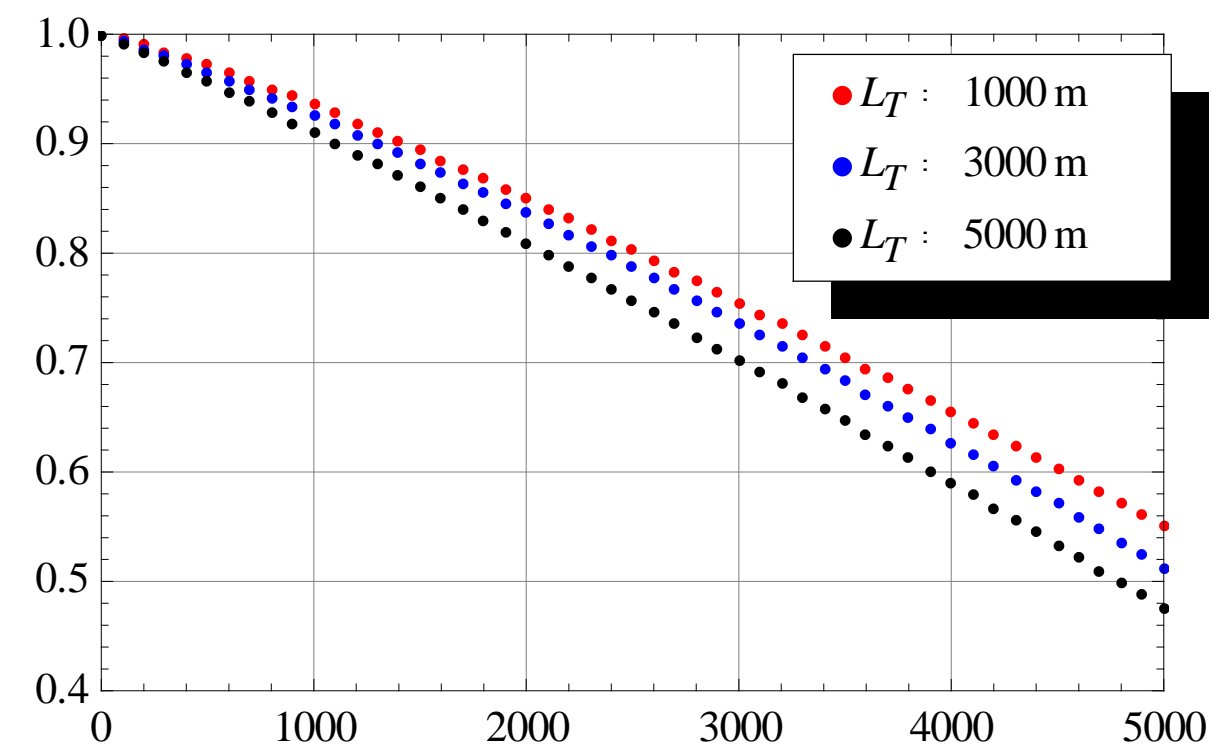

Figure 48. Same as Figure 47 but varying turbulence length scale. 


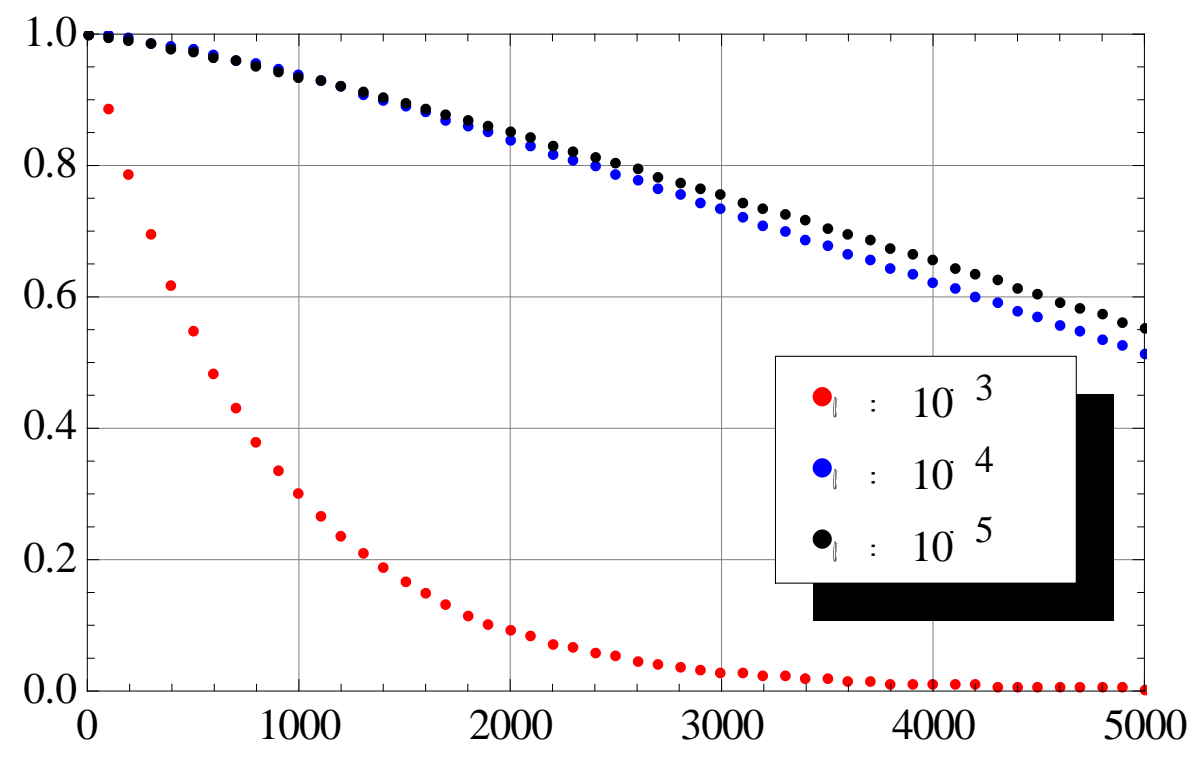

Figure 49. Same as Figure 47, but varying the absorption coefficient.

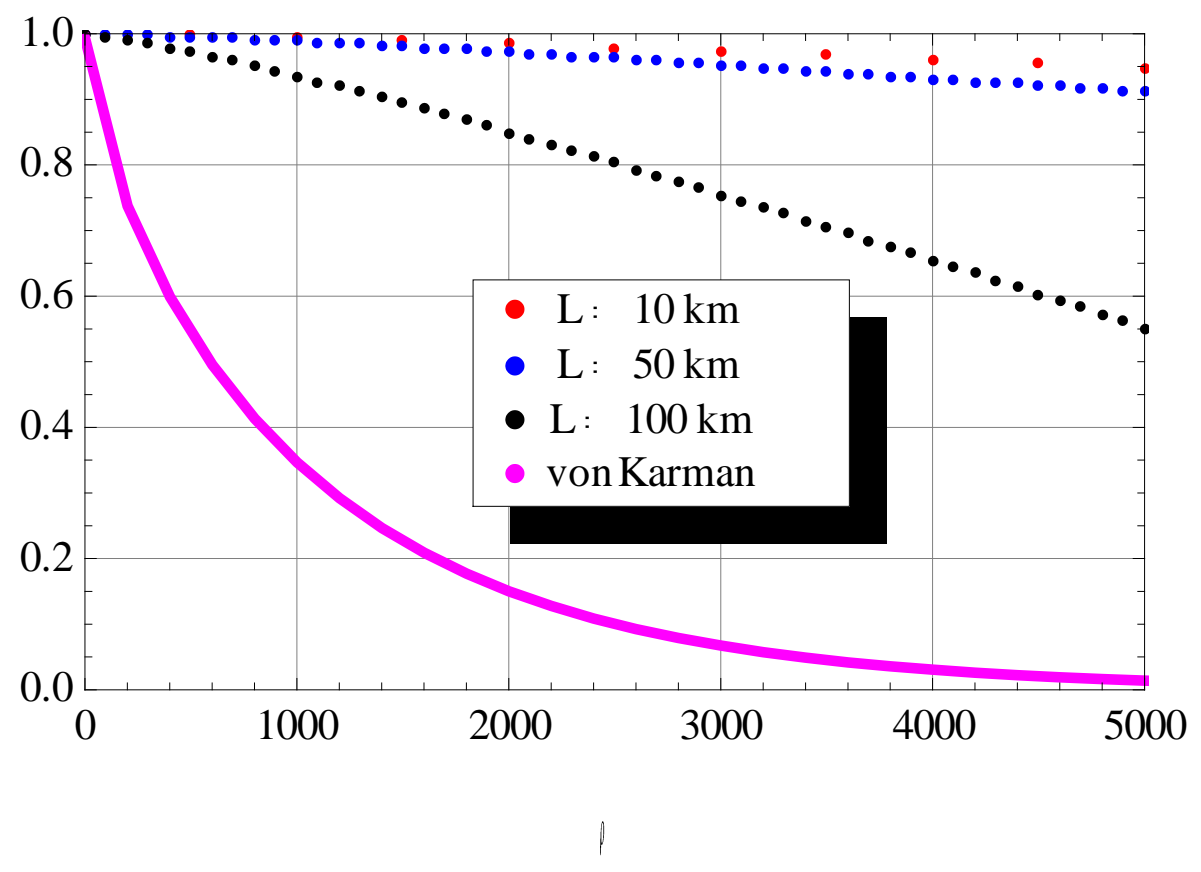

Figure 50. Same as Figure 47, but varying the total turbulence path length, $\mathrm{L}$.

\subsubsection{Model Fits to the Planck Function}

In the proceeding section, the analysis assumed that the Planck function could be approximated by a linear function of temperature. 
Linear and exponential fits to the Planck function as a function of temperature, were calculated for each wavenumber. The Planck function is given by:

$$
B_{v}(T)=\frac{k_{1} v^{3}}{e^{k_{2} v / T}-1}
$$

Where, $k_{1} \approx 1.2 \times 10^{-5}$ and $k_{2} \approx 1.44$, when the radiance is given in $\mathrm{mW} /\left(\mathrm{sr} \mathrm{cm}^{-1} \mathrm{~m}^{2}\right)$. Note that when $\mathrm{k}_{2} v / \mathrm{T}>>1, B_{v}(T)=k_{1} v^{3} e^{-k_{2} v / T}$, and when $\mathrm{k}_{2} v / \mathrm{T}<<1, B_{v}(T) \approx \frac{k_{1}}{k_{2}} v^{2} T$.

From the latter approximation, we choose a linear model of the form:

$$
B_{v}(T(x))=a_{v}+b_{v} T(x)
$$

Whereas, in the former case, we choose an exponential model of the form:

$$
B_{v}(T(x))=c_{v} e^{-d_{v} / T(x)}
$$

Note that all of the model coefficients - while containing the standard constants $\left(k_{1}, k_{2}\right)-$ are dependent on $v$.

Note that the linear function used in this analysis does not necessarily have to go through the origin, i.e., it is an approximation to the Planck function over the range of temperatures and wavenumbers that were considered. In the theoretical analysis given in the preceding section, the function, $B_{v}(T)=\frac{k_{1}}{k_{2}} v^{2} T$ was used, not the full linear function analyzed here.

First, let $T(x)=\bar{T}(x)+T^{\prime}(x)$. Assuming that the turbulent part of temperature, $T^{\prime}(x)$, is a zeromean process, i.e., $\left\langle T^{\prime}(x)\right\rangle=0$, and the expectation is a linear operator, it is easy to show that the correlation function of the temperature field is,

$$
\left\langle T\left(x_{1}\right) T\left(x_{2}\right)\right\rangle=\bar{T}\left(x_{1}\right) \bar{T}\left(x_{2}\right)+\left\langle T^{\prime}\left(x_{1}\right) T^{\prime}\left(x_{2}\right)\right\rangle
$$

This leads to,

$$
\begin{aligned}
\left\langle B_{v}\left(T\left(x_{1}\right)\right) B_{v}\left(T\left(x_{2}\right)\right)\right\rangle & =\left\langle a_{v}+b_{v} T\left(x_{1}\right) a_{v}+b_{v} T\left(x_{2}\right)\right\rangle \\
& =a_{v}^{2}+a_{v} b_{v}\left[\bar{T}\left(x_{1}\right)+\bar{T}\left(x_{2}\right)\right]+b_{v}^{2}\left[\bar{T}\left(x_{1}\right) \bar{T}\left(x_{2}\right)+\left\langle T^{\prime}\left(x_{1}\right) T^{\prime}\left(x_{2}\right)\right\rangle\right]
\end{aligned}
$$


If it is further assumed that $\bar{T}\left(x_{1}\right)=\bar{T}\left(x_{2}\right)=\bar{T}(x)$,

$$
\left\langle B_{v}\left(T\left(x_{1}\right)\right) B_{v}\left(T\left(x_{2}\right)\right)\right\rangle=\left[a_{v}+b_{v} \bar{T}(x)\right]^{2}+b_{v}^{2} R_{T}\left(x_{2}-x_{1}\right)
$$

This indicates that there is just a constant additive factor over the temperature correlation function used above.

The fits were calculated via a least-squares minimization algorithm. In the following, a set of fits to these models and error factors for different wavenumbers are presented.

It is clear that the linear fits only work well at the smaller wavenumbers, whereas the exponential fit is excellent over the entire range of wavenumbers. Unfortunately, the theoretical analysis of the exponential model is quite difficult, so for now we stick to the low wavenumber regime and use the linear model.

A plot of this linear fit is shown in Figure 51 for $v=400 \mathrm{~cm}^{-1}$. In this plot, the $\mathrm{x}$-axis units are temperature in degrees Kelvin and the y-axis as Planck function radiance in units $\mathrm{mW} /\left(\mathrm{sr} \mathrm{cm}^{-1}\right.$ $\left.\mathrm{m}^{2}\right)$. Subsequent plots, each computed at increments of $200 \mathrm{~cm}^{-1}$, are shown in Figure $52(v=$ $\left.600 \mathrm{~cm}^{-1}\right)$ through Figure $56\left(v=1400 \mathrm{~cm}^{-1}\right)$.

Error plots for the linear fits are shown in Figure 57 through Figure 62. As before, all of these plots have the same axes units, the $\mathrm{x}$-axis is temperature in units degrees Kelvin and the $y$-axis is Planck function in units $\mathrm{mW} /\left(\mathrm{sr} \mathrm{cm}^{-1} \mathrm{~m}^{2}\right)$.

A plot of the exponential fit is shown in Figure 63 for $v=400 \mathrm{~cm}^{-1}$. In this plot, the x-axis units are temperature in degrees Kelvin and the y-axis as Planck function radiance in units $\mathrm{mW} /\left(\mathrm{sr} \mathrm{cm}^{-}\right.$ $\left.{ }^{1} \mathrm{~m}^{2}\right)$. Subsequent plots, each computed at increments of $200 \mathrm{~cm}^{-1}$, are shown in Figure $64(v=$ $\left.600 \mathrm{~cm}^{-1}\right)$ through Figure $68\left(v=1400 \mathrm{~cm}^{-1}\right)$.

Error plots for the exponential fits are shown in Figure 69 through Figure 74. As before, all of these plots have the same axes units, the $\mathrm{x}$-axis is temperature in units degrees Kelvin and the $y$ axis is Planck function in units $\mathrm{mW} /\left(\mathrm{sr} \mathrm{cm}^{-1} \mathrm{~m}^{2}\right)$. 


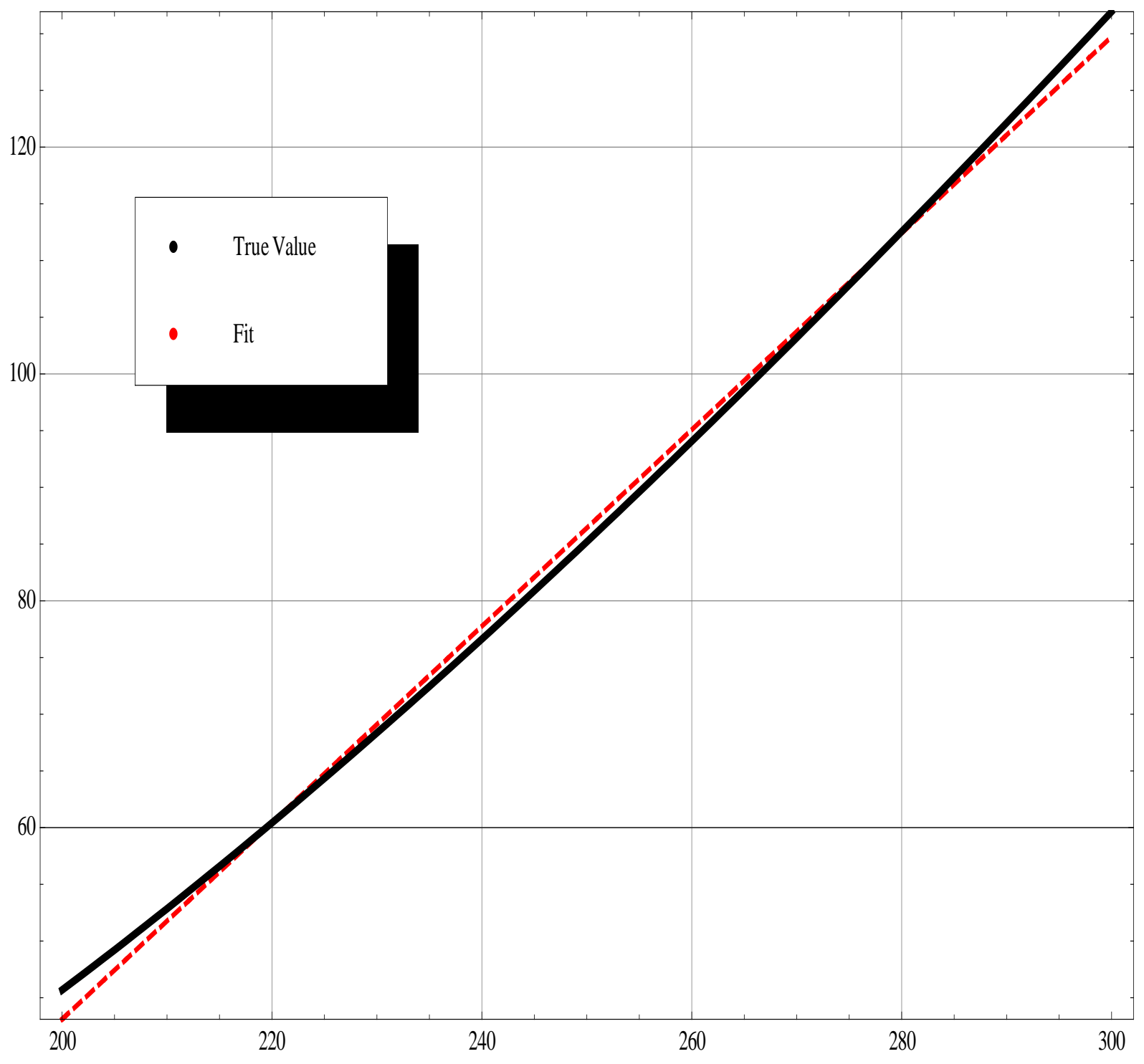

Figure 51. Linear fit to the Planck function for $v=400 \mathrm{~cm}^{-1}$. 


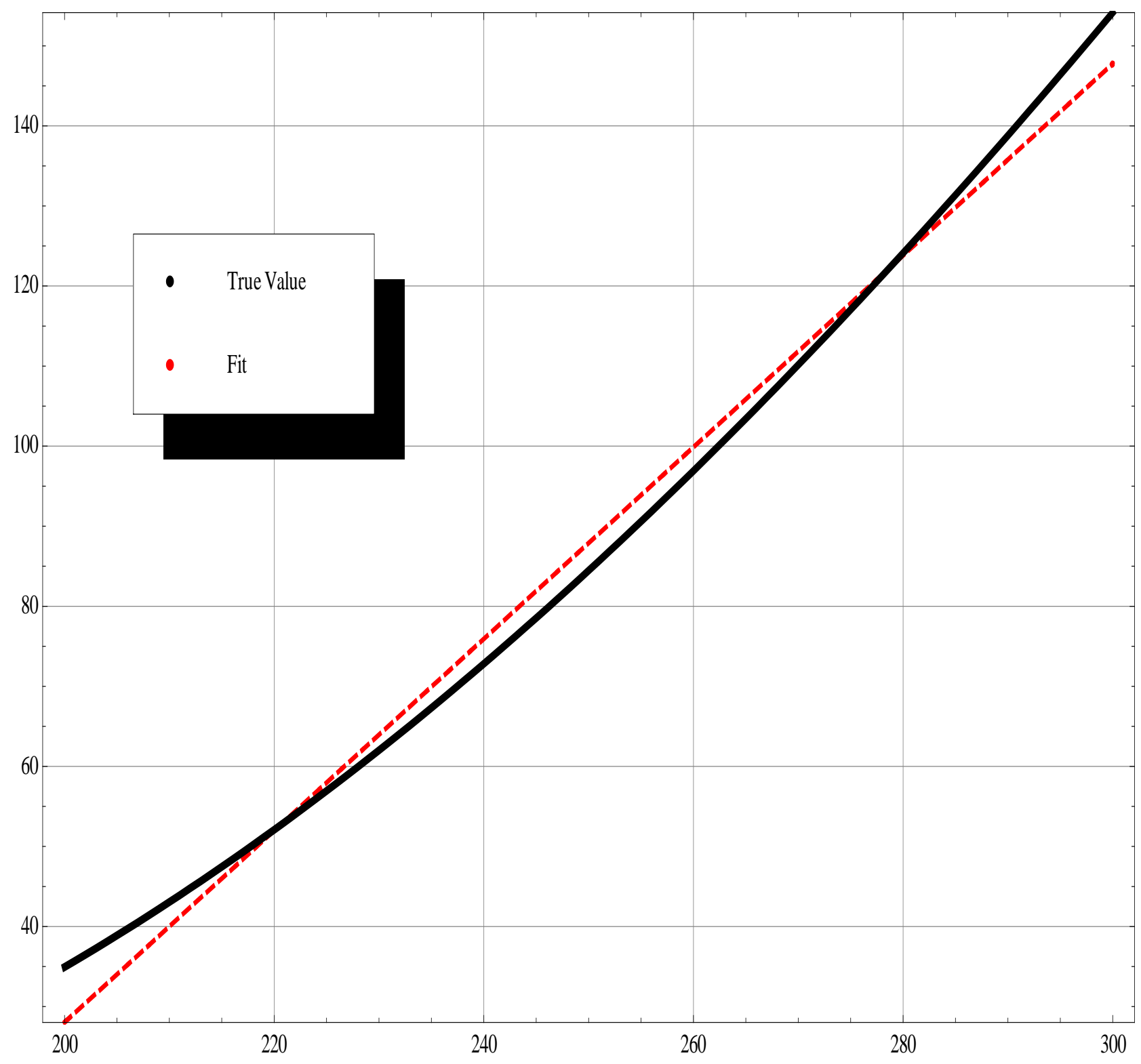

Figure 52. Linear fit to the Planck function for $v=600 \mathrm{~cm}^{-1}$. 


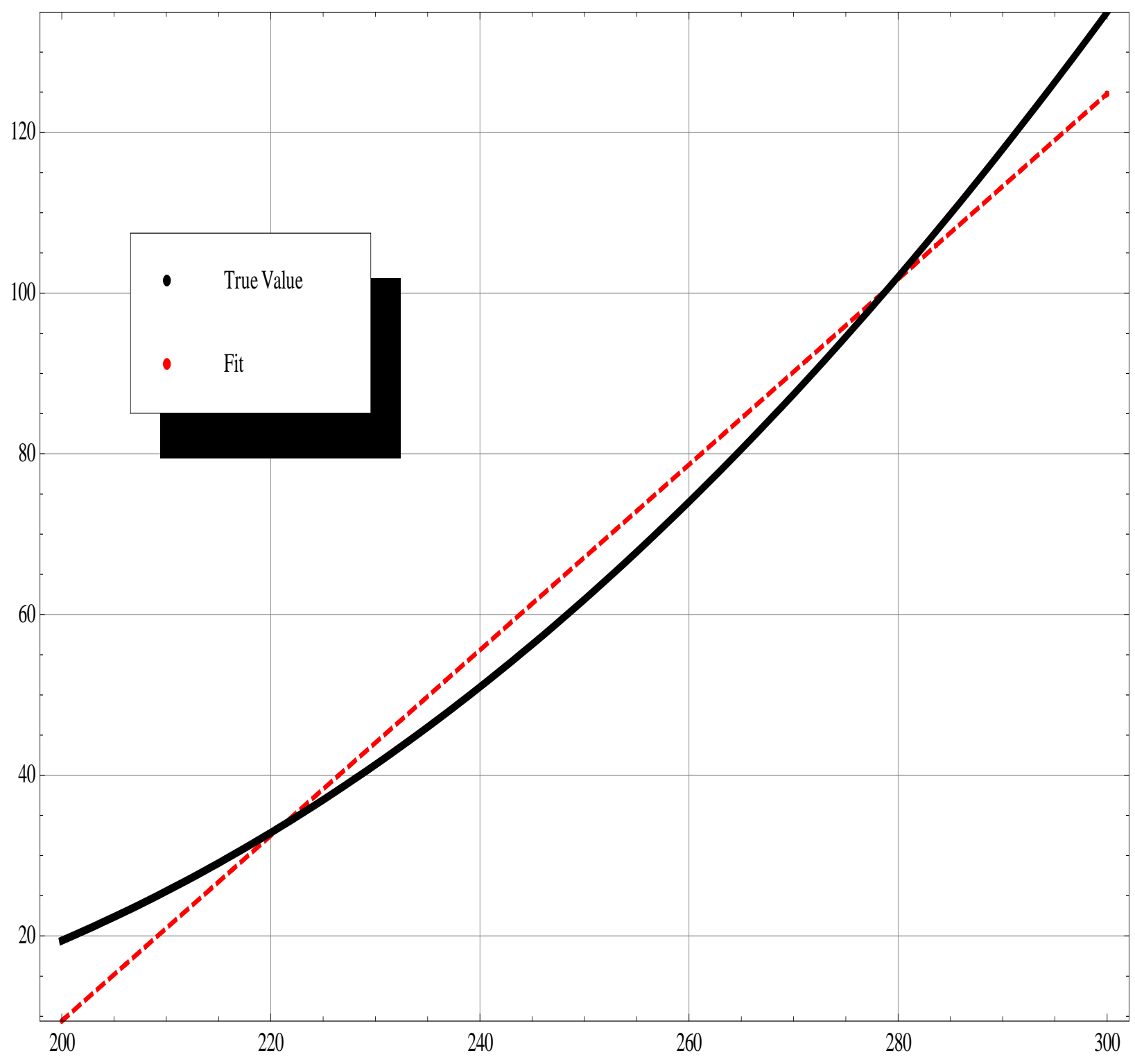

Figure 53. Linear fit to the Planck function for $v=800 \mathrm{~cm}^{-1}$. 


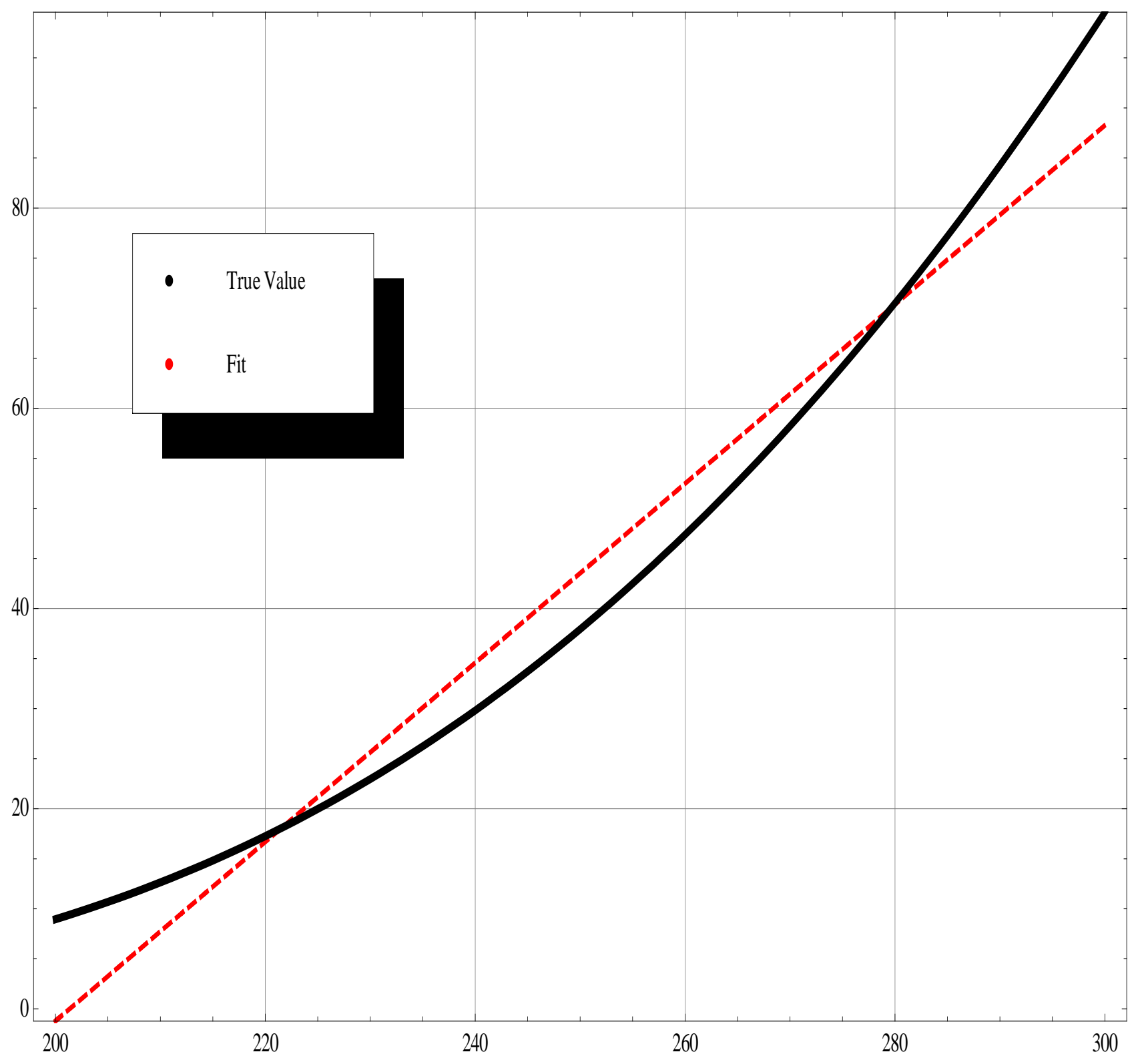

Figure 54. Linear fit to the Planck function for $v=1000 \mathrm{~cm}^{-1}$. 


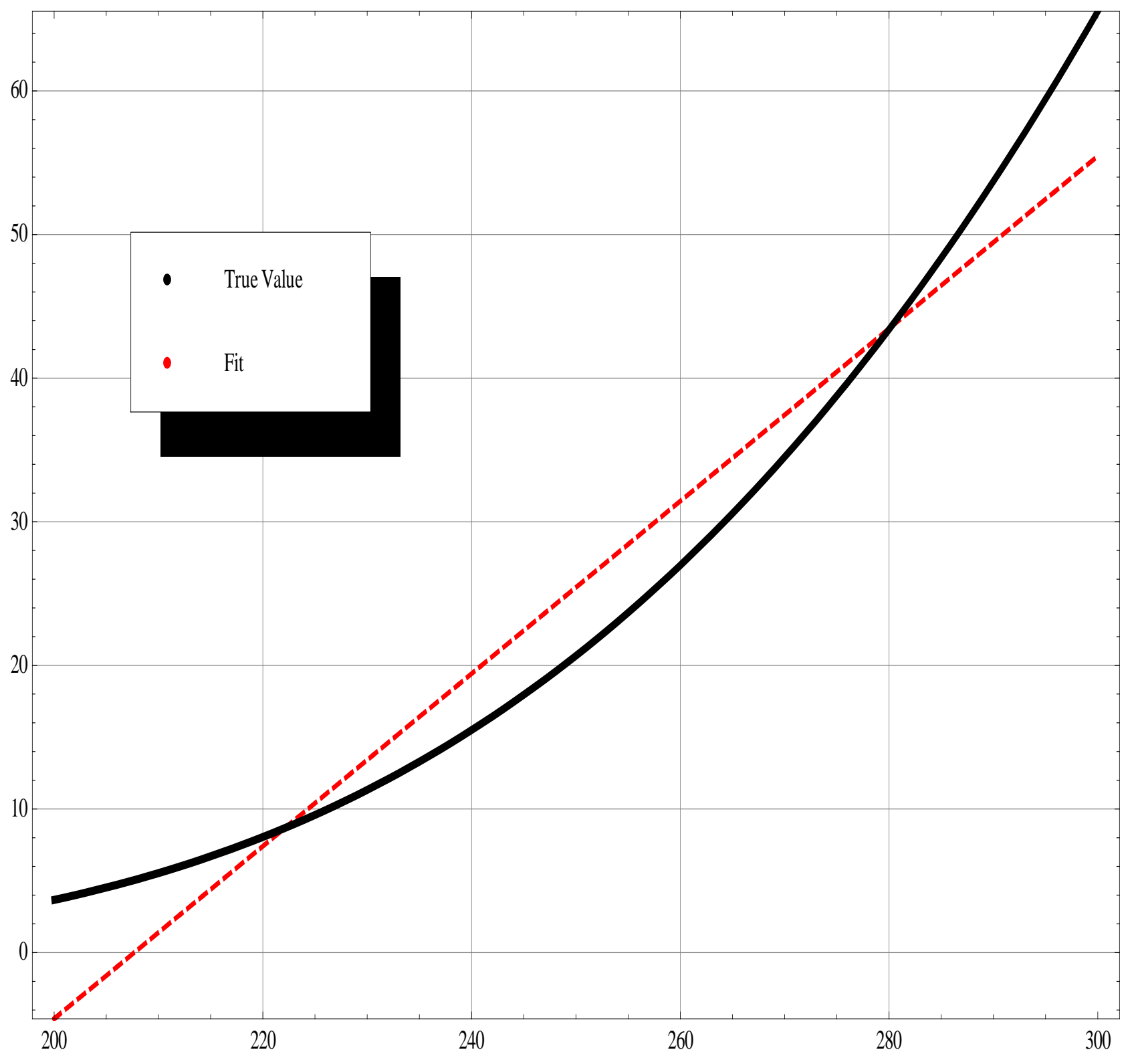

Figure 55. Linear fit to the Planck function for $v=1200 \mathrm{~cm}^{-1}$. 


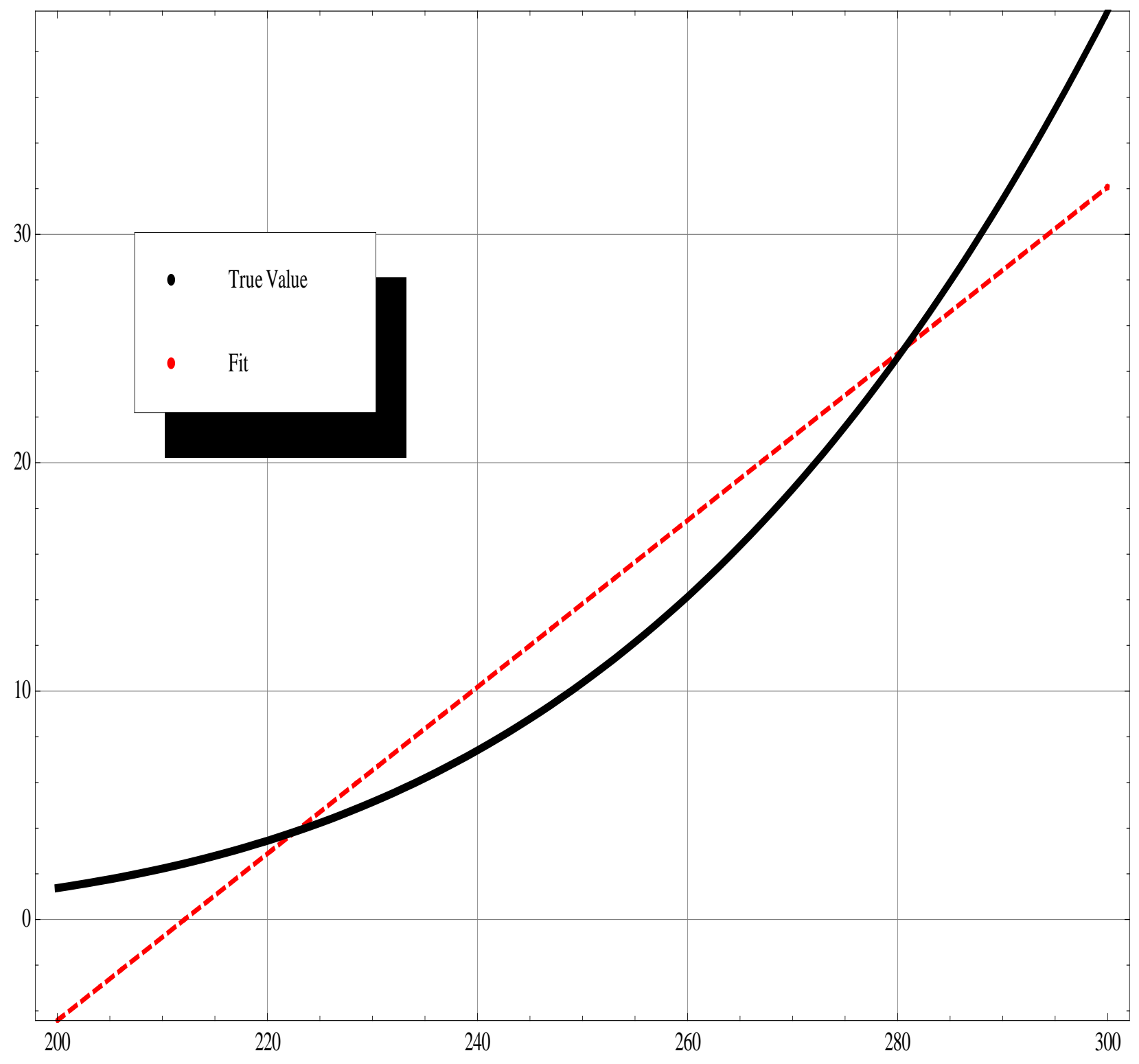

Figure 56. Linear fit to the Planck function for $v=1400 \mathrm{~cm}^{-1}$ 


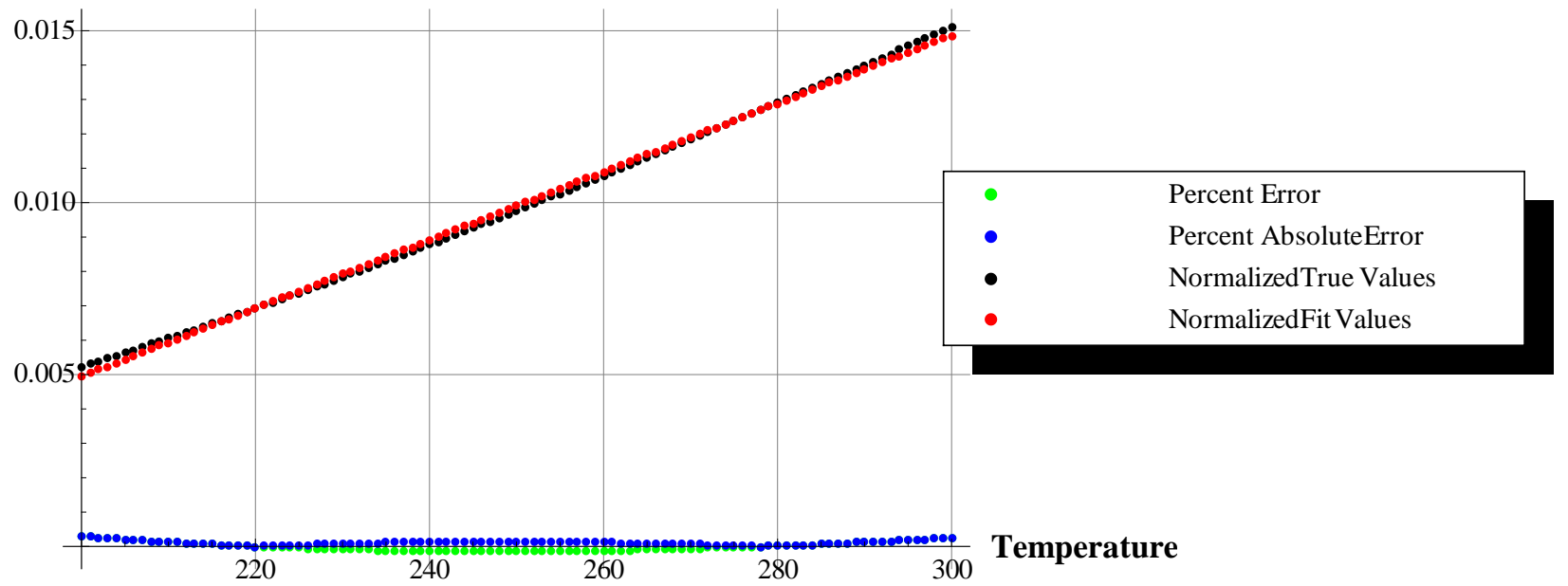

Figure 57. Errors for the linear model for $v=400 \mathrm{~cm}^{-1}$

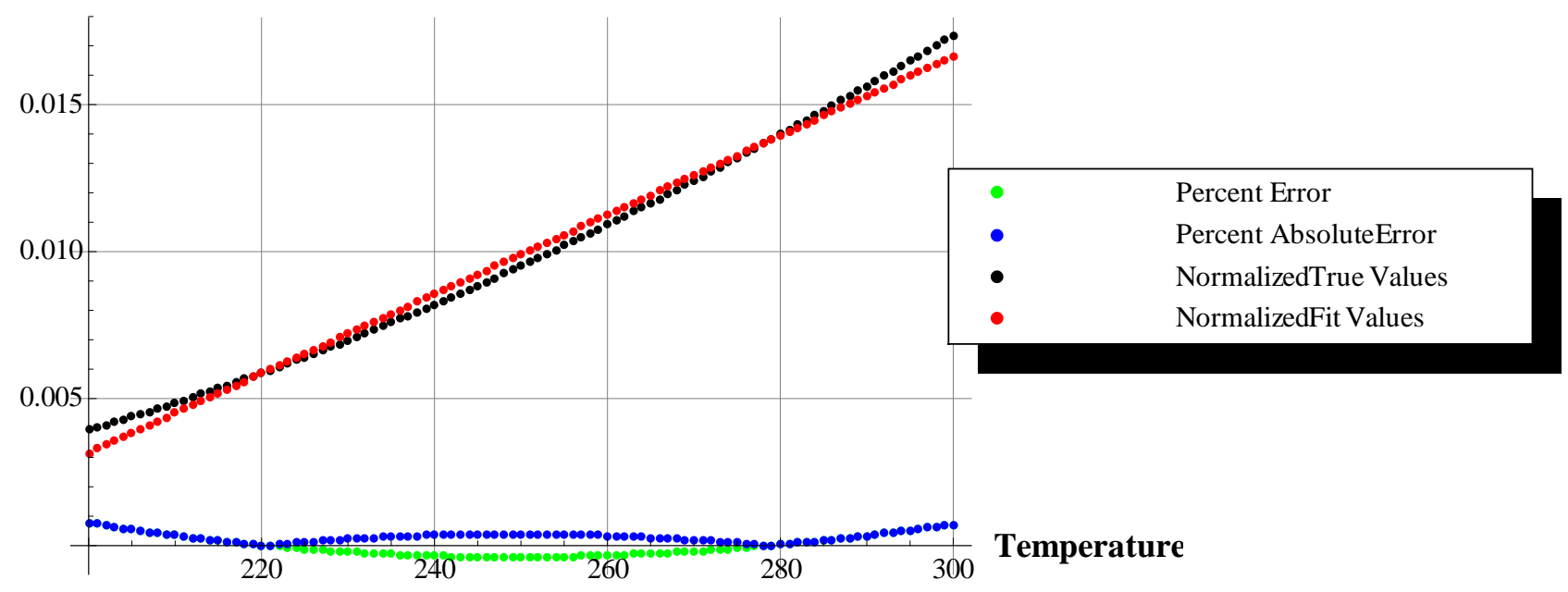

Figure 58. Errors for the linear model for $v=600 \mathrm{~cm}^{-1}$

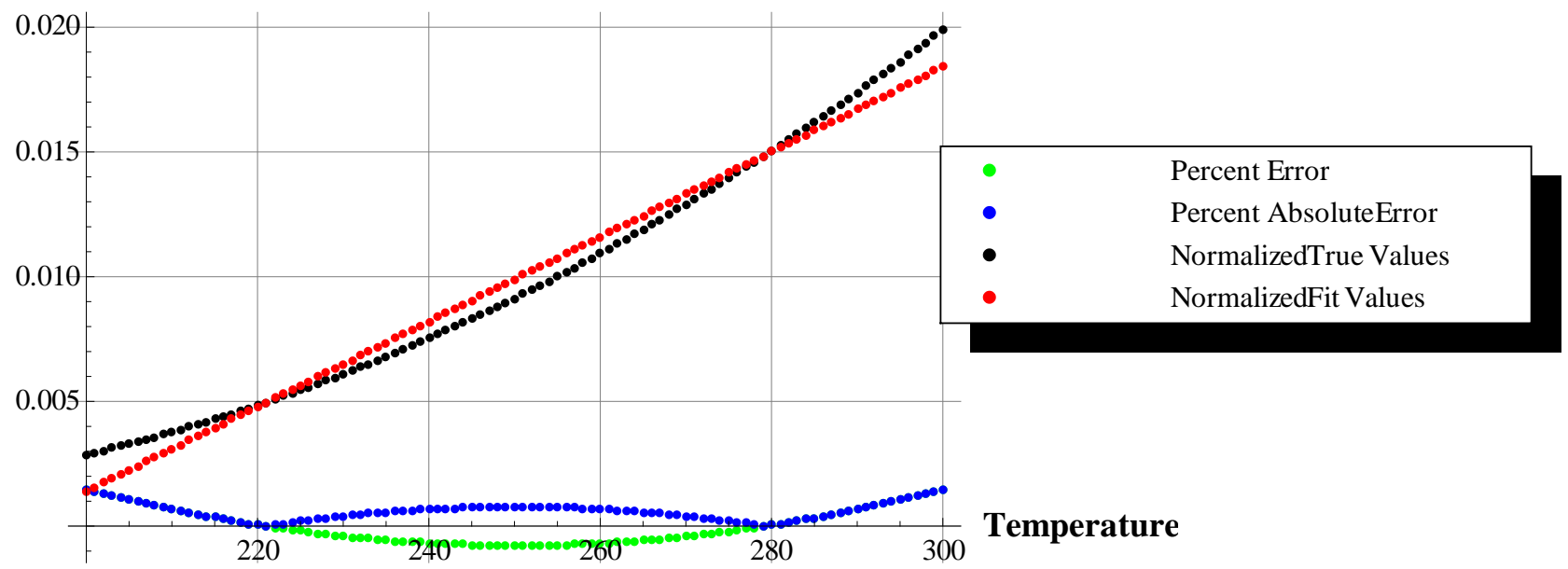


Figure 59. Errors for the linear model for $v=800 \mathrm{~cm}^{-1}$.

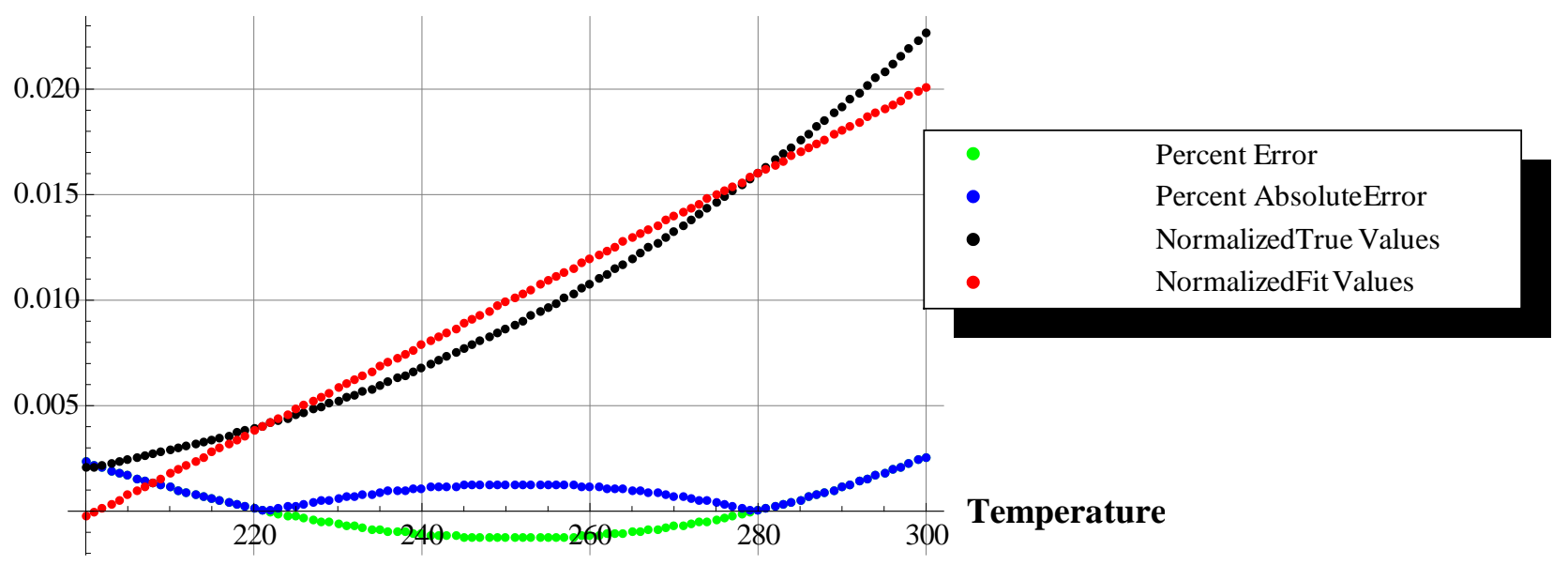

Figure 60. Errors for the linear model for $v=1000 \mathrm{~cm}^{-1}$.

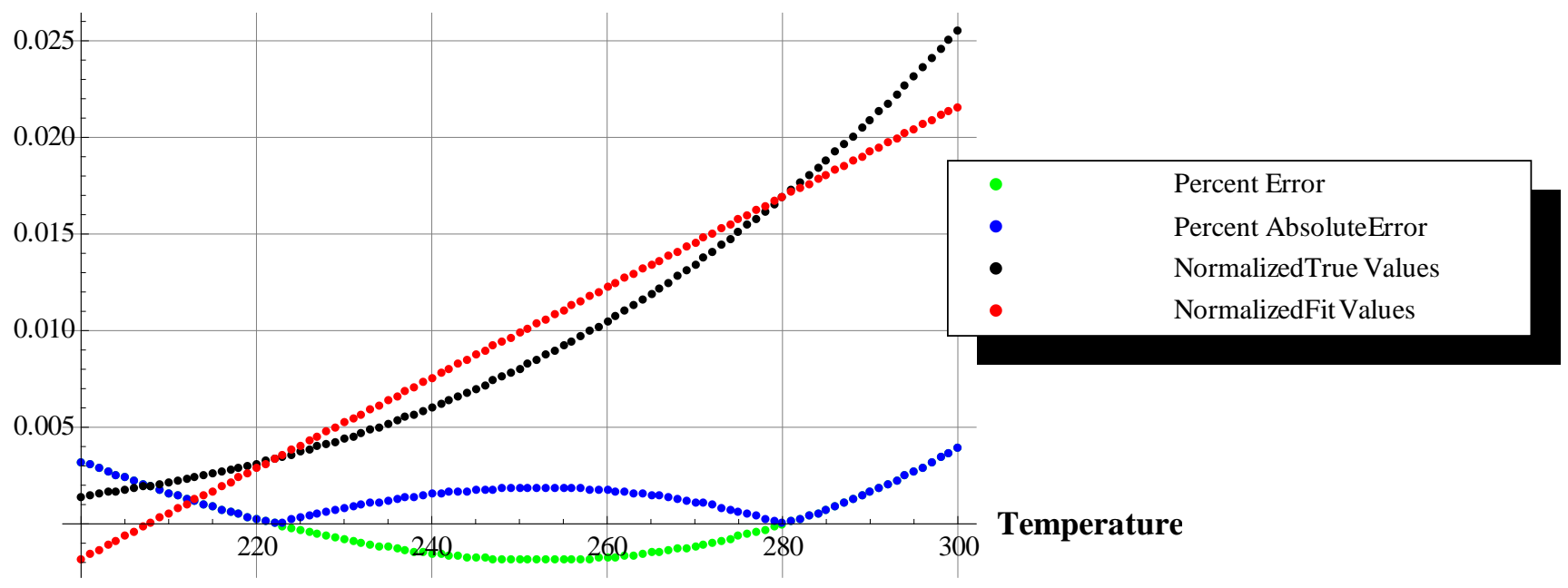

Figure 61. Errors for the linear model for $v=1200 \mathrm{~cm}^{-1}$ 


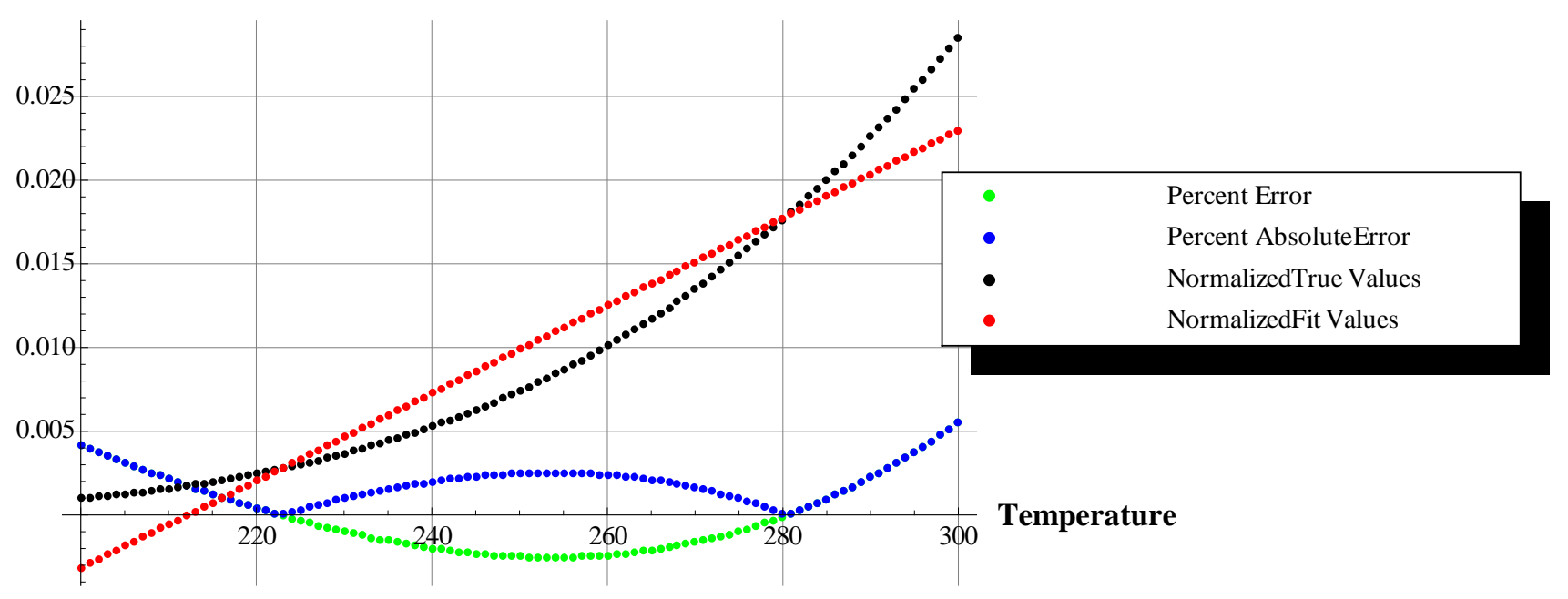

Figure 62. Errors for the linear model for $v=1400 \mathrm{~cm}^{-1}$ 


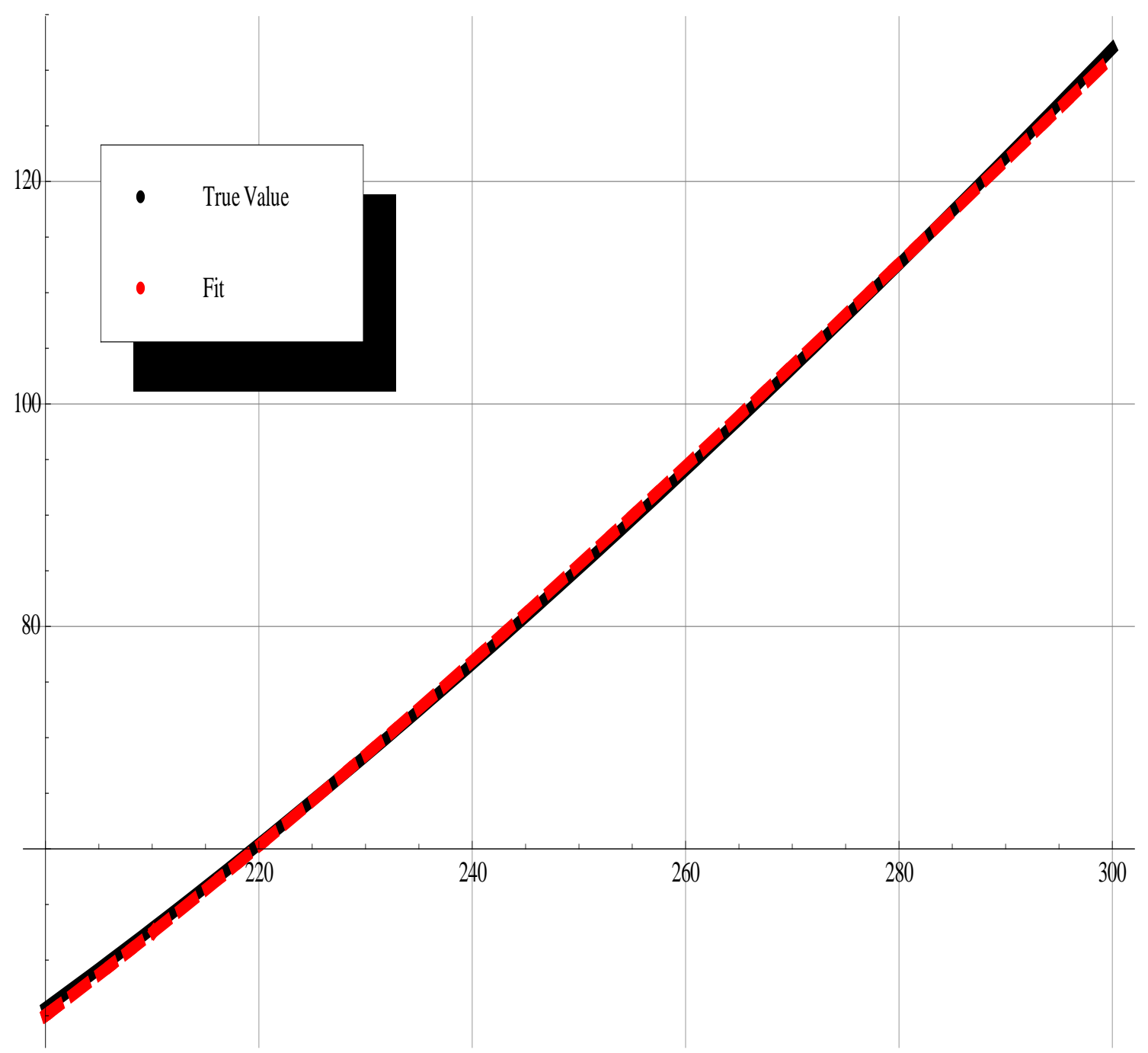

Figure 63. Exponential fit to the Planck function for $v=400 \mathrm{~cm}^{-1}$ 


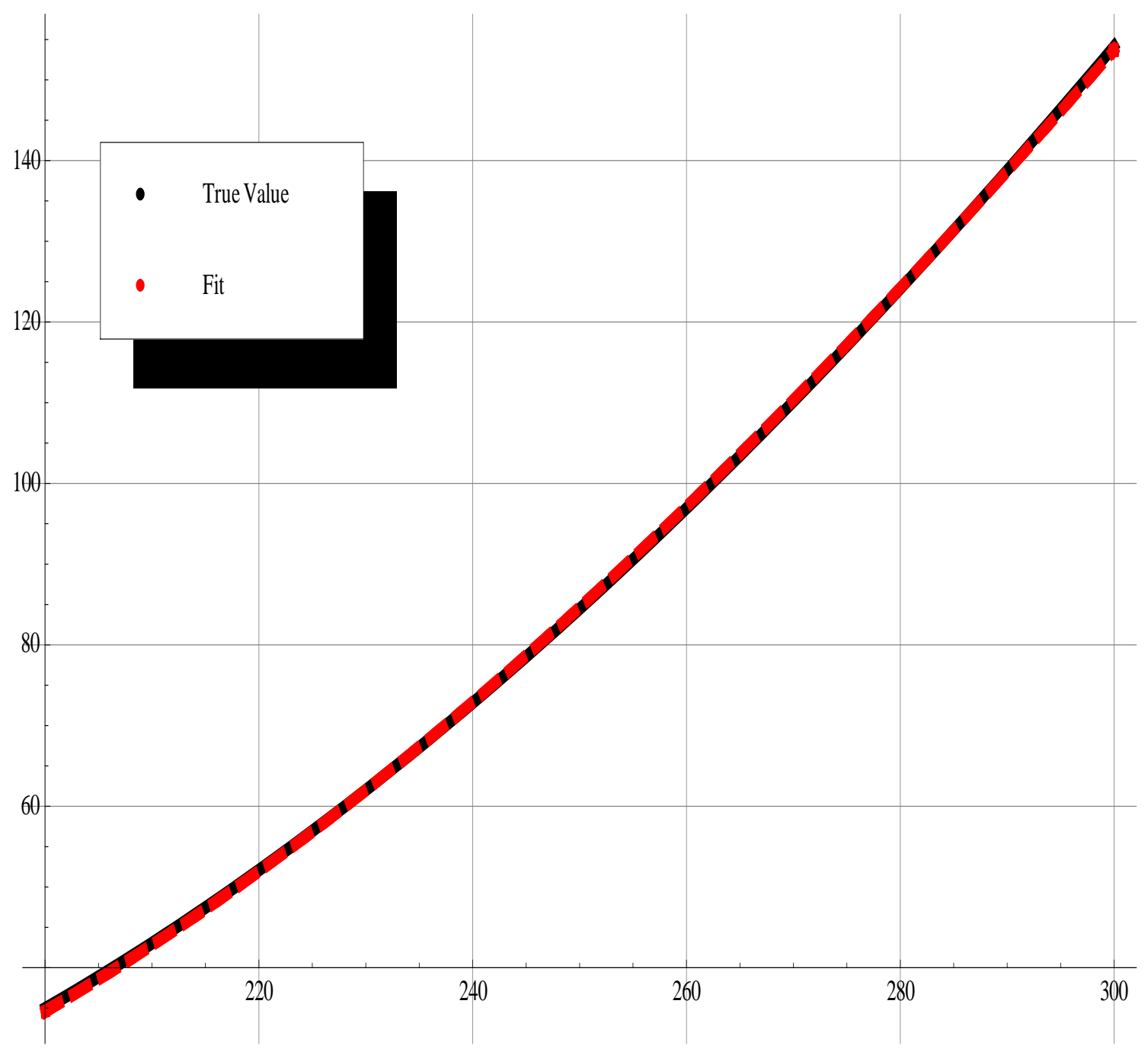

Figure 64. Exponential fit to the Planck function for $v=600 \mathrm{~cm}^{-1}$ 


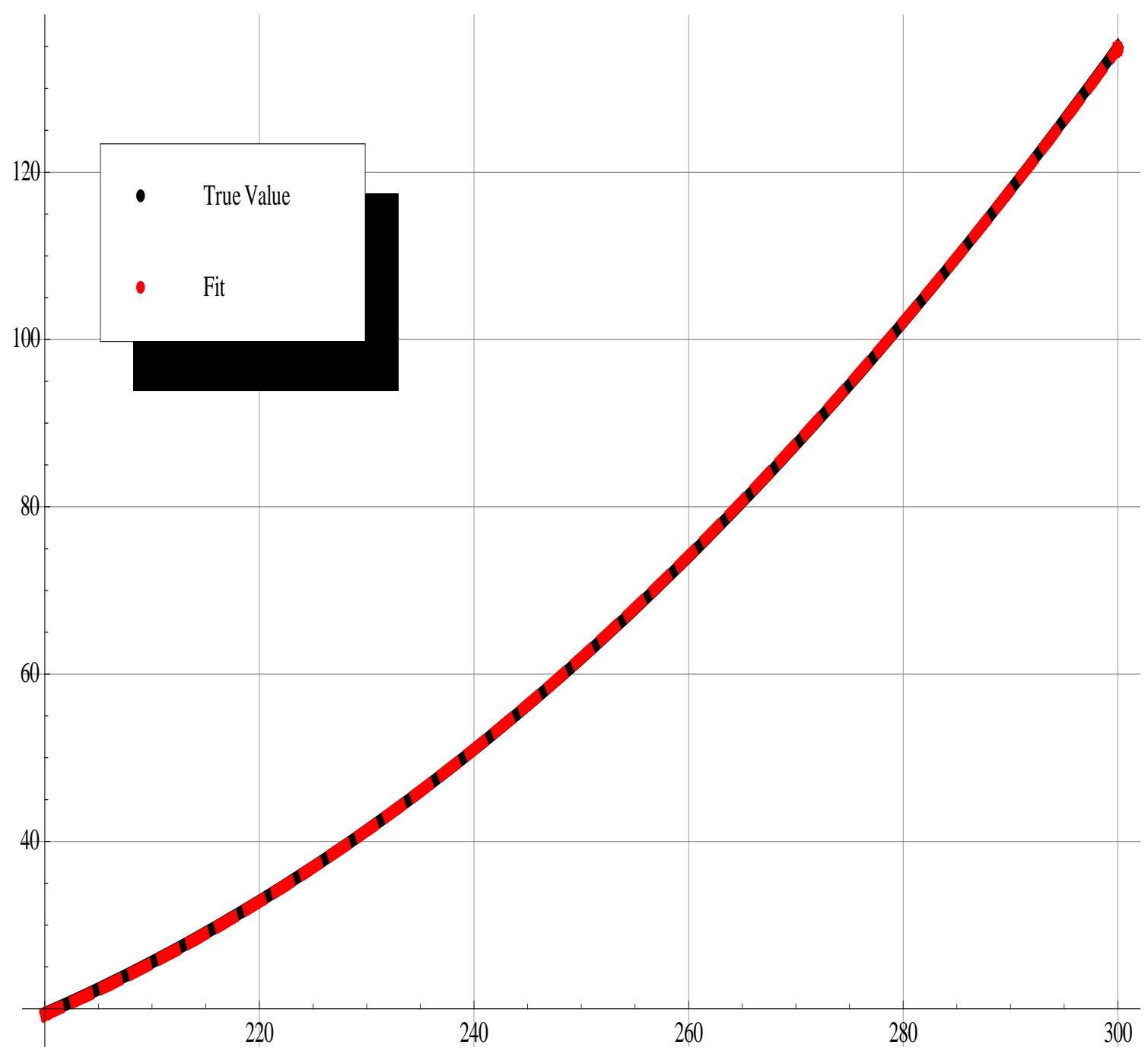

Figure 65. Exponential fit to the Planck function for $v=800 \mathrm{~cm}^{-1}$ 


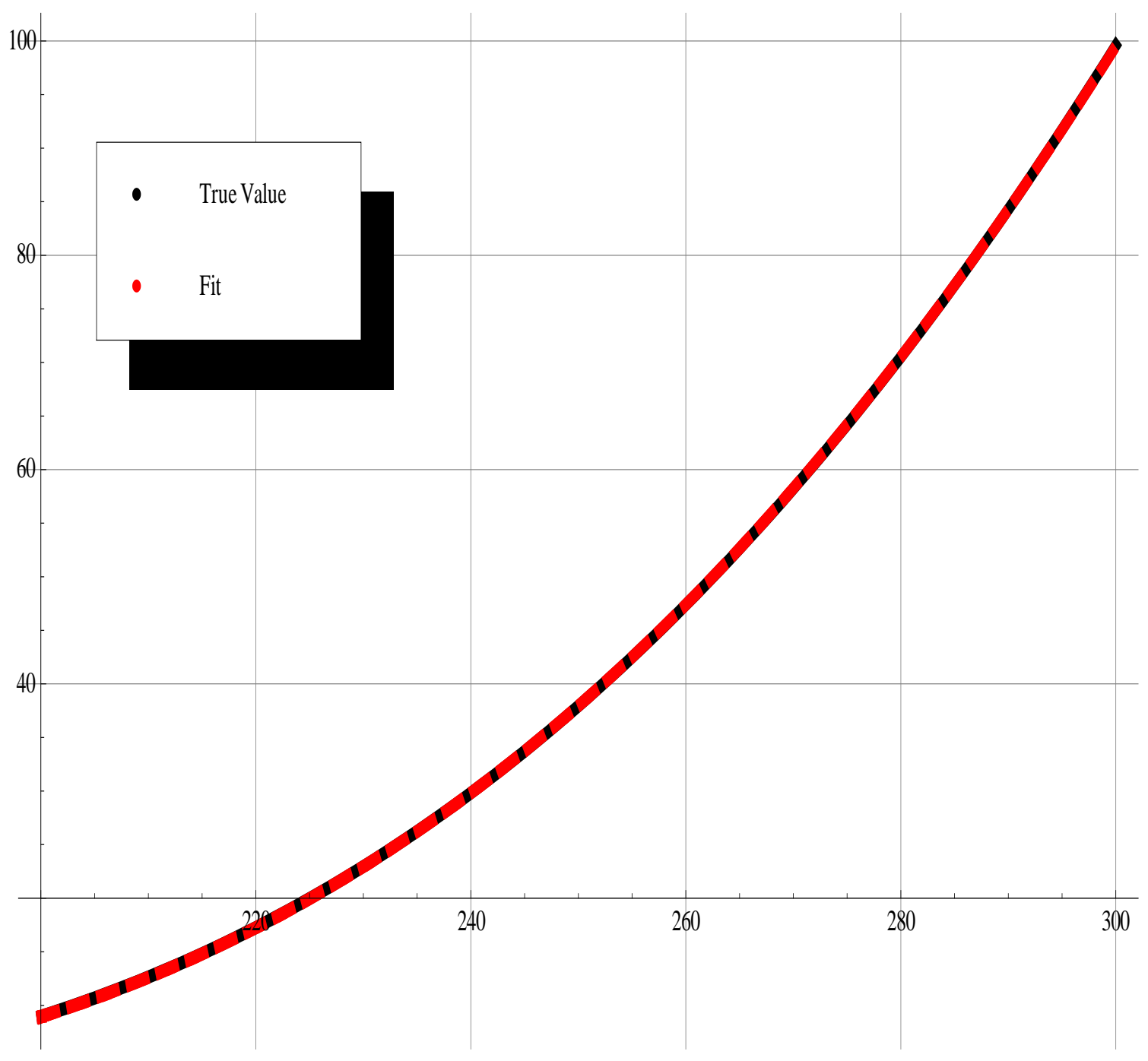

Figure 66. Exponential fit to the Planck function for $v=1000 \mathrm{~cm}^{-1}$ 


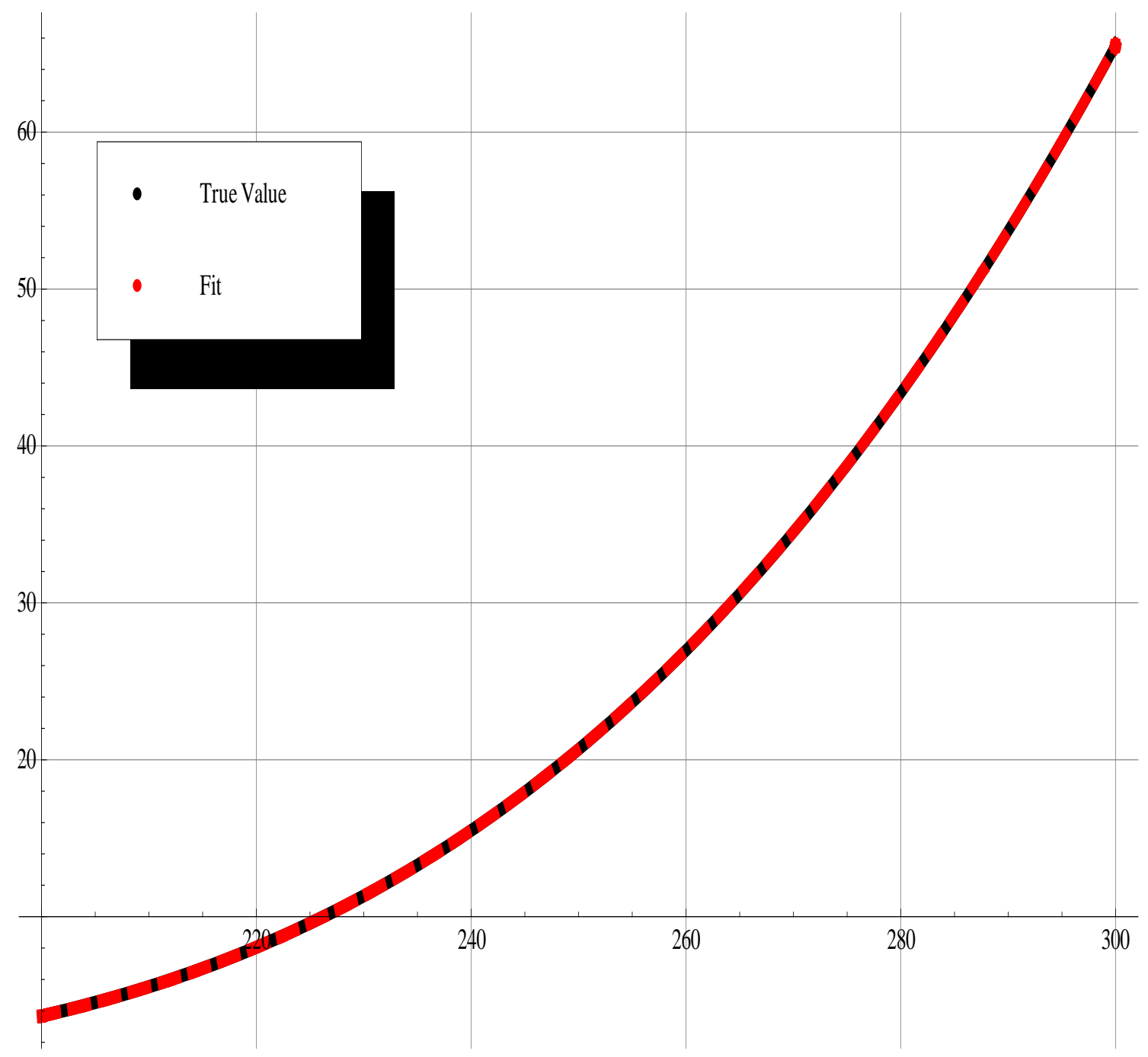

Figure 67. Exponential fit to the Planck function for $v=1200 \mathrm{~cm}^{-1}$ 


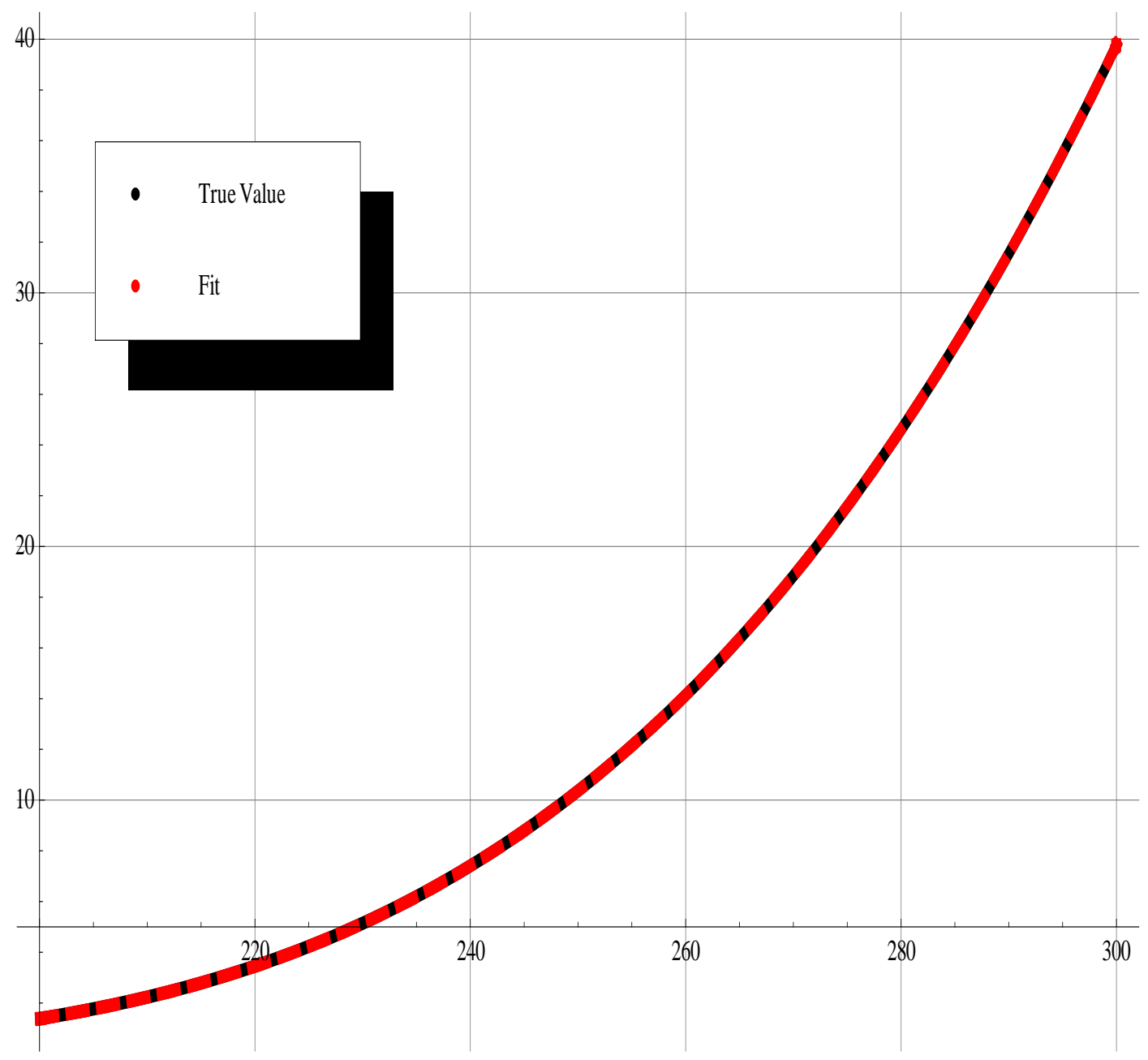

Figure 68. Exponential fit to the Planck function for $v=1400 \mathrm{~cm}^{-1}$

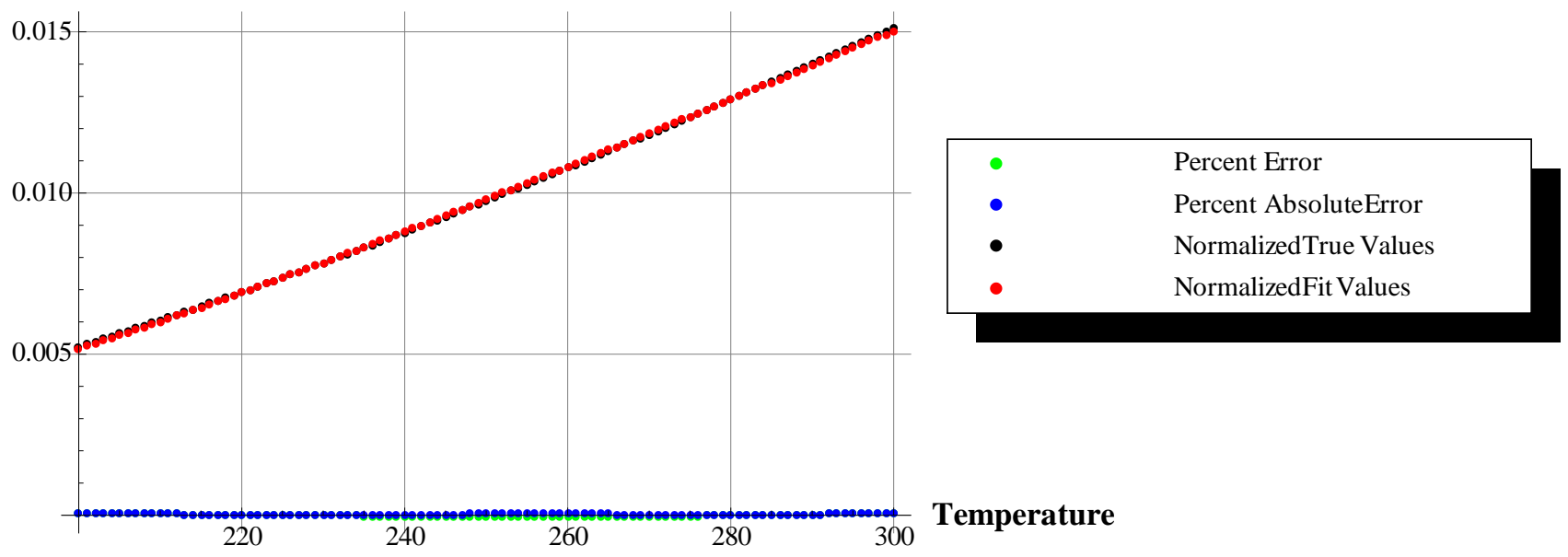

Figure 69. Errors for the exponential model for $v=400 \mathrm{~cm}^{-1}$ 


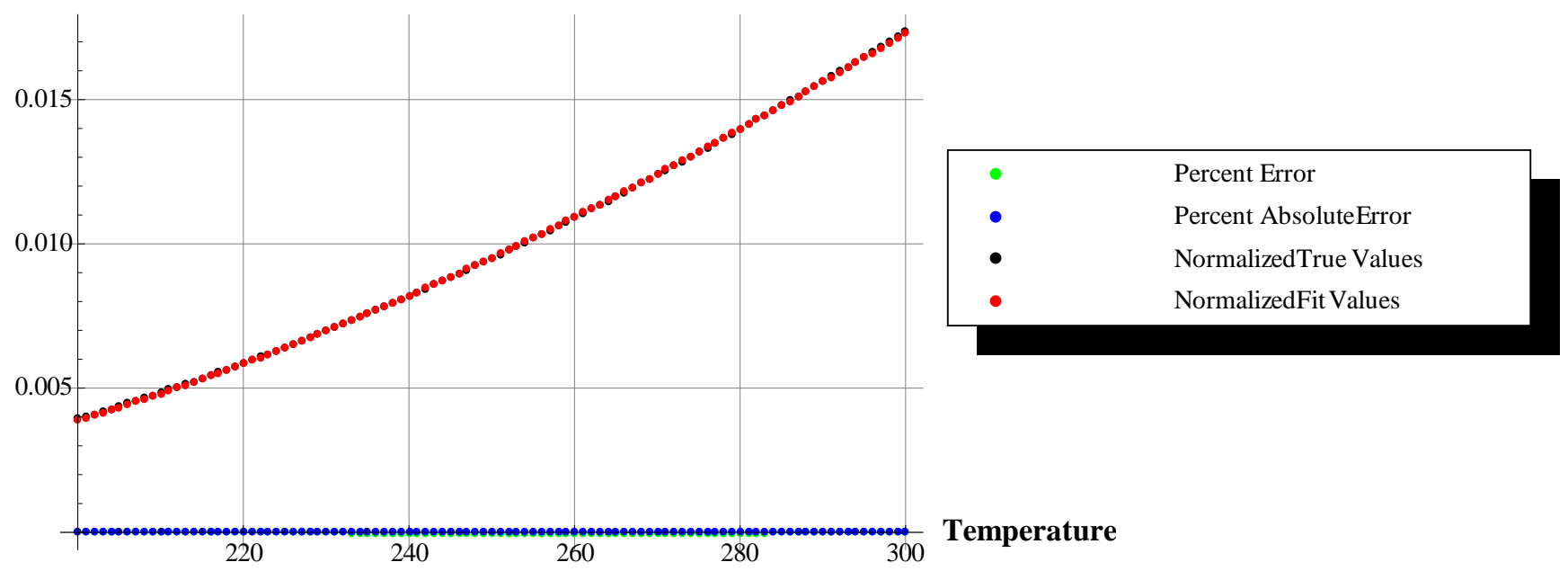

Figure 70. Errors for the exponential model for $v=600 \mathrm{~cm}^{-1}$

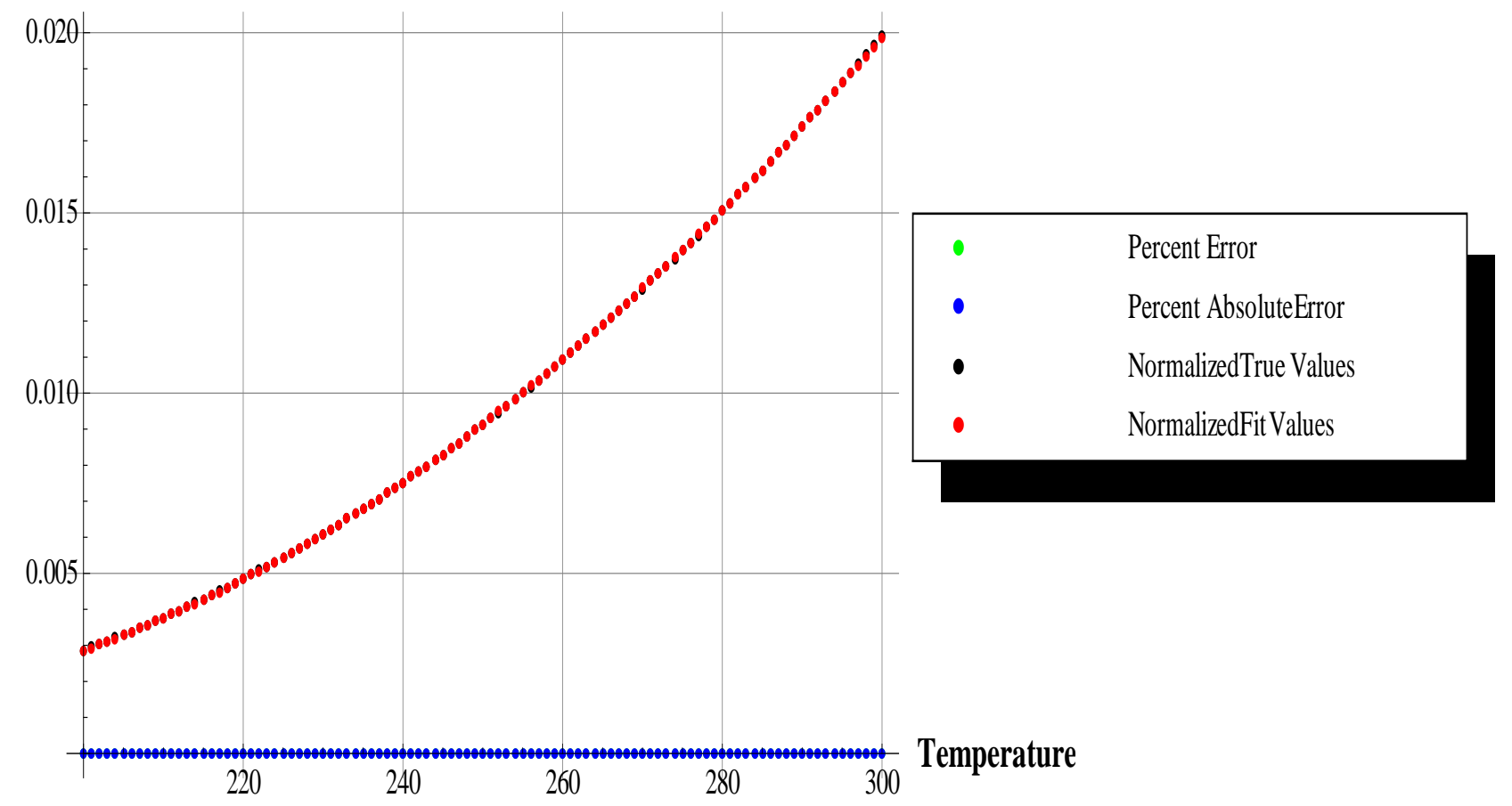

Figure 71. Errors for the exponential model for $v=800 \mathrm{~cm}^{-1}$ 


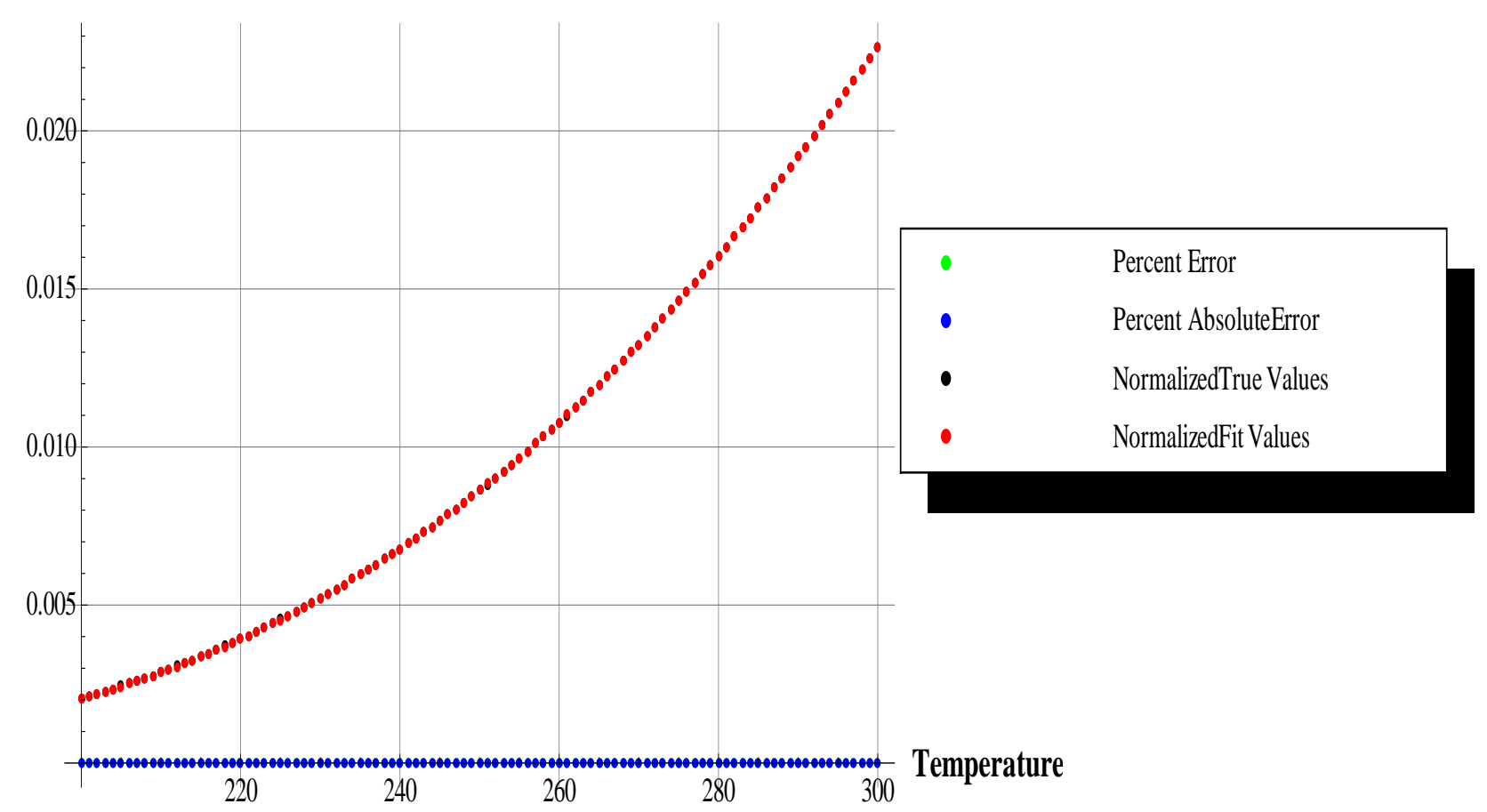

Figure 72. Errors for the exponential model for $v=1000 \mathrm{~cm}^{-1}$

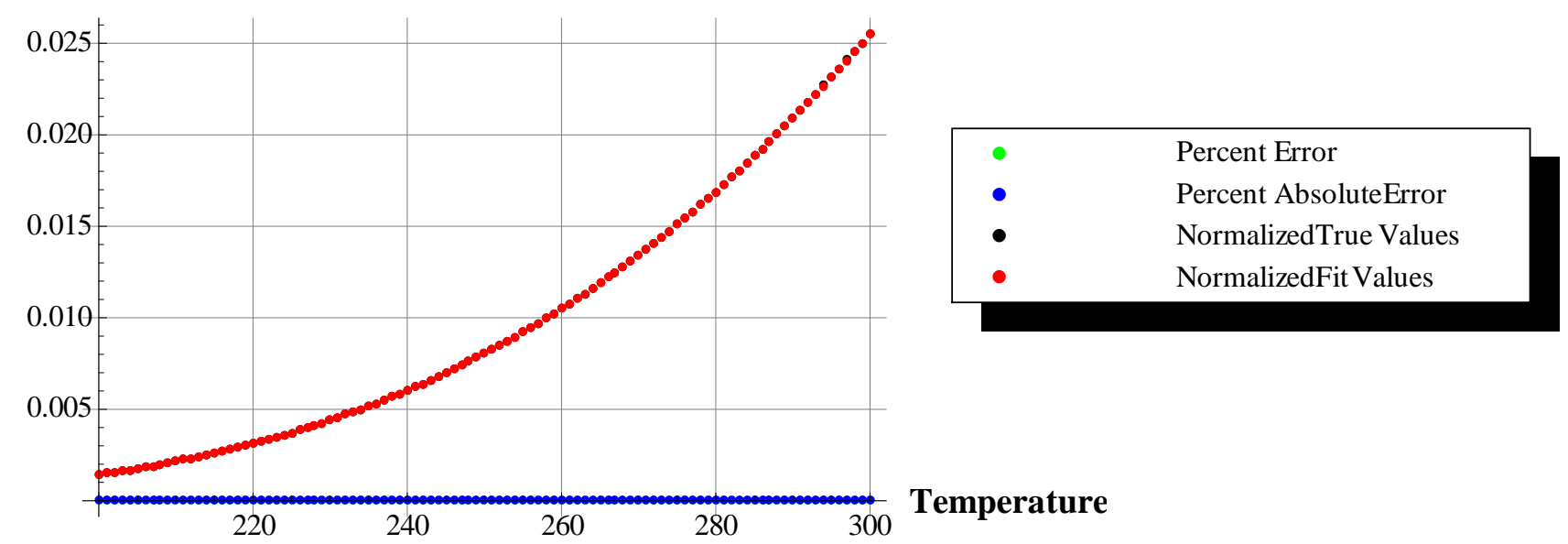

Figure 73. Errors for the exponential model for $v=1200 \mathrm{~cm}^{-1}$ 


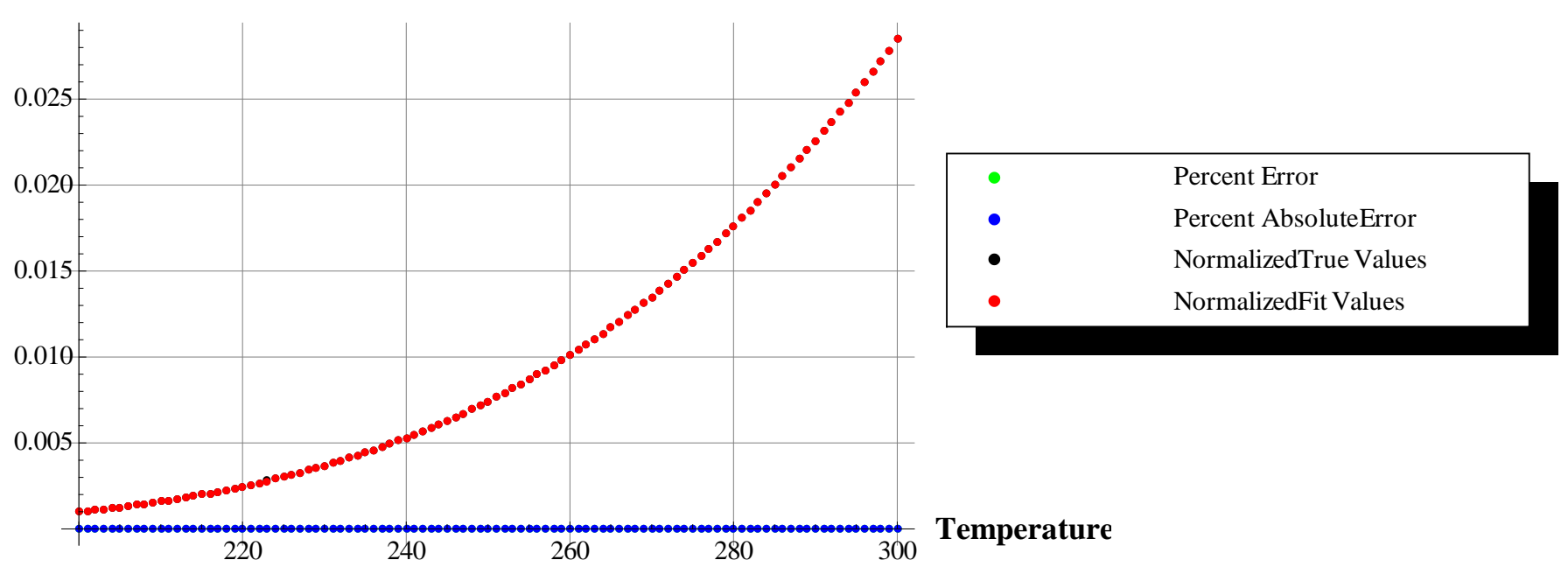

Figure 74. Errors for the exponential model for $v=1400 \mathrm{~cm}^{-1}$

\subsubsection{Sinusoidal Temperature Model}

It is very instructive to first analyze a simple, analytical model. For this we use a sinusoidal perturbation field, superimposed on a linear temperature field,

$$
T(x)=\left[\frac{T\left(x_{2}\right)-T\left(x_{1}\right)}{x_{2}-x_{1}}\right]\left(x-x_{1}\right)+T\left(x_{1}\right)+T_{0} \sin (k x)
$$

where $k=\frac{2 \pi}{\lambda}, \lambda$ being the wavelength, and $T_{0}$ the amplitude of the sinusoidal part. An example of this field and the corresponding radiance field (with constant absorption coefficient) are shown in Figure 75 where $\lambda=38 \mathrm{~km}, T_{0}=5^{\circ} \mathrm{K}$, slope $=3 \times 10^{-5}{ }^{\circ} \mathrm{K} \mathrm{m} \mathrm{m}^{-1}$ and $T\left(x_{1}\right)=247^{\circ} \mathrm{K}$. Figure 76 is a plot of the associated radiance field for the temperature field shown in Figure 75.

The plot shown in Figure 77 illustrates the radiance for the data shown in Figure 75 - without the linear trend. The red curve is the radiance, the black curve is a moving average of the radiance, and the blue curve is the residual radiance from the moving average. Figure 78 illustrates the same quantities shown in Figure 77, but with additive Gaussian noise.

The plots shown in Figure 79 are the radiance correlation functions for the residual curves (i.e., blue curves) in Figure 77 (black) and Figure 78 (brown), respectively. The red curve is the average of 6 realizations of the residuals with additive noise. Notice how the averaging helps in damping the effects of the noise. Furthermore, note that the noise power shows up at zero lag of the correlation functions.

A plot of the radiance correlation function for the residual of a sinusoidal temperature field with a wavelength of $25 \mathrm{~km}$ and with an average of six realizations is shown in Figure 80. A similar plot with a wavelength of $12.5 \mathrm{~km}$ is shown in Figure 81 . In both of these plots, the $\mathrm{x}$-axis is distance in units of meters and $y$-axis is correlation. These two plots illustrate the effects on the radiance correlation function when the wavelength of the sinusoidal temperature field is decreased. Notice how the damping increases with decreasing wavelength. 


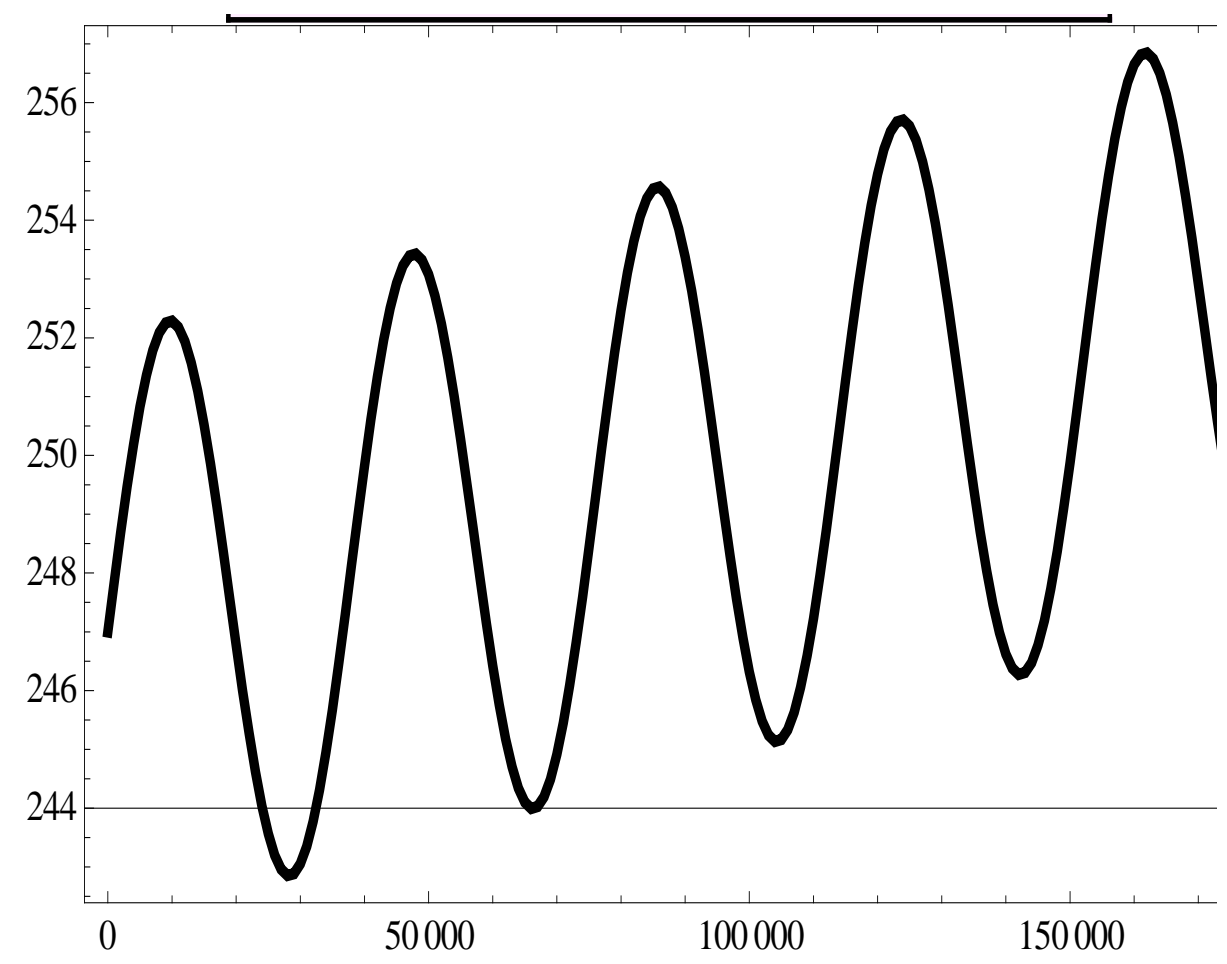

Figure 75. A linear-sinusoidal temperature field

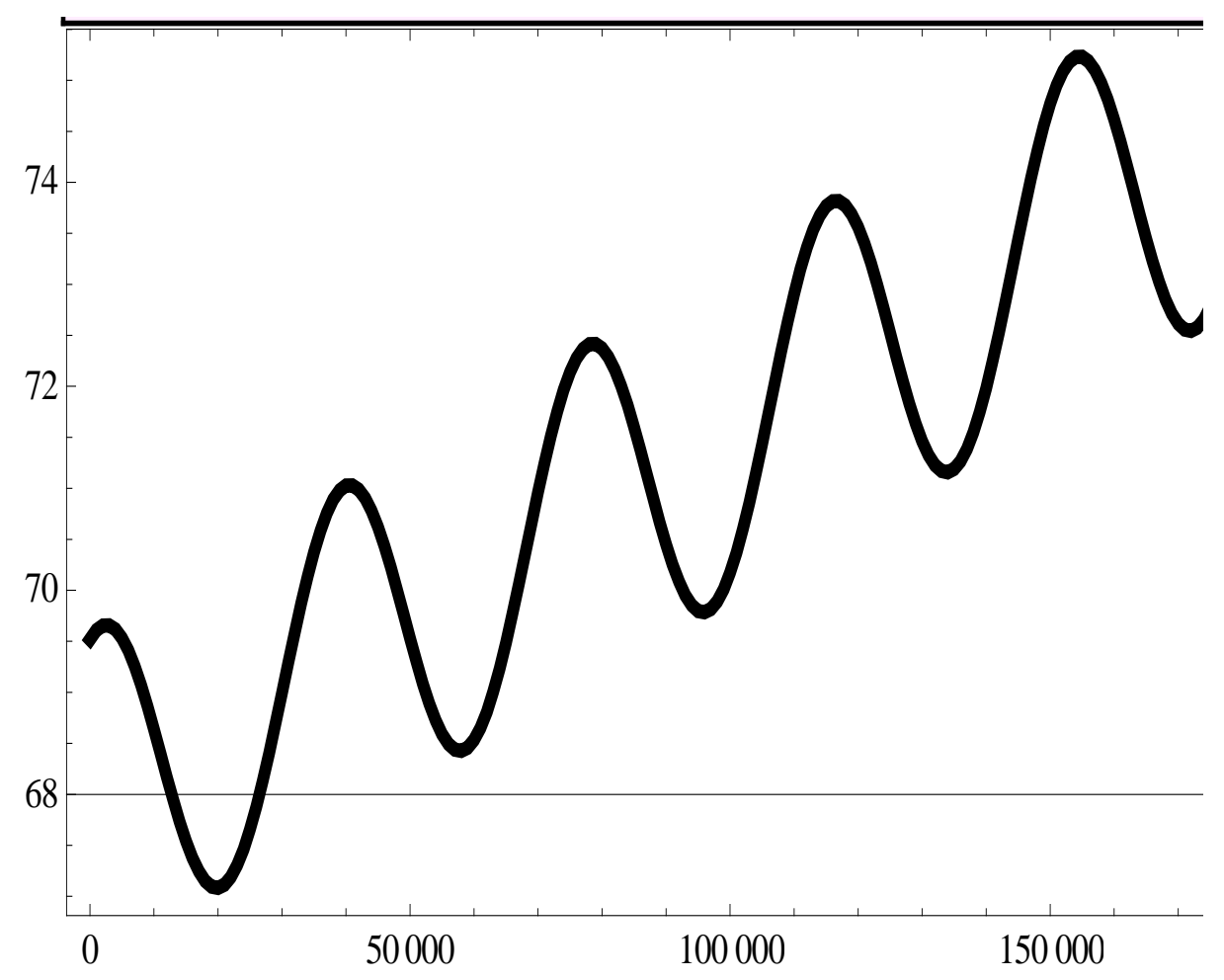

Figure 76. Radiance field for the linear-sinusoidal model shown in Figure 75. 


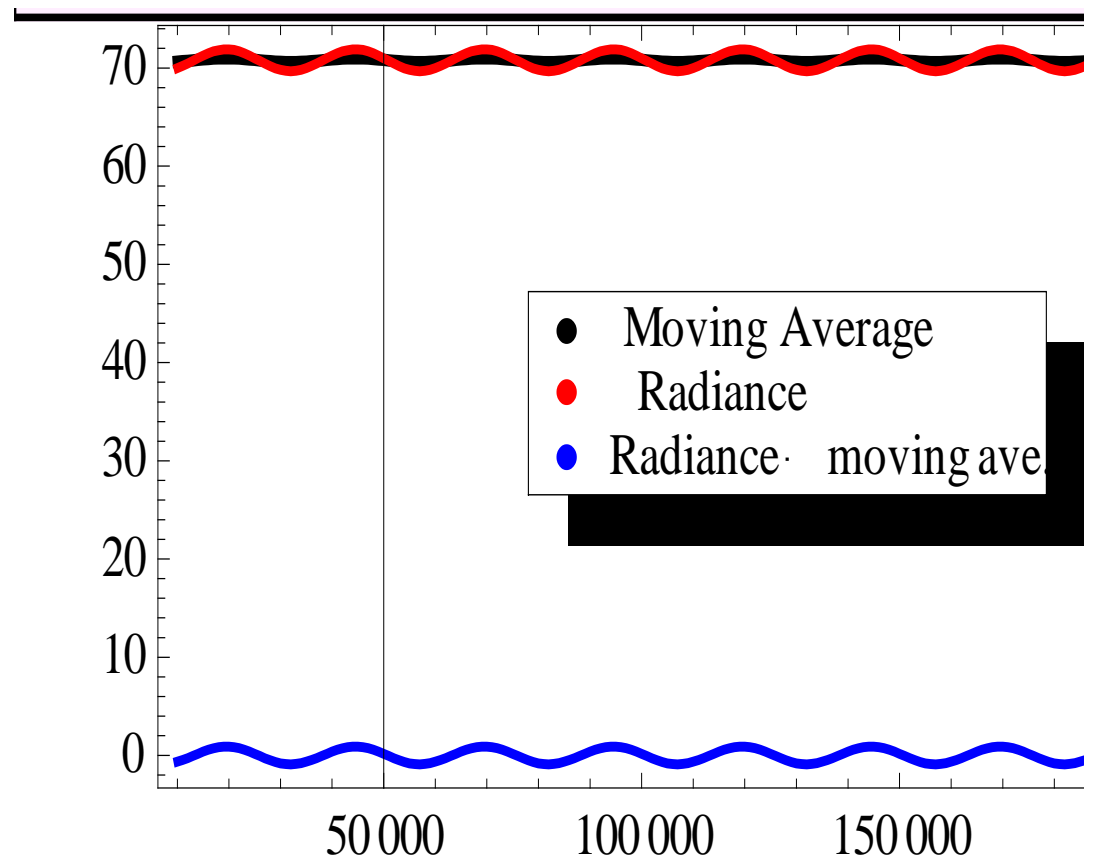

Figure 77. Radiance functions without the linear term.

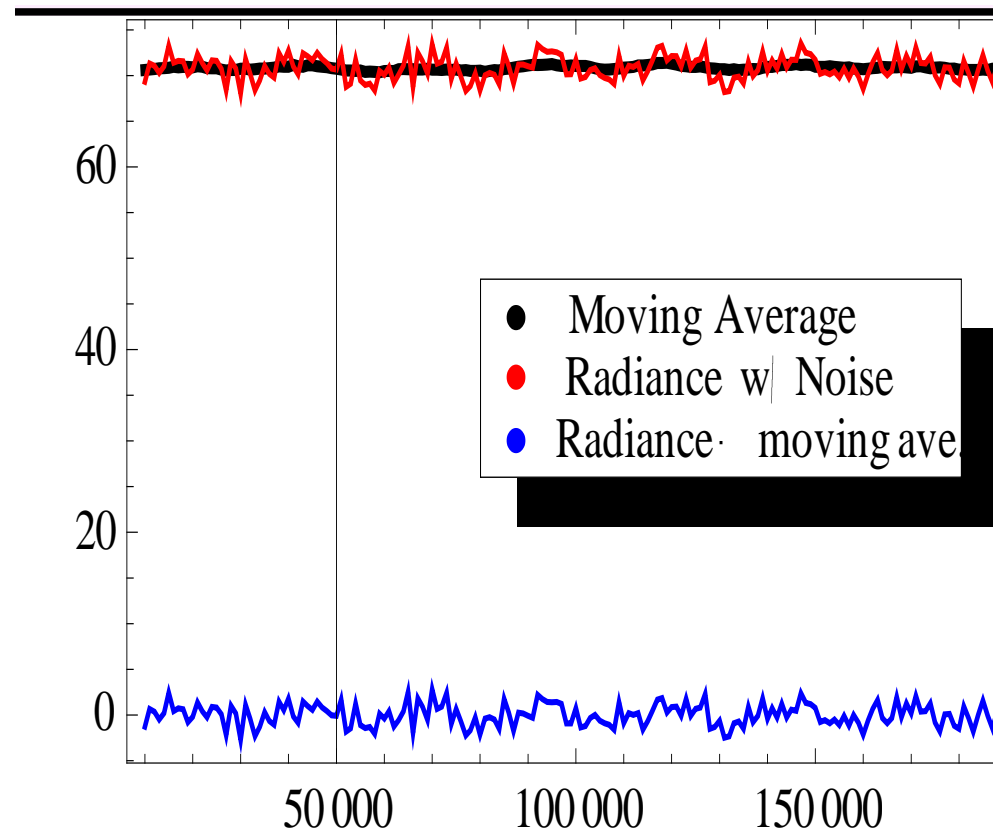

Figure 78. Same as in Figure 77, excepting with additive Gaussian noise 


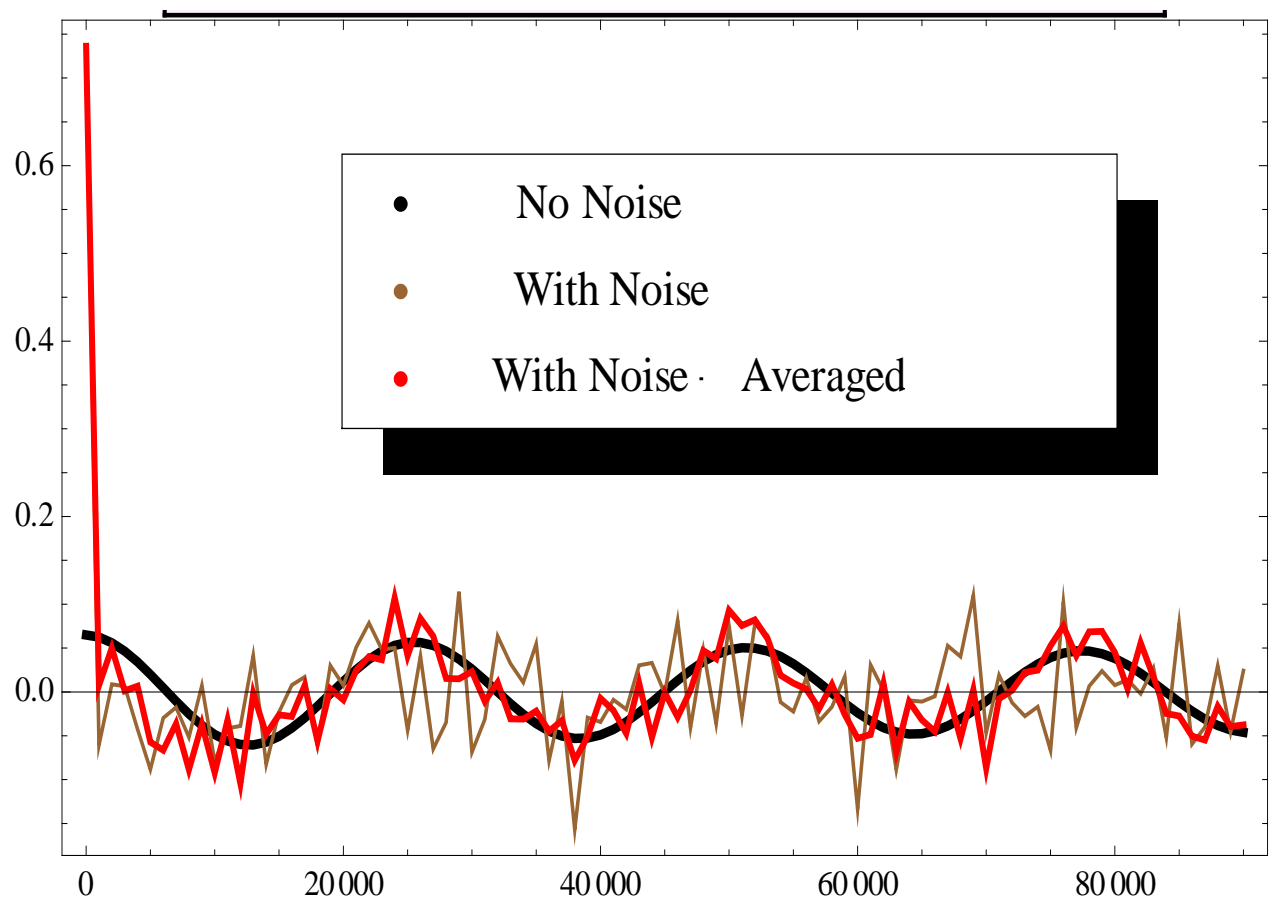

Figure 79. Radiance correlation functions for the residual curves.

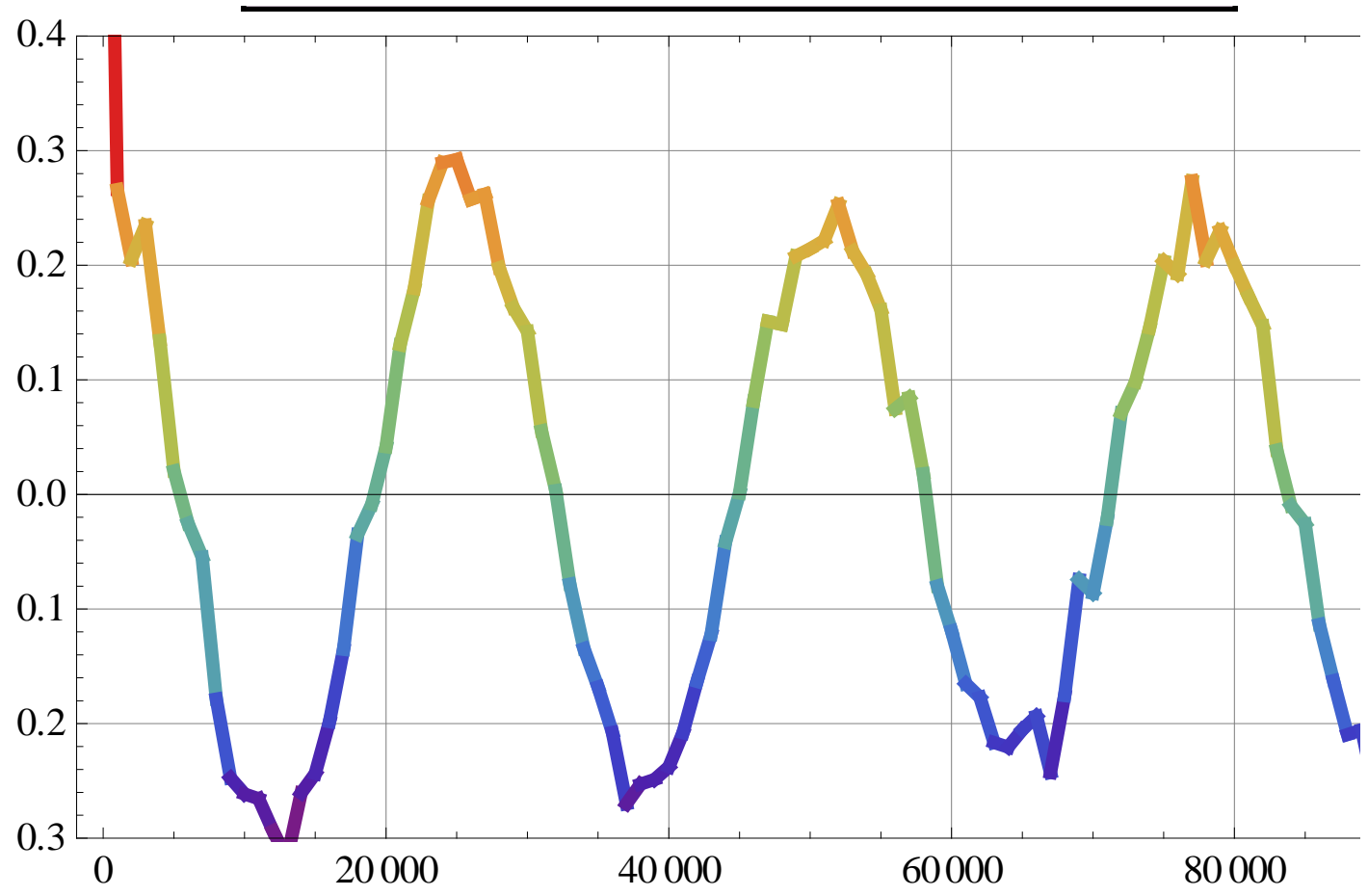

Figure 80. Radiance correlation function for the residual of a sinusoidal temperature field. 


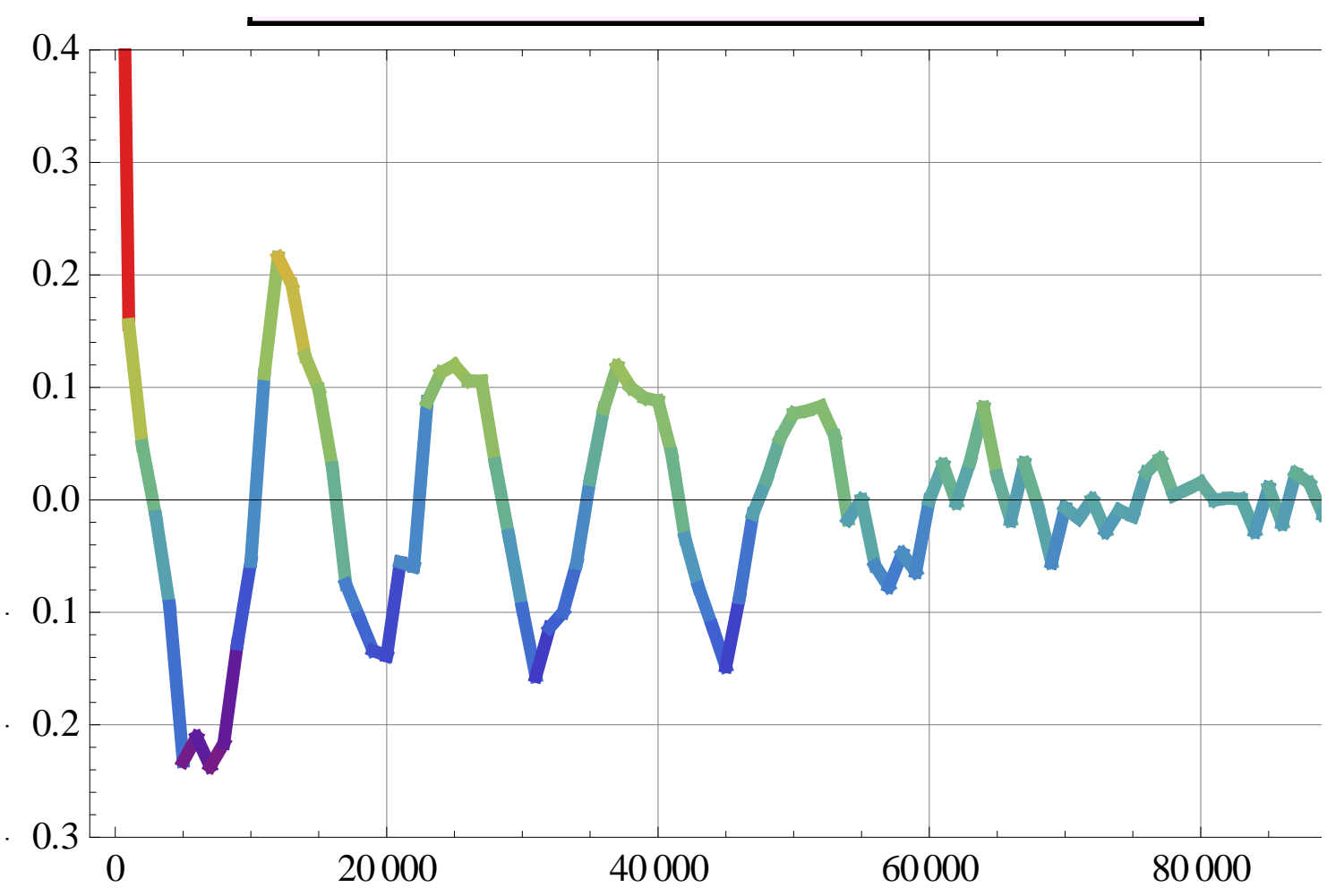

Figure 81. Same as for Figure 80, but with a $12.5 \mathrm{~km}$ wavelength temperature field

\subsubsection{WRF Model Case}

Simulated radiance data from a numerical weather model mountain wave simulation was analyzed. The mountain wave simulation used $1 \mathrm{~km}$ horizontal grid spacing. The radiance simulation modeled an aircraft starting at an $8 \mathrm{~km}$ altitude moving towards the mountain wave region with a $250 \mathrm{~m}$ step size. At each step, radiance that would have been measured at the aircraft location, looking through the mountain wave model grid and then continuing through a standard atmosphere to the top of the atmosphere is computed. Note the line-of-sight is along a horizontal line relative to the original location, and hence the height above the earth increases along the measurement path.

The temperature field and height above the earth, from the initial aircraft location through the end of the mountain wave simulation grid, is shown in Figure 82. In this plot, height is shown versus distance (black curve), starting at $8 \mathrm{~km}$ altitude and looking horizontally. The red curve is the associated temperature field from the WRF simulation. The large-scale wave structure - with wavelength can be seen in this figure. Figure 83 shows the temperature field as a function of height, from the initial aircraft location to the top of the atmosphere. In this plot, the temperature field as shown from the aircraft height at $8 \mathrm{~km}$ altitude looking horizontally to the top of the atmosphere. The values close-in are from the WRF model, the rest are from a standard atmosphere. Figure 84 shows the temperature field from the first $186.5 \mathrm{~km}$. The temperature field is a function of time (in seconds) along the flight path: $t=x / V$, where $V=250 \mathrm{~m}^{-1}$; hence the spatial domain is $187.5 \mathrm{~km}$. The horizontal scale is in seconds - illustrating how an approaching aircraft would measure the temperatures. Figure 85 shows the simulated radiances calculated from the temperature field shown in Figure 84. (Note: in this simulation, the absorption coefficients are not constant.) 


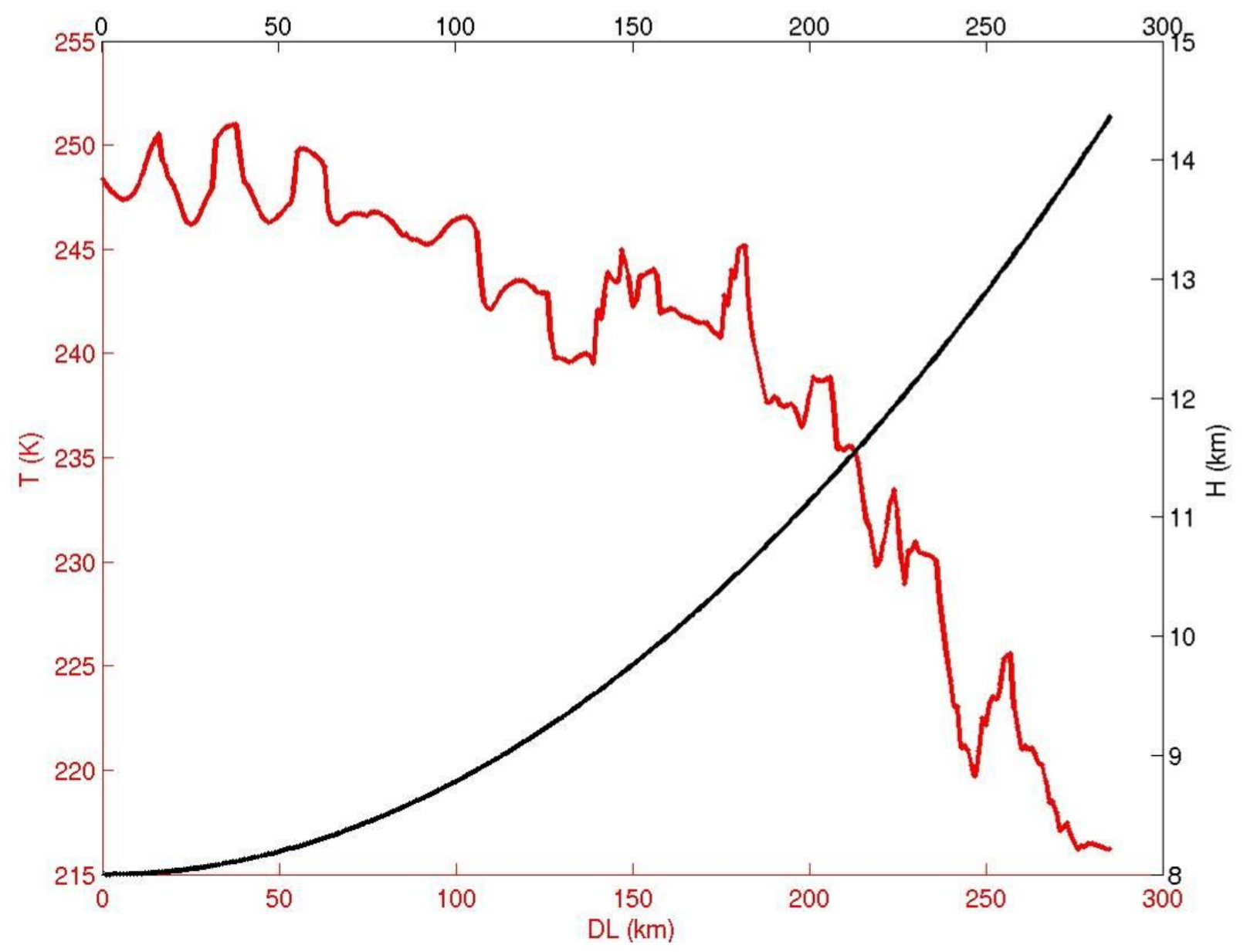

Figure 82. Height and associated temperature field versus distance.

Figure 86 illustrates the simulated radiance with the linear trend removed. Comparing these two figures with Figure 75 and Figure 76, respectively, it can be surmised that the structures that are visible are mountain waves with a wavelength of approximately $35 \mathrm{~km}$. Note that a smallerscale, small amplitude oscillation can be seen in Figure 86. It is believed that these smaller-scale oscillations are due to artifacts in the simulation's interpolation scheme. It should be noted that at a $1 \mathrm{~km}$ WRF grid spacing, the model is only resolving features that are on the order of $5 \mathrm{~km}$ and larger. Hence, there are really no turbulent features that would affect aircraft being resolved. This is assuming that the temperature fluctuations would be correlated to vertical velocity fluctuations. The scales of vertical velocity fluctuations that would impact an aircraft (via vertical accelerations) are on the order of 100-2000 m. Hence the WRF simulation illustrates that the larger scale mountain wave temperature variations can be seen via an IR sensor, but it does not imply that the important temperature fluctuations due to turbulence can be measured. This will be the subject of the following section. 


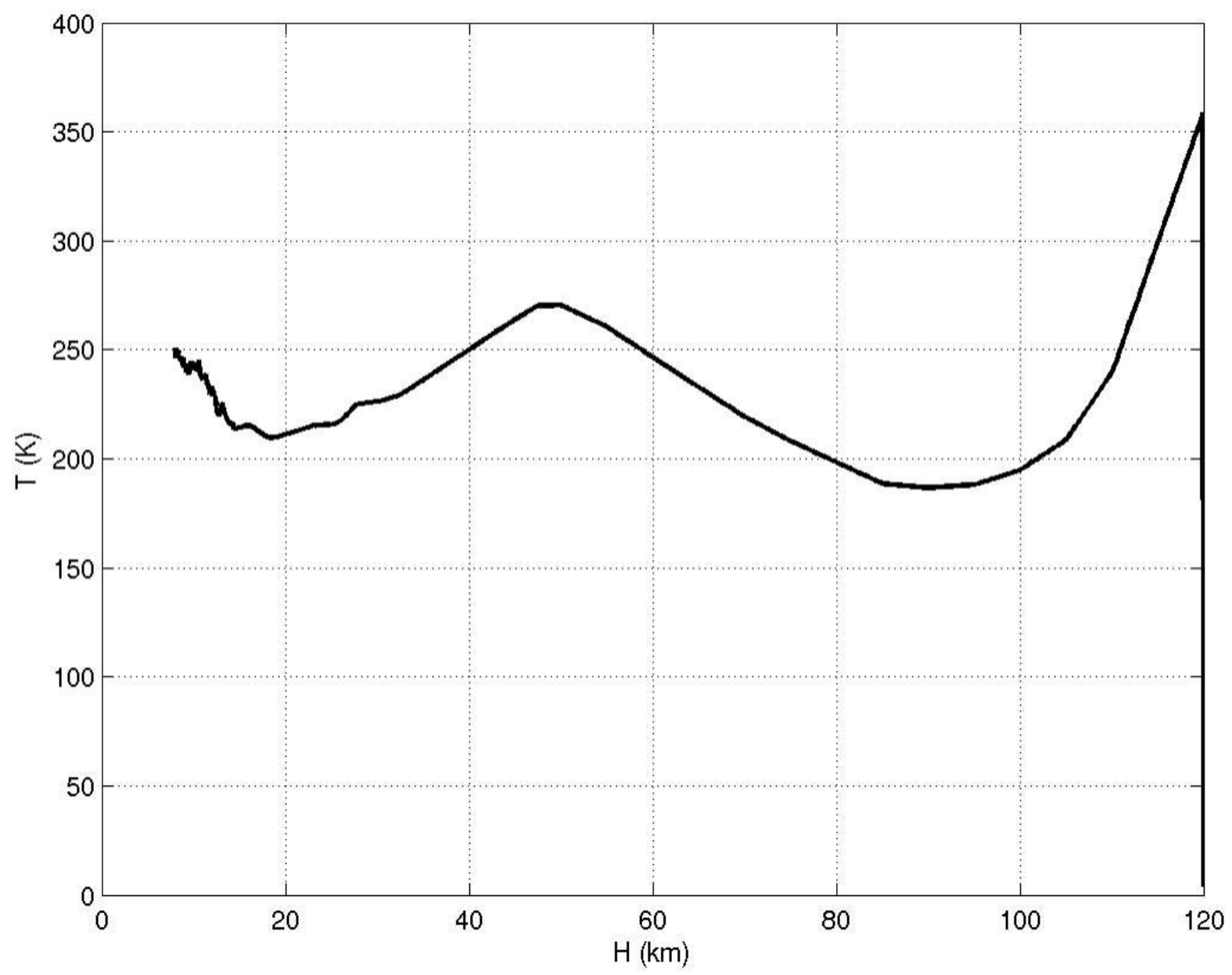

Figure 83. Temperature field as seen from aircraft location at $8 \mathrm{~km}$.

It should be noted that these small-scale oscillations are small in magnitude, but there was a concern about their affecting the radiance correlation calculations. Hence a data filtering scheme, based on wavelet signal deconstruction/reconstruction was studied. Wavelets are a good signal processing tool for this type of non-stationary data - not to mention their ability to handle the "sawtooth" pattern from the interpolation artifacts. Figure 87 illustrates the wavelet deconstruction/reconstruction process for increasing scale parameter. Think of this parameter as analogous to a wavelength, so larger scale parameters correspond loosely to increased wavelengths - or decreased spatial frequencies. Therefore, at each increasing scale value, one can think of the reconstruction as a low-pass filter for decreasing stop-band frequencies. From this figure, it can be seen that by scale level $\mathrm{jj}=4$ or 5 , the small-scale artifacts have been eliminated. 


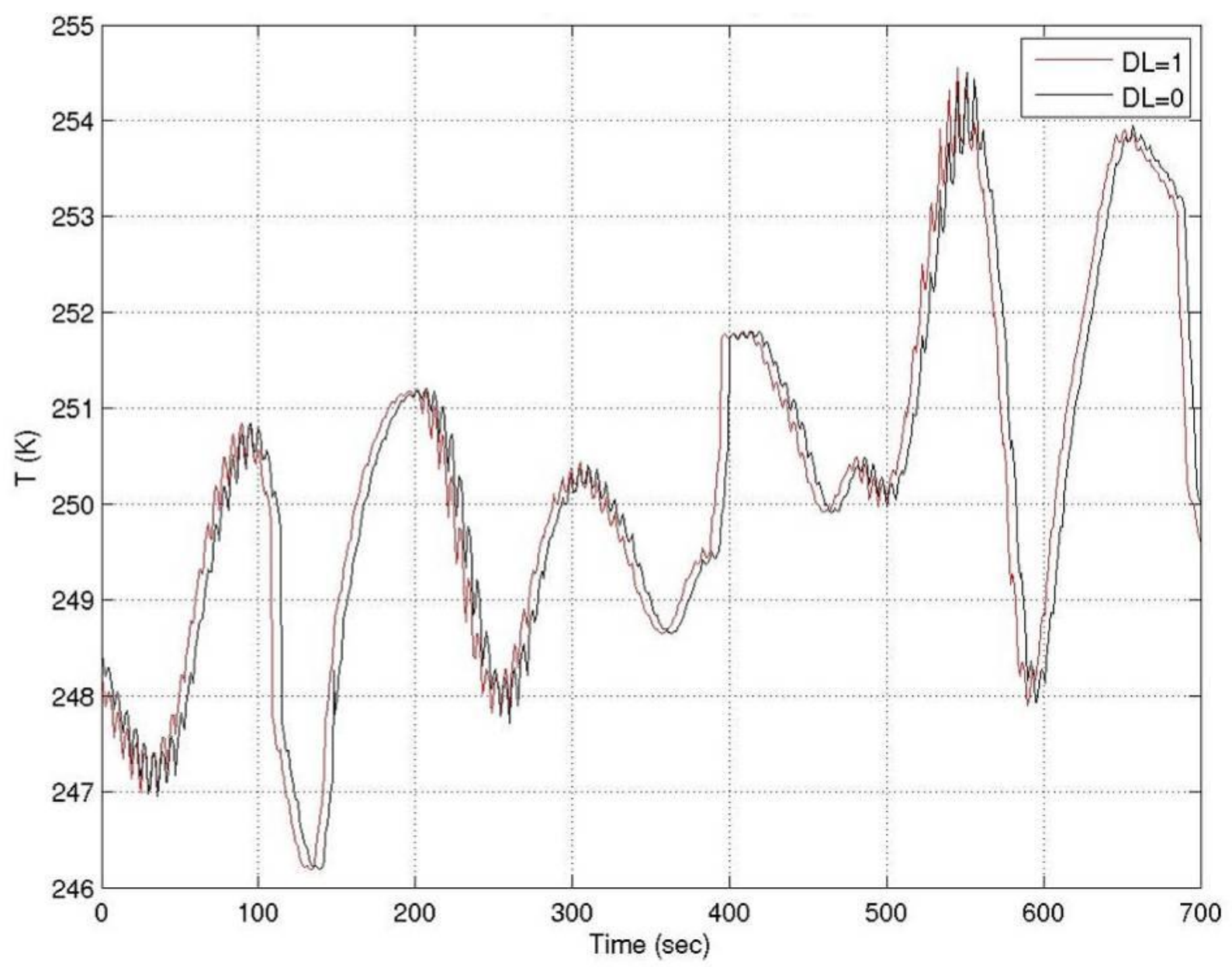

Figure 84. Temperature field as a function of time along the flight path.

The filtering procedure is illustrated in Figure 88. The bottom three panels show the so-called "detail coefficients" for the three smallest scales. These are analogous to extracting those portions of the signal that best match the wavelet basis function (here, the Harr, or "stepfunction" basis), at the respective scale. Hence, the second panel shows that portion of the data that best matches a two-point step-function. The filtering concept is simply to take these smallest-scale coefficients, set them to zero and reconstruct the signal. This result is shown in the top panel of this figure, where the dark blue points are the original signal and the light-blue points are the filtered data. It can be seen that this wavelet filter does an excellent job of removing the small-scale artifacts from the data.

The interesting time versus wavenumber decomposition is clearly shown in Figure 89. The plot shows the result of the filtering as a function of time and wavenumber for data from the segment shown in Figure 86 (where a single wavenumber was shown). The larger-scale features are retained as seen in the vertical fluctuations (dark red and dark blue bands on left side of plot) and the smaller-scale "noise" is removed. 


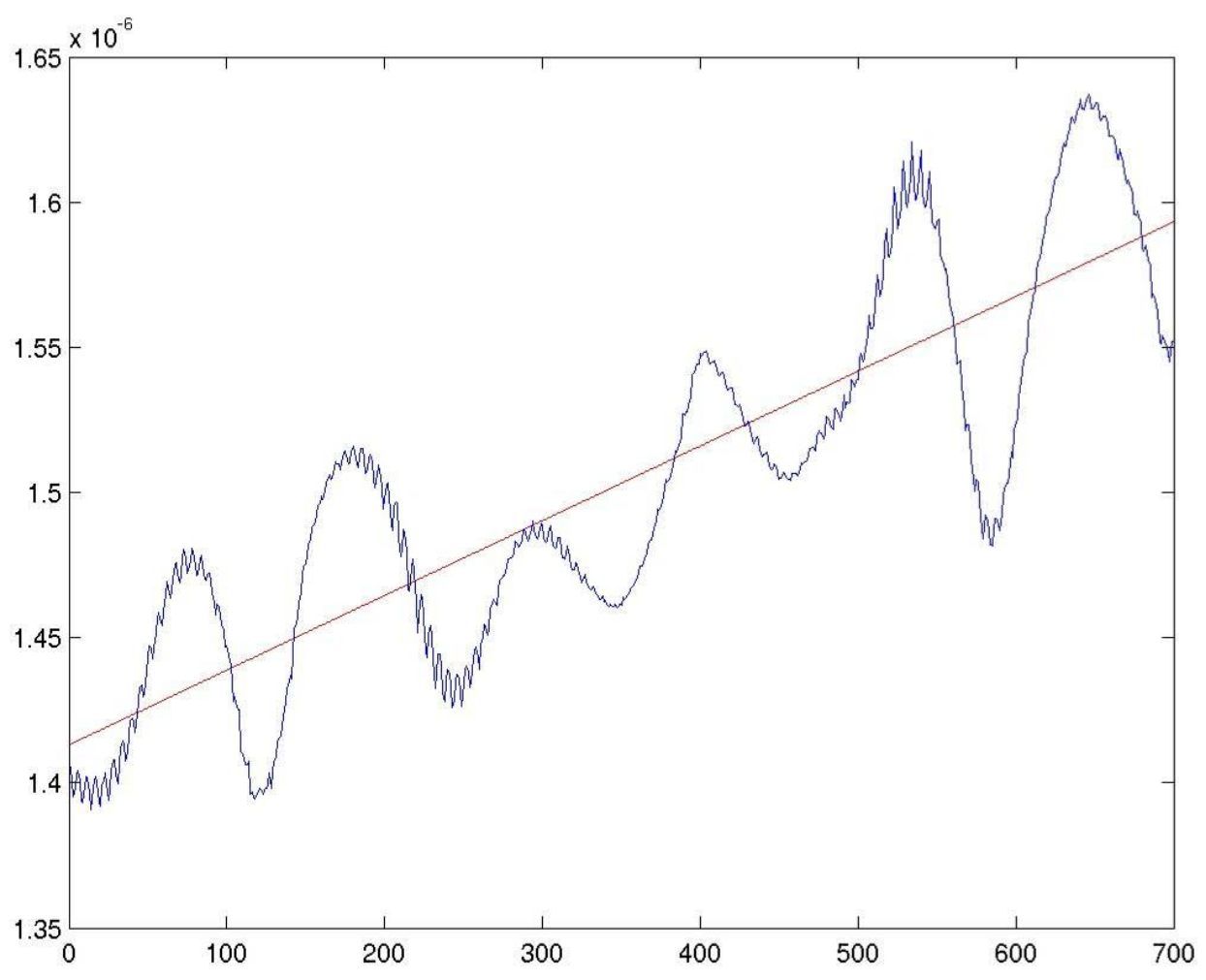

Figure 85. Simulated radiance field with linear trend line.

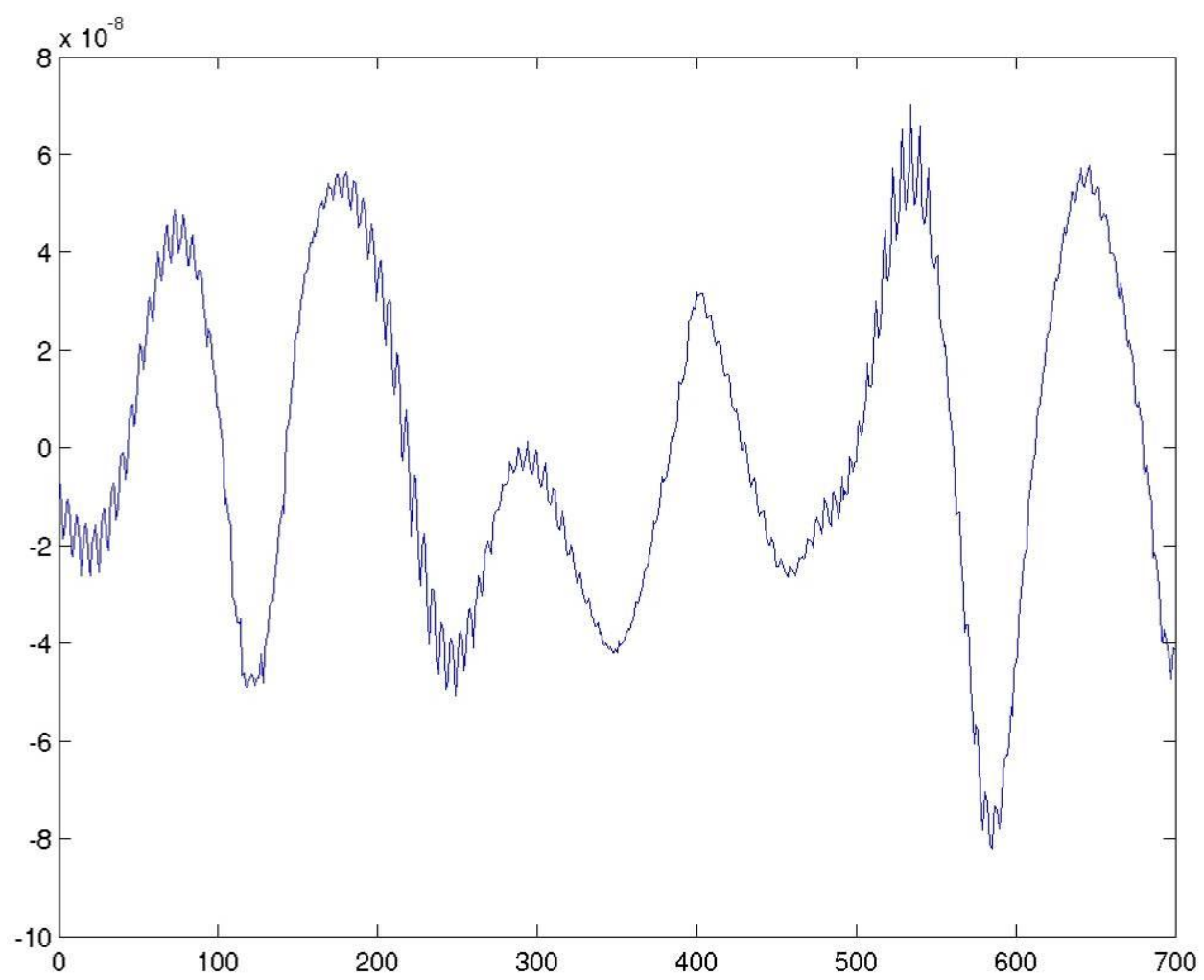

Figure 86. Same as Figure 85, but after trend removal. 

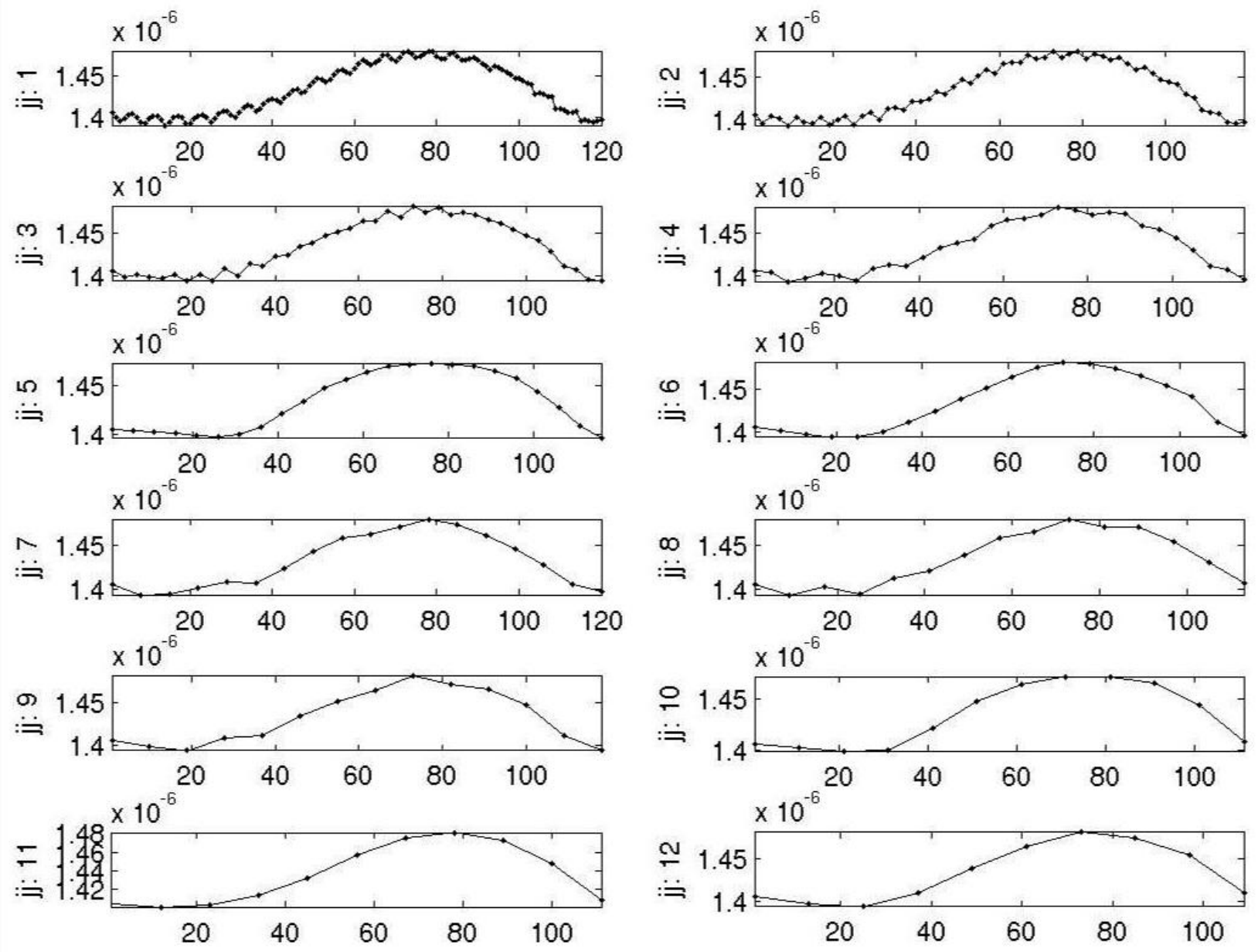

Figure 87. Wavelet filtering of the simulated radiance field. 

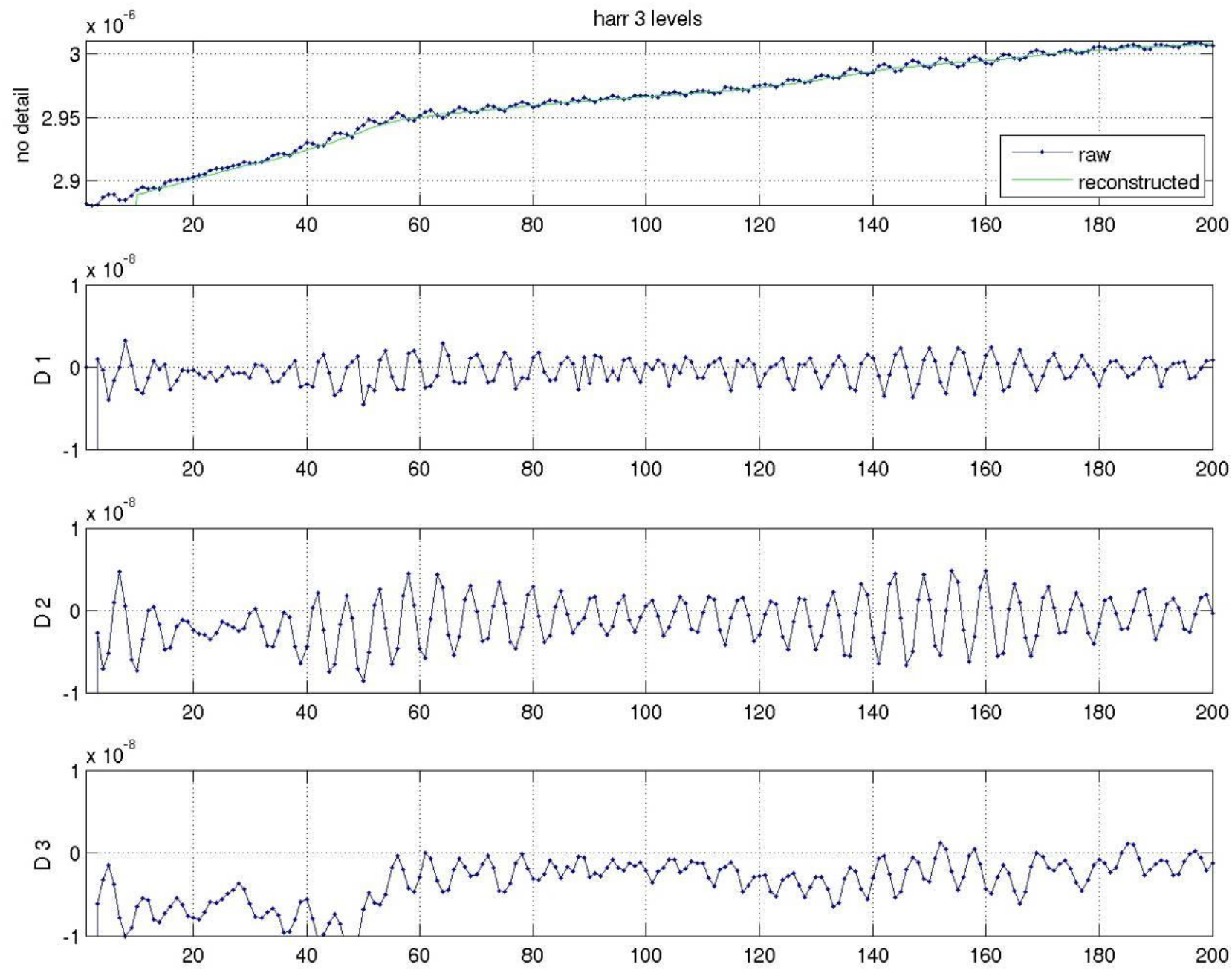

Figure 88. Wavelet deconstruction and filtering of the simulated radiance data. 


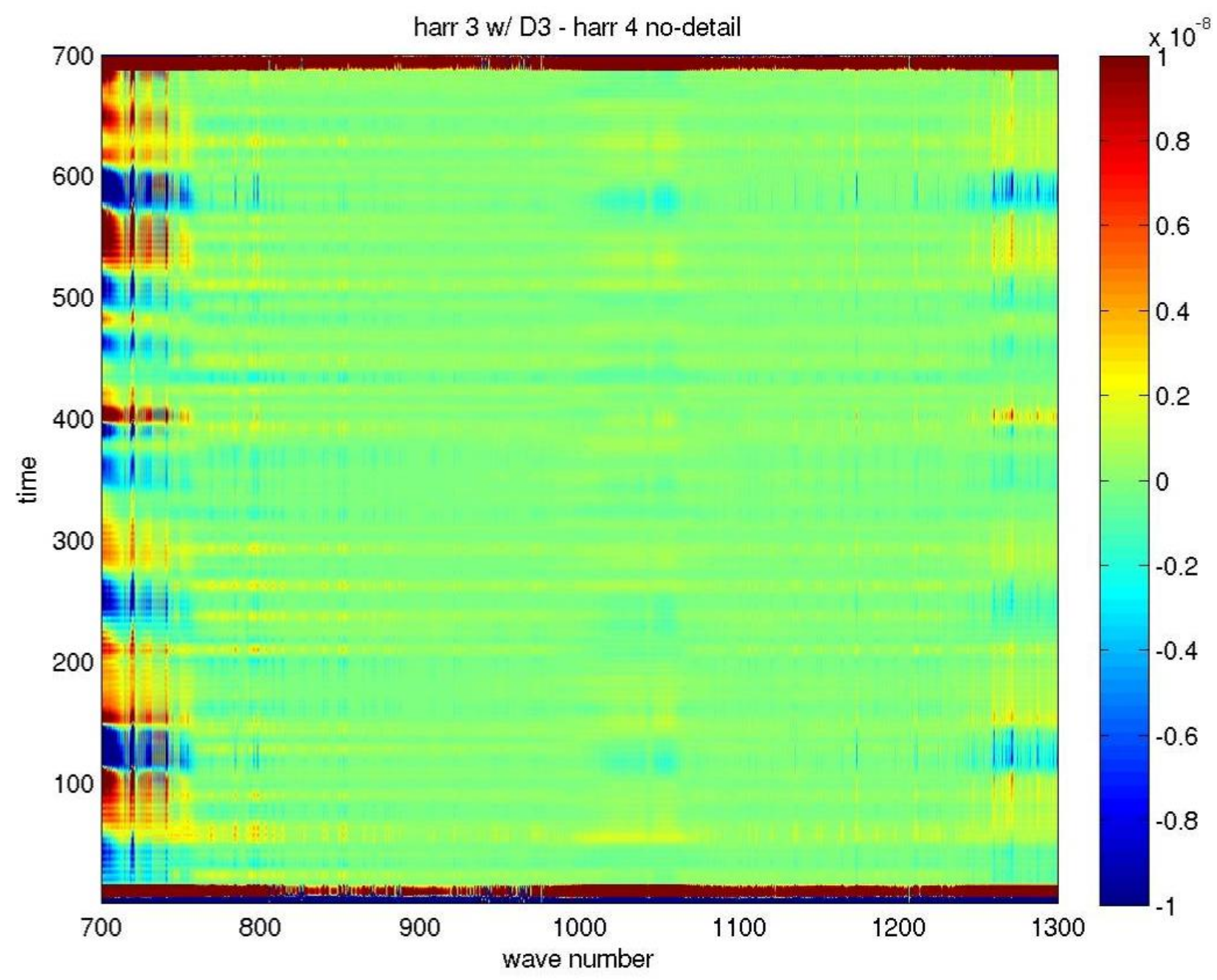

Figure 89. Wavelet-filtered simulated radiance values (color scale).

Results from the simulations showed that brightness temperature differences associated with mountain waves should be on the order of several K, with the largest signals in the relatively clear regions of the LWIR window, 700-800 cm-1 and 1000-1100 cm-1, and that the distance to the turbulence should be predictable from the spectrum. A simulation of mountain lee wave turbulence over Colorado on 6 March 2004 was used extensively to refine the instrument specifications for an airborne FLI.

An example of the modeling brightness temperature differences is shown in

Figure 90, which shows the variability at $800.8 \mathrm{~cm}-1$ in a westward-looking view of the modeled 6 March 2004 Mountain wave turbulence. The imaging field of view is 50 X 250 milliradians. 


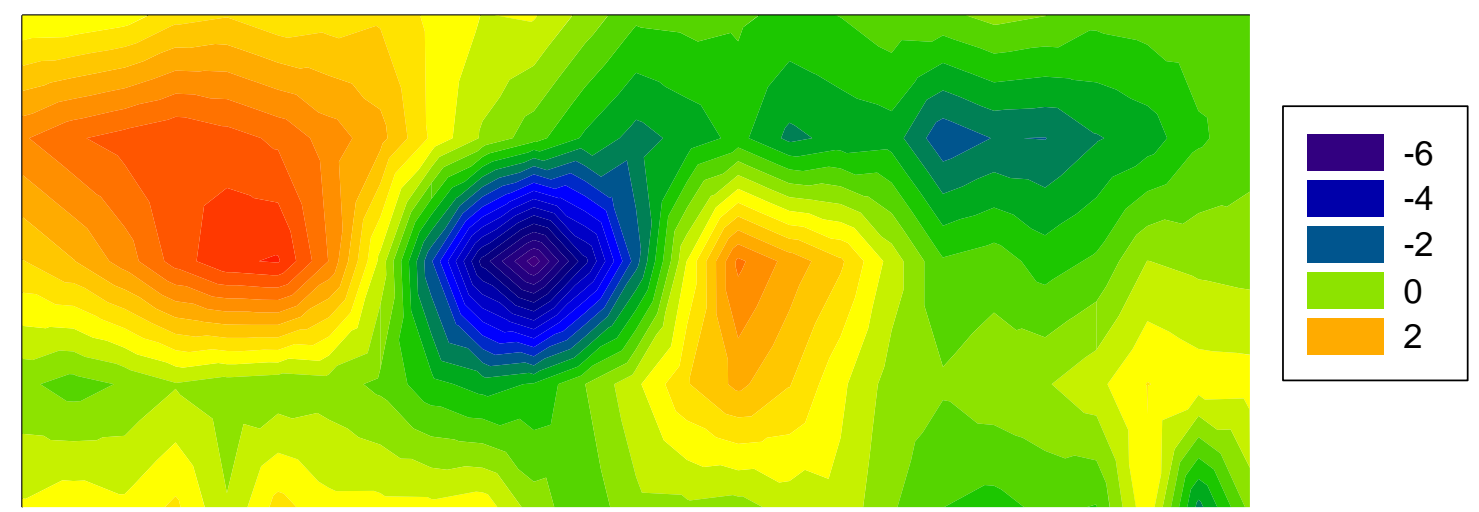

Figure 90. Brightness Temperature Differences

\subsection{4 von Karman Simulation Cases}

In these radiance simulations, a numerical method was used to generate random realizations of a spatial field of temperatures that on average have the theoretical correlation function. These fields were then used with the full HU simulation code, as well as a simpler Matlab version which calculated the radiances. This latter code was based on the constant absorption coefficient model presented above.

Figure 91 shows the spectra calculated from 69 realizations of a von Karman temperature field (blue lines). The simulated field was generated using the assumption that the temperature process is a Gaussian one; hence the statistics of the spectra will be exponential. The exponential probability density function is given by,

$$
P(x)=\frac{1}{\mu} e^{-x / \mu}
$$

where $\mu$ is the mean value for the distribution. For an exponential distribution, the standard deviation is equal to the mean. In this case, the theoretical spectrum level at a given frequency is the mean value. The probability distribution function is given by,

$$
P(x \leq b)=\int_{0}^{b} P(x) d x=\frac{1}{\mu} \int_{0}^{b} e^{-x / \mu} d x=1-e^{-b / \mu}
$$

If $b=\mu, P(x \leq \mu)=1-e^{-1} \approx 0.63$; hence, more often than not, samples from an exponential distribution will be less than its mean, as can be seen in Figure 91. The green curve shows the spectrum generated via averaging the spectra from all the realizations. The red curve is the theoretical spectrum. It appears that there is a slight negative bias in the simulated spectra. It is interesting to note that the averaged spectrum shows aliasing in the higher frequencies. This is due to the requirement that the spatial statistics (i.e., correlation structure) be exact. Therefore, when sampling this field aliasing occurs - just as would be the case for sampled real data. 
Figure 92 shows the averaged and theoretical correlation coefficient. The averaged correlation coefficient was generated from 120 random von Karman temperature fields.

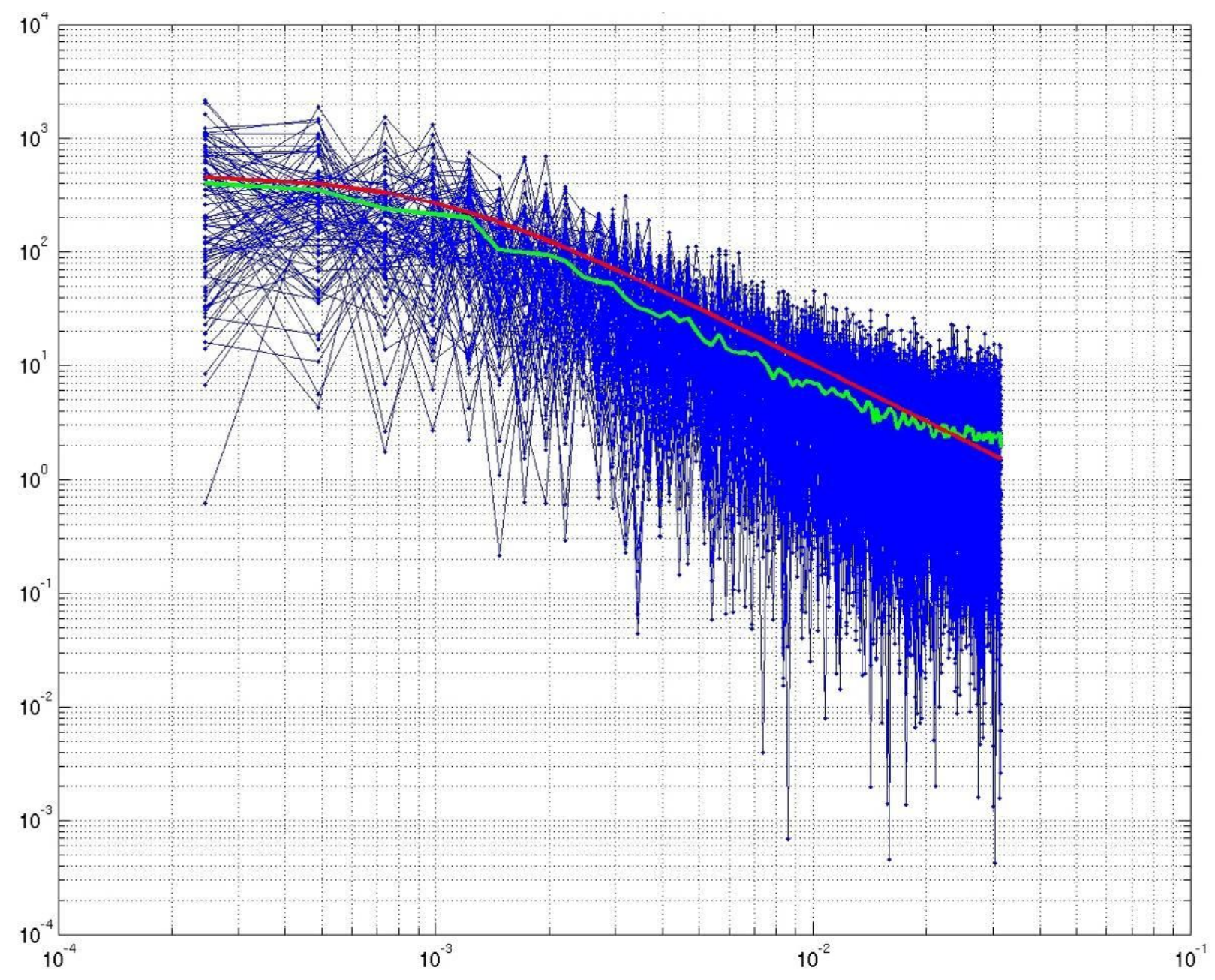

Figure 91. Power spectra of a simulated von Karman temperature field. 


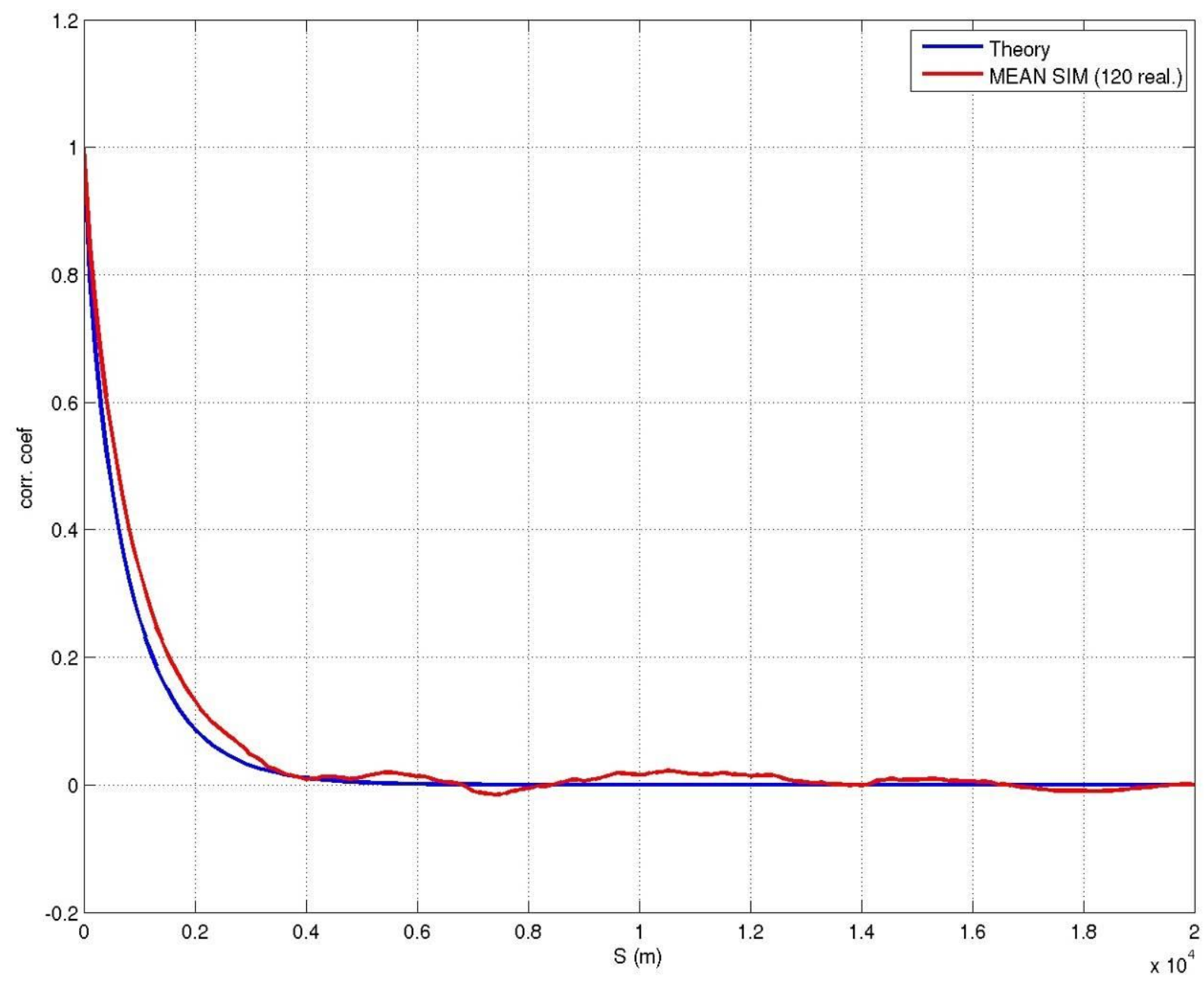

Figure 92. The averaged simulated and theoretical correlation coefficients.

For the theoretical analysis, as well as the von Karman simulations it was assumed that the absorption coefficient was a constant function of space. This quantity was estimated from the HU simulation code by using a constant altitude and Standard Atmosphere quantities for that altitude. This means that the temperature field was also constant function of space. This allows for the solution of the absorption coefficient by inverting the equation for the radiance. From above, we have:

$$
I(x)=\alpha e^{\alpha x} \int_{x}^{L} B\left(x^{\prime}\right) e^{-\alpha x^{\prime}} d x^{\prime}
$$

For constant $B(T(x))$, this can be solved for $\alpha$, 


$$
\alpha_{v}=\frac{-\ln \left[1-\frac{I_{v}(x)}{B_{v}(T)}\right]}{L-x}
$$

where the wavenumber dependence has been indicated explicitly. Figure 93 shows the results of this calculation for the von Karman temperature field. The upper figure shows the radiance calculated from the HU simulation (line connecting the red and blue dots). The saw tooth pattern may be an artifact from interpolation in the code. The straight line going through the sawtooth line is the average of the even and odd points, with 120 realizations used in the average. The bottom image shows the result of the absorption coefficient computation from the even (red), odd (black) and averaged radiances.
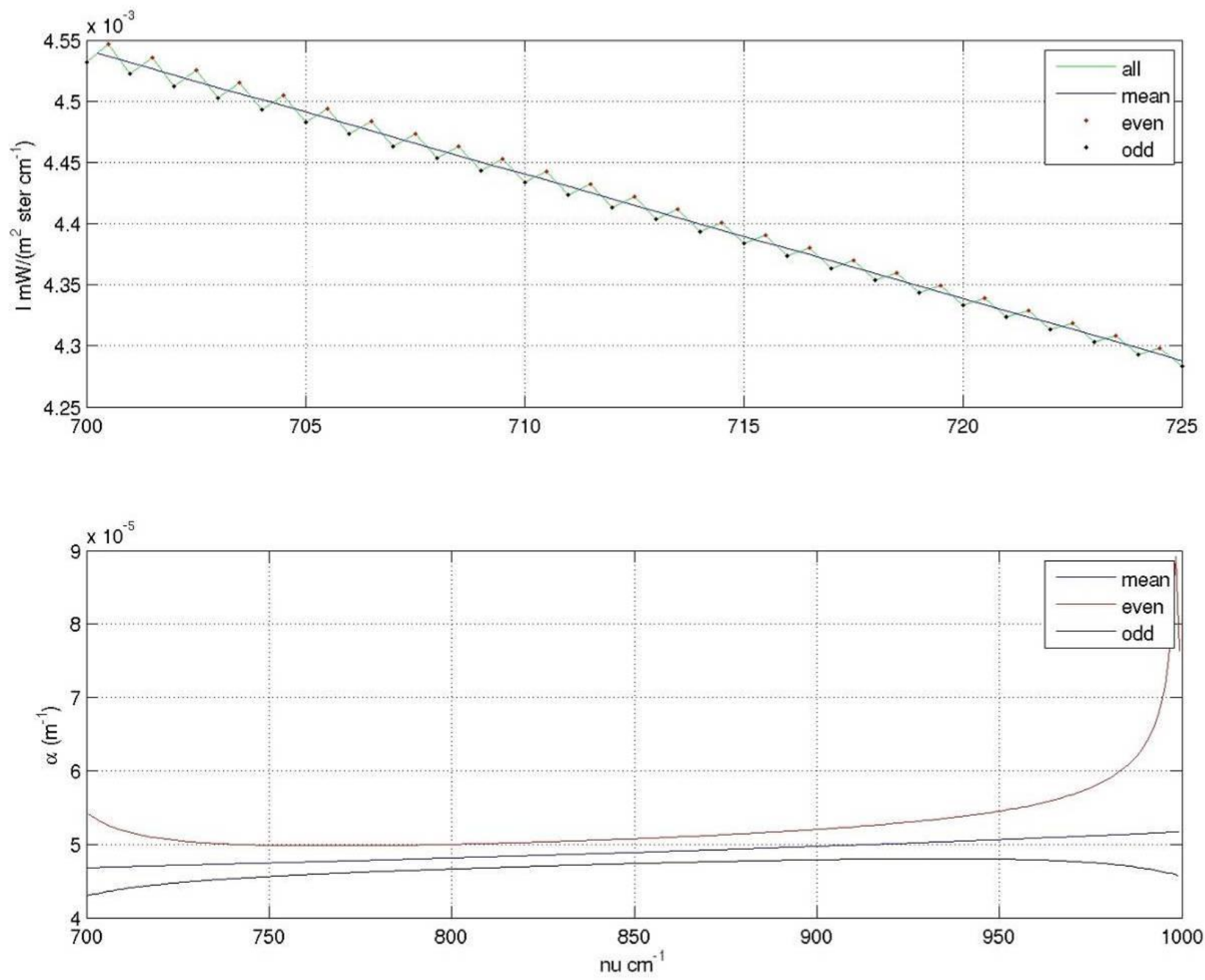

Figure 93. Estimation of absorption coefficient for Standard Atmosphere.

Figure 94 shows the result of the HU simulation applied to four simulated cases, with $\sigma_{T}=1$ and $5 \mathrm{~K}$, and $L_{T}=1000$ and $2000 \mathrm{~m}$. Note that the radiance of the constant $\mathrm{T}$ plus added von Karman $\mathrm{T}$ is subtracted from the radiance for constant $\mathrm{T}$. The turbulent temperature field 
was $100 \mathrm{~km}$ in length and the "aircraft" moved forward approximately $170 \mathrm{~m}$ per sample. The radiance for the constant altitude $(10 \mathrm{~km})$ standard atmosphere case with an added perturbation von Karman temperature field is calculated and then the radiances from the constant temperature case are subtracted. The figure shows these radiance differences as a function of wavenumber. The effect of varying the parameters is clear - especially the $\sigma_{T}=5 \mathrm{~K}, L_{T}=2000 \mathrm{~m}$ case. At first glance, this figure appears odd - in fact it looks like what one would get by inverting a standard absorption curve (modulo the sign, and absolute magnitude). So the values with smaller absorption appear to be larger, and vice versa. Figure 95 shows a detail view from the 725-760 $\mathrm{cm}^{-1}$ region of Figure 94.

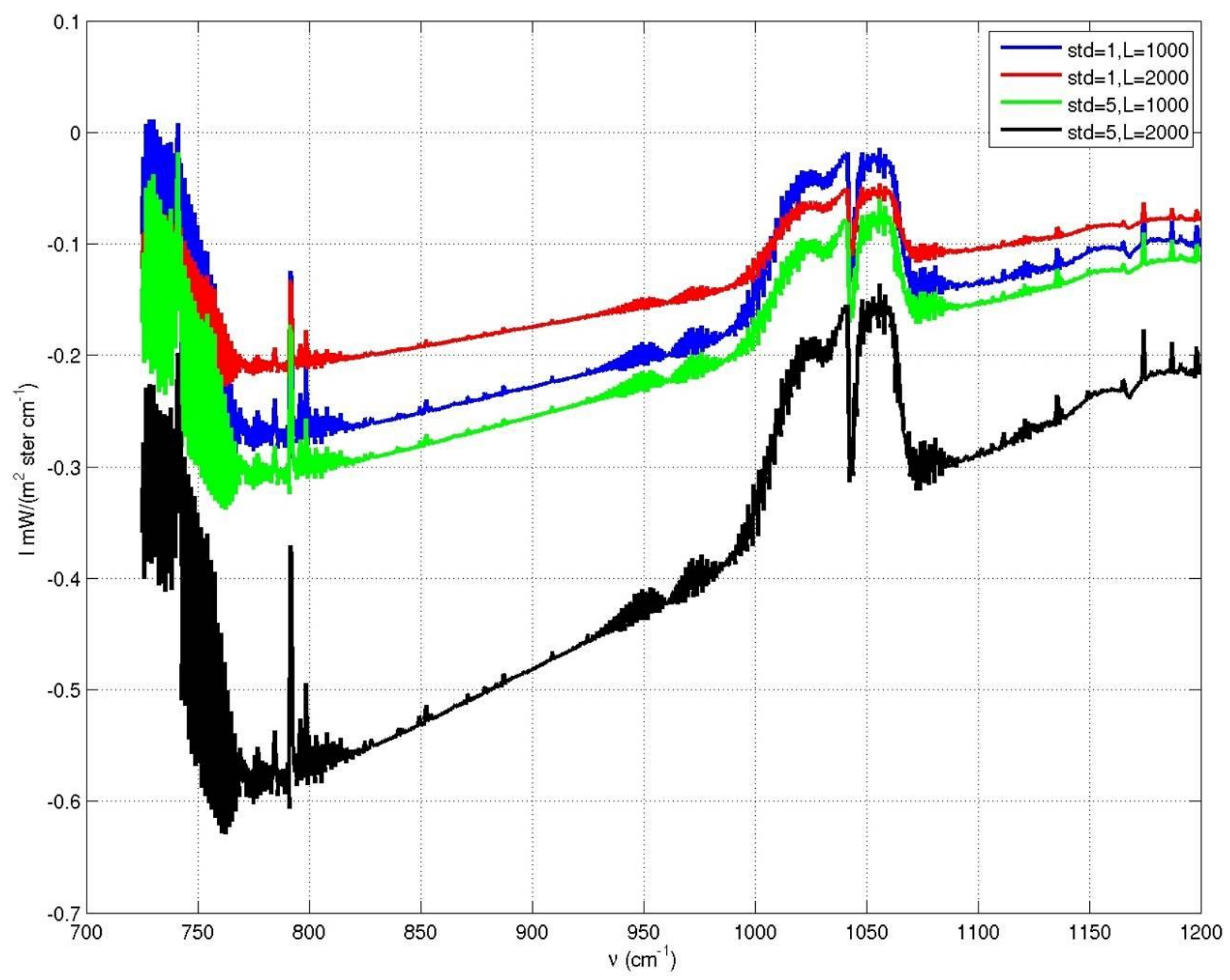

Figure 94. Simulated radiances as a function of wavenumber. 


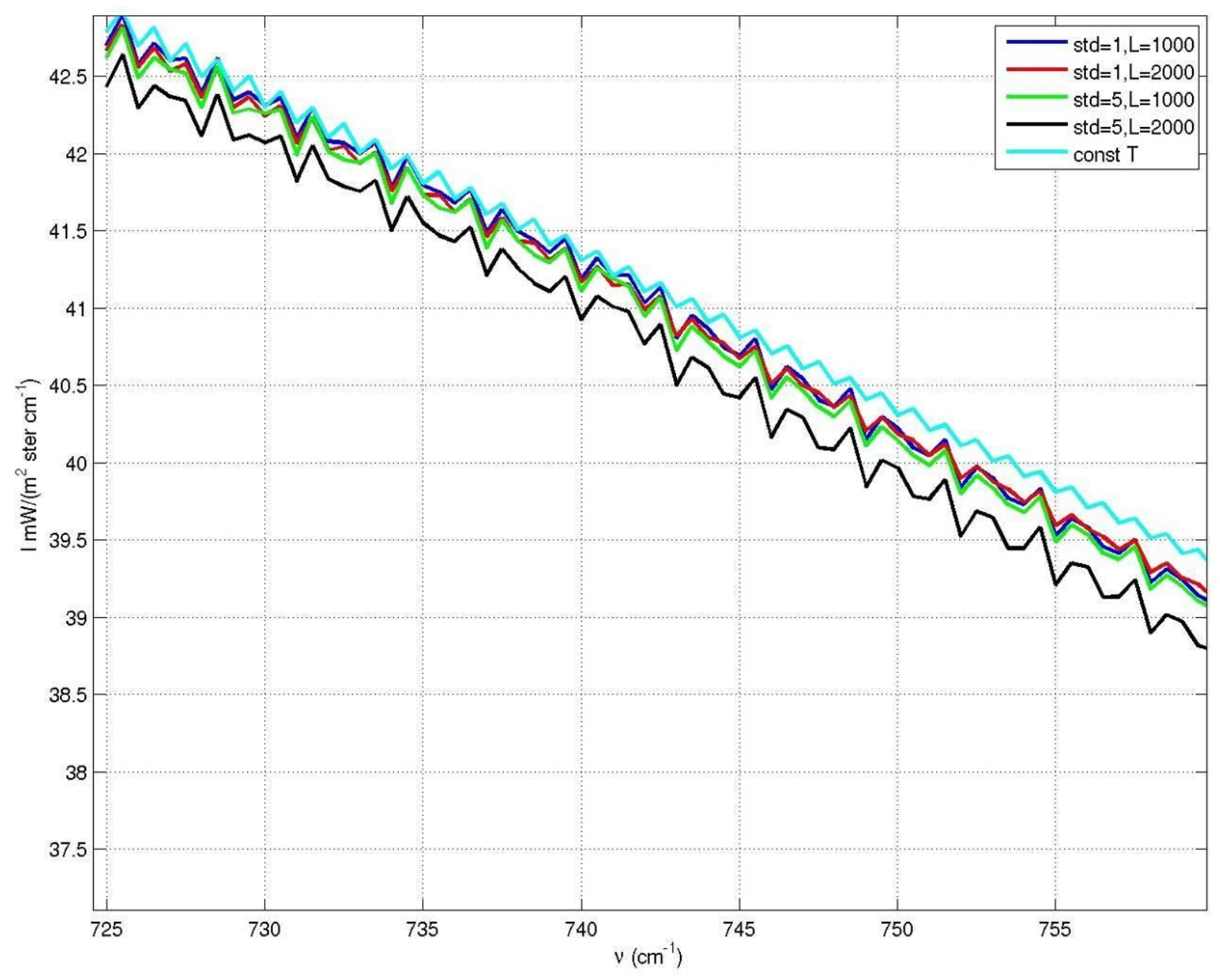

Figure 95. Detail of the data from Figure 94. 


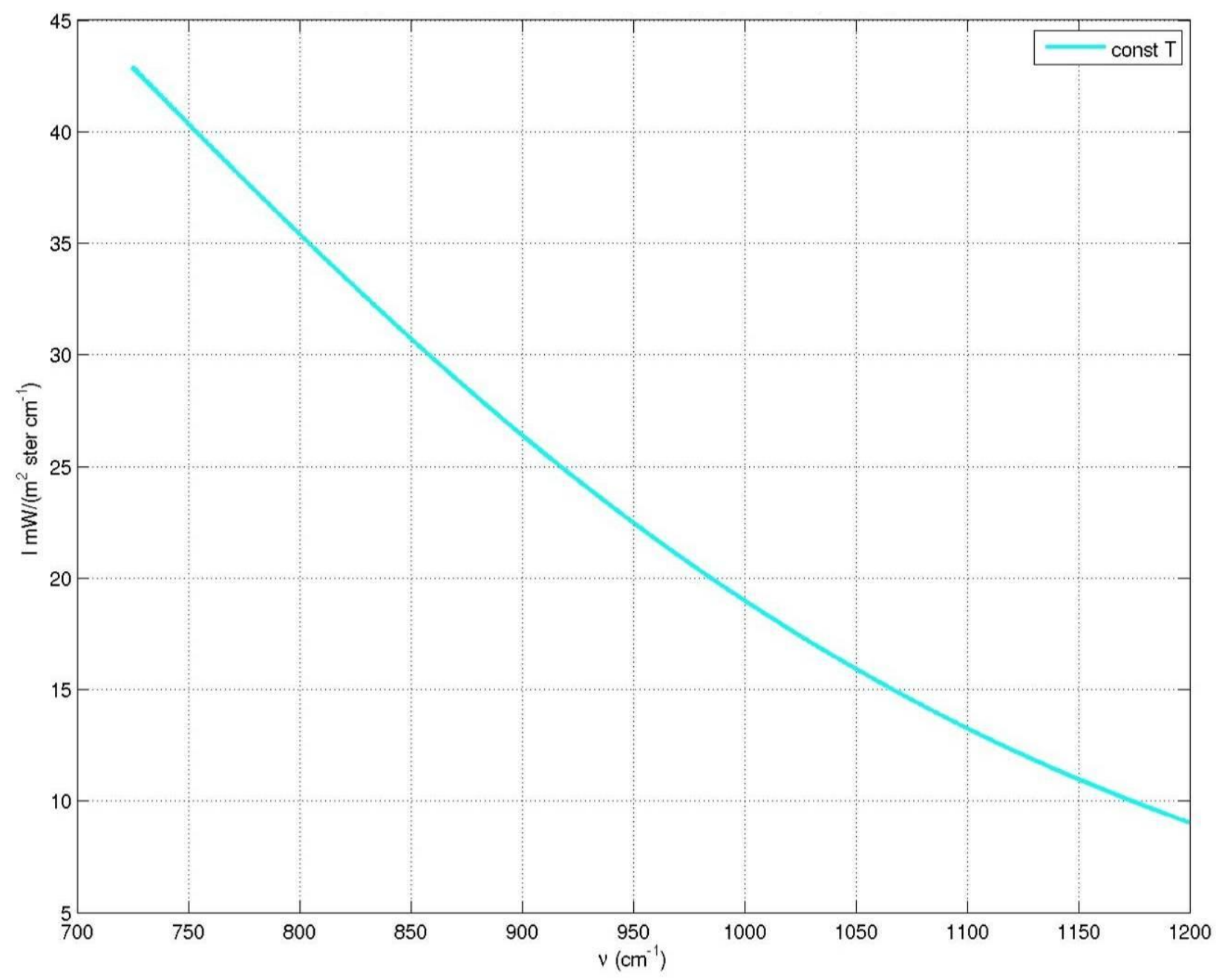

Figure 96. Radiance as a function of wavenumber for a constant temperature.

In Figure 96, just the radiance from the constant altitude (constant temperature) simulation is shown. Note the similarity to the Planck function for that temperature. Figure 97 is a plot of the data from Figure 96 along with the simulated radiance for the constant temperature plus the zeromean von Karman field. Radiance as a function of wavenumber from the Matlab simulation using a von Karman perturbation field added to a constant temperature field (dark blue). The light blue curve is the data from Figure 100. Note that the differences are quite small, and at this scale it is hard to discern any structure. The structure is revealed by the blue curve in Figure 94. 


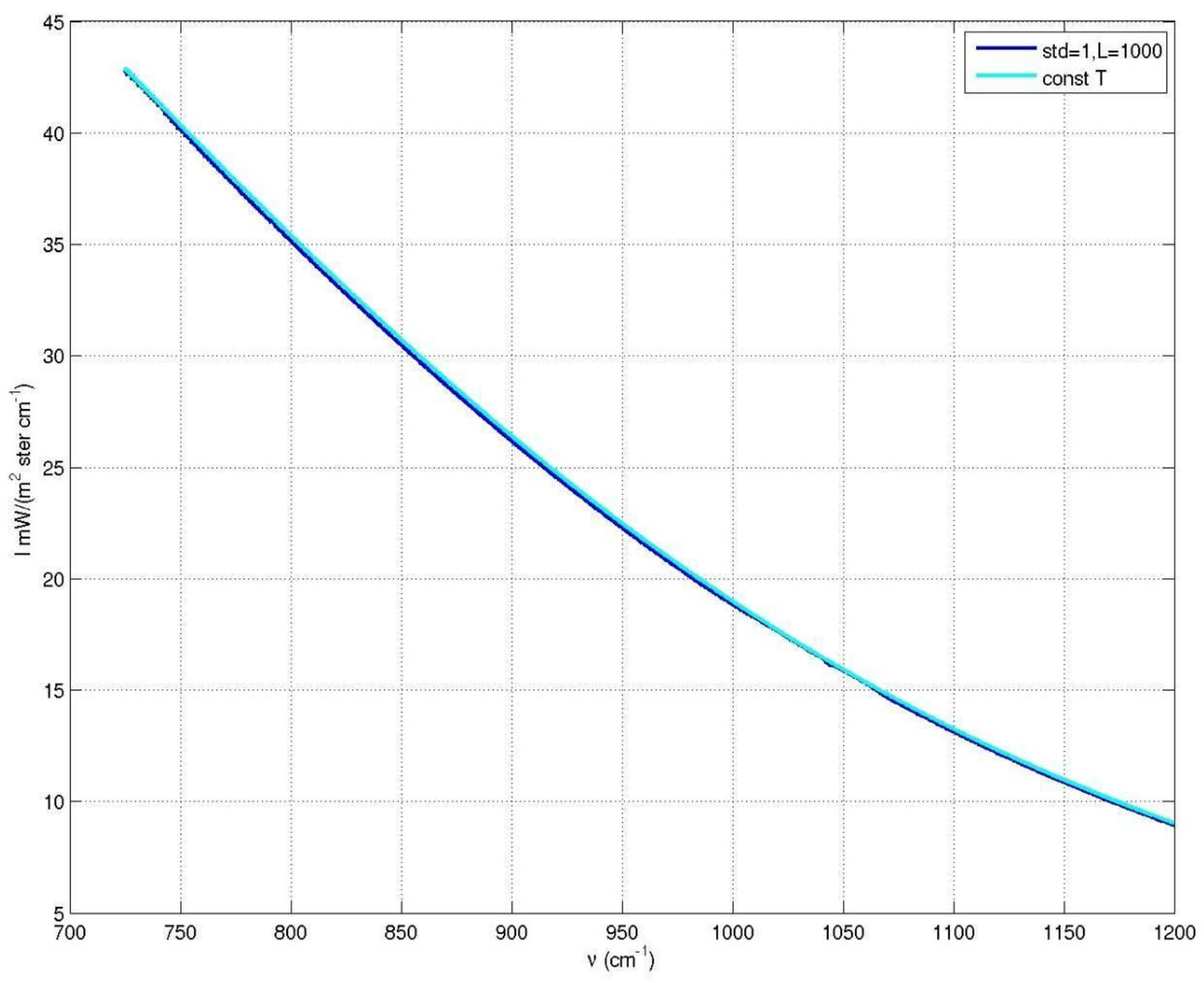

Figure 97. Radiance using a von Karman perturbation field 


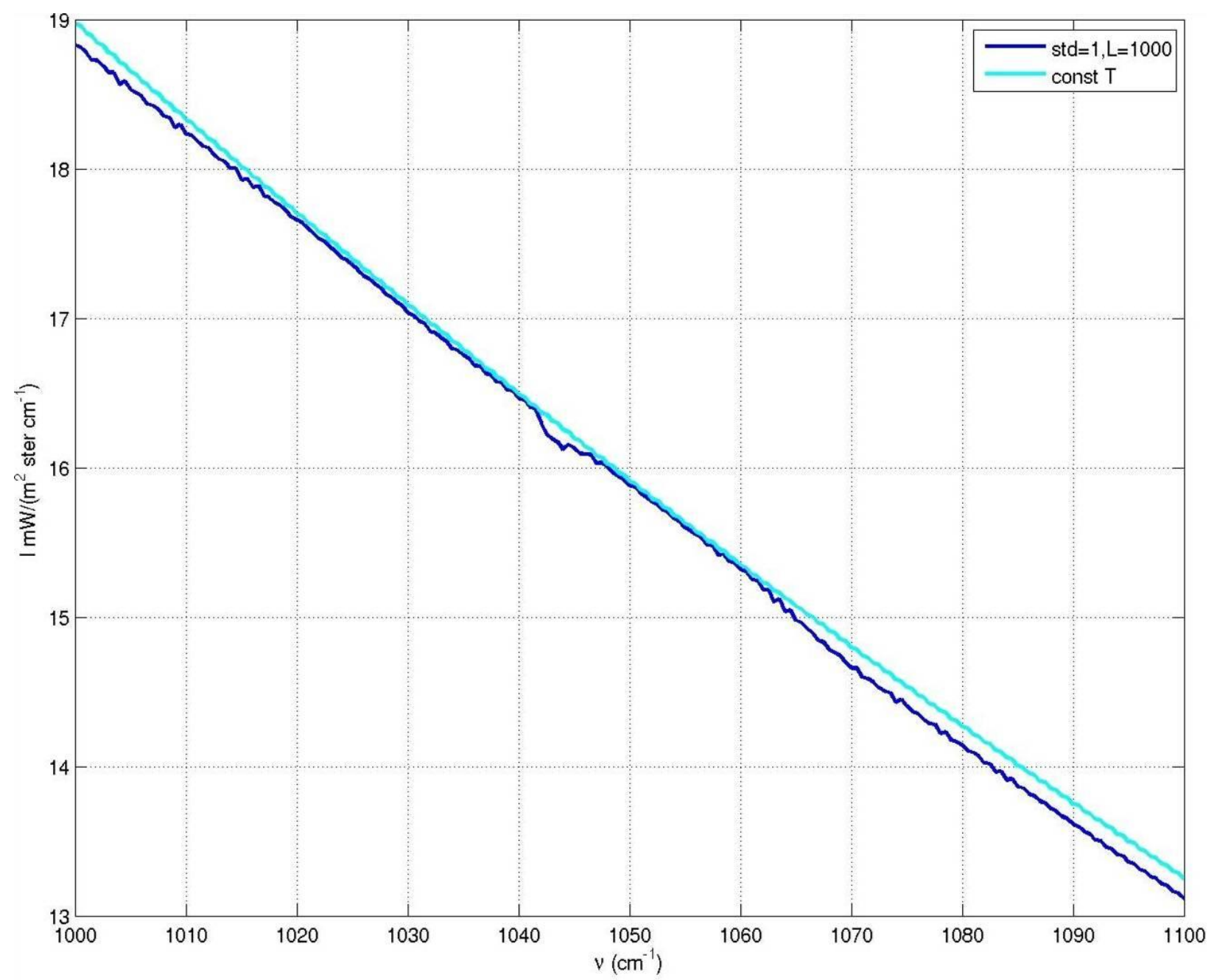

Figure 98. Detail of the data from Figure 97 between 1000 and $1100 \mathrm{~cm}^{-1}$.

Figure 99 illustrates the radiance differences (at a single wavenumber) for the four different cases shown in Figure 94. The upper panel shows the values from the HU simulation code, the lower from the Matlab code. There are clear differences, for which the source is unknown at this point. One possibility is - as mentioned above - that the Matlab code uses a constant absorption coefficient (taken from the constant temperature at the given altitude), whereas the HU code computes this value given the temperature (constant plus perturbation) at each point. 

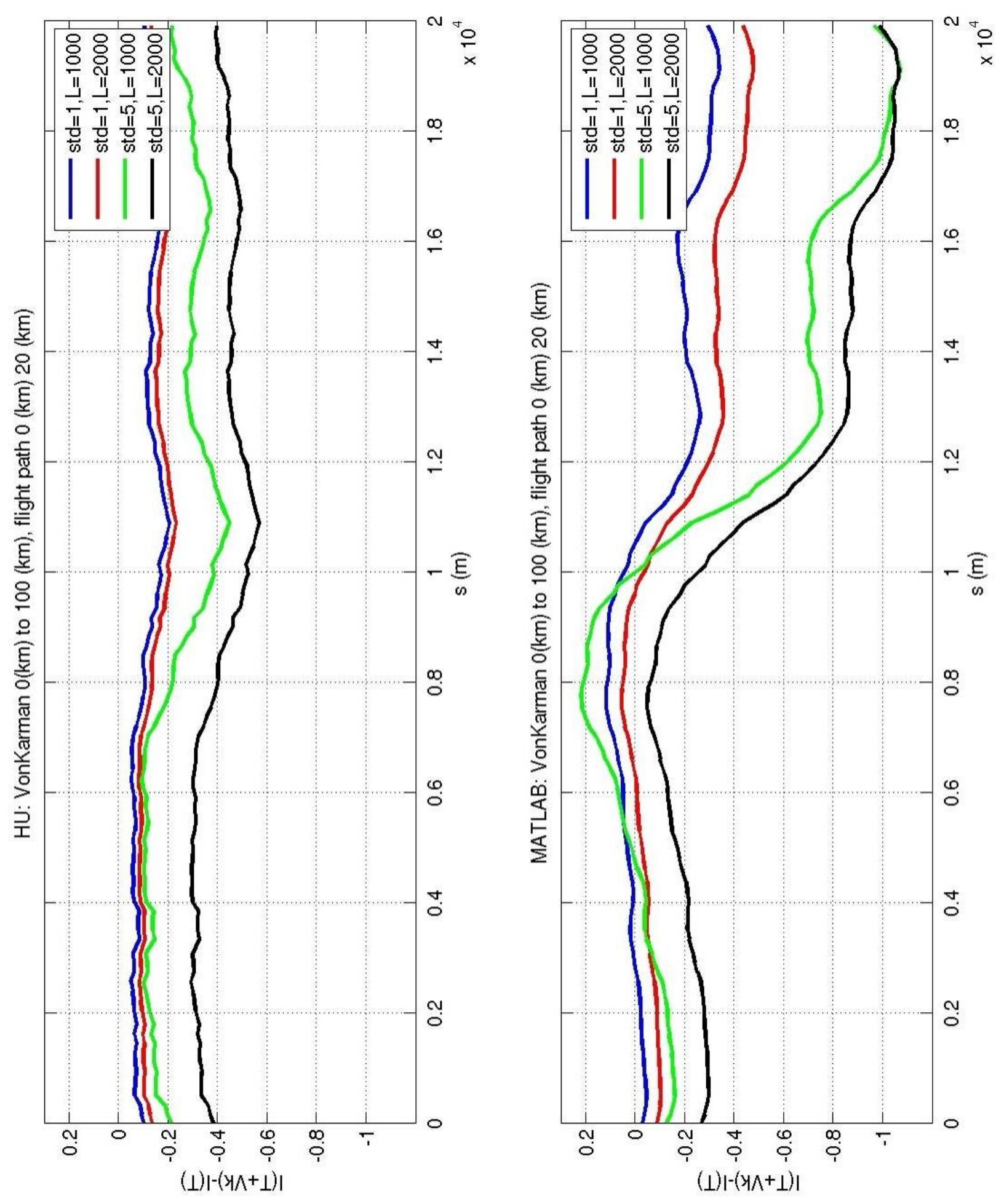

Figure 99. Comparison of four cases of a simulated radiance field. 

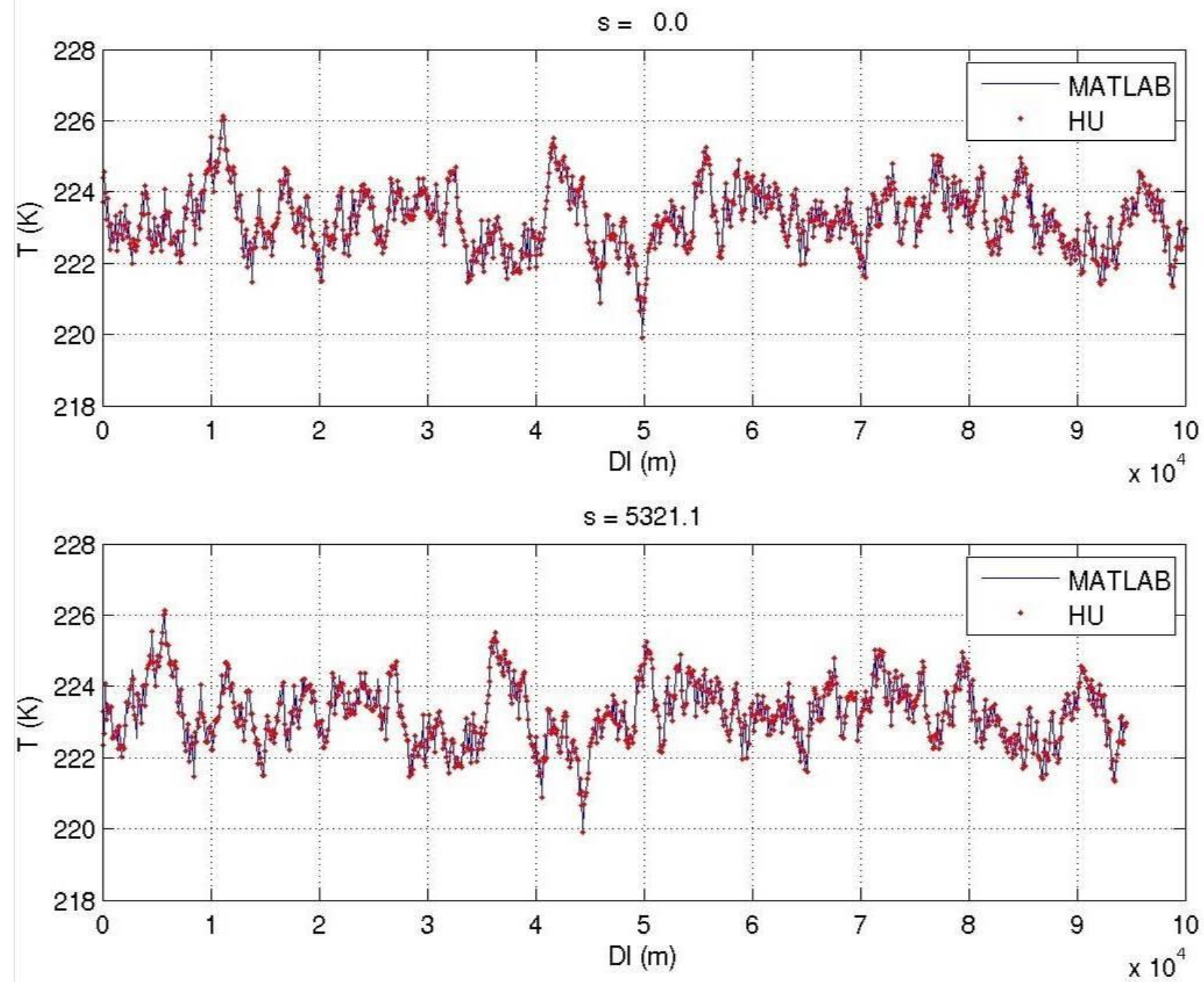

Figure 100. Comparison of von Karman temperature field used in the simulations.

Figure 100 shows the comparison of the temperature field (constant plus von Karman perturbation), as a function of aircraft motion along the flight path. The red dots are ones input to the HU simulation and the solid blue curve are those used in the Matlab simulation. The upper panel shows the data for the aircraft's initial position looking forward, whereas the lower panel shows the data for the aircraft at approximately $5300 \mathrm{~m}$ from the initial location. This confirms that the temperature data used in each simulation method is indeed the same; hence the discrepancy in the radiances does not come from a different set of temperatures being used.

The upper panel of Figure 101 shows the radiance difference for six different realizations of the von Karman temperature field, and the lower panel shows the corresponding correlation coefficient for the different realizations. It can be seen that the variation over realizations can be significant. Recall that the theoretical analysis presented above is for the ensemble average correlation functions and coefficients. 

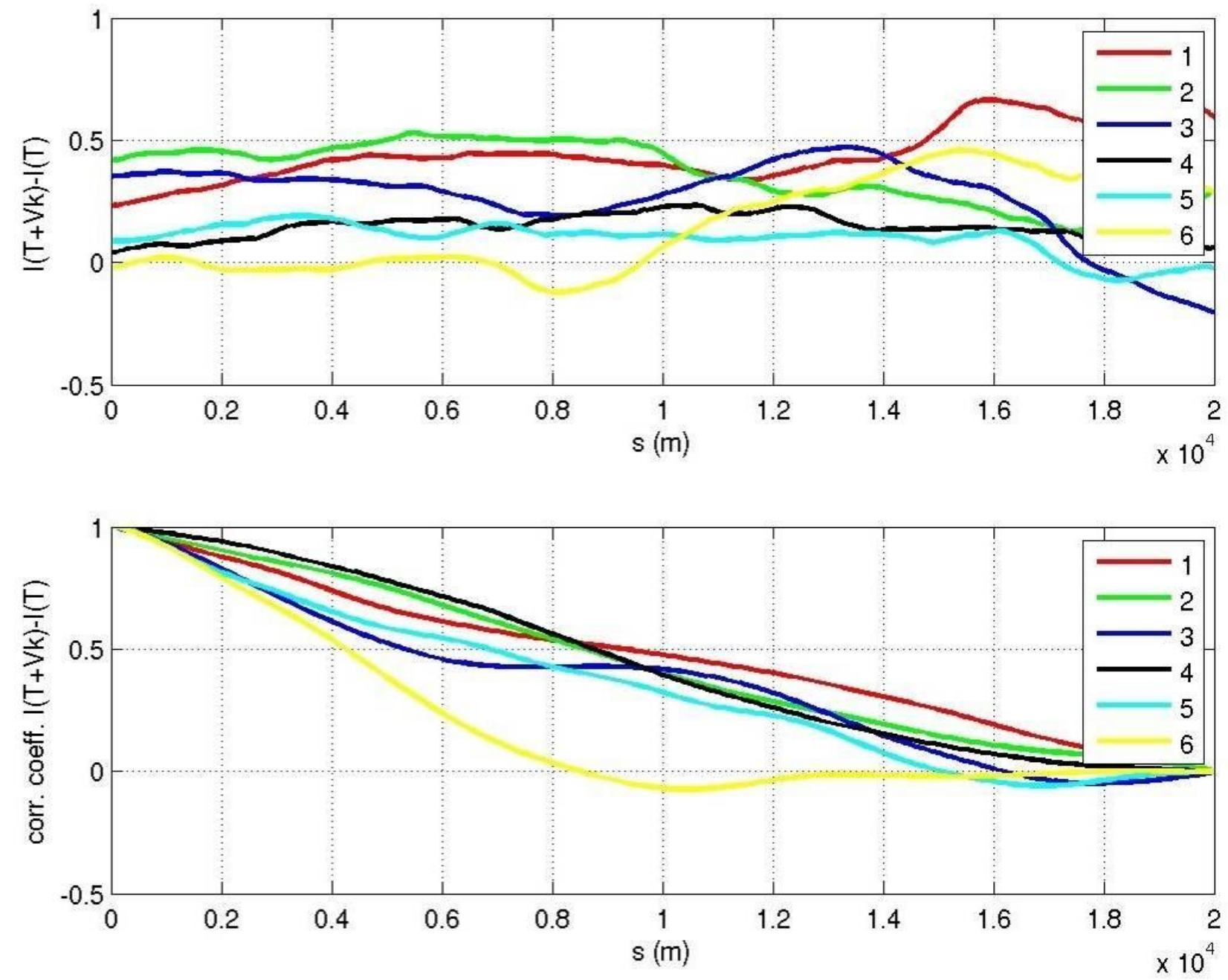

Figure 101. Simulated radiance difference field and associated correlation.

Figure 102 shows the radiance correlation coefficient as a function of aircraft displacement (lag) for the same four cases shown in Figure 94. Clearly, while the general trends are similar, there are distinct differences between the codes. Recall that the HU code computes the absorption coefficient for each position in space, and here the perturbed temperature field will have an effect on that quantity. It is not clear if that is the source of the difference. Since the HU simulation is much more similar to the theoretical calculations presented above, it is not clear whether this is a dominant effect. More analysis is needed on the effect of the spatially varying absorption coefficient. 

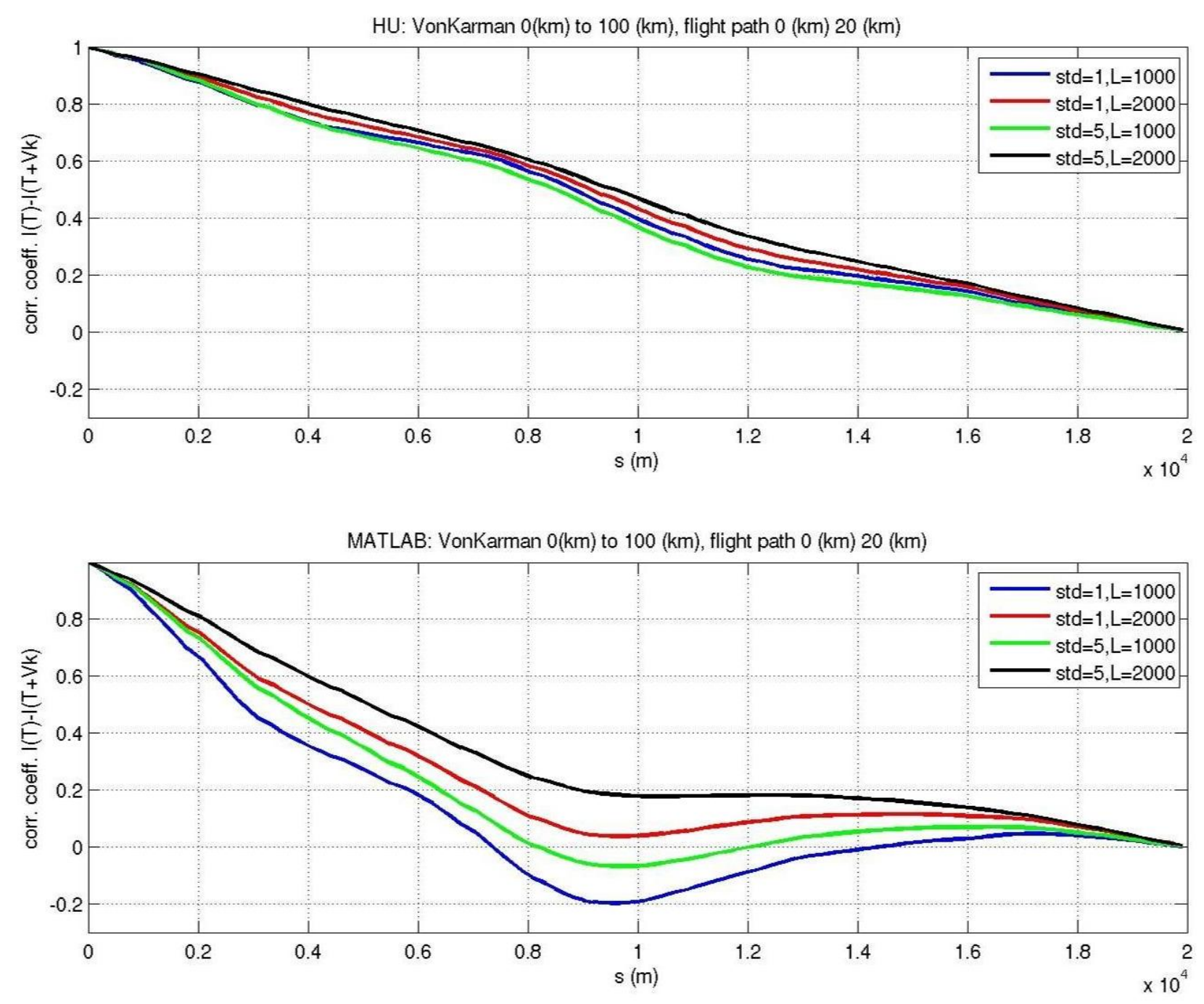

Figure 102. Comparison of the radiance correlation coefficient.

Finally, Figure 103 shows a comparison of the HU (blue) and Matlab (red) correlation coefficients for the average of the six realizations of the correlation functions used to create the correlation coefficient shown in Figure 101. After the averaging, these functions from the two simulation codes are more similar to each other, and compare favorably to the theoretical results derived above. It should be noted that the theoretical calculations were performed for just the ensemble average of the perturbation temperatures - not the differences between the radiances from the constant temperature with perturbed von Karman, and those from the constant temperature field. This is not the same as computing the radiations from the temperature differences (i.e., the perturbation temperature field). Also the Planck function model for the theoretical analysis was the linear temperature approximation, whereas the simulations used the full Planck function. Both of these items could be a source of discrepancy between the theoretical and simulated radiance correlation coefficients. Clearly, these are items for further analysis. 


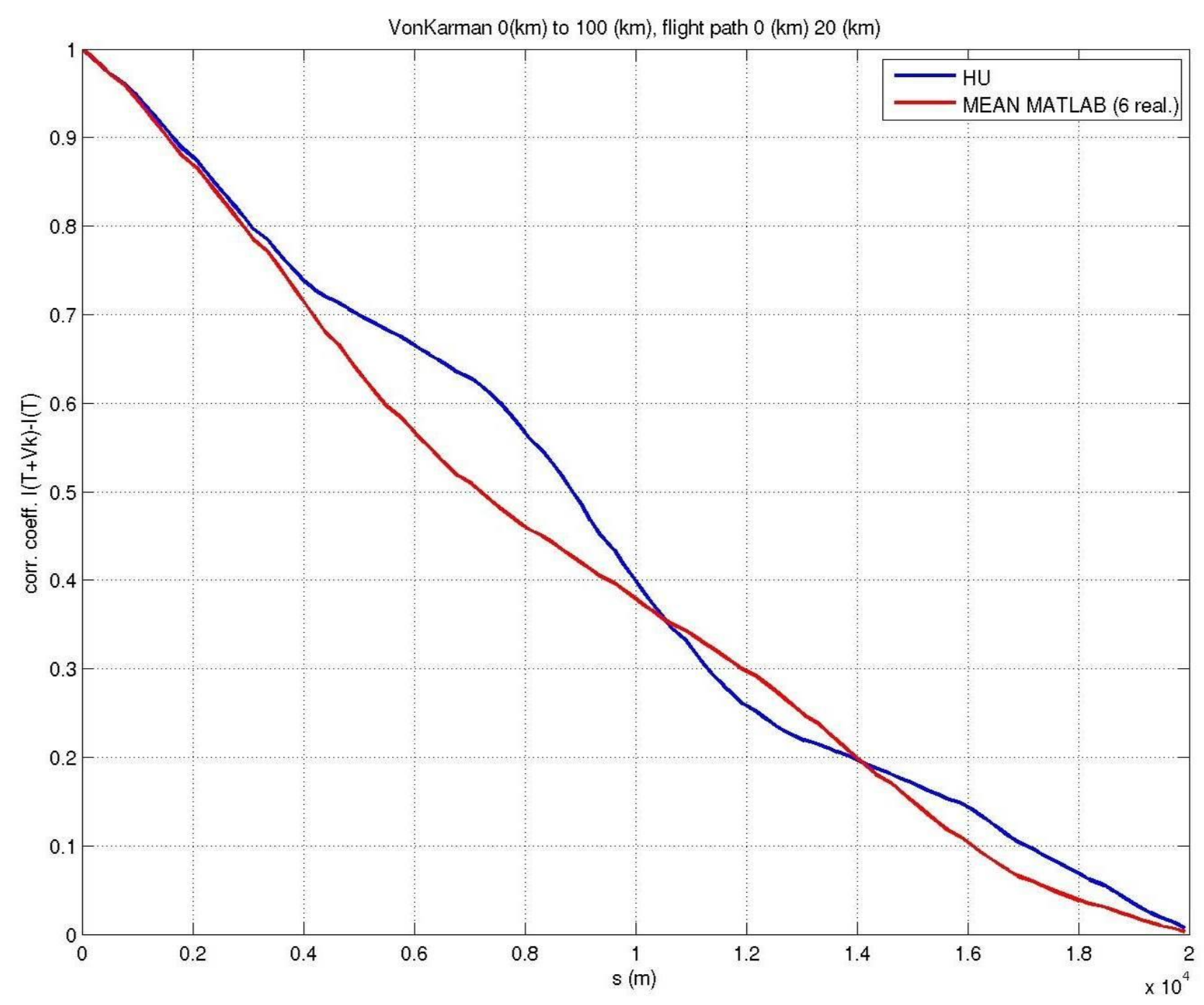

Figure 103. Comparison of the correlation coefficient calculated from simulations.

\section{Field Tests}

An empirical demonstration of the imaging FLI aviation hazard detection and hazard display capability was conducted as part of this study. The program included two field programs. The first field program occurred in Boulder, CO in January 2008. This field program focused on clear air / Lee wave turbulence. The second field program occurred in Madison, WI in June 2008. This field program focused on wake vortices. Both field programs were ground-based and included truth data. Site visits were conducted at each location prior to the field programs to assist in planning. Further details of the field programs are given below.

\subsection{Boulder Field Test}

A field program for Boulder, Colorado occurred on January 13 - 18, 2008. Due to delivery delays of an instrument, the November field program planned for Madison, WI, was delayed. 
The main purpose of these field programs was to evaluate the feasibility of an infrared interferometer to detect turbulence at a useful distance from the sensor. A secondary purpose of the tests was to evaluate turbulence detection and hazard prediction algorithms for r.m.s. vertical acceleration.

Radiometric measurements were conducted at the NCAR Foothills Laboratory in Boulder, Colorado. The instrument used was a D\&P spectral sensor, which is a spectral radiometer based on a rotary Michelson interferometer. The D\&P continuously recorded radiance spectra at a rate of four per second, covering the $2-18$ micron range at $4 \mathrm{~cm}^{-1}$ resolution. The D\&P was used with fore-optics that provided a 1.2 degree field of view, and a gold first-surface mirror was used to steer the field of view any angle from the zenith to the horizon.

The D\&P spectral sensor was used during the field test to gather the radiometric data over the full spectrum from 2 to 15 microns. The December time period was initially chosen as it is an active period for clear air turbulence, specifically, mountain wave turbulence generated over the Rocky Mountains. The location of the test is also important due to significant amount of air traffic associated with the Denver International Airport. The large amount of air traffic meant that pilot reports (PIREPS) and automated reports from United Airlines (UAL) 757 aircraft would be available as "ground truth" for the turbulence detection efforts.

The UAL aircraft are equipped with software to estimate and automatically report eddy dissipation rates (EDR) to the one-third power. These reports consist of peak and mean value of the EDR over the previous one minute (in cruise). These reports cover "none" to "extreme" levels of turbulence, which will be used as verification data. The truth data will allow for the evaluation of both true detection and false detection by the sensor and algorithms. PIREPS, on the other hand, are more subjective in nature. PIREPS are not always given for turbulence encounters (even severe ones); they rarely provide for null turbulence; the reported time and position can be in error; and the intensity level of the turbulence reported by the pilot is a subjective measure of the actual intensity level. 


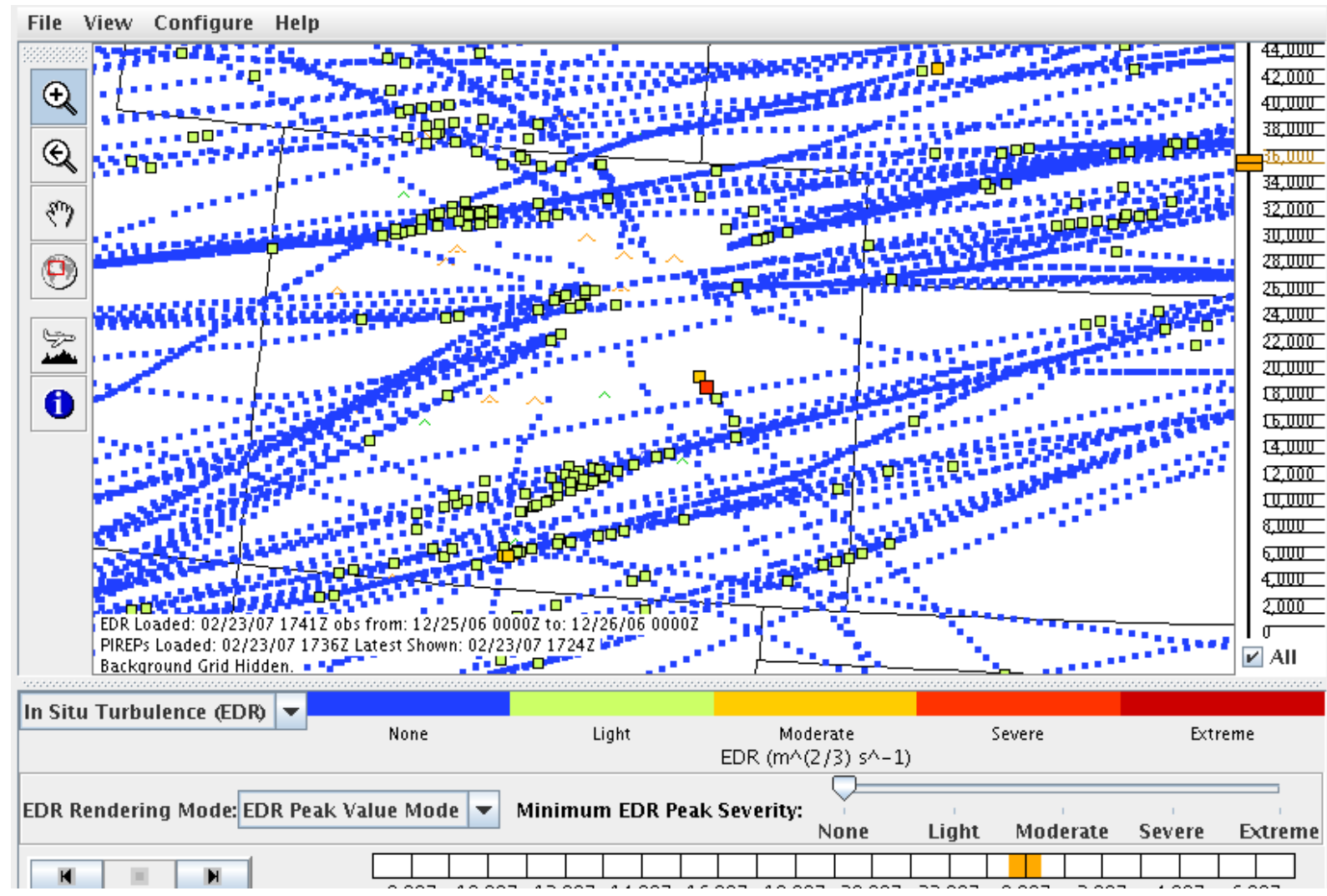

Figure 104. UAL EDR reports over Colorado for a 24 hour time period.

During the field test, the "EDR viewer" allowed for the display of real-time and archival EDR data sets. Figure 104 illustrates the viewer in archival mode, showing a 24 hour period of EDR reports, centered over the Colorado region. Each of the color-coded square dots indicates a oneminute EDR report for aircraft above 20,000 ft. Note that there a few designated air routes, along which a number of the flights come into and leave the Denver airport. Furthermore, the EDR viewer contains turbulence forecast information from the Graphical Turbulence Guidance (GTG) product. These data can be used in real time during a data collection period, or used to determine when good data collection periods might occur. Figure 105 illustrates a one-hour period during a severe encounter, showing the EDR reports and the GTG forecast valid at that time. In the figure, the solid colored regions are turbulence forecasts from GTG. 


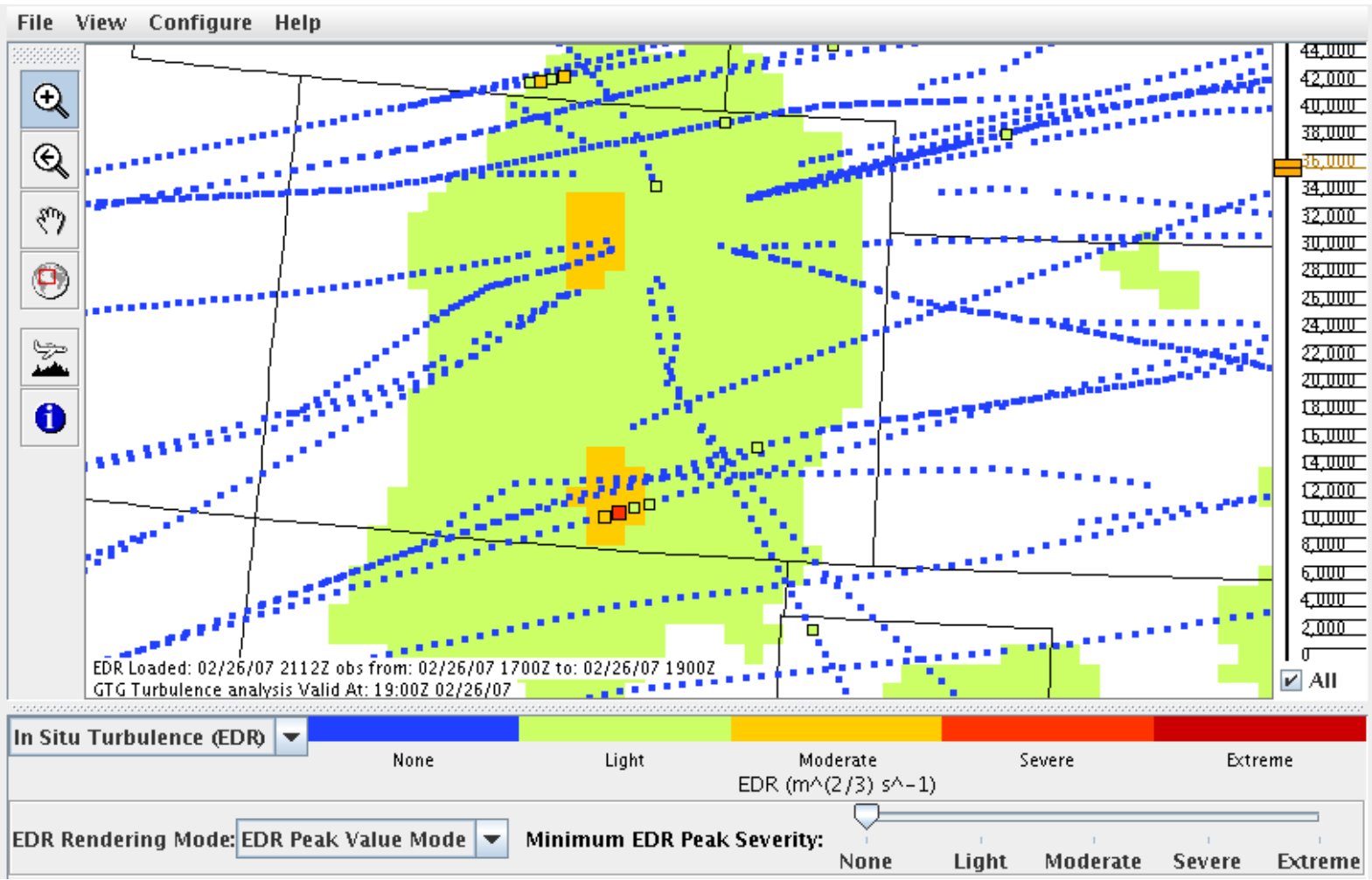

Figure 105. UAL EDR reports over Colorado, during a one hour time period.

The UAL reports also contain wind and temperature measurements from the time of the report. While these data are course in spatial scale, they can still provide useful information. Figure 106 shows a CAD drawing of the test equipment. Figure 107 illustrates time series plots of (from top to bottom) peak EDR, wind speed, wind direction, and temperature for a severe turbulence encounter from UAL EDR reports. It can be seen that there are changes in the wind prior to the encounter and temperature changes during the event. Furthermore, these data can be used to drive a UCAR-developed mountain wave turbulence forecast algorithm, and they can be used as input to a high-resolution model. These model results can then be used to drive sensor simulations.

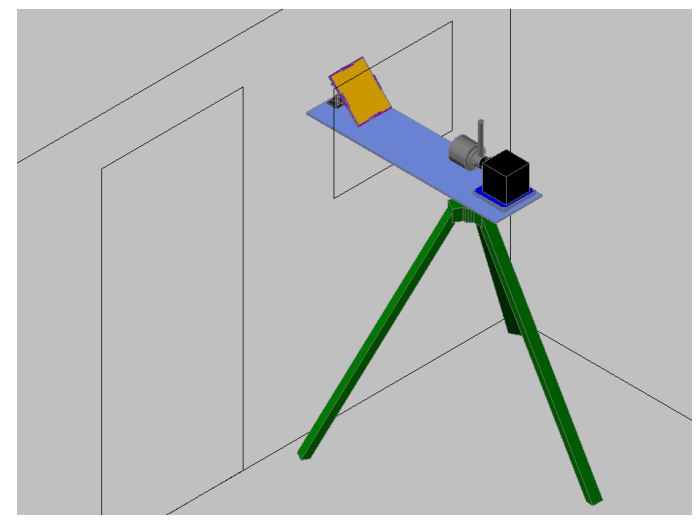

Figure 106. CAD drawing of the tripod-mounted D\&P system. 

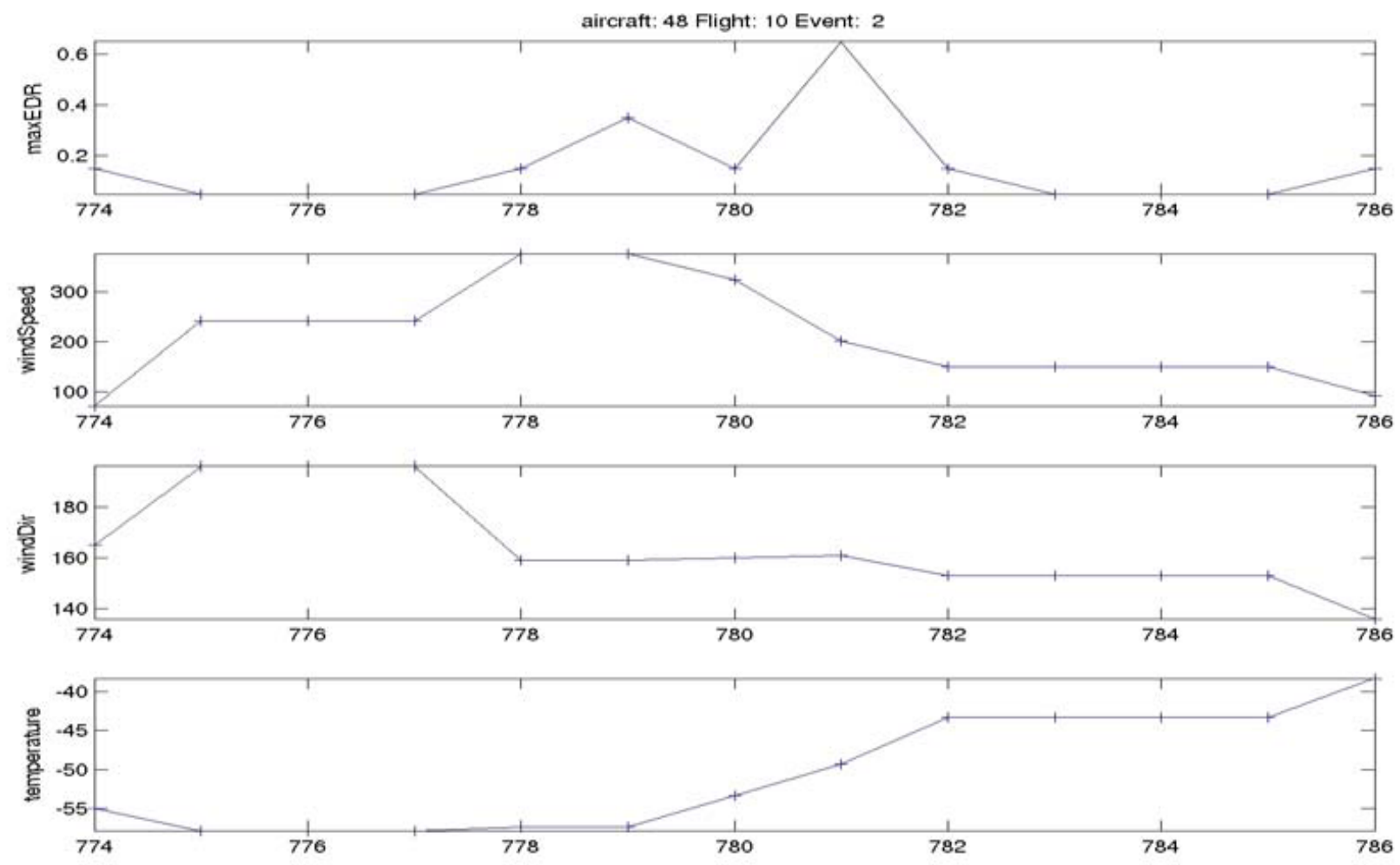

Figure 107. Time series plots from UAL EDR reports.

\subsubsection{Boulder Field Test Detail}

On January 13 - 18, 2008, field measurements were made of turbulent areas of the sky caused by mountain waves over the Rocky Mountains with a D\&P spectral sensor. The test was conducted at the NCAR Foothills Laboratory. The trailer was located at $40^{\circ} 02^{\prime} 18.01^{\prime \prime} \mathrm{N}, 105^{\circ} 14{ }^{\prime} 25.72^{\prime \prime} \mathrm{W}$ and $5287 \mathrm{ft}$ elevation, with the window side of the trailer facing 37 degrees south of west,

Figure 108 is an image from Google Maps ${ }^{\mathrm{TM}}$ with an arrow indicating the trailer site.

Figure 109 shows photos of the setup, including the tripod-mounted D\&P and the gold steering mirror.

Figure 110 shows a close up of the D\&P with parts labeled, including the HOBO Pro ${ }^{\mathrm{TM}}$ Series temperature and humidity monitor. 


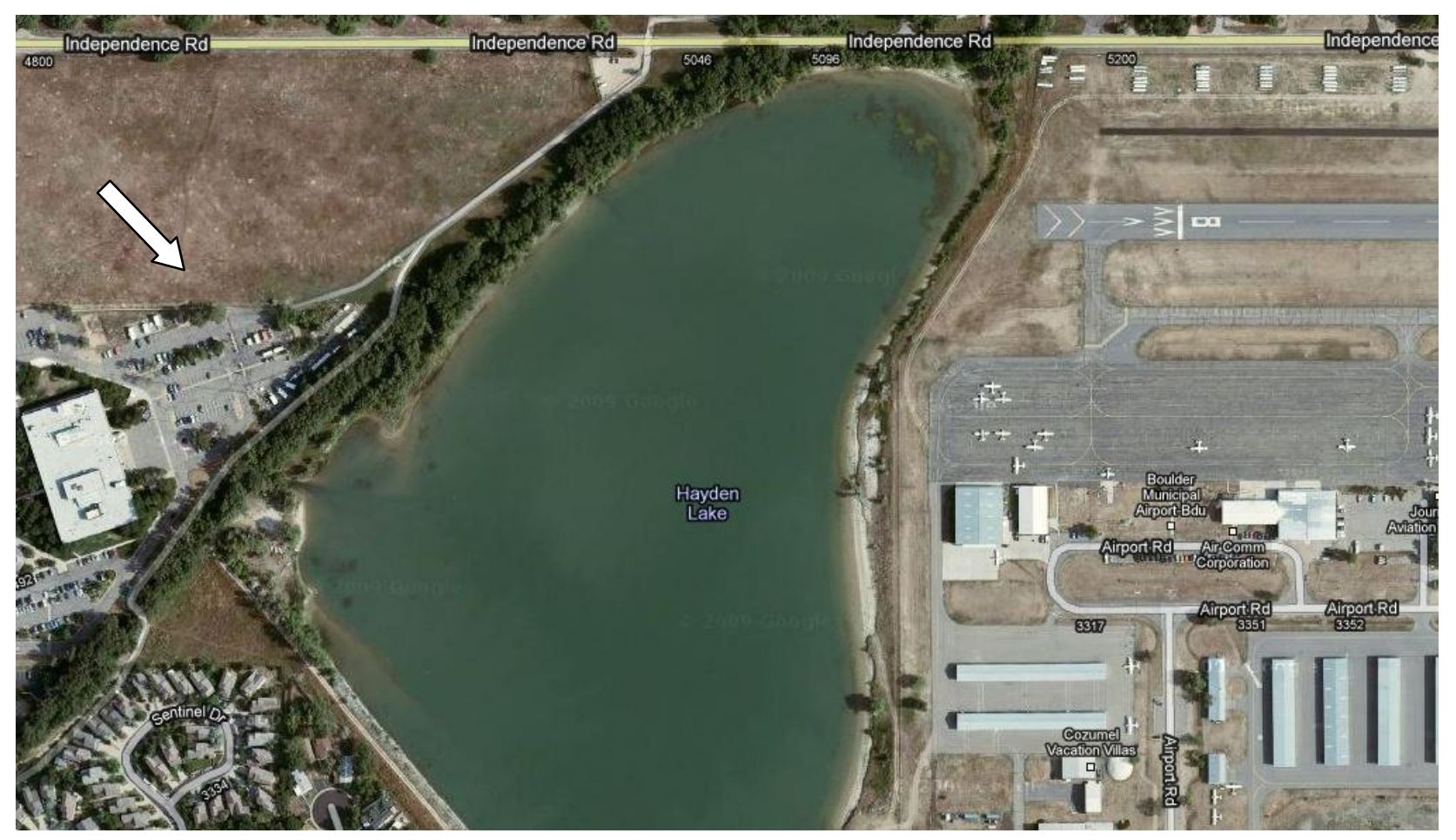

Figure 108. Google Earth image of trailer site.
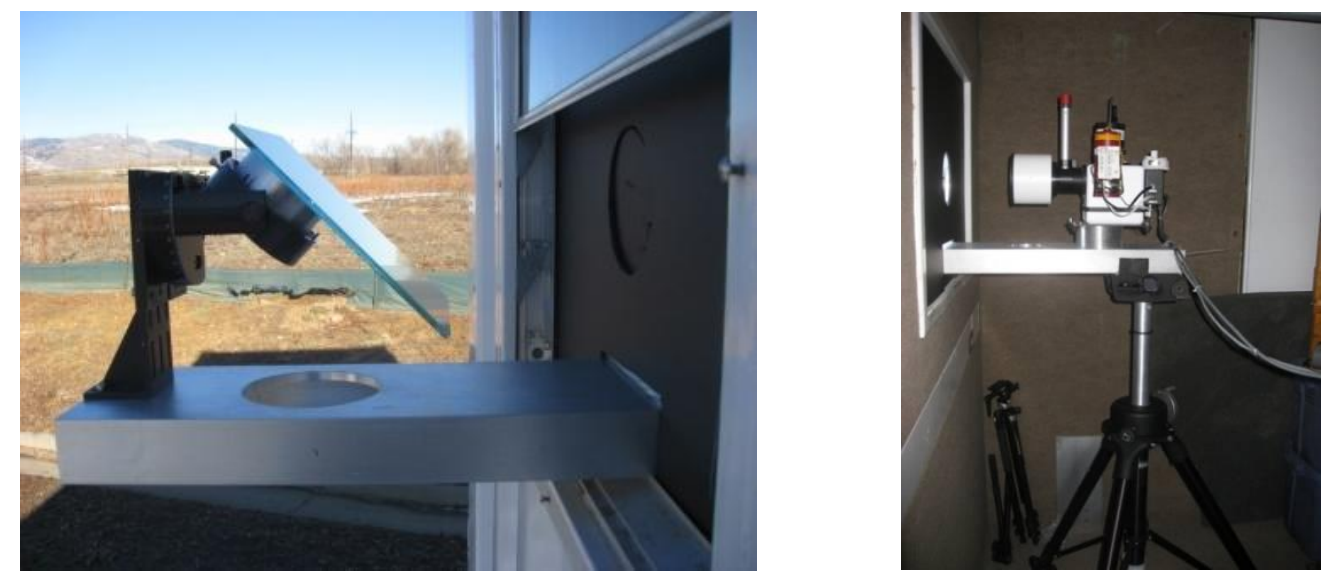

Figure 109. Gold mirror to control viewing angle, and the D\&P set up in the trailer.

The system was calibrated with cold and warm blackbodies before each measurement series. Blackbody measurements were repeated after each measurement series for completeness. Blackbody measurements were taken with 1000 coadds, and sky measurements were taken with 24 coadds unless otherwise stated. Interferograms are generated by the D\&P at a rate of 96 per second, so 24 coadds corresponds to $1 / 4$ second. A description of the measurements taken each day including pointing angle, sky conditions, sky images, and example data follows. 


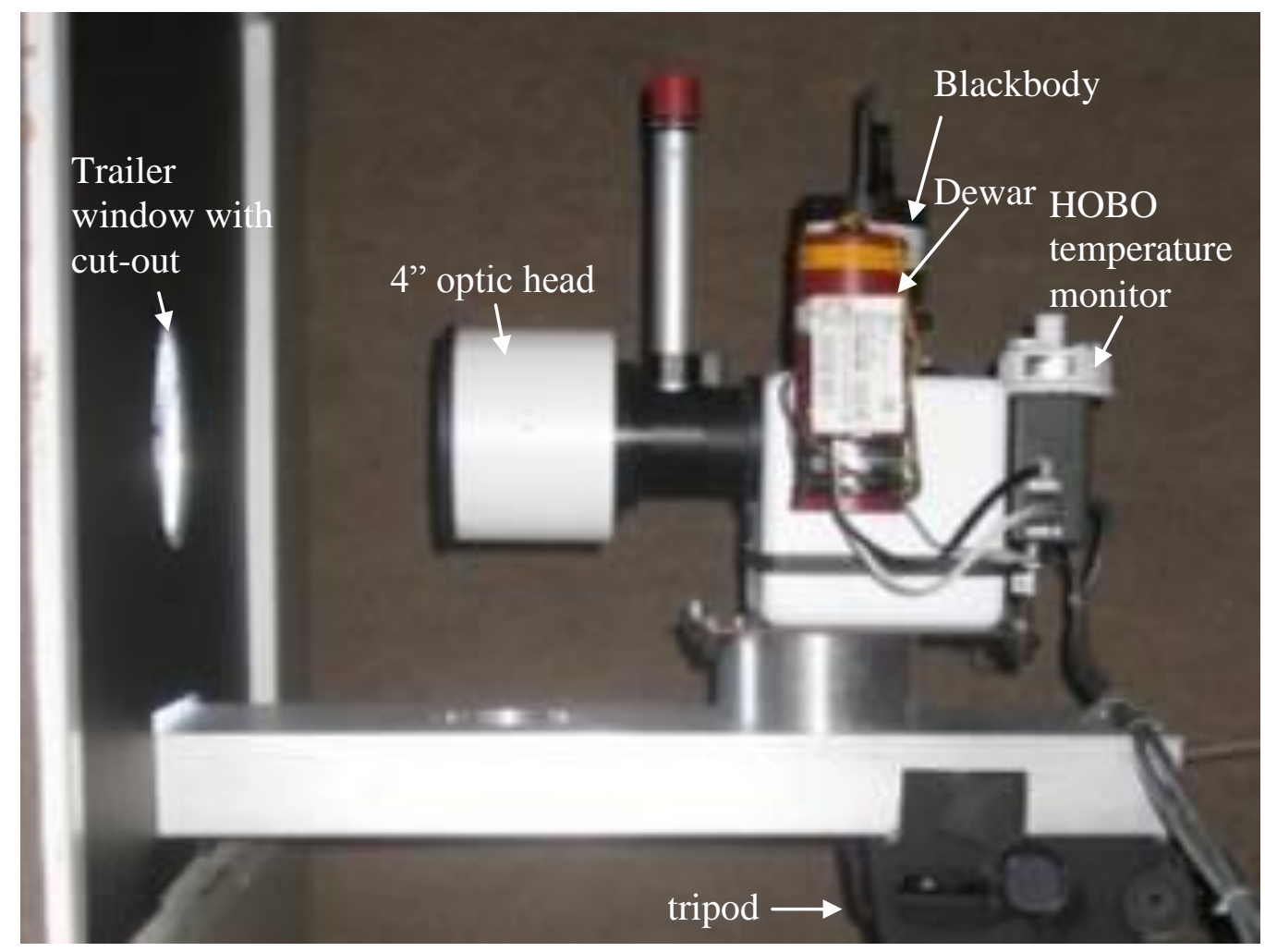

\subsubsection{Day 1, January 14, 2008}

The sky was clear all day, but unfortunately the winds were calm. The clear sky is evident in

Figure 111. Sky radiances were collected as a non-turbulent baseline at angles 90, 42, 30, 24, and 18 degrees, which correspond to $1,1.5,2,2.5$, and 3 airmasses, respectively.

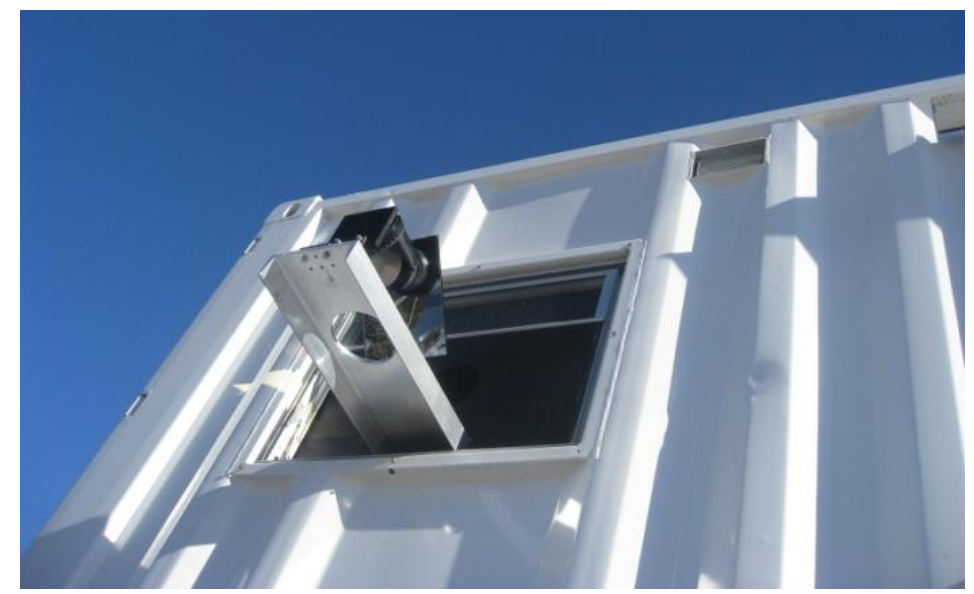

Figure 111. Image of the clear sky with trailer and mirror included.

This data was taken with 1000 coadds. In addition, noise measurements were taken as well: the D\&P was pointed towards a double layer of black foamcore. The spectrum is the shape of a blackbody curve, shown in Figure 112. The periodic spikes in the data, most noticeable around 6 microns, are due to a radar wind profiler next to the trailer. The profiler was turned off when the 
periodic features were seen in the data. No other data was affected by the profiler. Figure 112 Figure 117 are examples of the spectral radiance from each of the airmasses listed above. Note how the data changes around 14 microns as more airmasses enter the line of site, while spectral features in the $8-12$ micron window are still visible.

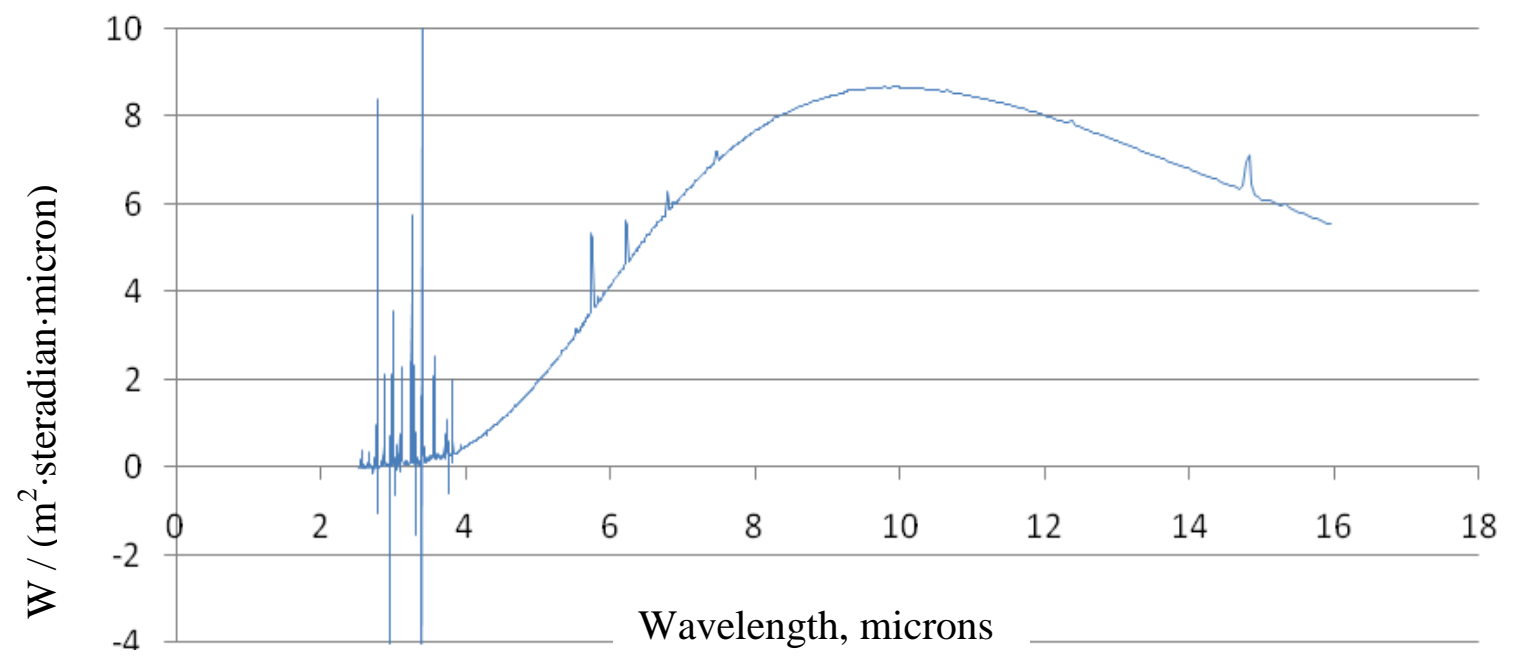

Figure 112. Radiance of black foamcore (for noise measurement)

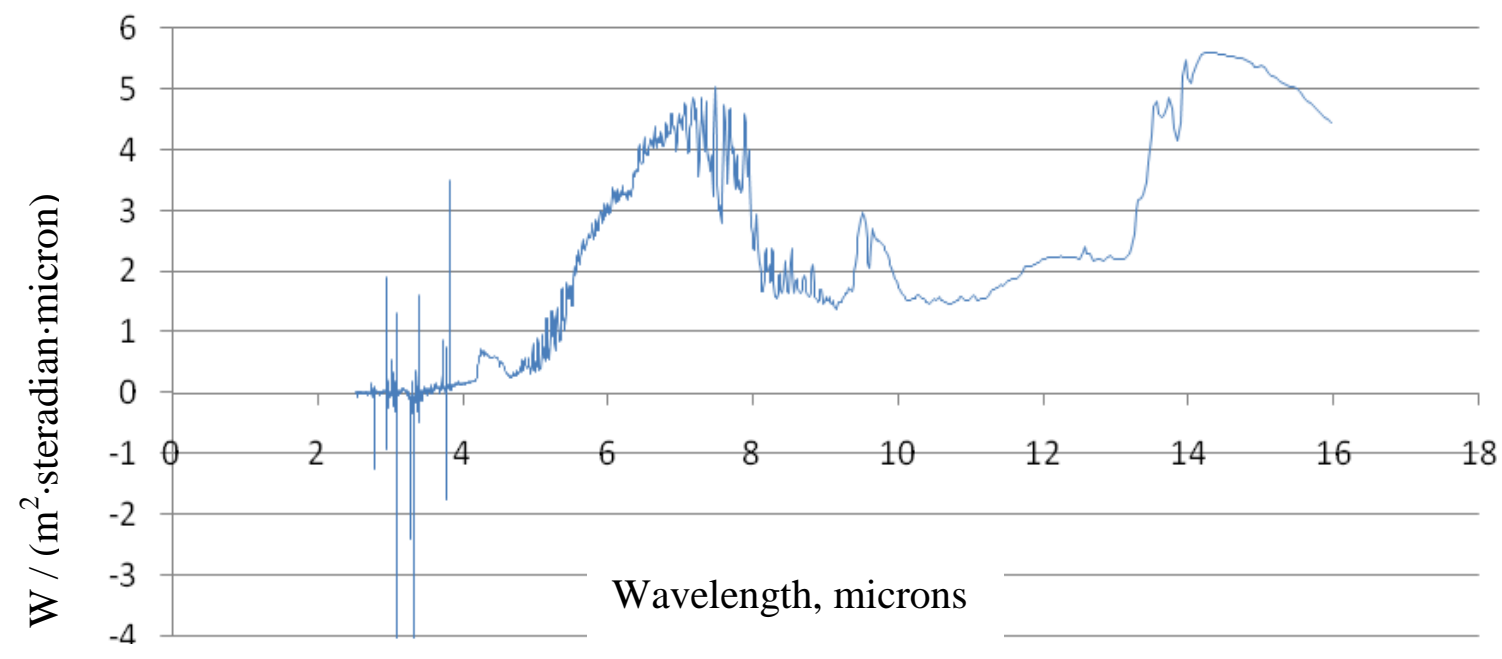

Figure 113. Radiance pointing at zenith. Clear Sky. 1 Airmass. 


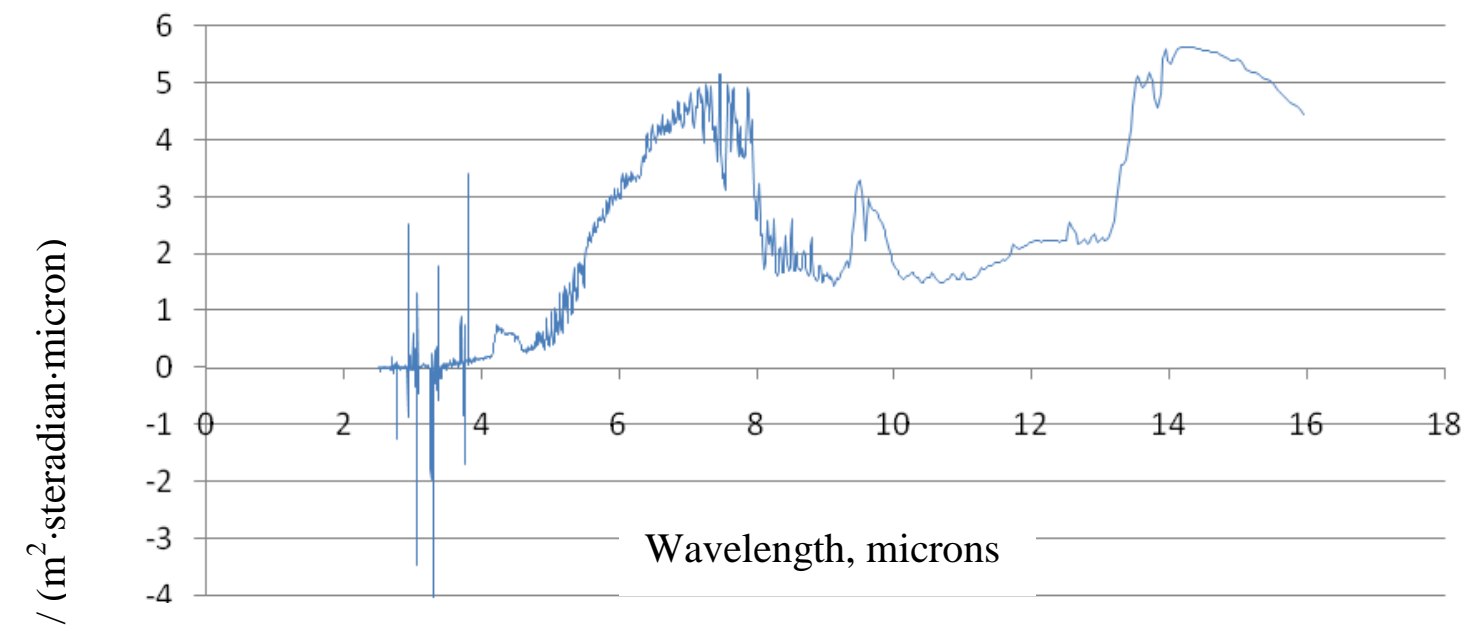

Figure 114. Radiance at $42^{\circ}$ elevation. Clear Sky. 1.5 airmasses.

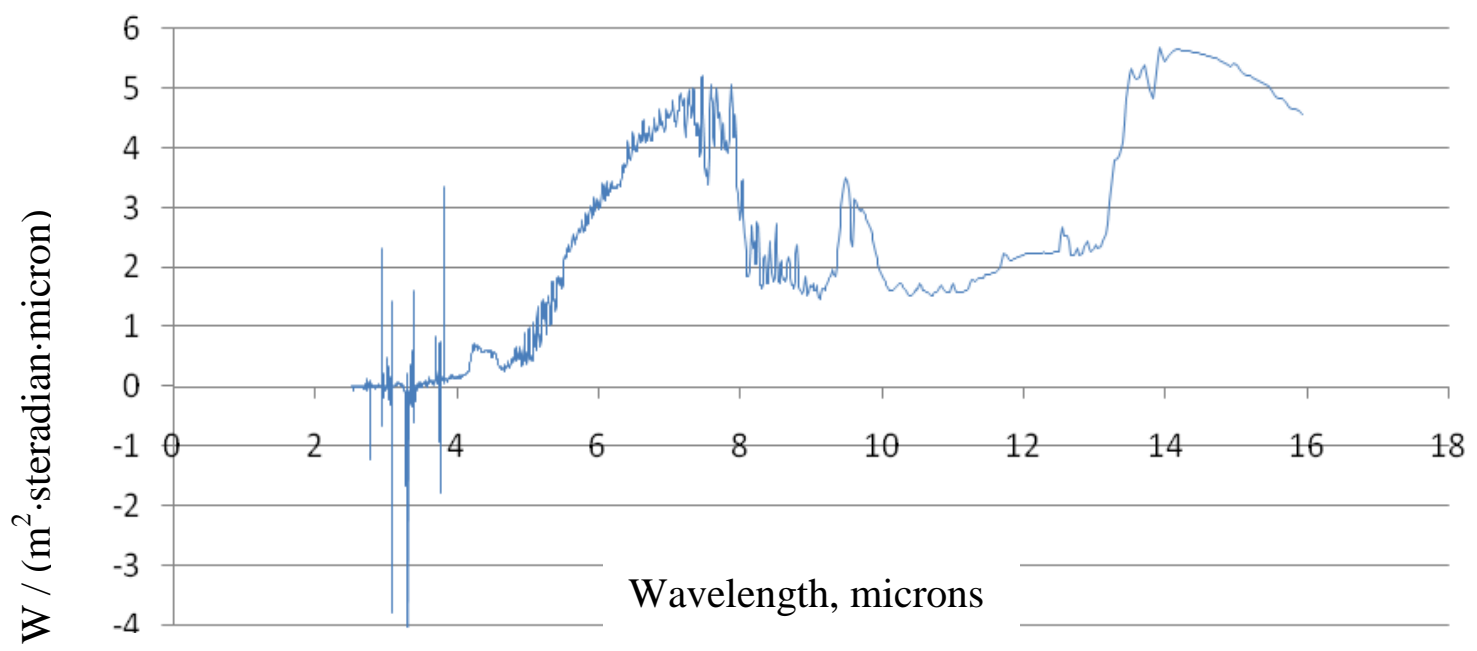

Figure 115. Radiance at $30^{\circ}$ elevation. Clear Sky. 2 airmasses.

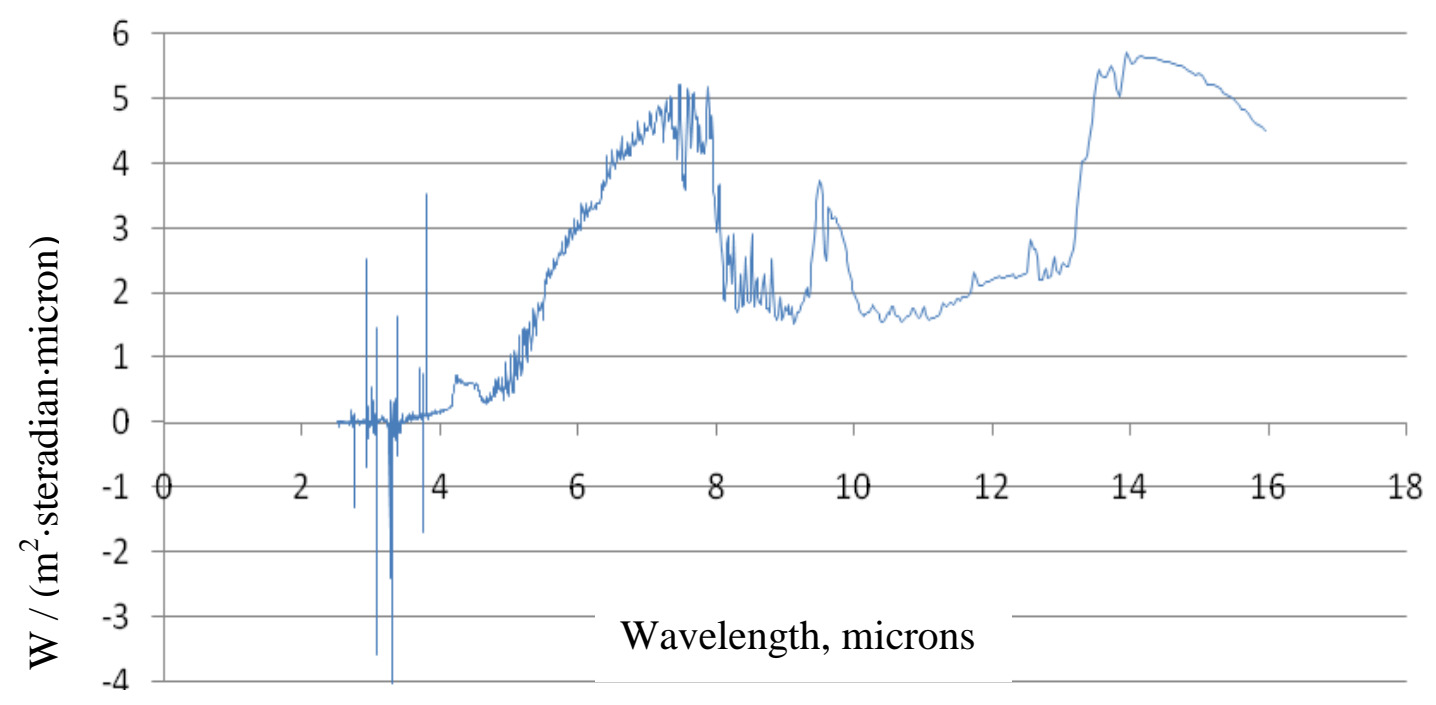

Figure 116. Radiance at $24^{\circ}$ elevation. Clear Sky. 2.5 airmasses. 


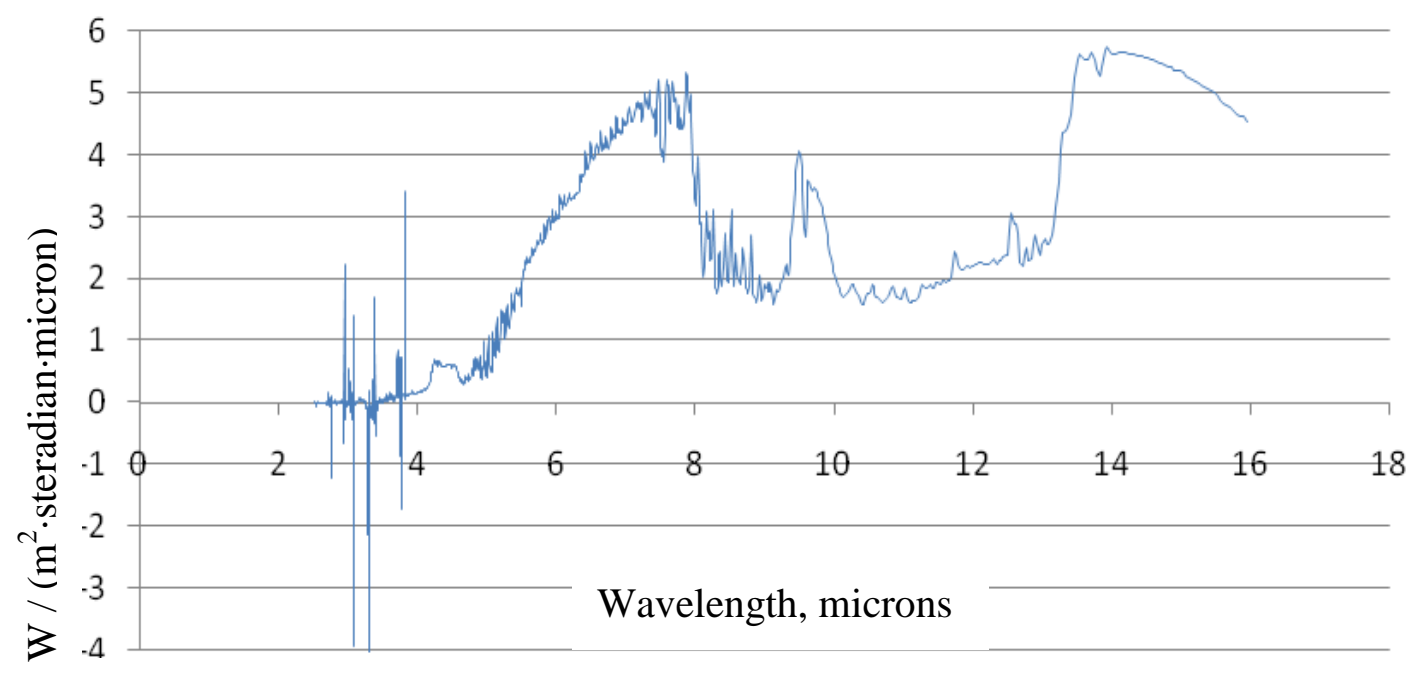

Figure 117. Radiance at $19^{\circ}$ elevation. Clear Sky. 3 airmasses.

\subsubsection{Day 2, January 15, 2008}

The day was overcast in the morning, sunny by the afternoon, with strong winds and turbulence reported. Figure 118 is an image of the skies in the morning. Before noon, the clouds opened over the mountains to the West. The D\&P setup was re-configured to allow viewing at such a shallow angle ( $5^{\circ}$ above the horizontal). Figure 119 (a) shows the tripod angles and (b) is an image taken through the window opening with the viewing position marked. Figure 120 is an example of the 24-coadd data collected at this angle.

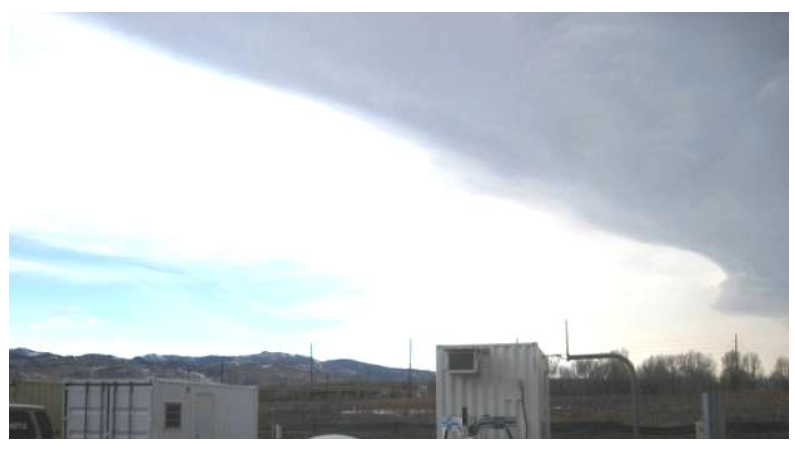

Figure 118. Image of the skies over the trailer Tuesday morning

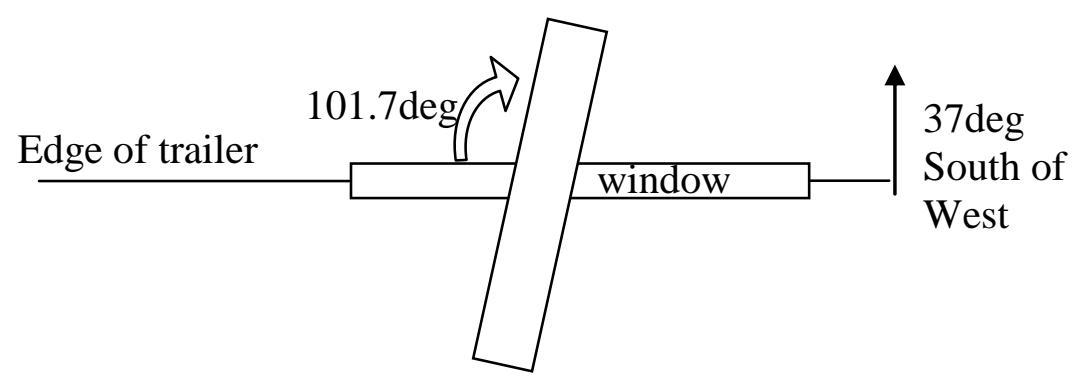

(a) D\&P tripod westward viewing angle at $5^{\circ}$ above the horizon 


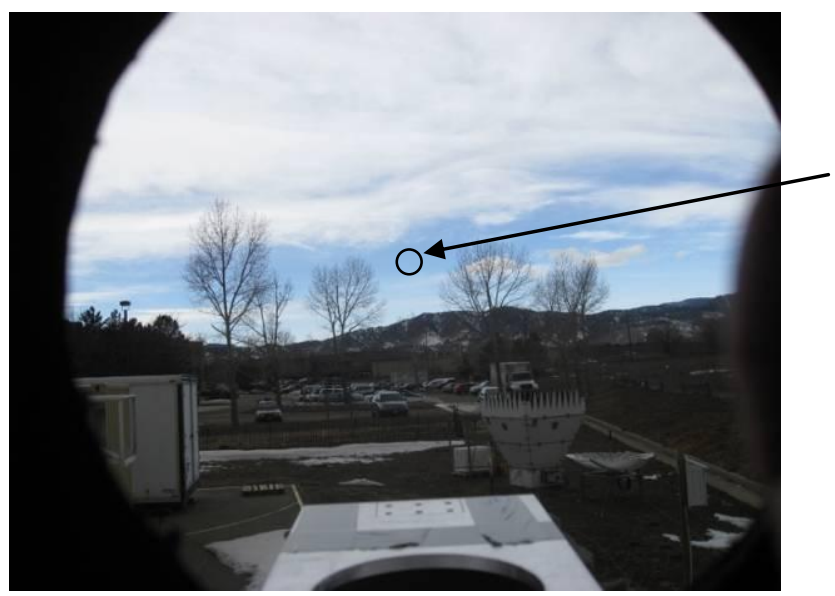

Figure 119 (b) Image taken with viewing position marked.

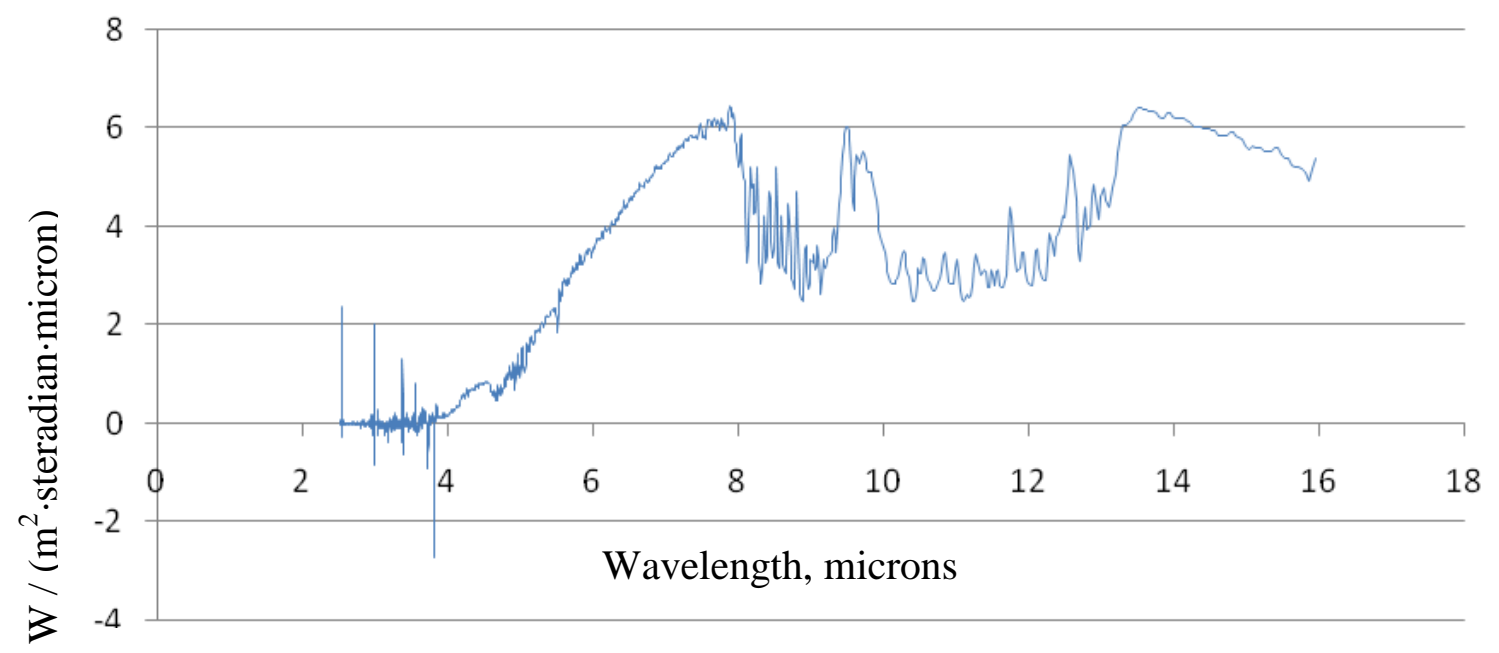

Figure 120. Sky radiance at $5^{\circ}$ above the horizontal. 24 coadds.

The skies cleared as the day went on. Figure 121 shows the sky at various times of the day. As the sky cleared at 1414 MST data collected at $18.5^{\circ}$ above the horizon is shown in Figure 122.
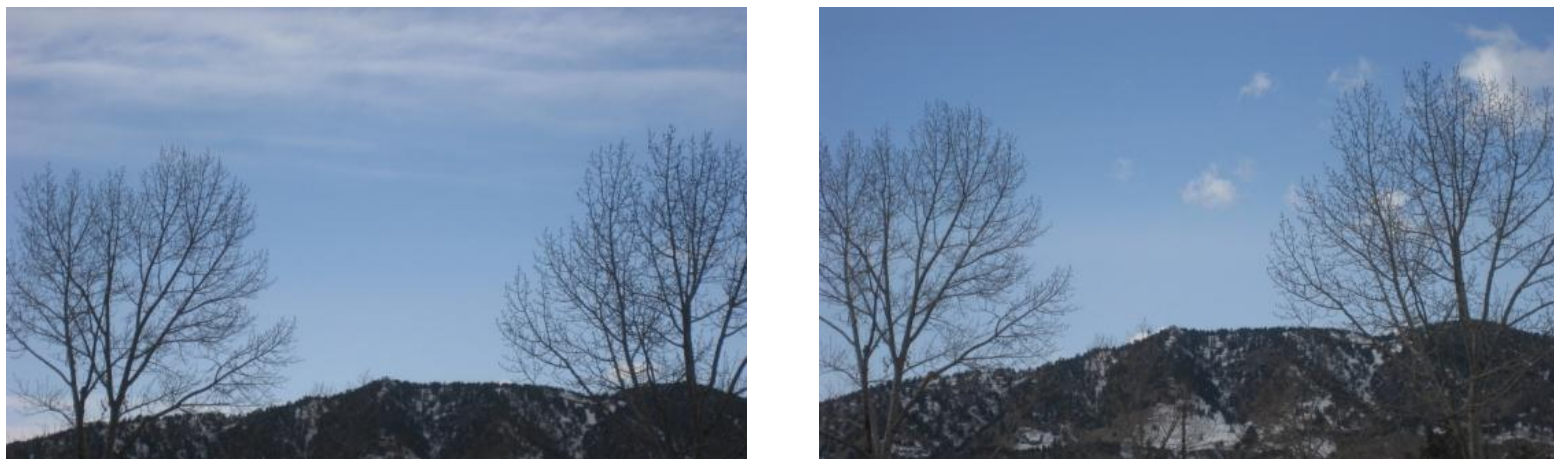

Figure 121. Images of the sky at 1151 MST (left) and 1305 MST (right). 


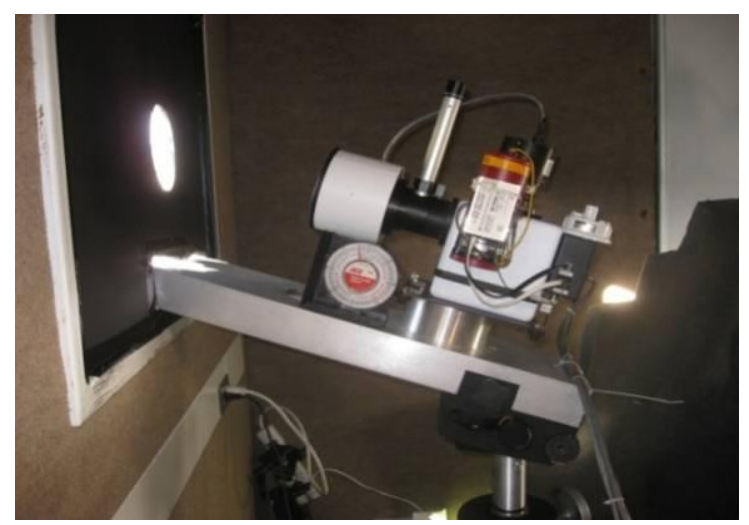

Figure 122. D\&P aimed at 18.5 degrees above horizon

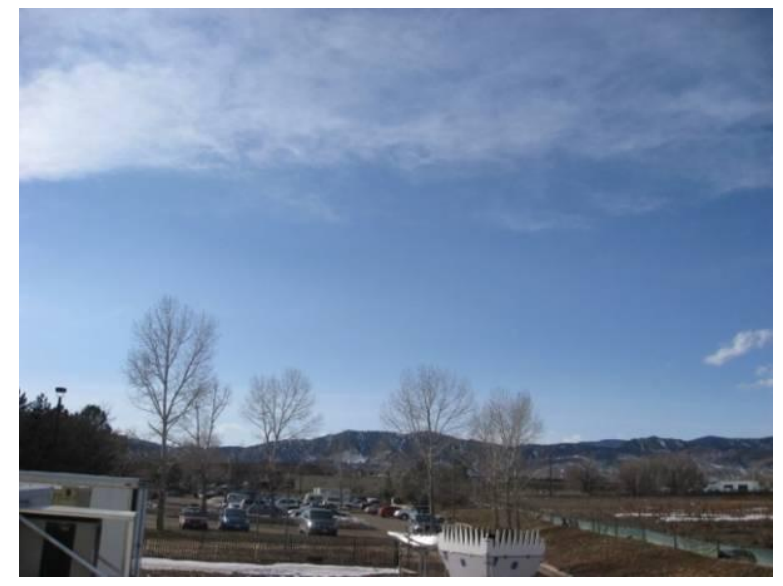

Figure 123. Image of the sky at 1414 MST

At $1642 \mathrm{MST}$, the mirror was added back into the system and the base plate was leveled. The mirror was tilted back $22.5^{\circ}$ to give a $45^{\circ}$ viewing angle ( $233^{\circ}$ azimuth, $45^{\circ}$ elevation). The skies were clear with some cirrus, as shown in Figure 124. Also shown in the figure (right) is an image of the sky at 1640 MST.
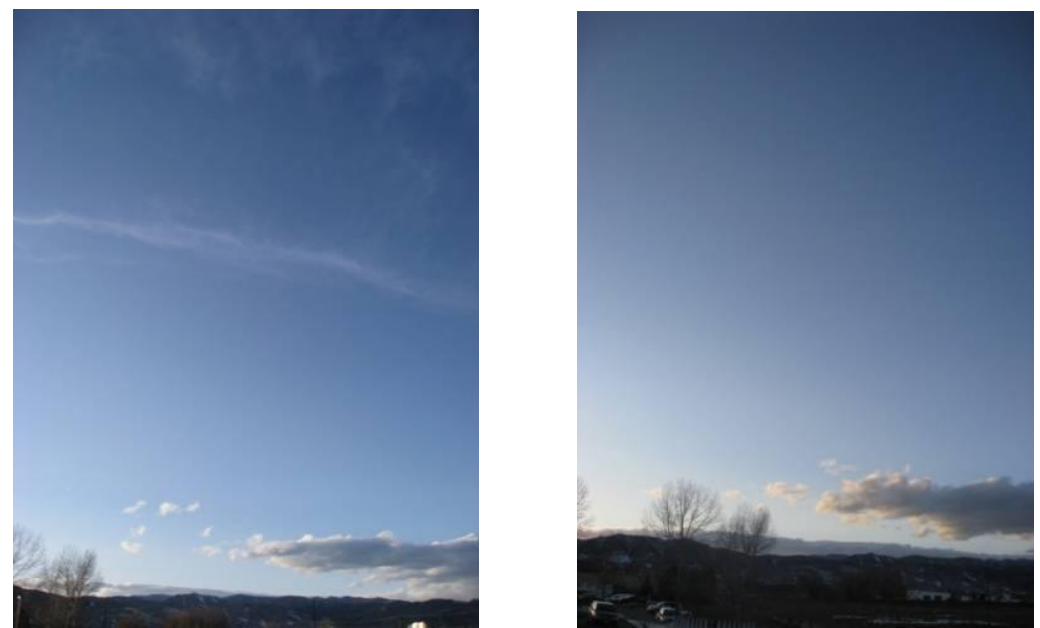

Figure 124. Image of the sky at 1611 MST (left) and 1640 MST (right) 
At 1707 MST, the mirror was angled to view straight up again. The sky was clear, as shown in Figure 125. At 1729 MST, the 11.7 angle was eliminated to make the D\&P channel perpendicular to the trailer edge and shifted the viewing angle from pointing vertically to pointing $45^{\circ}$ above the horizon. There were clear skies above with clouds coming in closer to the horizon, as shown in Figure 126. The D\&P was angled above the clouds. The last data collection effort for the day started at 1754 MST.

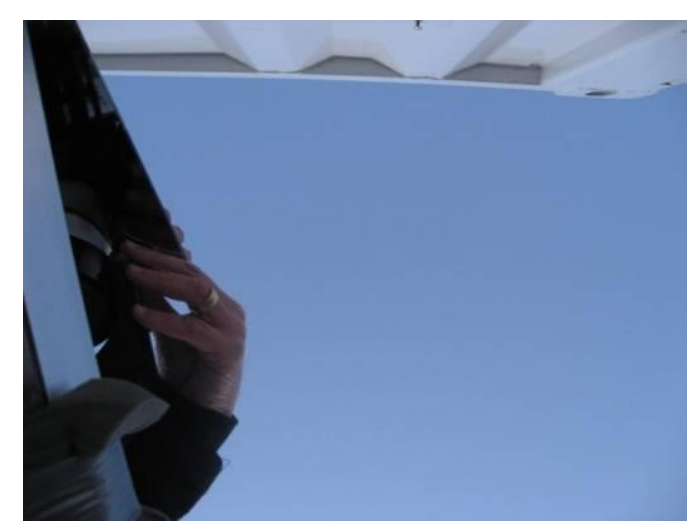

Figure 125. Clear sky above trailer at 1708 MST

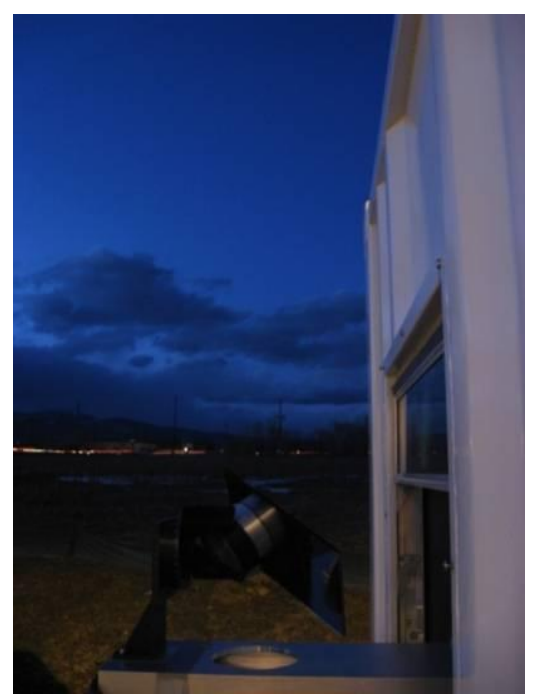

Figure 126. Picture of sky for the last data collection. Clouds visible just above the horizon.

\subsubsection{Day 3, January 16, 2008}

The day began as partly cloudy with thin, wispy cirrus. Data collection began at 1253 MST. The sky is pictured in Figure 127. At 1300, a cloud passed overhead, as shown in Figure 128. A spectral feature was produced around 3.5 -4.5 microns, which prompted the researchers to look at the sky initially. The feature is evident in the radiance spectra of Figure 129. Also note that the radiance is starting to resemble that of a blackbody, where the features in the $8-12$ micron range are not as evident. Clouds similar to the one in Figure 128 came and left often during this measurement cycle. 


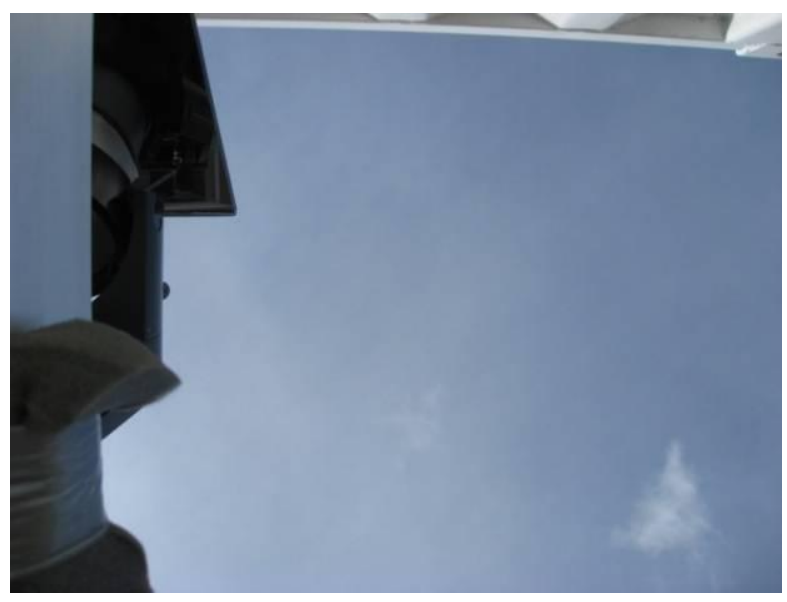

Figure 127. Picture of the sky at 1254 MST

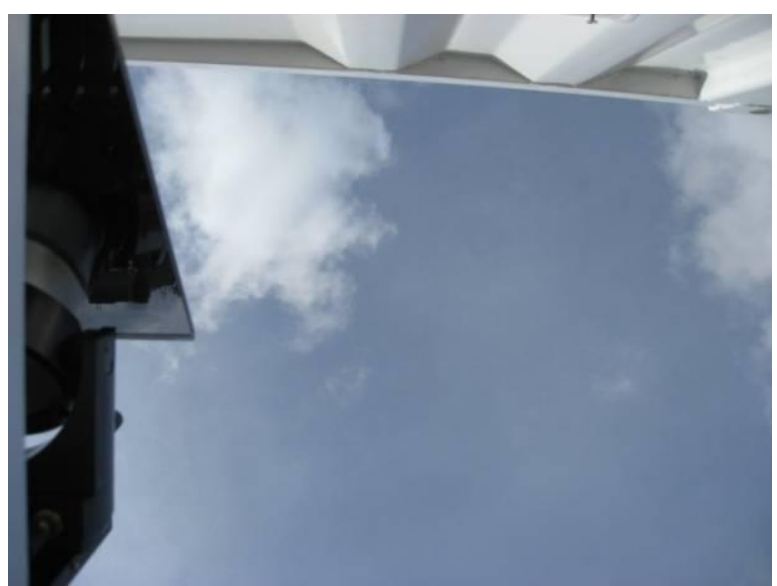

Figure 128. Picture of the sky at 1300 MST

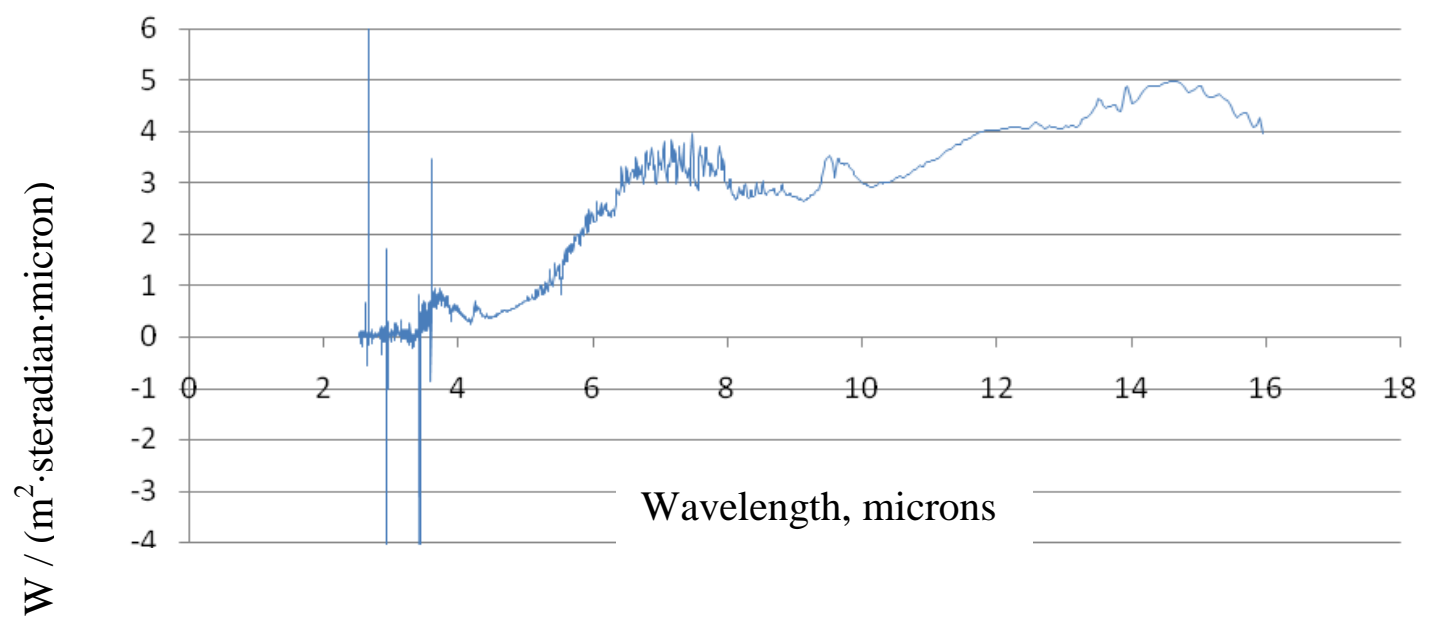

Figure 129. Radiance with a cloud present. 0116, Data Set 1, Data_077563. 
At 1333 MST, a new data collection cycle was initiated at $42^{\circ}$ elevation. The sky was partly cloudy. The D\&P was pointing approximately in the region marked with a circle in Figure 130. After 20 minutes of data collection at this angle, the D\&P was moved to $30^{\circ}$ elevation. The sky was still partly cloudy and the D\&P was pointed to a region just under the large cloud in Figure 131.

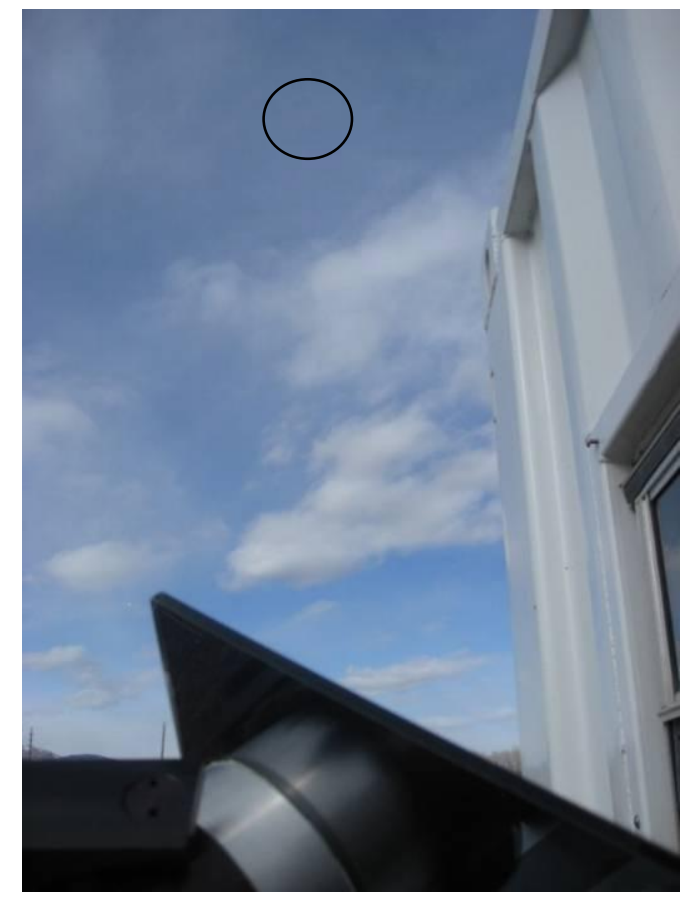

Figure 130. Sky image at 1336 MST. Viewing area marked by a circle.

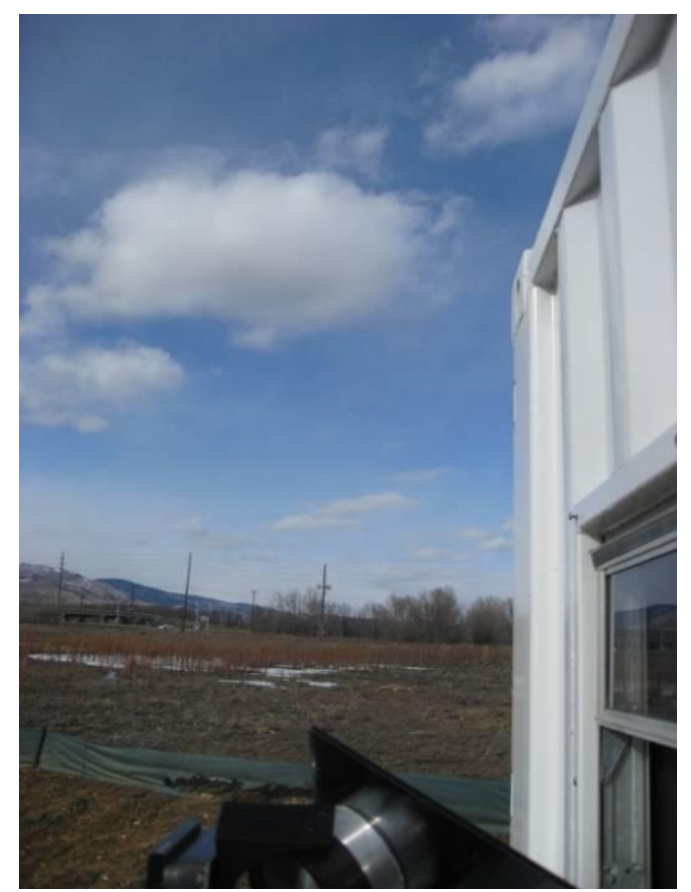

Figure 131. Sky image at 1354 MST 


\subsubsection{Day 4, January 17, 2008}

The day began with cirrus overcast and continued to get worse as the day progressed. Thin cirrus was directly overhead, and severe turbulence was predicted for about an hour. Figure 132 is a photograph of the thin cirrus overhead when the measurement started. Figure 133 is an example of the radiance data from this measurement period.

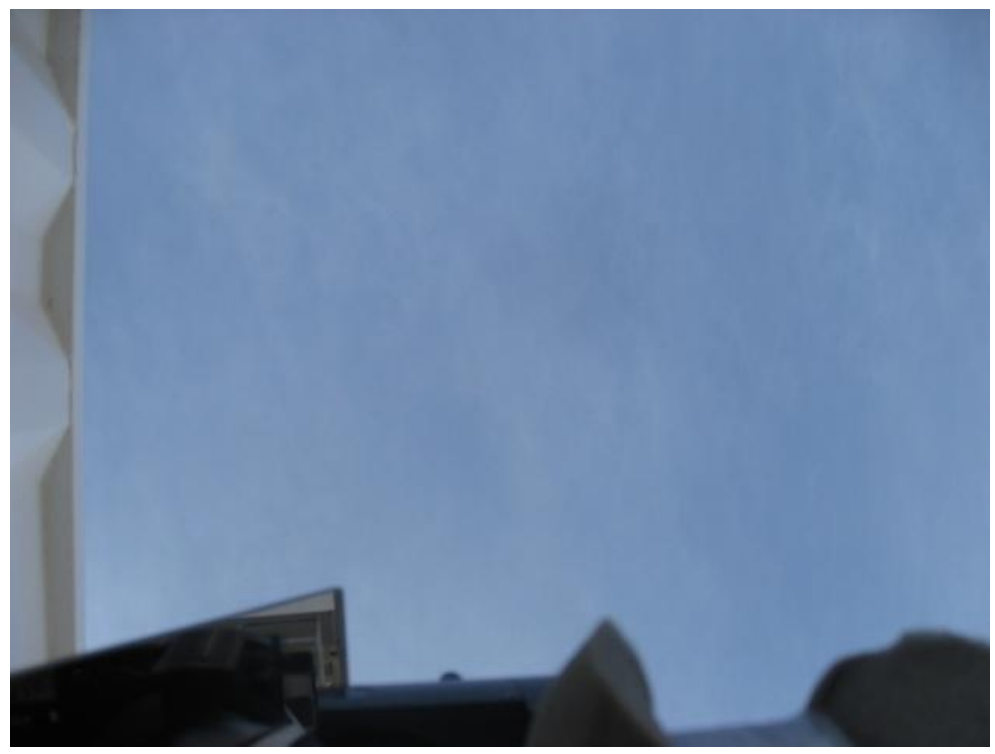

Figure 132. Thin cirrus overhead at 1017 MST

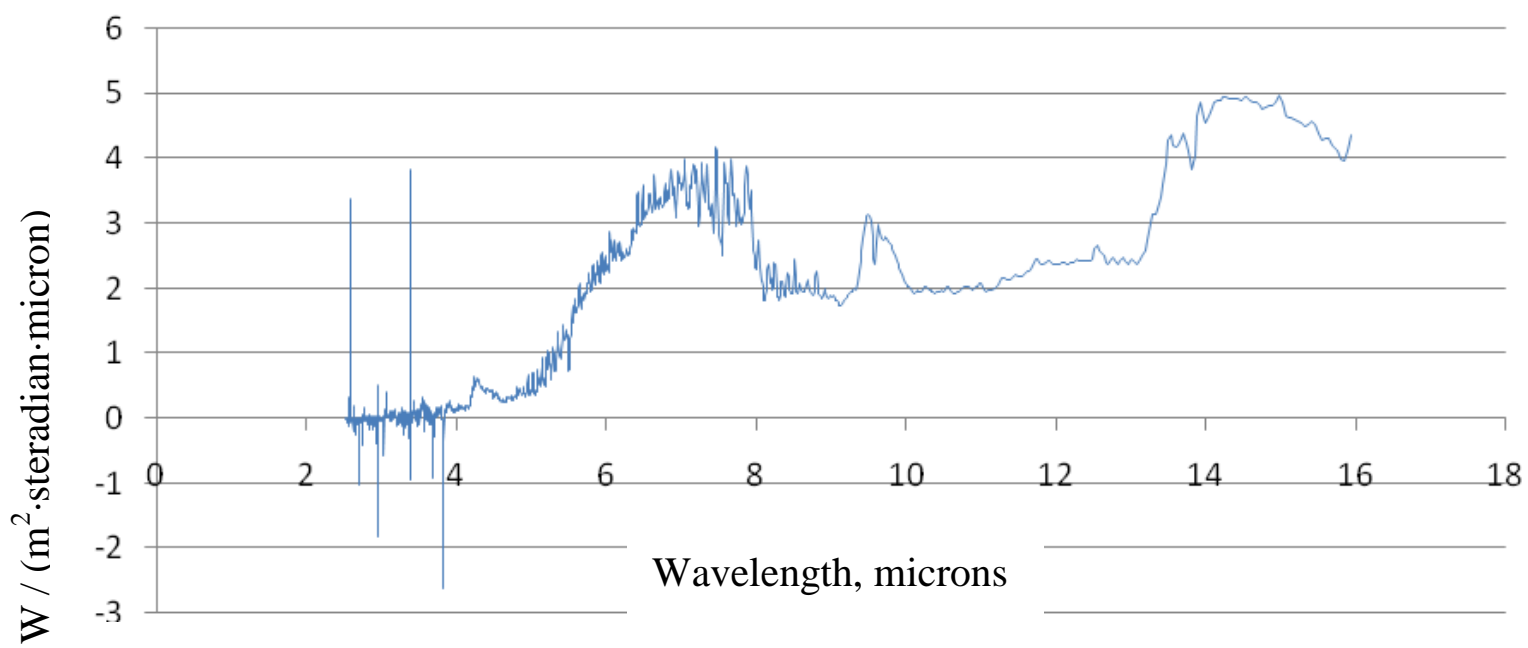

Figure 133. Radiance directly overhead. Thin cirrus. 0117, Data Set 1, Data_000003.

The thin cirrus was thicker towards the horizon, and thicker and more opaque than yesterday. The mirror was rotated to $30^{\circ}$ elevation to take more data. Figure 134 is an image of the sky at the new angle. 


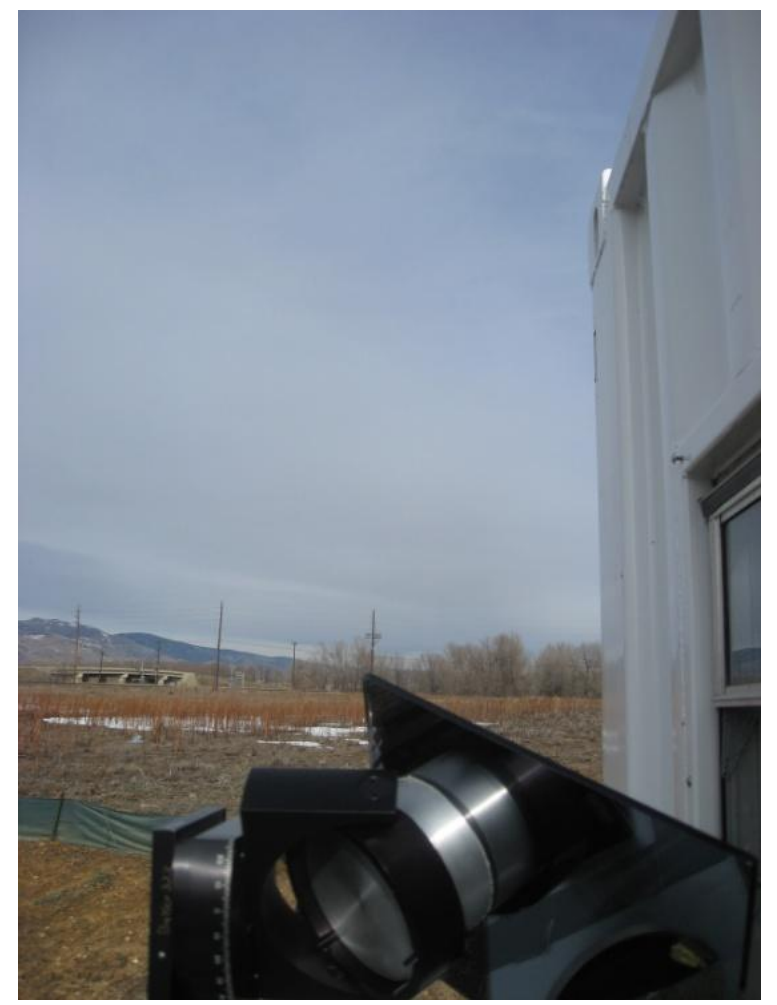

Figure 134. Thin cirrus at $30^{\circ}$ elevation at 1052 MST.

The mirror was turned to face more westward ( $291^{\circ}$ azimuth, $45^{\circ}$ elevation) since turbulence was predicted in that direction. The clouds became very thick in this direction, but data were collected for twelve minutes. Figure 136 is an example of the radiance data collected during this session.

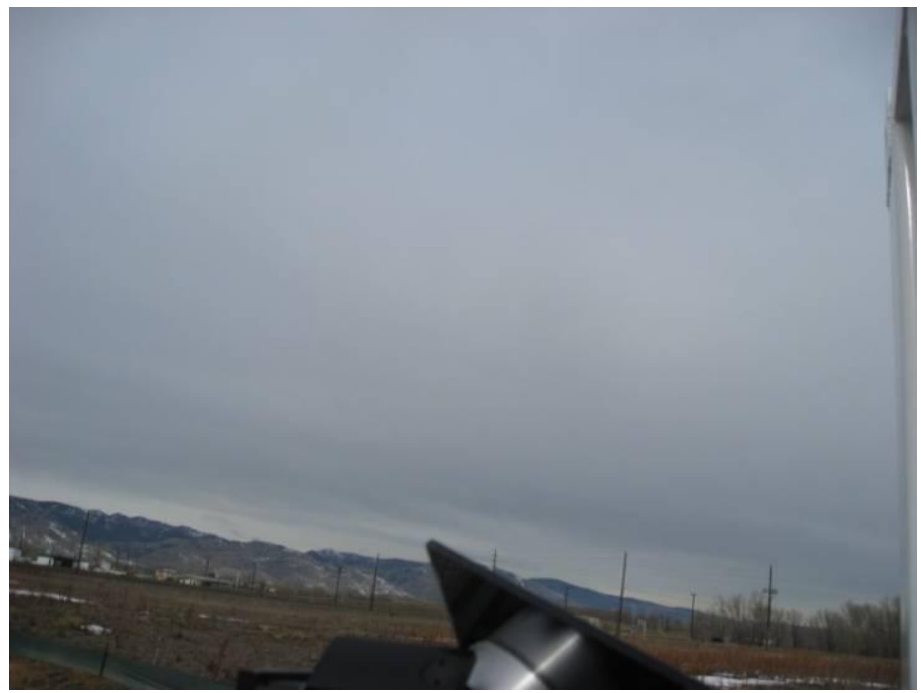

Figure 135 . Overcast at $1123 \mathrm{M}$ in the $291^{\circ}$ azimuth, $45^{\circ}$ elevation direction 


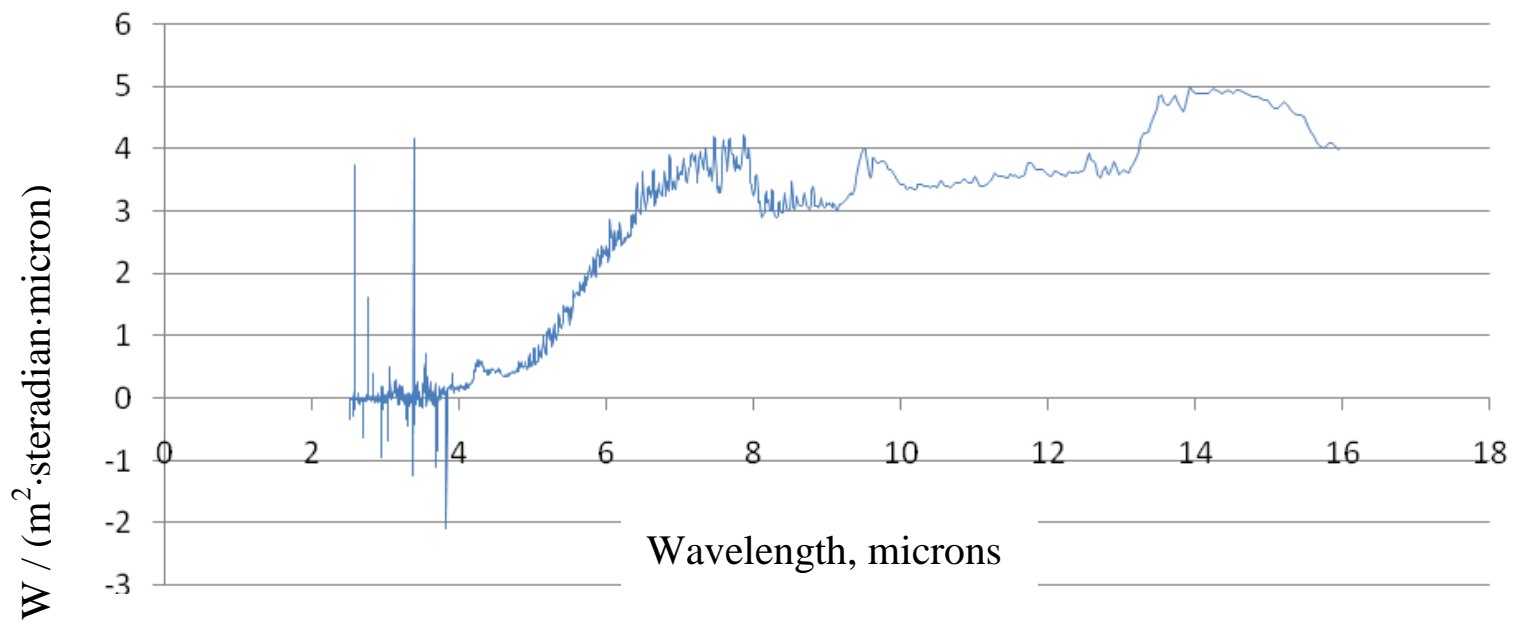

Figure 136. $291^{\circ}$ azimuth, $45^{\circ}$ elevation. Thick cirrus. 0117, Data Set 2, Data_006726.

The clouds became too thick for further measurements, until 1500 MST. At this time, there was a small opening in the clouds. The D\&P was pointed 20 degrees south of perpendicular to the trailer and 8 degrees up from the horizon. A circle in Figure 137 shows approximately where the D\&P was facing for this data set.

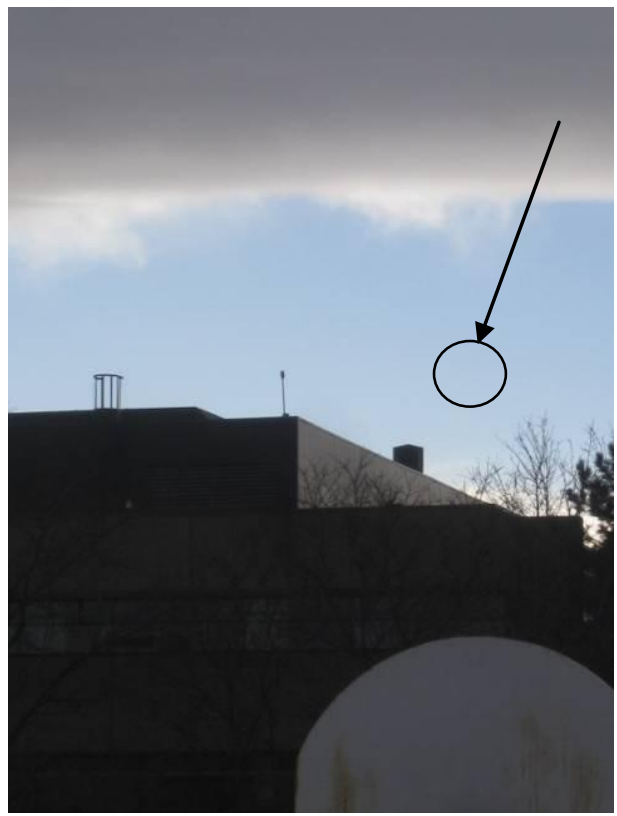

Figure 137. Break in the clouds at 1500 MST, 20 degrees south, 8 degrees elevation.

\subsubsection{Truth Data}

The truth data for the field test includes pilot reports (PIREPS) and automated reports from United Airlines $757 \mathrm{~s}$ of eddy dissipation rates (EDR), and ambient conditions at the test site recorded 100 meters from the trailer. Ambient condition reports include temperature, relative humidity, wind direction and speed, rain accumulation, pressure corrected to sea level, and dewpoint. This data, in addition to the analysis of the D\&P data is presented in Section 3.2.2. 


\subsubsection{Boulder Field Test Summary}

Table 1 gives a summary of the data collection effort, including direction, start time, duration of measurement, and sky conditions. Note that all times in the table are EST; subtract two hours to find MST. Azimuth (AZ) is measured in degrees in the convention manner, i.e. degrees clockwise from north. Elevation (EL) is measured in degrees up from the horizon. All calibrations were done with 1,000 coadds, as were the 1306 - 1313 EST scans on 1/14. All other sky radiance data were acquired with 24 coadds.

\section{Table 1. Summary of Data Collection Effort}

\begin{tabular}{|c|c|c|c|c|c|}
\hline Date & Start Time & $\mathrm{AZ}$ & EL & Length [min.] & Comments \\
\hline \multirow[t]{14}{*}{$1 / 14$} & & & & & Clear sky all day \\
\hline & & & & & Calibration \\
\hline & 1306 & 323 & 19 & - & Airmass 3 \\
\hline & 1308 & 323 & 24 & - & Airmass 2.5 \\
\hline & 1310 & 323 & 30 & - & Airmass 2 \\
\hline & 1312 & 323 & 42 & - & Airmass 1.5 \\
\hline & 1313 & - & 90 & - & Airmass 1 \\
\hline & 1333 & - & 90 & 15 & \\
\hline & & & & & Calibration \\
\hline & & & & & Calibration \\
\hline & 1629 & - & 90 & 90 & \\
\hline & & & & & Calibration \\
\hline & 1819 & - & 90 & 30 & \\
\hline & & & & & Calibration \\
\hline \multirow[t]{9}{*}{$1 / 15$} & & & & & Overcast \\
\hline & & & & & Calibration \\
\hline & $\sim 1335$ & 245 & 5 & 90 & Aiming over foothills to WSW \\
\hline & & & & & Calibration \\
\hline & & & & & Calibration \\
\hline & 1642 & 245 & 18.5 & 60 & Aiming over foothills to WSW \\
\hline & & & & & Calibration \\
\hline & 1811 & 233 & 45 & 30 & Problems with sunshine on D\&P optics \\
\hline & & & & & Calibration \\
\hline
\end{tabular}

Table 2. Summary of Data Collection Effort, cont.

\begin{tabular}{|l|l|l|l|l|l|}
\hline Date & Start Time & AZ & EL & Length [min.] & Comments \\
\hline & & & & & Sky clear by end of day \\
\hline $1 / 16$ & & & & & This cirrus, a few thin clouds \\
\hline & & & & & Calibration \\
\hline & 1453 & - & 90 & $\sim 30$ & \\
\hline & 1533 & 323 & 42 & 29 & Airmass 1.5 \\
\hline
\end{tabular}




\begin{tabular}{|l|l|l|l|l|l|}
\hline & 1554 & 323 & 30 & $\sim 18$ & Airmass 2 \\
\hline & 1615 & 323 & 19 & 18 & Airmass 3 \\
\hline & & & & & Calibration \\
\hline & 1651 & - & - & 6 & Viewing foam core (noise measurement) \\
\hline & 1703 & 143 & 20 & 5 & Opaque cloud to south \\
\hline $1 / 17$ & & & & & Cirrus overcast. Turbulence forecast. \\
\hline & & & & & Calibration \\
\hline & 1217 & - & 90 & 34 & \\
\hline & 1252 & 323 & 30 & 21 & Cirrus overcast, thicker today \\
\hline & 1321 & 291 & 45 & 12 & \\
\hline & 1334 & & & & Clouds are thick now \\
\hline & 1700 & 213 & 8 & 15 & Blue sky above NCAR roof \\
\hline & & & & & Calibration \\
\hline
\end{tabular}

\subsubsection{D\&P Data Analysis}

In this section, data from the Boulder field test are presented. Data selections from two days are analyzed. For each day, figures showing the measured radiances, as well as data from the UCAR EDR viewer are given. The latter set of figures show the turbulence reports that came from United Airlines aircraft during that time, and in the region. Underlain on those data are the forecast values from the GTN turbulence forecast system (including a mountain wave turbulence predictor). Another set of figures show winds and temperatures from the RUC model from the pertinent time and space region. On both days there were numerous reports of turbulence mainly to the east of the mountains. Unfortunately, during the measurement time period there was a very small cloud-free window over the mountains. The cloud pattern was typically of mountain wave activity: clear to the west, thin clouds over the mountains, and then clear again to the east of the mountains.

As will be seen below, there are data periods where the radiance correlation coefficients appear to be noise-like, but there are also many periods where a positive/negative correlation structure is seen. A large, contiguous time series of radiances were broken into sub-regions of approximately 75 second duration. A moving average for each wavenumber was subtracted from the radiances, and then the correlation coefficients were computed as a function of wavenumber for each subregion. Then these correlation coefficients for the sub-regions are averaged together. The number of averages is dependent on the length of the original radiance data. As an example of the results from this processing method, consider Figure 138. The upper panel shows the "raw" averaged correlation coefficients. It can be seen that there is a good deal of residual noise in this data; and hence a 2-d wavelet filter was applied to these data. The lower panel illustrates the beneficial effect of using the filter. The positive/negative structures are much more apparent, especially for the lower-signal wavenumbers.

Comparing Figure 138 to Figure 80 or Figure 86, for example, explains the positive/negative structures: they are most likely mountain waves. To further validate this conceptual model two different wavenumbers, $730 \mathrm{~cm}^{-1}$ and $1000 \mathrm{~cm}^{-1}$ (approximately), were chosen for further analysis. Figure 139 shows the "raw' correlation coefficient function for wavenumber $730 \mathrm{~cm}^{-1}$, and Figure 140 illustrates the effect of the wavelet filtering. Note that the coefficient values are very small, indicating a noise-like signal. In contrast, Figure 141 and Figure 142 show the same quantities, but at $1000 \mathrm{~cm}^{-1}$. The wave-like structure is clearly visible in these figures. For 
comparison, a sinusoidal temperature field with a $5^{\circ} \mathrm{K}$ amplitude and a wavelength of $12.5 \mathrm{~km}$ was used to simulate a radiance correlation coefficient function. An averaged simulated correlation coefficient for a sinusoidal temperature field with a $12.5 \mathrm{~km}$ wavelength and with added noise, shown in Figure 143. This simulation does a good job of approximating the correlation coefficient function shown in Figure 142. Figure 144 illustrates potential temperature cross-section over the Rocky Mountains on 17 February 1970. (Lilly 1973) Solid lines are isentropes $(\mathrm{K})$, dashed lines aircraft or balloon flight trajectories. Trapped lee waves are visible from $6-8 \mathrm{~km}$. It is most likely this type of structure that is seen in the measured radiance data.
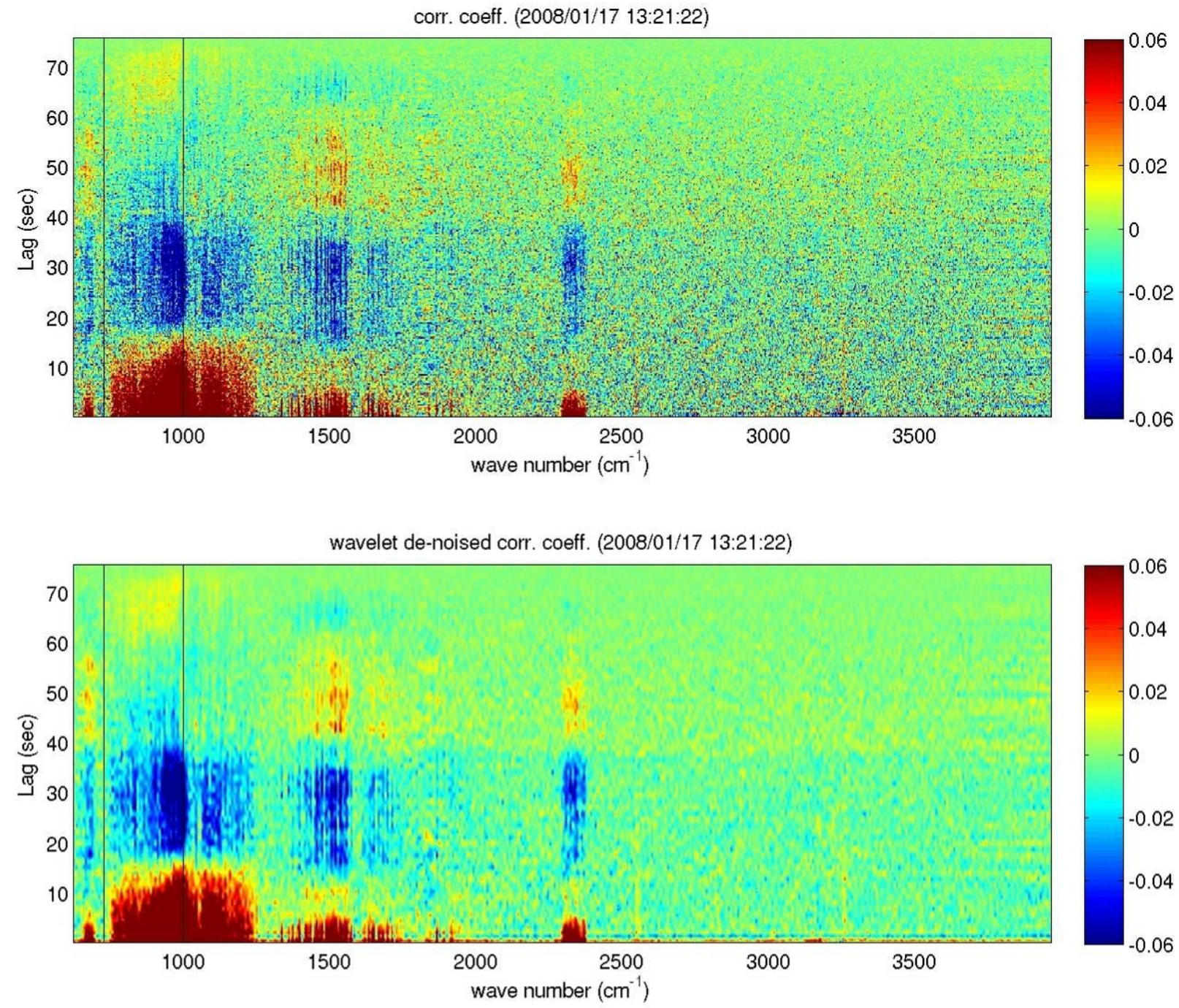

Figure 138. Example of positive/negative structure in the correlation coefficient field 


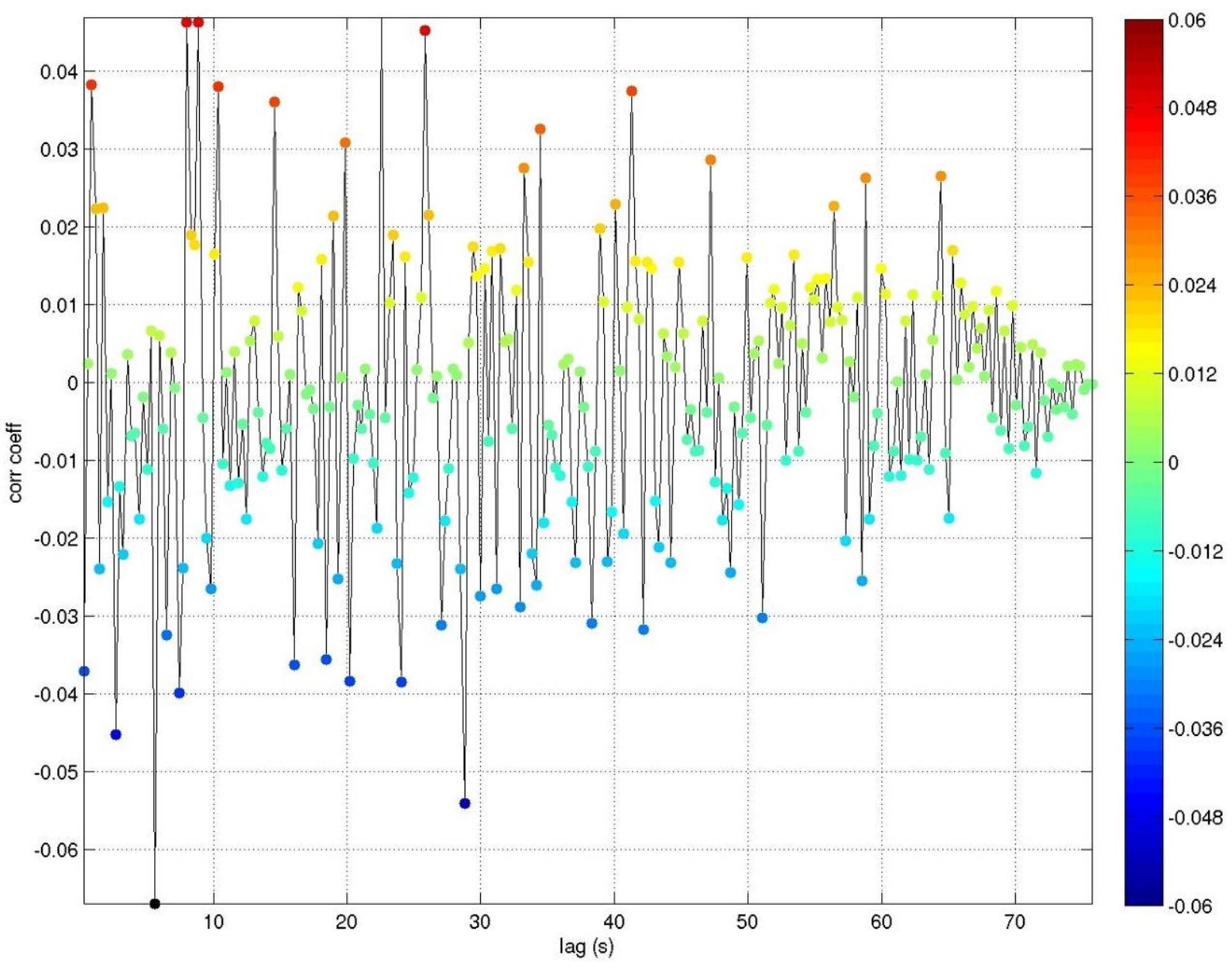

Figure 139. Raw correlation coefficient function for wavenumber $730 \mathrm{~cm}^{-1}$ 


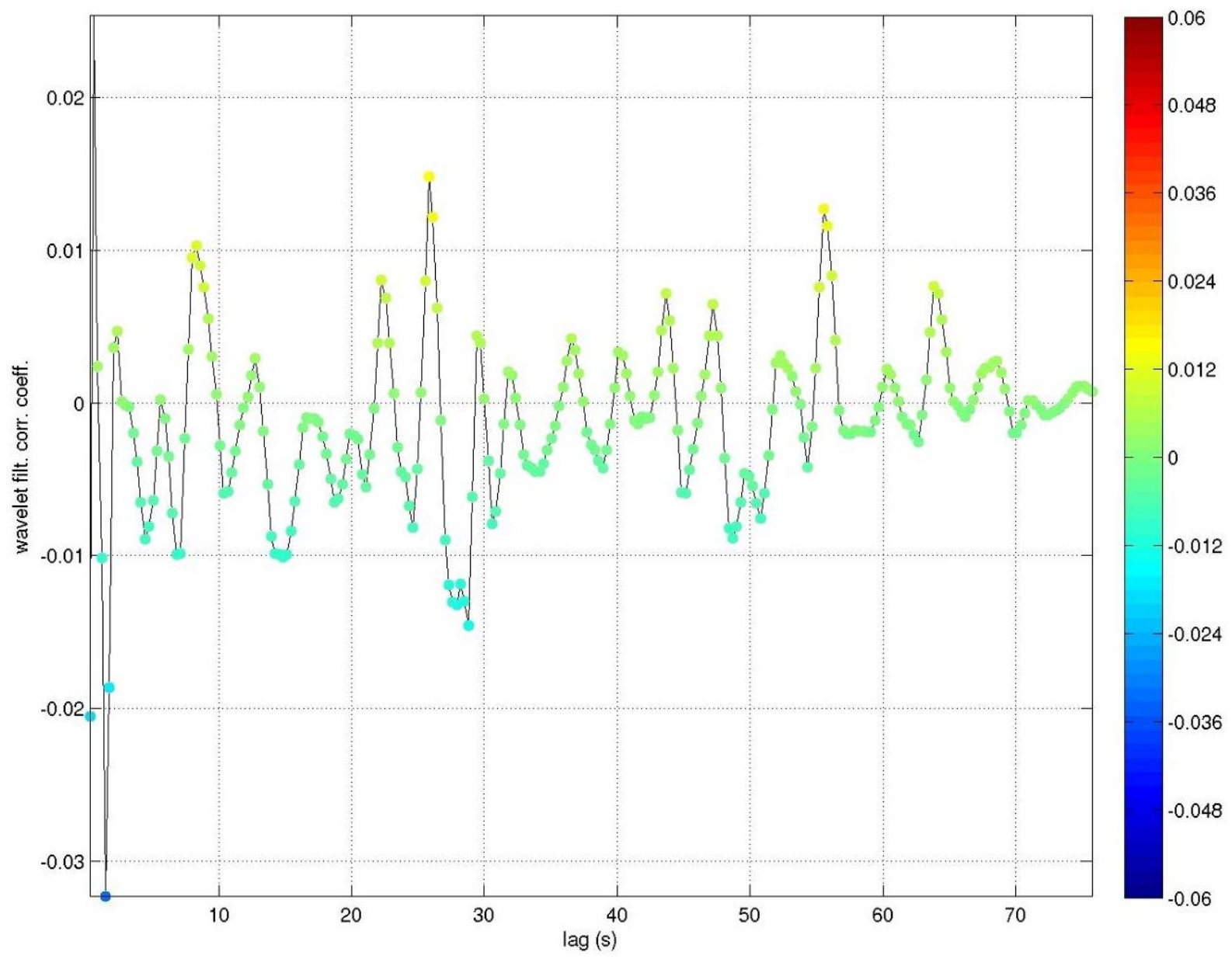

Figure 140. Same is in Figure 139, except after wavelet filtering 


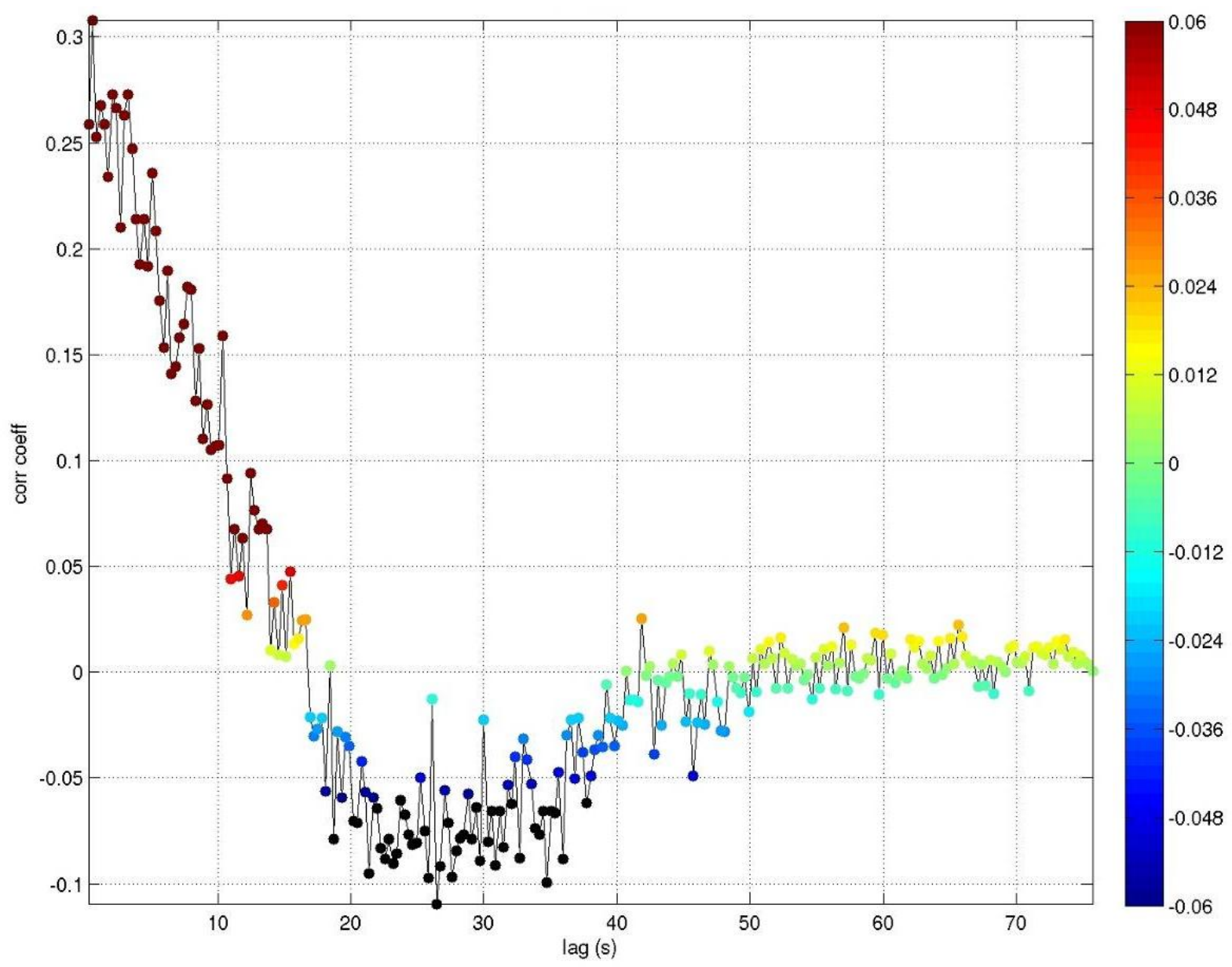

Figure 141. Same as for Figure 139, but for wavenumber $1000 \mathrm{~cm}^{-1}$ 


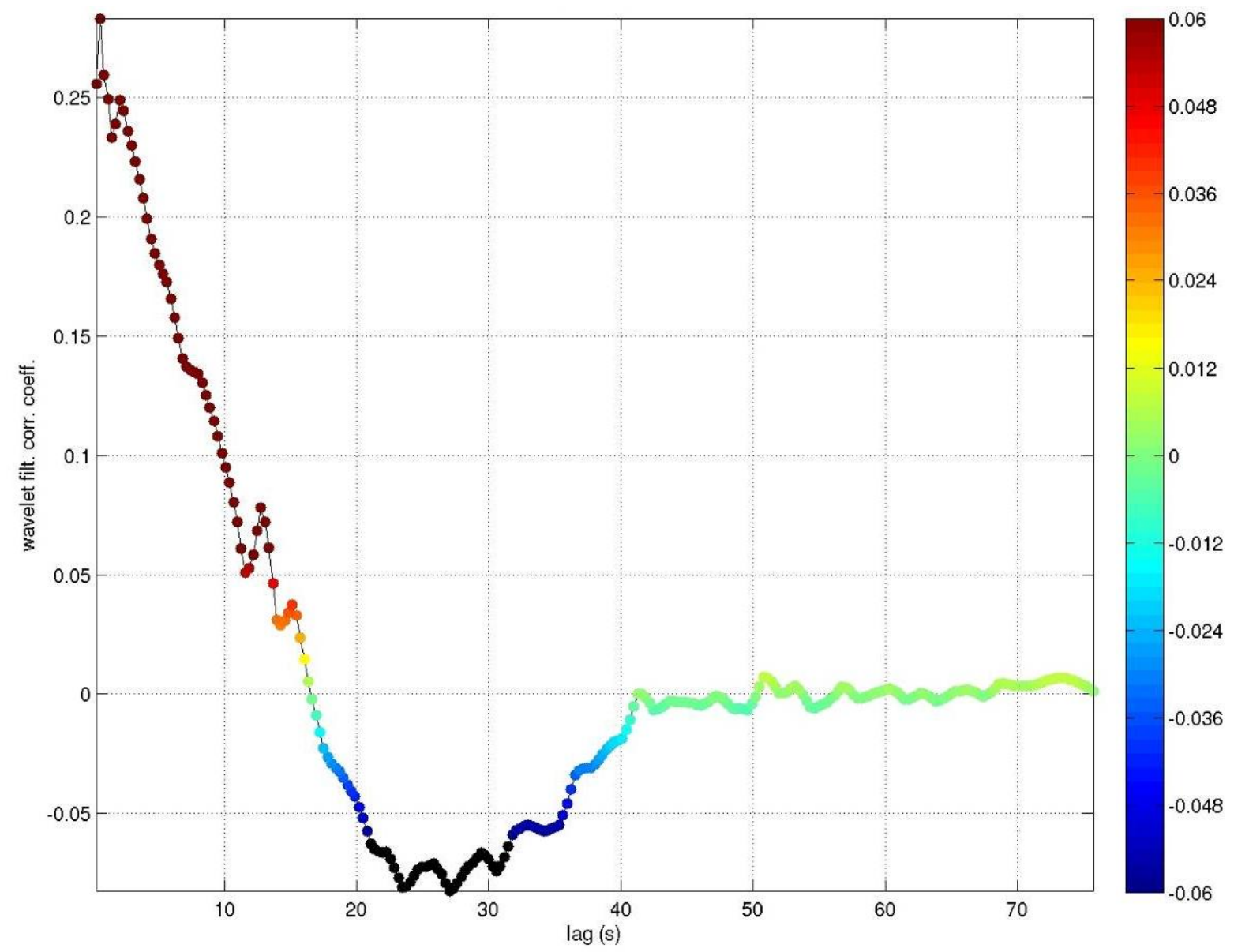

Figure 142. Same as for Figure 140, but for wavenumber $1000 \mathrm{~cm}^{-1}$ 


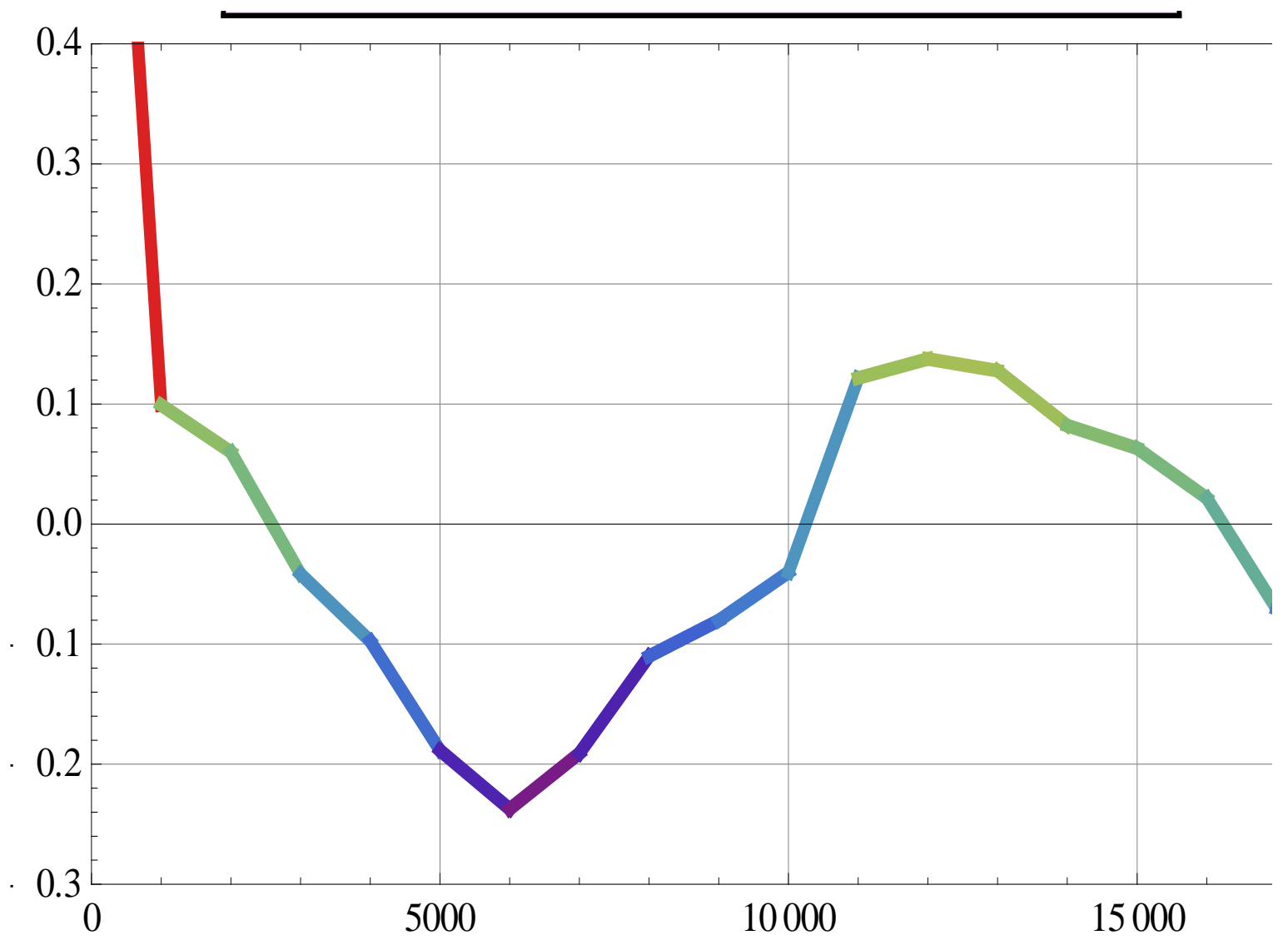

Figure 143. Averaged simulated correlation coefficient with noise. 


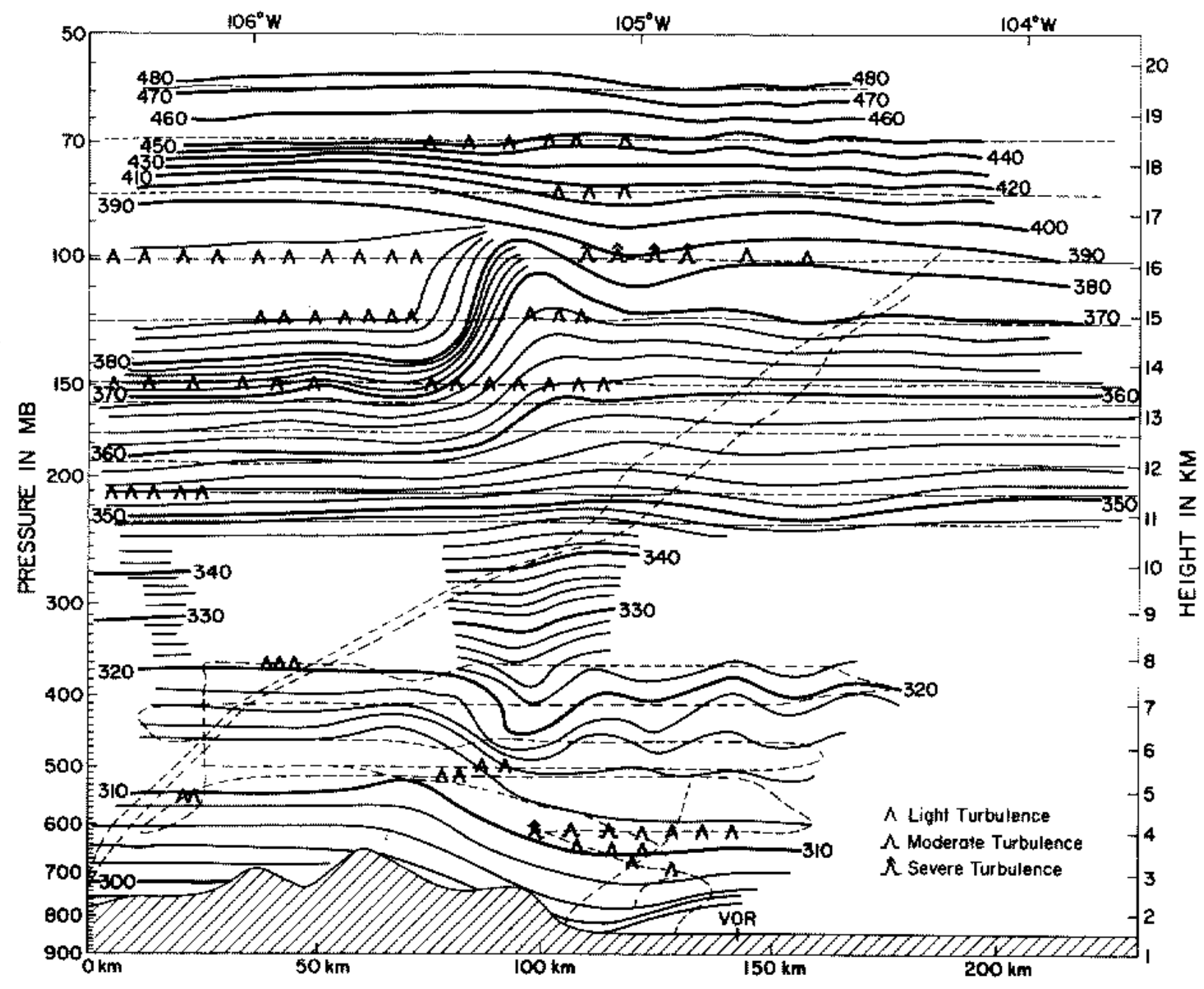

Figure 144. Potential temperature cross-section over the Rocky Mountains.

\subsubsection{Jan 15, 2008}

In this section, an analysis of the data collected on Jan 15, 2008 is presented. The first nine figures consist of three sets of three plots each (Figure 145 through Figure 153), showing the measured radiances (color scale) versus time and wavenumber; the associated correlation coefficient and power spectra, respectively. The upper panel in each of these plots show the average radiance at each wavenumber, taken over the given time period. Each set is for a different time segment. It can be seen that the measured radiances in this wavenumber region $\left(700-800 \mathrm{~cm}^{-1}\right)$ look fairly noisy. The correlation coefficients and spectra also indicate the noiselike nature. 

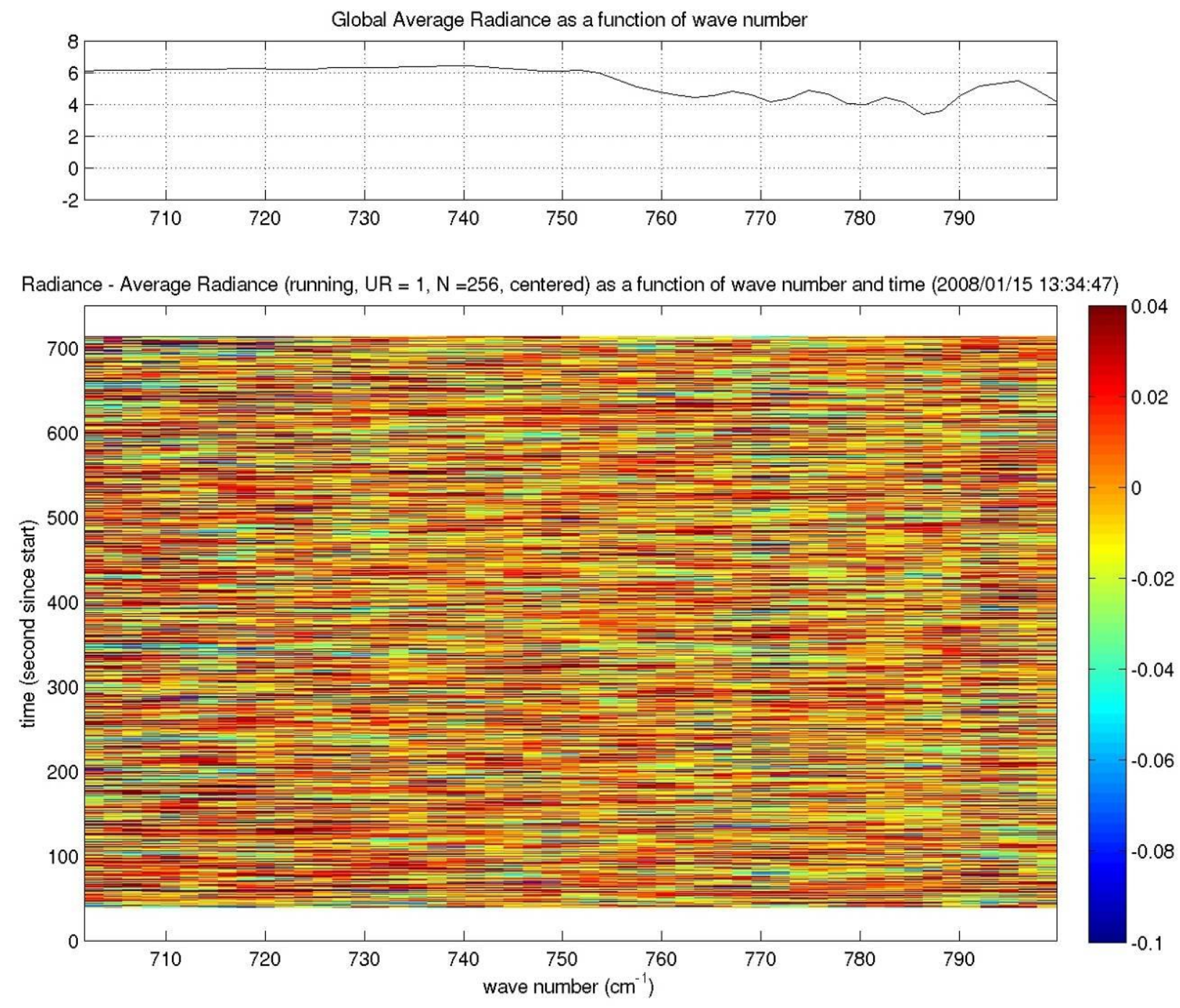

Figure 145. Radiance (color), as a function of time and wavenumber. Data is from a 700 second window starting at 13:34Z on Jan 15, 2008 

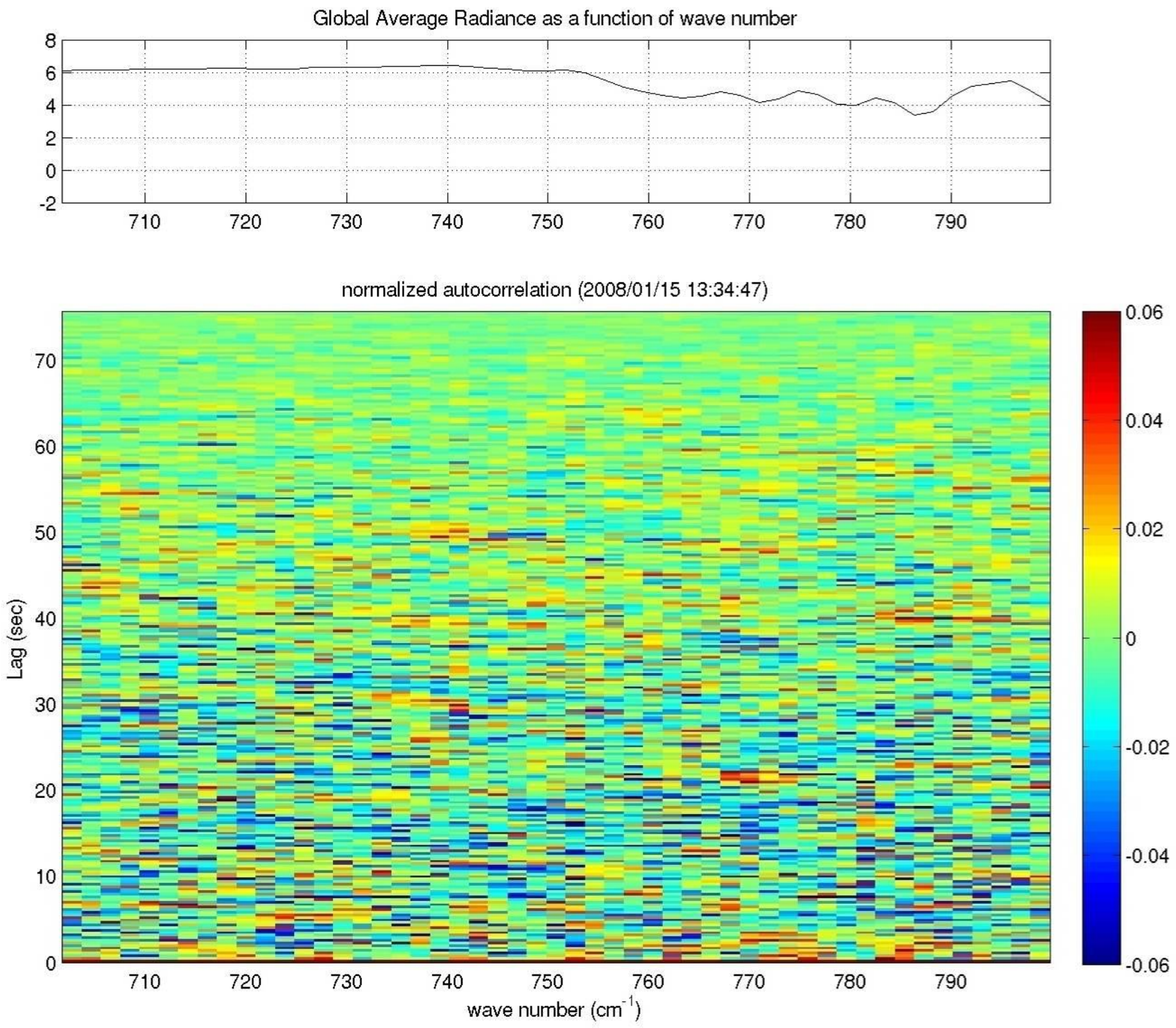

Figure 146. Correlation coefficient for data in Figure 145 

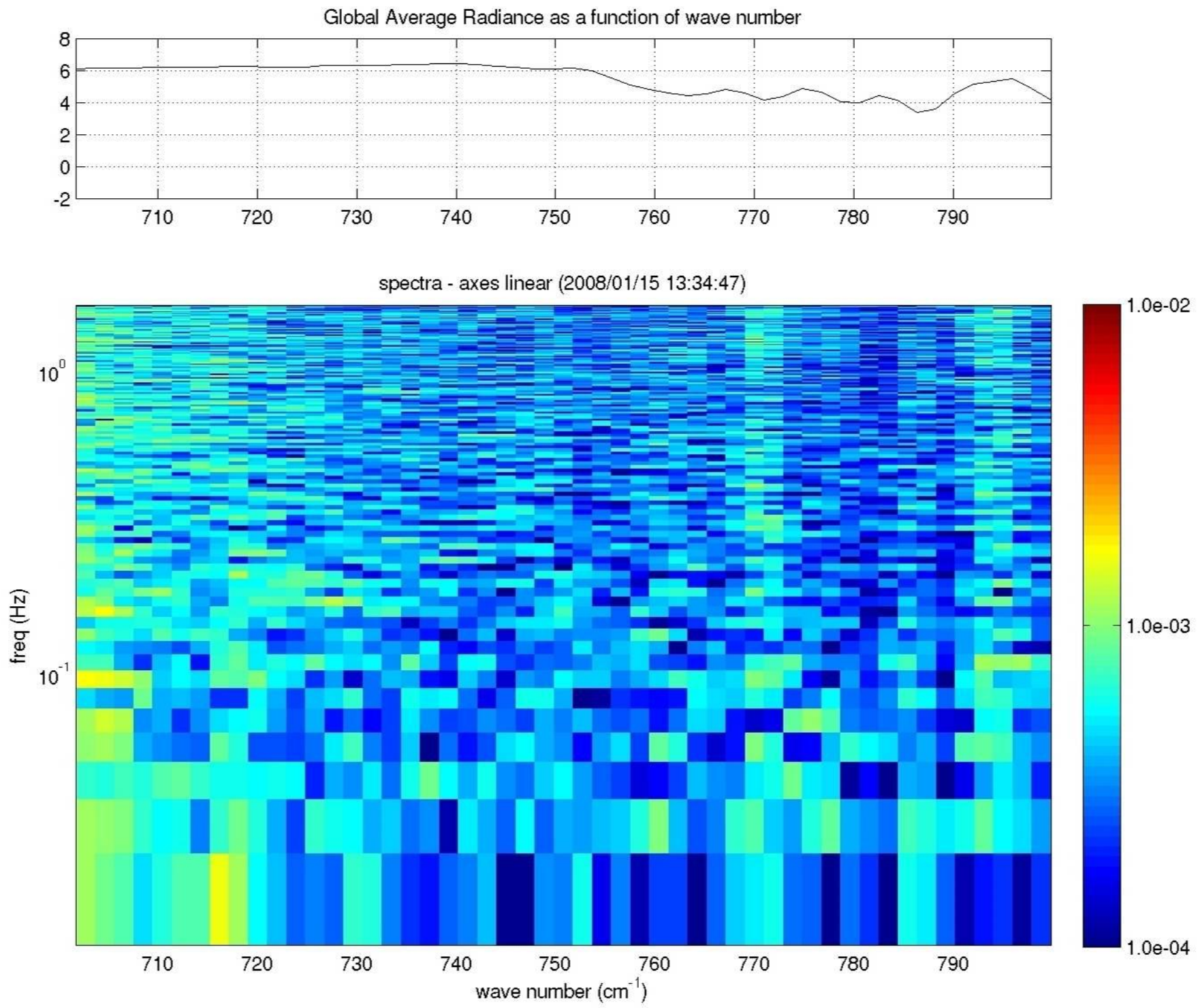

Figure 147. Power spectra of the data from Figure 145. 


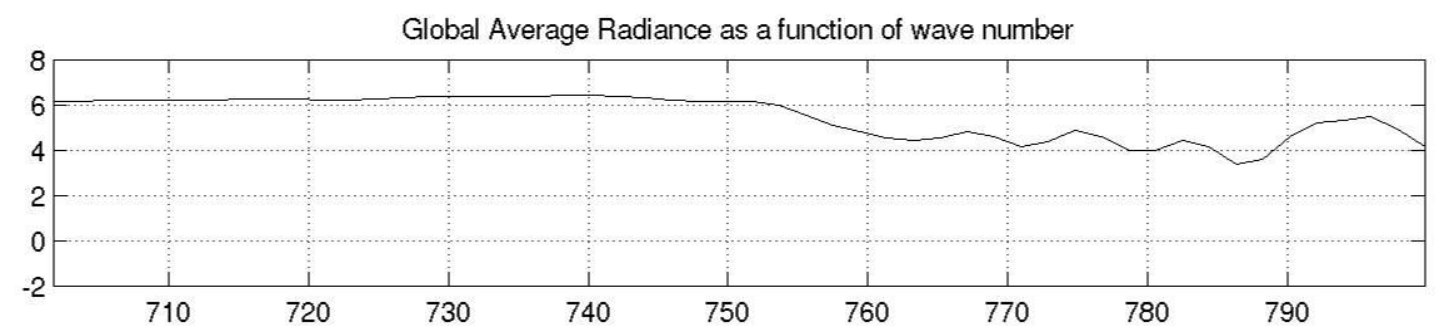

Radiance - Average Radiance (running, UR = 1, N =256, centered) as a function of wave number and time (2008/01/15 13:47:18)

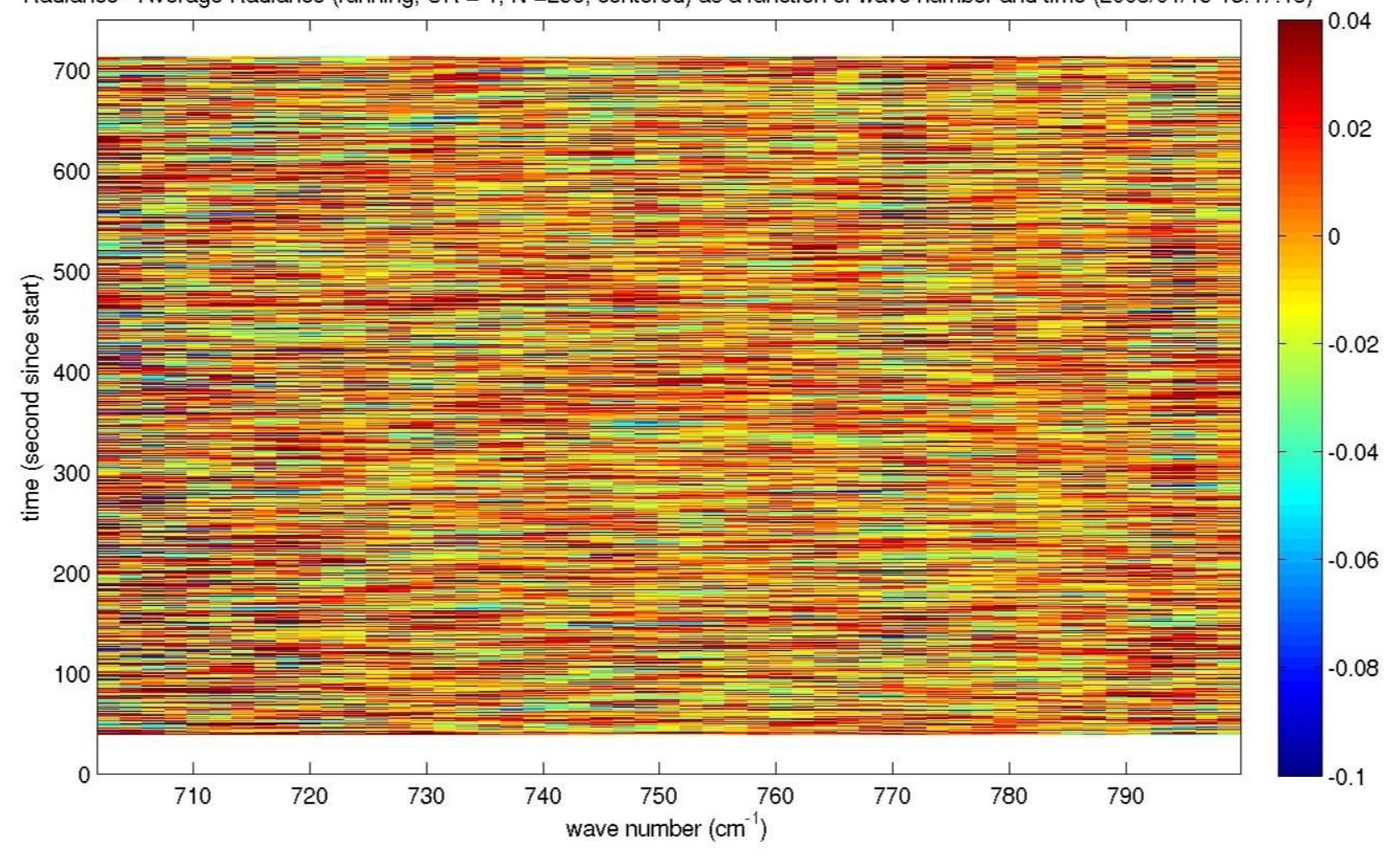

Figure 148. Same as for Figure 145, but showing a 700 second interval of data staring at 13:47Z on Jan 15, 2008 

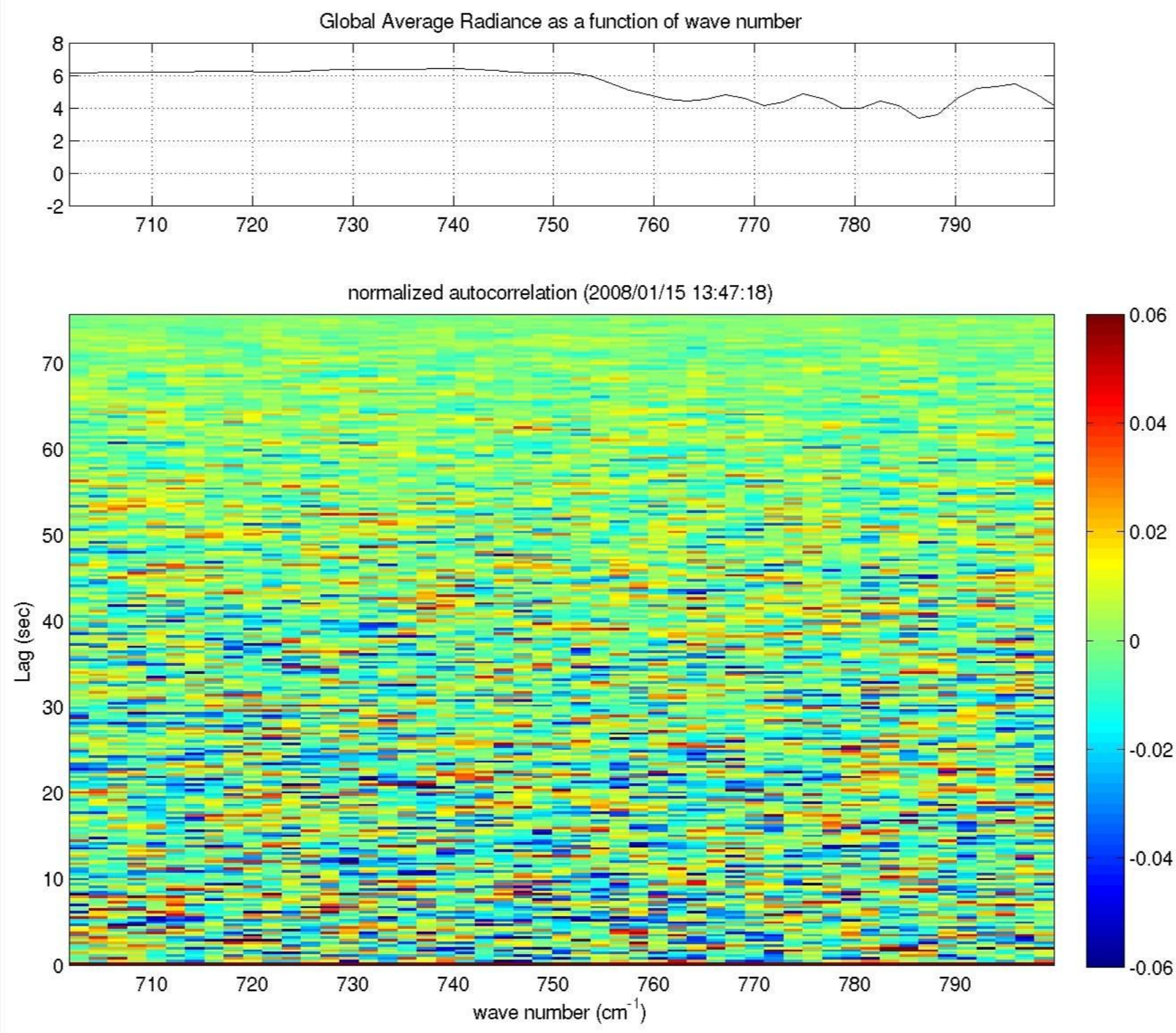

Figure 149. Correlation coefficient for the data shown in Figure 148 


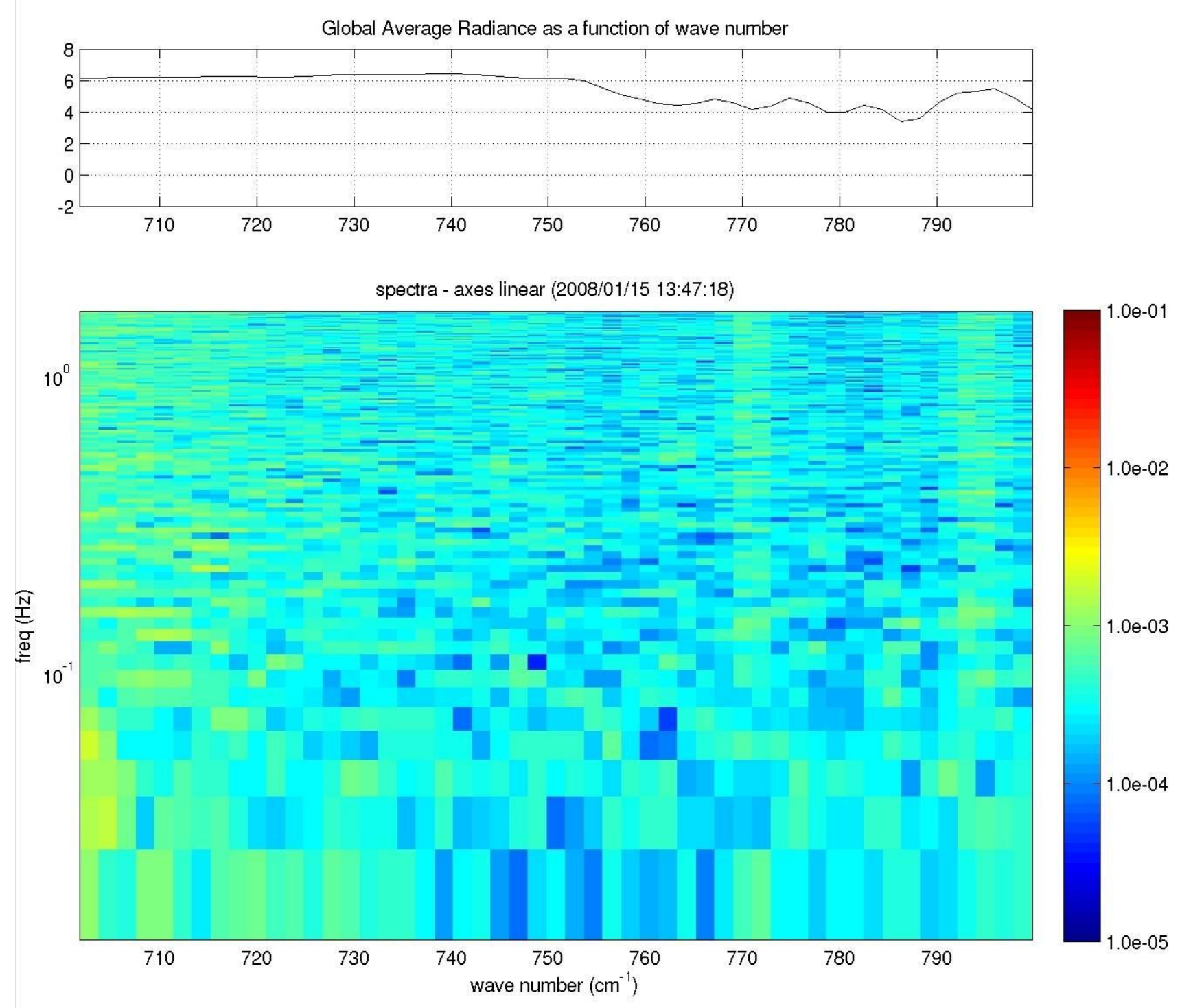

Figure 150. Power spectra of the radiances shown in Figure 148 


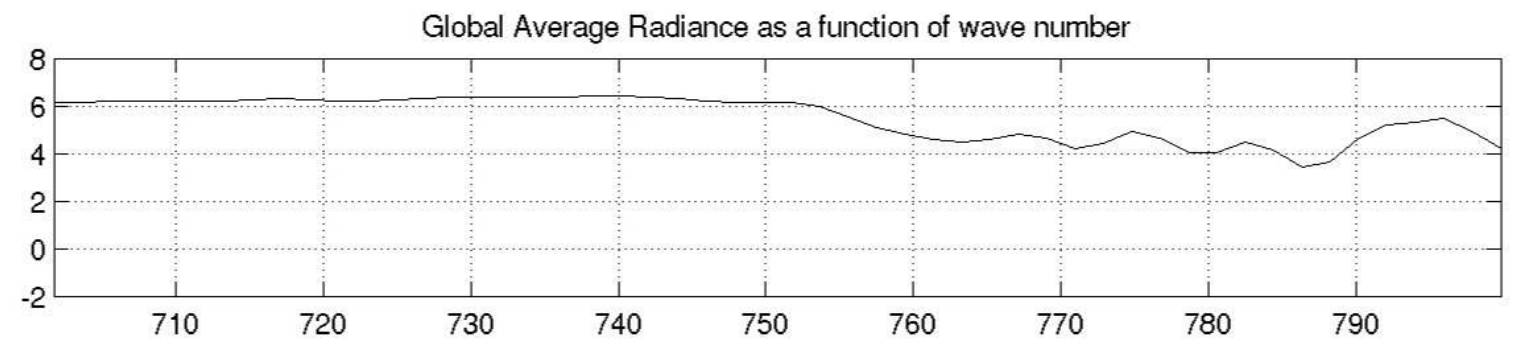

Radiance - Average Radiance (running, UR = 1, N =256, centered) as a function of wave number and time (2008/01/15 13:59:48)

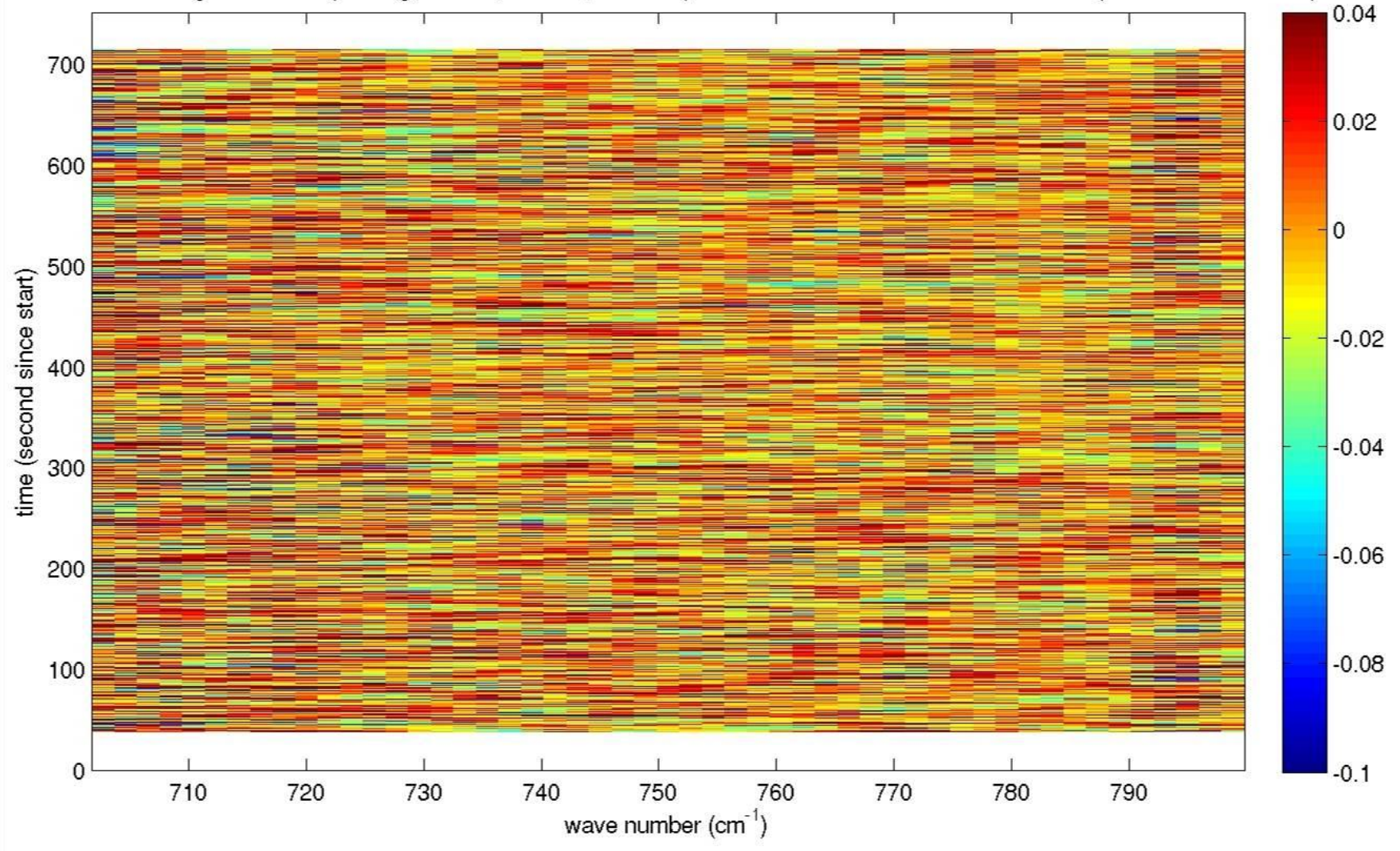

Figure 151. Same as for Figure 145. But for a 700 second section starting at 13:59Z, Jan 15. 2008 


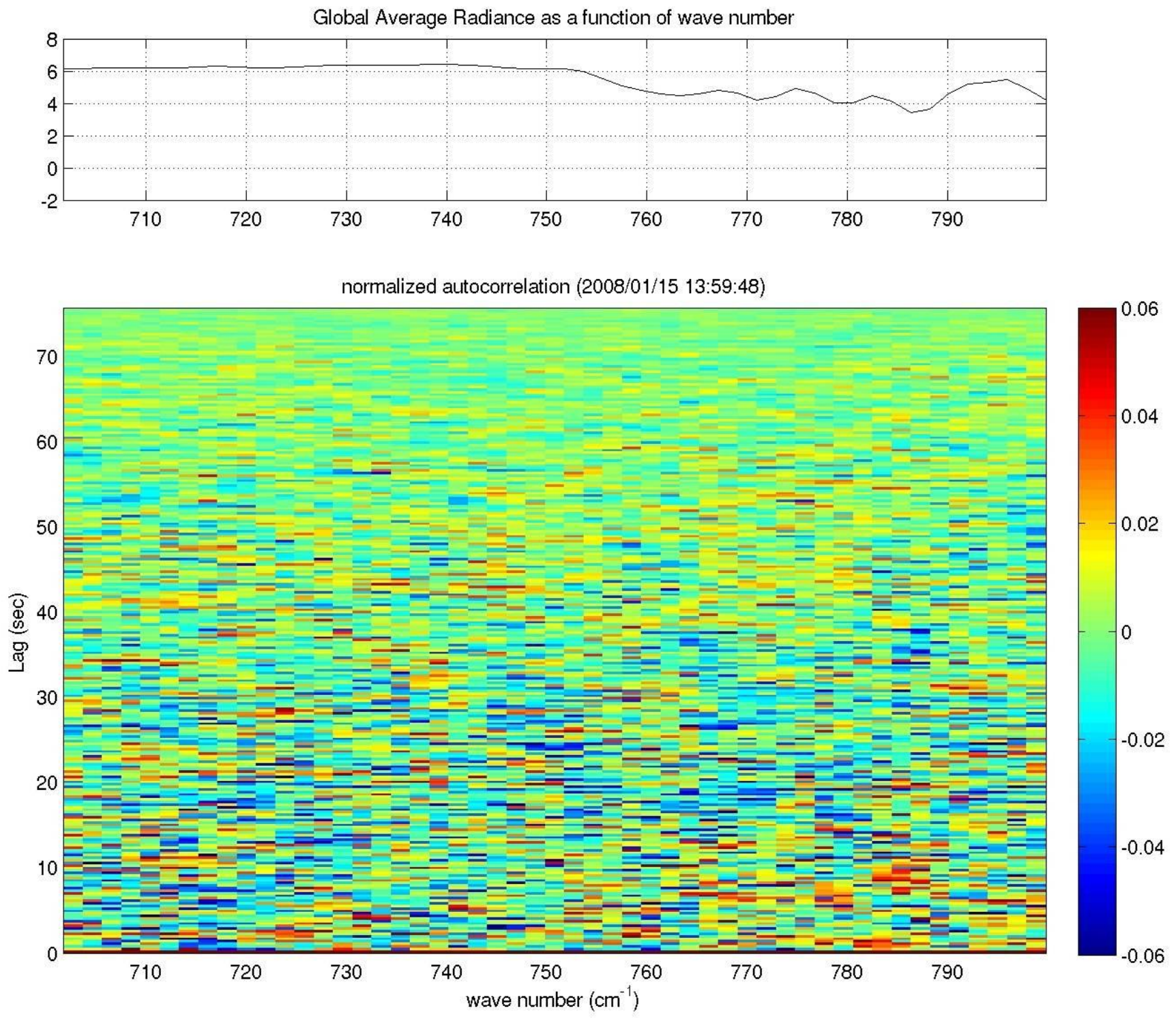

Figure 152. Correlation coefficient for the data shown in Figure 151 

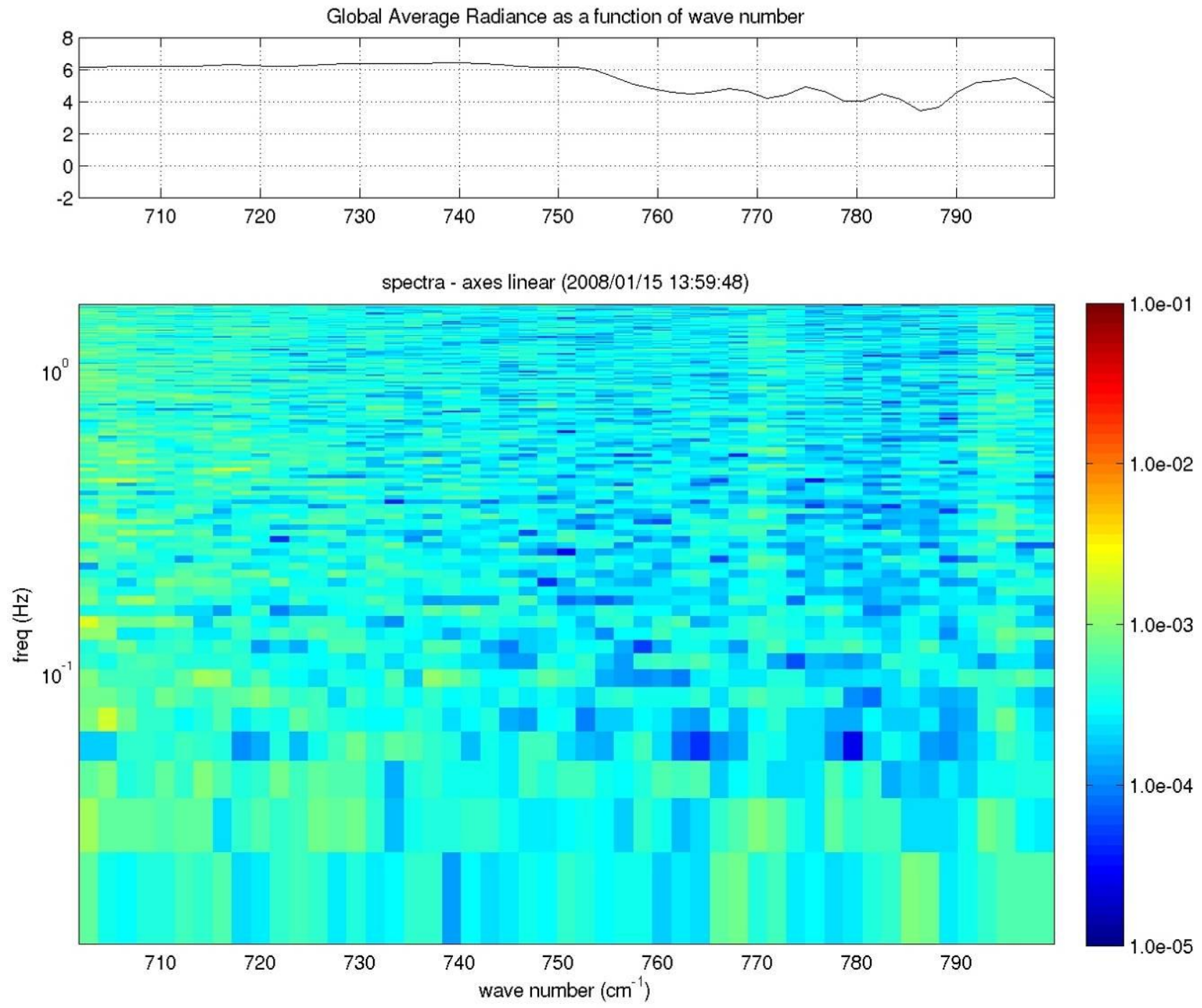

Figure 153. Power spectra for the data shown in Figure 151

Figure 154 through Figure 161 show the correlation coefficient functions over the different sampling periods, and also covering the full wavenumber range. The upper panels are the raw radiance correlation functions, whereas the bottom panels show the results of performing a wavelet filtering (described elsewhere). In distinction from the first set of figures (Figure 145 through Figure 153), the following data indicate a correlation structure that might be expected from turbulence, i.e., a fairly long segment (as a function of temporal lag) with positive correlation. A clear positive/negative/positive pattern can be seen. This pattern is quite consistent for the different time periods. There are four wavenumber regimes that show interesting signals, approximately $800-1000 \mathrm{~cm}^{-1}, 1100-1200 \mathrm{~cm}^{-1}, 1450-1550 \mathrm{~cm}^{-1}$, and $2350-2450 \mathrm{~cm}^{-1}$. A few general comments can be made regarding these figures: (1) the wavelet filtering improves the detection of the correlation coefficient structure - removing some of the noise-like features, (2) large positive correlations can be seen for the smaller lags, switching to negative correlations, (3) 
there are some fairly significant changes in relatively short time intervals, (4) the $800-1000 \mathrm{~cm}^{-1}$ wavenumber region seems to have the most interesting structure - having the largest positive correlations; (5) there is more negative correlation structure in the $1450-1550 \mathrm{~cm}^{-1}$, and 2350 $2450 \mathrm{~cm}^{-1}$ ranges, (6) excepting for the last time segment, the $1100-1200 \mathrm{~cm}^{-1}$ region does not contain much structure of interest. Figure 160 and Figure 161 show the most promising time segments, especially the latter one, showing a very strong positive correlation for the first 25 seconds. This indicates the likelihood that turbulence was present.
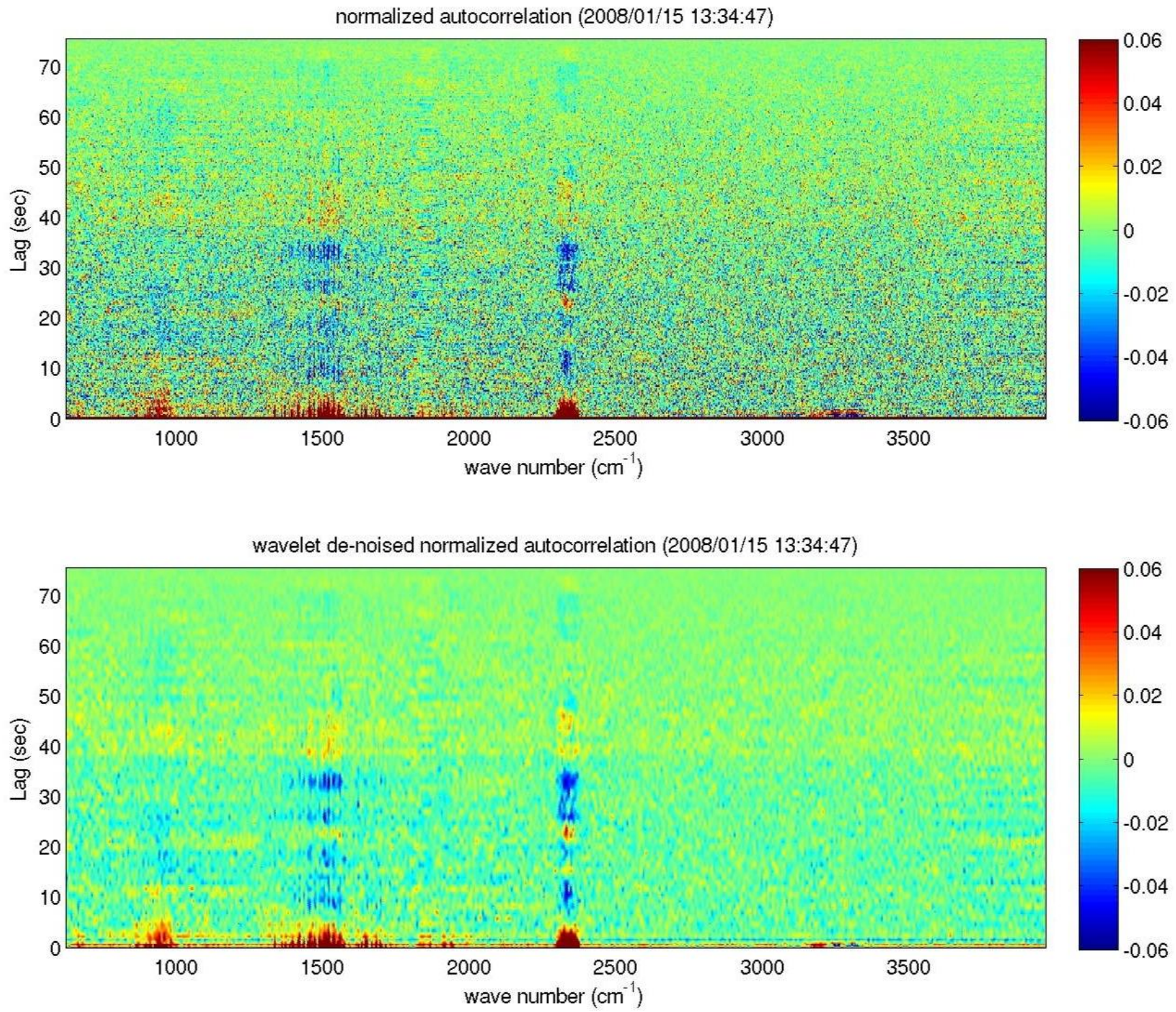

Figure 154. Radiance correlation coefficient as a function of lag and wavenumber. 

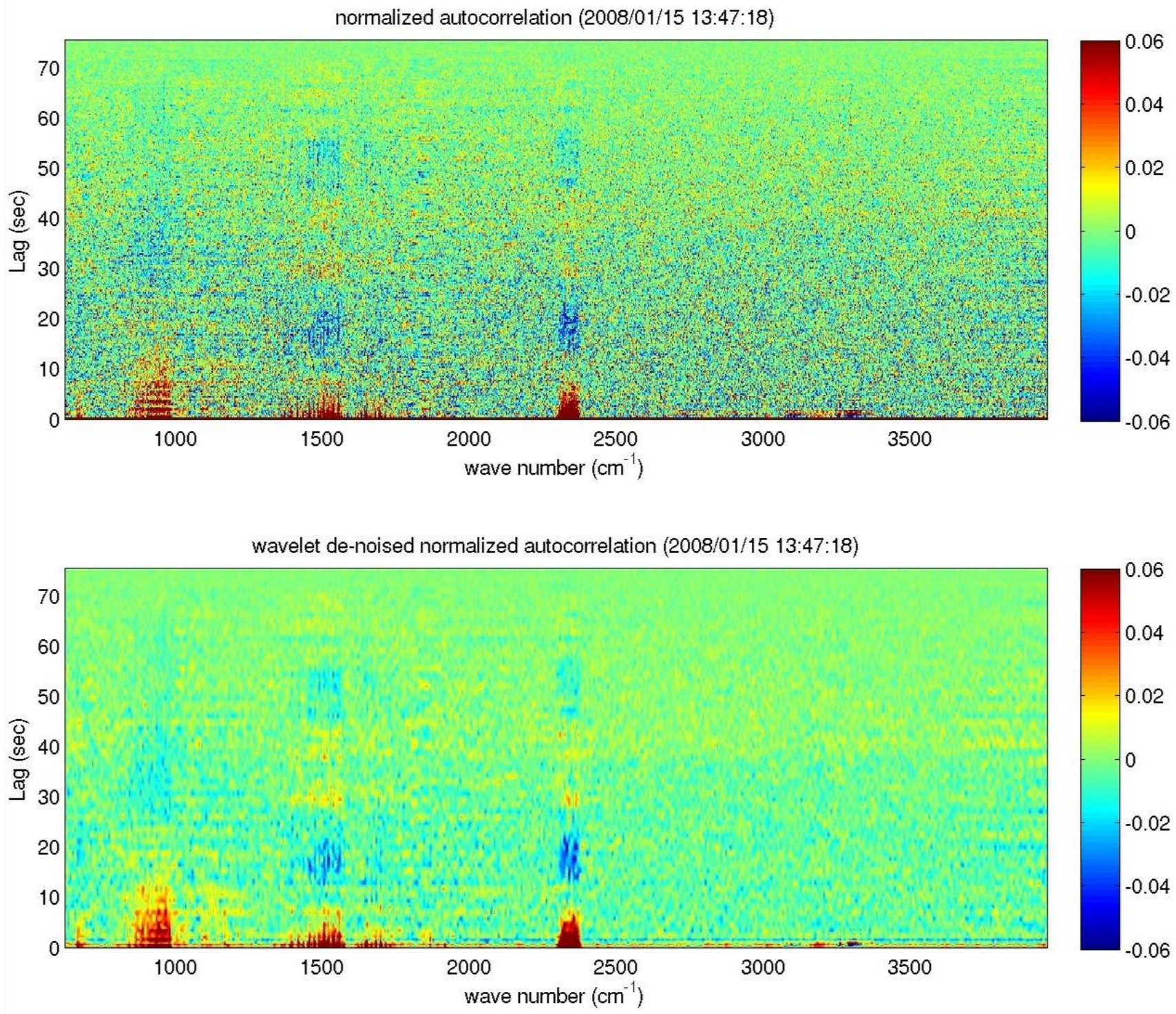

Figure 155. Same as Figure 154, but for time segment starting at 13:47 on Jan 15, 2008. 

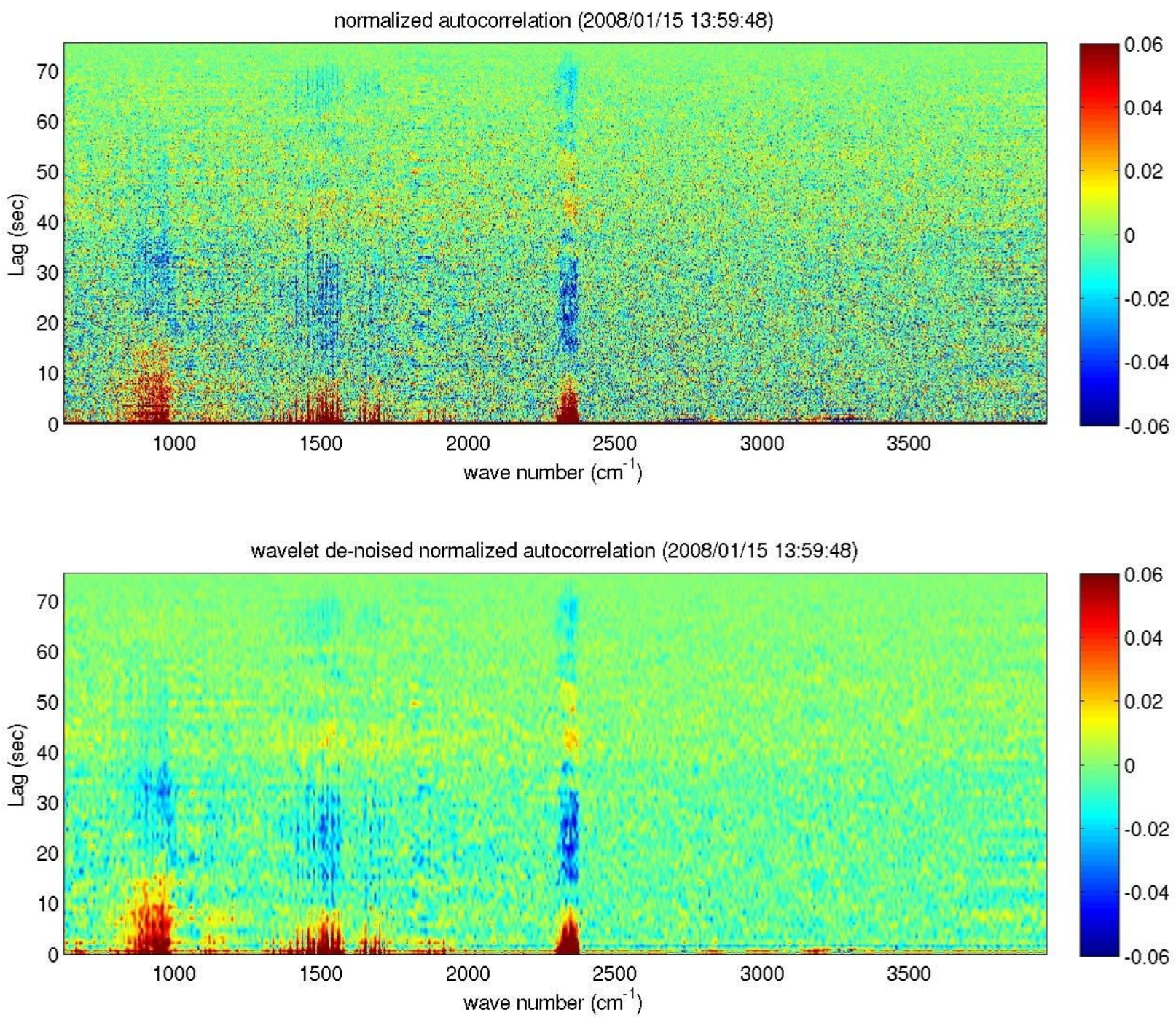

Figure 156. Same as Figure 154, but for time segment starting at 13:59Z on Jan 15, 2008. 

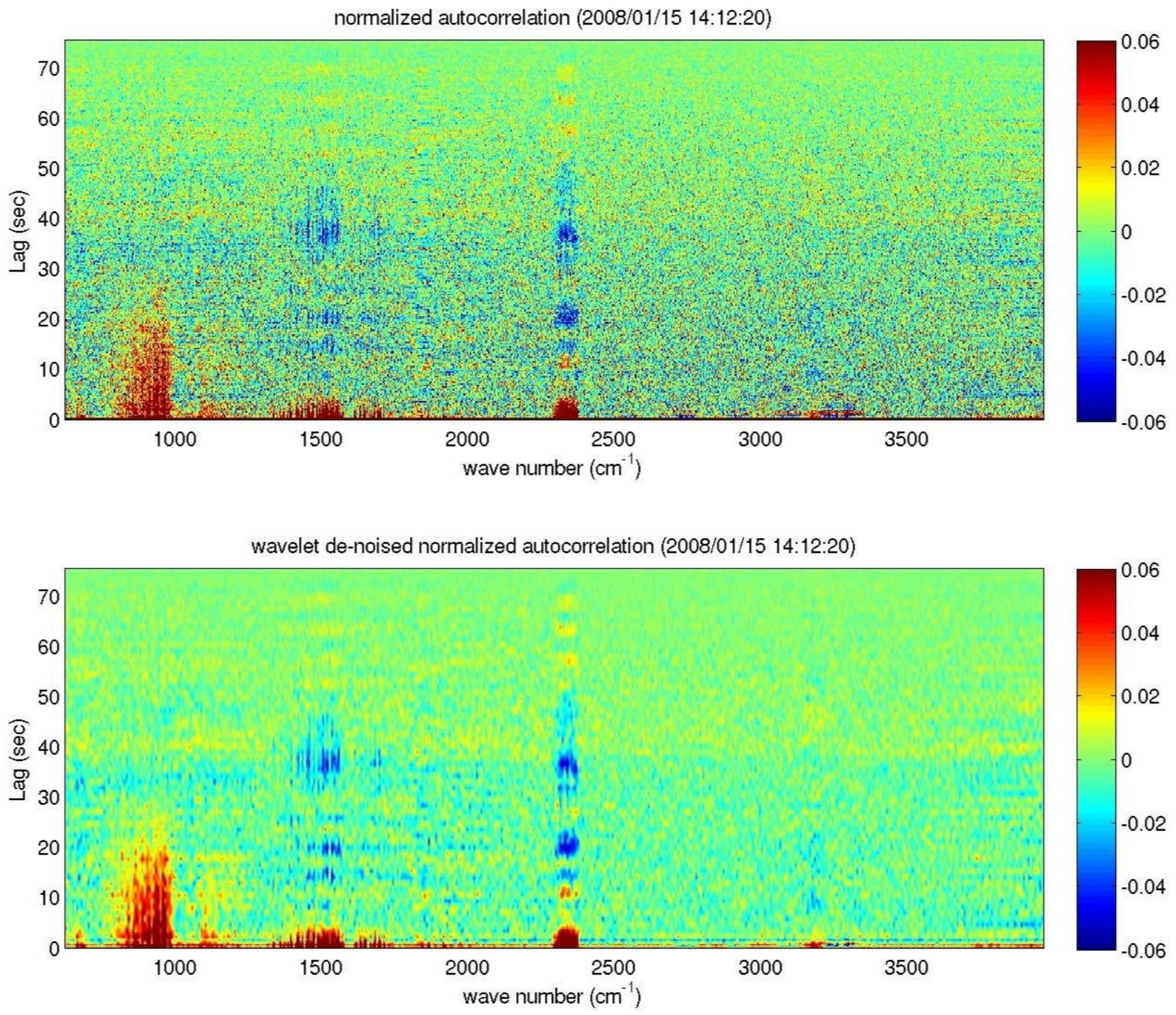

Figure 157. Same as Figure 154, but for time segment starting at 14:12Z on Jan 15, 2008 

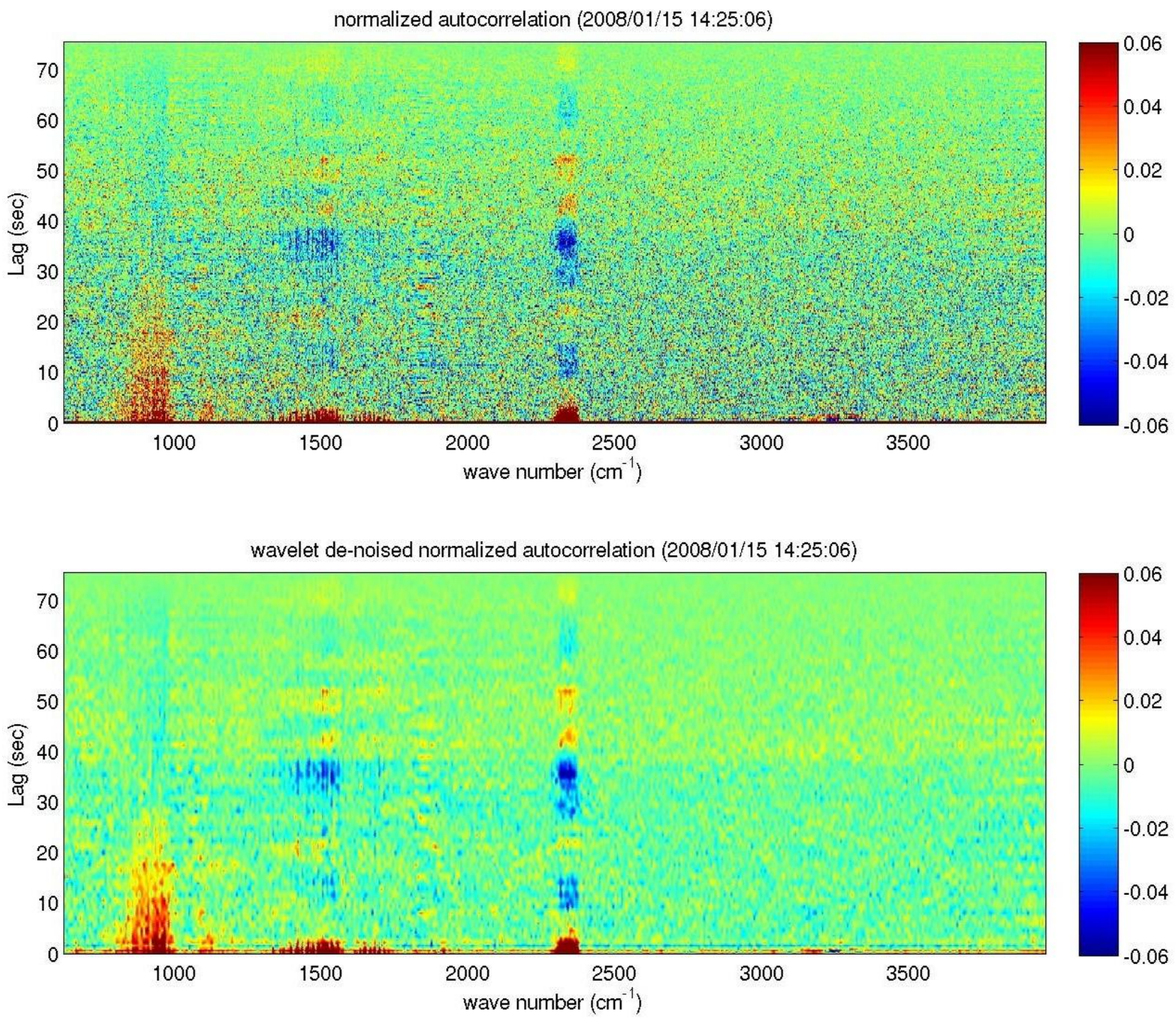

Figure 158. Same as Figure 154, but for time segment starting at 14:25Z on Jan 15, 2008 

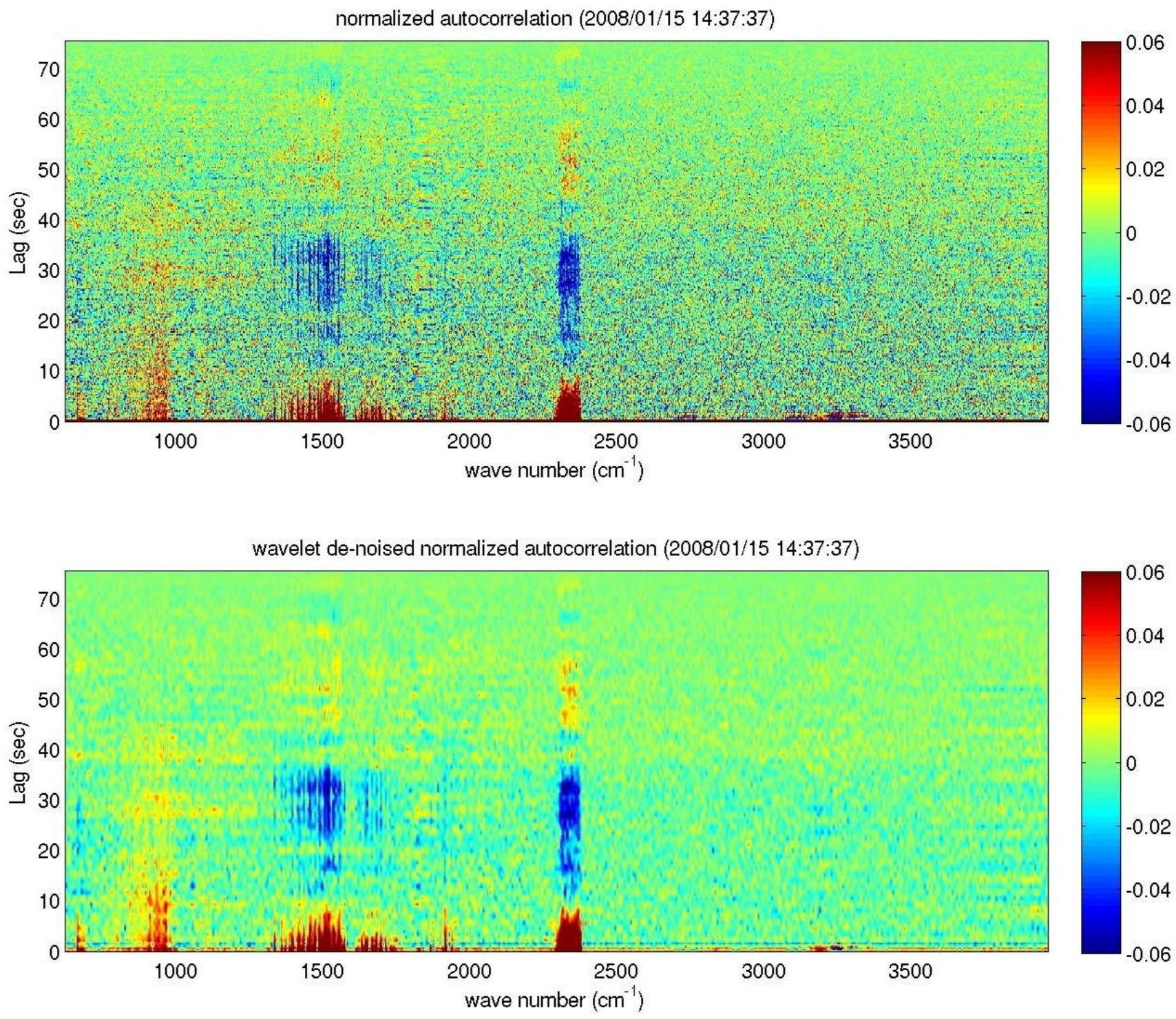

Figure 159. Same as Figure 154, but for time segment starting at 14:37Z on Jan 15, 2008 

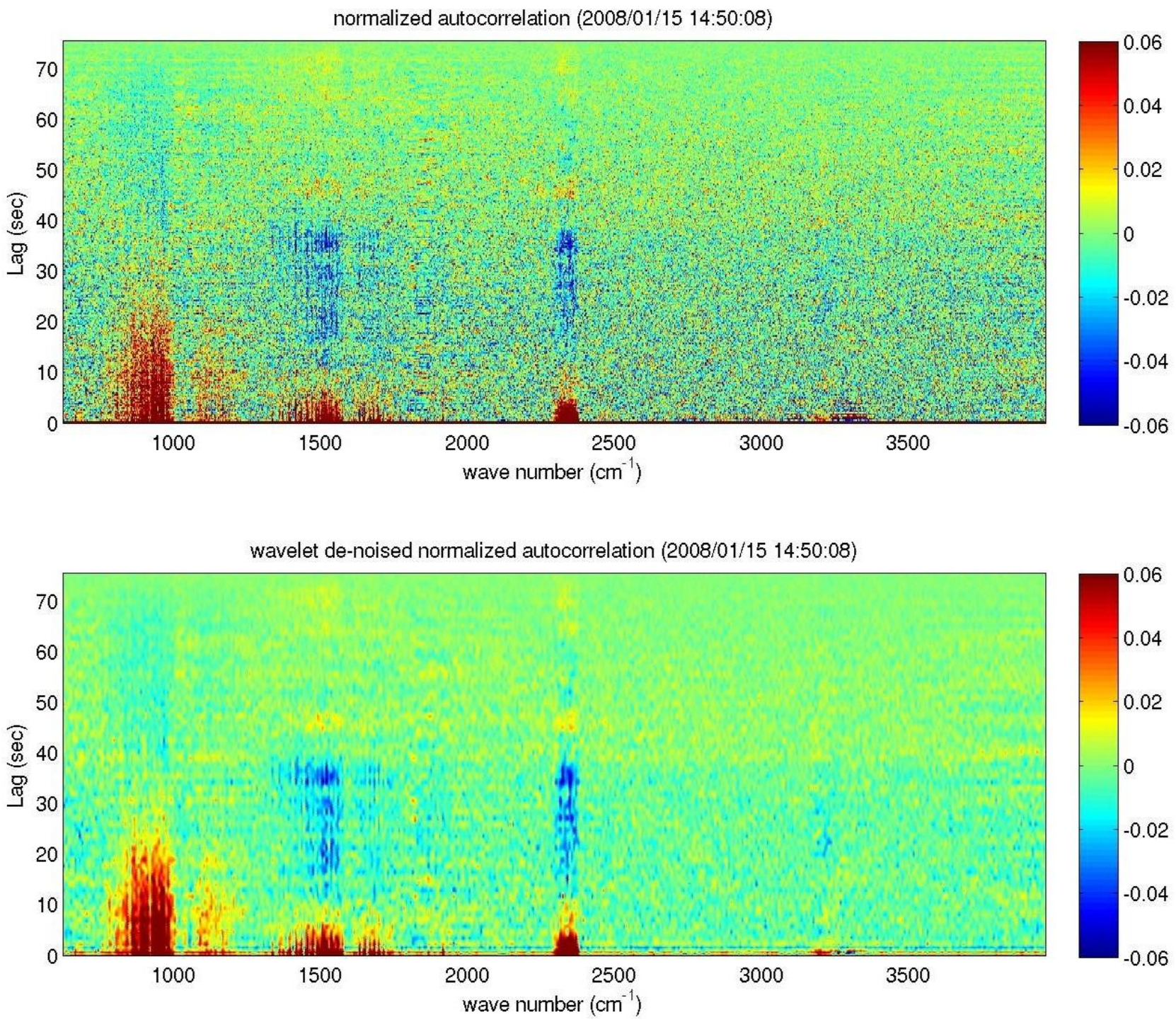

Figure 160. Same as Figure 154, but time segment starting at 14:50Z on Jan 15, 2008 

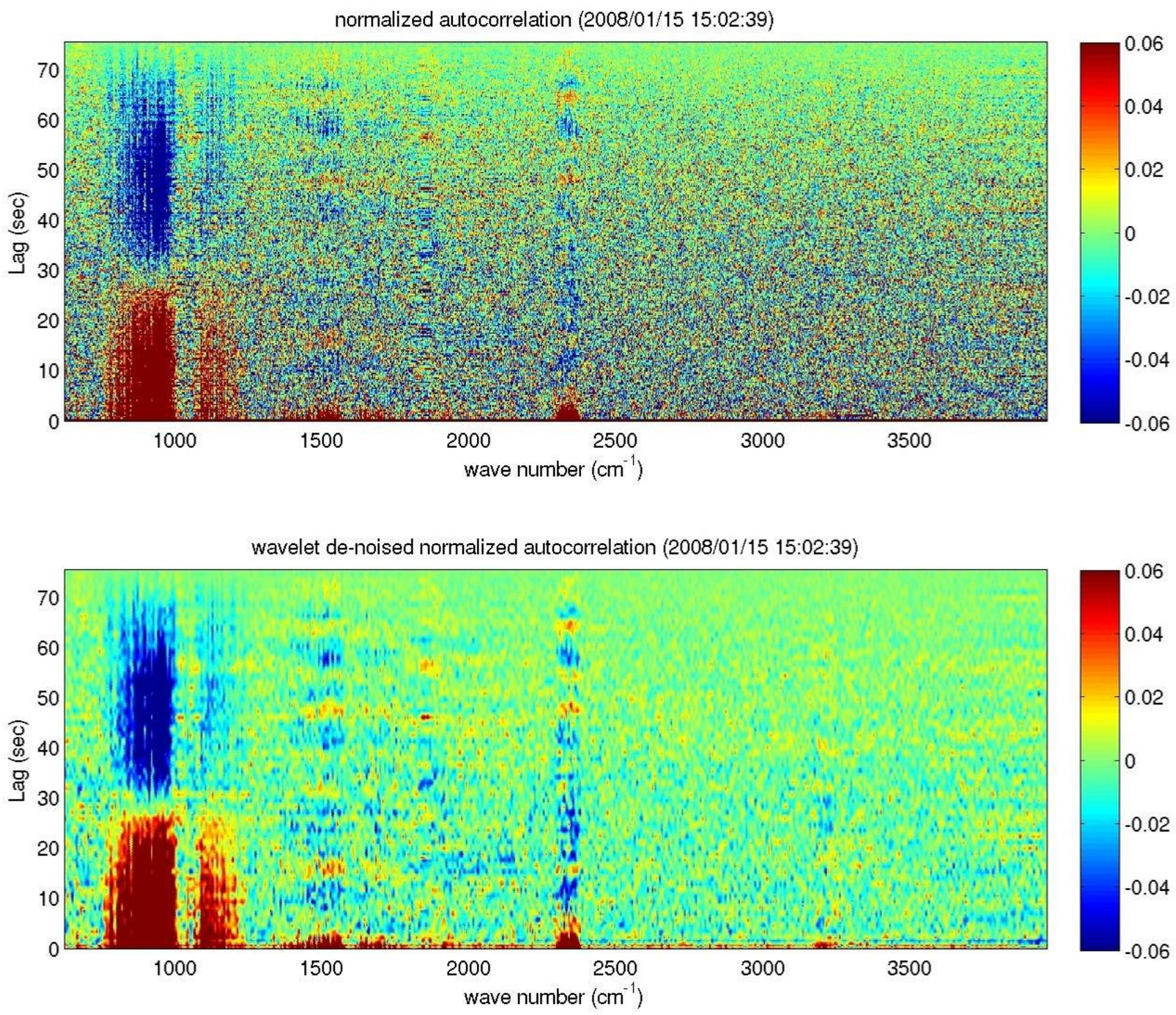

Figure 161. Same as Figure 154, but for time segment starting at 15:02Z on Jan 15, 2008.

Figure 162 through Figure 164 show the cross section lines used for subsequent EDR viewer vertical cross sections. The EDR reports are the color-coded square symbols, and the turbulence intensity increases as the color goes from cool to hot colors. These EDR viewer images show the data from the indicated time and going back 18 hours. The reports are typically one minute apart at the higher altitudes (approximately $12-15 \mathrm{~km}$ spacing). As the aircraft are climbing and descending - especially close to the surface - the reports are given at 1000 foot intervals. Each of the plan-view images shows different elevations levels - typically $4000 \mathrm{ft}$ apart. The EDR reports are given in a vertical window $+/-2000 \mathrm{ft}$. surrounding the indicated altitude. The spatial domain is approximately the state of Colorado. The brownish colors indicate the terrain height. The white star shown on Figure 162 is the approximate location of the IR sensor. The cluster of EDR reports towards the lower RHS of the lowest altitude figures indicates aircraft flying in and out of DIA. The GTG turbulence forecast product is shown as an underlain gridded color field (same color scale as for the EDR reports). Figure 162 is at 15:00 UTC and 10000 feet in altitude. 
The plan-view images are followed by vertical cross sections from the EDR viewer (Figure 172 through Figure 174). It can be seen that there was a fair amount of turbulence reports east of the mountains, from aircraft flying into DIA at lower altitudes. There are a few reports of light turbulence at higher altitudes over the mountains. Recall that the EDR reports are only available from United Airlines' 757 aircraft; hence it does not portray the complete picture in terms of turbulence during this time period, e.g., there were also positive turbulence pilot reports during this day. GTG was forecasting widespread moderate turbulence over the mountains.

There were relatively strong downslope winds on this day. Figure 175 through Figure 180 show wind data from the RUC model: contours of $\mathrm{u}$ - and v-components of the wind, respectively. It can be seen that there are moderately strong westerly winds, which are producing the downslope turbulence. Figure 181 and Figure 182 show cross sections of RUC temperatures - all with overlaid horizontal wind barbs.

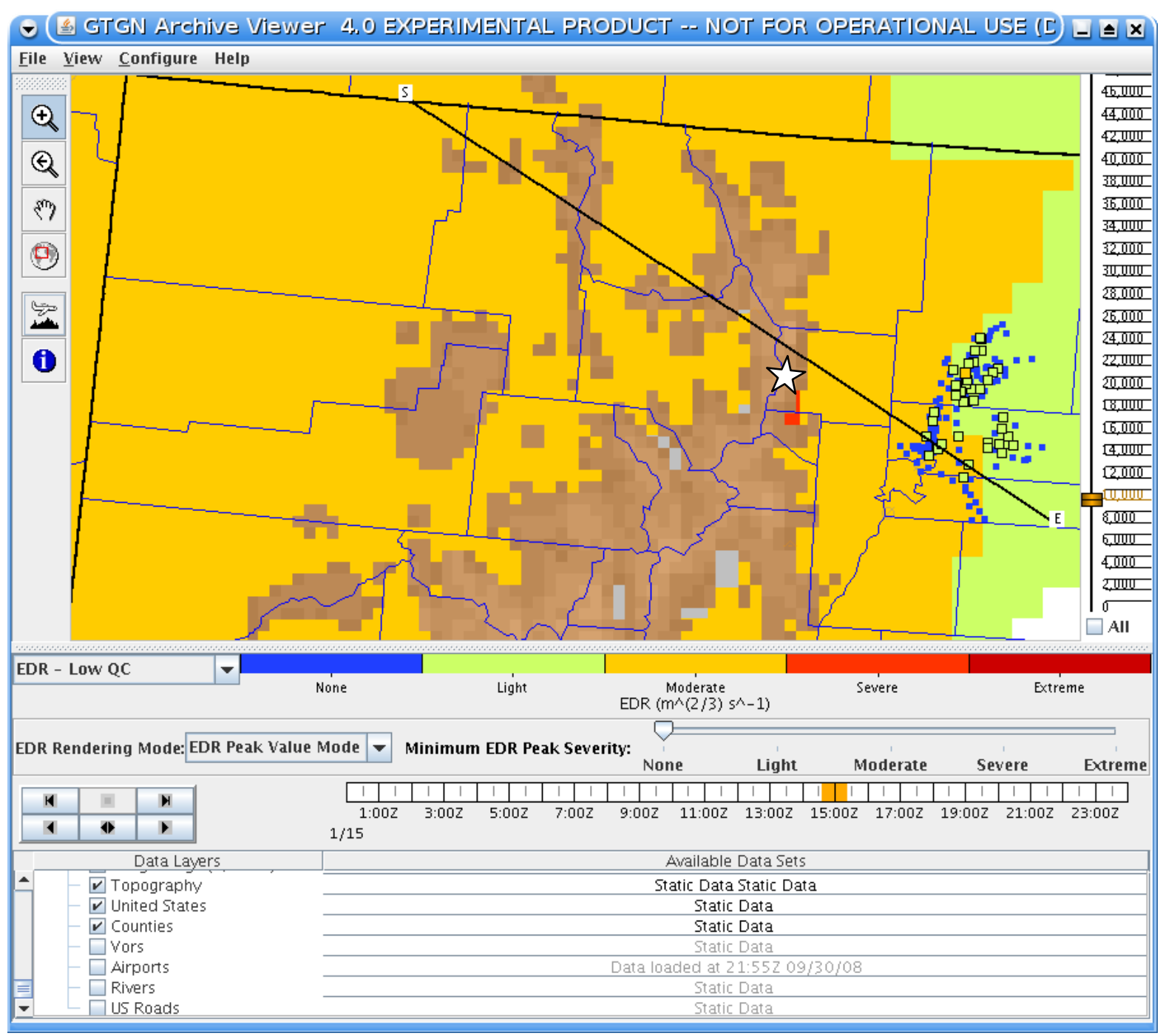

Figure 162. Northwest to Southeast cross section line. 


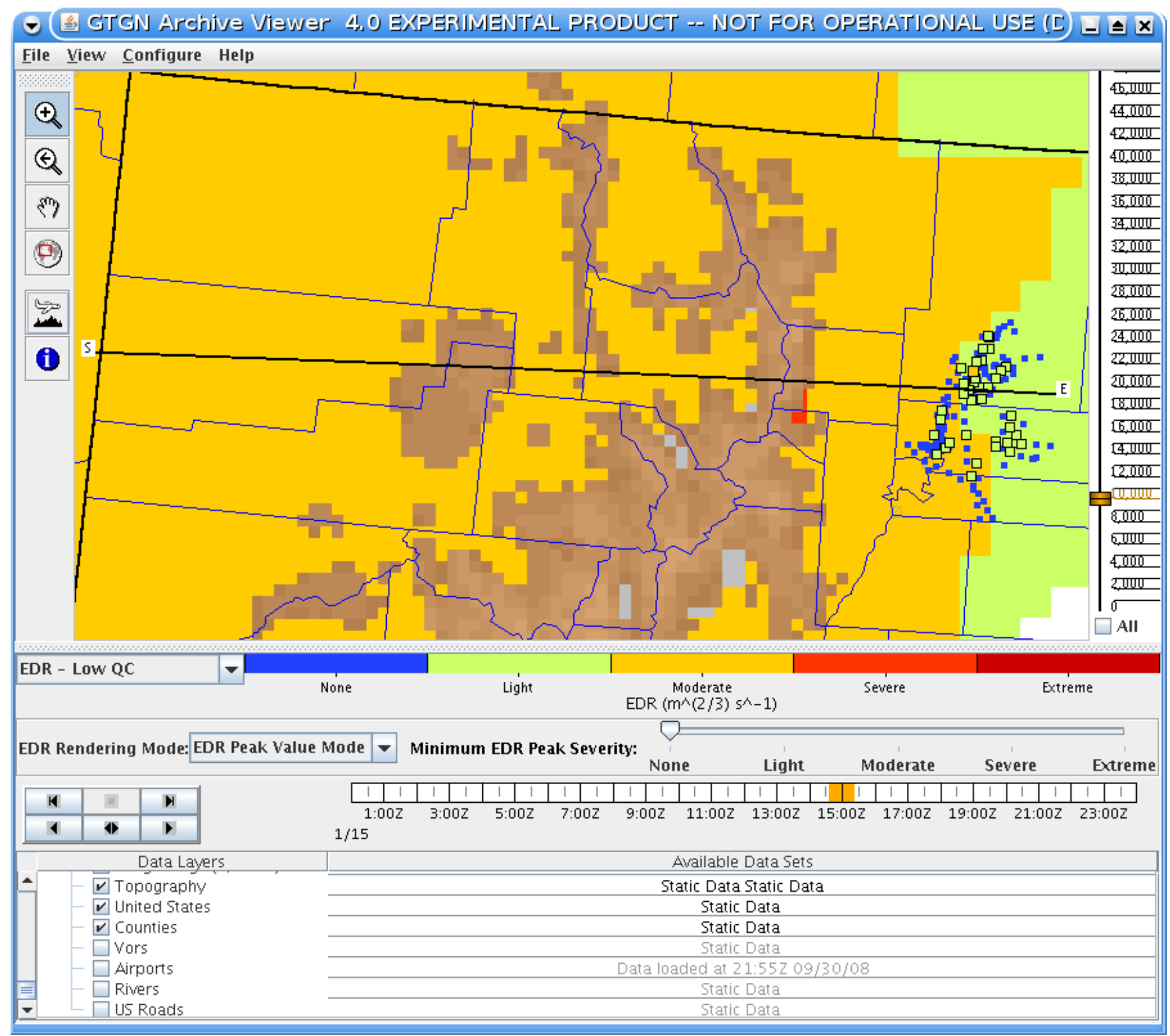

Figure 163. West to East cross section line. 


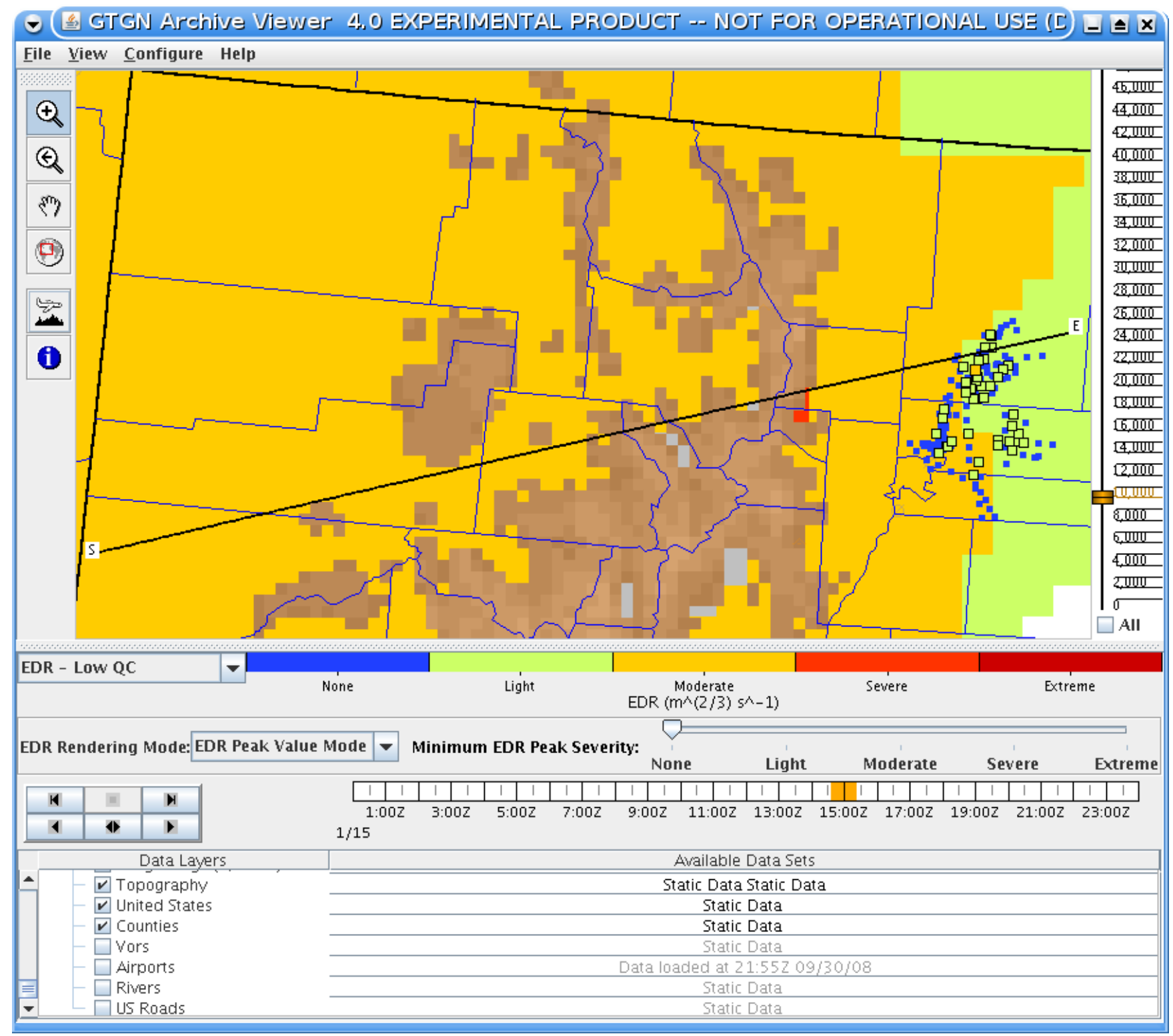

Figure 164. Southwest to Northeast cross section line. 


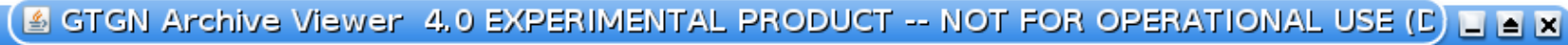

File $\underline{\text { View }}$ Configure Help

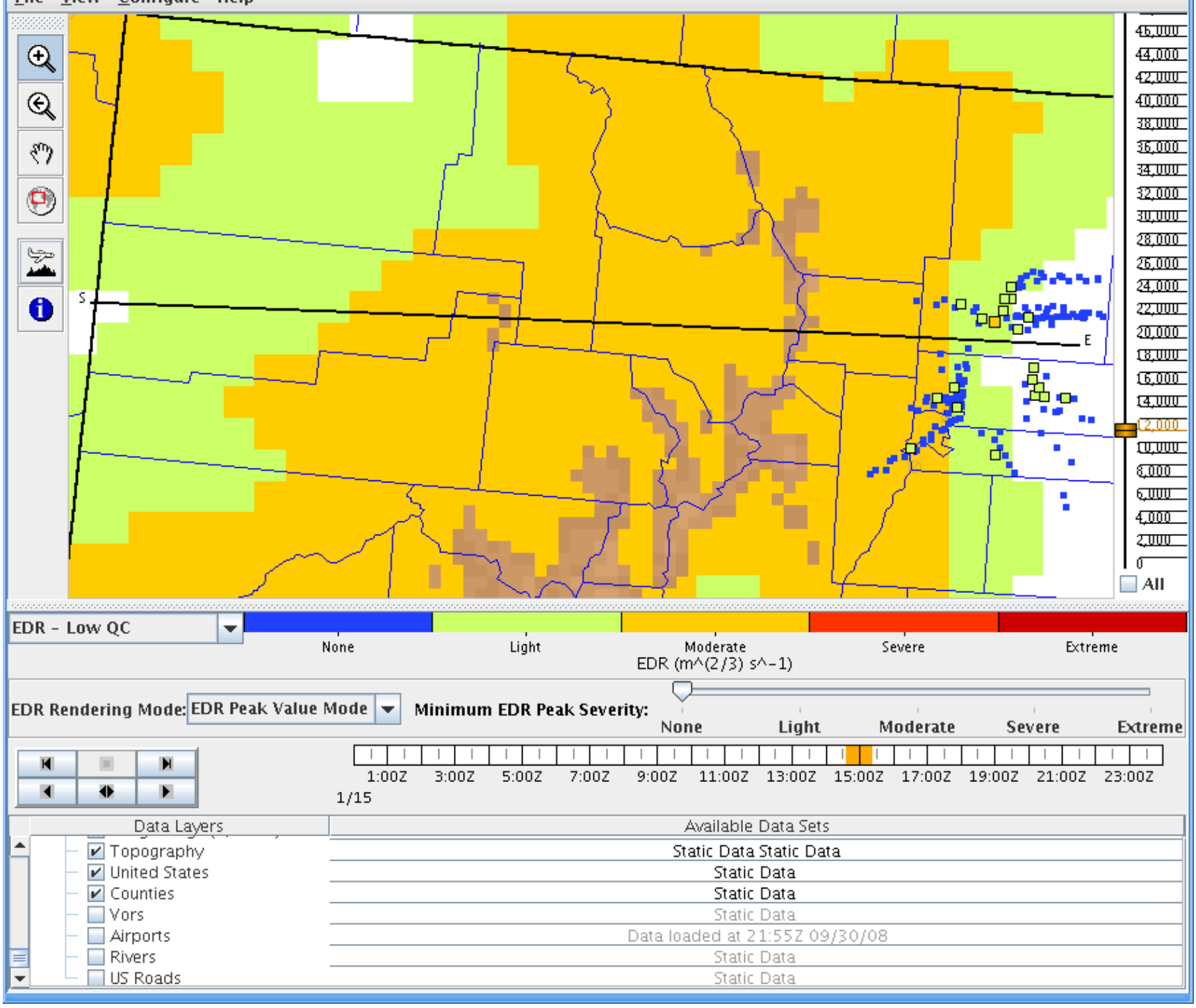

Figure 165. West to East cross section line at $12000 \mathrm{ft}$. 


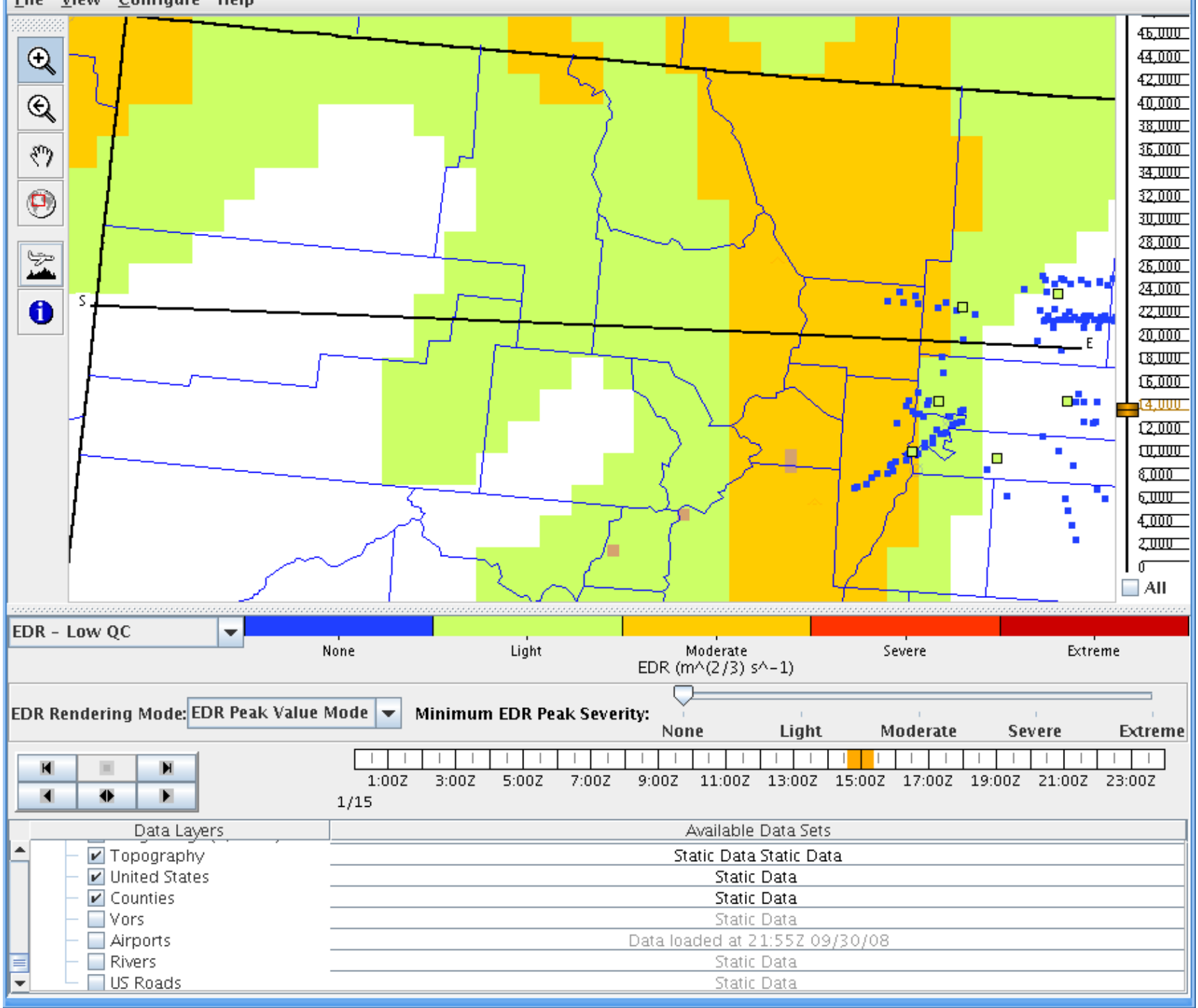

Figure 166. West to East cross section line at 14000 feet. 


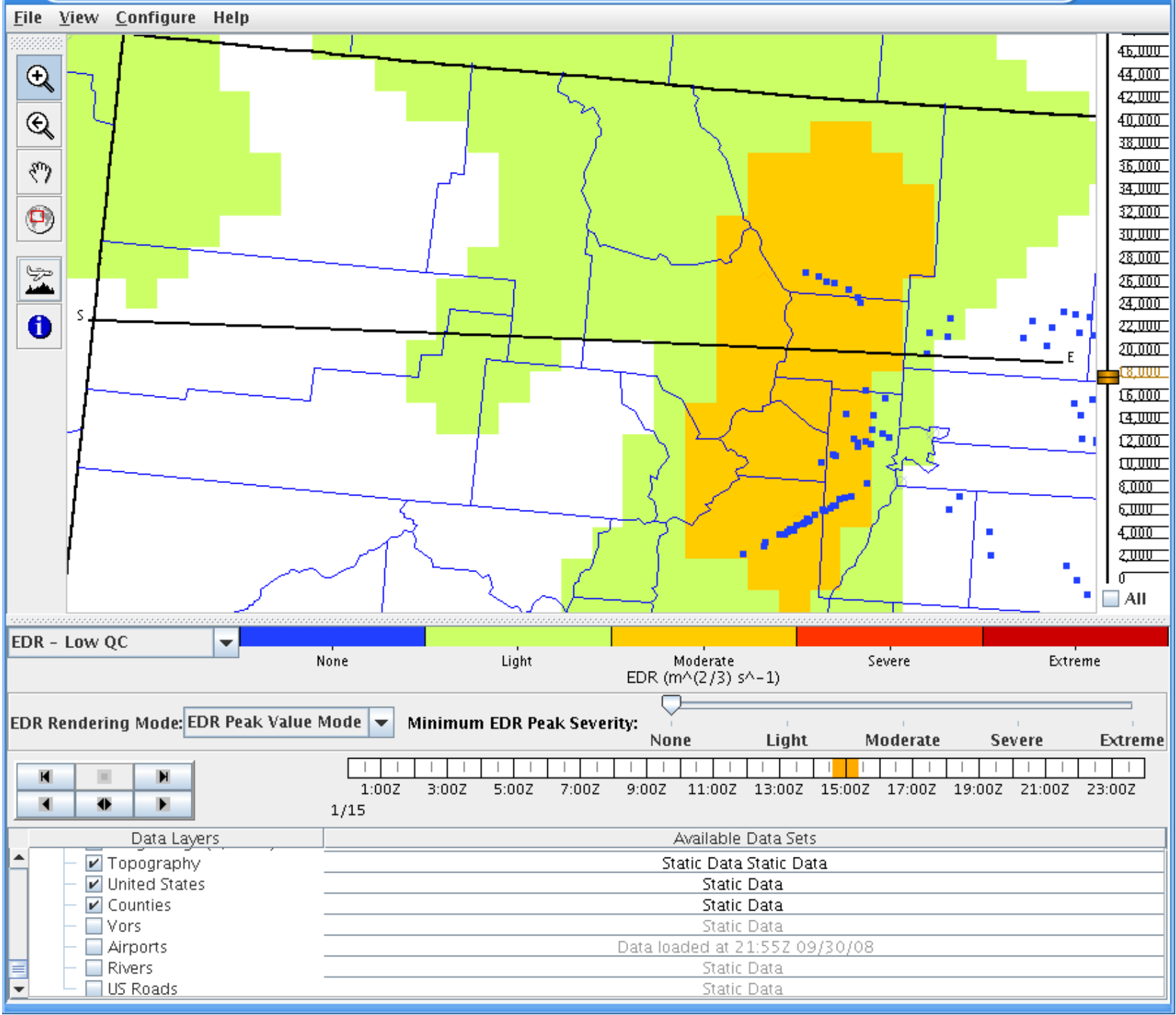

Figure 167. West to East cross section line at 18000 feet. 
$\nabla$ GTGN Archive VIEWEr 4, EXPERIMENTAL PRODUCT - NOT FOR OPERATIONAL USE (L) -

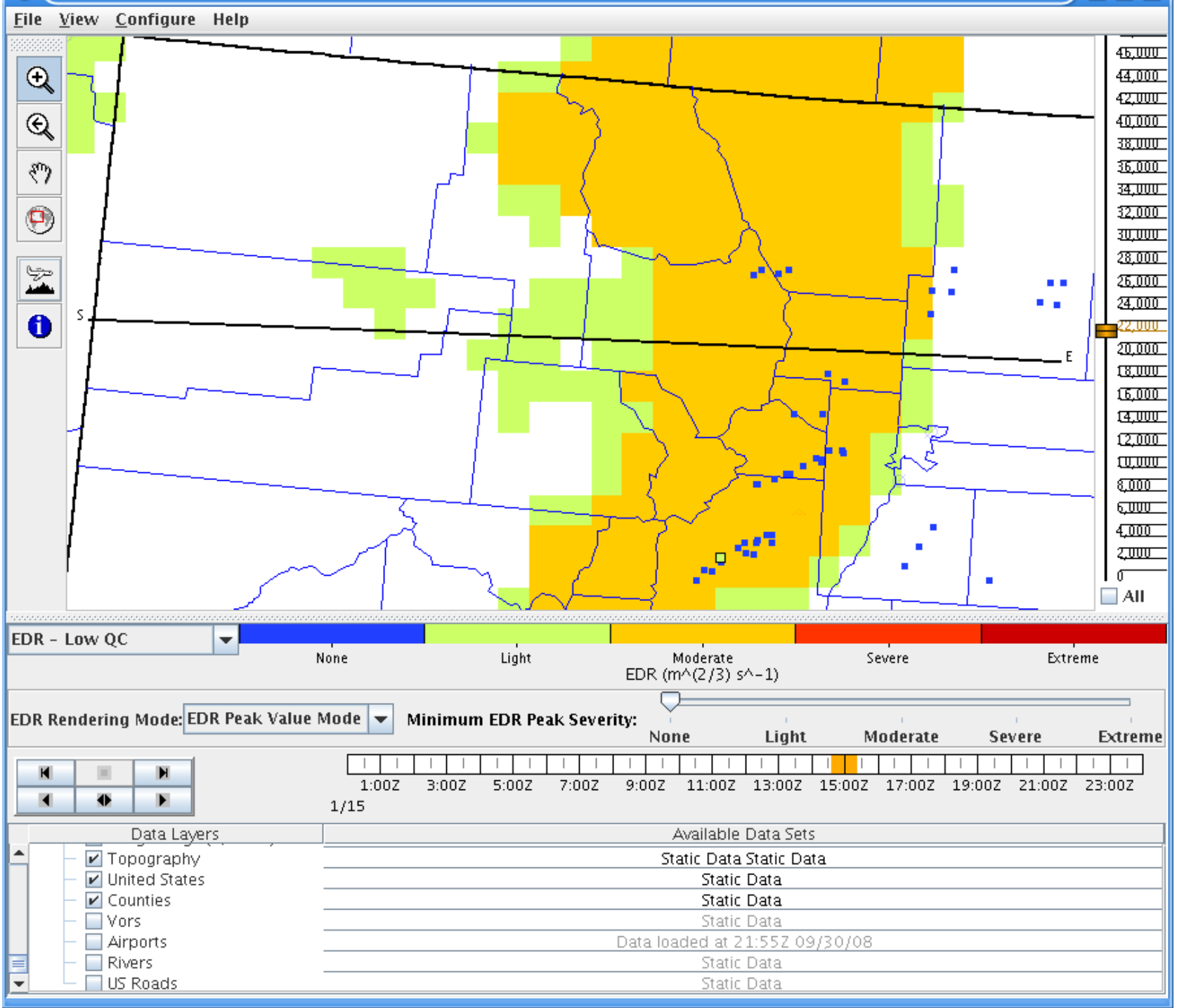

Figure 168. West to East cross section line at 22000 feet. 


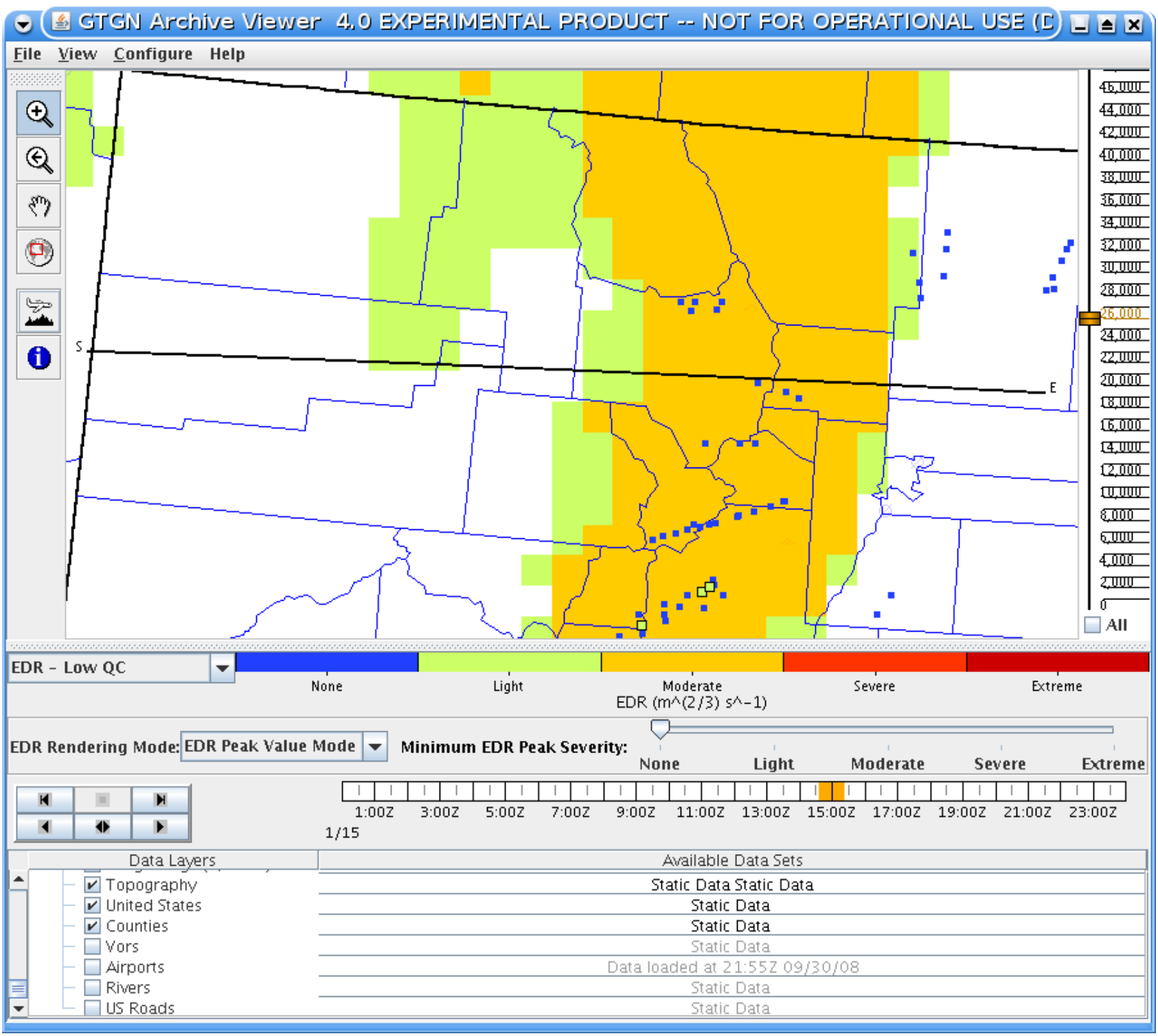

Figure 169. West to East cross section line at 26000 feet. 


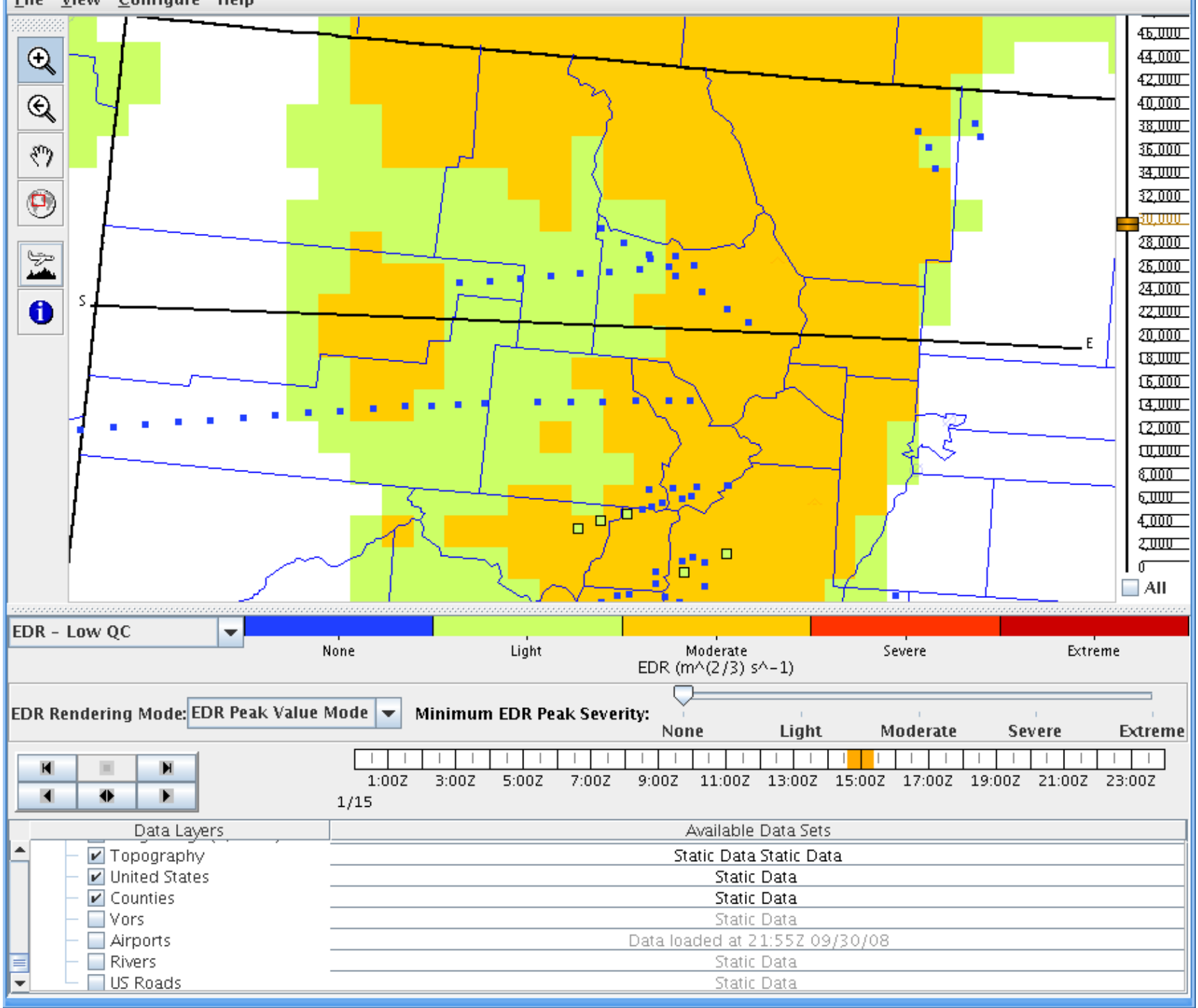

Figure 170. West to East cross section line at 30000 feet. 


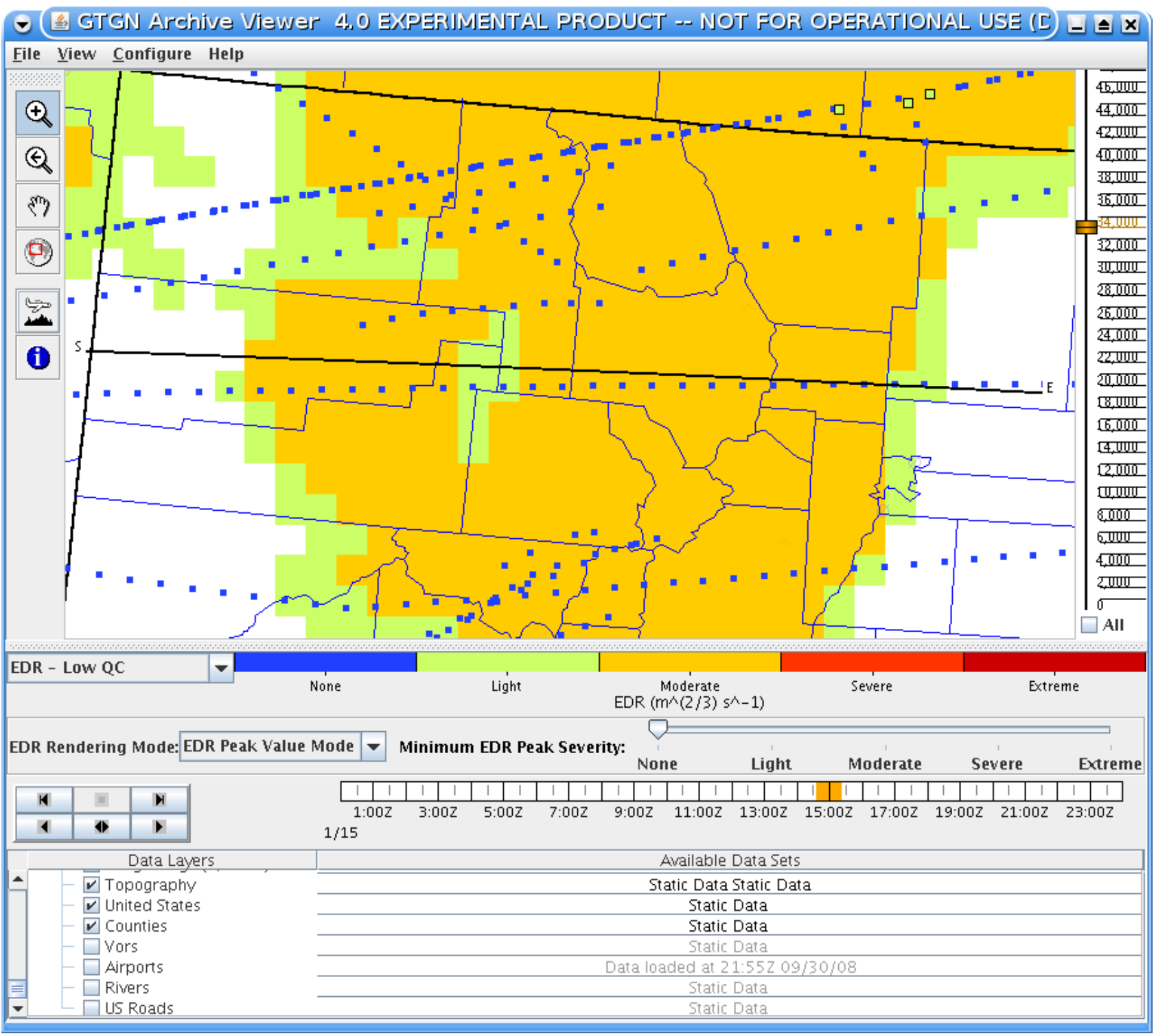

Figure 171. West to East cross section line at 34000 feet. 


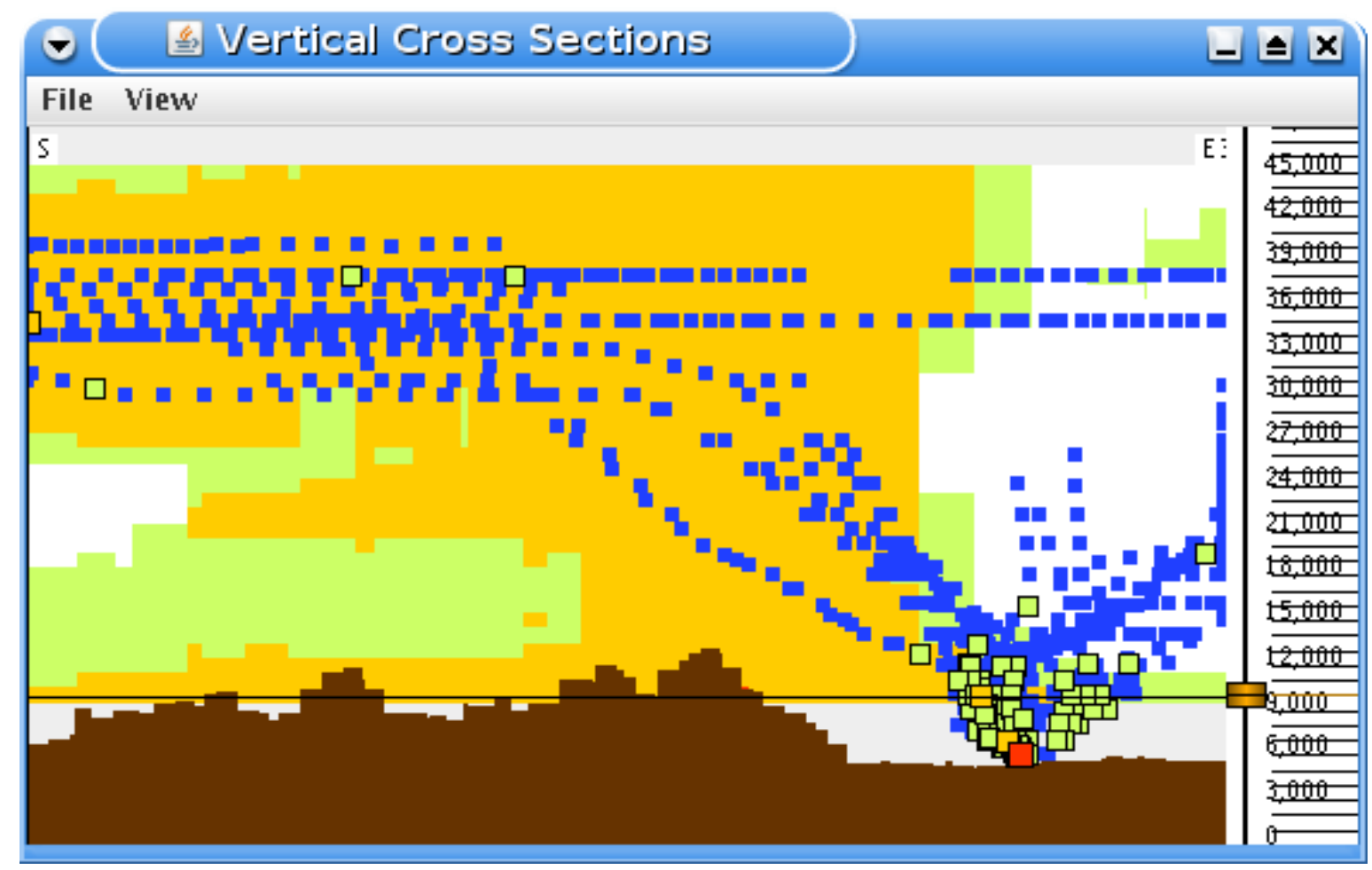

Figure 172. GTG vertical cross section looking Northwest.

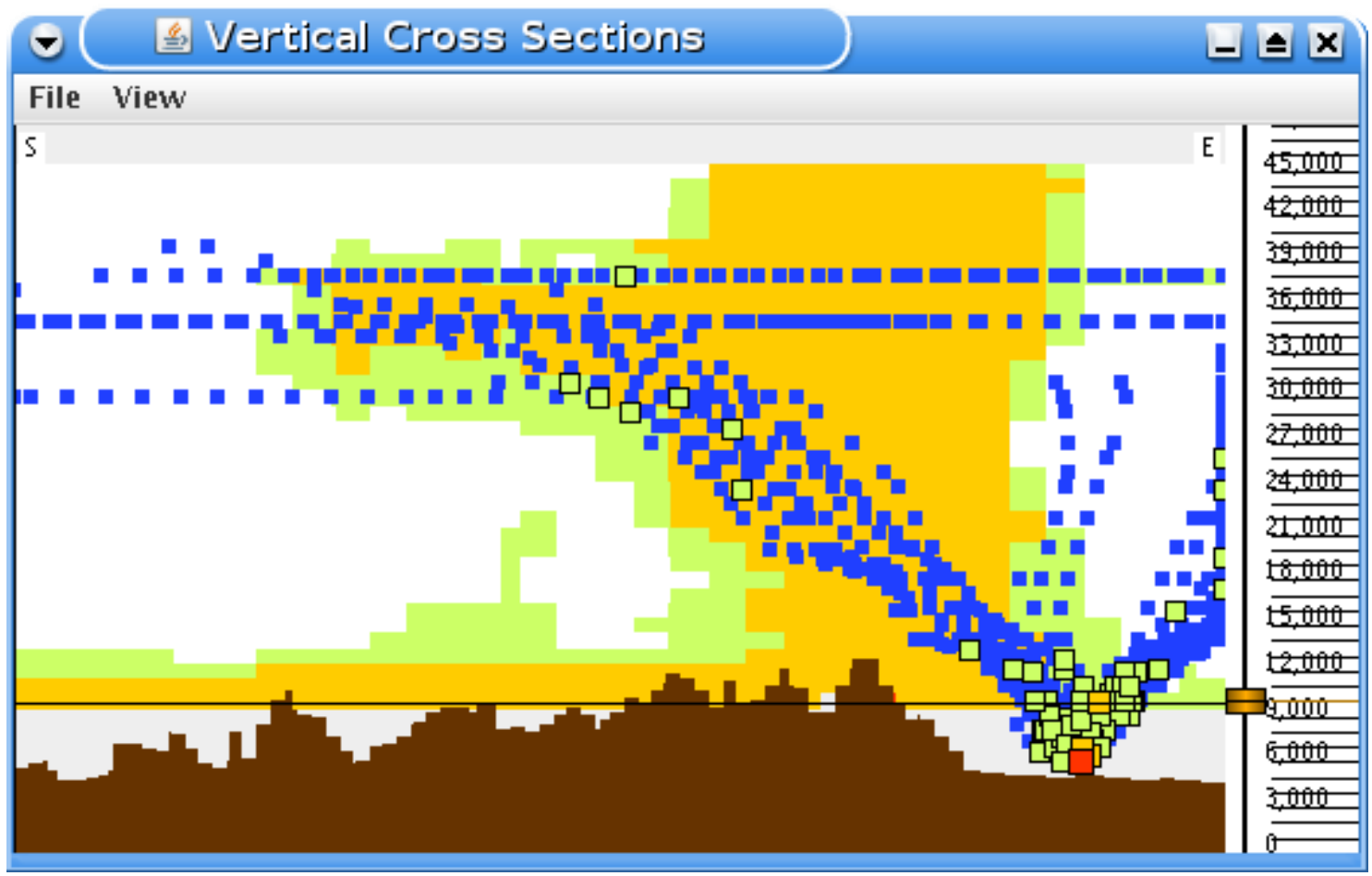

Figure 173. GTG vertical cross section looking Southwest. 


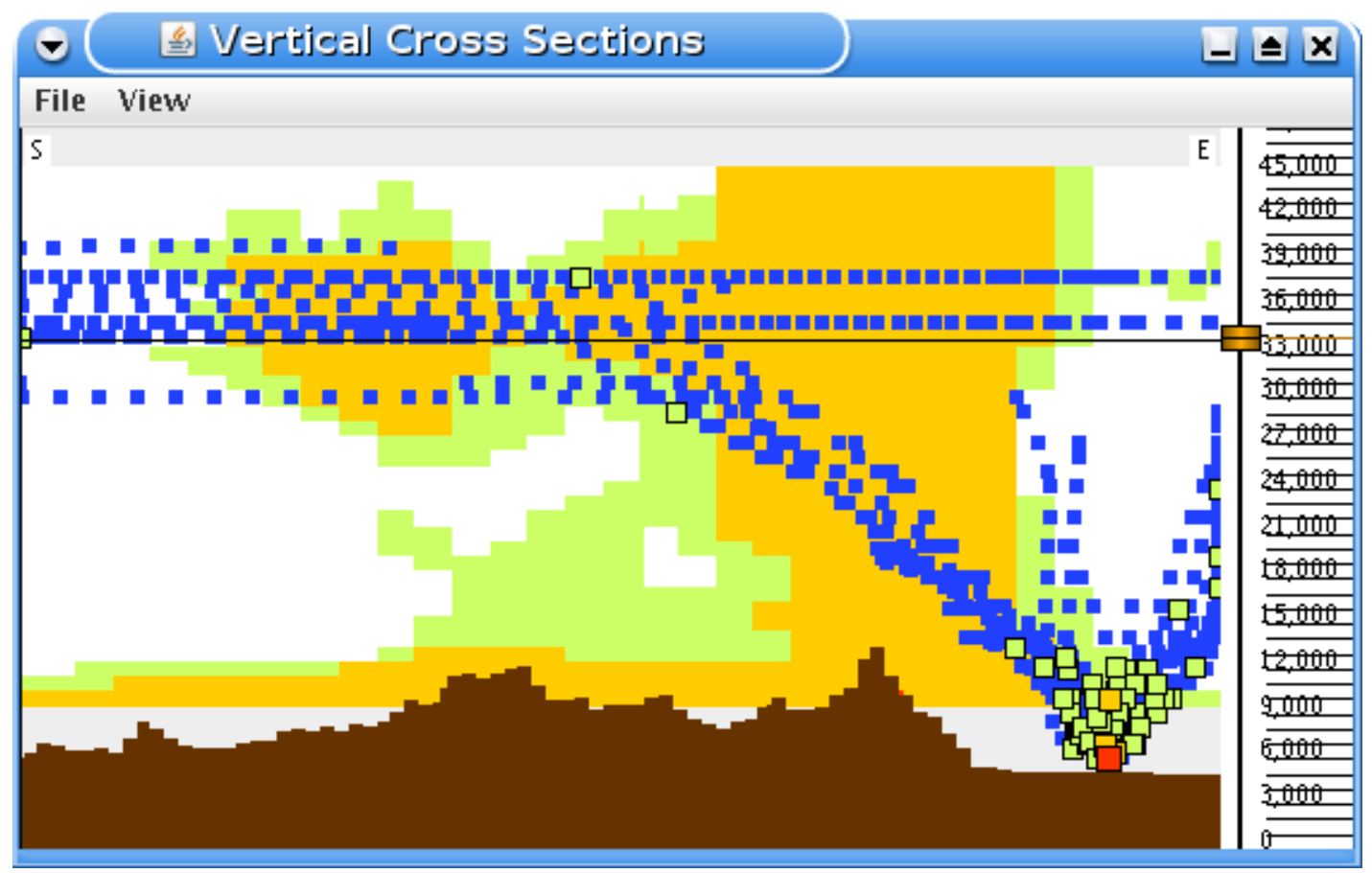

Figure 174. GTG vertical cross section looking West. 


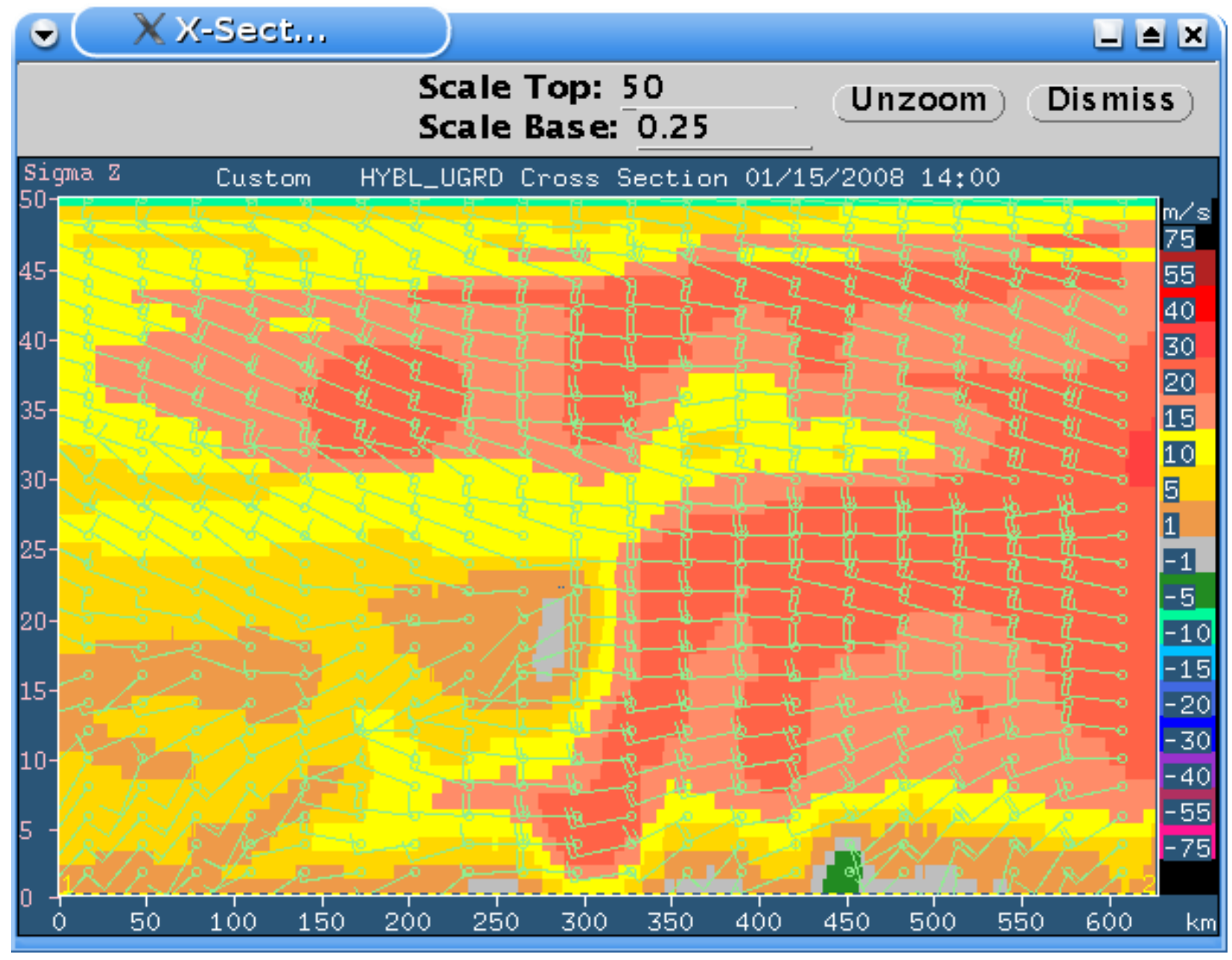

Figure 175. GTG vertical cross section with East-West wind barbs. 


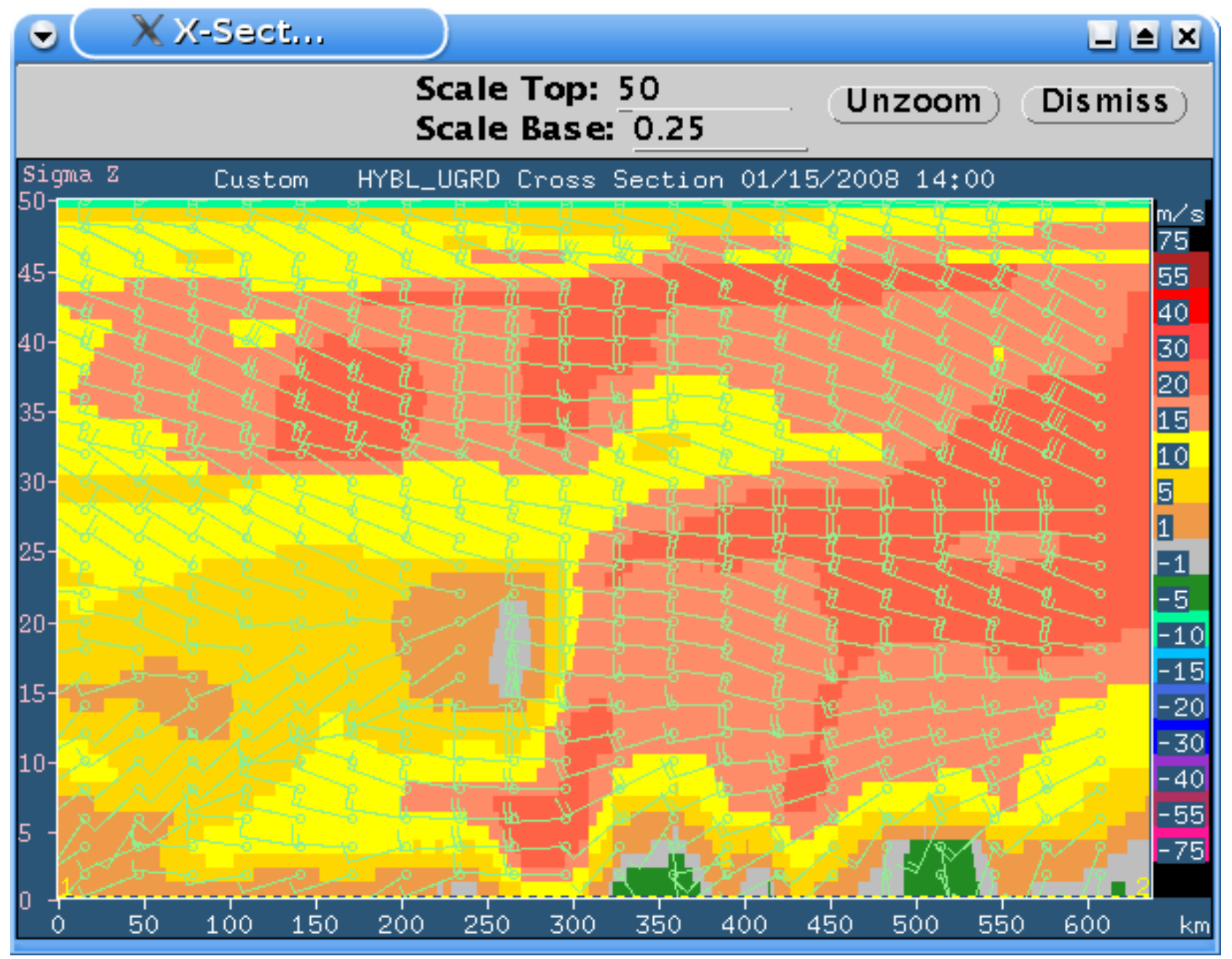

Figure 176. GTG vertical cross section with North-South wind barbs. 


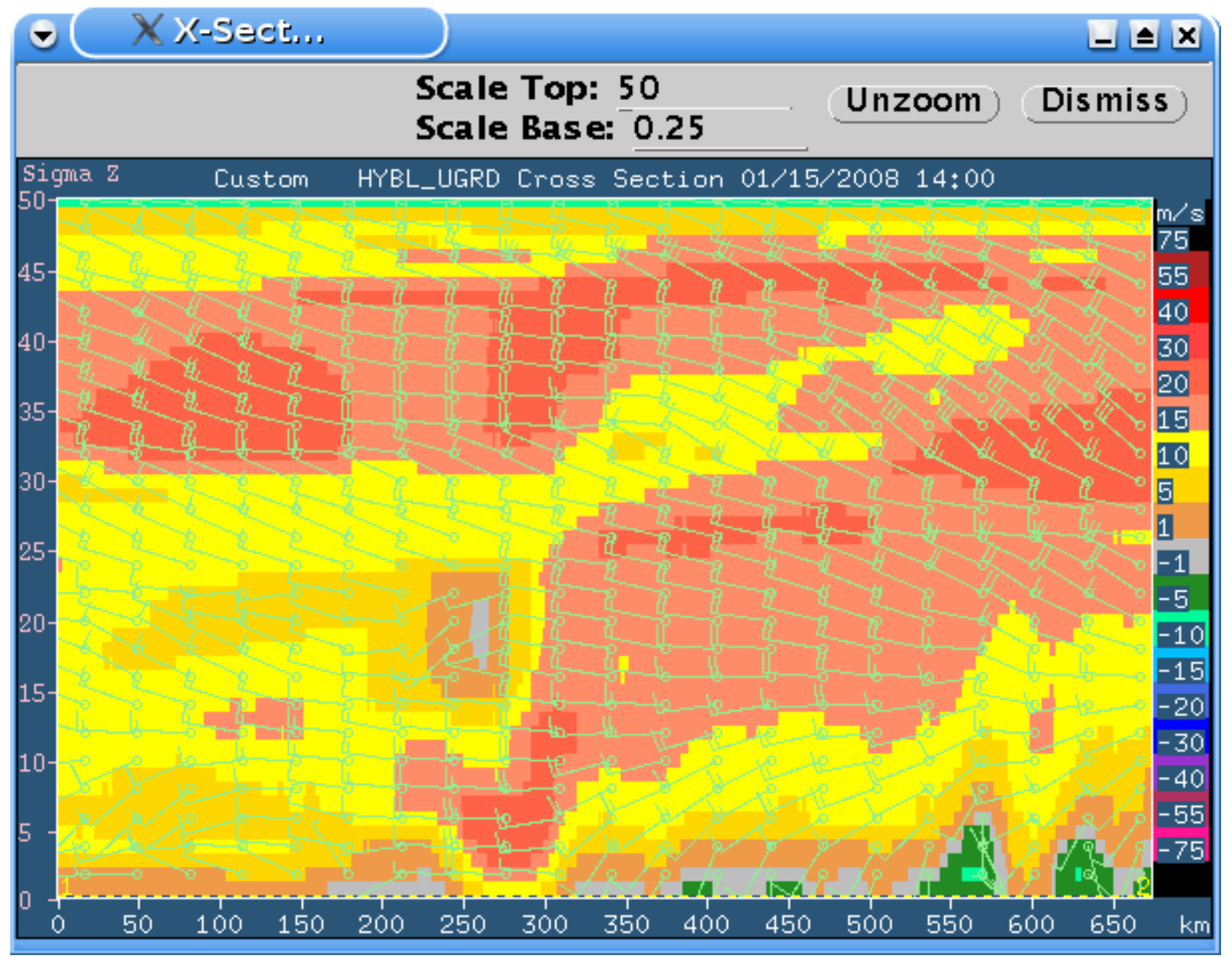

Figure 177. GTG vertical cross section with North-South wind barbs. 


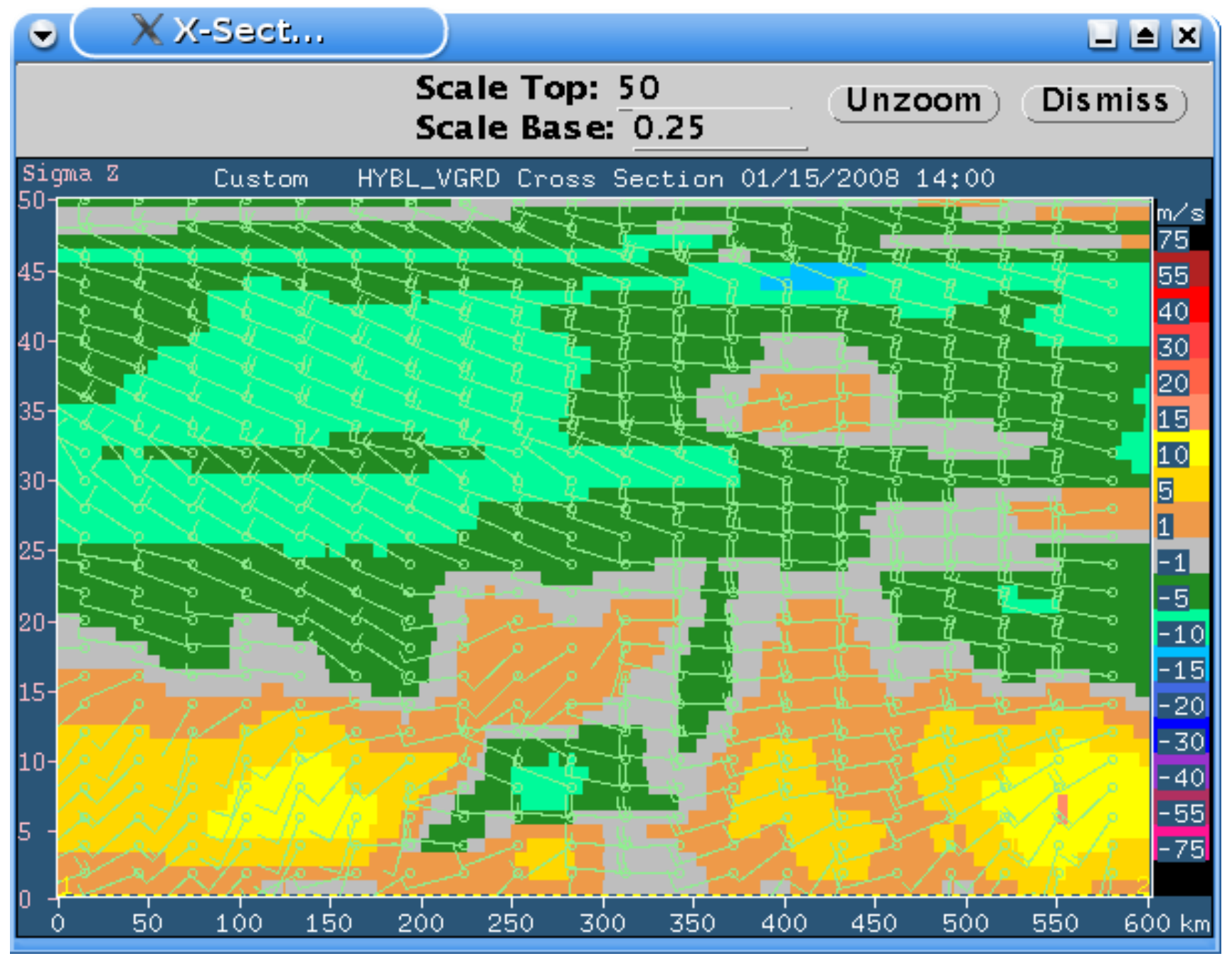

Figure 178. GTG vertical cross section with East-West wind barbs. 


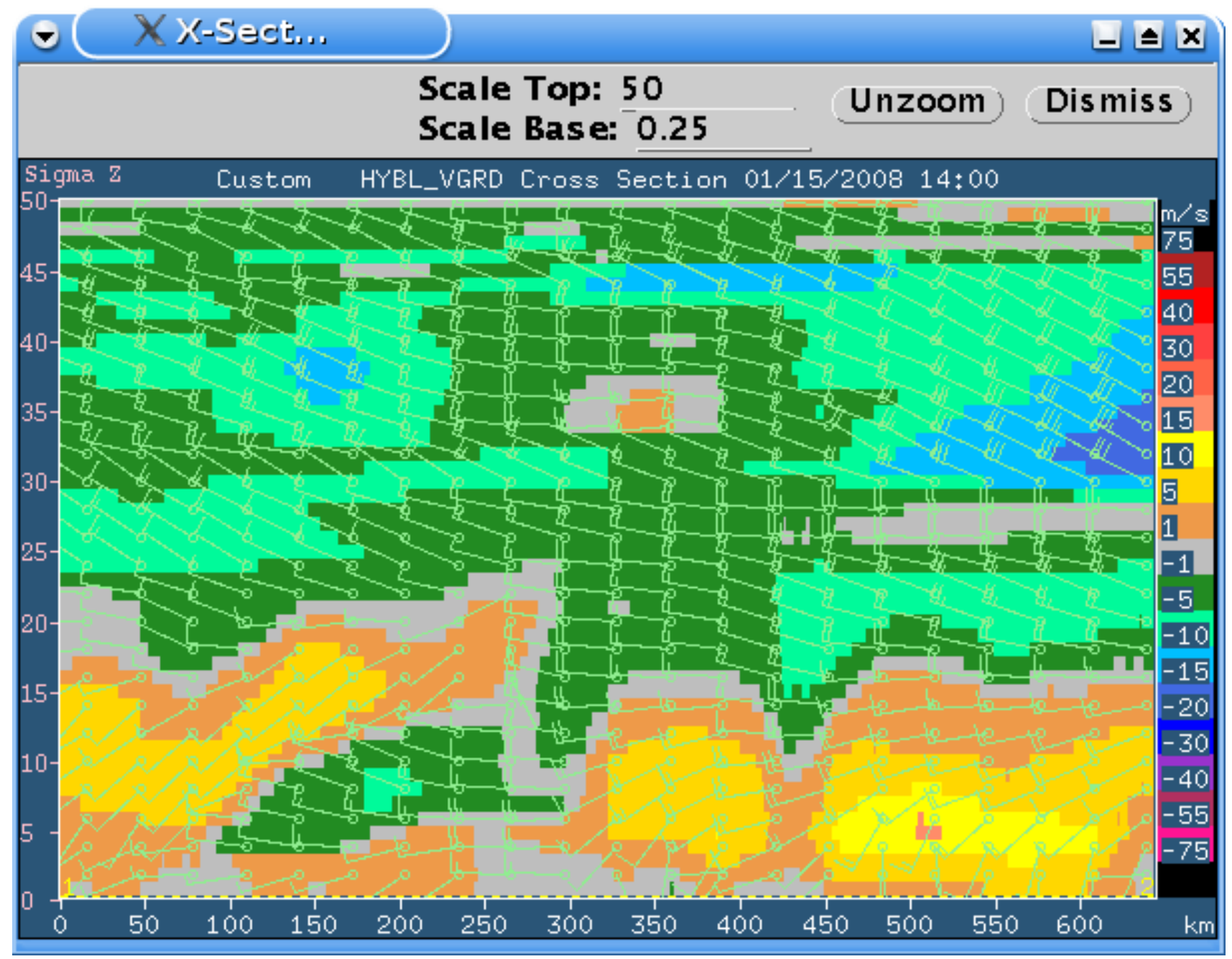

Figure 179. GTG vertical cross section of North-South wind barbs. 


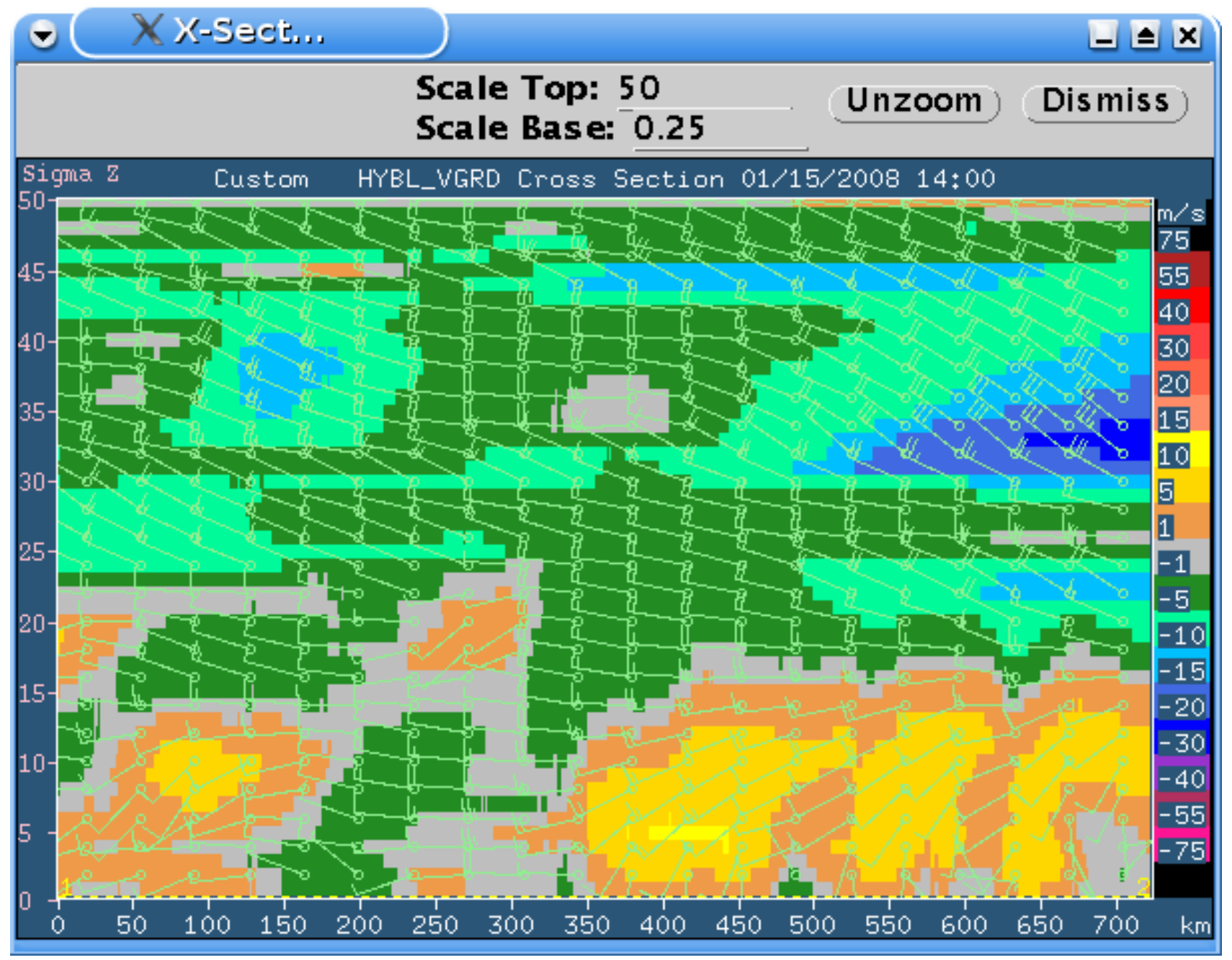

Figure 180. GTG vertical cross section of North-South wind barbs. 


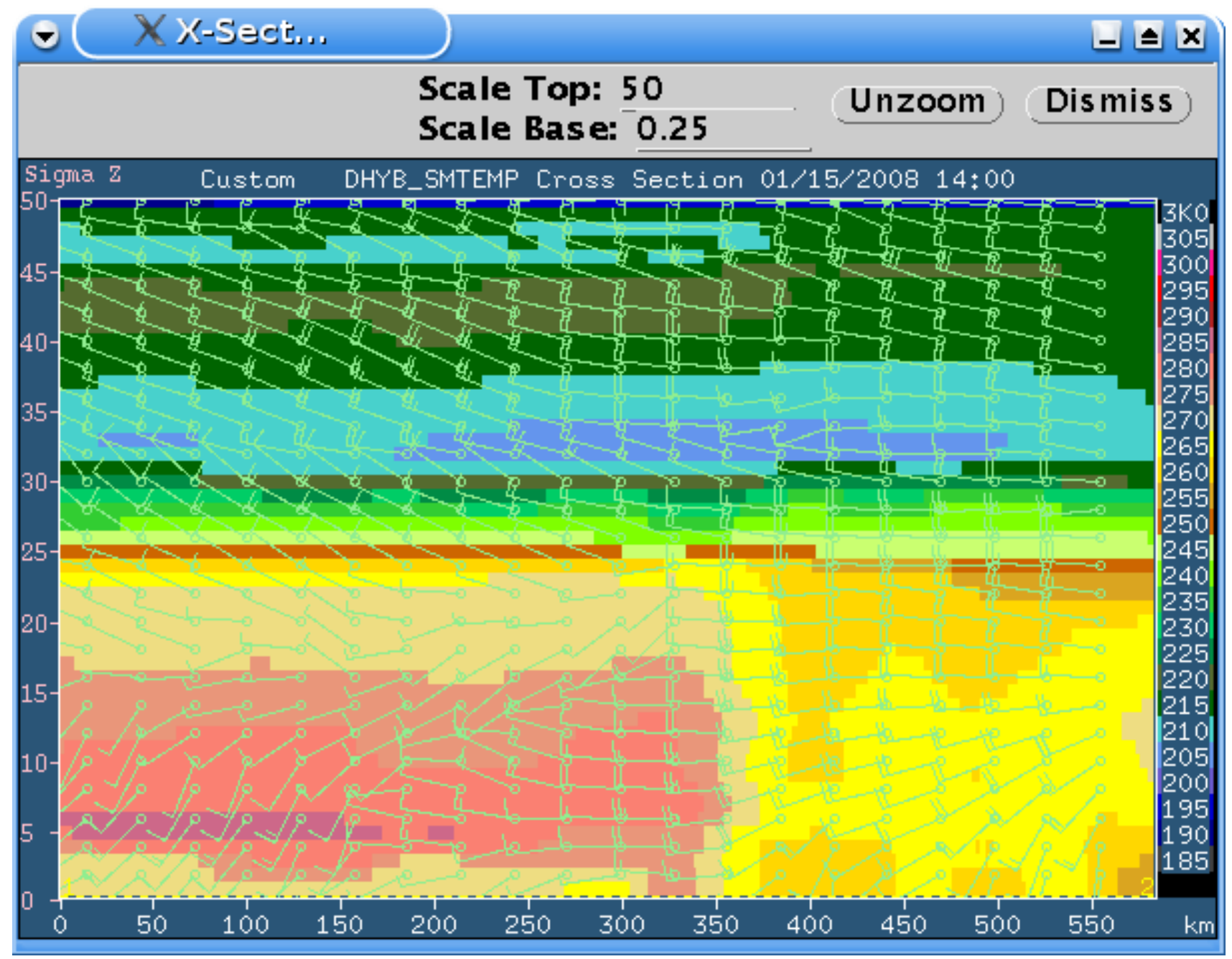

Figure 181. RUC temperature cross section looking Northwest. 


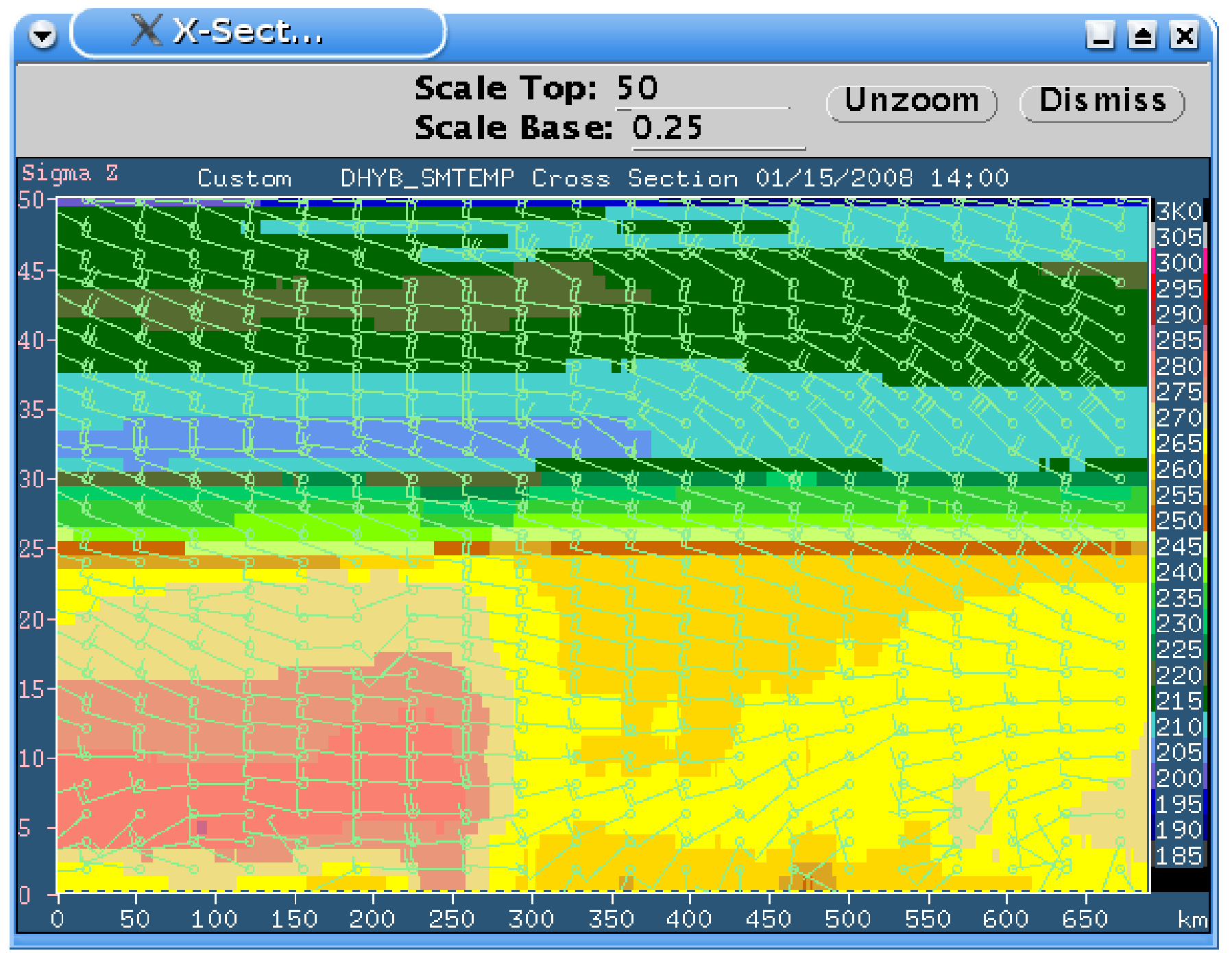

Figure 182. GTG vertical cross section of RUC temperature looking Southwest.

\subsubsection{January 17,2008}

Figure 183 shows the radiance correlation coefficient for a time period starting at 13:21Z on Jan 17,2008 . It can be seen that, in contrast to the data on the $15^{\text {th }}$, there is some positive correlation from $760-800 \mathrm{~cm}^{-1}$. Figure 184 shows the raw (upper) and wavelet-filtered radiance correlation coefficient for the same time period, but over the entire measured wavenumber range. Similar patterns to the data on the $15^{\text {th }}$ can be seen. There is a clear correlation structure. Figure 185 and Figure 186 show the correlation and power spectra for the same wavenumber range as in Figure 183, but at a time period approximately 38 minutes later. It can be seen that the data this latter time does not show any turbulence signatures.

Figure 187 through Figure 205 are a set of images which parallel those presented above for the Jan 15, 2008 case. Similar patterns of reported and forecast turbulence can be seen on this day. Figure 202 shows the vertical cross section v-component of the wind, overlaid with the horizontal wind barbs. The wind pattern on this day is quite different than that seen on the $15^{\text {th }}$. 
The winds have shifted from Westerly to Northerly, and the magnitudes are much larger on this day. This corresponds to a cold front moving into the area.
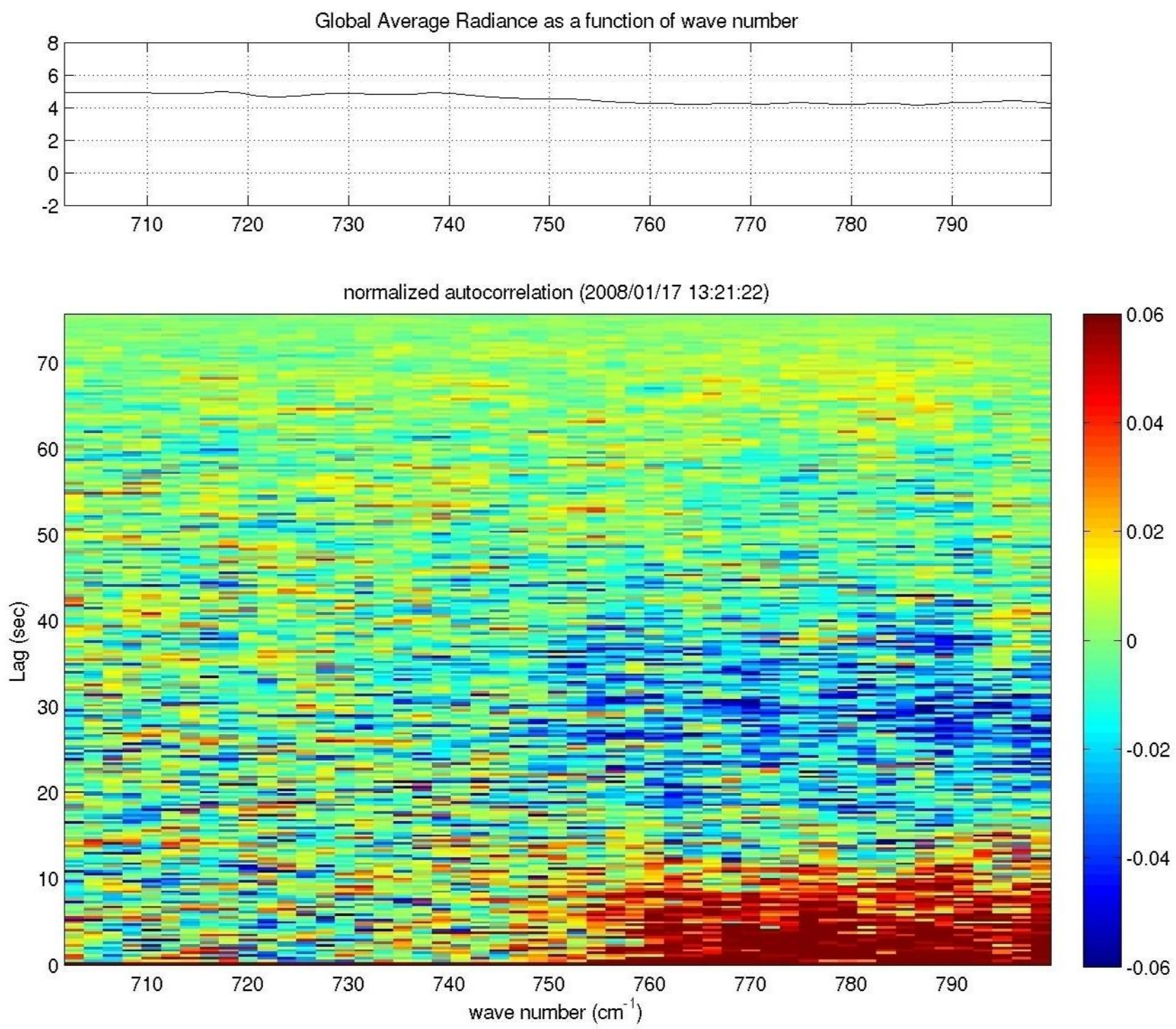

Figure 183. Radiance correlation coefficient showing positive correlation. 

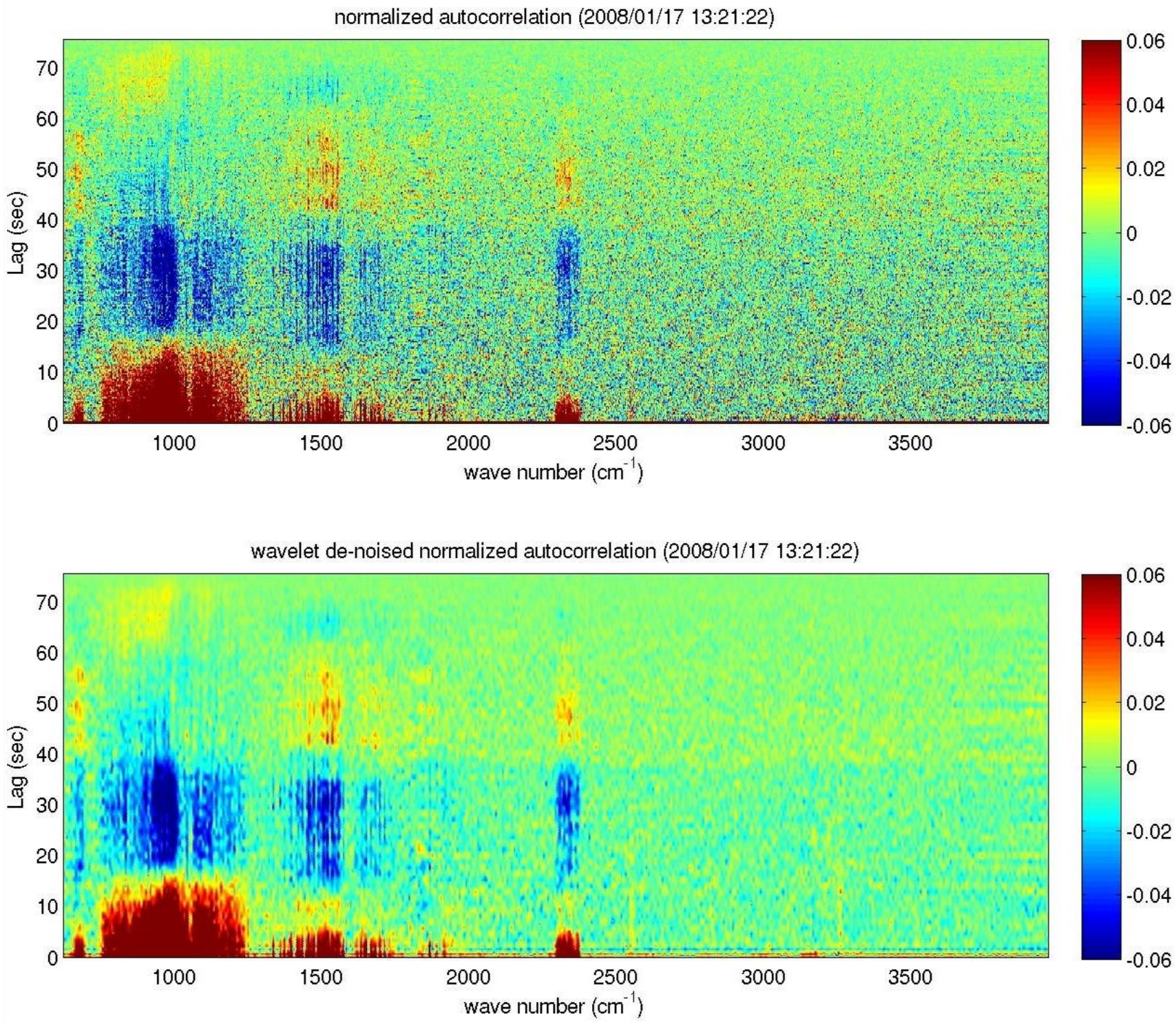

Figure 184. Raw (upper) and wavelet-filtered (lower) radiance correlation coefficient. 

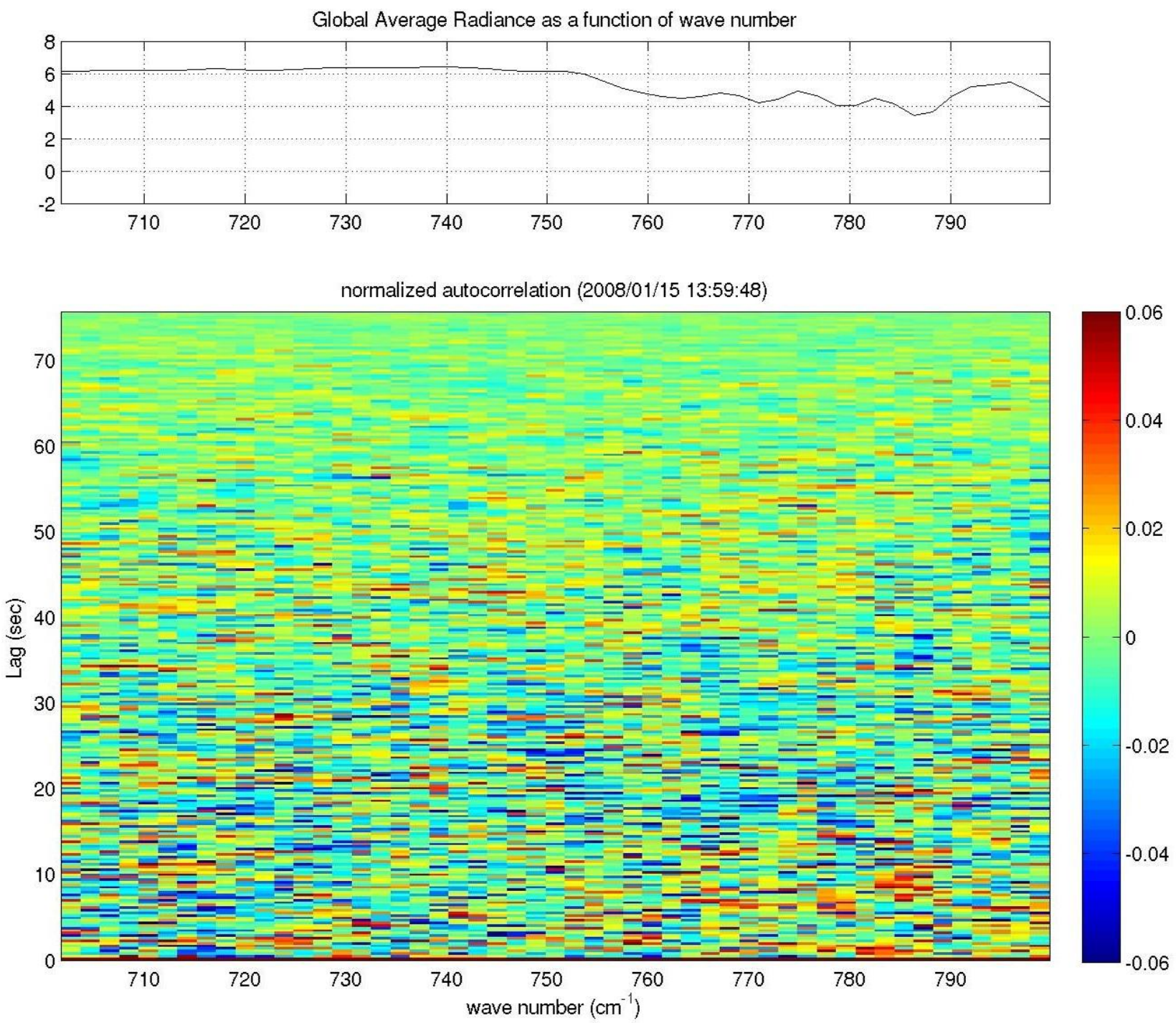

Figure 185. Same as Figure 183, but for a time segment starting at 13:59Z. 

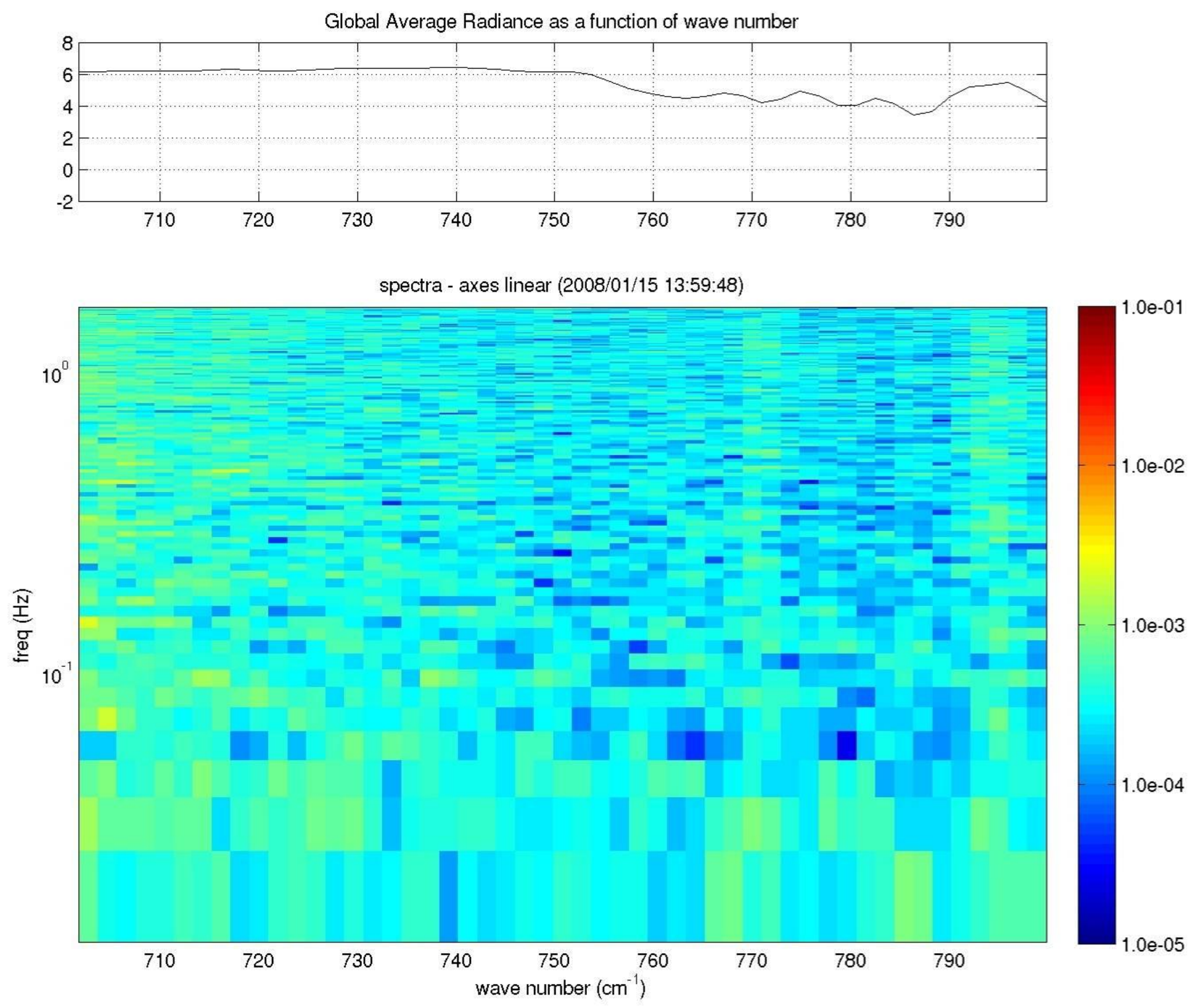

Figure 186. Power spectra for the data shown in Figure 185. 


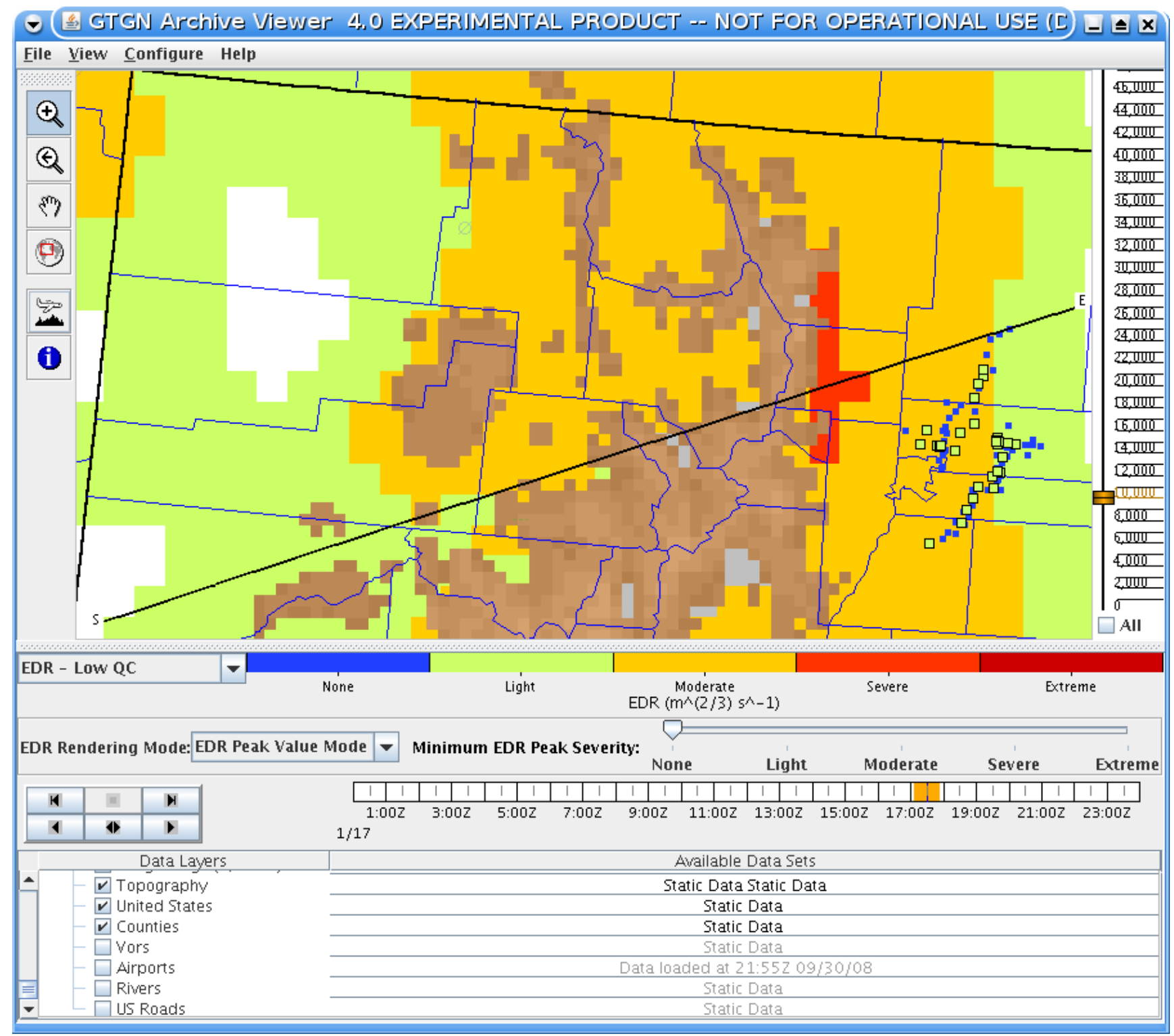

Figure 187. Northeast cross section line at $\mathbf{1 0 0 0 0}$ feet altitude. 


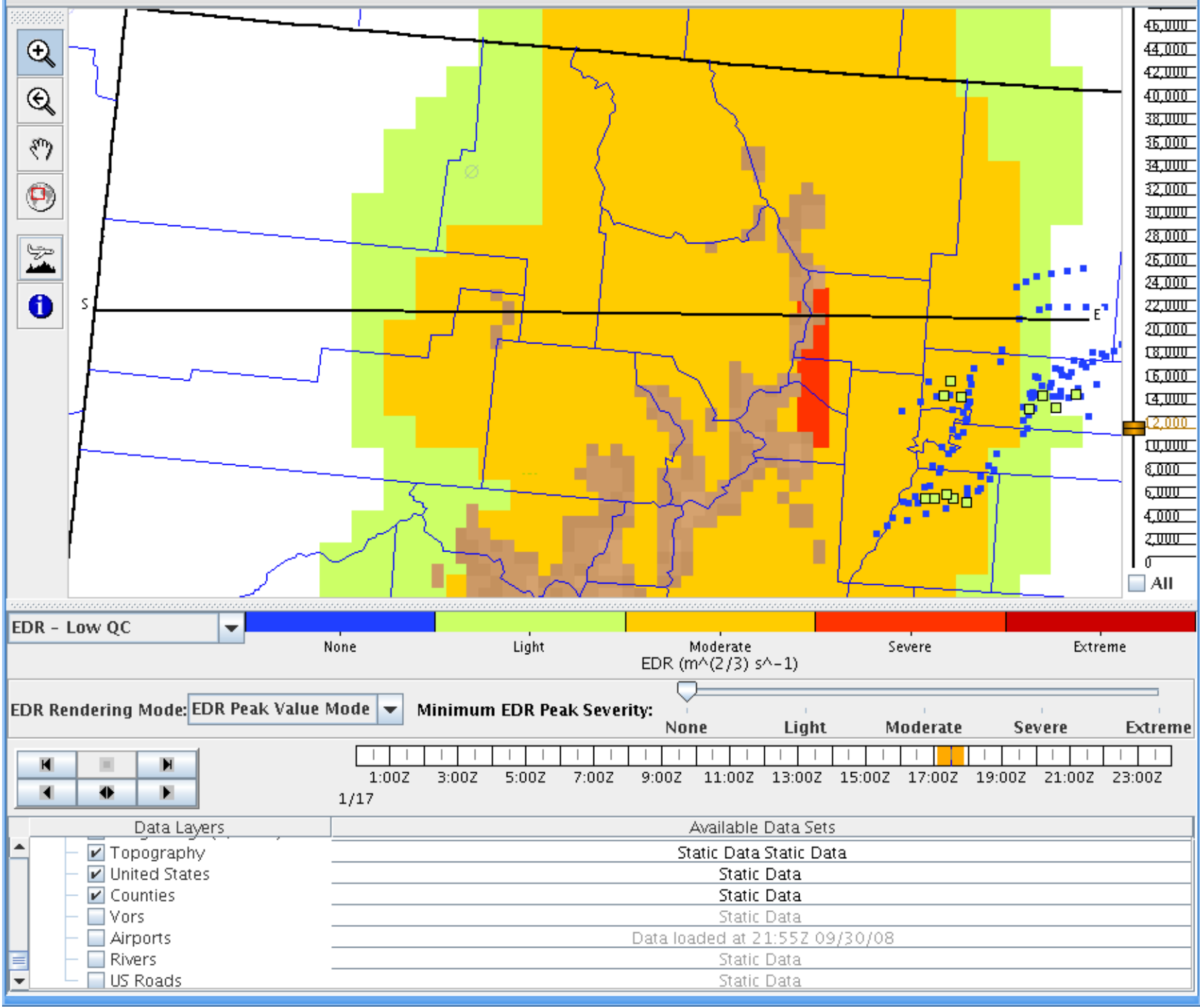

Figure 188. West to East cross section line at 12000 feet. 


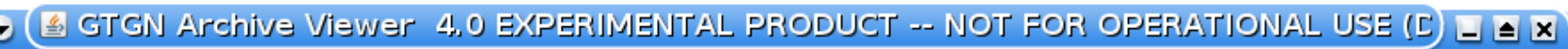

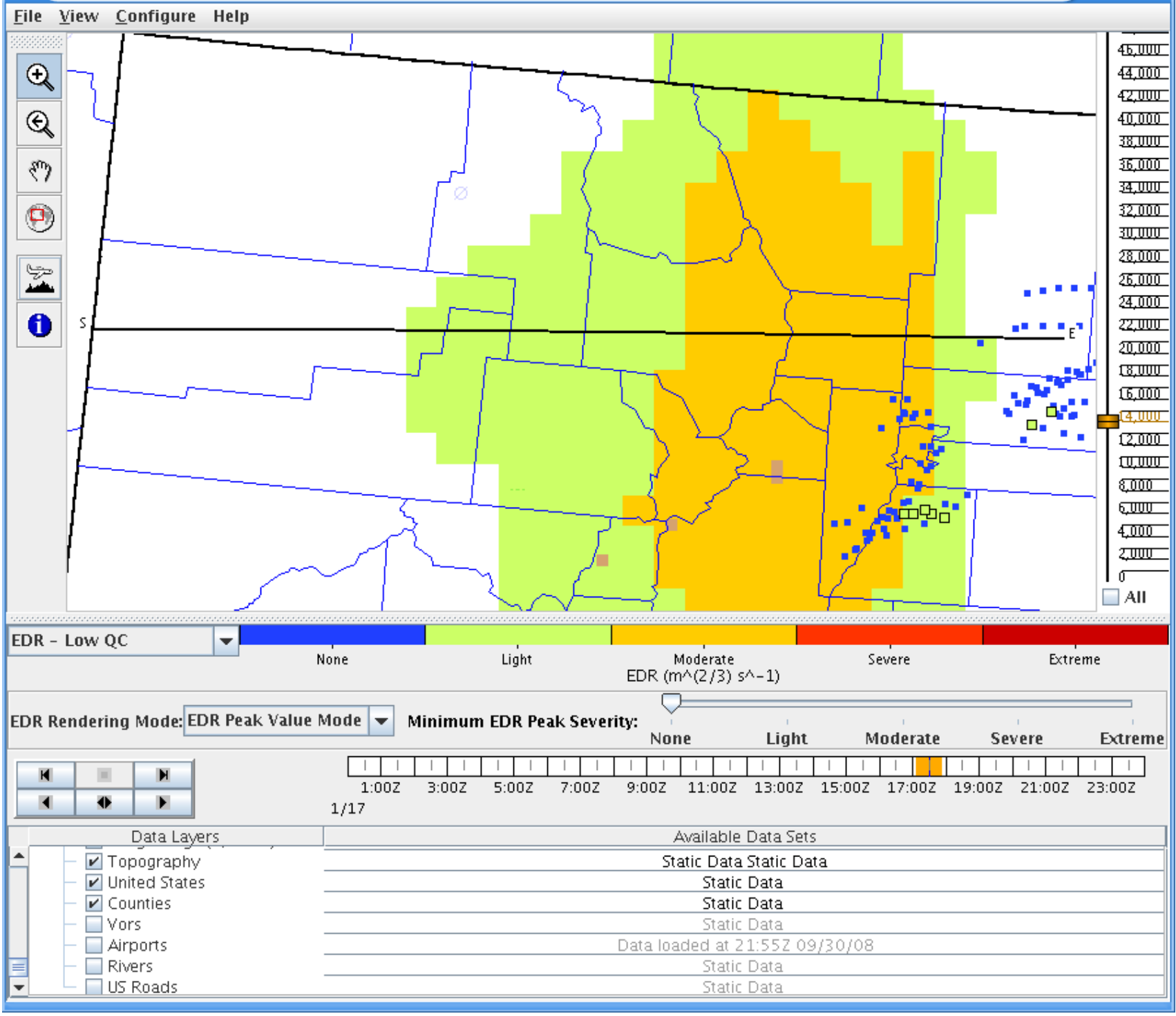

Figure 189. West to East cross section line at 14000 feet. 


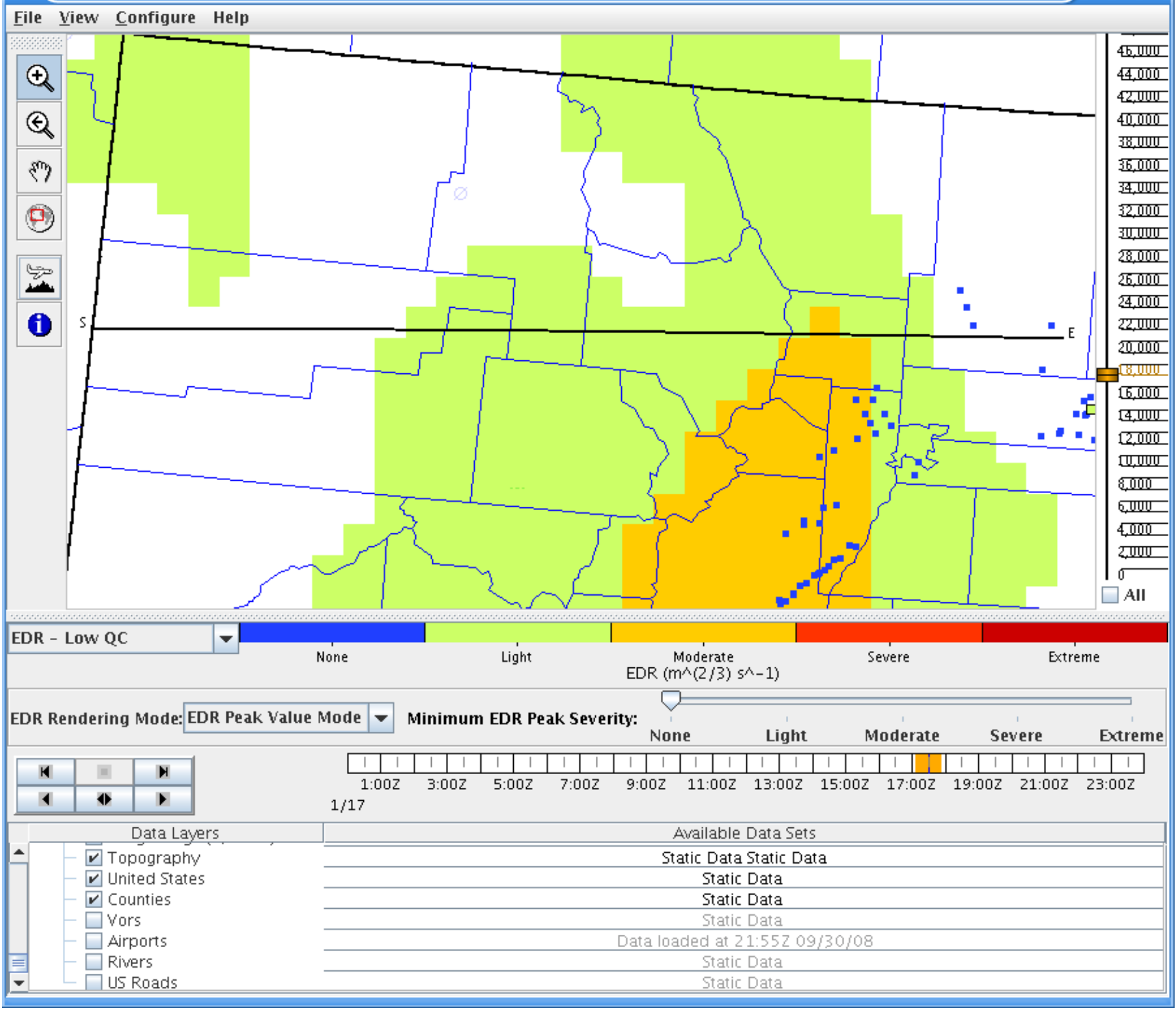

Figure 190. West to East cross section line at 18000 feet. 


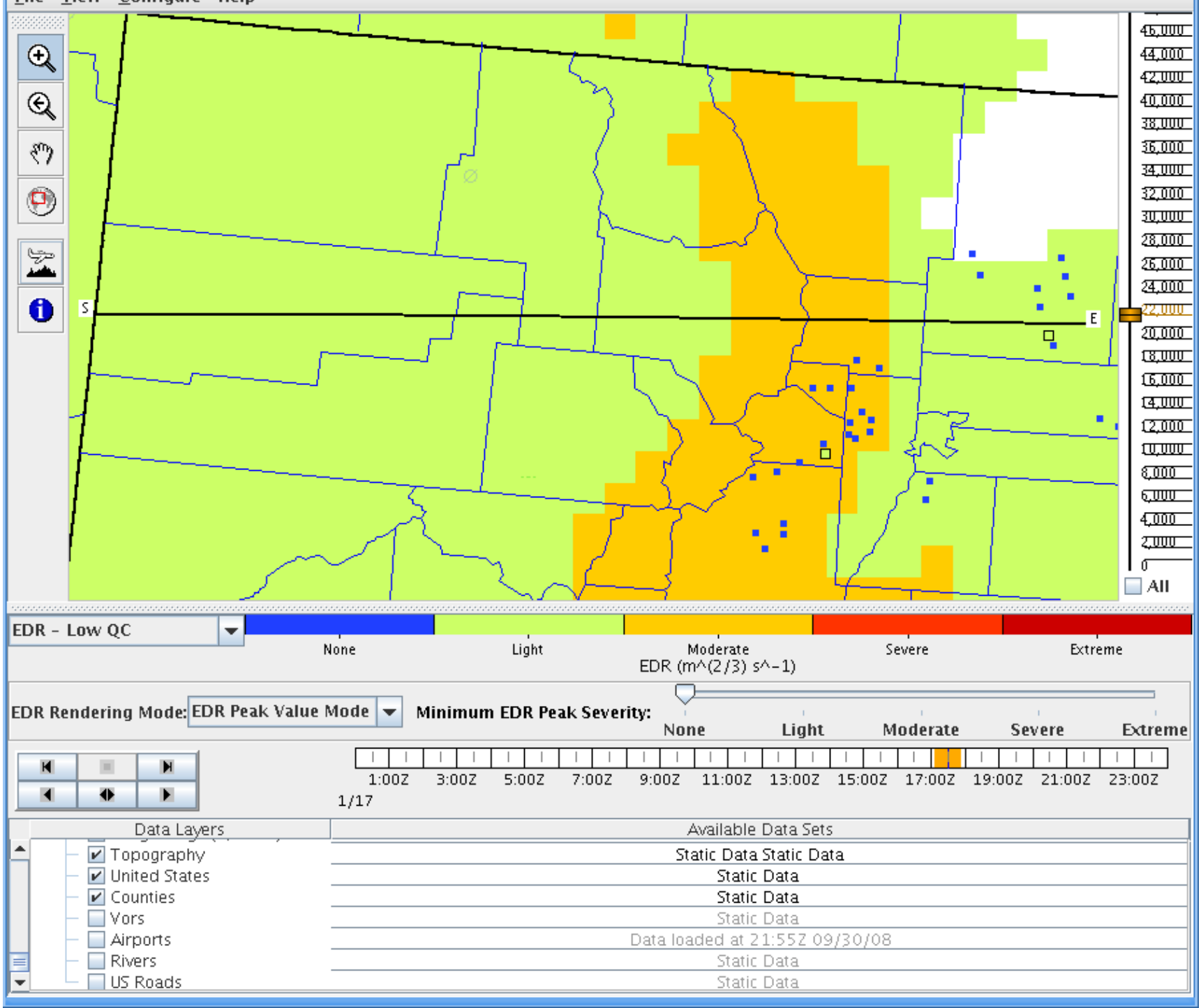

Figure 191. West to East cross section line at 22000 feet. 


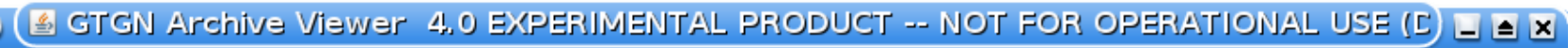

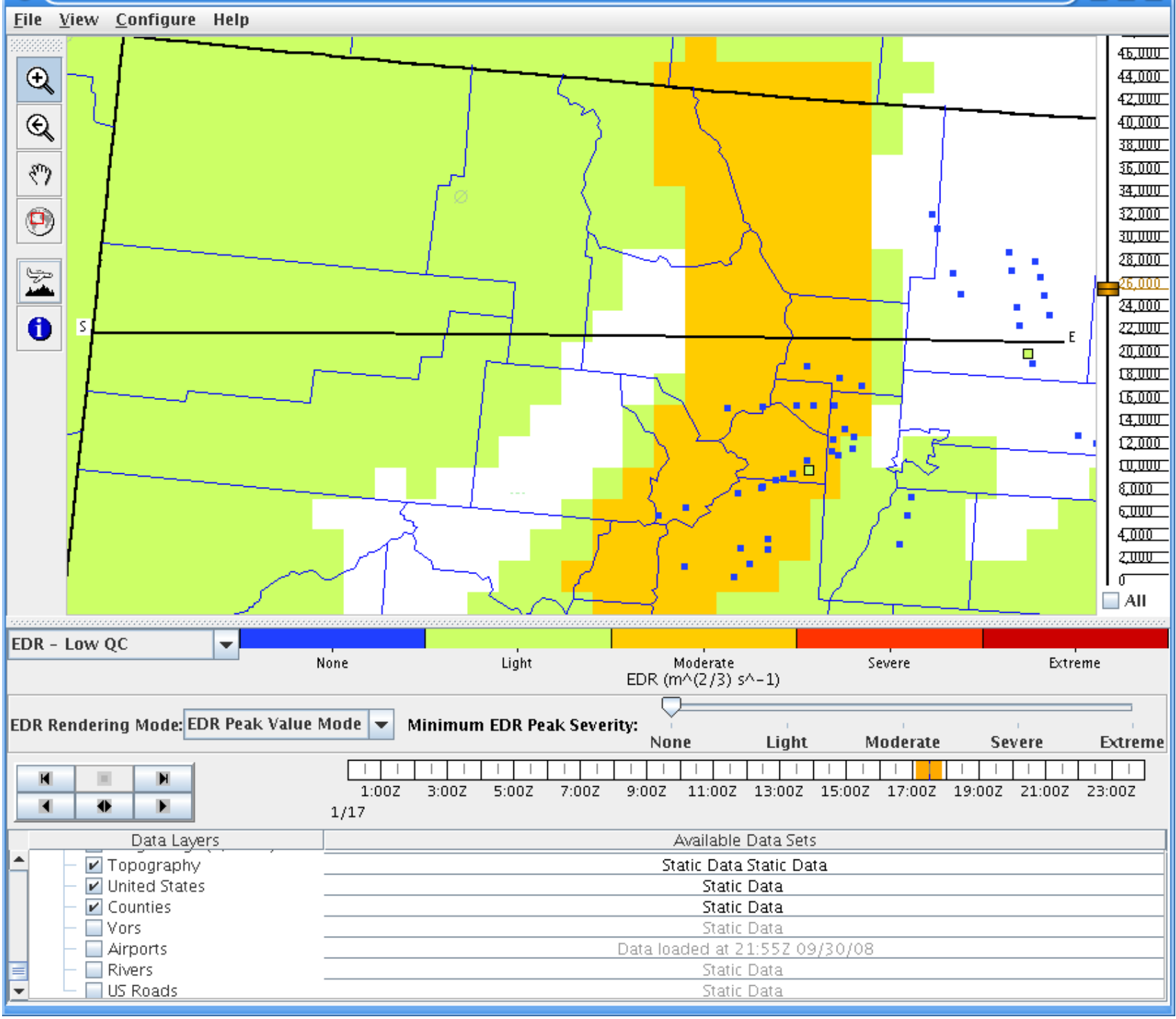

Figure 192. West to East cross section line at 26000 feet. 


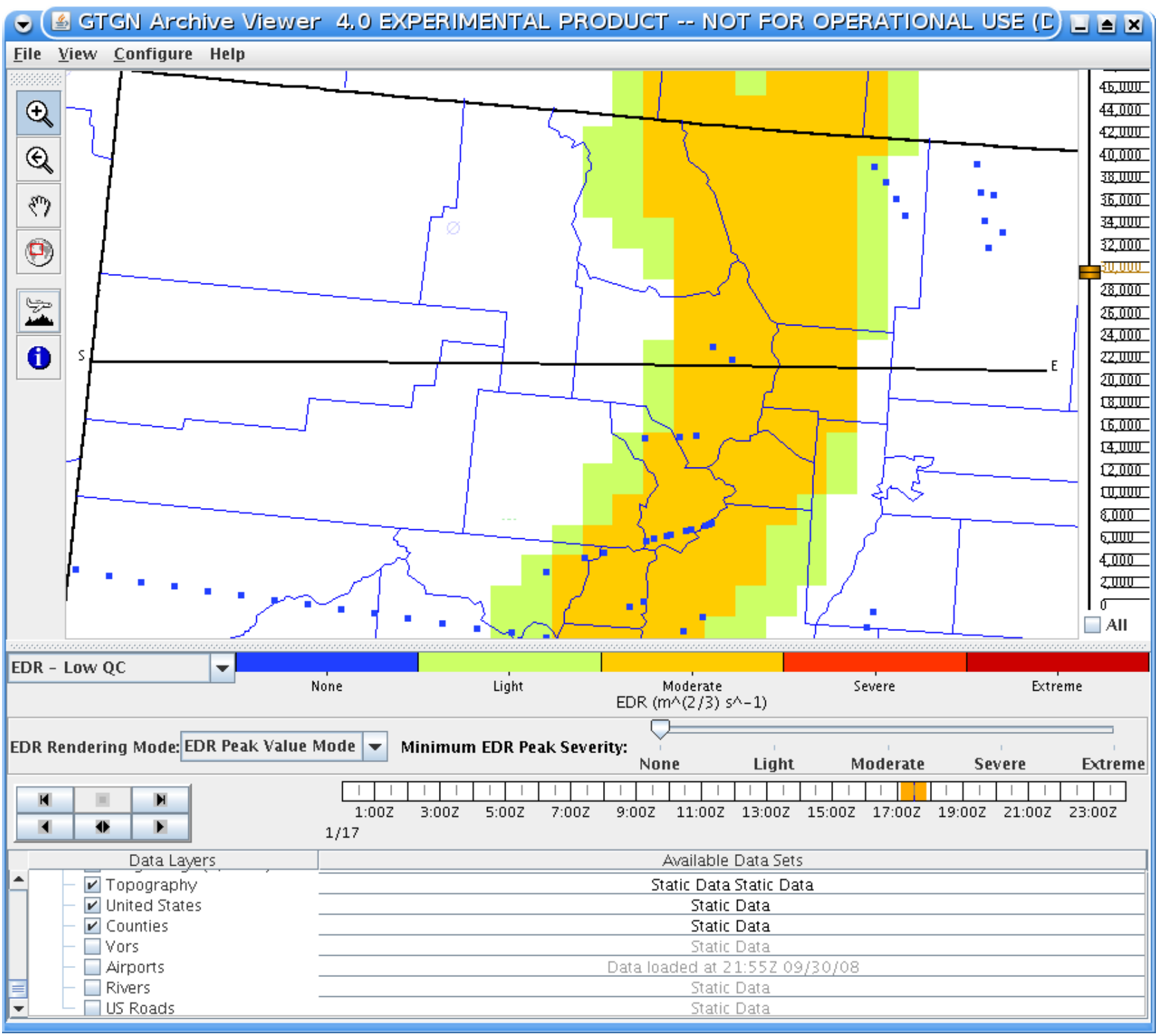

Figure 193. West to East cross section line at 30000 feet. 
$\checkmark$ GTGN Archive Viewer 4, EXPERIMENTAL PRODUCT - NOT FOR OPERATIONAL USE (L) - $-\mathbf{E}$

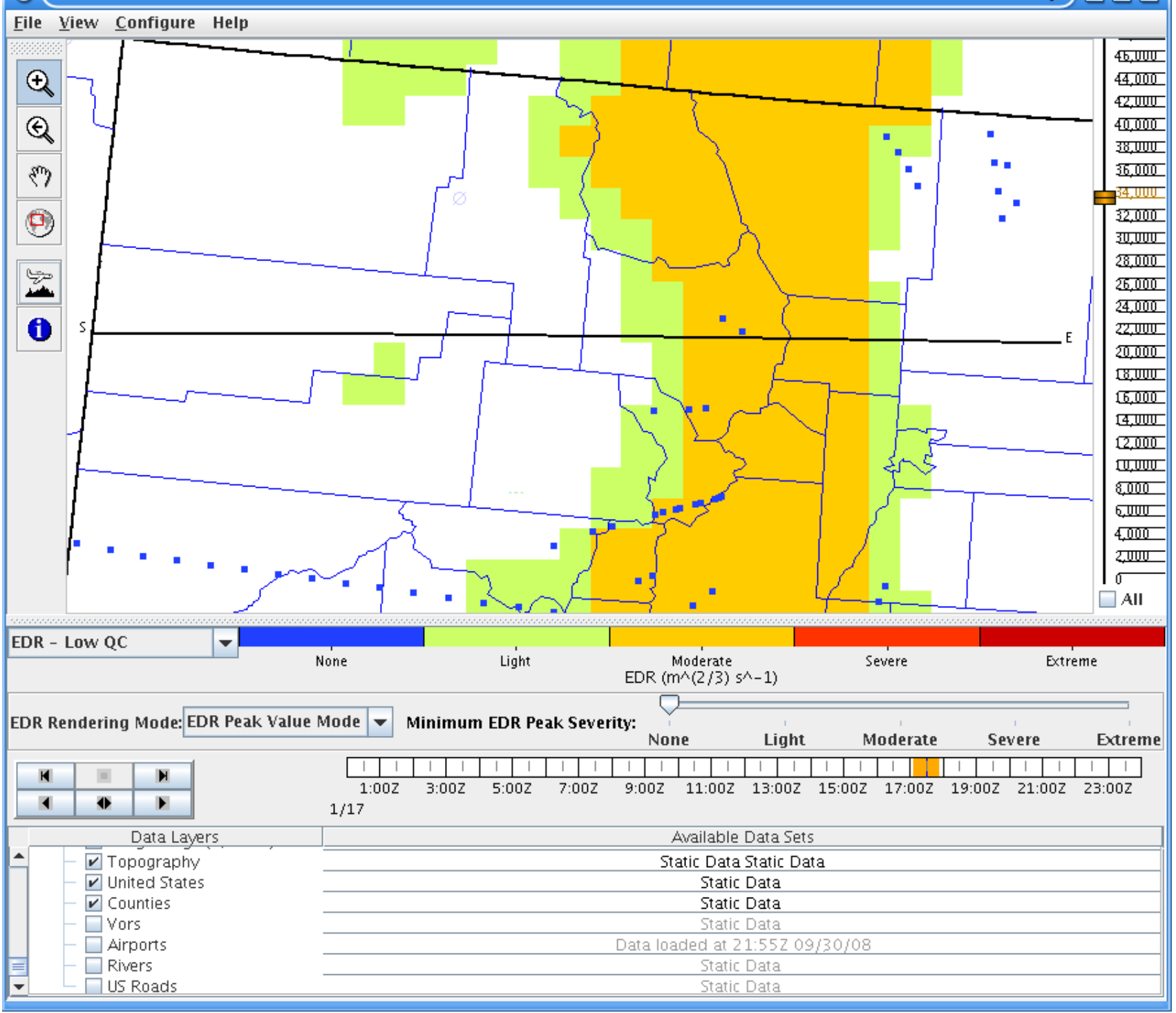

Figure 194. West to East cross section line at 34000 feet. 


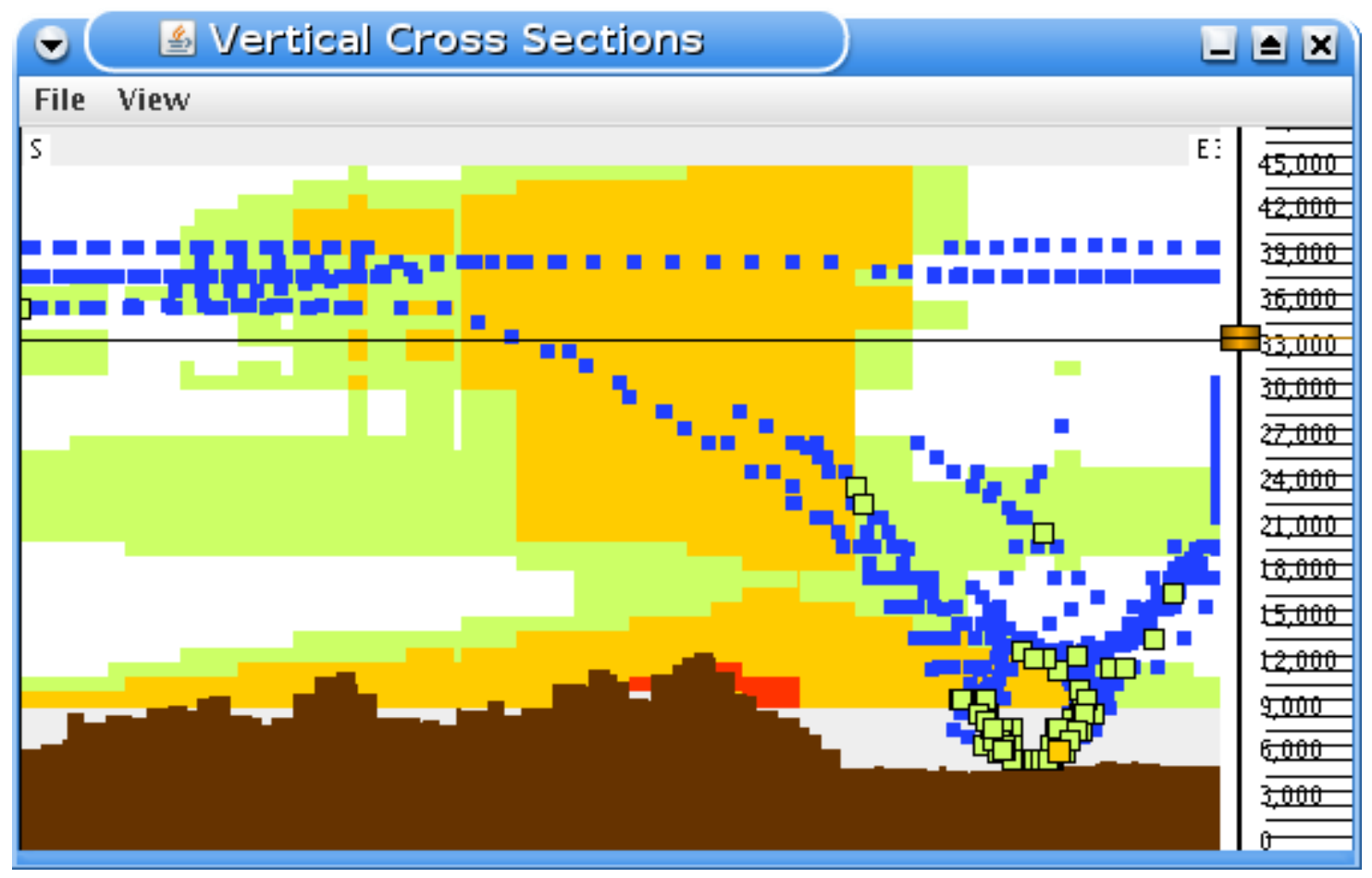

Figure 195. Vertical cross section looking north west on January 17th, 2008.

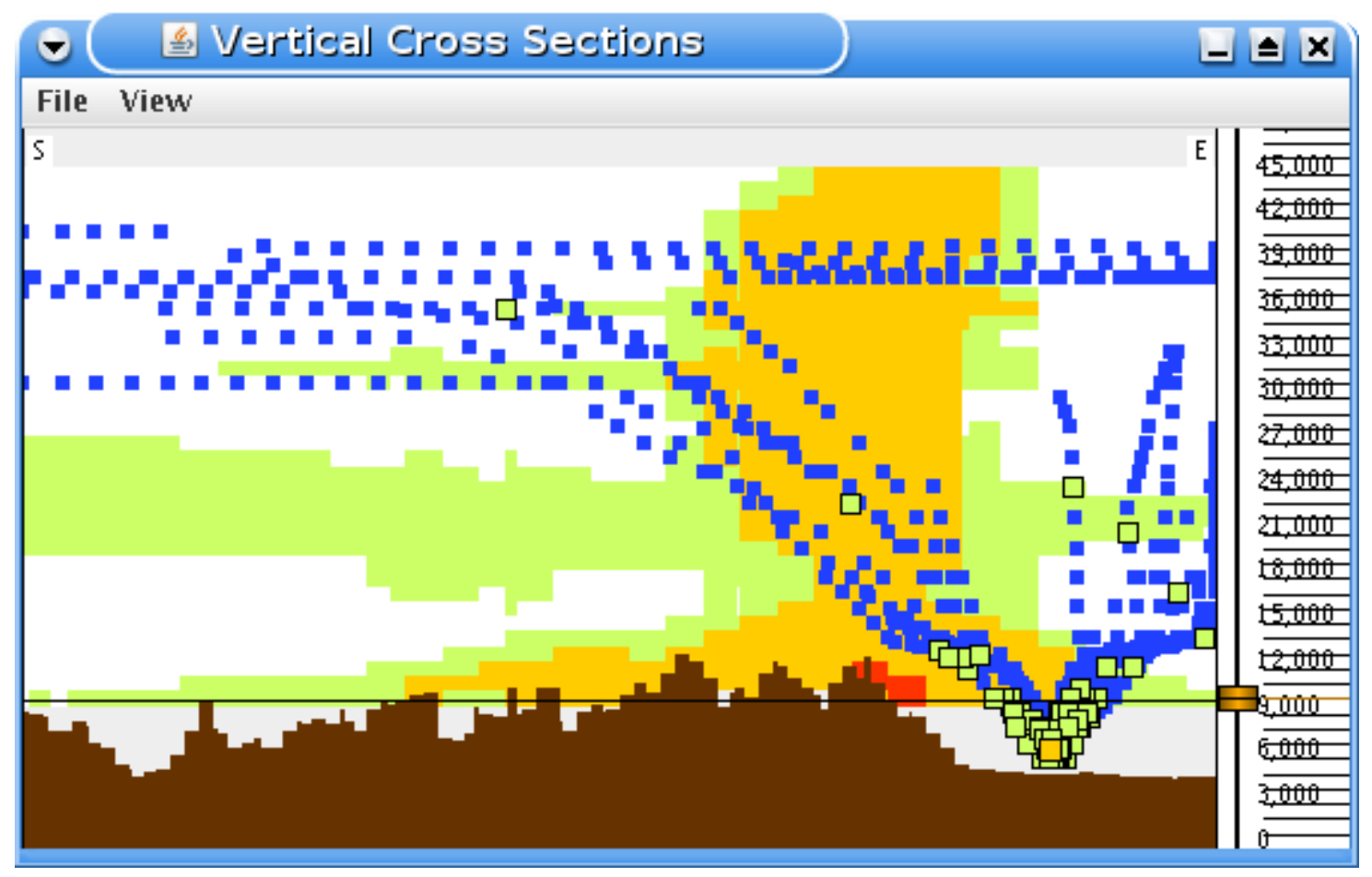

Figure 196. Vertical cross section looking Southwest. 


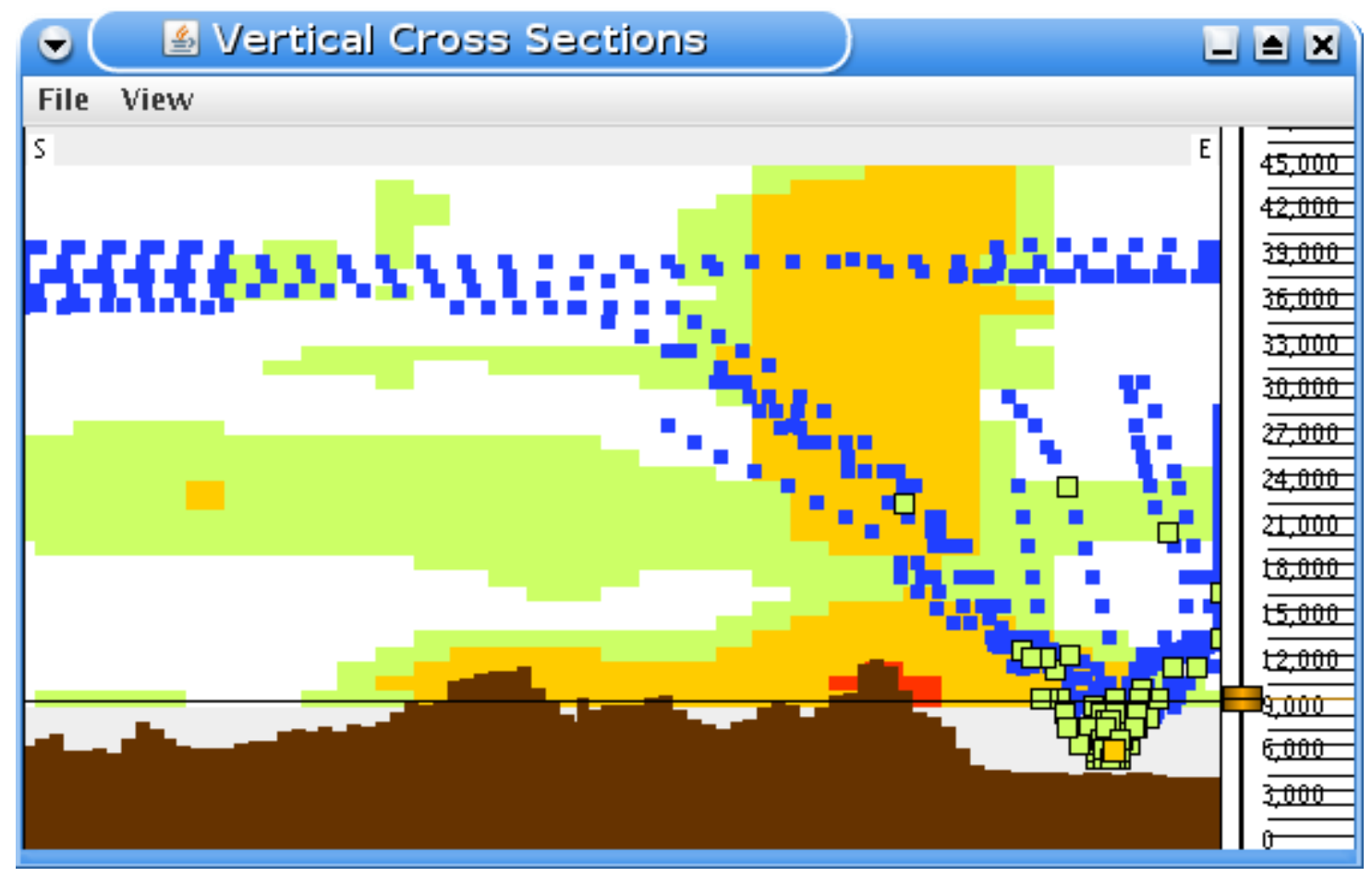

Figure 197. Vertical cross section looking West.

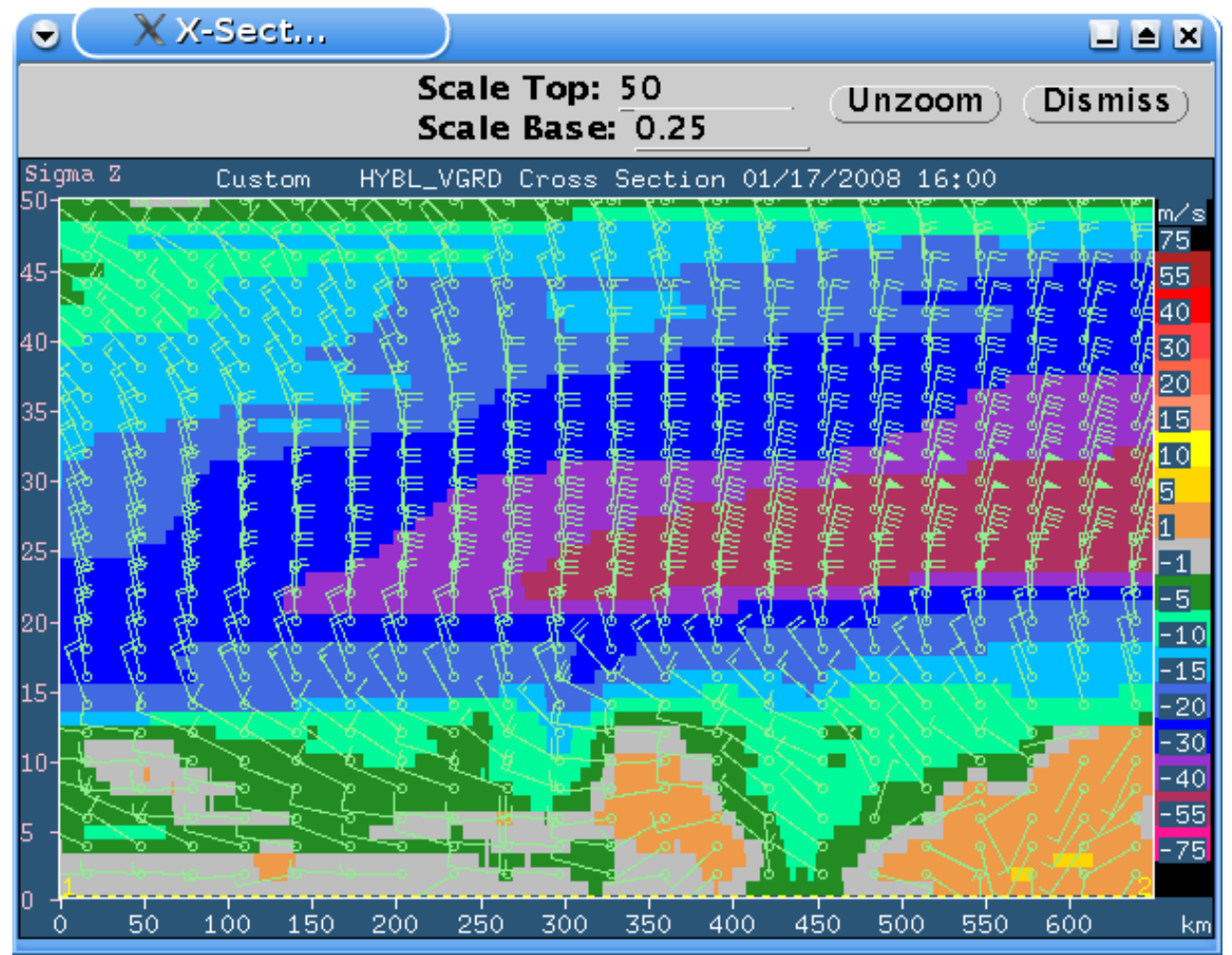

Figure 198. Vertical cross section of wind looking West barbs. 


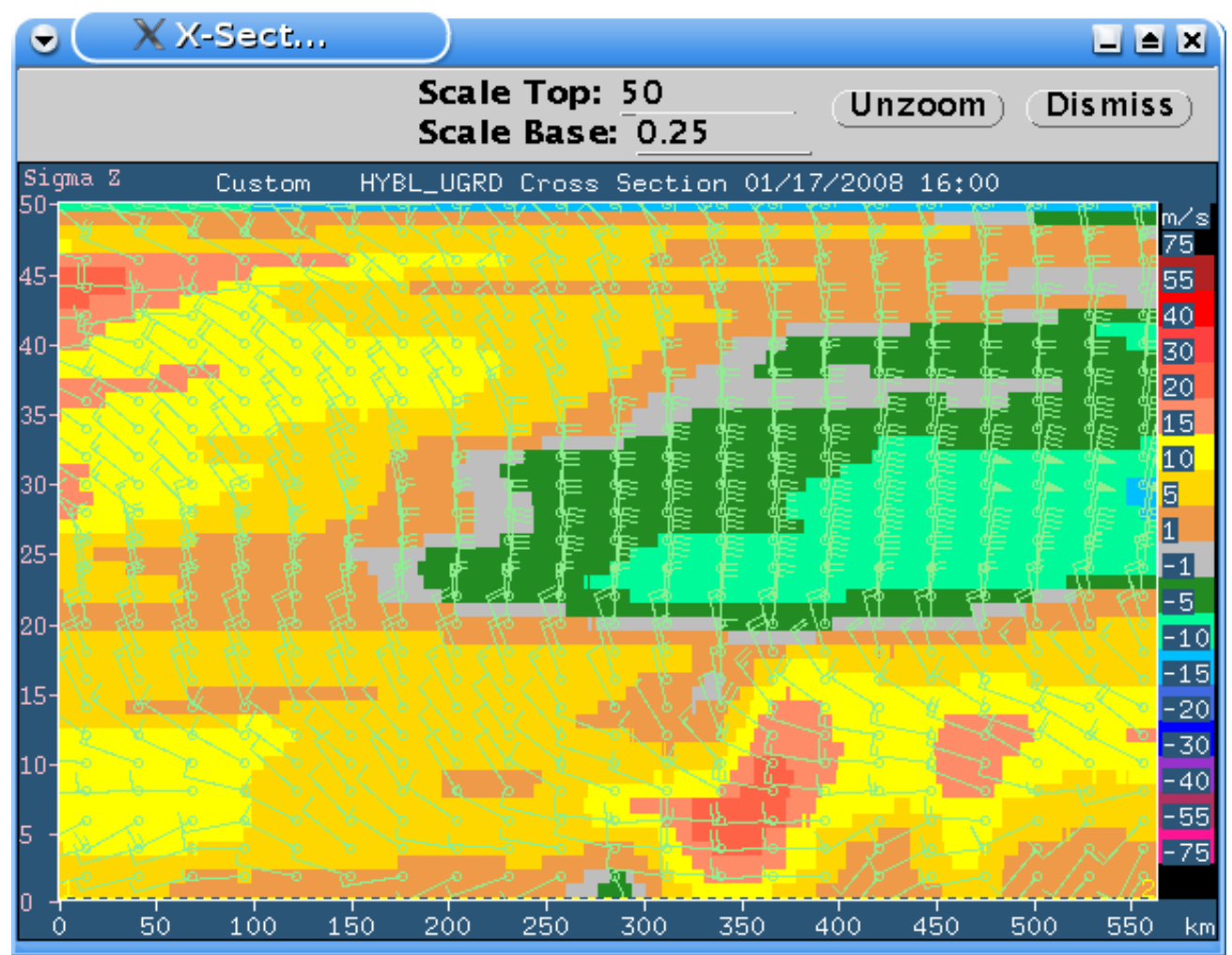

Figure 199.GTG vertical cross section with East-West wind barbs.

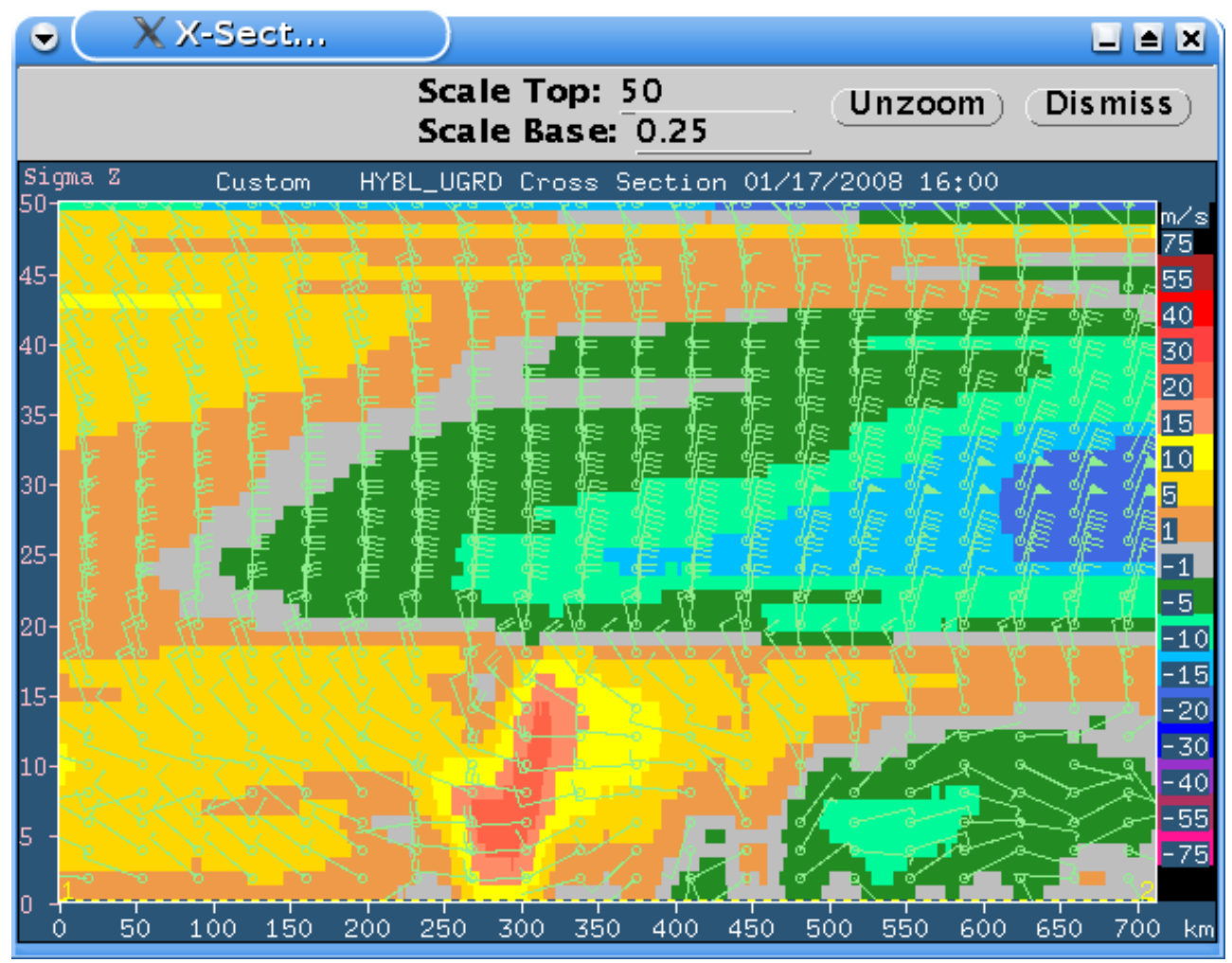

Figure 200. GTG vertical cross section of North-South wind barbs. 


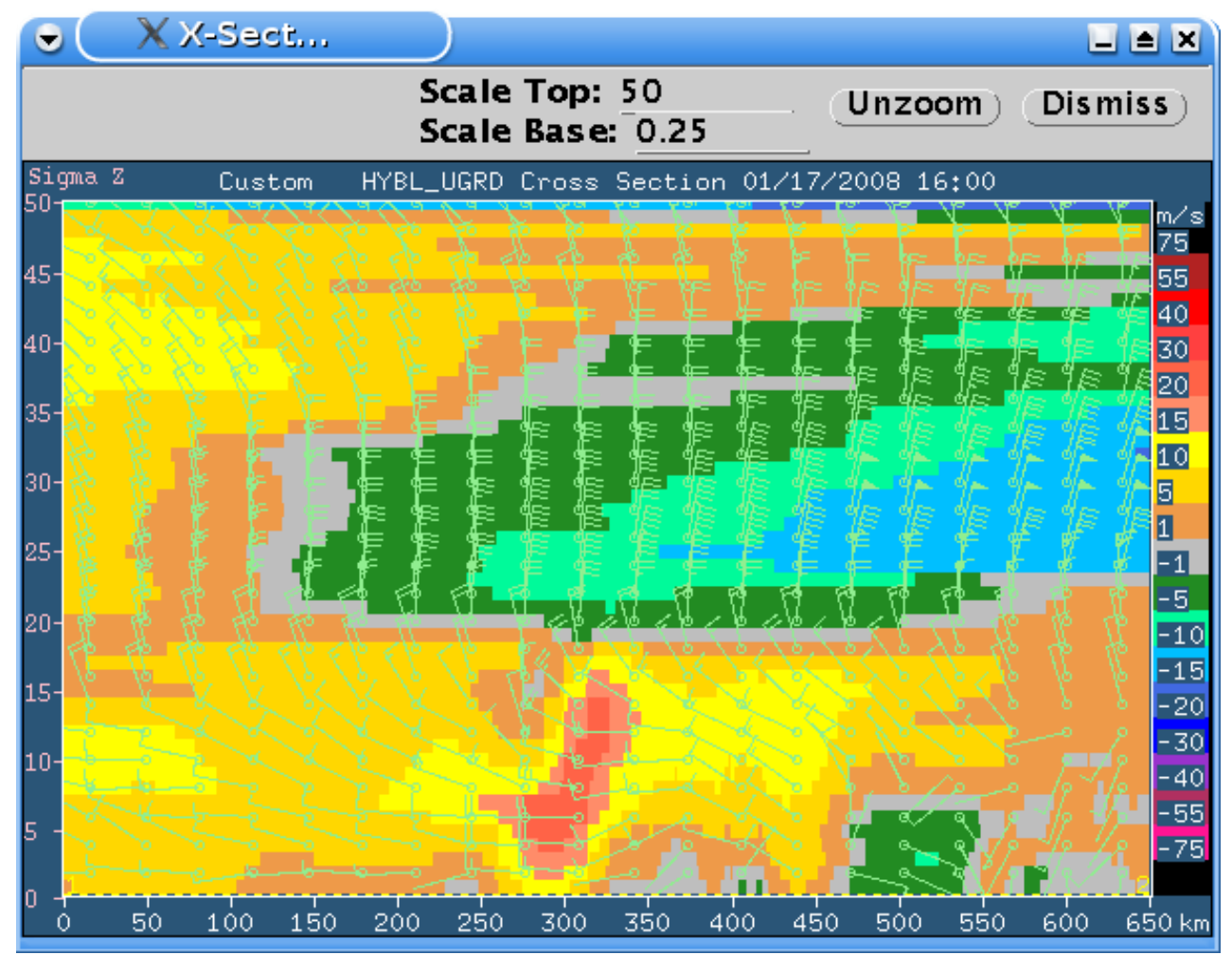

Figure 201. GTG vertical cross section and East-West wind barbs.

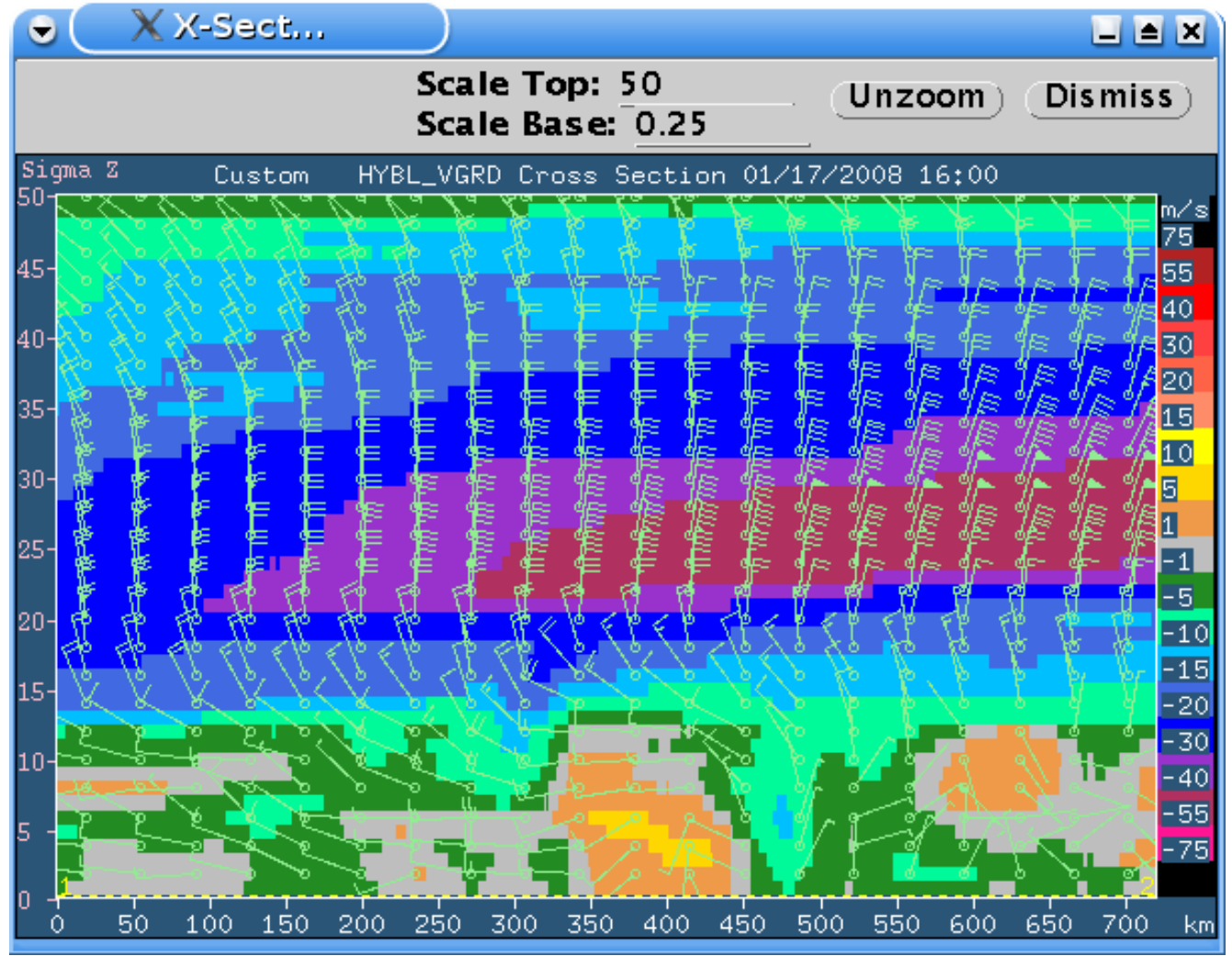

Figure 202. GTG vertical cross section with North-South wind barbs. 


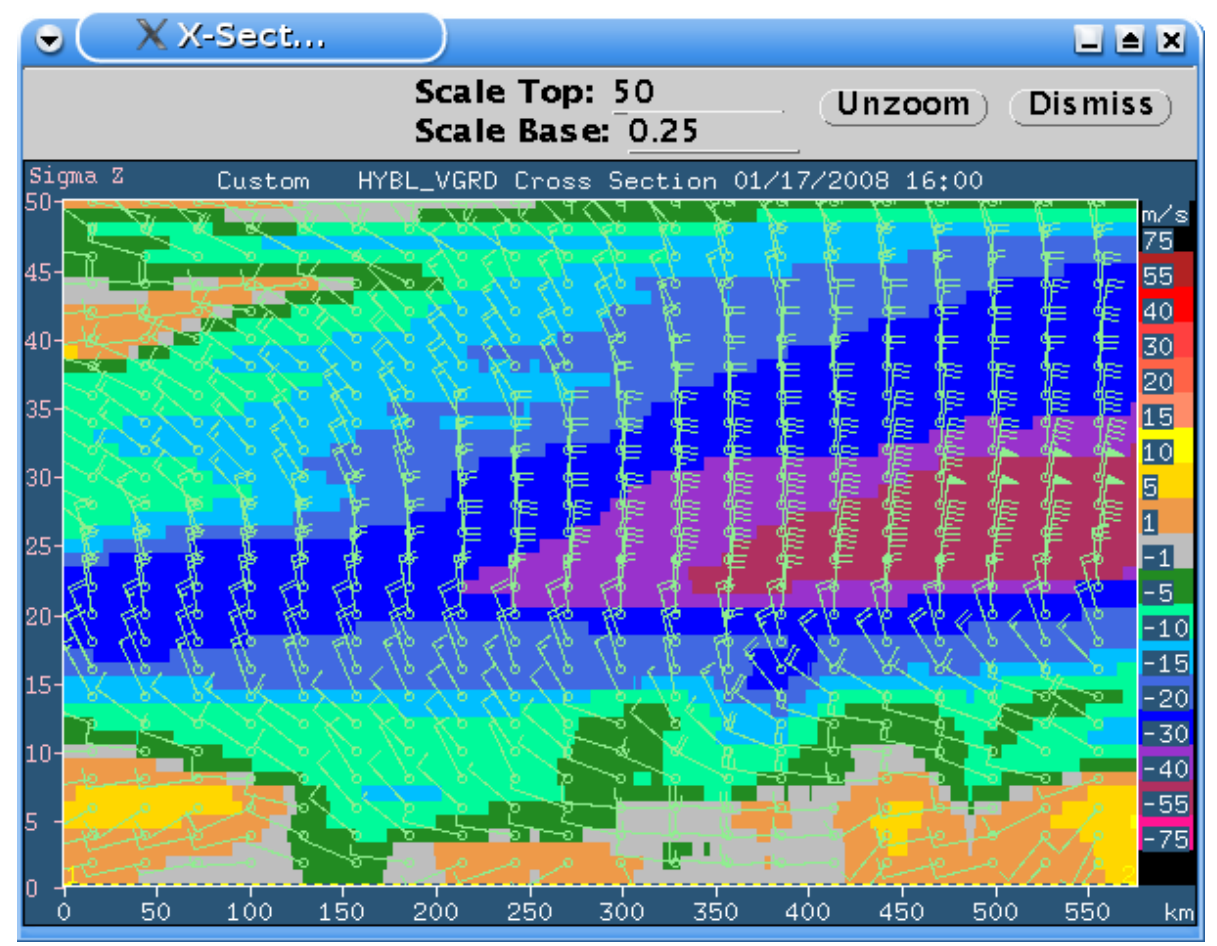

Figure 203. GTG vertical cross section with North-South wind barbs.

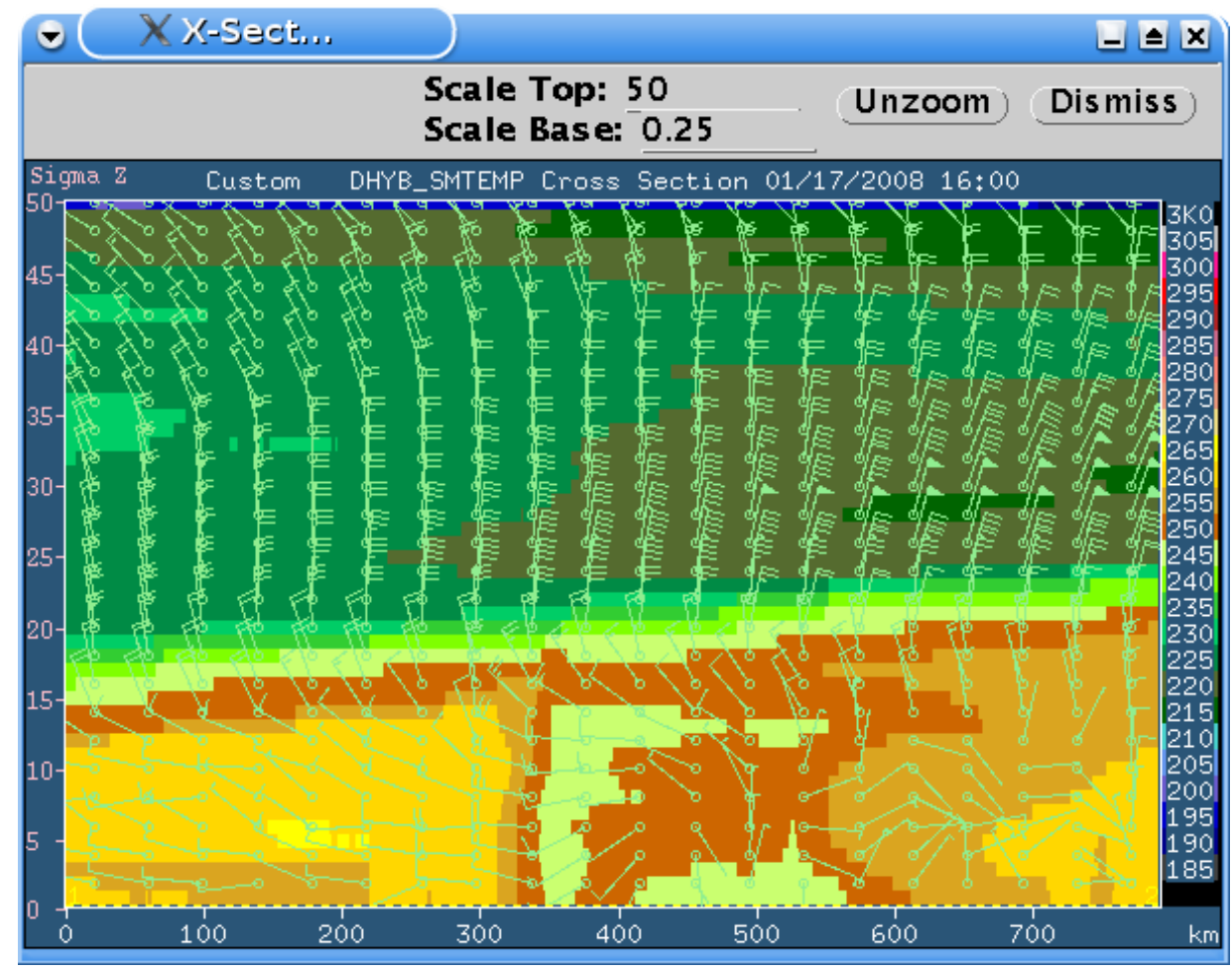

Figure 204. Temperature cross section looking Northwest. 


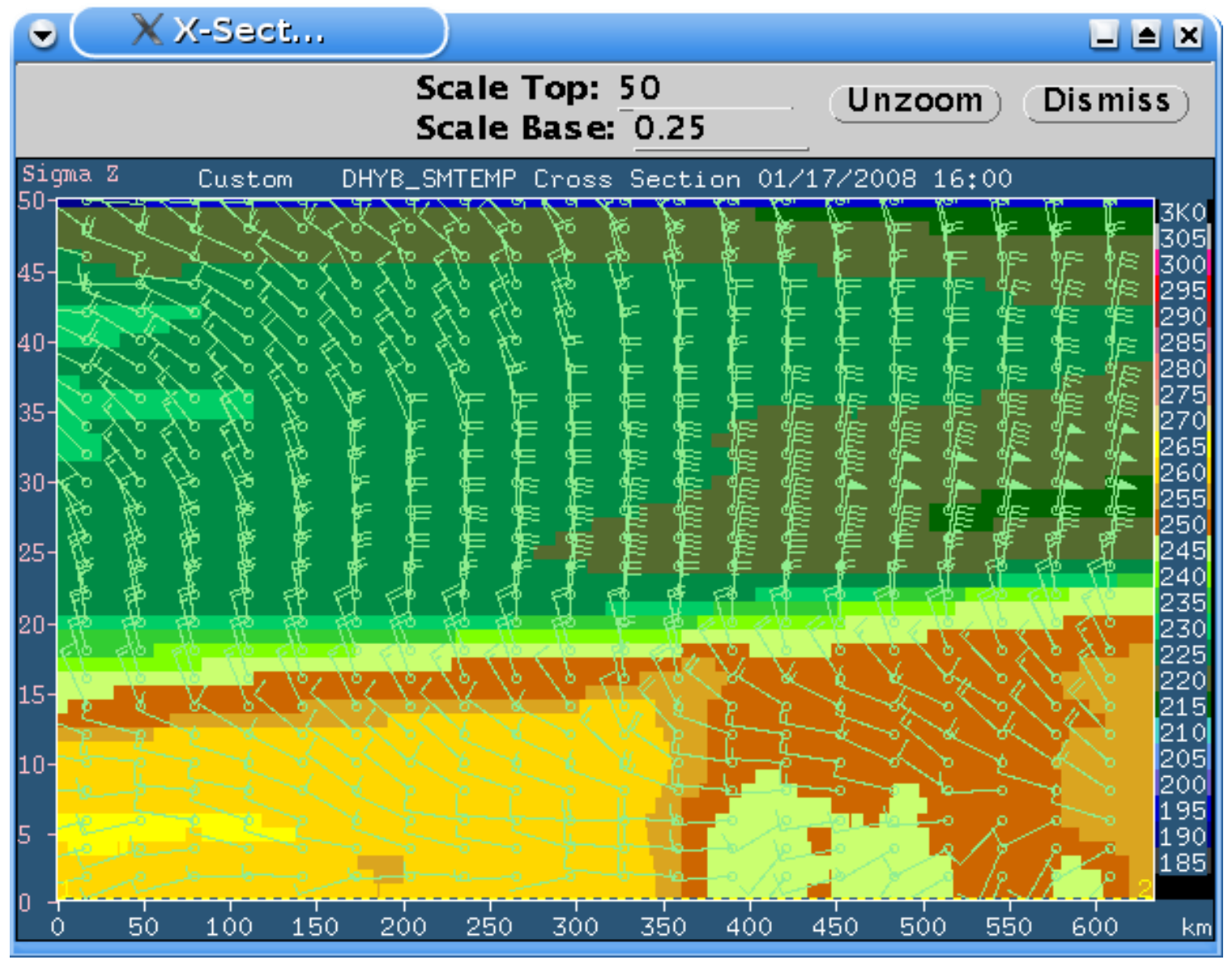

Figure 205. GTG vertical cross section of RUC temperature looking Northwest.

\subsection{Madison Field Test}

The second field measurement campaign is referred to as the Madison Wake Vortex Experiment (WAVEx). The program was conducted at Truax Field, site of the Dane County Regional Airport, on the northeast side of Madison. Figure 206 is a Google Earth image of the test site. The location of the test was chosen to allow the AERIbago to be utilized to provide truth data for the field campaign at minimum cost to the project. The program employed AFIT's MWIR FIRST and Bomem MR-154 instruments, GTRI's D\&P Spectrometer, in addition to the University of Wisconsin-Madison's AERIbago. The AERIbago is shown in Figure 207. Supporting data were also acquired, including weather data and a log of takeoffs and landings that included the aircraft type. 


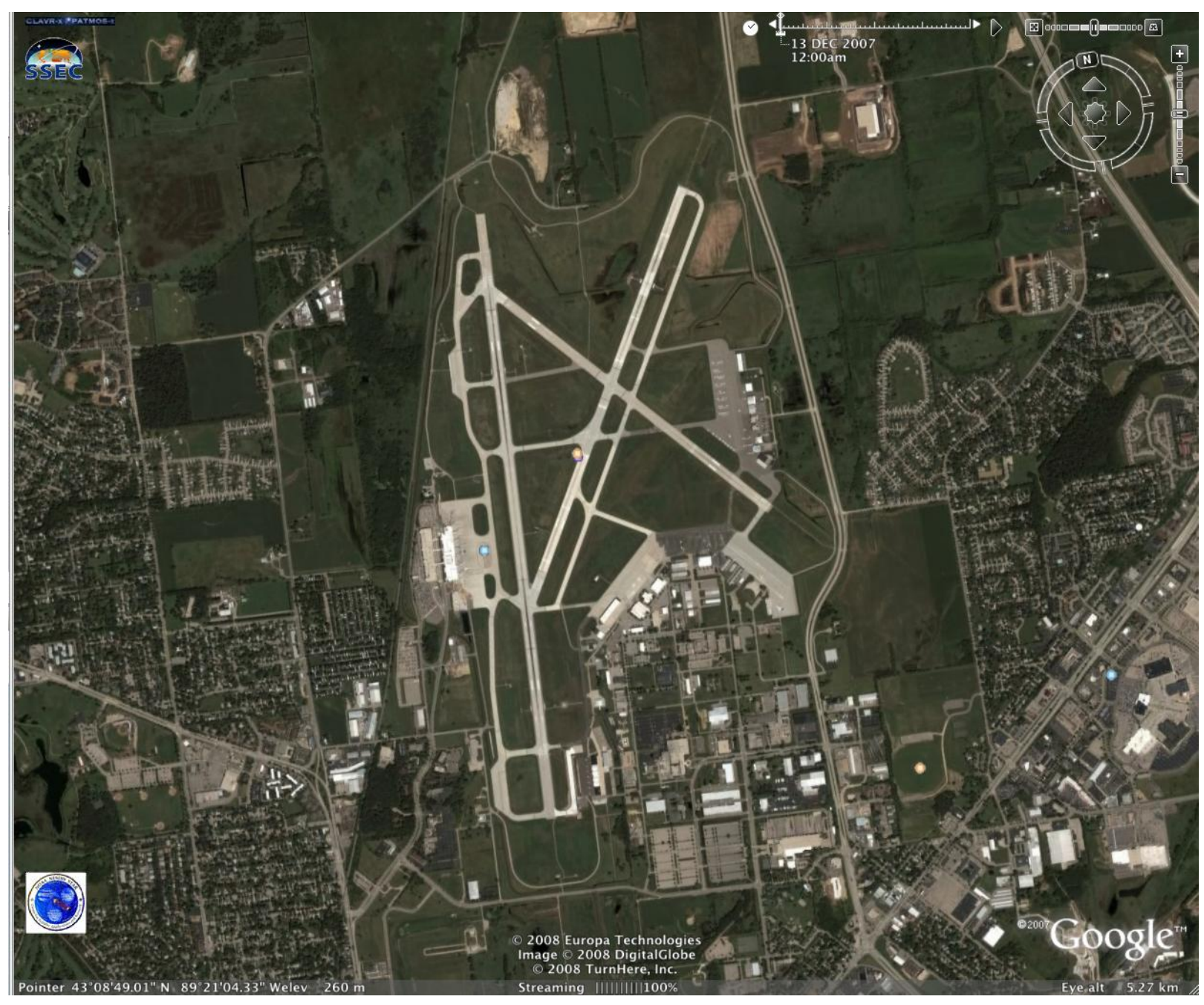

Figure 206. Google Earth Image of Truax Field. 


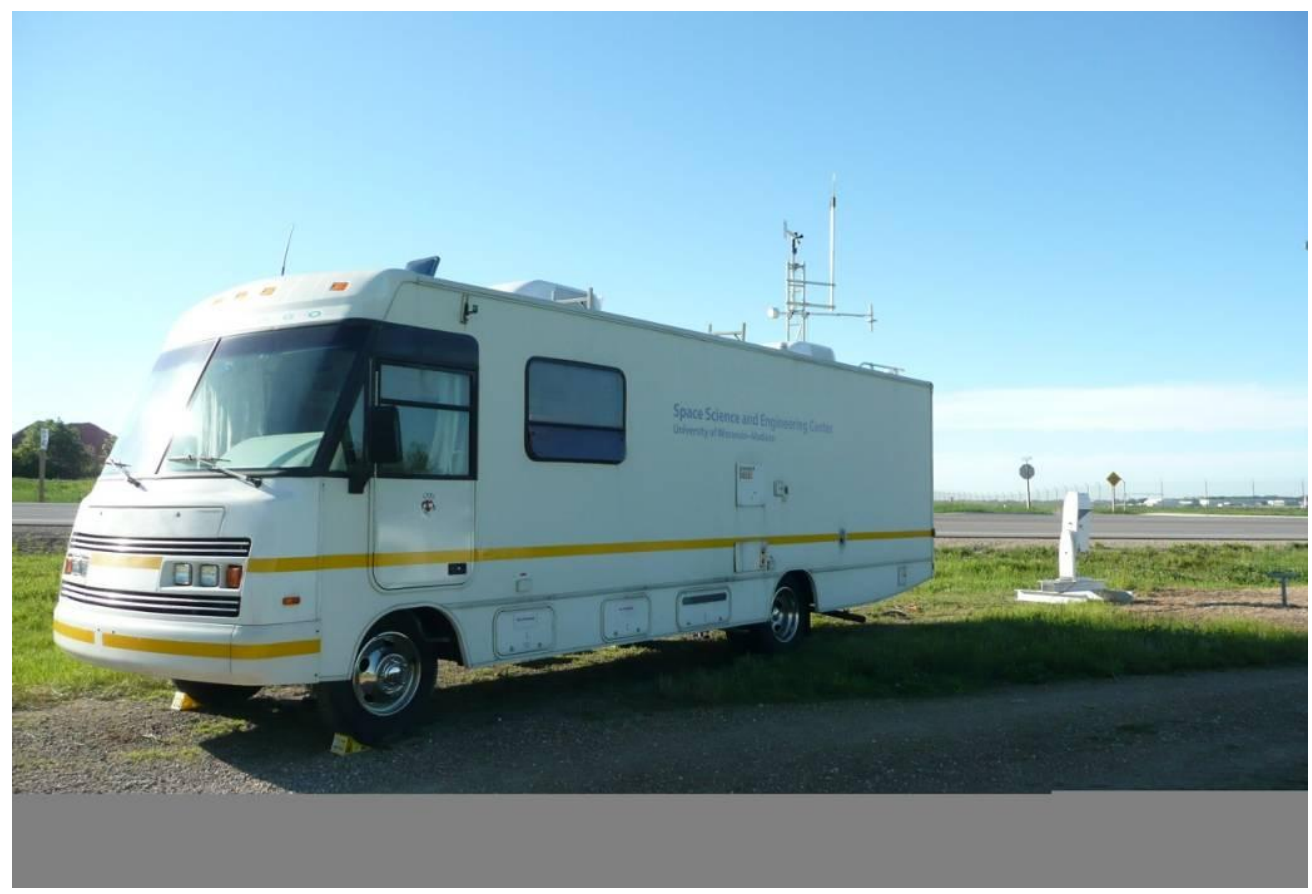

Figure 207. AERIbago deployed at Truax Field with suite of instruments.

It was believed that the Telops instrument was critical for the field program. Because of this fact, the field test, originally scheduled for the November timeframe, was moved to the June timeframe to accommodate delivery. The test occurred the week on June 1 - June 6, 2008.

Two types of measurements were performed: a) observations of airspace through which passenger jet aircraft had just flown, during both takeoffs and landings, for the purpose of finding radiometric signatures of wake vortices; and b) simultaneous observations of blue sky with the AERI and other instruments, for the purpose of comparison/calibration.

The Atmospheric Emitted Radiation Interferometer (AERI) is a fully automated ground-based passive infrared interferometer that measures downwelling atmospheric radiance from 3.3 - 18.2 $\mathrm{mm}\left(550-3000 \mathrm{~cm}^{-1}\right)$ at less than 10-minute temporal resolution with a spectral resolution of one wavenumber. Careful attention to calibration results in an absolute calibration accuracy of better than $1 \%$ of the ambient; the AERI system uses two internal blackbodies with calibration traceable to NIST temperature standards. The AERIbago is a modified 1994 Winnebago designed to deploy multiple weather instruments easily and quickly. The AERIbago currently contains an AERI instrument, ceilometer, surface station, radiosonde launch receiver, and GPS total precipitable water antennas. The AERIbago is spacious and provides clean electrical power to test instruments by means of a generator and inverter. The AERIbago provided space and security for all of the instruments used during the test. Figure 208 shows the AERI installed in the vehicle in a stowed configuration. During operation, it is mechanically elevated through an opening in the vehicle roof.

In the photograph, the Bomem spectrometer is on the right, the cylinder in front is the warm blackbody, and the cylinder to the left is the mirror rotation actuator. The ambient blackbody is on the back side, hidden from view. For temperature and water vapor profiling, the data 
acquisition cycle takes ten minutes, but much faster rates can be used, and the cycle is adjustable in the field. For this test, the acquisition cycle was set at three minutes.

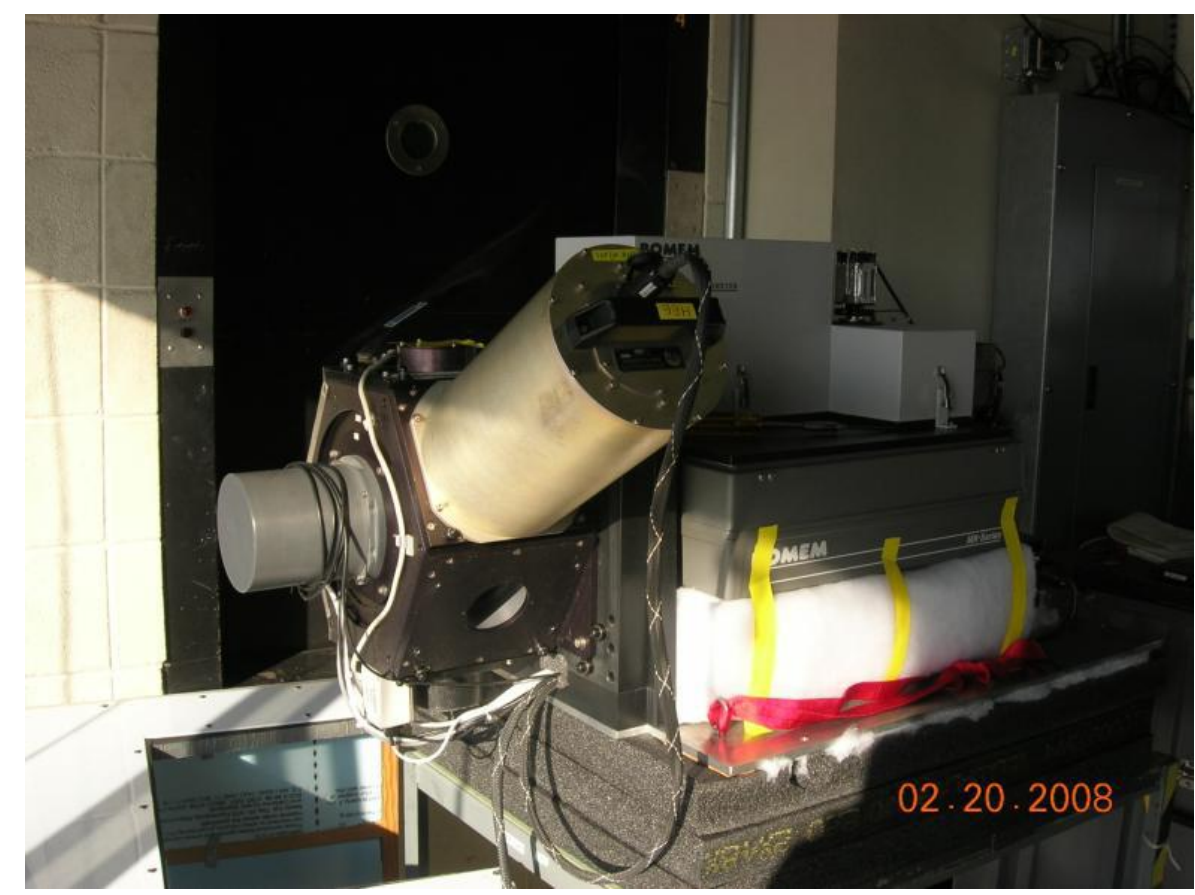

Figure 208. Measurement instrumentation for the AERIbago.

The main purpose of the field measurements in Madison was to verify that wake vortices have an observable signature in the high-resolution radiance spectrum that will provide a means to detect, and perhaps image, wake vortices associated with takeoffs and landings. Secondary objectives were to verify the EOF mitigation scheme for low slant range visibility and to detect icing conditions, i.e. supercooled water droplets in clouds. However, there was a consensus during the initial field test teleconference that the test should be postponed until delivery of the Telops instrument because it is more important to the outcome of the program than catching icing/fog conditions. Therefore, due to the delay of the field test to the June timeframe, conditions such as low slant range visibility and icing were unavailable.

Figure 209 - Figure 210 are Google Earth images of the proposed setup sites. These sites were chosen because about $90 \%$ of the commercial flights use Runway $18-36$. Takeoffs are $60 \%$ to the north and 40\% to the south. Tower hours are from 6:00 a.m. to 11:00 p.m. The takeoffs by large aircraft are mostly in the morning with another (smaller) set in the afternoon. The ground-based data was collected on the first day at the proposed north site outside of the airport runway perimeter to avoid security clearance and have a better slant angle on wake vortex occurrence. On Wed June $4^{\text {th }}$, the suite of instruments was moved to a slightly different site marked on Figure 209 as "field test location" and remained there for the duration of the test. Proper placement of the equipment allowed images of aircraft during take-off and landing. Figure 211 is an example of a landing aircraft from the location of the test instrumentation. 


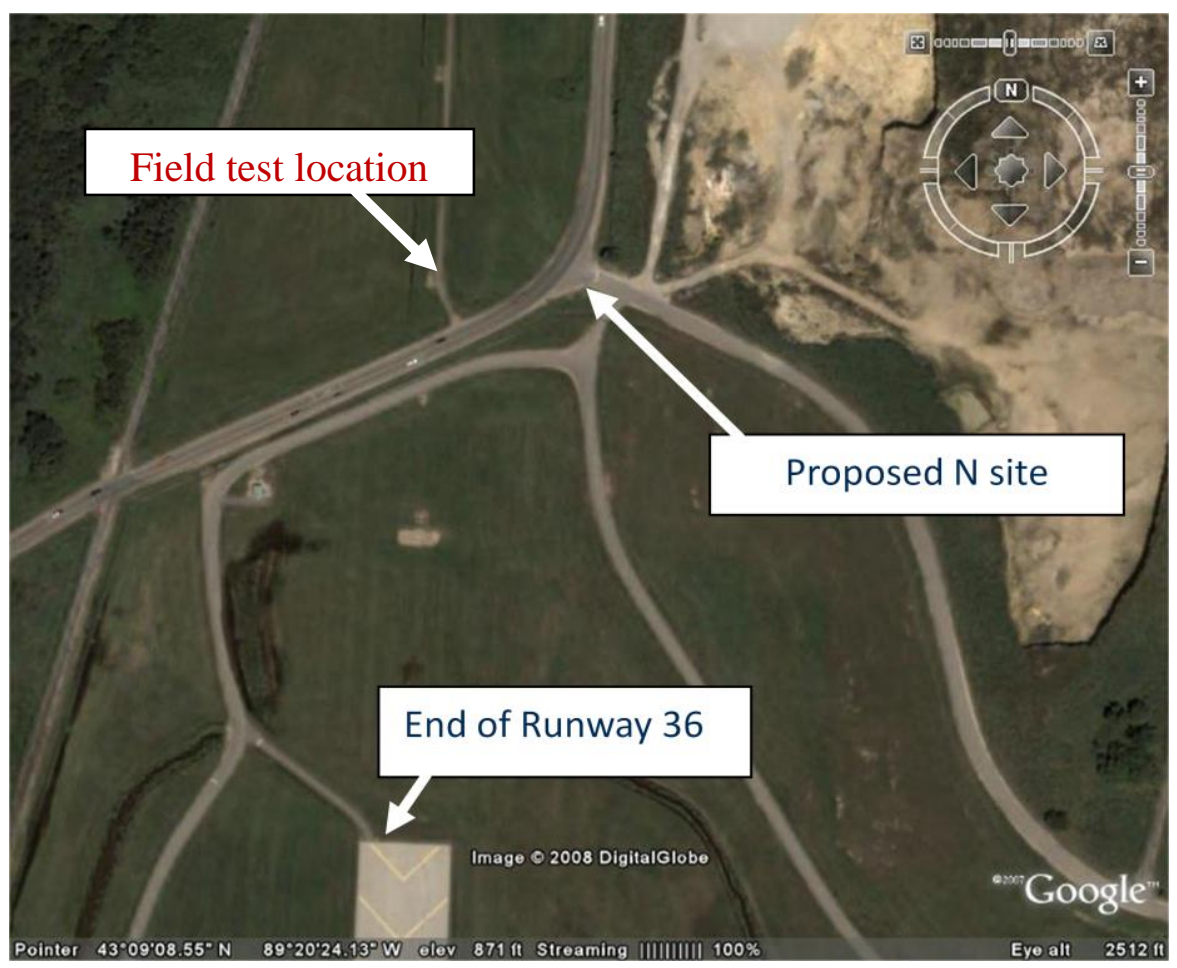

Figure 209. Proposed North site.

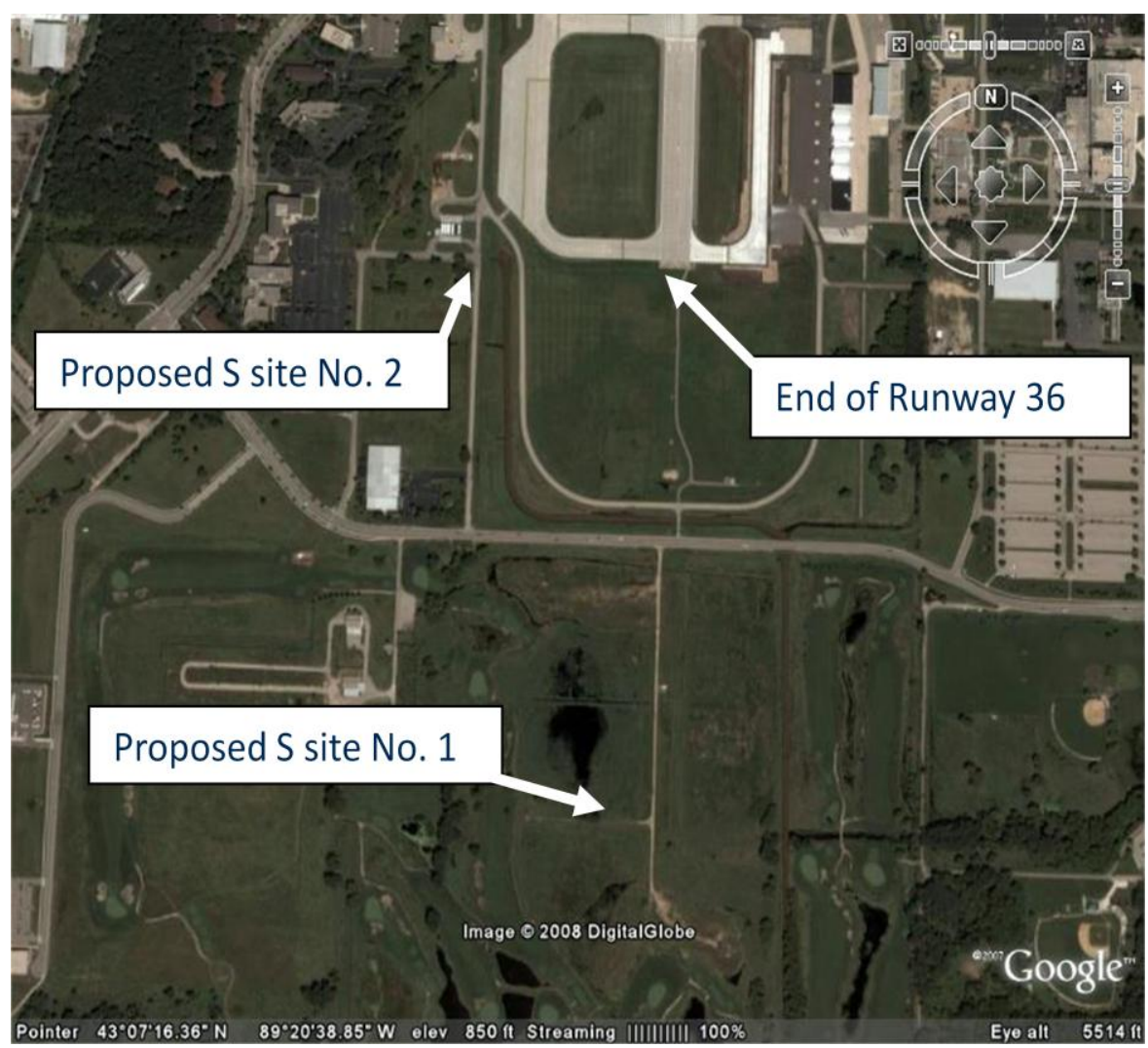

Figure 210. Proposed South sites. 


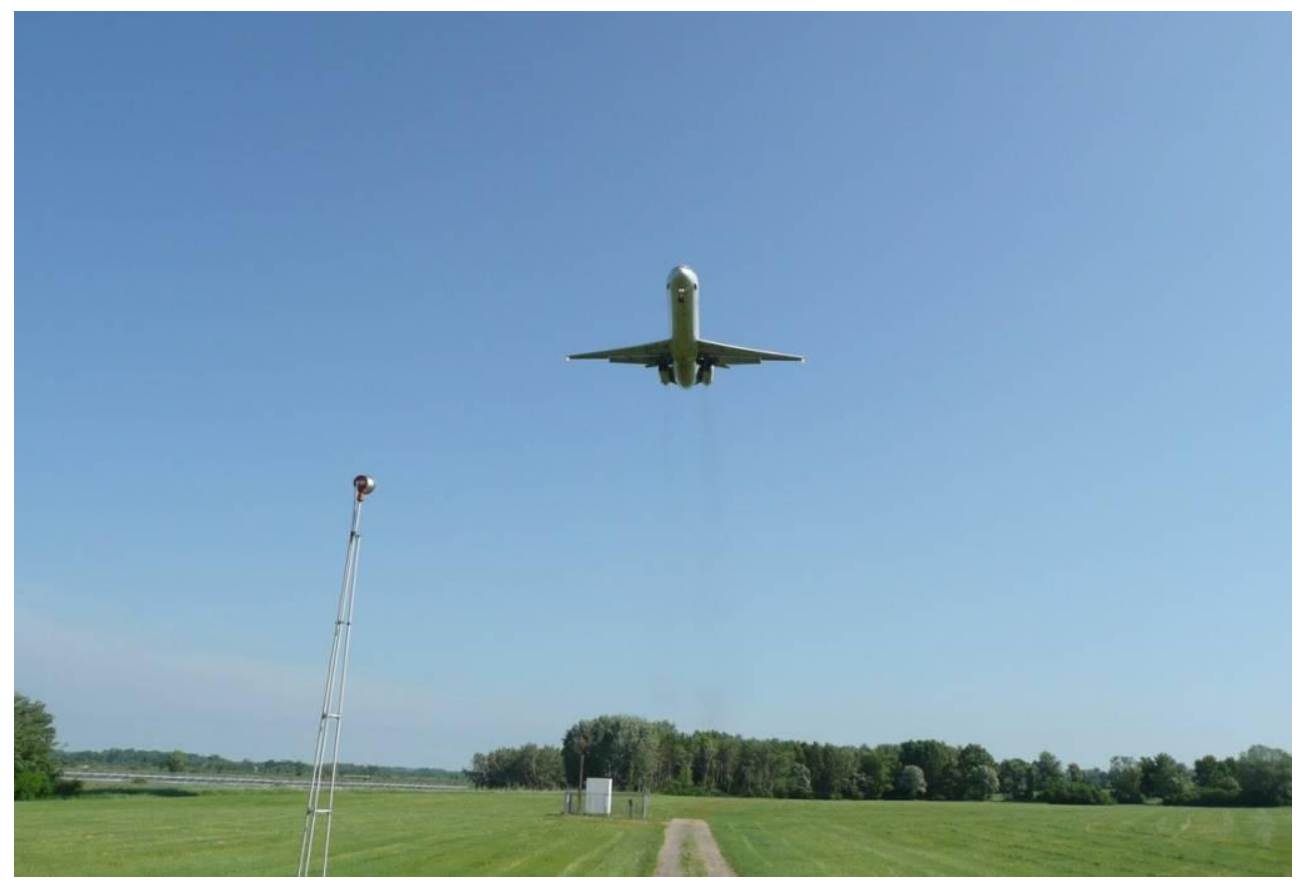

Figure 211. Landing aircraft from North Field Test Site Location.

\subsubsection{Telops MWIR FIRST Data Analysis}

PC based Enhancement of Measurement Signal to Noise: In order to resolve the wake vortex signature from the Telops MWIR FIRST measurements, the following procedure was investigated:

(a) Transform the calculations for the spectra of wake vortex brightness signatures, a portion of which were shown in Figure 4 through Figure 6, above, to the spectral range and spectral resolution of the MWIR FIRST data.

(b) Use wake vortex radiance spectra to compute Principal Components (PCs) of the wake vortex radiance signal.

(c) To the wake vortex FIRST data frames with embedded aircraft, as well as subsequent frames apply the PCs computed in (b) above to the first radiance spectra for each pixel. This will yield a set of PC coefficients (i.e., PC scores for each pixel and each frame).

(d) Construct an image for each of the PC coefficients as well as for the reconstructed radiant brightness temperature signal for various spectral bands (e.g., 2200-2235 wavenumbers) using 1, 1-2,1-3,1-4, 1-5, to 1-total number of the PCs for the reconstruction. Since the vortex radiance signals do not have noise included, this process should filter the noise and the vortex might be revealed, if there is any vortex signal in the radiance data. The vortex may be resolved in either the images of each of the PCs or in the reconstructed radiance for the spectral bands, which have the highest vortex signals.

PC Computation: Principal Components were computed for the wake vortex simulations described above. For these calculations the following parameters were assumed:

- Viewing angle $=20^{\circ}$ over horizon 
- $\quad$ Training set of spectra:

○ 5 distances along the line of view: $300 \mathrm{~m}, 400 \mathrm{~m}, 500 \mathrm{~m}, 600 \mathrm{~m}, 700 \mathrm{~m}$

- 4 thicknesses (projected to vertical): $1 \mathrm{~m}, 2 \mathrm{~m}, 3 \mathrm{~m}, 4 \mathrm{~m}$

- 6 temperature perturbations at the core: $0 \mathrm{~K},-2 \mathrm{~K},-4 \mathrm{~K},-6 \mathrm{~K},-8 \mathrm{~K},-10 \mathrm{~K}$

- $5 \mathrm{CO}_{2}+\mathrm{N}_{2} \mathrm{O}+\mathrm{CO}$ factors of $\mathrm{x} 1, \mathrm{x} 5, \mathrm{x} 10, \mathrm{x} 20, \mathrm{x} 50$

- Total number of spectra $=5 \times 4 \times 6 \times 5=600$

The high spectral resolution $\left(\delta v=0.25 \mathrm{~cm}^{-1}\right)$ LBLRTM-simulated spectra were convolved into 60 FIRST bands $\left(\sim 1802-3574 \mathrm{~cm}^{-1}, \delta v=\sim 30 \mathrm{~cm}^{-1}\right)$.

Figure 212 shows a comparison between a FIRST radiance spectrum, observed near the wingtip of a landing aircraft, and the mean simulated wake vortex radiance spectrum. The standard deviation of the 600 simulated vortex radiance spectra, used to compute the wake vortex radiance PCs, is also shown. There is surprisingly good agreement between the absolute radiances of the measured and calculated radiance spectra. However, the measured spectrum does not show the abnormally low wake vortex radiance signatures in the $2100-2200 \mathrm{~cm}^{-1}$ and $2500-2950 \mathrm{~cm}^{-1}$ regions depicted in the calculated spectra.

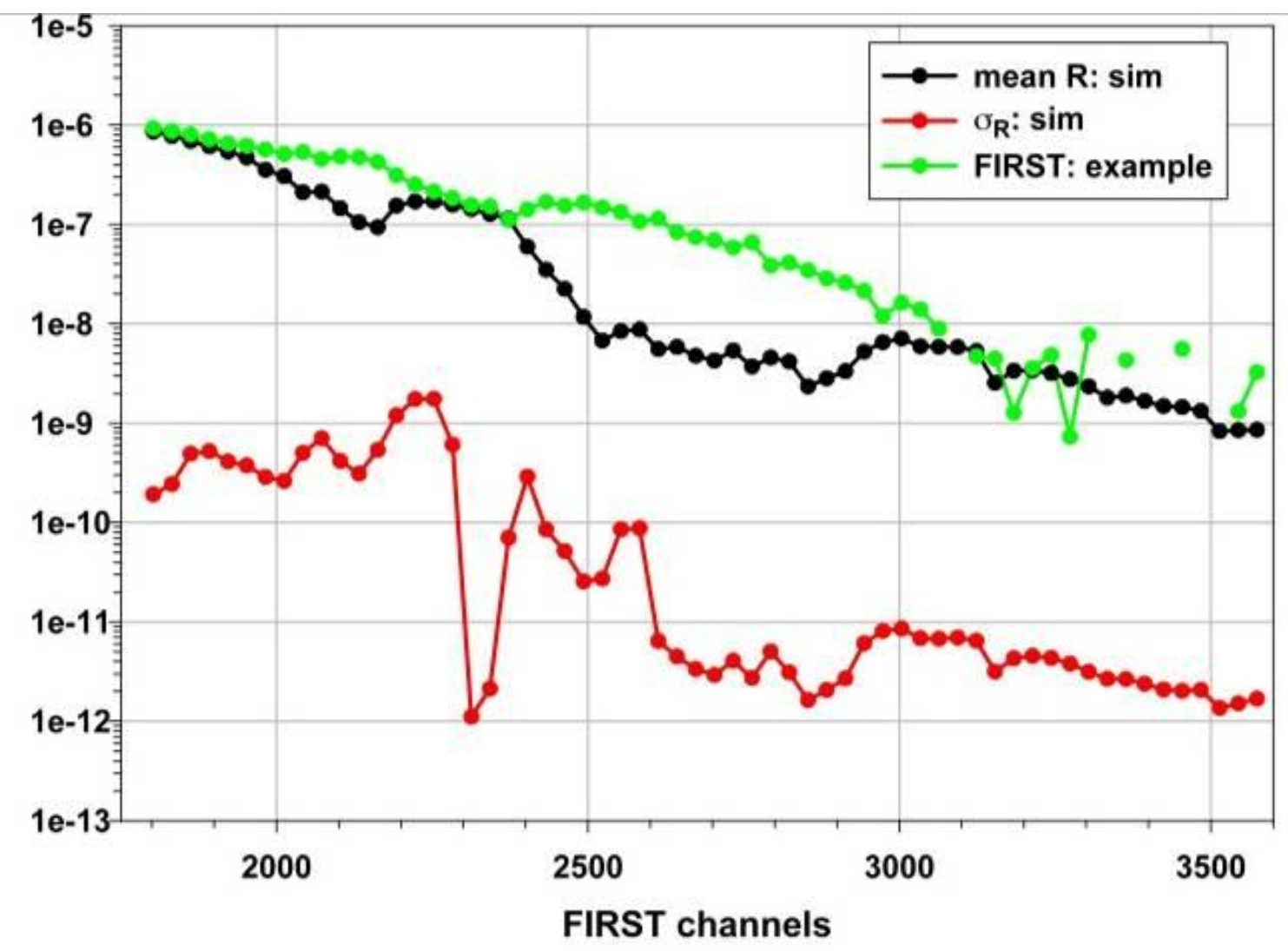

Figure 212. Mean simulated radiance spectrum compared to measured radiance spectrum. 
The relative magnitude of the relative variation of the wake vortex radiance signals is shown more clearly in Figure 213. As can be seen, the largest signal is only about $1 \%$, as expressed in terms of the standard deviation of the wake vortex signals relative to the mean wake vortex radiance signal. As discussed earlier, this result implies that a very high signal to noise ratio measurement (e.g., 1000:1) is needed to be able to detect these vortices in actual infrared spectral radiance observations.

Figure 214 shows the first five PCs of the wake vortex radiance spectra. It can be seen that most of the variable wake vortex induced spectral structure lies in the $1800-2250 \mathrm{~cm}^{-1}$ spectral region. How, in the analysis to follow, it is shown that the wake vortex structure could not be resolved by Telops MWIR FIRST data acquired in Madison WI during the WAVEx.

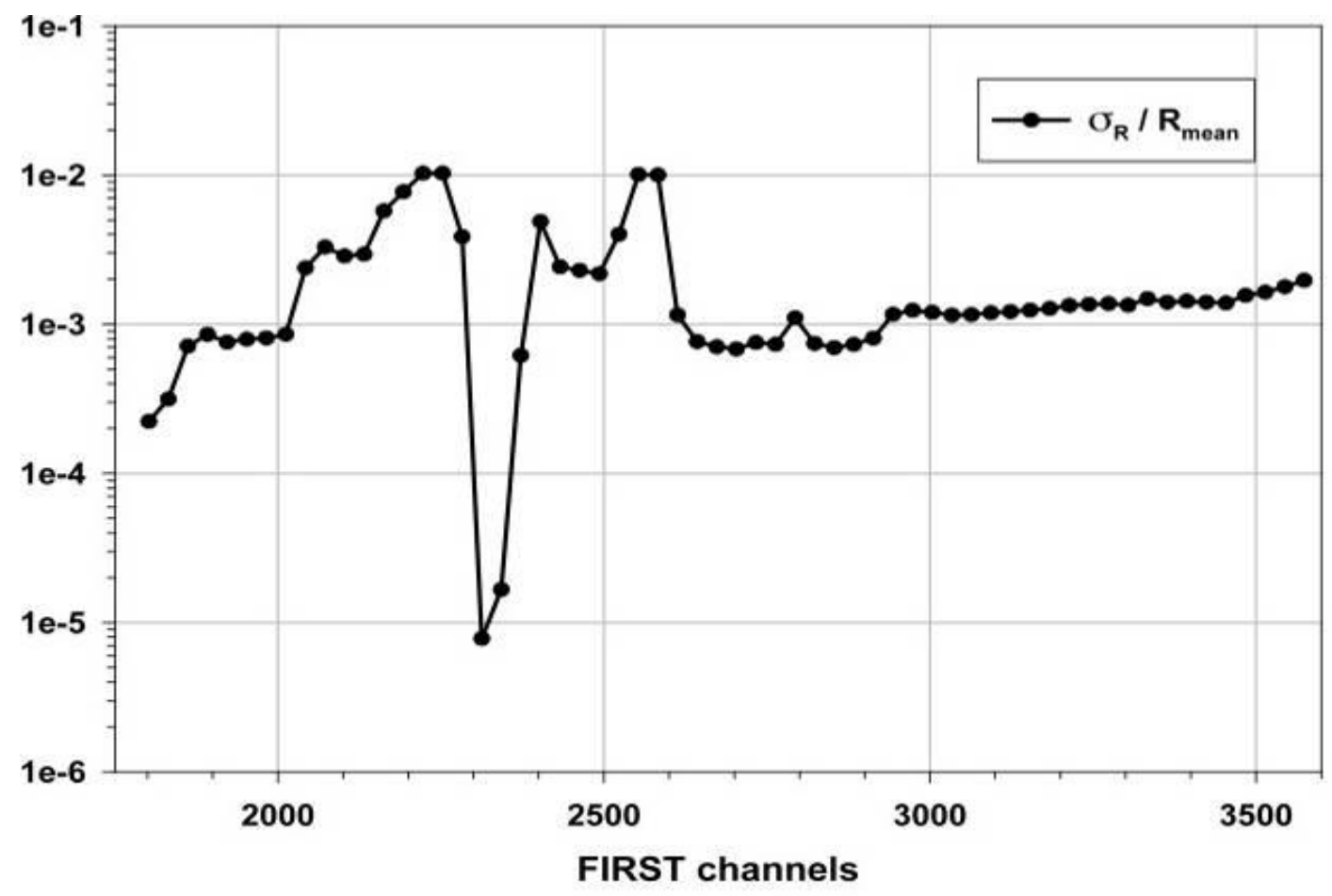

Figure 213. Ratio of the radiance standard deviation to the mean. 


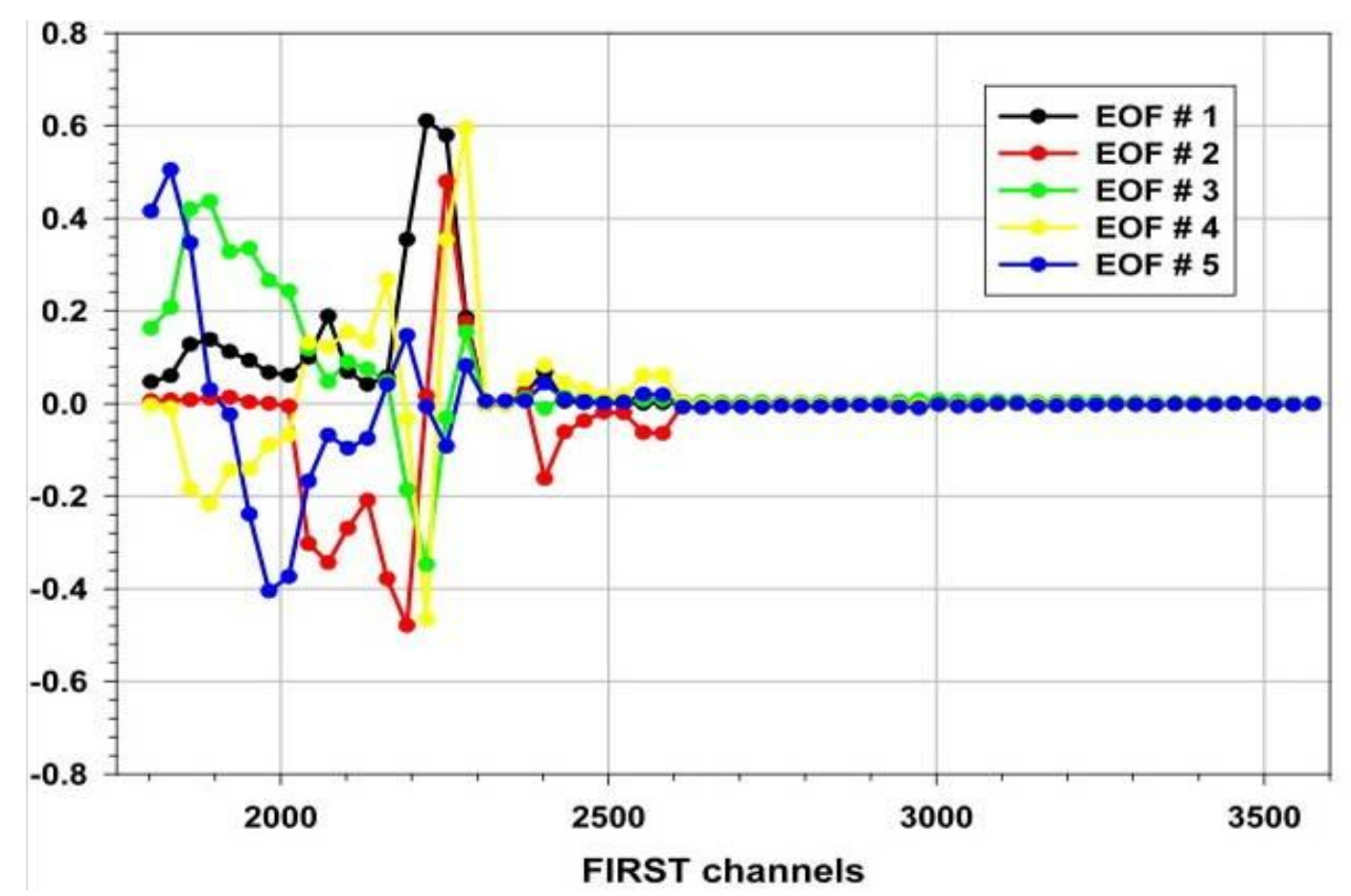

Figure 214. The first five PCs used to characterize the wake vortex radiance spectrum.

\subsubsection{Signal Processing for Analysis of MWIR FIRST WAVEx Data}

Background: The goal of the analysis was to determine if a thermal signature was apparent within the hyperspectral data from the Telops MWIR FIRST instrument. As a result of the theoretical computations described above, there was an expectation that the thermal infrared imagery would reveal a cool core within the warmer environmental air ring of the wake vortex. If the sky was clear with no cloud interference, and the instrument was aligned properly on the wingtip, then a wake vortex thermal image may have been captured by the instrument. Each pixel contains 60 samples of the IR spectrum from $1800 \mathrm{~cm}^{-1}$ to $3600 \mathrm{~cm}^{-1}$, and the field of view was constrained to 70 pixels in the vertical and 256 pixels in the horizontal. The resulting "hypercubes" or "cubes" were captured at a rate of about $4 \mathrm{~Hz}$. This rate was a function of the number of captured pixels and the integration time per pixel.

The final day of the WAVEx, June $7^{\text {th }}$, was fortuitous in that the ambient conditions favored successful data collection. There was a clear sky and there were three early morning flights landing over the selected deployment runway. Of the three flights, L0836, L0847, and L0923, only the first was properly aligned such that the center of the $70 \times 256$ pixels captured the tip of the left wing of American Eagle 3922, a CRJ from Chicago-O'Hare.

Figure 215 below shows a differential image of the broadband $\left(1800-3600 \mathrm{~cm}^{-1}\right)$ spectral radiance during a DC-9 landing on 7 June 2008. The four images in the figure are subsequent in time. In Figure 215, the exhaust gases are clearly evident in these images. Unfortunately the wing tips are not visible in these frames so that the vortex may not be visible.

In Figure 216, the same data from the previous figure was decomposed using Principal Component Analysis. Each subplot in the figure is of one of the first twelve principal 
components, illustrating how the analysis is able to extract the orthogonal components of the original data.
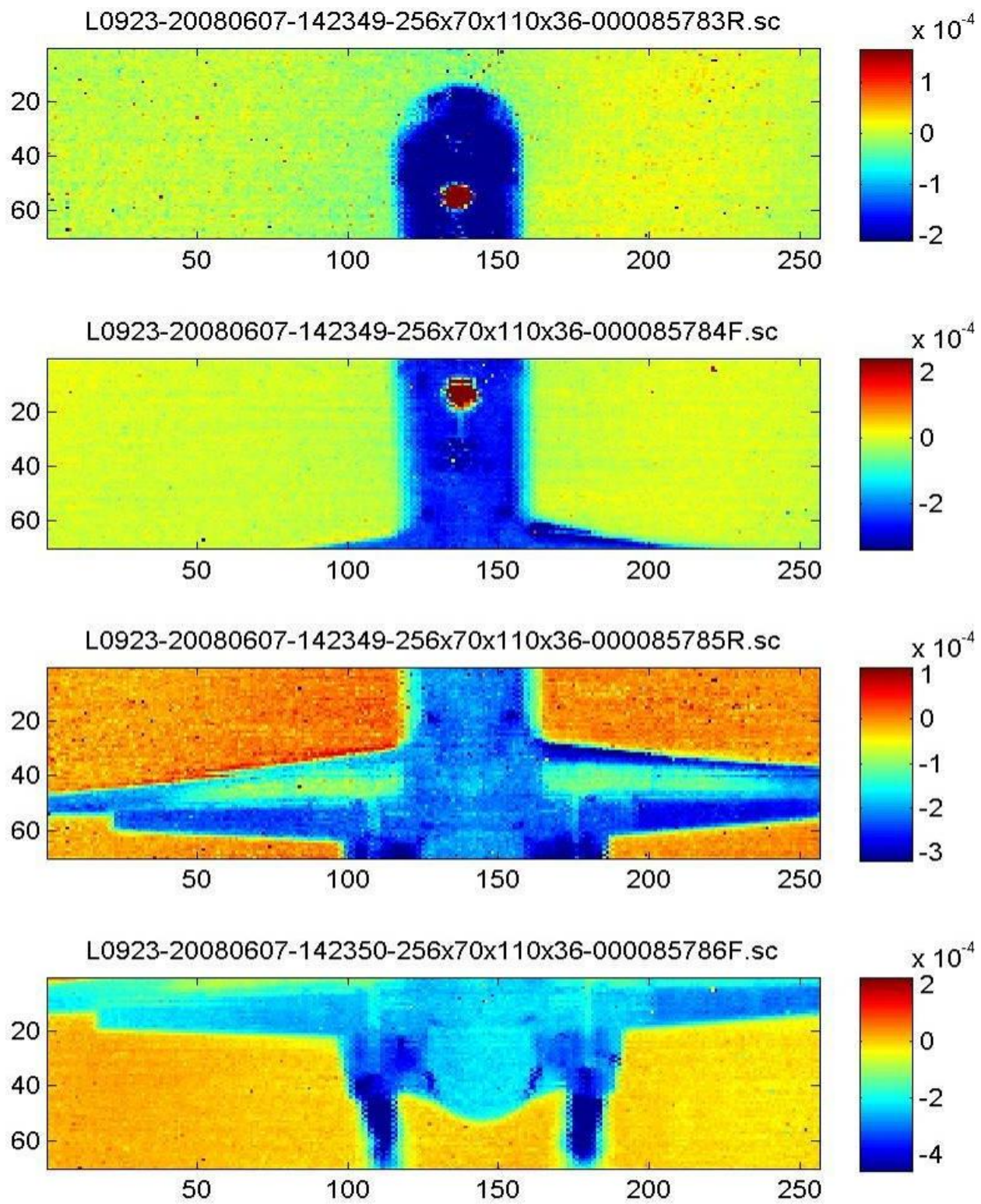

Figure 215. Differential image of the broadband spectral radiance. 

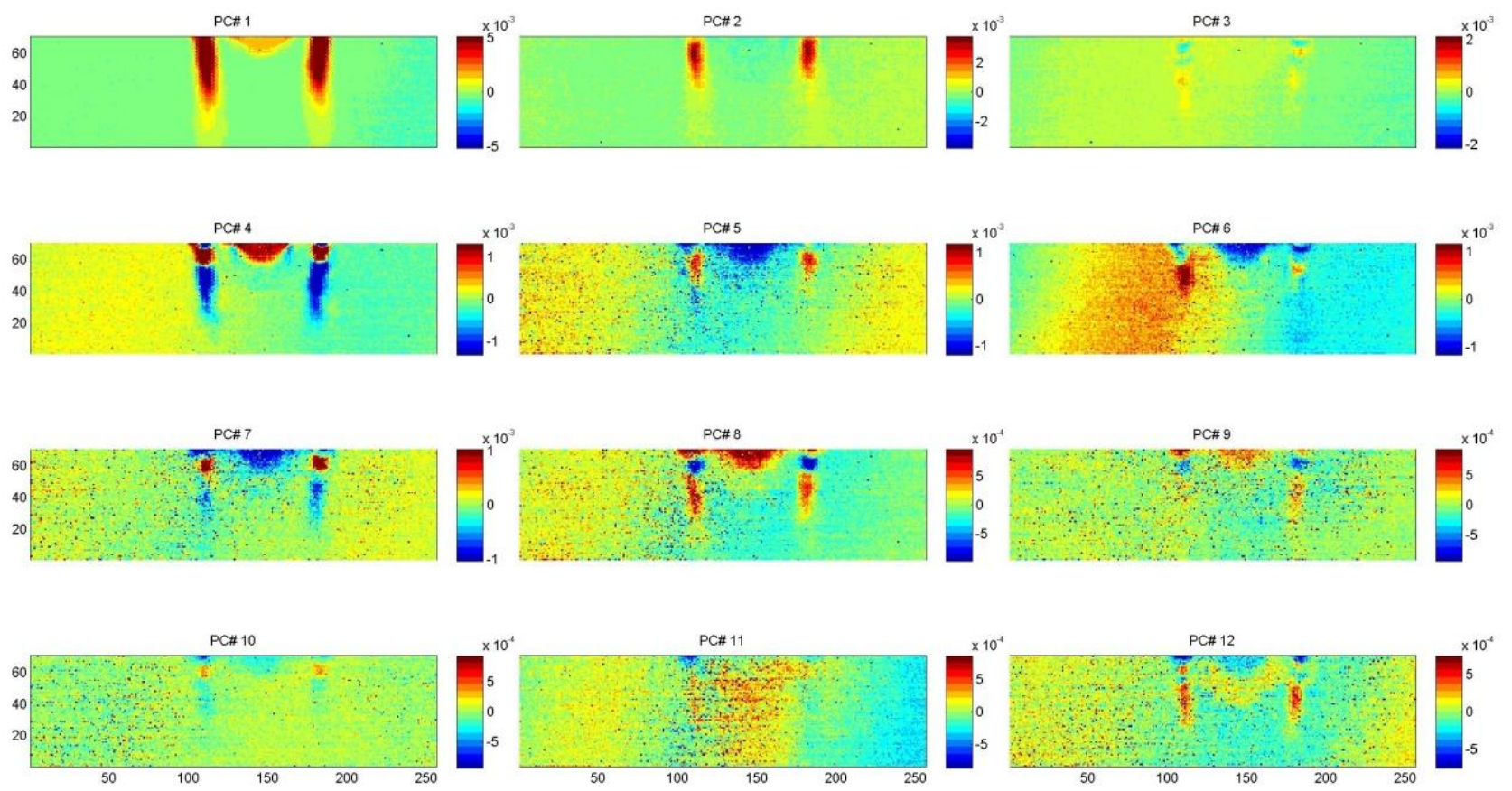

Figure 216. First twelve PCA decomposed fields.

Radiometric Calibration: The first step in the signal processing was calibration of the raw data cubes. The MWIR FIRST instrument collects two sets of 100 cubes each of two different blackbody sources. Telops provided calibration software with the instrument to perform a twopoint linear regression for each pixel from the 400 cubes of calibration data. The result is a gain and offset value for each pixel. These gain and offset values must then be applied to the raw cubes to generate calibrated cubes of known radiance.

One caveat should be noted here. The instrument acquires two types of cubes, based on the direction of the internal instrument scanning mirror, either "Forward" or "Reverse". Telops processing software treats the two types separately, with calibrations performed for both, individually.

Background Computation: The wake vortex signature for this particular IR spectral region was known to be at a very low signal to noise ratio. As such, the second step in the signal processing is to compute two cubes (one forward, one reverse) that represent the calibrated scene prior to the aircraft pass. In order to be statistically significant, all available forward (reverse) scanned calibrated cubes were averaged together to create a forward (reverse) background cube.

Figure 217 below shows the images of the total band spectral brightness temperatures (i.e., integrated over the IR spectrum resolved by the MWIR FIRST instrument) resulting from the calibration of the raw MWIR FIRST interferogram for the forward and backward interferogram scan directions. As can be seen, the radiance images for the forward and backward mirror scans are very similar, as they should be. Note that the background radiance field has a warm spot near pixel $(140,50)$ that might be misinterpreted as a wake vortex signature in subsequent images. 

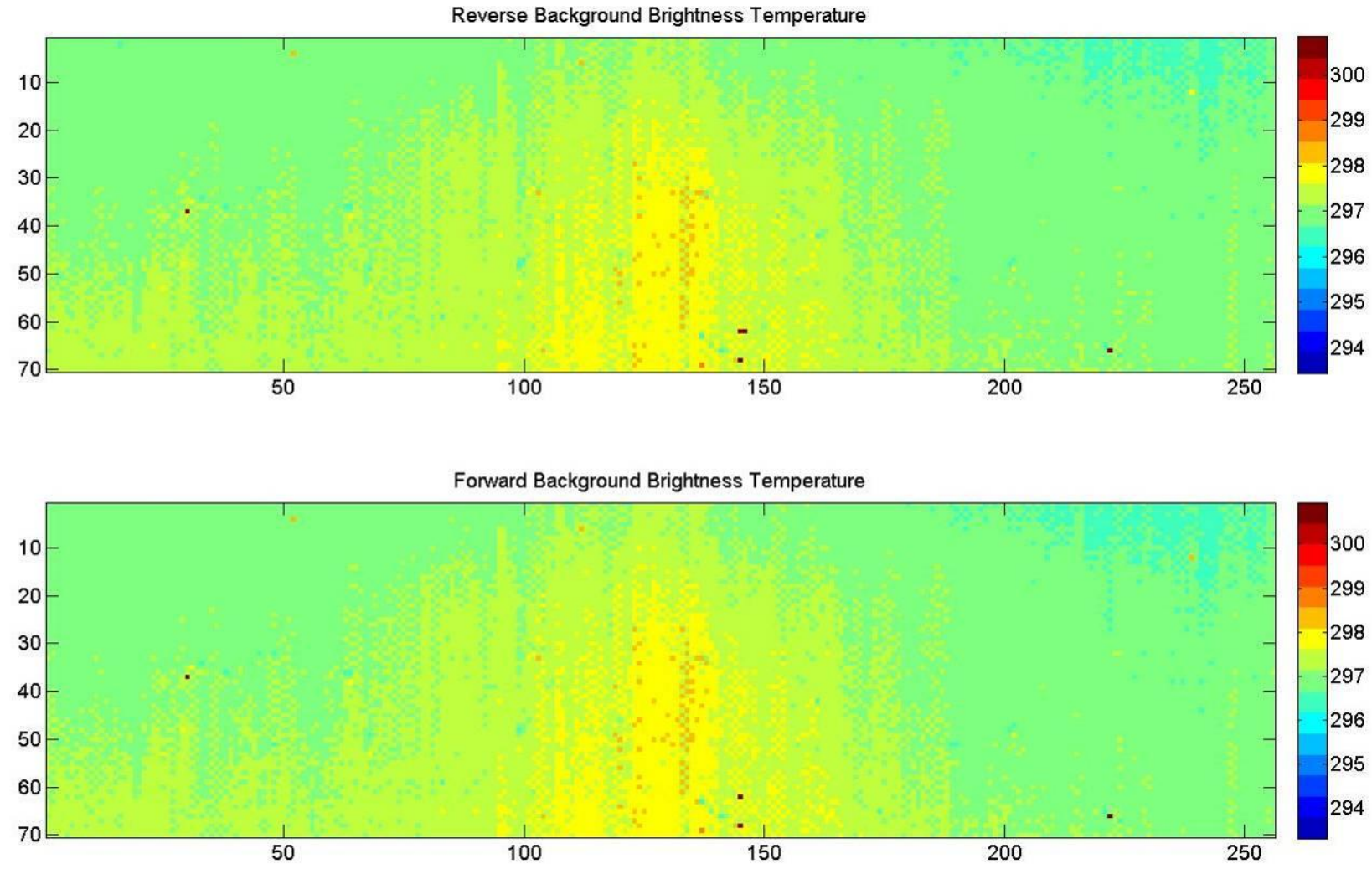

Figure 217. Average background of MWIR FIRST brightness temperature.

Using the background cubes, a "difference" cube was computed between each forward (reverse) cube after aircraft passage and the forward (reverse) background cube. So far, all the cubes mentioned have dimensions $70 \times 256$ × 60. To create a simple difference image, each cube was integrated over the spectral domain to create a $70 \times 256$ image. Other images are possible, including only integration over a band of interest, such as CO $\left(2000 \mathrm{~cm}^{-1}\right.$ to $\left.2100 \mathrm{~cm}^{-1}\right)$.

Figure 218 and Figure 219 show the background removed differential total spectral band radiance images obtained with the MWIR FIRST instrument as an aircraft passed through the instrument's field of regard. As can be seen, the existence of the aircraft passing through the instruments field of regard is easily seen. Unfortunately, however, there was no evidence of a wake vortex radiance feature in these radiance image frames. 

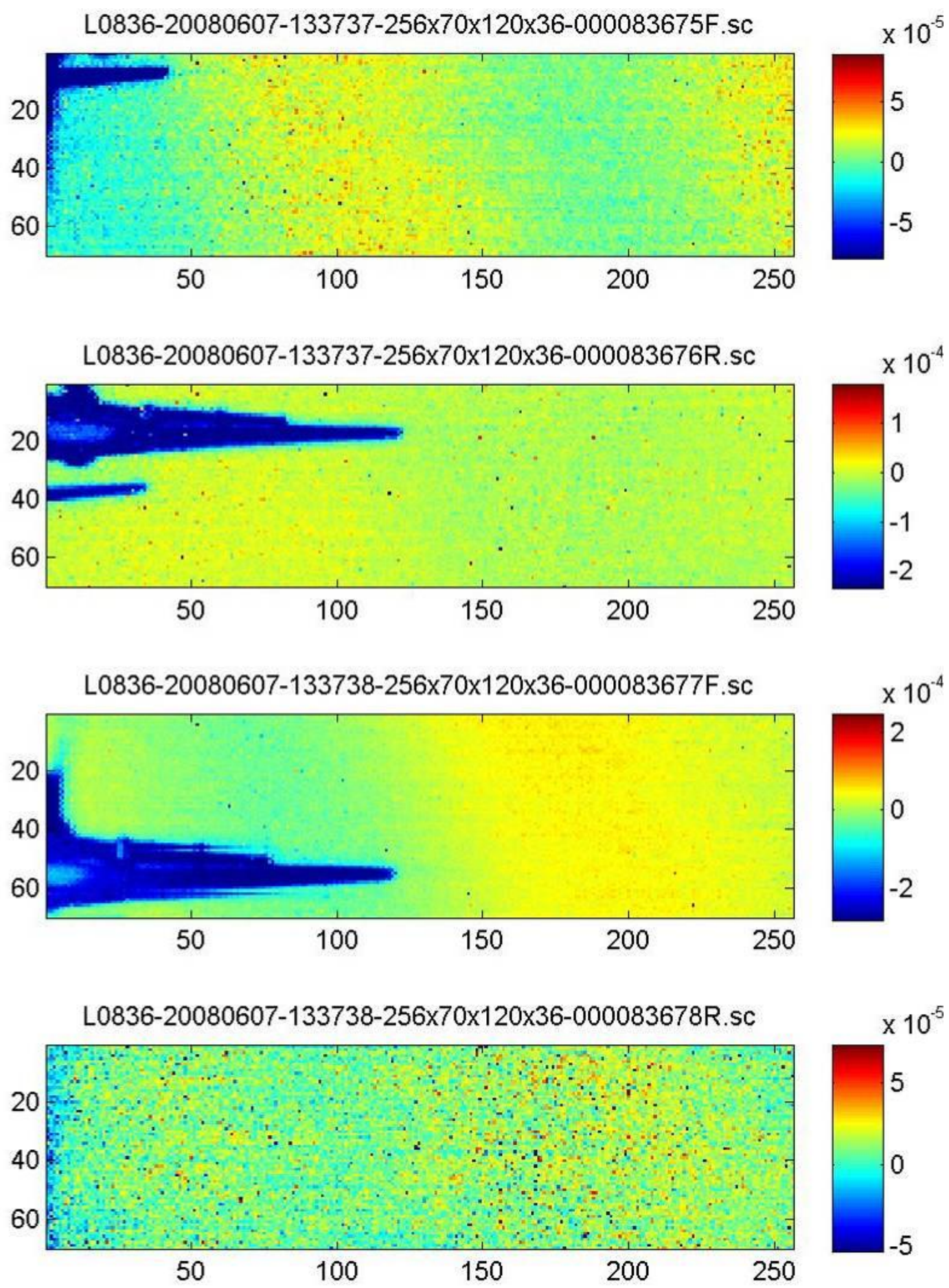

Figure 218. Background removed differential total spectral band radiance. 

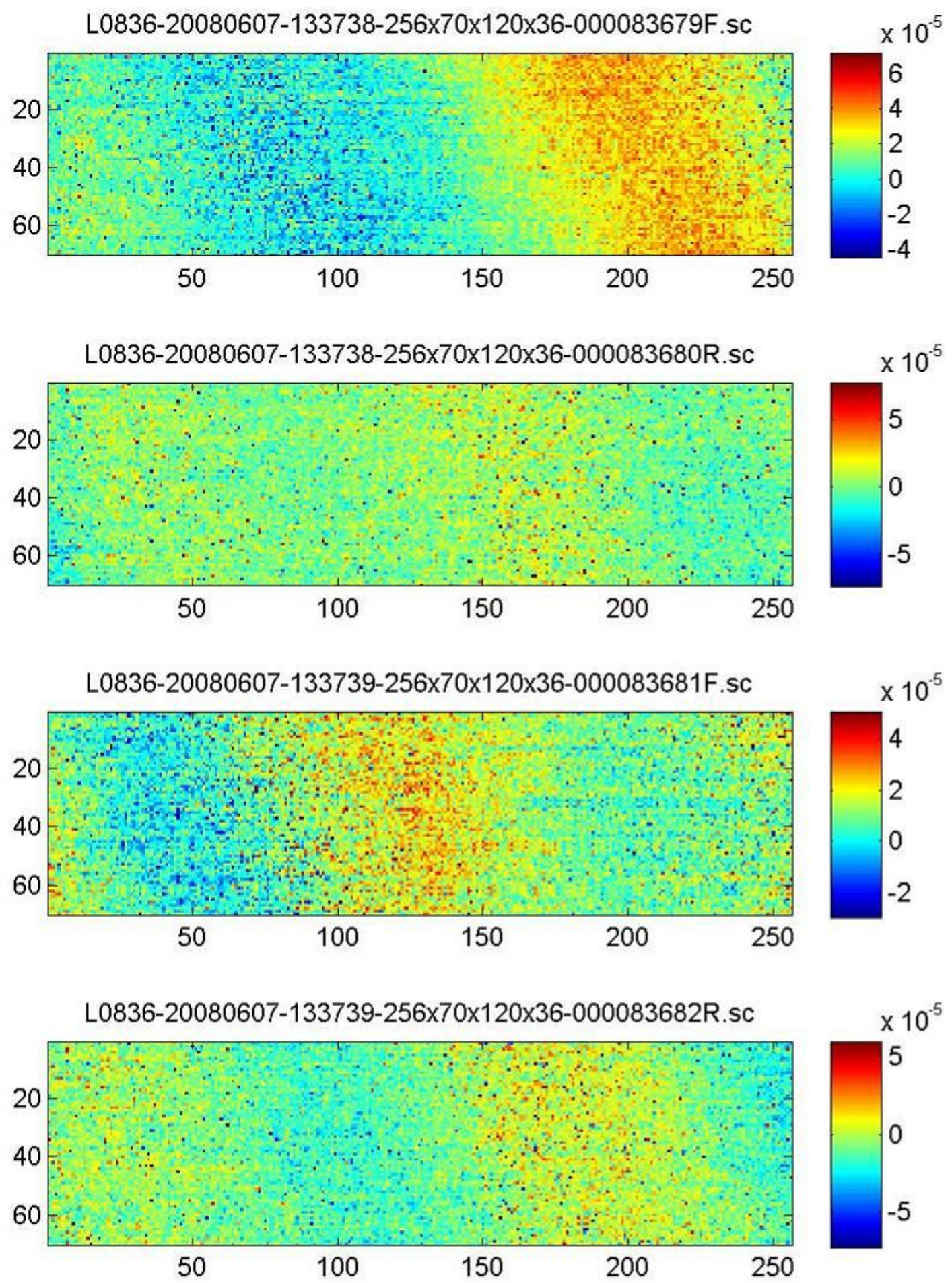

Figure 219. Background removed differential total spectral band radiance images.

The MWIR FIRST instrument calibrated radiance cubes have engineering units of radiance that can be converted to brightness temperature using the Planck function. All of the computed radiances for this signal processing are given in brightness temperature with units in degrees Kelvin. 
Principal Component Analysis: The next step in the signal processing involves this well-known data analysis technique. The goal was to reduce the dimensionality and the inherent redundancy of the data. Each pixel in the cube is adjacent to another pixel that captures essentially the same scene radiance. This redundancy can be effectively removed via PCA, which involves the eigenvalue decomposition of the covariance matrix. Specifically, the covariance matrix for each difference cube was computed from its reshaped 2-D matrix $(70 X 256=17920$ by 60$)$. An eigenvalue decomposition results in a matrix of eigenvectors, known as "Empirical Orthogonal Functions" or EOFs; an array of eigenvalues; and a matrix of eigenvectors projected onto the original data, known as "PC Scores". The original data can be reconstructed by a matrix multiplication of the PC Scores and the EOFs.

Figure 220 shows the efficiency of the PC representation of the MWIR FIRST radiance measurements for a sampling of different detector elements. The logarithm of the eigenvalues (unexplained variance) for the first 30 principal components of observed MWIR FIRST spectra for different focal plane array detector elements is shown. The abscissa is the PC number while the ordinate is the logarithm of the eigenvalue associated with the use of a given number of PCs. The red curve refers to the forward scan and the blue curve pertains to the reverse scan.

As can be seen, it only takes about 5-10 PCs to capture all of the significant variability of the MWIR FIRST observations. The authors have no explanation why the reverse scan (i.e., the blue curves) seem to have much more variance to explain than the forward scans (i.e., the red curves).

Since the method reduces the data redundancy, the first few EOFs contain the majority of variance. Various techniques exist in the literature for selecting how many EOFs to use. The simplest is to plot the eigenvalues in a "scree plot" and note where the "knee" of the curve occurs. Noise in the data can be "filtered" by reconstructing the data using these first few EOFs. This method was applied to the difference cubes, with reconstruction using only the first 12 EOFs.

While very effective with most data, the PCA filtering did not reveal the expected thermal wake vortex signature. The signal to noise ratio was simply too low for this application in this IR band (spectrum from $1800 \mathrm{~cm}^{-1}$ to $3600 \mathrm{~cm}^{-1}$ ). 


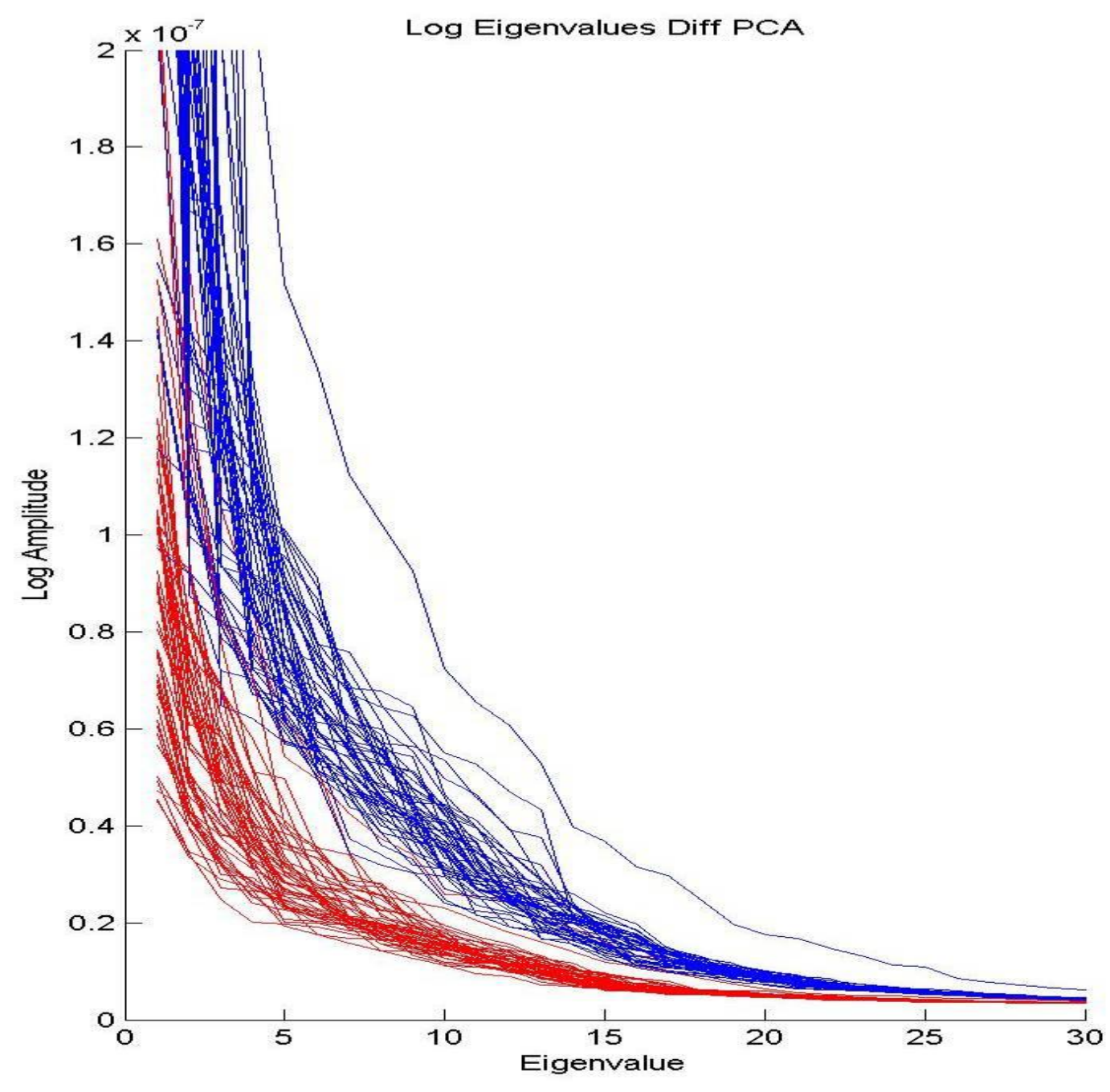

Figure 220. Unexplained variance for the first 30 principal components.

Figure 221 and Figure 222 show the same image sequence shown in Figure 218 and Figure 219 obtained from the first 12 PC filtered radiance spectra. The data are plotted in terms of brightness temperature, rather than radiance. The total range of brightness temperature deviation shown was $\pm 2^{\circ} \mathrm{K}$. Unfortunately, the simple PC noise filtering of the data did not enhance the signal to noise ratio of the MWIR FIRST data to the level needed to resolve the wake vortex brightness temperature signatures in the imaging spectrometer frames obtained during an aircraft landing. 

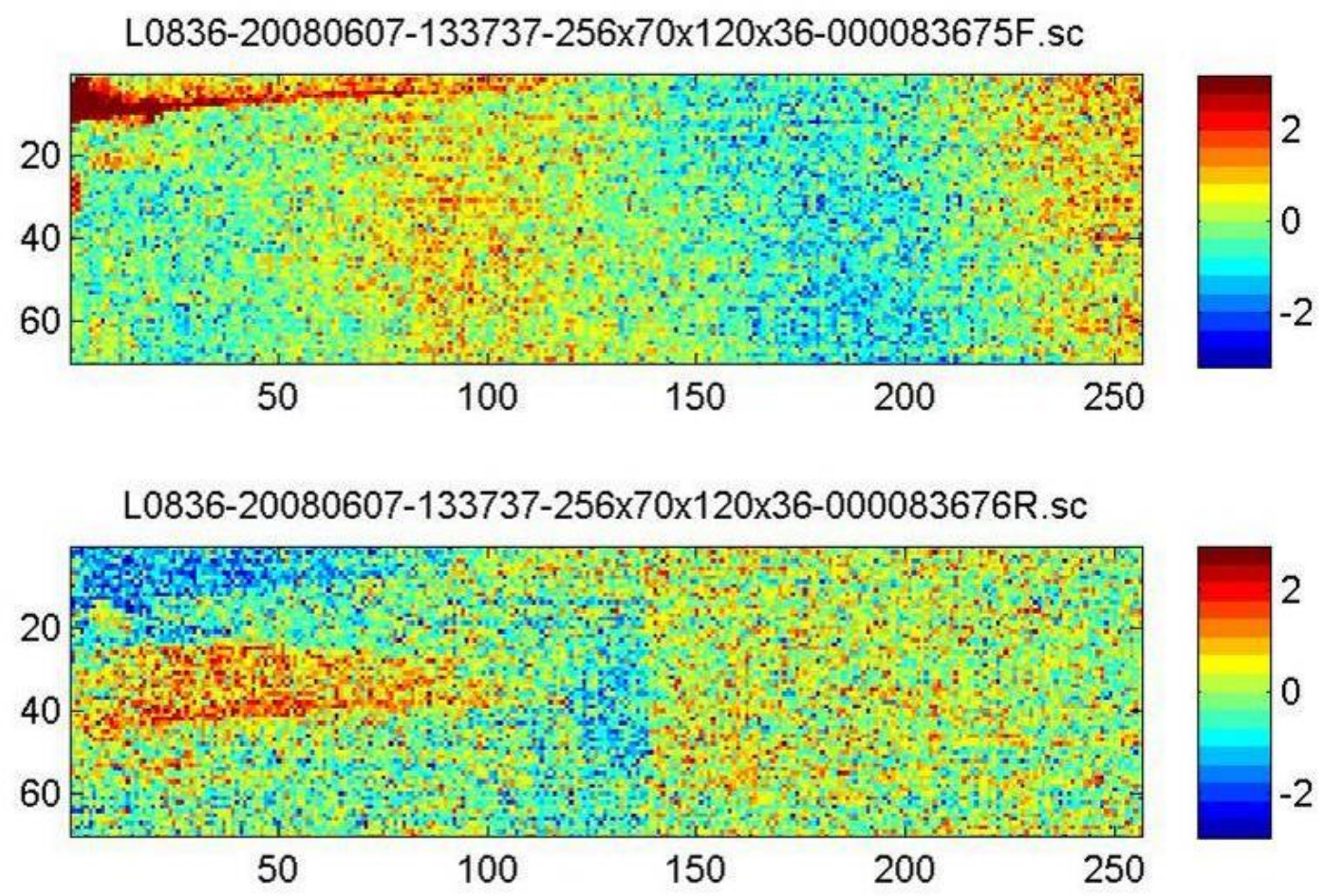

L0836-20080607-133738-256x70x120x36-000083677F.SC

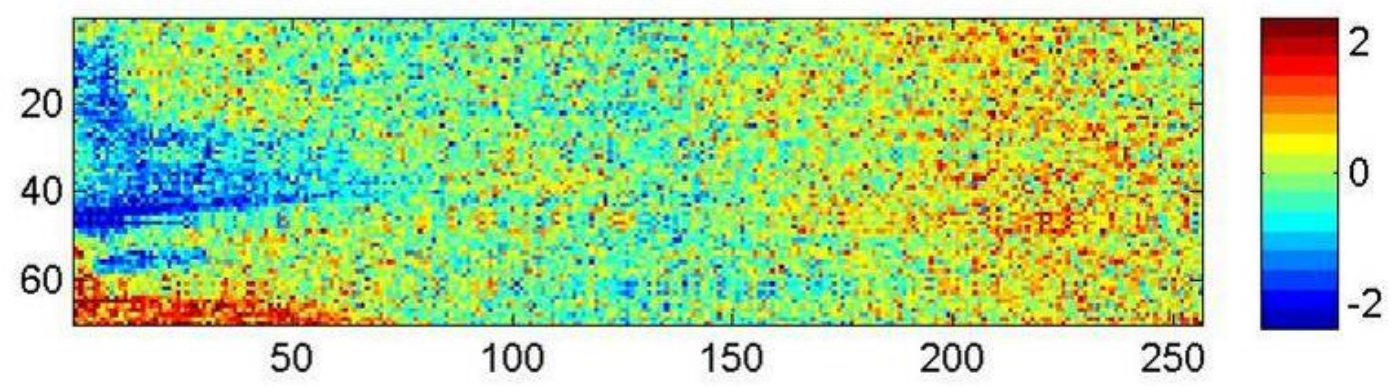

L0836-20080607-133738-256x70x120x36-000083678R.sc

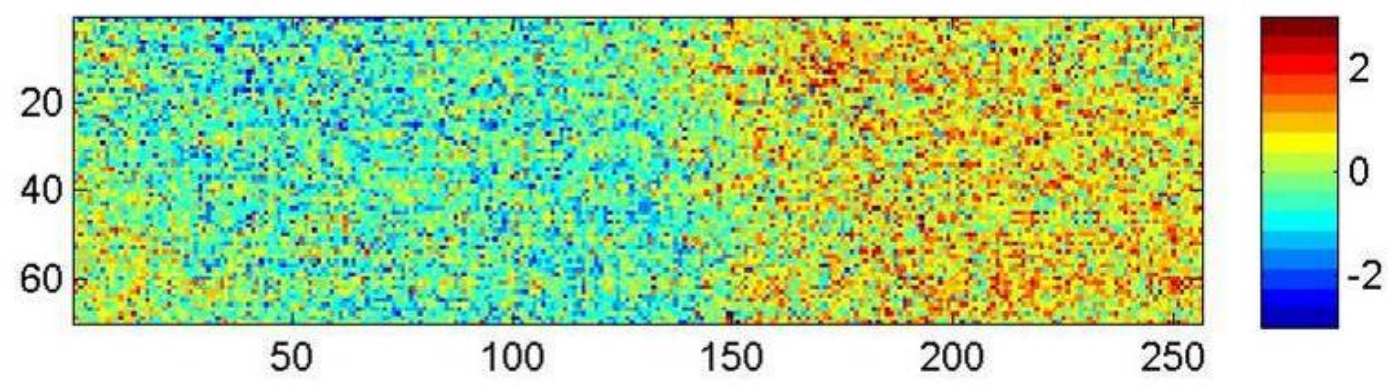

Figure 221. PC filtered differential brightness temperature. 

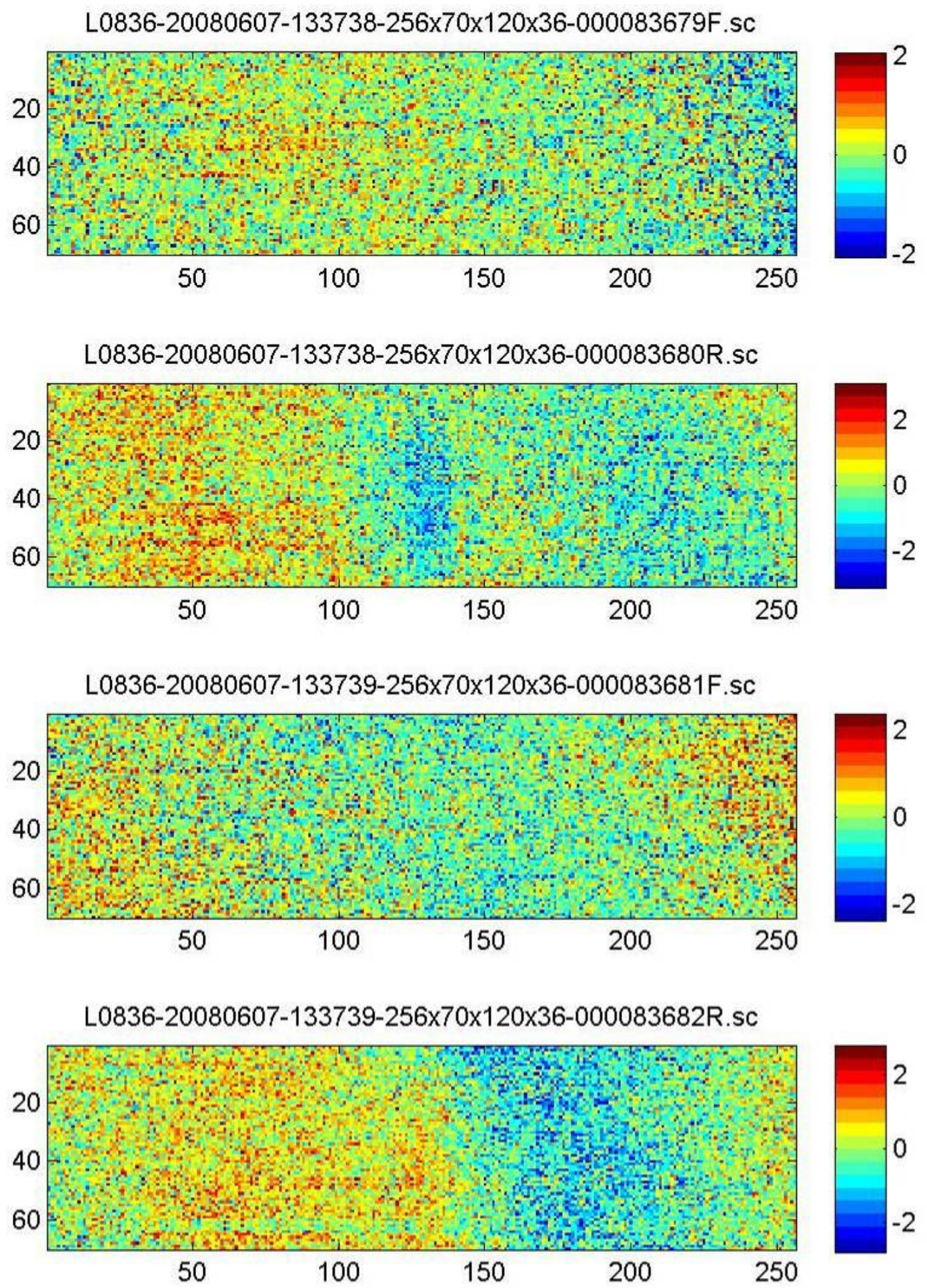

Figure 222. PC filtered differential brightness temperature.

Refined PCA: Since the PCA noise filtering was unable to extract the expected thermal signature, an alternate method was needed. The idea was to use PCs computed from the wake vortex radiance model data to perform the PCA filtering, as described earlier in Section 2.1.4. 
In that section, the LBLRTM radiance model was used to calculate the spectra of a wake vortex and transform it to the spectral range and spectral resolution of the MWIR FIRST data. These spectra were then used to compute PCs of the wake vortex radiance signal.

These PCs were then applied to the radiance spectra of the MWIR FIRST difference cubes for each pixel. The result was a new set of PC scores. Filtering was then performed by reconstructing with only a limited number of PCs. Again; images for each of the PC scores as well as for the reconstructed radiance signal were generated.

Since the modeled PCs for the vortex radiance signal do not have noise included, the noise should be filtered by this process and the vortex should be revealed. It was anticipated that the vortex might be resolved in either the images of each of the PCs or in the reconstructed radiance for the spectral bands, which have the highest vortex signals.

$\mathrm{CO}$ and $\mathrm{CO}_{2} / \mathrm{N}_{2} \mathrm{O}$ band Analysis: After reviewing the results of the radiance simulations, it was determined that the $\mathrm{CO}$ and $\mathrm{CO}_{2} / \mathrm{N}_{2} \mathrm{O}$ bands within the $2000-2300 \mathrm{~cm}^{-1}$ spectral region might contain a detectable wake vortex signature. The possibility did exist that this signature was being overwhelmed by noise in adjacent IR channels. All of the processing that was performed on the cubes was repeated, but with only the limited subset of cube data for the $\mathrm{CO}$ and $\mathrm{CO}_{2} / \mathrm{N}_{2} \mathrm{O}$ bands. These cubes had dimension $70 \times 256 \times 10$. Unfortunately, this analysis did not reveal a wake vortex signature.

Figure 223 and Figure 224 show $\mathrm{CO}$ and $\mathrm{CO}_{2} / \mathrm{N}_{2} \mathrm{O}$ spectral band brightness temperature images obtained with the MWIR FIRST instrument as the aircraft passed through the instruments field of regard. These data are the same as the prior coupled image sets. Note that the total range of the differential brightness temperature images is less than $1 \mathrm{~K}$. In spite of the very large enhancement of the brightness temperature range and the restriction of the spectral band pass to the spectral regions where the largest vortex signature should be observed, there is still no evidence of a wake vortex resolved by the MWIR FIRST data. Thus, it is concluded that the wake vortex signature, if it exists, is below the signal to noise ratio of the MWIR FIRST measurements. 

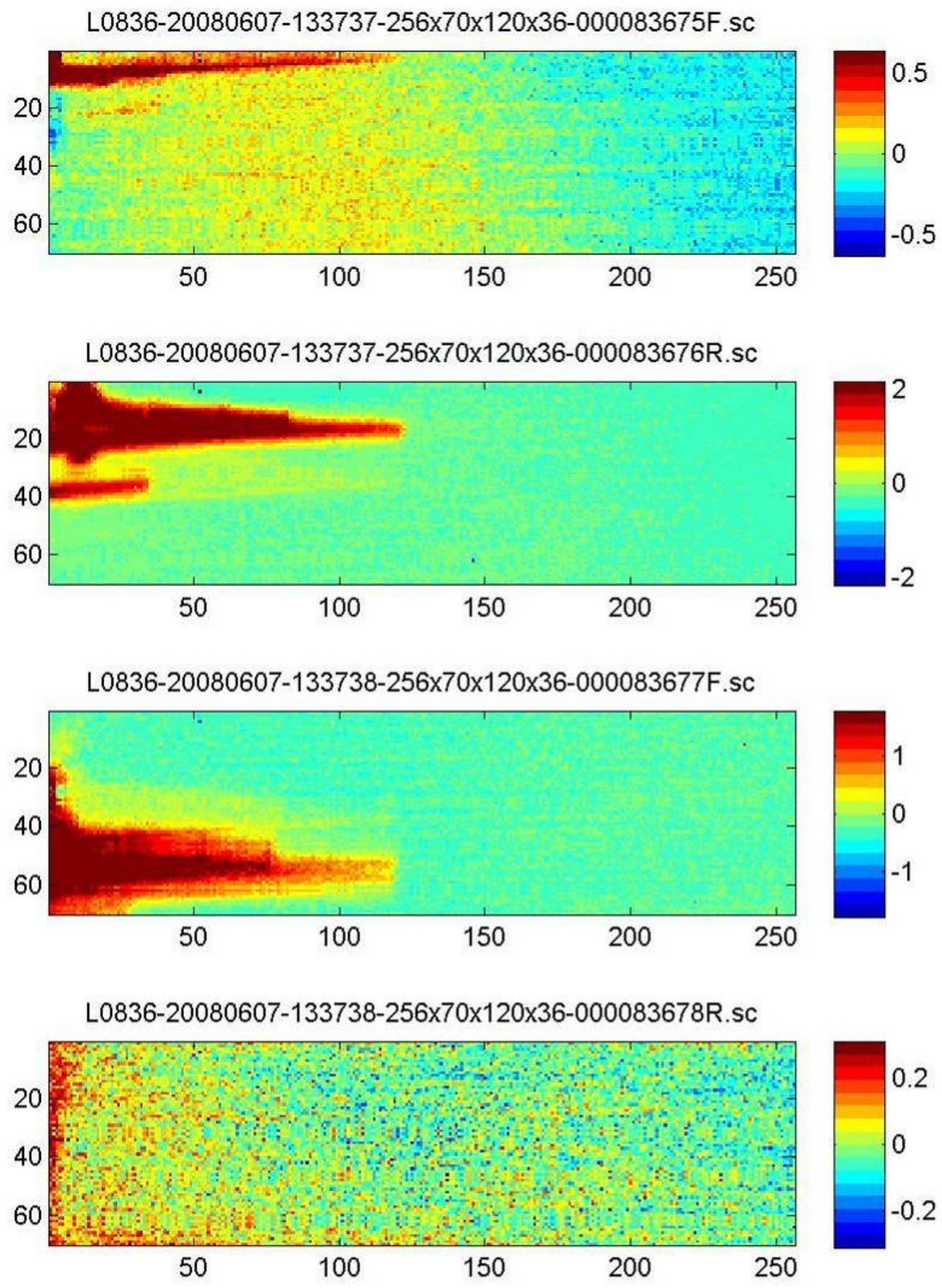

Figure 223. PC filtered differential $\mathrm{CO}$ and $\mathrm{CO}_{2} / \mathrm{N}_{2} \mathrm{O}$ band brightness temperatures. 

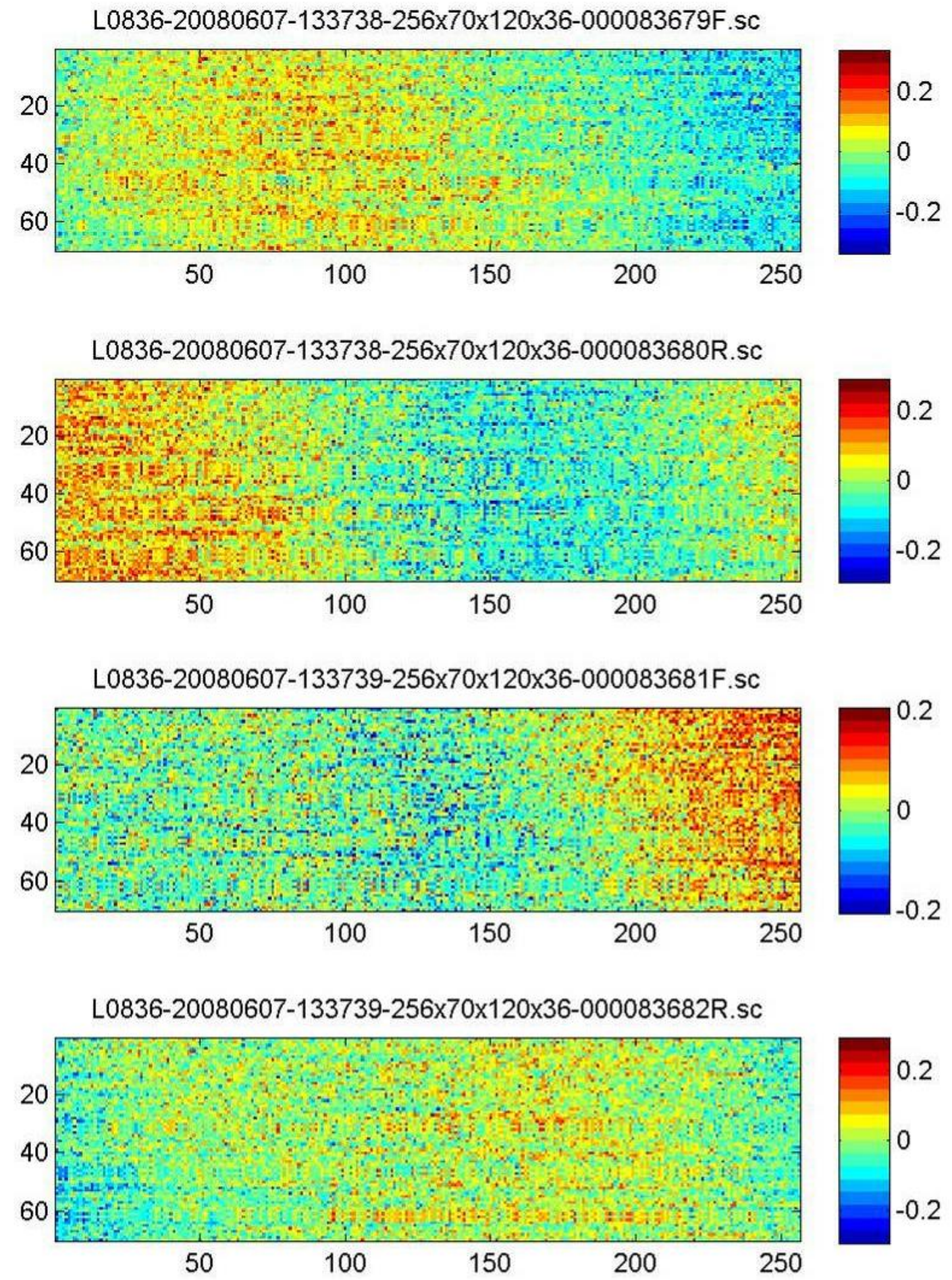

Figure 224. PC filtered differential $\mathrm{CO}$ and $\mathrm{CO}_{2} / \mathrm{N}_{2} \mathrm{O}$ band brightness temperatures.

Noise Floor Estimation: In order to quantify a threshold for the minimum detectable bounds for the wake vortex thermal signature, a simple statistical analysis was performed on the difference cubes for a representative pixel. 
Figure 225 shows the mean and standard deviation (STD) of the brightness temperatures observed for all the pixels and frames associated with the aircraft passage sequence, plotted versus cube number or time. As can be seen the standard deviation of the brightness temperature measurements is near the $0.5 \mathrm{~K}$ level for most detector elements (i.e., image pixels). Since the theoretical calculations presented above show that the wake vortex brightness temperature signal has an amplitude generally much less than this apparent noise level, it is no wonder that wake vortices in the WAVEx MWIR FIRST thermal imagery cannot be resolved. In the figure, the "A/C" notation indicates the "time" of the landing aircraft.

Figure 226 and Figure 227 show imagery of the mean and standard deviation of the spectral band for each pixel in the wing tip region. As the aircraft moved thru the instrument FOV, the wing tip traversed a vertical line at about horizontal pixel number 110 . The wing tip region was thus selected to be $110 \pm 40$ pixels (central portion of the $70 \times 256$ pixel array) or $70 \times 80$. The images of the mean and standard deviation statistics clearly show the impact of the aircraft on the observed radiances but there is no sign of a wake vortex. Thus it is concluded from all the analyses performed thus far, that the signal to noise ration of the MWIR FIRST measurements is inadequate for resolving the very small spectral radiance features associated with a wake vortex.

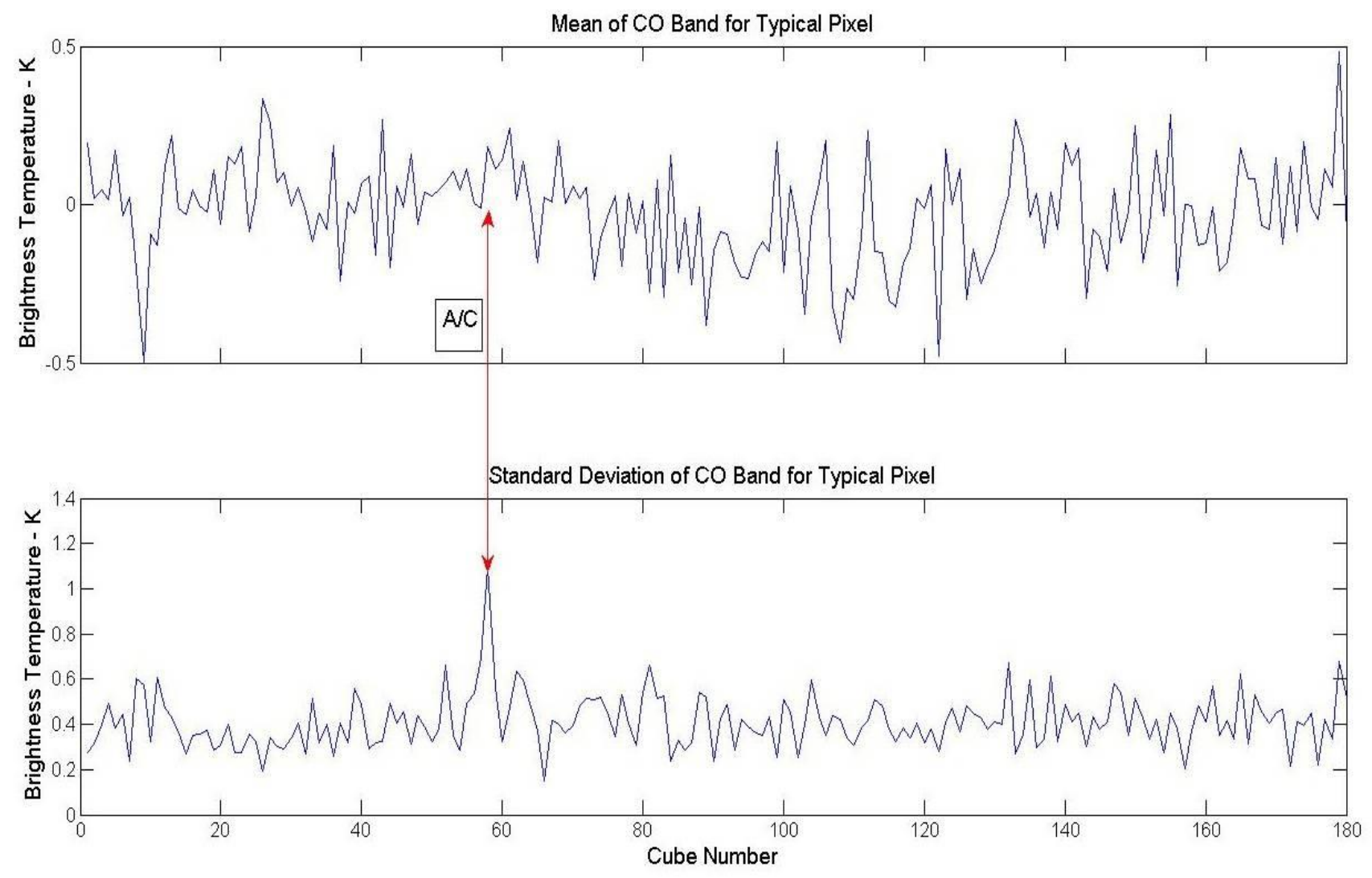

Figure 225. Mean and STD of $\mathrm{CO} \& \mathrm{CO}_{2} / \mathrm{N}_{2} \mathrm{O}$ band brightness temperatures. 

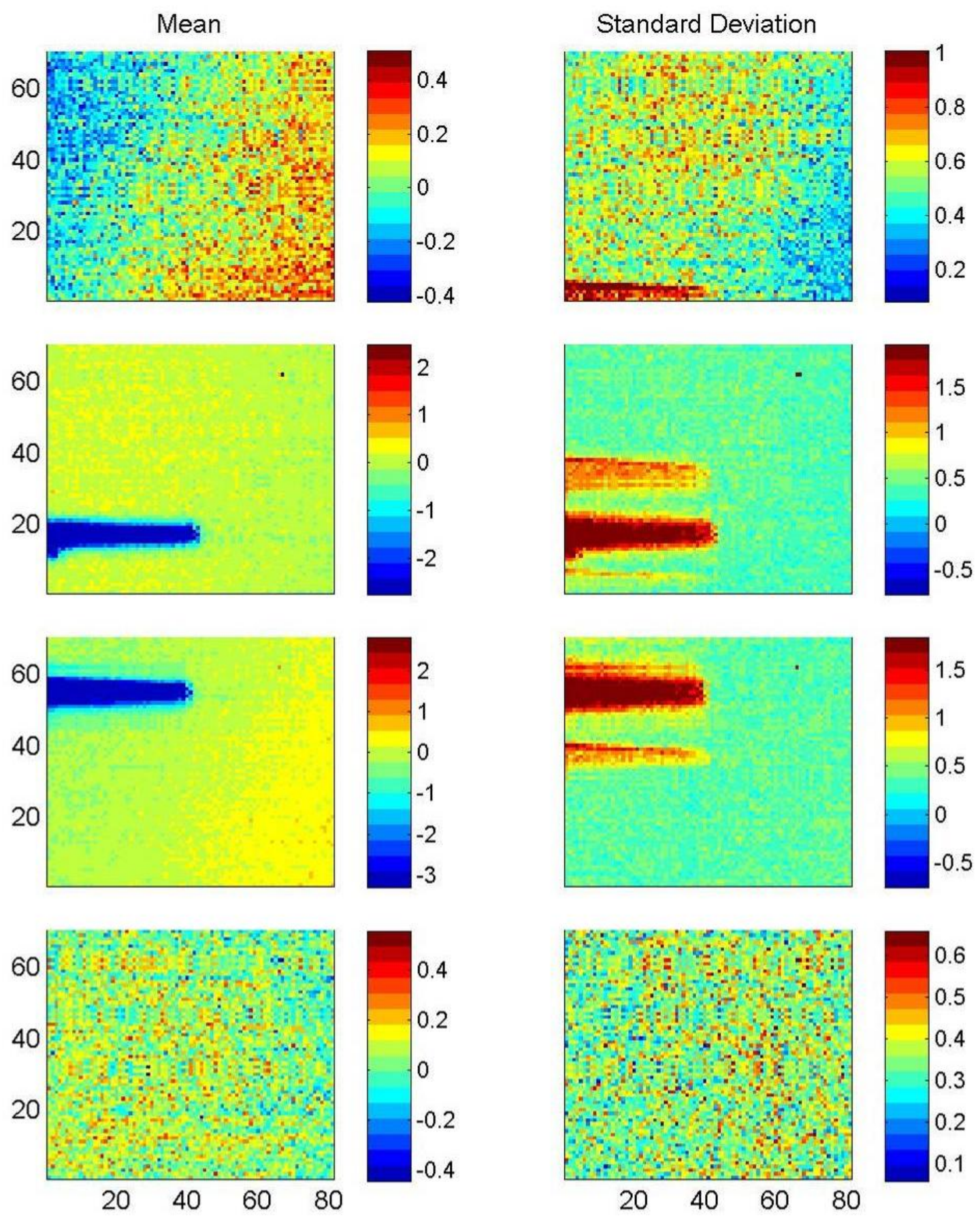

Figure 226. Mean and STD imagery for the $\mathrm{CO}$ and $\mathrm{CO}_{2} / \mathrm{N}_{2} \mathrm{O}$ spectral band. 

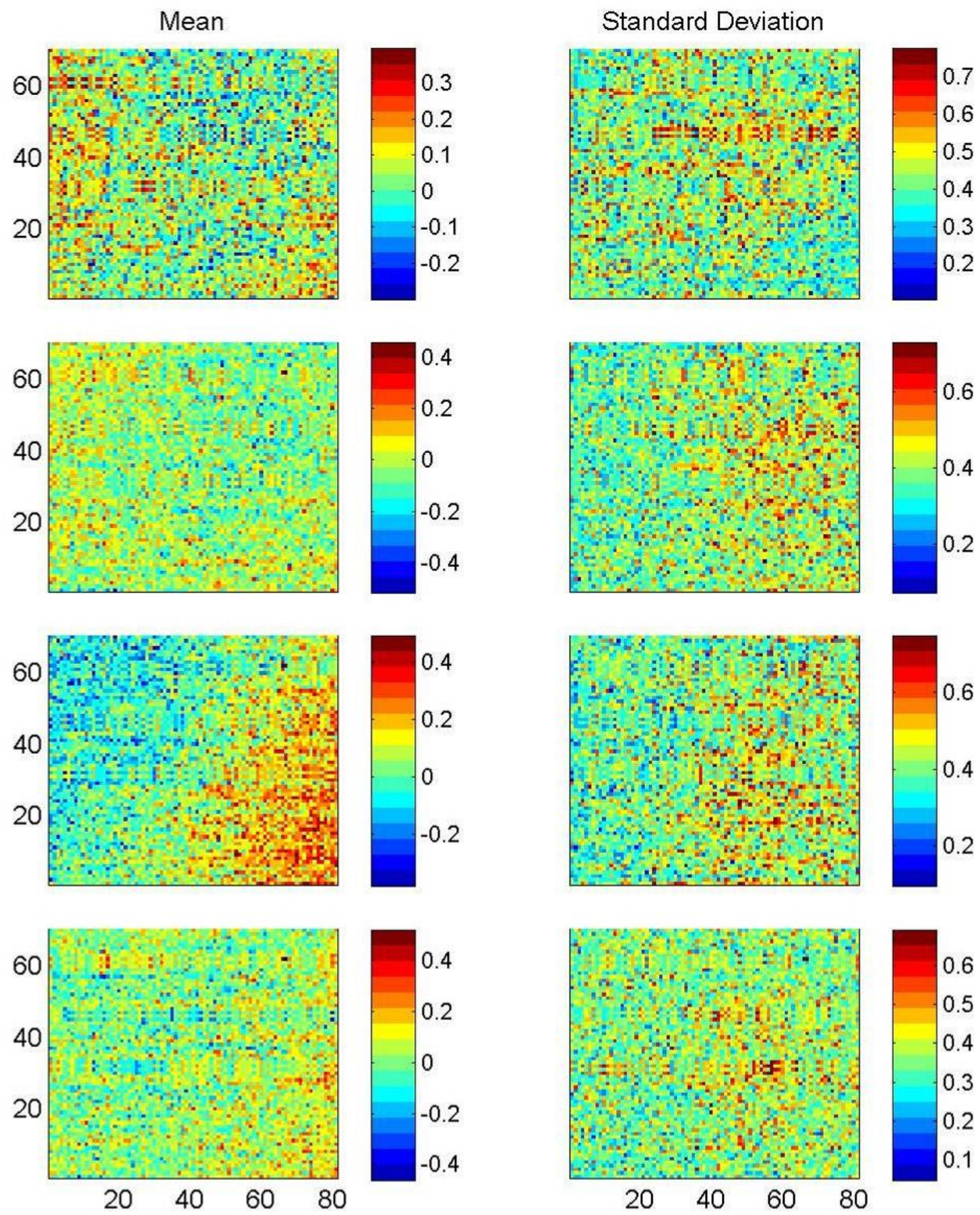

Figure 227. Mean and STD imagery for the $\mathrm{CO}$ and $\mathrm{CO}_{2} / \mathrm{N}_{2} \mathrm{O}$ spectral band.

\subsubsection{D\&P Data Analysis Results}

As mentioned above, the modeled radiometric signatures of aircraft wake vortices are small, on the order of $0.1 \mathrm{~K}$, so a clear, blue sky was desired as the coldest and most constant background. The weather was uncooperative during the test week, with frequent clouds and rain. The test was extended an extra day in order to operate with a clear sky background for one morning, during which several landing aircraft flew through the instrument fields of view. Analysis of the 
resulting data did show the aircraft itself in the MWIR FIRST imagery and in the D\&P spectra, the standard deviation of the brightness temperature image measurements, for the operating conditions used in the field measurements, was about $0.5 \mathrm{~K}$, which is significantly larger than the expected signatures.

The D\&P was able to resolve what could potentially be a wake vortex about 80 seconds after the passage of a DC9 aircraft. Figure 228 shows radiance (in color) versus wavenumber in the $750-$ $900 \mathrm{~cm}^{-1}$ region (horizontal axis), with time increasing upward on the vertical axis. The numbers on the time axis are the number of spectra recorded since the aircraft passed overhead, and the interval between spectra is $300 \mathrm{~ms}$. The mean radiance spectrum has been subtracted in order to highlight the variations, and the plot has been smoothed by five-point boxcar averaging on both axes. The blue band at about 200 spectra ( $80 \mathrm{~s}$ after the aircraft passage) could be interpreted as the passage of a cold vortex core through the D\&P's field of view, however, background data from the same day shows similar variations, though on a larger timescale.

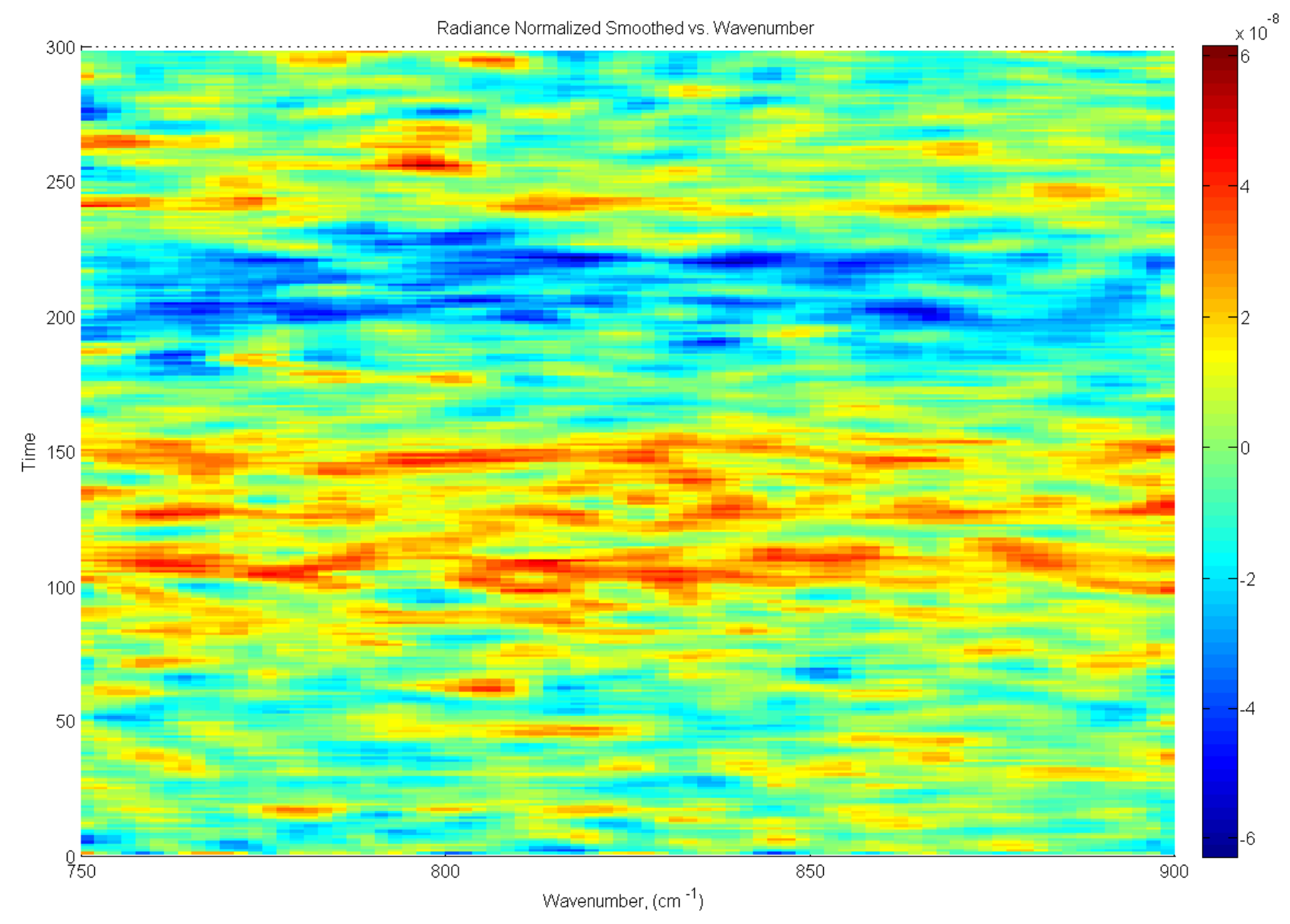

Figure 228. D\&P DC9 060708 radiance versus wavenumber.

The D\&P data was higher resolution and had less noise than the Telops MWIR FIRST, therefore it was very important to the field program to analyze this data, though it was originally intended to be a part of the field test for intercomparison purposes only. While the Telops MWIR FIRST 
is an imager that looked 20 degrees above the horizon, the D\&P is a single line of sight instrument that was pointing vertically.

The analysis focuses on the DC9 aircraft that flew through the field of view on Saturday June 7, 2008, after beginning other WAVEx data. June $7^{\text {th }}$ was the only clear day, and therefore offered the most uniform background. In addition, the DC9 is the largest aircraft in any data set, and therefore has the largest wake vortex. Lastly, the DC9 fuselage flew directly in the line of sight, offering the best chance to catch a wake vortex drifting into the field of view due to cross winds. GTRI performed some simple analysis to help visualize the data.

Data visualization tools were developed to view the data taken with the D\&P Spectrometer. These tools consisted of Matlab scripts to read in the D\&P data, convert to appropriate units, and generate different types of plots so that times and wavenumbers of interest could be selected for further analysis.

To ensure that the D\&P data had good fidelity and was converted appropriately, a script to generate 2D plots of radiance versus wavenumber for times of interest was created. Planck blackbody radiation curves at the ambient air temperature are shown in the plot of Figure 229. The black curve in the plot is the blackbody radiation curve and each of the other curves in the plot corresponds to the radiances at the time stamp in the D\&P data file (shown in the plot legend). As can be seen in the plot, the D\&P data matches well with the blackbody curve for the wavenumbers where atmospheric absorption is high. 


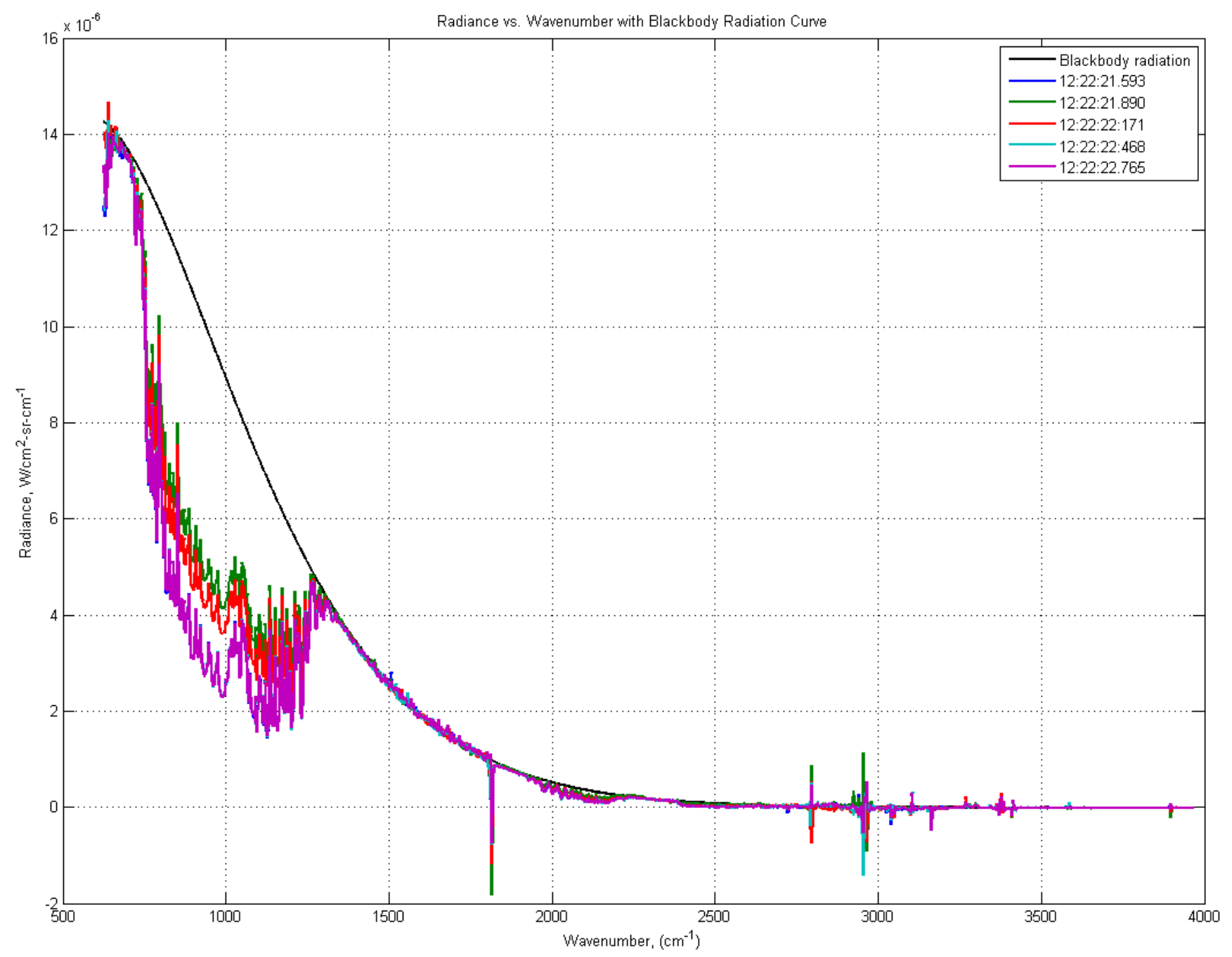

Figure 229. 2D Plot of Radiance vs. Wavenumber

During WAVEx, the D\&P was set in continuous acquisition mode and took measurements approximately every $\sim 300 \mathrm{~ms}$. In order to view minutes of data at the same time, a script to generate 3D surface plots of radiance vs. wavenumber over time was developed. The script has the ability to limit the plotted wavenumbers and data files to those that are requested to facilitate zooming into areas of interest. Figure 230 is an example 3D surface plot where a "bump" in the radiance values occurs just after 100 time samples into the sequence. This bump is the aircraft of interest flying through the D\&P field of view (FOV). Figure 231 is a plot that is zoomed in on the "bump." 


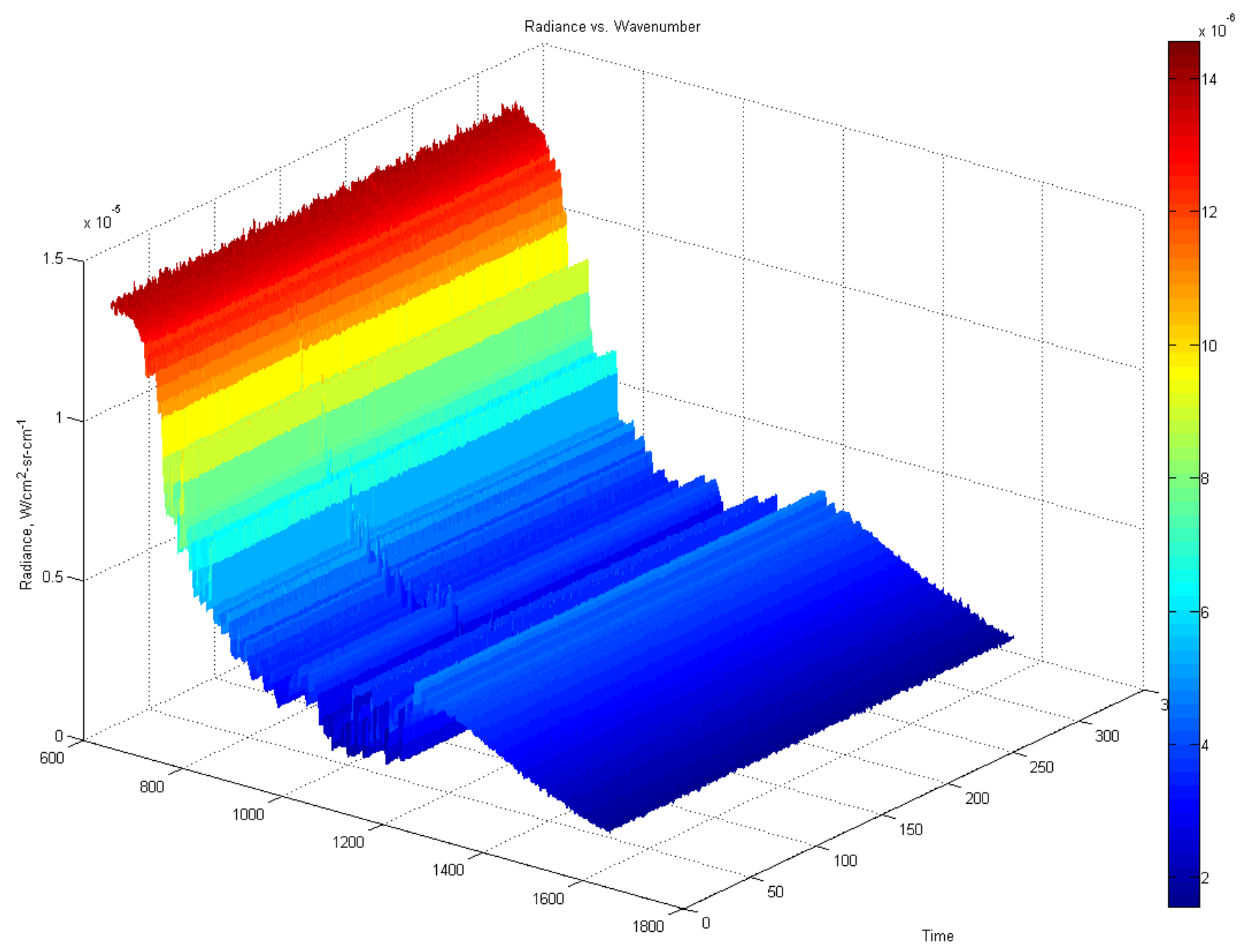

Wavenumber, $\left(\mathrm{cm}^{-1}\right)$

Figure 230. 3D Surface Plot of Radiance versus Wavenumber. 


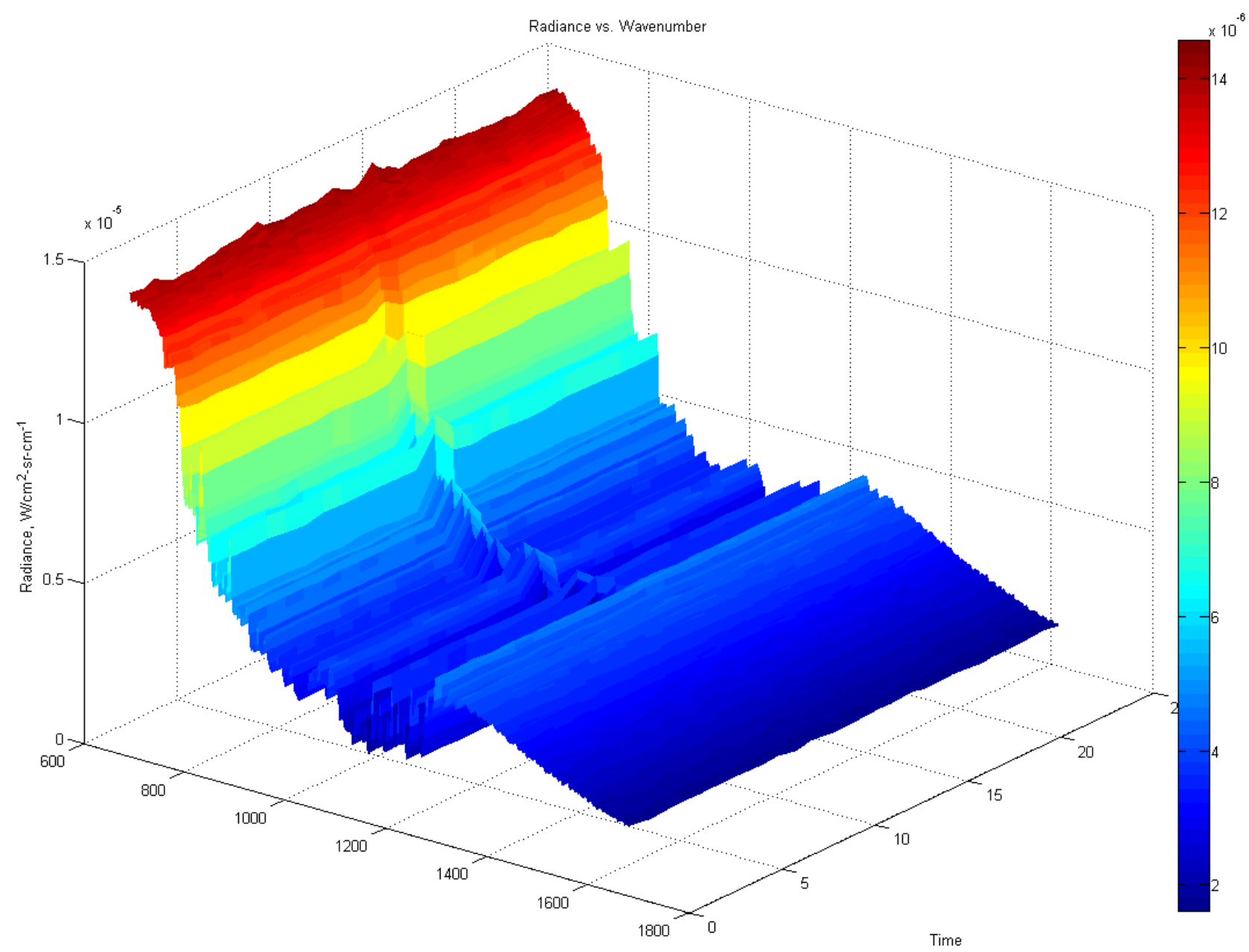

Wavenumber, $\left(\mathrm{cm}^{-1}\right)$

Figure 231. Detail of previous 3D Surface Plot

For looking at sudden radiance changes in time, another useful plot is a $2 \mathrm{D}$ plot of wavenumber vs. time with the radiances plotted on a color scale within the plot. Figure 232 shows the data from Figure 230 plotted in this manner. The aircraft is seen as a stripe in the data just after the time of 100 samples mark. 


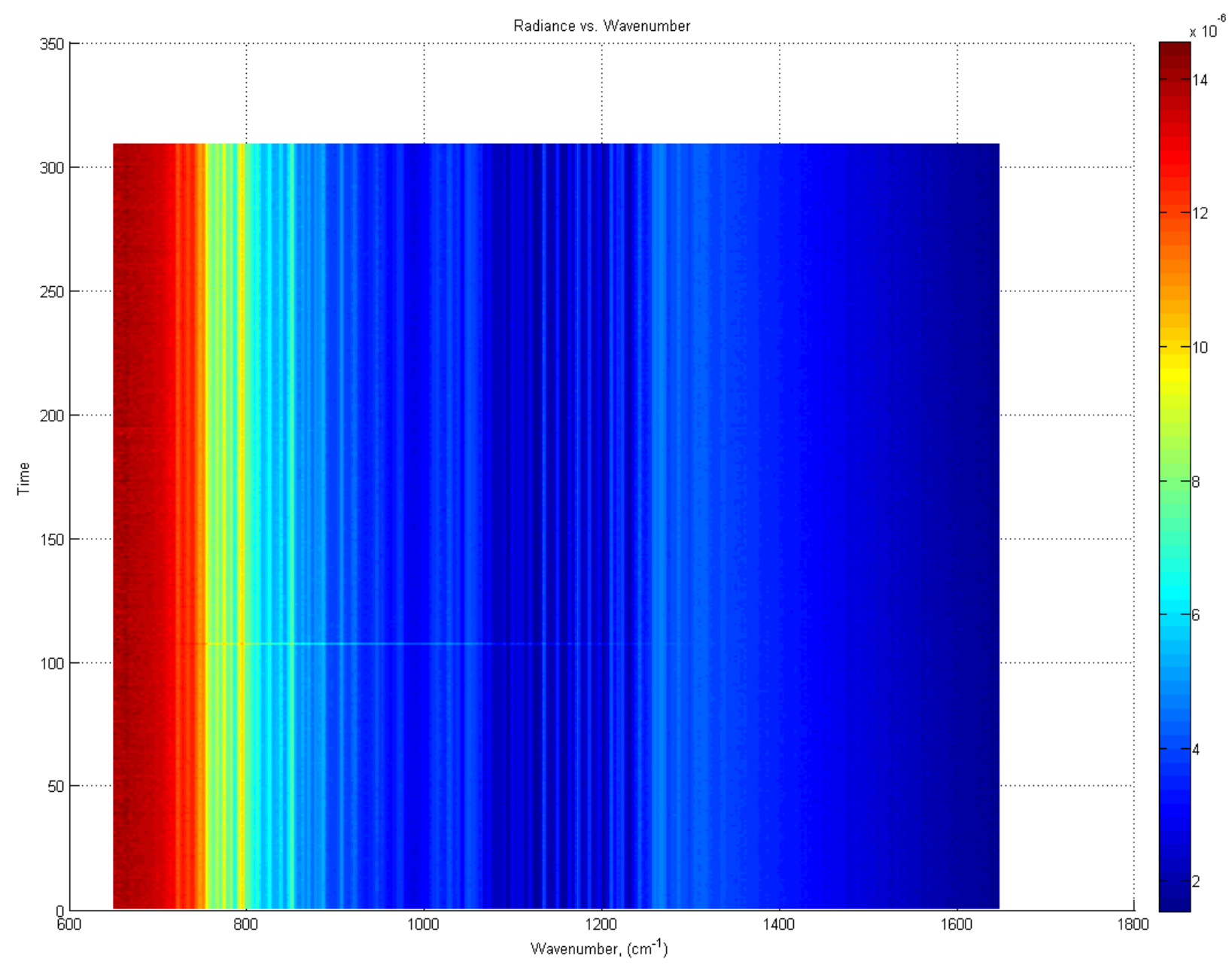

Figure 232. 2D Plot of Radiance, Wavenumber vs. Time

To make these radiance changes even more evident, a script for a radiance difference plot, which takes each time sample and subtracts it from its neighbor, was created. These plots are useful because areas where the radiance is not changing show up as a single color. Figure 233 shows the data from Figure 232 plotted in this manner. 


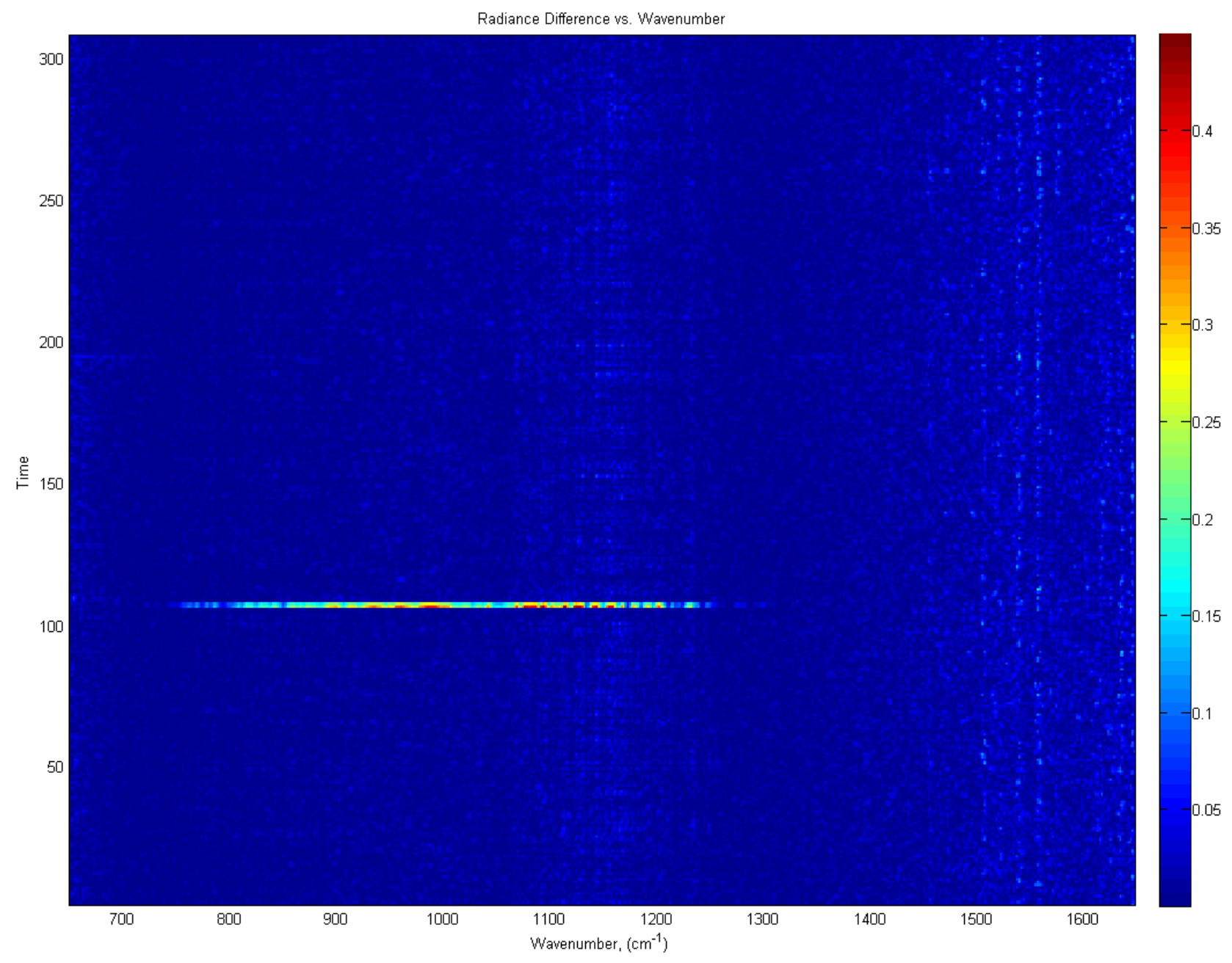

Figure 233. Radiance Difference Plot

Figure 234 shows the same difference plot zoomed in to the same amount of time used in Figure 231. 


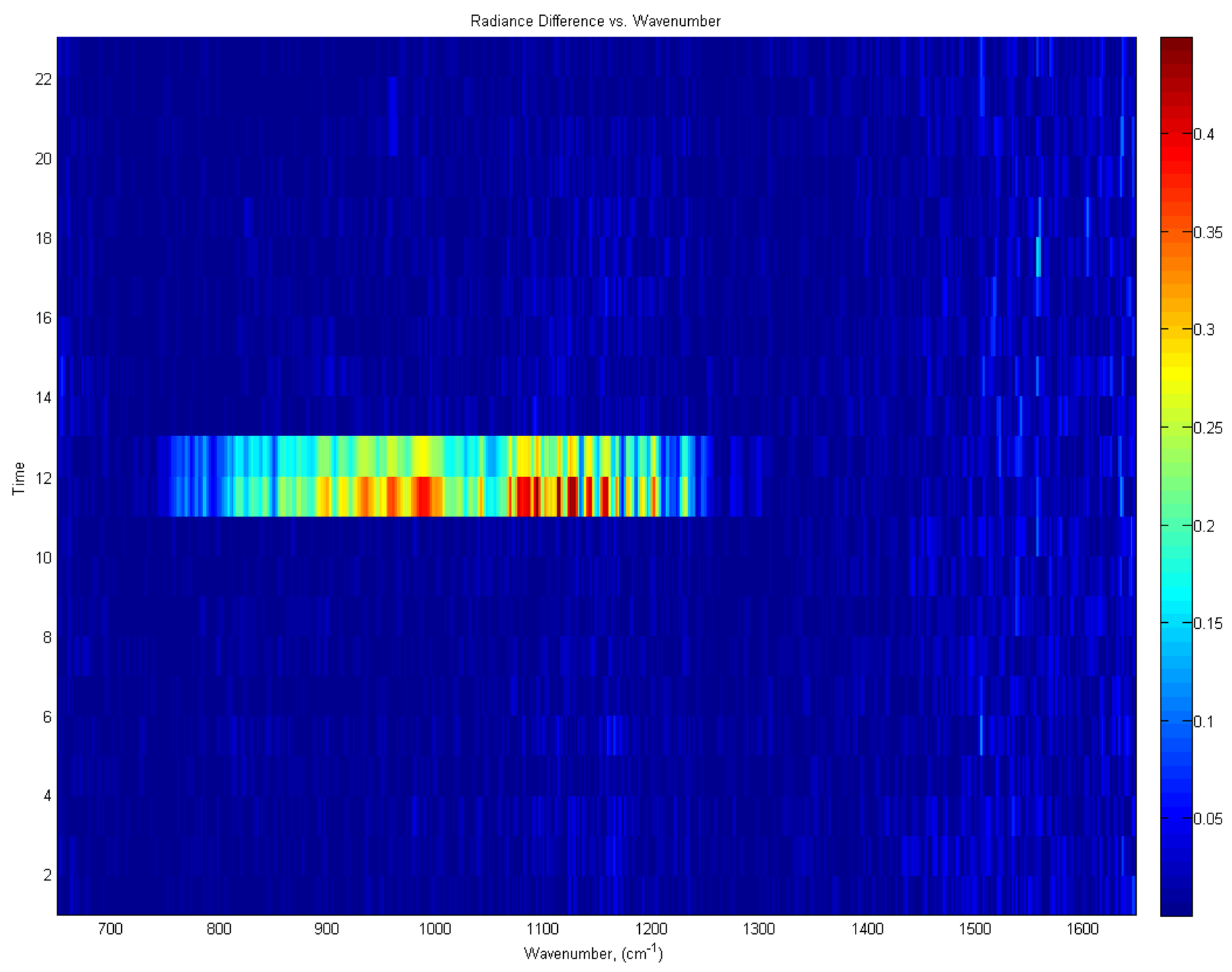

Figure 234. Zoomed in Radiance Difference Plot

As expected, one issue that became evident from the WAVEx data was that cloud cover in the atmosphere had a huge impact on the ability to look for radiance changes in the D\&P data. Figure 235 shows radiance data from Data Set 2 on June 7, 2008. The notes for this day describe that this data set was taken during a period of perfect sky (no clouds). 


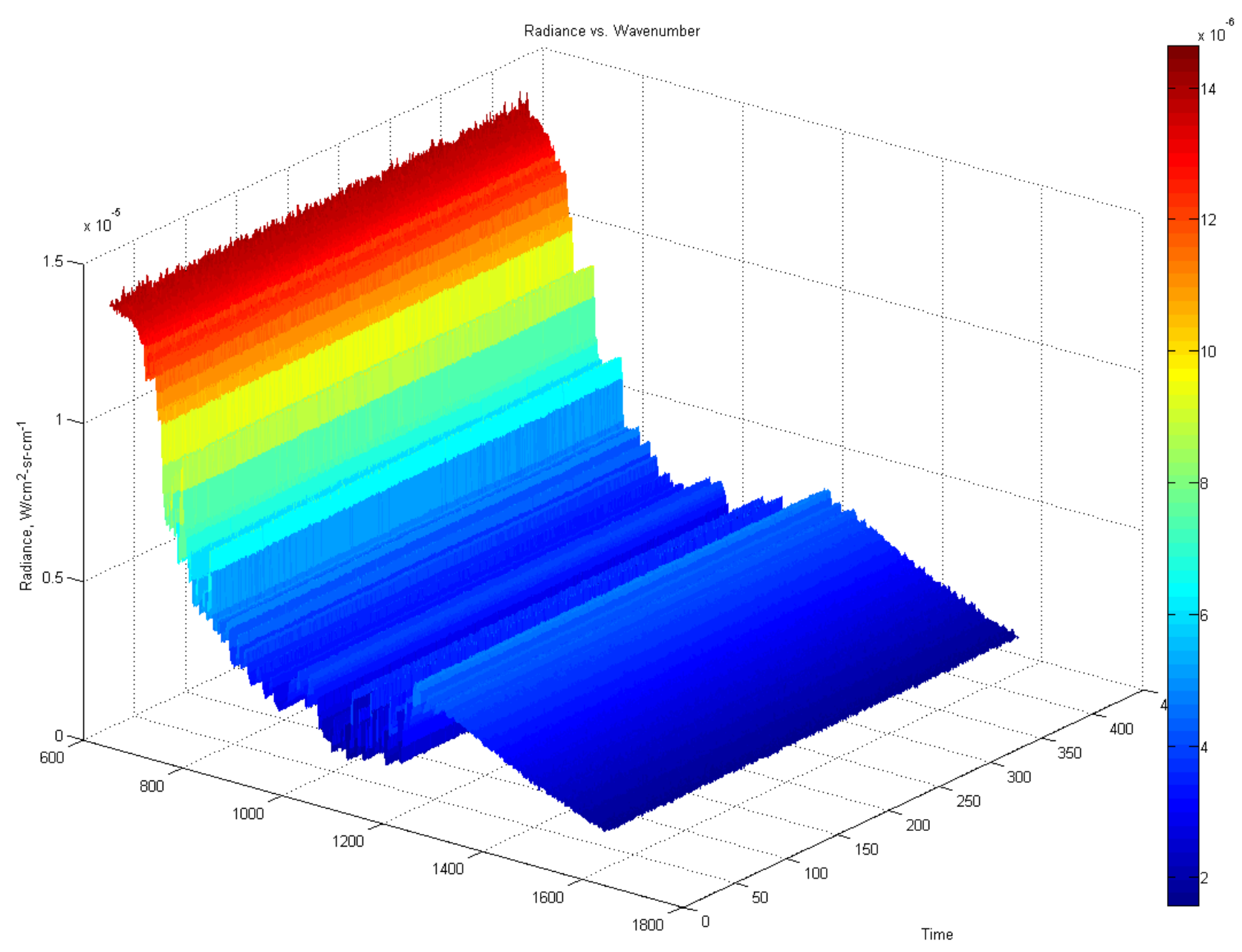

Wavenumber, $\left(\mathrm{cm}^{-1}\right)$

Figure 235. Clear Sky Radiance Surface Plot 
Contrasting this is Figure 236, which shows radiance data from Data Set 5 on the same day. The notes for this data say that it was taken when the sky was overcast. The change in radiance data from the clear sky condition earlier in the day is readily apparent from blackbody shape of the data in Figure 236.

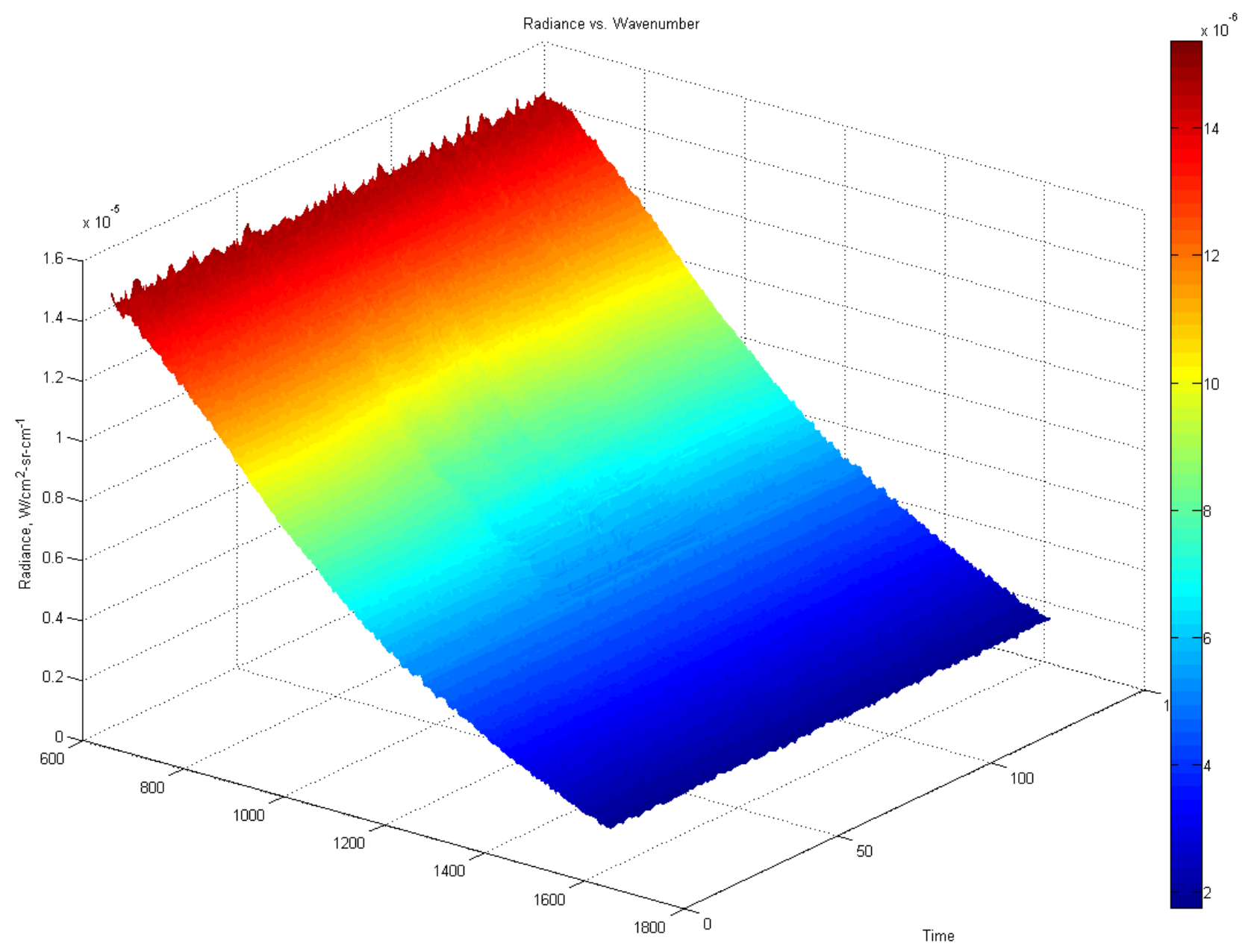

Wavenumber, $\left(\mathrm{cm}^{-1}\right)$

Figure 236. Overcast Radiance Surface Plot 
Between these two extremes (clear sky and overcast) are periods of time where clouds appeared, but the sky was not totally overcast. Data Set 9 on June 2, 2007 was a long data collection that began with a few cirrus clouds and ended with thicker cloud cover. Figure 237 and Figure 238 show radiance surface plots from the beginning and end of this data set, respectively. The clouds are evident in Figure 238 from the waviness of the data over time as clouds pass through the field of view. Although the cloud cover was not nearly as thick or consistent as the overcast sky in Figure 236. Figure 240 zooms in on the time intervals just before and after the DC9 passes through the FOV. Based on the length of time that the aircraft was in the FOV, in addition to body length and typical airspeeds at landing, it is concluded that the signal is from the fuselage of the DC9.

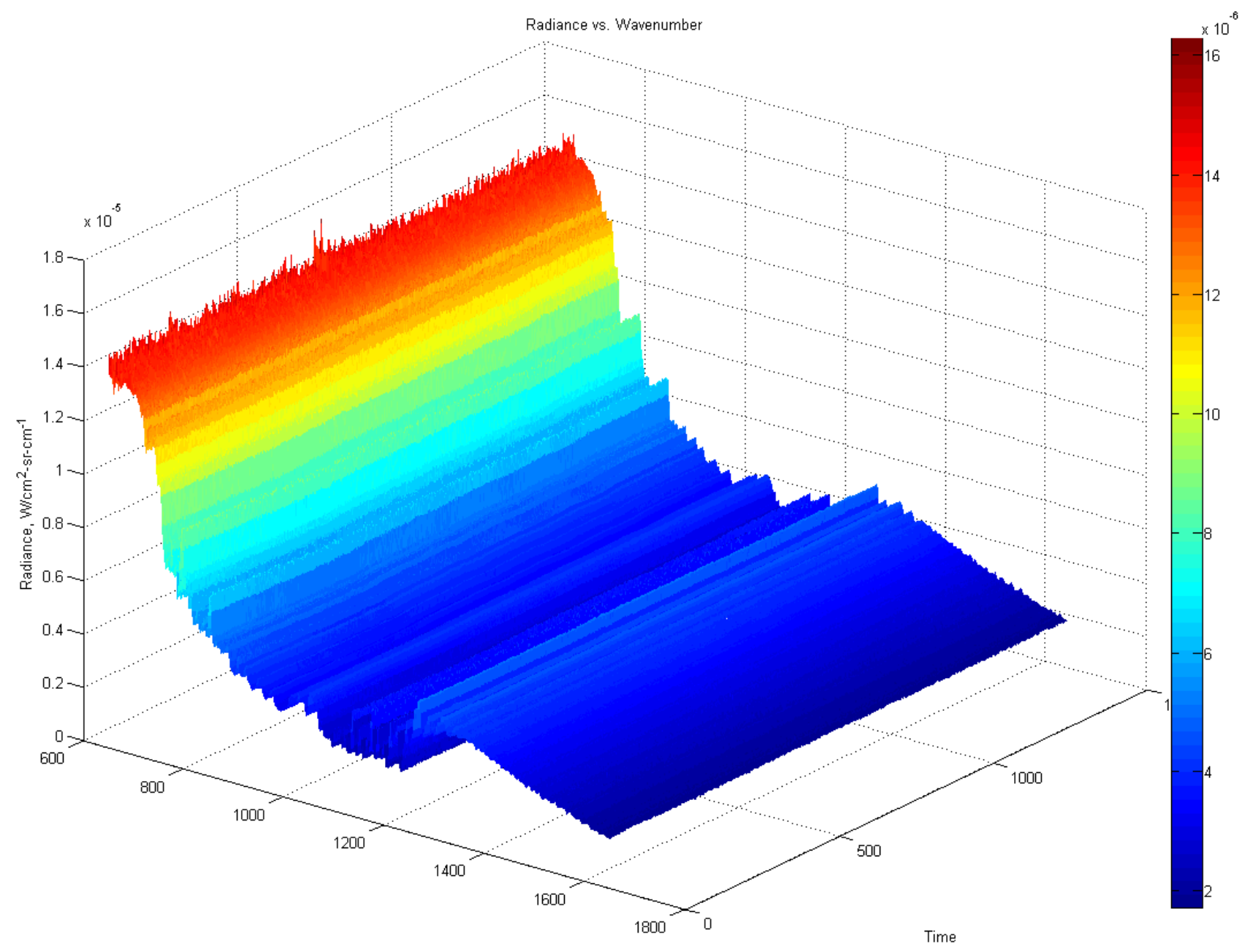

Wavenumber, $\left(\mathrm{cm}^{-1}\right)$

Figure 237. Data Set 9 Beginning Radiance Surface Plot 


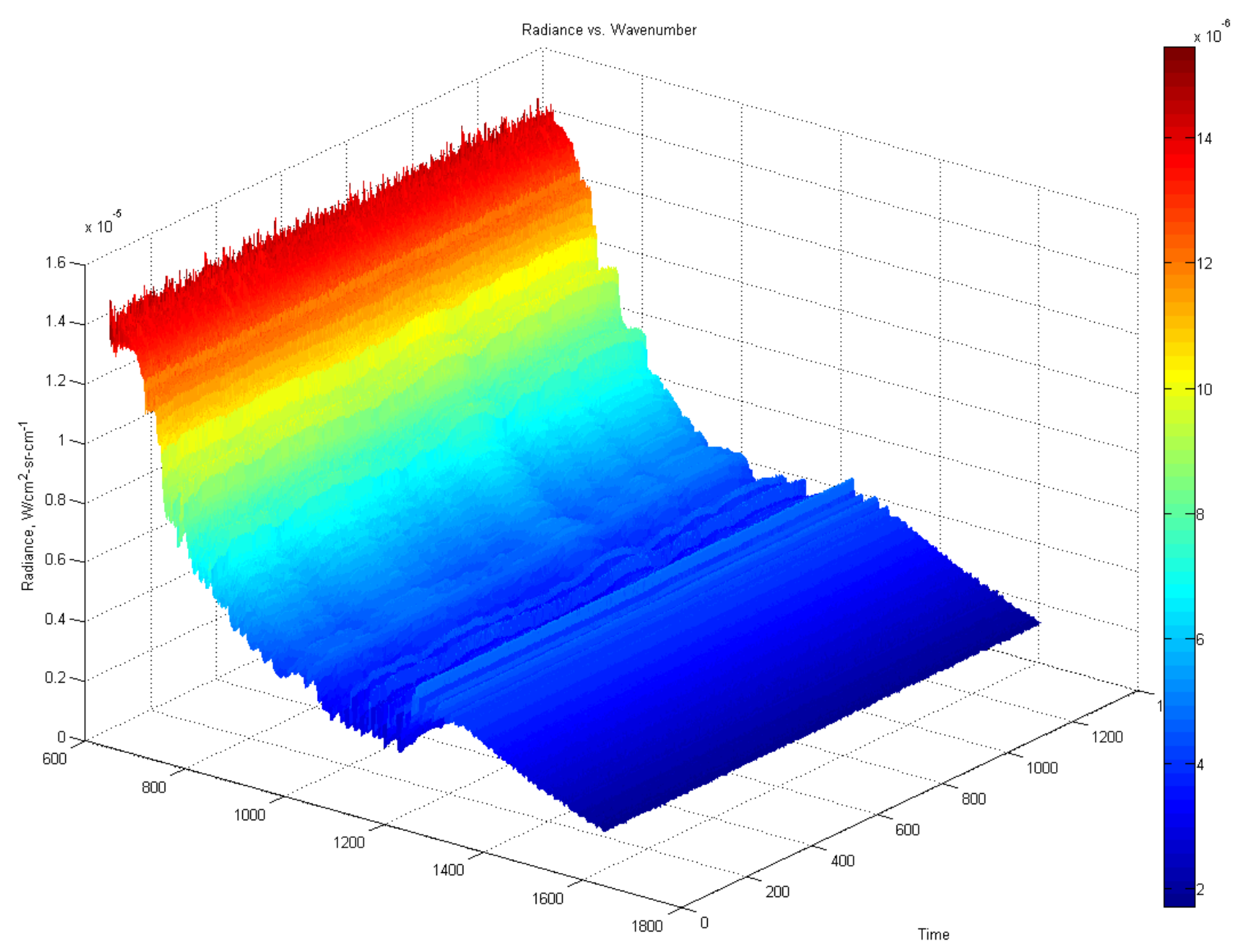

Wavenumber, $\left(\mathrm{cm}^{-1}\right)$

Figure 238. Data Set 9 End Radiance Surface Plot

A process was used to choose which of the D\&P data sets should be selected for further analysis. It required finding a clear sky time period in the data where an aircraft flew through the Field of View (FOV) of the instrument. Figure 239 shows the radiances for Data Set 4 on June 7, 2008. The background radiances are similar to those seen in the clear sky plot above; however, there are a few consecutive time intervals where the radiances are much larger than the rest. This increase in radiances coincides with the time that the fuselage of a DC9 passed through the D\&P FOV. 


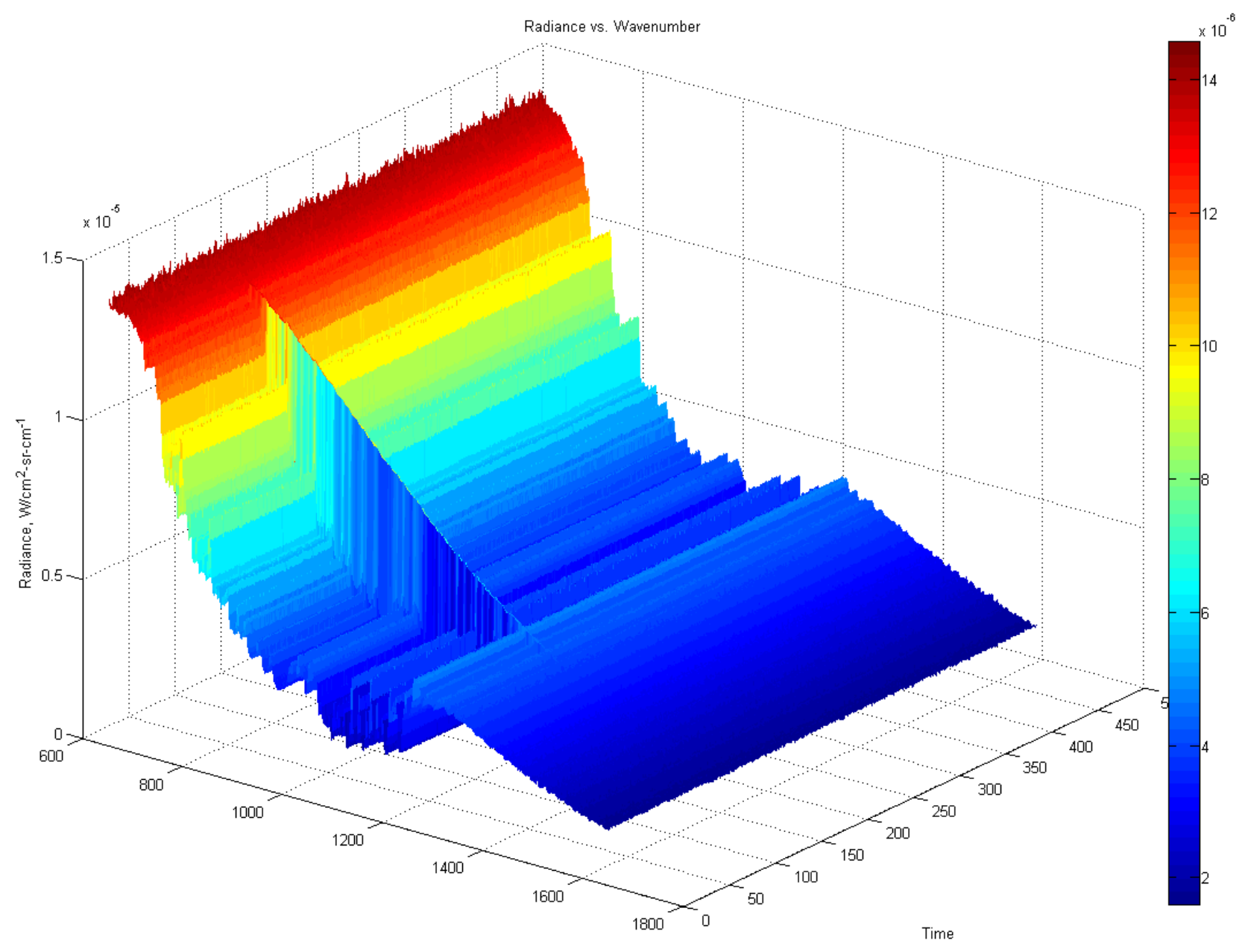

Wavenumber, $\left(\mathrm{cm}^{-1}\right)$

Figure 239. DC9 Passing Through FOV 


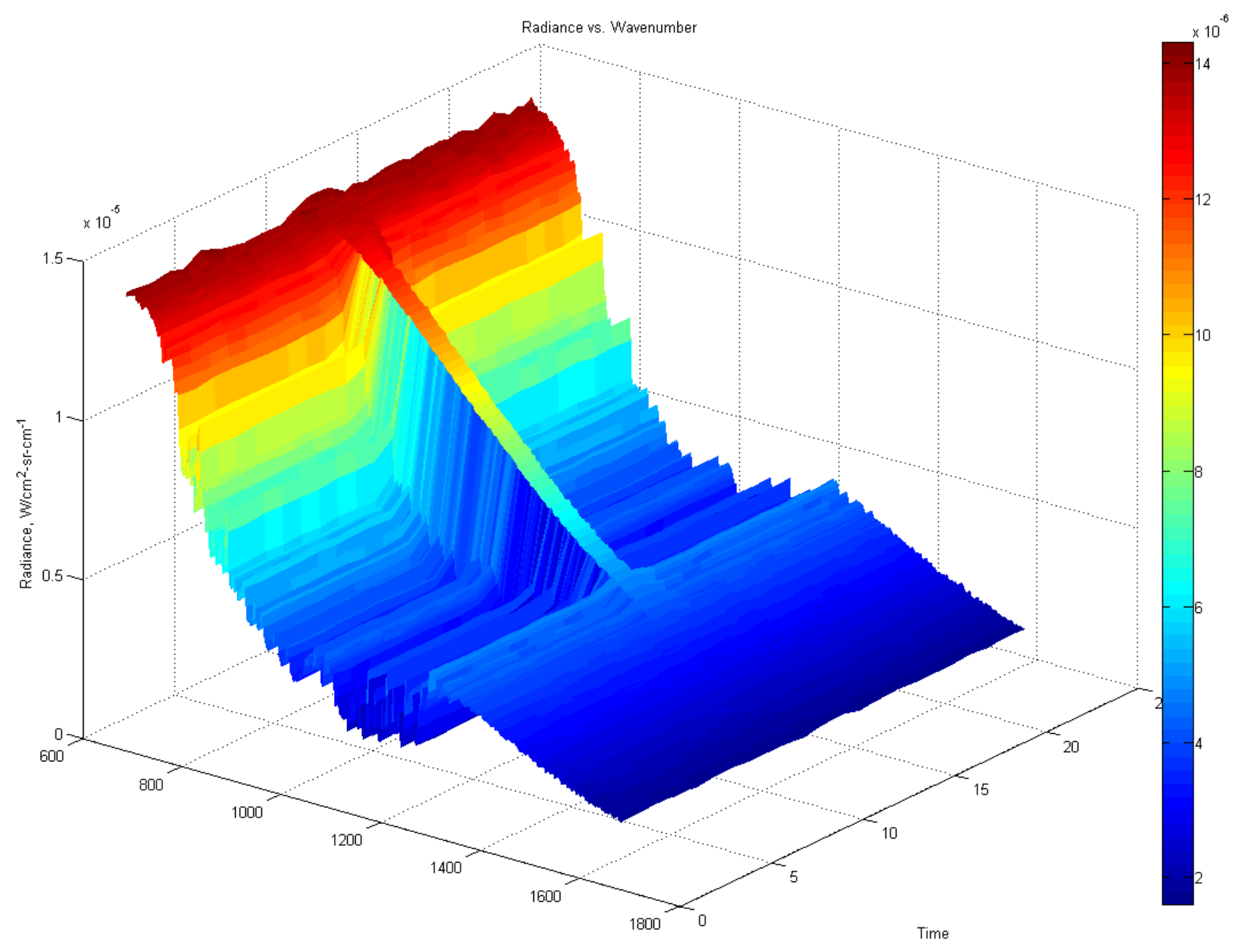

Wavenumber, $\left(\mathrm{cm}^{-1}\right)$

Figure 240. Large Aircraft in FOV Zoomed In 
Figure 241 shows a radiance difference plot that corresponds to the time intervals of Figure 240. Note that a plateau is seen in this plot (solid blue line at time interval 12) because the radiances are nearly identical for the two time intervals that the aircraft is directly in the FOV.

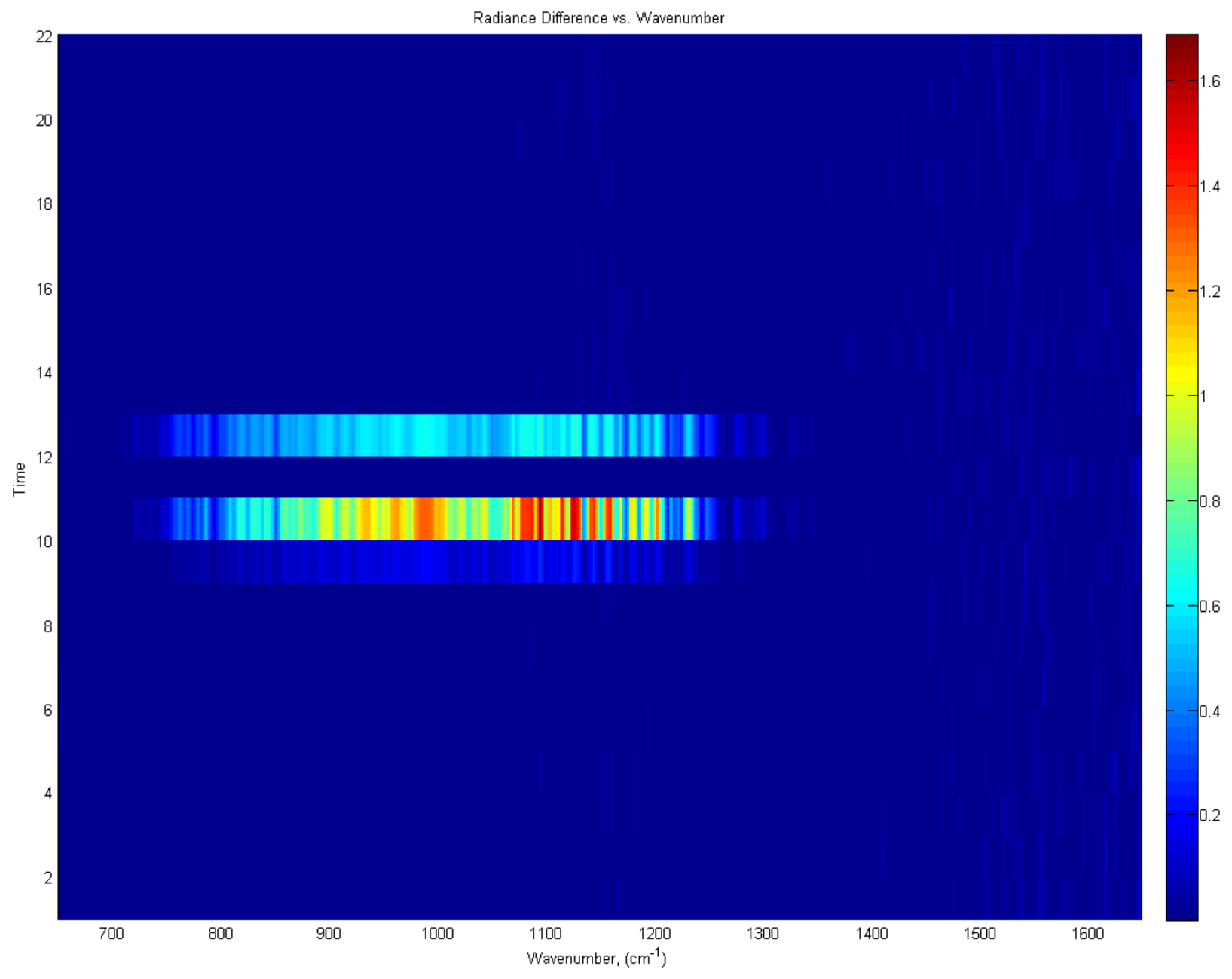

Figure 241. Radiance Difference Plot for Large Aircraft in FOV 
Finally, Figure 242 shows the radiances in a 2D plot for the time intervals immediately around when the large aircraft passed through the FOV. Also included is a blackbody radiation curve at the approximate air temperature at that time. The time stamps for the data are shown in the legend. This plot shows that the radiances when the aircraft is in the FOV are nearly equivalent to the blackbody radiation curve.

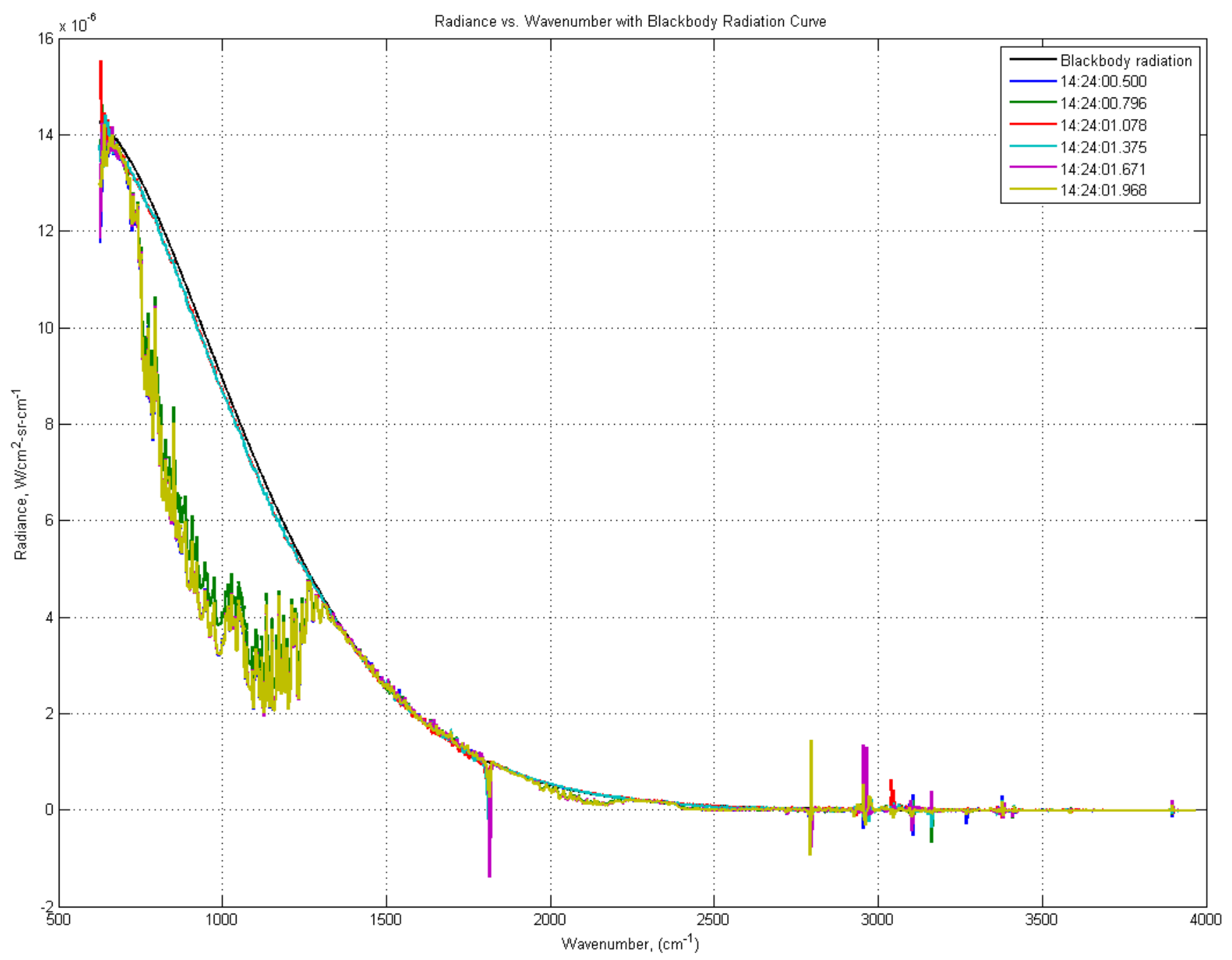

Figure 242. Time Sequenced 2D Radiance Plot 
Next, the authors chose to specifically look at wavenumbers 700-800. Figure 243 is a standard surface plot of the DC9 data set for wavenumbers 700-800. The aircraft is clearly evident around 260 on the Time scale (which is actually the number of files. To get time, multiply by $300 \mathrm{msec}$ for each file.) Looking farther out in the data ( $\sim 85$ seconds after the aircraft) there is a dip that occurs in the data. This dip is circled on Figure 243 where it is the most evident. To obtain a better view of this dip, the data in Figure 243 was plotted two dimensionally in number of files (time) versus radiance in Figure 244.

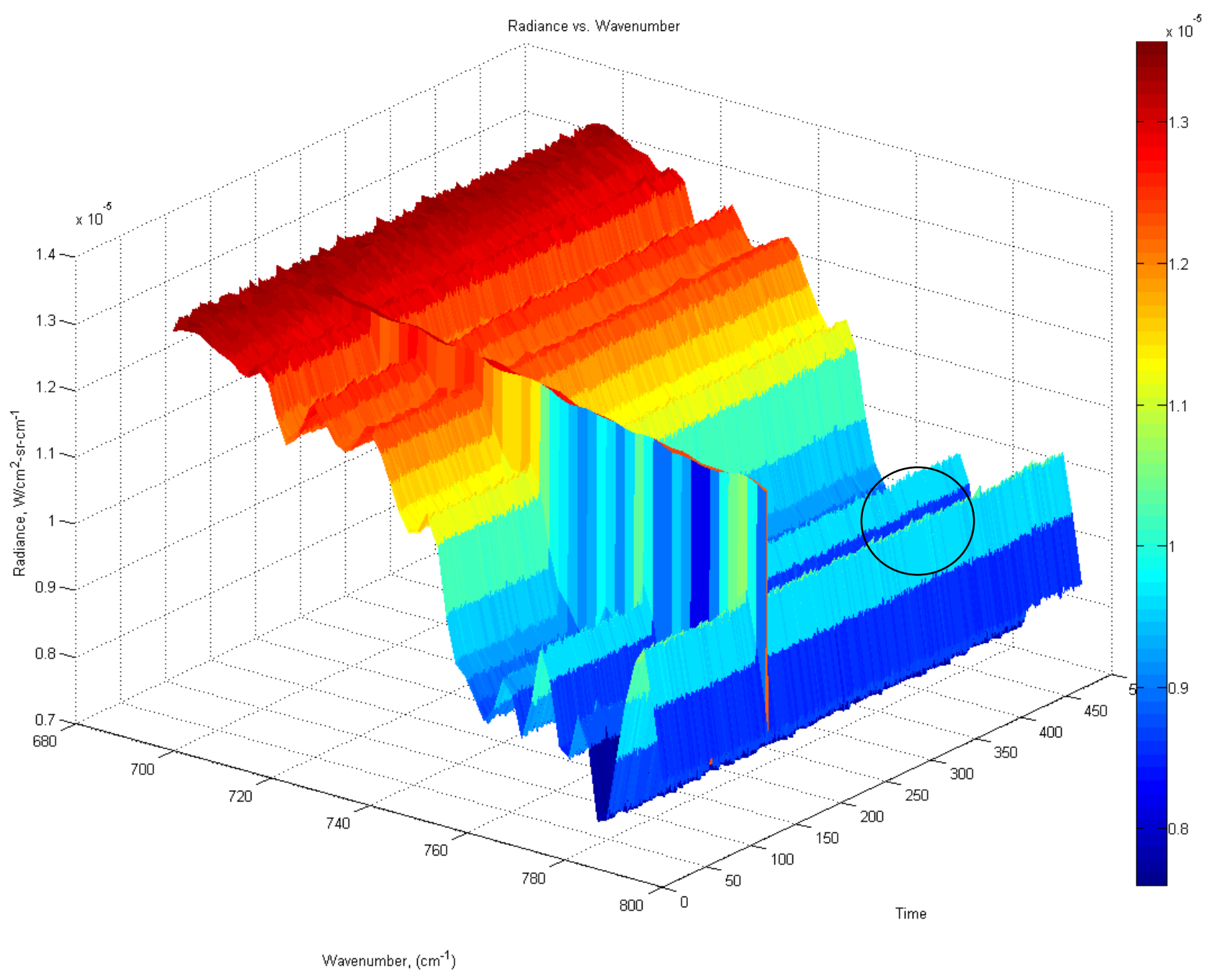

Figure 243. Surface plot of DC9 data from 060708 


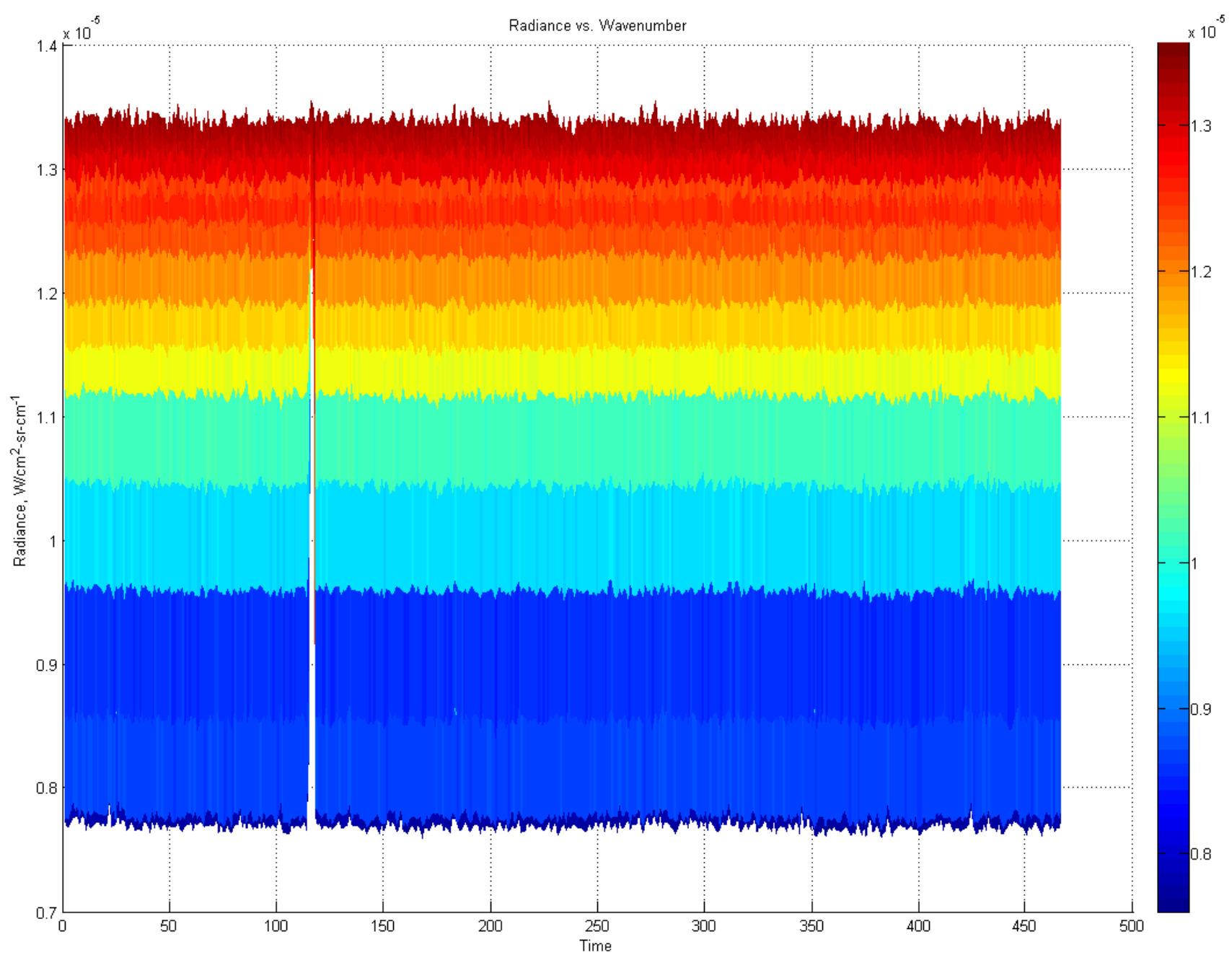

Figure 244. Two-dimensional plot of number of files (Time) versus Radiance of DC9 data

In Figure 244, the dip in the data is more evident around 380 files at radiances between 0.9 and 1.1. More analysis is needed, but the research team believes there is a possibility that this could be due to the aircraft's wake vortex. The timing is appropriate for viewing a vortex caused by the DC9, and the core of a vortex is colder than the surrounding air.

Next, the data was normalized by subtracting the averaged background (excluding the aircraft passage) from the data. The data was then smoothed using a sliding average of five data points. As seen in Figure 245, the colder area at approximately 80 seconds ("time" 200) after the aircraft passes through the FOV is clearly evident. Note that the feature lasts for $40-50$ files on the "Time" scale, which is approximately $13-17$ seconds in time. 


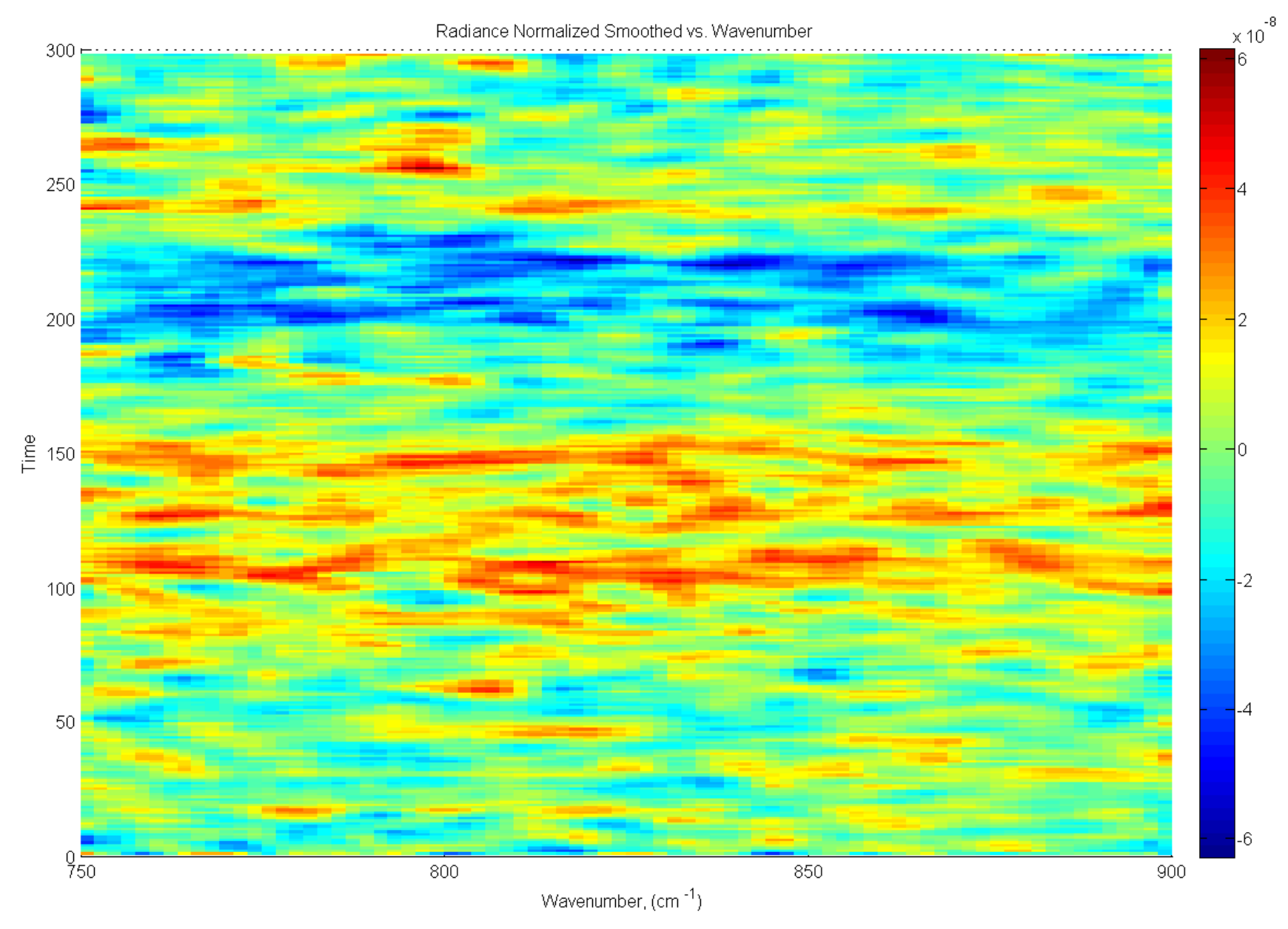

Figure 245. Normalized and smoothed DC9 radiance

For comparison, several minutes of data of "clear blue sky" were acquired earlier that day. Again, the conditions were extremely windy and there was high humidity. Figure 246 is a compilation of files showing same normalized and smoothed analysis as performed on the time period following the aircraft. Again, the "Time" scale is actually the number of files represented in the data set, and in this case there are 4000 files $^{1}$. To convert to time, each file represents $\sim 300$ msec. The four figures create a time sequence, with the bottom figure representing the first 1000 files in the series, the next figure representing the next 1000 files in the series and so on. Each tick mark represents 100 files.

A wave pattern is present in this background data as well. This pattern represents the variability in the spectral sky radiance during the time of the measurements. While no clouds were visible, sub-visual cirrus may have been present. The peak of the additional radiance is in frequencies less than 1000 wavenumbers, which is consistent with observations of cirrus.

\footnotetext{
${ }^{1}$ The "Time" scale goes from $0-1000$ and then repeats. The repetition happens because the graphs for each 1000 files had to be generated separately. The graphs were then combined into one image to better see what was being represented.
} 


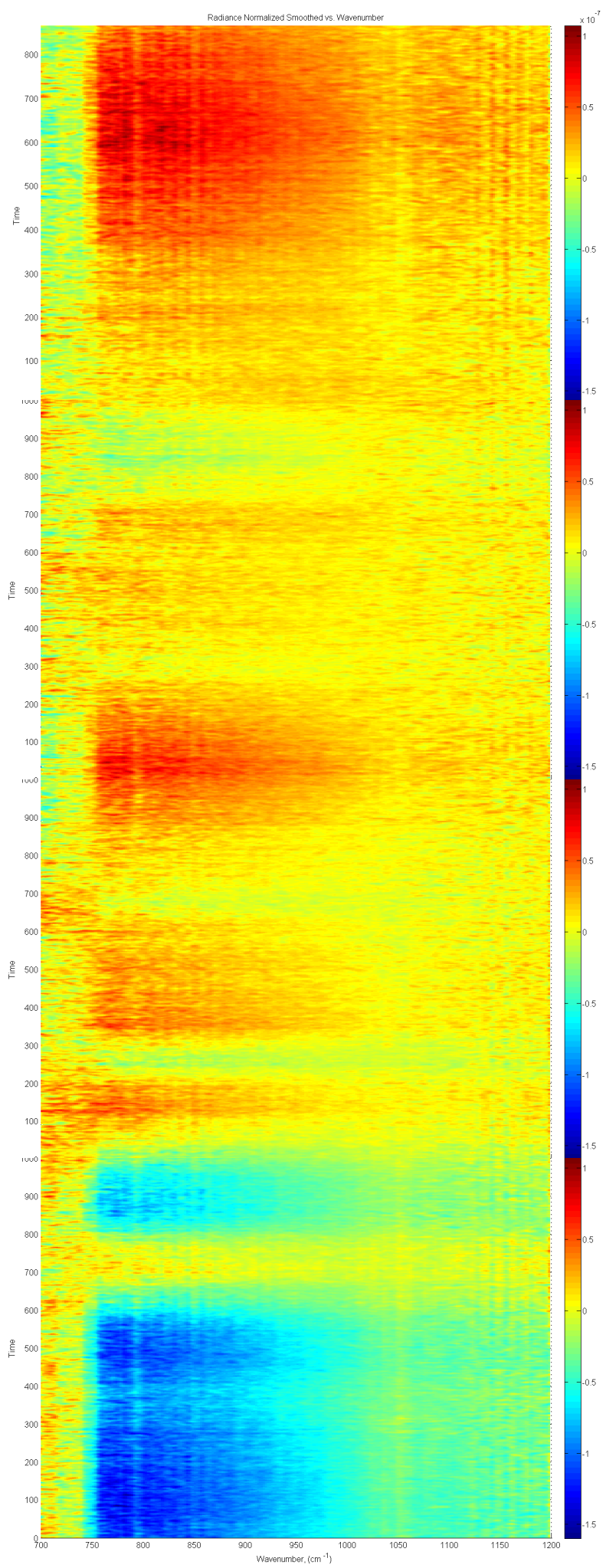

Figure 246. Data Set 1 060708, first 1000 files normalized and smoothed 


\subsubsection{Data Intercomparison with AERI}

Intercomparisons were planned for both the D\&P spectrometer and the Telops MWIR FIRST imager with the AERI instrument that is calibrated to NIST standards.

\subsubsection{Telops MWIR FIRST}

The AFIT MWIR FIRST data for the AERI - FIRST intercomparison was delivered to UWSSEC along with AFIT Matlab routines for accessing and working with the data and a draft AFIT document "Post-Test Report for the WAVEx Field Test". The data consisted of raw, uncalibrated interferograms for an $8 \times 8$ region of the array. Only one blackbody calibration reference was included with the dataset, so it was necessary to also use a second blackbody calibration reference taken earlier in the day in order to calibrate the dataset.

Prior to proceeding with calibration it was discovered that a focal plane array anomaly, particular to the AFIT MWIR FIRST instrument, needed to be corrected in order to remove a systematic error from the interferogram data. Software for applying the correction was provided by AFIT, but repeated attempts to apply the correction were unsuccessful and it was later confirmed that there were problems with the correction software. Given the state of the delivered FIRST data, and the extensive work required to properly correct and calibrate the data, the AFIT FIRST AERI intercomparison could not be completed.

\subsubsection{D\&P}

The D\&P data set consisted of calibrated radiances, zero filled to $2 \mathrm{~cm}^{-1}$ resolution. Brightness Temperature timeseries of the mean radiances in two window regions and a 'surface' region for both the AERI and D\&P instruments were used to define the optimum comparison period. Mean radiances for each instrument were determined for this comparison period, and the AERI calibrated radiances were reduced to the D\&P native spectral resolution $\left(4 \mathrm{~cm}^{-1}\right)$. Zero-padding in the interferogram domain was then used to produce an oversampled version of each spectrum, and the oversampled spectra were interpolated to a common wavenumber grid. The results are shown in Figure 247, which is a comparison of zenith sky radiance spectra recorded simultaneously with the D\&P spectrometer and the AERI instrument during the time interval 12:03:31 - 12:22:16 UTC on 7 June 2008. On this full scale, the radiance intercomparison appears to be satisfactory in spectral regions where the atmosphere is opaque, such as near 700 $\mathrm{cm}^{-1}$ and at $1300-1900 \mathrm{~cm}^{-1}$.

However, in semi-transparent regions there appears to be a large radiometric calibration error in the D\&P calibration, with the D\&P warmer by $\sim 10$ radiance units $\left(\mathrm{mW} / \mathrm{m}^{2} \mathrm{sr} \mathrm{cm}^{-1}\right)$ in the LWIR window. It should be noted that during the duration of the WAVEX experiment, conditions were not ideal for a clear-sky intercomparison. Such tests are best conducted for extremely clear sky conditions with low relative humidity, which helps ensure low atmospheric temporal variability during the test. The full resolution AERI spectra show high $\mathrm{RH}$ for the intercomparison data collection period. 


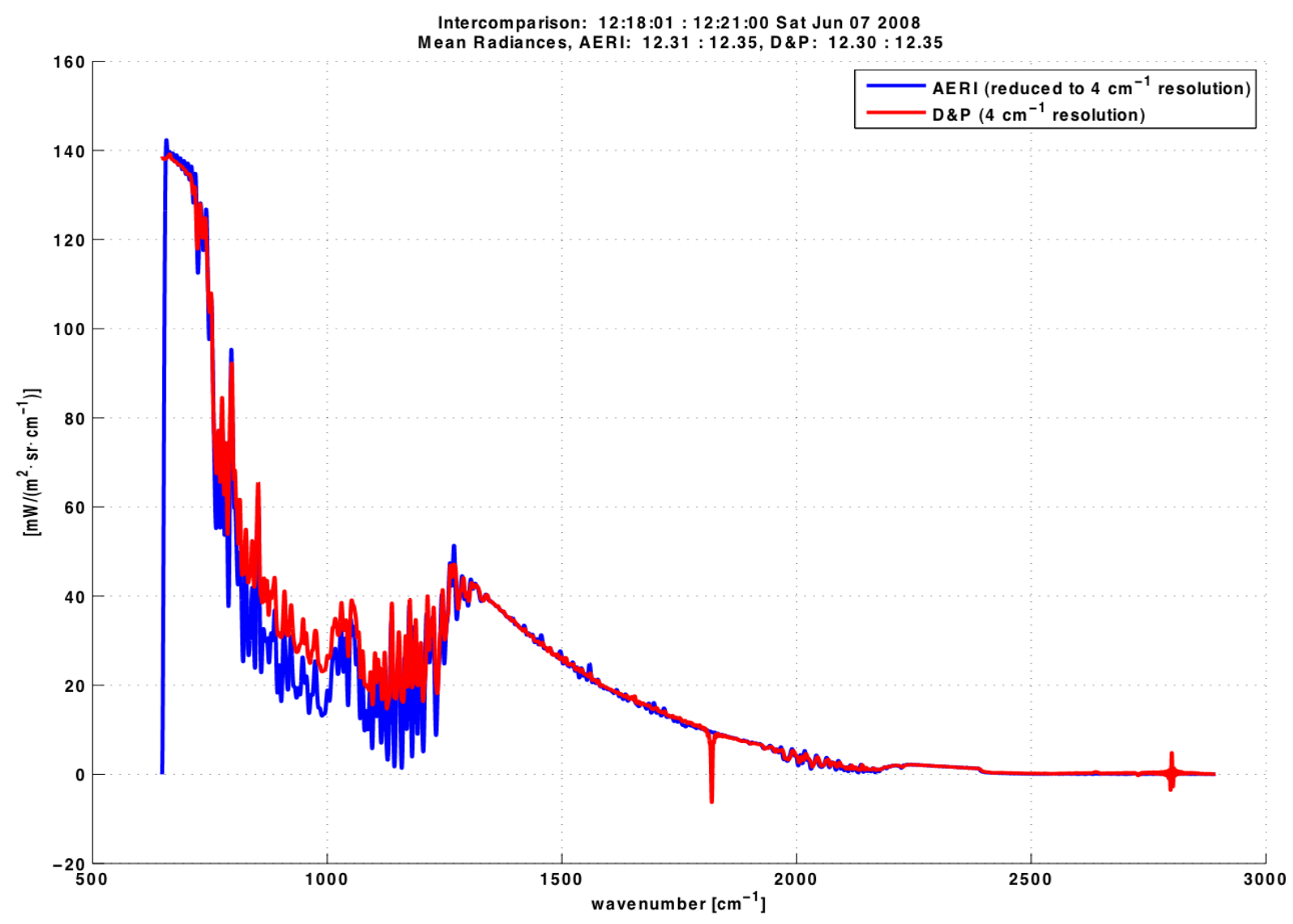

Figure 247. AERI - D\&P Intercomparison, Radiance Units

The radiance spectra from the two instruments were then converted to brightness temperatures. Figure 248 shows the spectral brightness temperatures from the two instruments as well as the differences between the two. The spectral radiance differences are shown as percentages in Figure 249. A successful intercomparison between two well-calibrated instruments, under optimum comparison conditions, should show agreement within $1 \mathrm{~K}$. The agreement meets this criterion in most of the spectral regions where the atmosphere is opaque, but in the semitransparent regions the D\&P data shows significantly higher brightness temperatures. 

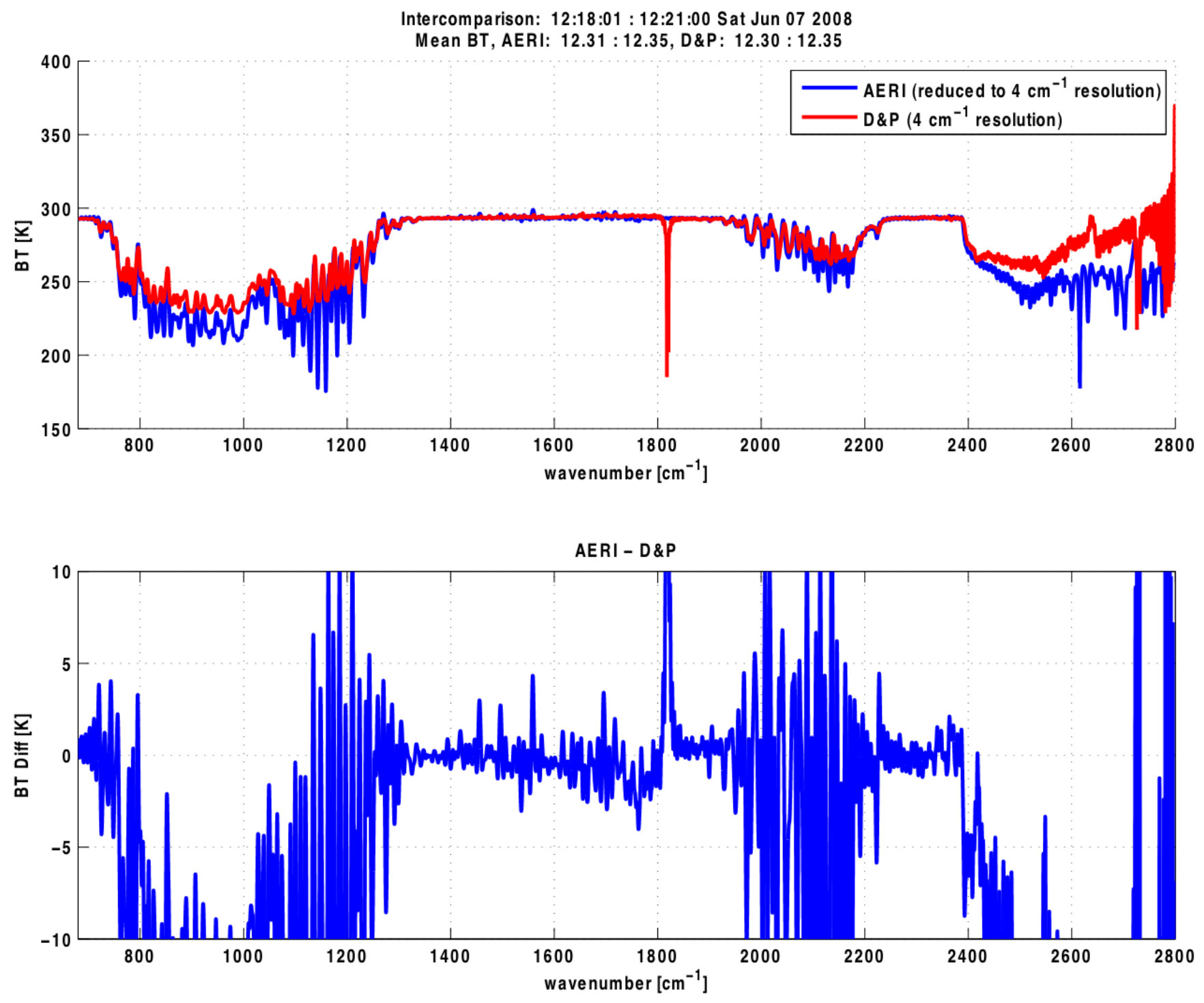

Figure 248. AERI - D\&P comparison spectra in brightness temperature. 


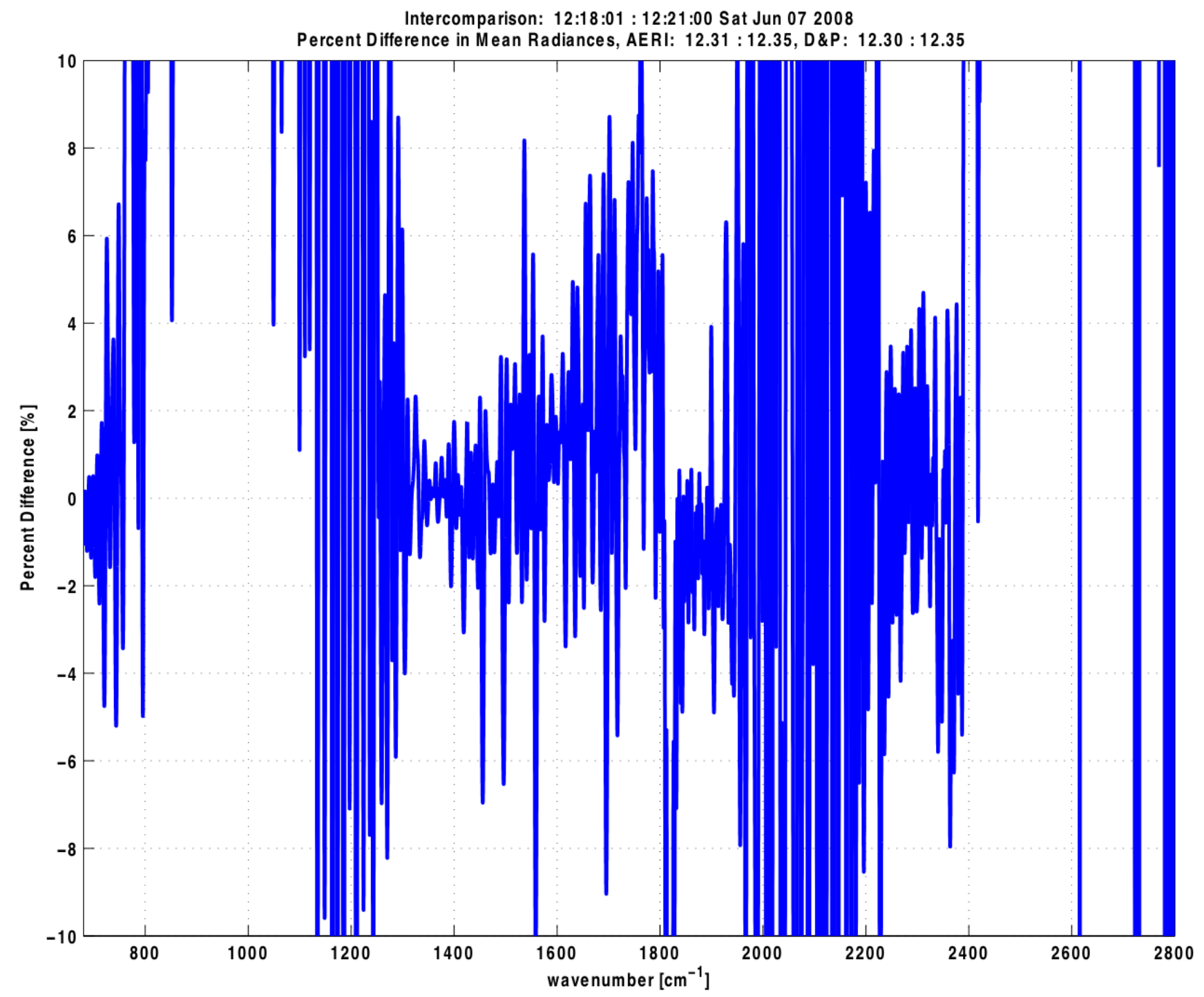

Figure 249. AERI - D\&P comparison, radiance percent difference.

\section{Conclusions}

There were several major accomplishments during the FLI Phase 2 Program regarding measurements, simulations/analysis, and algorithm development. The most significant accomplishment was that the research team was able to detect clear air turbulence associated with mountain waves. Data collected during the Boulder field campaign clearly showed a damped sinusoidal wave effect associated with clear air turbulence from mountain waves (Figure 184). The data matched modeled data as well as theoretical data. This effect has never been detected with infrared radiometry before and will be valuable not only to the aviation safety community, but the existing turbulence nowcasting and forecasting communities as well.

Data collected during the WAVEx field campaign had mixed results. Data collected by the Telops MWIR FIRST was unfortunately too noisy to resolve any vortices, but data collected by the non-imaging D\&P had higher resolution and was less noisy. The D\&P was able to resolve what might be a wake vortex about 80 seconds after the passage of a DC9 aircraft (Figure 245). 
If proven, this accomplishment is significant and has direct application to enhanced aviation safety as well as Next Generation Air Transportation System (NextGen) terminal operations.

Simulations and analyses performed to date on the FLI program have included the hazards of clear air turbulence, wake vortices, icing during all phases of flight, volcanic ash, and low slant range visibility. The simulations and analyses performed during this effort have shown that all of these hazards should be detectable with relevant warning times. The detection and avoidance of these hazards will enhance aviation safety and NextGen operations.

Using CAT as an example, the simulation sensitivity studies employed the EOF regression technique. The EOF technique requires a large number of data sets, and three types of data sets were used: idealized simulations based on a von Kármán representation of the atmospheric turbulence spectrum; measured data from commercial aircraft flight data recorders and from research aircraft; and four-dimensional simulations of actual severe turbulence encounters. The data sets were used to simulate the radiance that would be observed by an airborne FLI instrument for different aircraft altitudes and positions, over a wide range of azimuth and elevation observing angles.

Results from the simulations showed that brightness temperature differences associated with mountain waves should be on the order of $1-2 \mathrm{~K}$, with the largest signals in the relatively clear regions of the LWIR window, 700-800 $\mathrm{cm}^{-1}$ and $1000-1100 \mathrm{~cm}^{-1}$, and that the distance to the turbulence should be predictable from the spectrum. A simulation of mountain lee wave turbulence over Colorado on 6 March 2004 was used extensively to refine the instrument specifications for an airborne FLI.

Finally, there have been accomplishments in algorithm development as well. Algorithms are key to the final implementation of a FLI system. The final system must be able to perform calculations in real time to be of use as an airborne hazard sensor. The system must be able to analyze the data to positively identify a hazard and relate it to the intensity of the effect that the aircraft might experience. It must also be able to detect the range to the hazard and to minimize false alarms. The two main accomplishments towards algorithm development include 1) the physically and statistically based use of EOFs and 2) relating atmospheric turbulence structures to the radiance signals of the FLI instrument. Both of these approaches have provided efficient means of analysis to provide real-time feedback for the pilot, and together, these algorithms are on the path forward to create a system to detect a hazard and its range, while relating the hazard to the effect on the aircraft, thereby minimizing false alarms.

In addition to the accomplishments towards the development of a FLI system, the project and findings have been widely publicized at events such as AIAA, OSA, and SPIE meetings and conferences. Appendix $\mathrm{A}$ is a listing of the publications and presentations made to date on the project, including Phase 1 and Phase 2. The Appendix also lists planned future publications and presentations.

Modeling and simulations have shown that the proposed FLI system is capable of detecting a wide range of hazards, including clear air turbulence, wake vortices, icing during all phases of flight, volcanic ash, and low slant range visibility. In addition, detections ranges are expected to provide sufficient time to avoid the hazard or minimize its impact. Algorithms have been developed to relate the physical hazard to the radiance spectrum that is collected by the FLI system. Finally, field measurement campaigns provided empirical demonstrations of radiometric hazard detection, for mountain wave turbulence and potentially a wake vortex. 
Even with the success of detecting mountain wave turbulence and potentially a wake vortex associated with a DC9 aircraft, there are several high priority tasks remaining in order to validate the utility of an airborne FLI instrument with good detection and ranging capabilities as well as a low false alarm rate. Based on all of the research and findings of the program to date, the authors recommend three high priority tasks to continue with the validation of the FLI concept as a hazard detection sensor. These tasks include 1) airborne interferometric measurements, 2) simulations and analysis, and 3) further development of algorithms.

Collecting airborne interferometric measurements along with truth data is critical. Ground-based measurements were conducted in this phase of the program instead of airborne measurements for cost reasons. It is much cheaper to conduct a ground-based field study as opposed to an airborne field study. However, the ground-based measurement geometry is not ideal for the radiometric detection of hazards because the radiometric background and atmospheric transparency are quite different from those at flight altitudes. In addition, the measurements do not correspond to the project simulations, which have almost all been created and analyzed for the airborne environment. The goal of the airborne tests will be empirical demonstrations of hazard detection and ranging along with truth data. The flights are expected to result in a large number of data sets for further algorithm development and performance evaluation.

Simulations and analysis covers two important subtasks - simulating the atmospheric environment during field measurements and further analysis of WAVEx data. Simulating the atmospheric environment from the airborne truth data enables a physical understanding of the environment in addition to the optimization of algorithms and instrumentation. Further analysis of WAVEx data is also a high priority subtask. The research team was able to analyze a significant amount of hyperspectral data from WAVEx. However, there is also high quality data from a Bomem MR-154 instrument that was not originally intended to be a part of the field test. This data should be analyzed in order to capitalize on the investment already made in the program. The Bomem is non-imaging, like the D\&P Spectrometer, but it was aimed at 20 degrees above the horizon in the same direction as the Telops MWIR FIRST, whereas the D\&P was aimed vertically. In addition, visible spectrum cameras were boresighted with the Bomem to collect a video record of the flight of each aircraft through the field of view. The D\&P and Bomem should have seen similar phenomena, and this data should be analyzed for confirmation as well as detection of additional vortices.

An operational FLI sensor will provide multiple hazard detection in all phases of flight, and so the main payoff will be enhanced safety. However, there are other benefits of such a sensor: first, detection of terminal-area hazards such as wake vortices may enable reduced aircraft separations in dense traffic, which would increase airport capacity. Second, data from such a sensor could be used to enhance the accuracy of nowcast/forecast models. Lastly, such a sensor could play a key role in the comprehensive monitoring, sharing, and analysis of data for aircraft safety, collaborative air traffic management, and an integrated weather prediction system.

Finally, there have been accomplishments in algorithm development as well. Algorithms are key to the final implementation of a FLI system. The final system must be able to perform calculations in real time to be of use as an airborne hazard sensor. The system must be able to analyze the data to positively identify a hazard and relate it to the intensity of the effect that the aircraft might experience. It must also be able to estimate the range to the hazard and to minimize false alarms. The two main accomplishments towards algorithm development include a) the physically and statistically based use of EOFs and b) relating atmospheric turbulence 
structures to the radiance signals of the FLI instrument. Both of these approaches have provided efficient means of analysis to provide real-time feedback for the pilot, and together, these algorithms are on the path forward to create a system to detect a hazard and its range, while relating the hazard to the effect on the aircraft, thereby minimizing false alarms.

Even with the success of detecting mountain wave turbulence and potentially a wake vortex associated with a DC9 aircraft, there are several high priority tasks remaining in order to validate the utility of an airborne FLI instrument with good detection and ranging capabilities as well as a low false alarm rate. Based on all of the research and findings of the program to date, we recommend three high priority tasks to continue with the validation of the FLI concept as a hazard detection sensor. These tasks include 1) airborne interferometric measurements, 2) simulations and analysis, and 3) further development of algorithms.

An operational FLI sensor will provide multiple hazard detection in all phases of flight, and so the main payoff will be enhanced safety. However, there are other benefits of such an airborne sensor: first, detection of terminal-area hazards such as wake vortices may enable reduced aircraft separations, which would increase airport capacity. Second, data from such a sensor could be used to enhance the accuracy of nowcast/forecast models. 


\section{Bibliography}

Clark, T. L., 1977: A small-scale numerical model using a terrain following co-ordinate system. J. Comput. Phys., 24, 186-215.

Clark, T. L., Hall, W. D., Kerr, R. M., Middleton, D., Radke, L., Ralph, F. Martin, Nieman, P. J., and D. Levinson, 2000: Origins of aircraft-damaging clear air turbulence during the 9 December 1992 Colorado downslope windstorm: Numerical simulations and comparison to observations. $J$. Atmos. Sci., 57, 1105-1131.

Clark, T.L.,W. D. Hall, and J. L. Coen, 1996: Source code documentation for the Clark-Hall cloudscale model: Code version G3CH01. NCAR Technical Note NCAR/TN-426+STR, 137pp.

Gimmestad G.G., C.D. Papanicolopoulos, M.A. Richards, D.L. Sherman, and L.L. West, "Feasibility Study of Radiometry for Airborne Detection of Aviation Hazards", (NASA/CR2001-210855 under contract NAS1-99073).

Lane, T. P., R. D. Sharman, H.-M. Hsu, W. D. Hall, M. A. Shapiro, R. Plougonven, and J. J. Murray, 2005: Numerical simulations of gravity waves and turbulence during the ATReC campaign. Proceedings of the 43rd Aerospace Sciences Meeting and Exhibit, AIAA 2003-194.

Lilly, D.K. and Kennedy, P. J. (1973). Observations of a stationary mountain wave and its associated momentum flux and energy dissipation. J. Atmos. Sci., 30, 1135-1152.

Murrow, H. N., 1987: Measurements of atmospheric turbulence. In Atmospheric Turbulence Relative to Aviation, Missile, and Space Programs, NASA Conference Publication 2468, 137154.

Murrow, H. N., W. E. McCain, and R. H. Ryne, 1982: Power spectral measurements of clear-air turbulence to long wavelengths for altitudes up to 14000 meters. NASA Technical Paper 1979, $161 \mathrm{pp}$.

West L.L., G.G. Gimmestad, W.L. Smith, S. Kireev, "Applications of a Forward-Looking Interferometer (FLI) for the On-board Detection of Aviation Weather Hazards", NASA Task NNL06AB99T, Feb 2007.

West L.L., G.G. Gimmestad, W.L. Smith, S. Kireev, L.B. Cornman, P.R. Schaffner, G. Tsoucalas, "Applications of a Forward-Looking Interferometer (FLI) for the On-board Detection of Aviation Weather Hazards", NASA Technical Publication NASA/TP-2008-215536, 2008

Whiteway, J. A., G. P. Klaasen, N. G. Bradshaw, and J. Hacker, 2004: Transition to turbulence in shear above the tropopause. Geophys. Res. Lett., 31, L02118, doi:10.1029/2003GL018509. 


\section{Appendix A: Publications and Presentations}

Airborne Radiometric Detection of Aviation Hazards Workshop, National Institute of aerospace, Hampton, VA, 14-15 August 2006.

"Airborne Forward Looking Interferometer Turbulence Investigation," G. Gimmestad, L. West, W. Smith, S. Kireev, Xu Liu, P. Schaffner, and J. Murray, proceedings of the $45^{\text {th }}$ AIAA Aerospace Sciences meeting AIAA 2007-78, Reno, NV, 8-11 January 2007.

"Airborne Forward Looking Interferometer Turbulence Investigation," W. Smith, S. Kireev, GG. Gimmestad, L. West, X. Liu, P. Schaffner, and J. Murray, presented at the OSA Topical Meeting on Fourier Transform Spectroscopy, Santa Fe, NM, 15 February 2007.

"Radiometric Sensing of Wake Vortices," G. Gimmestad, L. West, W. Smith, S. Kireev, Xu Liu, P. Schaffner, and J. Murray, presented at the Wake Vortex Meeting, Flight Research Laboratory, Ottawa, Canada, 23 March 2007.

"Hazard Detection Analysis for a Forward Looking Interferometer," L. West, NASA Aviation Safety Technical Conference, St. Louis, MO, 10-12 October 2007.

"Applications of a Forward-Looking Interferometer for the On-board Detection of Aviation Weather Hazards," L. West, G. Gimmestad, W. Smith, S. Kireev, L. Cornman, P. Schaffner, and G. Tsoucalas, NASA Technical Publication, 2008-215536.

"Interferometric radiometer for in-flight detection of aviation hazards," W. Smith, S. Kireev, L. West, G. Gimmestad, L. Cornman, W. Feltz, G. Perram, and T. Daniels, Proceedings of SPIE 7088, San Diego, CA 12-14 August 2008.

“Applications of Airborne Forward Looking Imaging FTS," W. Smith, S. Kireev, T. Daniels, L. West, G. Gimmestad, K. Gross, and G. Smith, Telops Science Applications Workshop, Washington DC, 25-26 September 2008.

"Hazard Detection Analysis for a Forward Looking Interferometer," L. West and G. Gimmestad, NASA Aviation Safety Technical Conference, Denver, CO, 21-23 October 2008.

"Hazard Detection Analysis for a Forward-Looking Interferometer," L. West, G. Gimmestad, R. Herkert, W. Smith, S. Kireev, T. Daniels, L. Cornman, B. Sharman, A. Weekley, G. Perram, K. Gross, G. Smith, W. Feltz, J. Taylor, E. Olson, AIAA-2009-3635, 1st AIAA Atmospheric and Space Environments Conference, San Antonio, Texas, June 22-25, 2009 


\section{Appendix B: WAVEx Auxiliary Data}

The chronology of the WAVEx field test is best left in the two trip reports that were assembled after the test. These reports are titled "WavexTripReport.doc" and "WAVExTestReport-AFITDRAFT.pdf'. These reports are included with this final report as additional reading. Each report delineates the instrumentation, the weather conditions, and the data collected by the team members. The supporting data listed below includes flight schedules and meteorological data for the project.

As a summary, the weather in Madison during the test time period (and the alternate test period) was extremely bad. Cloudy conditions, wind and rain plagued the experiment. However, the team stayed one extra day (Saturday June 7, 2008) to acquire data during a blue sky period. The weather was still extremely windy $(6-10 \mathrm{~m} / \mathrm{s})$ and the atmosphere had high humidity $(>80 \%)$. (See Appendix B) In addition, severe weather, including tornados, was rolling in. The research team was able to collect data on one large aircraft, a DC9, before having to tear down the field set up. In addition, four CRJs also landed within the test set up field of view.

The weather was not very cooperative during most of the week of WAVEx (i.e., low clouds limited the sensitivity of the measurements to viewed wake vortices), but imaging spectrometry of scenes with wake vortices against a clear sky background were obtained on the last day of the experiment, Saturday, June 7, 2008. The selected back-up week for the field experiment encountered even worse weather conditions than the week of the experiment.

The following data was collected during WAVEx. The data was collected from the UW/SSEC roof weather station. (http://rig.ssec.wisc.edu/)

2-Jun-08

Local 6:00: Pretty clear, some clouds on the horizon

$\begin{array}{ccccccccc}\text { Time } & \mathrm{T} & \begin{array}{c}\text { Station } \\ \mathrm{P}\end{array} & \begin{array}{c}\text { Alti- } \\ \text { meter }\end{array} & \begin{array}{c}\text { Dew } \\ \text { Point }\end{array} & \begin{array}{c}\text { Wind } \\ \text { Dir }\end{array} & \begin{array}{c}\text { Wind } \\ \text { Speed }\end{array} & \begin{array}{c}\text { Precip } \\ \text { since } \\ \text { 00Z }\end{array} & \begin{array}{c}\text { Solar } \\ \text { Flux }\end{array} \\ \text { local-h] } & {[\mathrm{C}]} & \begin{array}{c} \\ {[\mathrm{hPa}]}\end{array} & \text { inHg } & {[\mathrm{C}]} & {[\mathrm{deg}]} & \begin{array}{c} \\ {[\mathrm{m} / \mathrm{s}]}\end{array} & {[\mathrm{mm}]} & {[\mathrm{W} / \mathrm{m} 2]} \\ 10: 46 & 18 & 975.3 & 29.94 & 12.3 & 347 & 1.3 & 0 & 25 \\ 10: 48 & 16.6 & 975.2 & 29.93 & 12.7 & 348 & 0.9 & 0 & 26.6 \\ 10.5 & 17.6 & 975.2 & 29.93 & 12.2 & 333 & 1.9 & & 37.5 \\ 10: 52 & 17.7 & 975.3 & 29.94 & 12.1 & 320 & 2.1 & & 38.5 \\ 10: 54 & 18 & 975.5 & 29.94 & 12.3 & 320 & 1.5 & & 43.6 \\ 10: 56 & 17.5 & 975.5 & 29.94 & 12.4 & 306 & 1.5 & & 44.4 \\ 10: 58 & 17.9 & 975.5 & 29.94 & 11.9 & 304 & 1.7 & & 51.6 \\ 11: 00 & 18.7 & 975.5 & 29.94 & 11.4 & 318 & 1.8 & & 50.2 \\ 11: 05 & 18.1 & 975.7 & 29.95 & 11.7 & 284 & 1.7 & & 42.4 \\ 11: 10 & 18.3 & 975.8 & 29.95 & 11.6 & 294 & 1.5 & & 62.5 \\ & & & & & 237 & & & \end{array}$




$\begin{array}{cccccccc}11: 15 & 18.4 & 975.7 & 29.95 & 11.4 & 298 & 1.1 & 96.9 \\ 11: 20 & 18.3 & 975.9 & 29.95 & 11.5 & 299 & 1.3 & 113 \\ 11: 25 & 18.7 & 975.8 & 29.95 & 11.3 & 315 & 1.3 & 123.8 \\ 11: 30 & 19.1 & 975.8 & 29.95 & 10.8 & 316 & 1.1 & 135.7 \\ 11: 35 & 19.3 & 975.9 & 29.95 & 10.6 & 322 & 1.1 & 149.4 \\ 11: 40 & 19.1 & 975.9 & 29.95 & 10.6 & 338 & 1.2 & 173.8 \\ 11: 45 & 19.2 & 976 & 29.96 & 11 & 322 & 1 & 195.9 \\ 11: 50 & 19 & 975.9 & 29.95 & 11.6 & 345 & 0.9 & 201.3 \\ 11: 55 & 19.1 & 975.9 & 29.95 & 11.3 & 349 & 0.6 & 220.8 \\ 12: 00 & 18.5 & 976 & 29.96 & 12.3 & 8 & 0.6 & 247.3 \\ 12: 05 & 18.7 & 976 & 29.96 & 12.6 & 343 & 0.6 & 253.4 \\ 12: 10 & 18.8 & 976 & 29.96 & 13 & 1 & 0.4 & 263.9 \\ 12: 15 & 18.7 & 976 & 29.96 & 13 & 25 & 0.6 & 265.2 \\ 12: 20 & 18: 09 & 976.1 & 29.96 & 13.1 & 353 & 0.3 & 223.5 \\ 13: 45 & 20.9 & 976.8 & 29.98 & 11.9 & 199 & 2.4 & 438.1 \\ 13: 50 & 21.3 & 976.6 & 29.97 & 12.6 & 181 & 2.2 & 453.6 \\ 14: 00 & 21.6 & 976.5 & 29.97 & 13 & 172 & 1.7 & 410.8 \\ 14: 05 & 22.1 & 976.5 & 29.97 & 12.9 & 152 & 0.9 & 420.1 \\ 14: 10 & 22.1 & 976.4 & 29.97 & 13.1 & 155 & 2.2 & \end{array}$

10:00 local:

more cirrus

clouds

coming

$\begin{array}{cccccccc}14: 20 & 22 & 976.3 & 29.97 & 12.3 & 180 & 1.5 & 431.6 \\ 14: 35 & 22.3 & 976.2 & 29.96 & 12.5 & 158 & 2.1 & 437.4 \\ 14: 45 & 22.4 & 976.2 & 29.96 & 12.3 & 181 & 2 & 475 \\ 14: 55 & 22.4 & 976.3 & 29.97 & 12.7 & 141 & 2.1 & 495.4 \\ 15: 05 & 22.6 & 976.2 & 29.96 & 12.7 & 161 & 2.5 & 499 \\ 15: 10 & 23.1 & 976.2 & 29.96 & 12.6 & 196 & 1.4 & 477.8 \\ 15: 20 & 23.1 & 976.1 & 29.96 & 12.4 & 198 & 2.3 & 537 \\ 15: 30 & 23.1 & 976 & 29.96 & 11.8 & 175 & 1.7 & 585.4 \\ 15: 40 & 23.5 & 976 & 29.96 & 12.1 & 192 & 2.3 & 526.5 \\ 15: 50 & 23.5 & 975.9 & 29.95 & 11.5 & 191 & 1.9 & 560.3 \\ 15: 55 & 23.6 & 975.9 & 29.95 & 10.7 & 201 & 3.2 & 559.1\end{array}$

11:00 local: the

whole sky in

cirrus; no direct

sun

$\begin{array}{llllllll}16: 00 & 23.5 & 975.8 & 29.95 & 10.7 & 207 & 2.6 & 538.2\end{array}$ 


$\begin{array}{cccccccc}16: 10 & 23.9 & 975.8 & 29.95 & 10.9 & 145 & 1 & 518 \\ 16: 25 & 23.8 & 975.4 & 29.94 & 11.5 & 184 & 2.4 & 617.5 \\ 16: 40 & 24.6 & 975.3 & 29.94 & 10.9 & 218 & 2.3 & 584.8 \\ 16: 50 & 24.7 & 975.2 & 29.93 & 10.2 & 191 & 3.3 & 524.5 \\ 17: 00 & 24.6 & 975.1 & 29.93 & 8.7 & 181 & 2.4 & 649.1 \\ 17: 10 & 24.8 & 975.2 & 29.93 & 9.6 & 215 & 2.6 & 788.1\end{array}$

12:00 local:

Mainly cloudy;

the sun appears

sometimes

$\begin{array}{cccccccc}17: 20 & 24.6 & 975.2 & 29.93 & 9 & 187 & 2 & 566.1 \\ 17: 30 & 25 & 975.1 & 29.93 & 9 & 193 & 2.4 & 606 \\ 17: 40 & 25.3 & 974.9 & 29.92 & 8.1 & 189 & 3 & 664.3 \\ 17: 50 & 25.2 & 975 & 29.93 & 7.7 & 190 & 2.5 & 455.5 \\ 18: 00 & 25.3 & 975.1 & 29.93 & 7.7 & 150 & 1.4 & 382.1 \\ 18: 10 & 25 & 975.1 & 29.93 & 7.6 & 201 & 4.2 & 336.3 \\ 18: 30 & 24.8 & 975.2 & 29.93 & 8.5 & 220 & 4.1 & 272.2 \\ 18: 40 & 24.7 & 975.4 & 29.94 & 8.9 & 216 & 3.8 & 259.2 \\ 18: 50 & 24.7 & 975.3 & 29.94 & 9.4 & 210 & 4.3 & 282.4 \\ 19: 00 & 24.8 & 975.3 & 29.94 & 9.4 & 202 & 3.4 & 332.6 \\ 19: 15 & 24.8 & 975 & 29.93 & 9.6 & 226 & 1.9 & 355.7 \\ 19: 25 & 25.1 & 974.9 & 29.92 & 10.1 & 213 & 2.6 & 390.4\end{array}$

2:00 local:

mostly

cloudy

$\begin{array}{cccccccc}19: 40 & 24.9 & 974.7 & 29.92 & 10.6 & 186 & 4.5 & 316.9 \\ 19: 50 & 25.2 & 974.5 & 29.91 & 10.9 & 220 & 2.9 & 357.5 \\ 20: 00 & 25 & 974.5 & 29.91 & 10.6 & 210 & 2.7 & 284.2 \\ 20: 10 & 24.9 & 974.3 & 29.9 & 11 & 204 & 2.6 & 214.8 \\ 20: 20 & 25 & 974.2 & 29.9 & 11 & 225 & 3.3 & 192 \\ 20: 30 & 24.9 & 974 & 29.9 & 10.7 & 213 & 3.3 & 170.8\end{array}$

June 3:

rain, no

measureme

nts

4-Jun-

08

Local

6:00: low 
clouds,

foggy

\begin{tabular}{|c|c|c|c|c|c|c|c|c|}
\hline $\begin{array}{l}\text { Time } \\
\text { [Zlocal }\end{array}$ & $\mathrm{T}$ & $\begin{array}{c}\text { Station } \\
\text { P }\end{array}$ & $\begin{array}{l}\text { Altimet } \\
\text { er }\end{array}$ & DewP & $\begin{array}{l}\text { WindDi } \\
\mathrm{r}\end{array}$ & WindSp & $\begin{array}{l}\text { Precipsi } \\
\text { nce00Z }\end{array}$ & $\begin{array}{c}\text { SolarFl } \\
\text { ux }\end{array}$ \\
\hline$-5 \mathrm{~h}]$ & {$[\mathrm{C}]$} & {$[\mathrm{hPa}]$} & inHg & {$[\mathrm{C}]$} & [deg] & {$[\mathrm{m} / \mathrm{s}]$} & {$[\mathrm{mm}]$} & {$[\mathrm{W} / \mathrm{m} 2]$} \\
\hline $11: 15$ & 13.1 & 964.5 & 29.61 & 13 & 87 & 4.9 & 0 & 18.2 \\
\hline $11: 35$ & 13.1 & 964.9 & 29.62 & 13.1 & 82 & 3.4 & & 43 \\
\hline 11:45 & 13.2 & 964.8 & 29.62 & 13.2 & 36 & 2 & & 35.3 \\
\hline $11: 55$ & 13.1 & 964.6 & 29.61 & 13.1 & 67 & 4.3 & & 43.4 \\
\hline $12: 10$ & 13.1 & 964.2 & 29.6 & 13.1 & 53 & 3.6 & & 47.4 \\
\hline $12: 20$ & 13.2 & 964.1 & 29.59 & 13.2 & 73 & 3 & & 66.3 \\
\hline $12: 50$ & 13.4 & 964.6 & 29.61 & 13.3 & 70 & 1.7 & & 33.4 \\
\hline
\end{tabular}

8:00 local:

still heavy

fog

$\begin{array}{cccccccc}13: 00 & 13: 03 & 964.6 & 29.61 & 13.2 & 37 & 3.5 & 53.6 \\ 13: 20 & 13.1 & 964.4 & 29.6 & 13.1 & 55 & 3.1 & 77.1 \\ 13: 30 & 13.4 & 964.7 & 29.61 & 13.4 & 64 & 2.6 & 67.1 \\ 13: 45 & 13.6 & 965 & 29.62 & 13.6 & 95 & 4.1 & 94.1 \\ 14: 10 & 13.9 & 965.1 & 29.62 & 13.9 & 47 & 3.2 & 152.8 \\ 14: 30 & 14.3 & 965.1 & 29.62 & 14.2 & 103 & 5.1 & 179.1 \\ 14: 50 & 14.6 & 965.2 & 29.63 & 14.4 & 100 & 3.4 & 95.1\end{array}$

10:00 local:

fog rising a

bit, but still

heavy

$\begin{array}{cccccccc}15: 10 & 15 & 965 & 29.62 & 14.5 & 63 & 3.2 & 85.4 \\ 15: 30 & 14.8 & 965.1 & 29.62 & 14.3 & 68 & 3.6 & 226.6 \\ 15: 45 & 15.3 & 965.3 & 29.63 & 14.7 & 85 & 3.7 & 189 \\ 16: 00 & 15.6 & 965.9 & 29.65 & 14.8 & 133 & 1.8 & 130.6 \\ 16: 25 & 16.2 & 966.2 & 29.66 & 15 & 100 & 2.3 & 141.9 \\ 16: 50 & 16.2 & 965.8 & 29.65 & 15 & 68 & 2.9 & 305.2 \\ 17: 05 & 16.7 & 965.7 & 29.64 & 15.3 & 112 & 4 & 232.7 \\ 17: 20 & 16.9 & 965.6 & 29.64 & 15.4 & 76 & 2.7 & 250 \\ 17: 40 & 17.1 & 965.5 & 29.64 & 15.6 & 83 & 2.6 & 271 \\ 17: 50 & 17.5 & 965.4 & 29.63 & 16 & 113 & 3.2 & 374\end{array}$


13:00 local:

fog is lifting,

but still

there, no sun

$\begin{array}{llllllll}18: 00 & 17.4 & 965.1 & 29.62 & 15.9 & 115 & 4.6 & 444.9\end{array}$

13:20 local:

sun appears

first time

over day

$\begin{array}{cccccccc}18: 20 & 17.9 & 964.9 & 29.62 & 16.1 & 75 & 2.1 & 452 \\ 19: 00 & 18.8 & 964.9 & 29.62 & 16.5 & 117 & 2.8 & 557.5 \\ 19: 15 & 19.2 & 964.8 & 29.62 & 16.4 & 113 & 3.7 & 478.6 \\ 19: 35 & 18.6 & 964.7 & 29.61 & 16.6 & 92 & 2.1 & 391.6 \\ 19: 50 & 19.1 & 964.9 & 29.62 & 16.5 & 113 & 3.2 & 340.9\end{array}$

15:00

local:

mostly

cloudy

$\begin{array}{cccccccc}20: 30 & 20.1 & 965.1 & 29.62 & 16.8 & 141 & 1.9 & 214.2 \\ 20: 40 & 20.1 & 965.1 & 29.62 & 16.9 & 91 & 1.5 & 201.5 \\ 21: 00 & 20.4 & 965 & 29.62 & 17.1 & 129 & 1.1 & 173\end{array}$

5-Jun-08

Local 11:00: mostly cloudy, but clouds are not too close to the surface; rain stopped about 10 local

\begin{tabular}{|c|c|c|c|c|c|c|c|c|}
\hline $\begin{array}{c}\text { Time } \\
\text { [Z,loca } \\
1-5 \mathrm{~h}]\end{array}$ & $\begin{array}{c}\mathrm{T} \\
{[\mathrm{C}]}\end{array}$ & $\begin{array}{c}\text { Station } \\
\mathrm{P} \\
{[\mathrm{hPa}]}\end{array}$ & $\begin{array}{l}\text { Altime } \\
\text { ter } \\
\text { inHg }\end{array}$ & $\begin{array}{c}\text { DewP } \\
{[\mathrm{C}]}\end{array}$ & $\begin{array}{c}\text { Wind } \\
\text { Dir } \\
{[\mathrm{deg}]}\end{array}$ & $\begin{array}{c}\text { WindS } \\
\mathrm{p} \\
{[\mathrm{m} / \mathrm{s}]}\end{array}$ & $\begin{array}{c}\text { Precip } \\
\text { since0 } \\
0 Z \\
\\
{[\mathrm{~mm}]}\end{array}$ & $\begin{array}{c}\text { SolarF } \\
\text { lux } \\
{[\mathrm{W} / \mathrm{m} 2} \\
]\end{array}$ \\
\hline $16: 00$ & 19.5 & 966.6 & 29.67 & 19.4 & 93 & 2.8 & 30.2 & 87.3 \\
\hline $16: 15$ & 19.4 & 966.9 & 29.68 & 19.4 & 112 & 3.4 & 30.2 & 111.7 \\
\hline $16: 35$ & 19.6 & 967.1 & 29.69 & 19.6 & 79 & 3 & 30.2 & 89.9 \\
\hline $16: 45$ & 19.7 & 967.1 & 29.69 & 19.7 & 92 & 3.1 & & 78.3 \\
\hline 17:00 & 19.8 & 967.1 & 29.69 & 19.8 & 67 & 1.9 & & 68.9 \\
\hline $17: 30$ & 19.5 & 967.2 & 29.69 & 19.3 & 89 & 3.4 & & 46.1 \\
\hline $17: 50$ & 19.6 & 967.1 & 29.69 & 19.1 & 72 & 5.3 & & 59.2 \\
\hline 18:10 & 20 & 967.2 & 29.69 & 19.7 & 100 & 4.3 & & 65 \\
\hline
\end{tabular}




$\begin{array}{lccccccc}\text { 18:45 } & 19.9 & 967.2 & 29.69 & 19.3 & 78 & 3.6 & 218.1 \\ 19: 30 & 20.7 & 966.9 & 29.68 & 20 & 87 & 2.6 & 171 \\ 20: 05 & 21 & 967.2 & 29.69 & 20.3 & 50 & 2.3 & 112.5 \\ 20: 20 & 21.3 & 967.1 & 29.69 & 20.5 & 72 & 2 & 127.3 \\ \text { 20:25 } & 21.3 & 967.2 & 29.69 & 20.5 & 81 & 2.6 & 140.7 \\ \begin{array}{l}\text { Shutdown: } \\ \text { sprinkles }\end{array} & & & & & & \\ \text { again. } & & & & & & & \end{array}$

The following data was collected by the AERIbago Met Tower. Detailed data below is from June 7, 2008. Figure 250 and Figure 251 show plots the pressure, temperature, relative humidity, and the mixing ratio from June 6 and June 7, respectively. Figure 252 and Figure 253 show the wind direction and wind speed for the same dates and timeframes. Figure 254 and Figure 255 give the AERI mean radiances from 060708 in the wavenumber ranges $550-1800$ and $1800-3000$, respectively. Figure 260 gives the AERIbago Vaisala Backscatter for all days data was collected during WAVEx.

TIME 1212839207

TIME 1212839267

TIME 1212839327

TIME 1212839387

TIME 1212839447

TIME 1212839507

TIME 1212839567

TIME 1212839627

TIME 1212839687

TIME 1212839747

TIME 1212839807

TIME 1212839867

TIME 1212839927

TIME 1212839987

TIME 1212840047

TIME 1212840107

TIME 1212840167

TIME 1212840227

TIME 1212840287

TIME 1212840347

TIME 1212840407

TIME 1212840467

TIME 1212840527

TIME 1212840587

TIME 1212840647

TIME 1212840707

TIME 1212840767

TIME 1212840827

TIME 1212840887

TIME 1212840947

TIME 1212841007

TIME 1212841067

TIME 1212841127

TIME 1212841187

TIME 1212841247

TIME 1212841307
$\mathrm{RH}$ 86.4 TEMP 18.66

$\mathrm{RH}$ 86.7 TEMP 18.69

$\mathrm{RH}$ 86.9 TEMP 18.69

$\mathrm{RH}$ 86.1 TEMP 18.75

$\mathrm{RH}$ 86.1 TEMP 18.87

$\mathrm{RH}$ 85.3 TEMP 18.94

$\mathrm{RH}$ 84.9 TEMP 18.99

$\mathrm{RH}$ 85.4 TEMP 18.95

$\mathrm{RH}$ 85.9 TEMP 18.93

$\mathrm{RH}$ 85.6 TEMP 18.92

$\mathrm{RH}$ 85.1 TEMP 18.95

$\mathrm{RH}$ 84.9 TEMP 18.97

$\mathrm{RH}$ 85.7 TEMP 19.01

$\mathrm{RH}$ 85.4 TEMP 19.01

$\mathrm{RH}$ 85.0 TEMP 19.1

$\mathrm{RH}$ 85.3 TEMP 19.12

$\mathrm{RH}$ 84.6 TEMP 19.15

$\mathrm{RH}$ 83.9 TEMP 19.17

$\mathrm{RH}$ 83.9 TEMP 19.22

$\mathrm{RH}$ 84.3 TEMP 19.26

$\mathrm{RH}$ 84.4 TEMP 19.27

$\mathrm{RH}$ 83.8 TEMP 19.3

RH 83.9 TEMP 19.34

$\mathrm{RH}$ 84.2 TEMP 19.38

$\mathrm{RH}$ 84.0 TEMP 19.46

$\mathrm{RH}$ 83.3 TEMP 19.5

$\mathrm{RH}$ 83.2 TEMP 19.52

$\mathrm{RH}$ 83.9 TEMP 19.55

$\mathrm{RH}$ 83.2 TEMP 19.62

$\mathrm{RH}$ 83.0 TEMP 19.65

RH 82.6 TEMP 19.7

RH 83.1 TEMP 19.77

$\mathrm{RH}$ 82.7 TEMP 19.81

$\mathrm{RH}$ 81.9 TEMP 19.85

$\mathrm{RH}$ 82.6 TEMP 19.89

RH 82.7 TEMP 19.94

\begin{tabular}{|c|c|c|c|}
\hline WDIR & 181.8 WSPD & 4.17 & PRES 980.31 \\
\hline WDIR & 184.9 WSPD & 3.572 & \\
\hline WDIR & 184.6 WSPD & 3.546 & RES 980.34 \\
\hline NDIR & 180.5 WSPD & 4.701 & PRES \\
\hline NDIR & 178.4 WSPD & 3.595 & 80.37 \\
\hline NDIR & 185.7 WSPD & 4.464 & 980. \\
\hline WDIR & 187.9 WSPD & 4.341 & PRE \\
\hline VDIR & 189.9 WSPD & 3.958 & PRES 980.36 \\
\hline WDIR & 186.9 WSPD & 4.002 & PRES 98 \\
\hline WDIR & 185.5 WSPD & 3.846 & ES $\subseteq$ \\
\hline WDIR & 192.1 WSPD & 4.617 & PRES 9 \\
\hline NDIR & 185.4 WSPD & 4.621 & PRES \\
\hline VDIR & 183.5 WSPD & 3.802 & 80.5 \\
\hline VDIR & 181.5 WSPD & 4.124 & 80.46 \\
\hline WDIR & 184.9 WSPD & 4.408 & PRE \\
\hline WDIR & 180.8 WSPD & 4.034 & PRES 980.5 \\
\hline WDIR & 184.8 WSPD & 4.64 & PRES \\
\hline WDIR & 184.0 WSPD & 5.218 & PRES \\
\hline WDIR & 183.2 WSPD & 4.92 & PRES \\
\hline WDIR & 184.2 WSPD & 4.363 & 980.48 \\
\hline WDIR & 186.7 Ws & 4.286 & 80.4 \\
\hline NDIR & $184.8 \mathrm{~W}$ & 5.006 & $\mathrm{P}$ \\
\hline WDIR & 181.6 WSPD & 4.598 & 80.5 \\
\hline DIR & $73.3 \quad$ WSPD & 4.59 & 980.5 \\
\hline WDIR & 182.3 WSPD & 4.756 & 980.51 \\
\hline WDIR & 177.5 WSPD & 4.557 & 980.48 \\
\hline WDIR & 180.2 WSPD & 4.709 & 980.48 \\
\hline WDIR & 177.1 WSPD & 4.124 & 980.51 \\
\hline WDIR & 183.0 WSPD & 4.214 & 980.51 \\
\hline WDIR & 181.5 WSPD & 4.189 & 980.5 \\
\hline WDIR & 177.5 WSPD & 4.712 & ES 980.52 \\
\hline WDIR & 174.9 WSPD & 3.984 & S 980.45 \\
\hline WDIR & 171.1 WSPD & 4.67 & ES 980.46 \\
\hline WDIR & 174.2 WSPD & 4.938 & ES 980.51 \\
\hline WDIR & 183.4 WSPD & 4.565 & 80.49 \\
\hline & & & \\
\hline
\end{tabular}

242 
TIME 1212841367

TIME 1212841427

TIME 1212841487

TIME 1212841547

TIME 1212841607

TIME 1212841667

TIME 1212841727

TIME 1212841787

TIME 1212841847

TIME 1212841907

TIME 1212841967

TIME 1212842027

TIME 1212842087

TIME 1212842147

TIME 1212842207

TIME 1212842267

TIME 1212842327

TIME 1212842387

TIME 1212842447

TIME 1212842507

TIME 1212842567

TIME 1212842627

TIME 1212842687

TIME 1212842747

TIME 1212842807

TIME 1212842867

TIME 1212842927

TIME 1212842987

TIME 1212843047

TIME 1212843107

TIME 1212843167

TIME 1212843227

TIME 1212843287

TIME 1212843347

TIME 1212843407

TIME 1212843467

TIME 1212843527

TIME 1212843587

TIME 1212843647

TIME 1212843706

TIME 1212843767

TIME 1212843826

TIME 1212843886

TIME 1212843946

TIME 1212844006

TIME 1212844066

TIME 1212844126

TIME 1212844186

TIME 1212844246

TIME 1212844306

TIME 1212844366

TIME 1212844426

TIME 1212844486

TIME 1212844546

TIME 1212844606

TIME 1212844666
$\mathrm{RH}$ 83.1 TEMP 19.99 $\mathrm{RH}$ 82.5 TEMP 20.03 RH 81.2 TEMP 20.08 $\mathrm{RH} 81.6$ TEMP 20.07 $\mathrm{RH}$ 81.5 TEMP 20.12 $\mathrm{RH}$ 82.1 TEMP 20.13 $\mathrm{RH}$ 82.3 TEMP 20.17 $\mathrm{RH} 81.7$ TEMP 20.22 $\mathrm{RH}$ 81.0 TEMP 20.27 RH 82.1 TEMP 20.33 $\mathrm{RH}$ 82.2 TEMP 20.37 RH 81.9 TEMP 20.42 $\mathrm{RH} 81.0$ TEMP 20.45 RH 81.8 TEMP 20.47 $\mathrm{RH}$ 81.2 TEMP 20.55 $\mathrm{RH}$ 80.4 TEMP 20.6 $\mathrm{RH}$ 80.4 TEMP 20.65 $\mathrm{RH} 79.9$ TEMP 20.69 RH 79.9 TEMP 20.75 $\mathrm{RH} 78.7$ TEMP 20.81 $\mathrm{RH} 79.2$ TEMP 20.82 $\mathrm{RH} 78.0$ TEMP 20.89 $\mathrm{RH} 79.5$ TEMP 20.95 $\mathrm{RH}$ 78.6 TEMP 21.0 $\mathrm{RH} 78.4$ TEMP 21.04 RH 78.3 TEMP 21.1

$\mathrm{RH} 78.1$ TEMP 21.11 $\mathrm{RH} 77.7$ TEMP 21.12 $\mathrm{RH} 77.9$ TEMP 21.16 RH 77.9 TEMP 21.21 $\mathrm{RH} 77.1$ TEMP 21.23 $\mathrm{RH} 76.8$ TEMP 21.26 $\mathrm{RH}$ 76.2 TEMP 21.3

$\mathrm{RH} 76.4$ TEMP 21.34 $\mathrm{RH} 77.1$ TEMP 21.38 $\mathrm{RH} 77.6$ TEMP 21.48 $\mathrm{RH} 76.7$ TEMP 21.52 $\mathrm{RH} 76.1$ TEMP 21.53 $\mathrm{RH} 75.8$ TEMP 21.55 $\mathrm{RH} 75.8$ TEMP 21.56 $\mathrm{RH} 75.9$ TEMP 21.6 $\mathrm{RH} 75.5$ TEMP 21.62 $\mathrm{RH} 75.9$ TEMP 21.69 $\mathrm{RH} 74.9$ TEMP 21.75 RH 74.9 TEMP 21.76 $\mathrm{RH}$ 76.2 TEMP 21.76 RH 75.9 TEMP 21.8 $\mathrm{RH} 75.3$ TEMP 21.87 $\mathrm{RH} 75.3$ TEMP 21.92 $\mathrm{RH} 74.3$ TEMP 21.91 RH 75.2 TEMP 21.9 $\mathrm{RH} 76.3$ TEMP 21.95 $\mathrm{RH}$ 74.7 TEMP 22.01 $\mathrm{RH} 75.3$ TEMP 22.03 $\mathrm{RH} 75.2$ TEMP 22.09 $\mathrm{RH} 74.7$ TEMP 22.14
WDIR 177.6 WSPD 3.912 WDIR 182.0 WSPD 4.351 WDIR 184.9 WSPD 4.978 WDIR 177.3 WSPD 4.554 WDIR 179.2 WSPD 4.688 WDIR 178.1 WSPD 4.266 WDIR 180.3 WSPD 4.003 WDIR 177.3 WSPD 4.63 WDIR 177.2 WSPD 5.439 WDIR 176.3 WSPD 4.211 WDIR 175.2 WSPD 4.462 WDIR 175.1 WSPD 4.559 WDIR 176.3 WSPD 5.181 WDIR 178.8 WSPD 4.305 WDIR 173.1 WSPD 4.756 WDIR 183.8 WSPD 5.334 WDIR 174.9 WSPD 5.148 WDIR 179.9 WSPD 5.401 WDIR 177.4 WSPD 5.548 WDIR 181.1 WSPD 6.399 WDIR 181.1 WSPD 5.971 WDIR 180.5 WSPD 6.77 WDIR 184.6 WSPD 4.663 WDIR 183.7 WSPD 5.463 WDIR 181.5 WSPD 5.628 WDIR 179.5 WSPD 6.096 WDIR 171.1 WSPD 6.306 WDIR 178.8 WSPD 6.72 WDIR 182.1 WSPD 5.831 WDIR 182.0 WSPD 5.493 WDIR 181.4 WSPD 6.831 WDIR 180.8 WSPD 7.33 WDIR 182.0 WSPD 7.13 WDIR 185.1 WSPD 7.07 WDIR 179.0 WSPD 6.205 WDIR 181.7 WSPD 5.062 WDIR 182.5 WSPD 6.368 WDIR 180.2 WSPD 6.541 WDIR 178.2 WSPD 6.283 WDIR 174.4 WSPD 6.845 WDIR 177.8 WSPD 6.793 WDIR 184.5 WSPD 6.863 WDIR 179.9 WSPD 6.568 WDIR 184.3 WSPD 6.834 WDIR 174.7 WSPD 7.0 WDIR 185.5 WSPD 5.831 WDIR 178.2 WSPD 5.922 WDIR 173.5 WSPD 6.133 WDIR 177.8 WSPD 6.092 WDIR 180.1 WSPD 6.499 WDIR 176.9 WSPD 6.172 WDIR 181.7 WSPD 5.446 WDIR 181.0 WSPD 6.12 WDIR 182.3 WSPD 5.762 WDIR 180.9 WSPD 6.159 WDIR 178.3 WSPD 6.14
PRES 980.5 PRES 980.5 PRES 980.46 PRES 980.46 PRES 980.5

PRES 980.46 PRES 980.45 PRES 980.43 PRES 980.46 PRES 980.46 PRES 980.46 PRES 980.41 PRES 980.42 PRES 980.36 PRES 980.39 PRES 980.36 PRES 980.37 PRES 980.29 PRES 980.34 PRES 980.32 PRES 980.33 PRES 980.36 PRES 980.38 PRES 980.35 PRES 980.38 PRES 980.35 PRES 980.33 PRES 980.32 PRES 980.3 PRES 980.31 PRES 980.31 PRES 980.34 PRES 980.31 PRES 980.34 PRES 980.39 PRES 980.32 PRES 980.29 PRES 980.24 PRES 980.24 PRES 980.26 PRES 980.26 PRES 980.23 PRES 980.25 PRES 980.18 PRES 980.23 PRES 980.19 PRES 980.17 PRES 980.19 PRES 980.21 PRES 980.18 PRES 980.19 PRES 980.18 PRES 980.16 PRES 980.18 PRES 980.21 PRES 980.22 
TIME 1212844726

TIME 1212844786

TIME 1212844846

TIME 1212844906

TIME 1212844966

TIME 1212845026

TIME 1212845086

TIME 1212845146

TIME 1212845206

TIME 1212845266

TIME 1212845326

TIME 1212845386

TIME 1212845446

TIME 1212845506

TIME 1212845566

TIME 1212845626

TIME 1212845686

TIME 1212845746

TIME 1212845806

TIME 1212845866

TIME 1212845926

TIME 1212845986

TIME 1212846046

TIME 1212846106

TIME 1212846166

TIME 1212846226

TIME 1212846286

TIME 1212846346

TIME 1212846406

TIME 1212846466

TIME 1212846526

TIME 1212846586

TIME 1212846646

TIME 1212846706

TIME 1212846766

TIME 1212846826

TIME 1212846886

TIME 1212846946

TIME 1212847006

TIME 1212847066

TIME 1212847126

TIME 1212847186

TIME 1212847246

TIME 1212847306

TIME 1212847366

TIME 1212847426

TIME 1212847486

TIME 1212847546

TIME 1212847606

TIME 1212847666

TIME 1212847726

TIME 1212847786 979.95

TIME 1212847846 TIME 1212847906

TIME 1212847966
$\mathrm{RH} 74.7$ TEMP 22.14 RH 75.4 TEMP 22.17 $\mathrm{RH} 75.4$ TEMP 22.25 $\mathrm{RH} 75.0$ TEMP 22.36 $\mathrm{RH} 74.4$ TEMP 22.41 RH 73.9 TEMP 22.41 $\mathrm{RH}$ 73.6 TEMP 22.39 $\mathrm{RH} 73.5$ TEMP 22.4 $\mathrm{RH} 74.4$ TEMP 22.43 $\mathrm{RH} 75.2$ TEMP 22.5 $\mathrm{RH} 73.9$ TEMP 22.51 $\mathrm{RH} 74.8$ TEMP 22.57 $\mathrm{RH} 74.4$ TEMP 22.66 $\mathrm{RH} 73.7$ TEMP 22.68 $\mathrm{RH} 73.4$ TEMP 22.73 $\mathrm{RH} 73.9$ TEMP 22.74 $\mathrm{RH} 73.7$ TEMP 22.74 $\mathrm{RH} 73.6$ TEMP 22.84 $\mathrm{RH} 72.9$ TEMP 22.88 $\mathrm{RH} 73.1$ TEMP 22.89 RH 72.3 TEMP 22.9 $\mathrm{RH} 72.7$ TEMP 22.89 $\mathrm{RH} 73.1$ TEMP 22.93 $\mathrm{RH}$ 73.6 TEMP 22.95 $\mathrm{RH} 73.3$ TEMP 22.98 $\mathrm{RH} 73.8$ TEMP 22.99 $\mathrm{RH}$ 73.6 TEMP 23.05 $\mathrm{RH} 73.2$ TEMP 23.07 $\mathrm{RH}$ 74.0 TEMP 23.1 $\mathrm{RH}$ 73.0 TEMP 23.11 $\mathrm{RH} 73.7$ TEMP 23.17 $\mathrm{RH} 72.3$ TEMP 23.16 $\mathrm{RH} 73.3$ TEMP 23.18 $\mathrm{RH} 74.4$ TEMP 23.27 $\mathrm{RH}$ 73.3 TEMP 23.34 $\mathrm{RH} 73.0$ TEMP 23.36 $\mathrm{RH} 72.9$ TEMP 23.37 $\mathrm{RH} 72.6$ TEMP 23.42 $\mathrm{RH} 72.6$ TEMP 23.44 $\mathrm{RH} 72.8$ TEMP 23.45 $\mathrm{RH}$ 73.4 TEMP 23.5 $\mathrm{RH} 73.0$ TEMP 23.55 $\mathrm{RH} 73.0$ TEMP 23.58 RH 71.9 TEMP 23.72 RH 71.8 TEMP 23.71 $\mathrm{RH} 73.3$ TEMP 23.69 RH 73.0 TEMP 23.7 $\mathrm{RH} 72.8$ TEMP 23.69 $\mathrm{RH}$ 73.0 TEMP 23.69 $\mathrm{RH} 74.1$ TEMP 23.77 $\mathrm{RH} 73.5$ TEMP 23.83 $\mathrm{RH} 73.2$ TEMP 23.87 $\mathrm{RH} 73.8$ TEMP 23.88 $\mathrm{RH} 74.4$ TEMP 23.94 $\mathrm{RH}$ 73.6 TEMP 24.0
WDIR 179.7 WSPD 5.988

WDIR 184.1 WSPD 5.274

WDIR 179.3 WSPD 5.557

WDIR 177.3 WSPD 5.263

WDIR 184.1 WSPD 5.909

WDIR 183.1 WSPD 6.453

WDIR 183.6 WSPD 6.602

WDIR 174.2 WSPD 6.84

WDIR 182.8 WSPD 6.181

WDIR 179.5 WSPD 5.563

WDIR 177.0 WSPD 6.837

WDIR 179.7 WSPD 5.968

WDIR 190.6 WSPD 5.648

WDIR 181.4 WSPD 5.787

WDIR 179.1 WSPD 6.737

WDIR 178.3 WSPD 6.28

WDIR 178.0 WSPD 6.546

WDIR 182.6 WSPD 6.46

WDIR 179.9 WSPD 7.56

WDIR 177.4 WSPD 7.42

WDIR 183.6 WSPD 7.83

WDIR 182.2 WSPD 7.22

WDIR 174.2 WSPD 7.55

WDIR 179.1 WSPD 6.623

WDIR 179.7 WSPD 7.71

WDIR 185.0 WSPD 6.633

WDIR 176.0 WSPD 7.16

WDIR 179.8 WSPD 7.29

WDIR 179.7 WSPD 6.385

WDIR 195.0 WSPD 7.35

WDIR 190.5 WSPD 7.05

WDIR 187.4 WSPD 9.48

WDIR 182.2 WSPD 7.51

WDIR 187.6 WSPD 6.429

WDIR 187.8 WSPD 7.45

WDIR 182.2 WSPD 7.26

WDIR 187.1 WSPD 7.39

WDIR 181.8 WSPD 7.97

WDIR 182.7 WSPD 7.35

WDIR 184.0 WSPD 7.75

WDIR 178.6 WSPD 6.742

WDIR 189.2 WSPD 6.764

WDIR 180.9 WSPD 7.16

WDIR 183.2 WSPD 8.31

WDIR 184.1 WSPD 8.27

WDIR 189.9 WSPD 6.769

WDIR 183.5 WSPD 7.29

WDIR 195.7 WSPD 7.5

WDIR 193.8 WSPD 7.05

WDIR 191.3 WSPD 6.017

WDIR 189.2 WSPD 6.573

WDIR 196.1

WSPD

WDIR 195.3 WSPD 6.76

WDIR 192.1 WSPD 6.671

WDIR 183.9 WSPD 7.98
PRES 980.2

PRES 980.21

PRES 980.18

PRES 980.21

PRES 980.22

PRES 980.27

PRES 980.31

PRES 980.3

PRES 980.25

PRES 980.22

PRES 980.22

PRES 980.24

PRES 980.19

PRES 980.23

PRES 980.26

PRES 980.23

PRES 980.31

PRES 980.24

PRES 980.23

PRES 980.25

PRES 980.17

PRES 980.16

PRES 980.19

PRES 980.18

PRES 980.18

PRES 980.18

PRES 980.18

PRES 980.14

PRES 980.16

PRES 980.15

PRES 980.15

PRES 980.17

PRES 980.05

PRES 979.99

PRES 979.95

PRES 979.96

PRES 979.96

PRES 979.96

PRES 979.93

PRES 979.88

PRES 979.9

PRES 979.86

PRES 979.86

PRES 979.89

PRES 979.9

PRES 979.93

PRES 979.89

PRES 979.94

PRES 979.96

PRES 979.97

PRES 979.98

7.05 PRES

PRES 979.88

PRES 979.84

PRES 979.84 
TIME 1212848026

TIME 1212848086

TIME 1212848146

TIME 1212848206

TIME 1212848266

TIME 1212848326

TIME 1212848386

TIME 1212848446

TIME 1212848506

TIME 1212848566

TIME 1212848626

TIME 1212848686

TIME 1212848746

TIME 1212848806

TIME 1212848866

TIME 1212848926

TIME 1212848986

TIME 1212849046

TIME 1212849106

TIME 1212849166

TIME 1212849226

TIME 1212849286

TIME 1212849346

TIME 1212849406

TIME 1212849466

TIME 1212849526

TIME 1212849586

TIME 1212849646

TIME 1212849706

TIME 1212849766

TIME 1212849826

TIME 1212849886

TIME 1212849946

TIME 1212850006

TIME 1212850066

TIME 1212850126

TIME 1212850186

TIME 1212850246

TIME 1212850306

TIME 1212850366

TIME 1212850426

TIME 1212850486

TIME 1212850546

TIME 1212850606

TIME 1212850665

TIME 1212850726

TIME 1212850785

TIME 1212850845

TIME 1212850905

TIME 1212850965

TIME 1212851025

TIME 1212851085

TIME 1212851145

TIME 1212851205

TIME 1212851265

TIME 1212851325
$\mathrm{RH} 74.0$ TEMP 23.98 $\mathrm{RH} 74.2$ TEMP 24.0 $\mathrm{RH} 74.9$ TEMP 23.99 $\mathrm{RH} 75.0$ TEMP 24.02 $\mathrm{RH} 75.2$ TEMP 24.04 $\mathrm{RH} 75.1$ TEMP 24.14 $\mathrm{RH}$ 75.3 TEMP 24.2

$\mathrm{RH} 75.5$ TEMP 24.21 $\mathrm{RH} 74.7$ TEMP 24.25 RH 74.3 TEMP 24.27 RH 75.2 TEMP 24.25 $\mathrm{RH} 74.1$ TEMP 24.26 $\mathrm{RH} 75.3$ TEMP 24.29 $\mathrm{RH} 75.0$ TEMP 24.3

$\mathrm{RH} 75.1$ TEMP 24.28 $\mathrm{RH} 75.4$ TEMP 24.29 $\mathrm{RH}$ 76.0 TEMP 24.3

$\mathrm{RH} 75.5$ TEMP 24.38 $\mathrm{RH} 75.2$ TEMP 24.37 $\mathrm{RH}$ 76.2 TEMP 24.4

$\mathrm{RH} 76.8$ TEMP 24.52 $\mathrm{RH} 75.9$ TEMP 24.74 $\mathrm{RH} 74.7$ TEMP 24.77 $\mathrm{RH} 75.5$ TEMP 24.68 $\mathrm{RH} 75.9$ TEMP 24.66 $\mathrm{RH} 76.1$ TEMP 24.62 $\mathrm{RH} 77.3$ TEMP 24.66 $\mathrm{RH} 77.2$ TEMP 24.71 RH 77.0 TEMP 24.76 $\mathrm{RH} 77.5$ TEMP 24.8 $\mathrm{RH} 77.0$ TEMP 24.81 $\mathrm{RH} 77.4$ TEMP 24.81 $\mathrm{RH} 77.2$ TEMP 24.86 $\mathrm{RH} 76.6$ TEMP 24.77 RH 77.9 TEMP 24.69 $\mathrm{RH} 78.4$ TEMP 24.66 $\mathrm{RH} 79.3$ TEMP 24.75 $\mathrm{RH} 78.1$ TEMP 24.83 RH 78.7 TEMP 24.85 $\mathrm{RH} 77.9$ TEMP 24.81 $\mathrm{RH} 78.8$ TEMP 24.79 $\mathrm{RH} 79.3$ TEMP 24.76 $\mathrm{RH} 79.4$ TEMP 24.82 $\mathrm{RH} 79.1$ TEMP 24.77 RH 79.6 TEMP 24.73 $\mathrm{RH} 79.8$ TEMP 24.72 $\mathrm{RH} 79.6$ TEMP 24.69 RH 79.0 TEMP 24.62 $\mathrm{RH}$ 80.7 TEMP 24.59 $\mathrm{RH}$ 81.0 TEMP 24.58 RH 81.3 TEMP 24.47 $\mathrm{RH}$ 81.8 TEMP 24.43 RH 81.6 TEMP 24.39 $\mathrm{RH}$ 82.4 TEMP 24.35 $\mathrm{RH}$ 82.4 TEMP 24.35 $\mathrm{RH} 82.3$ TEMP 24.33
WDIR 193.9 WSPD 7.5

WDIR 186.4 WSPD 8.49

WDIR 192.4 WSPD 8.33

WDIR 189.4 WSPD 9.28

WDIR 195.1 WSPD 8.57

WDIR 191.8 WSPD 8.28

WDIR 188.3 WSPD 7.94

WDIR 186.0 WSPD 7.23

WDIR 185.8 WSPD 8.76

WDIR 189.7 WSPD 8.78

WDIR 190.6 WSPD 8.13

WDIR 181.8 WSPD 8.77

WDIR 193.0 WSPD 6.795

WDIR 187.3 WSPD 7.75

WDIR 187.3 WSPD 8.11

WDIR 190.2 WSPD 7.91

WDIR 189.1 WSPD 6.675

WDIR 196.8 WSPD 7.2

WDIR 197.1 WSPD 6.759

WDIR 194.3 WSPD 6.311

WDIR 179.9 WSPD 6.009

WDIR 187.3 WSPD 6.594

WDIR 190.3 WSPD 6.883

WDIR 188.4 WSPD 7.18

WDIR 185.6 WSPD 7.57

WDIR 186.4 WSPD 7.34

WDIR 186.5 WSPD 6.827

WDIR 188.1 WSPD 6.167

WDIR 180.0 WSPD 6.769

WDIR 178.6 WSPD 6.362

WDIR 184.2 WSPD 6.429

WDIR 179.8 WSPD 6.52

WDIR 184.7 WSPD 7.04

WDIR 180.5 WSPD 8.04

WDIR 182.3 WSPD 6.8

WDIR 185.5 WSPD 6.515

WDIR 187.3 WSPD 5.802

WDIR 185.6 WSPD 6.708

WDIR 190.0 WSPD 6.703

WDIR 179.5 WSPD 7.79

WDIR 177.1 WSPD 7.13

WDIR 179.3 WSPD 6.496

WDIR 174.6 WSPD 7.81

WDIR 179.3 WSPD 7.82

WDIR 178.9 WSPD 7.68

WDIR 181.7 WSPD 7.18

WDIR 176.0 WSPD 7.9

WDIR 188.4 WSPD 9.14

WDIR 173.5 WSPD 6.933

WDIR 172.4 WSPD 7.31

WDIR 174.9 WSPD 7.74

WDIR 172.5 WSPD 6.623

WDIR 171.9 WSPD 8.03

WDIR 176.2 WSPD 6.726

WDIR 174.4 WSPD 8.0

WDIR 177.5 WSPD 8.0
PRES 979.77

PRES 979.79

PRES 979.76

PRES 979.74

PRES 979.68

PRES 979.65

PRES 979.7

PRES 979.73

PRES 979.72

PRES 979.75

PRES 979.75

PRES 979.68

PRES 979.63

PRES 979.63

PRES 979.62

PRES 979.68

PRES 979.71

PRES 979.73

PRES 979.7

PRES 979.66

PRES 979.66

PRES 979.68

PRES 979.74

PRES 979.61

PRES 979.7

PRES 979.68

PRES 979.68

PRES 979.68

PRES 979.63

PRES 979.63

PRES 979.58

PRES 979.51

PRES 979.52

PRES 979.52

PRES 979.56

PRES 979.55

PRES 979.53

PRES 979.52

PRES 979.58

PRES 979.56

PRES 979.51

PRES 979.54

PRES 979.6

PRES 979.57

PRES 979.59

PRES 979.59

PRES 979.57

PRES 979.61

PRES 979.58

PRES 979.55

PRES 979.57

PRES 979.58

PRES 979.57

PRES 979.64

PRES 979.67

PRES 979.66 
TIME 1212851385

TIME 1212851445

TIME 1212851505

TIME 1212851565

TIME 1212851625

TIME 1212851685

TIME 1212851745

TIME 1212851805

TIME 1212851865

TIME 1212851925

TIME 1212851985

TIME 1212852045

TIME 1212852105

TIME 1212852165

TIME 1212852225

TIME 1212852285

TIME 1212852345

TIME 1212852405

TIME 1212852465

TIME 1212852525

TIME 1212852585

TIME 1212852645

TIME 1212852705

TIME 1212852765

TIME 1212852825

TIME 1212852885

TIME 1212852945

TIME 1212853005

TIME 1212853065

TIME 1212853125

TIME 1212853185

TIME 1212853245

TIME 1212853305

TIME 1212853365

TIME 1212853425

TIME 1212853485

TIME 1212853545

TIME 1212853605

TIME 1212853665

TIME 1212853725

TIME 1212853785

TIME 1212853845

TIME 1212853905

TIME 1212853965

TIME 1212854025

TIME 1212854085

TIME 1212854145

TIME 1212854205

TIME 1212854265

TIME 1212854325

TIME 1212854385

TIME 1212854445
RH 82.7 TEMP 24.32 $\mathrm{RH}$ 82.6 TEMP 24.31 $\mathrm{RH}$ 82.3 TEMP 24.28 $\mathrm{RH}$ 82.6 TEMP 24.22 $\mathrm{RH} 82.9$ TEMP 24.15 $\mathrm{RH}$ 83.5 TEMP 24.13 $\mathrm{RH}$ 83.5 TEMP 24.26 $\mathrm{RH}$ 82.5 TEMP 24.37 $\mathrm{RH}$ 83.4 TEMP 24.4 $\mathrm{RH}$ 83.2 TEMP 24.56 $\mathrm{RH} 82.0$ TEMP 24.58 $\mathrm{RH}$ 82.1 TEMP 24.58 $\mathrm{RH}$ 82.2 TEMP 24.6

$\mathrm{RH}$ 83.1 TEMP 24.62 $\mathrm{RH}$ 82.7 TEMP 24.73 RH 81.7 TEMP 24.75 $\mathrm{RH}$ 82.0 TEMP 24.73 $\mathrm{RH}$ 82.3 TEMP 24.77 RH 82.6 TEMP 24.92 $\mathrm{RH}$ 81.4 TEMP 25.0

$\mathrm{RH}$ 81.6 TEMP 25.01 $\mathrm{RH}$ 82.2 TEMP 25.09 $\mathrm{RH}$ 81.0 TEMP 25.19 $\mathrm{RH} 82.1$ TEMP 25.26 $\mathrm{RH}$ 80.6 TEMP 25.3 RH 81.0 TEMP 25.31 $\mathrm{RH}$ 80.8 TEMP 25.35 $\mathrm{RH}$ 80.3 TEMP 25.31 RH 80.9 TEMP 25.24 $\mathrm{RH} 81.0$ TEMP 25.26 $\mathrm{RH}$ 80.4 TEMP 25.24 $\mathrm{RH} 81.1$ TEMP 25.19 $\mathrm{RH}$ 81.4 TEMP 25.21 $\mathrm{RH}$ 81.6 TEMP 25.28 RH 81.5 TEMP 25.38 RH 81.4 TEMP 25.59 $\mathrm{RH} 79.2$ TEMP 25.75 $\mathrm{RH} 79.7$ TEMP 25.65 $\mathrm{RH}$ 80.1 TEMP 25.66 $\mathrm{RH}$ 80.1 TEMP 25.66 $\mathrm{RH}$ 80.4 TEMP 25.81 $\mathrm{RH} 79.6$ TEMP 25.94 $\mathrm{RH} 79.8$ TEMP 26.01 $\mathrm{RH} 78.6$ TEMP 26.03 $\mathrm{RH} 78.3$ TEMP 26.08 $\mathrm{RH} 77.9$ TEMP 26.08 $\mathrm{RH} 78.9$ TEMP 26.15 $\mathrm{RH}$ 78.2 TEMP 26.3 RH 79.1 TEMP 26.44 $\mathrm{RH} 77.6$ TEMP 26.59 RH 77.1 TEMP 26.62 RH 77.1 TEMP 26.59
WDIR 177.4 WSPD 7.53

WDIR 173.1 WSPD 7.68

WDIR 175.1 WSPD 8.64

WDIR 177.2 WSPD 8.05

WDIR 179.2 WSPD 8.22

WDIR 185.1 WSPD 6.239

WDIR 182.6 WSPD 6.136

WDIR 183.8 WSPD 7.14

WDIR 176.7 WSPD 6.292

WDIR 179.2 WSPD 6.368

WDIR 177.1 WSPD 6.435

WDIR 182.8 WSPD 7.3

WDIR 170.1 WSPD 6.798

WDIR 180.0 WSPD 5.473

WDIR 181.0 WSPD 5.782

WDIR 173.6 WSPD 6.845

WDIR 184.3 WSPD 6.845

WDIR 186.0 WSPD 5.985

WDIR 175.7 WSPD 5.712

WDIR 175.5 WSPD 6.076

WDIR 182.4 WSPD 5.762

WDIR 188.9 WSPD 5.009

WDIR 183.1 WSPD 6.001

WDIR 176.2 WSPD 4.322

WDIR 167.1 WSPD 4.63

WDIR 166.9 WSPD 6.092

WDIR 173.9 WSPD 6.59

WDIR 185.3 WSPD 6.226

WDIR 171.7 WSPD 5.962

WDIR 167.5 WSPD 5.975

WDIR 159.7 WSPD 6.084

WDIR 170.0 WSPD 6.094

WDIR 166.2 WSPD 5.414

WDIR 167.1 WSPD 5.397

WDIR 171.1 WSPD 5.522

WDIR 182.0 WSPD 5.201

WDIR 187.6 WSPD 5.651

WDIR 184.1 WSPD 5.207

WDIR 173.4 WSPD 5.341

WDIR 180.4 WSPD 4.92

WDIR 179.0 WSPD 4.577

WDIR 173.8 WSPD 5.74

WDIR 173.3 WSPD 5.963

WDIR 177.5 WSPD 6.13

WDIR 185.8 WSPD 7.3

WDIR 182.7 WSPD 7.25

WDIR 182.2 WSPD 5.988

WDIR 179.0 WSPD 6.512

WDIR 182.2 WSPD 5.236

WDIR 186.8 WSPD 5.914

WDIR 190.9 WSPD 5.588

WDIR 185.0 WSPD 5.382
PRES 979.65

PRES 979.65

PRES 979.59

PRES 979.59

PRES 979.65

PRES 979.67

PRES 979.7

PRES 979.7

PRES 979.67

PRES 979.64

PRES 979.56

PRES 979.56

PRES 979.55

PRES 979.53

PRES 979.6

PRES 979.66

PRES 979.62

PRES 979.6

PRES 979.58

PRES 979.52

PRES 979.52

PRES 979.56

PRES 979.55

PRES 979.58

PRES 979.61

PRES 979.62

PRES 979.63

PRES 979.59

PRES 979.51

PRES 979.46

PRES 979.41

PRES 979.42

PRES 979.46

PRES 979.44

PRES 979.47

PRES 979.5

PRES 979.52

PRES 979.52

PRES 979.47

PRES 979.51

PRES 979.52

PRES 979.56

PRES 979.47

PRES 979.49

PRES 979.48

PRES 979.44

PRES 979.41

PRES 979.4

PRES 979.39

PRES 979.43

PRES 979.46

PRES 979.47 

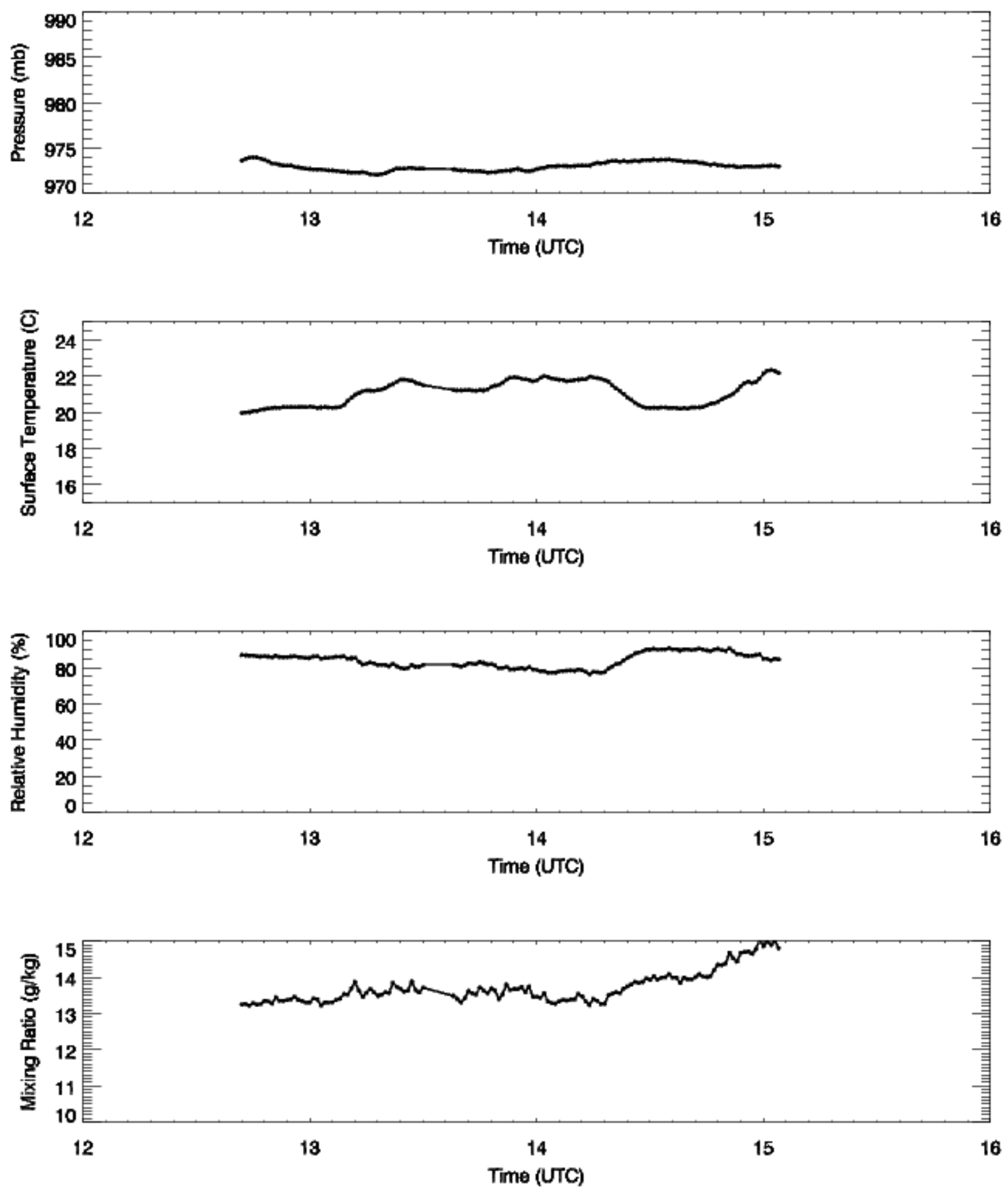

Figure 250. AERIbago surface observations 060608. 

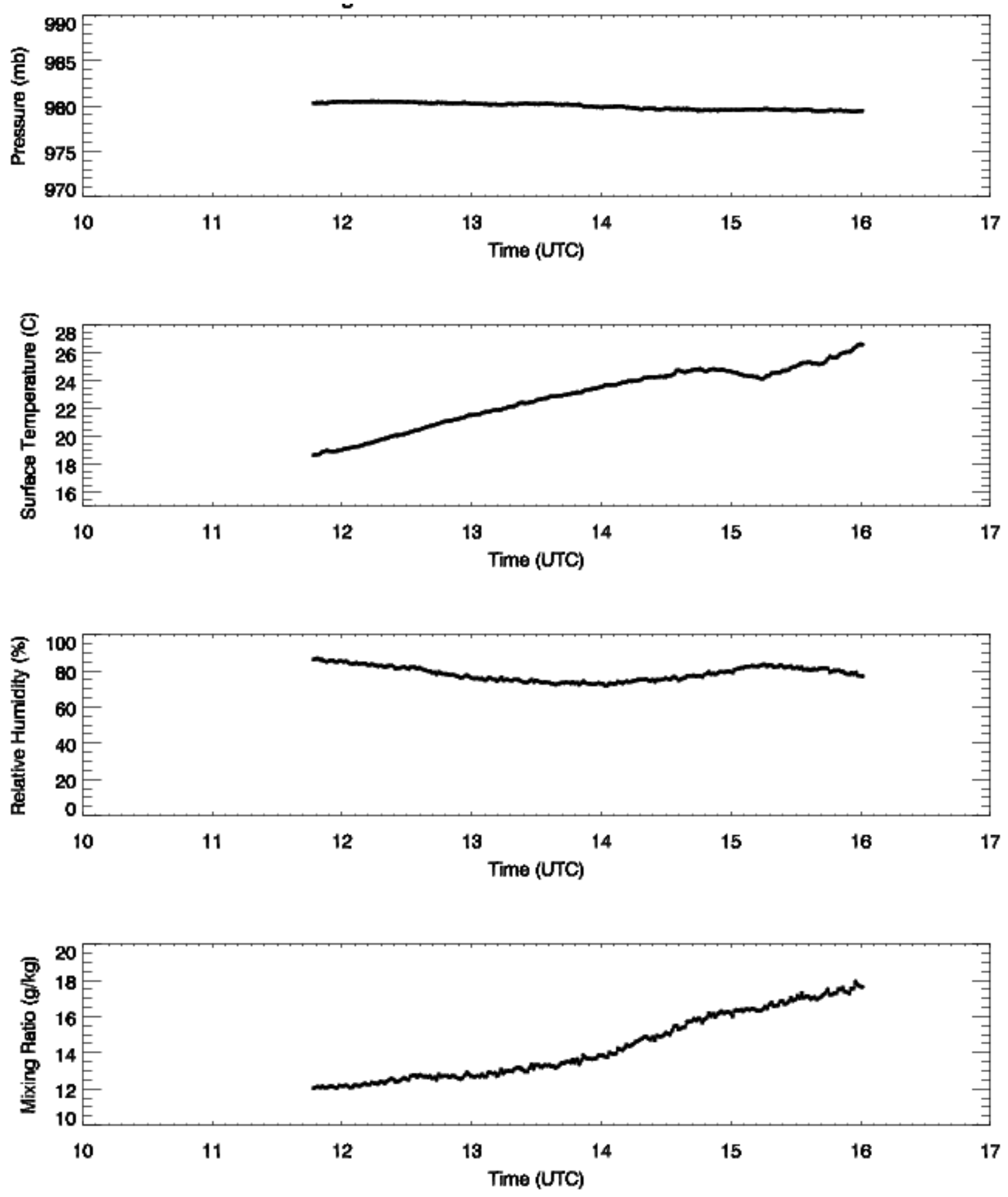

Figure 251. AERIbago surface observations 060708. 

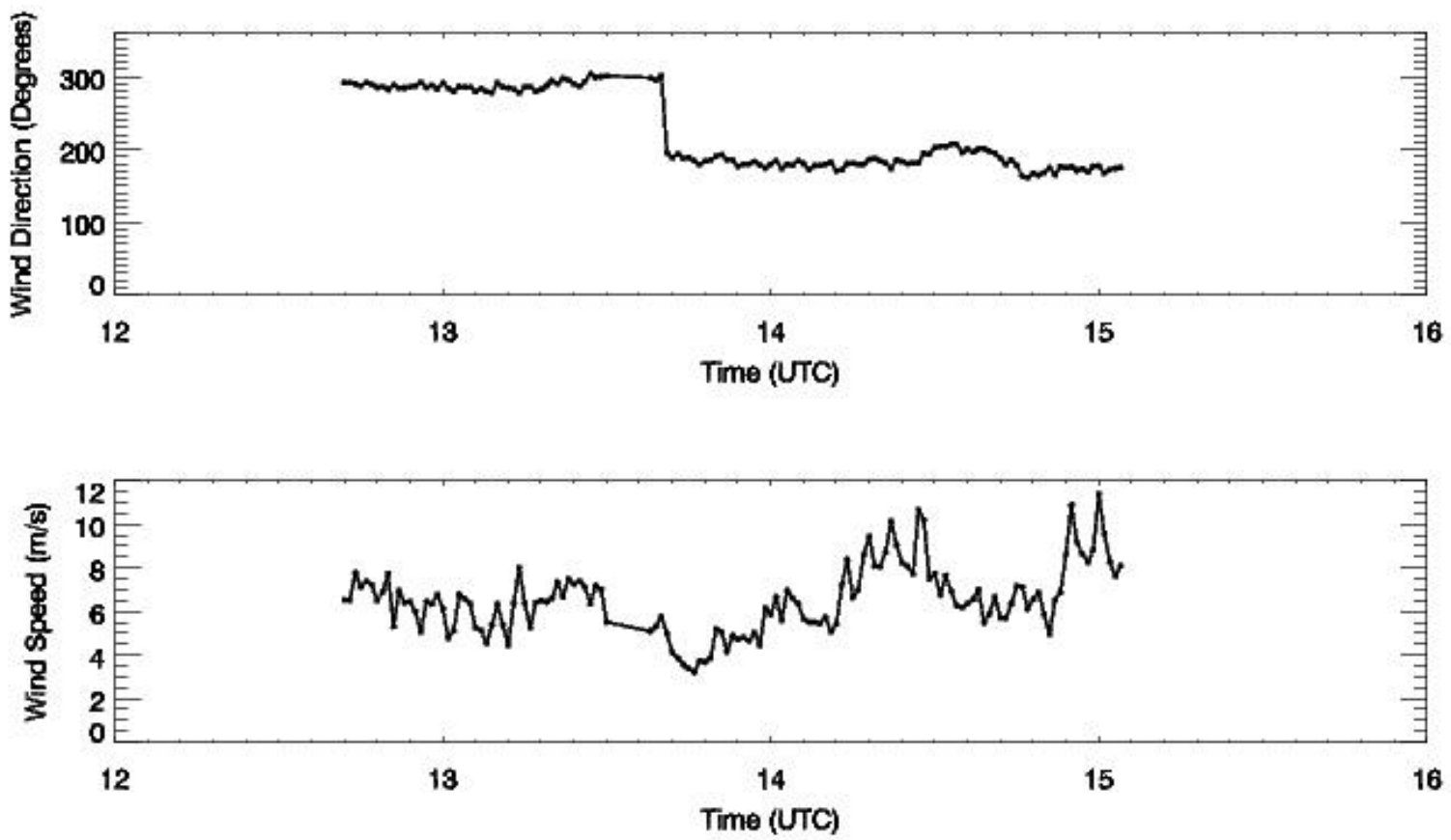

Figure 252. Wind direction and speed for 060608 .
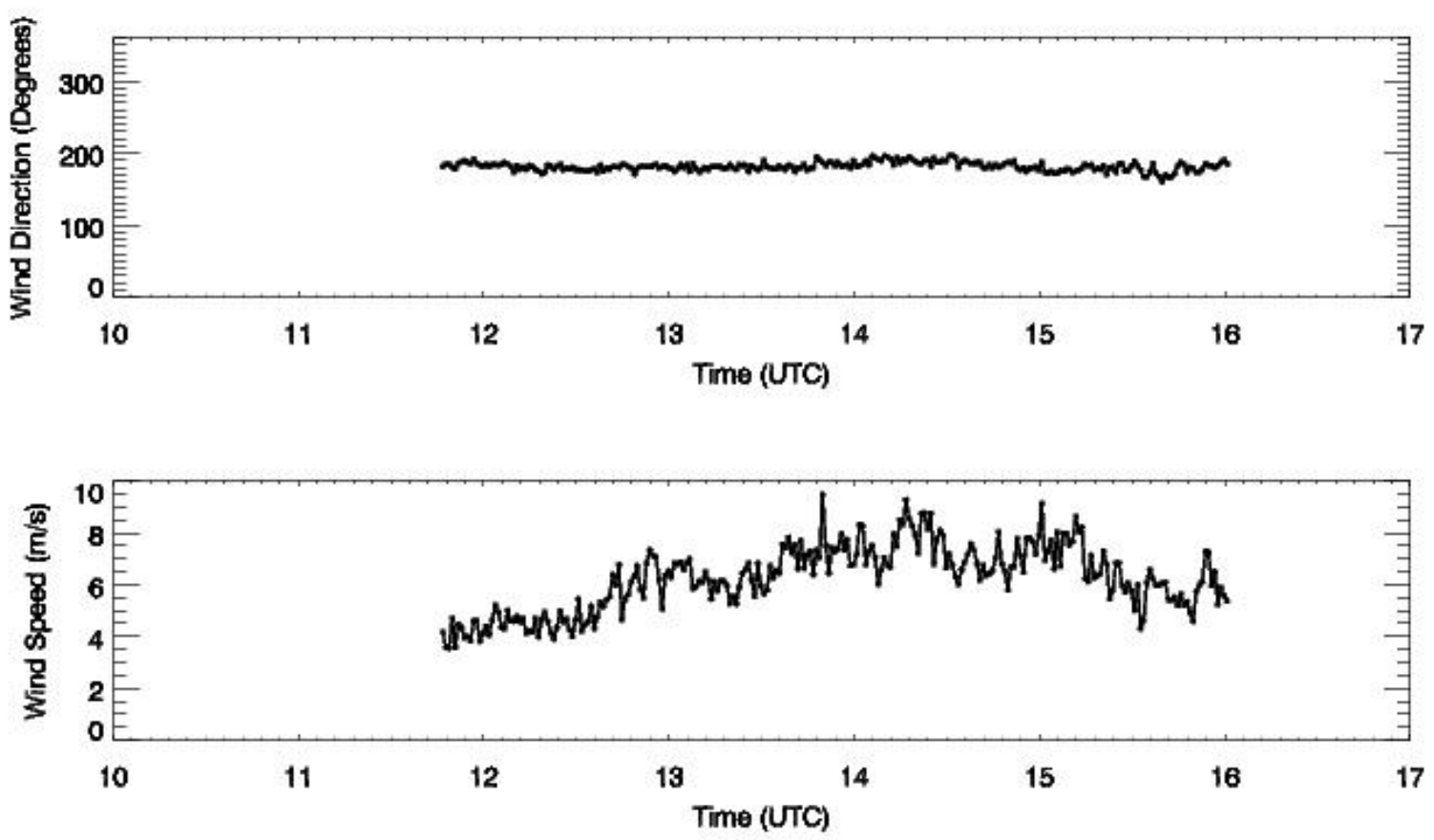

Figure 253. Wind direction and speed for 060708. 


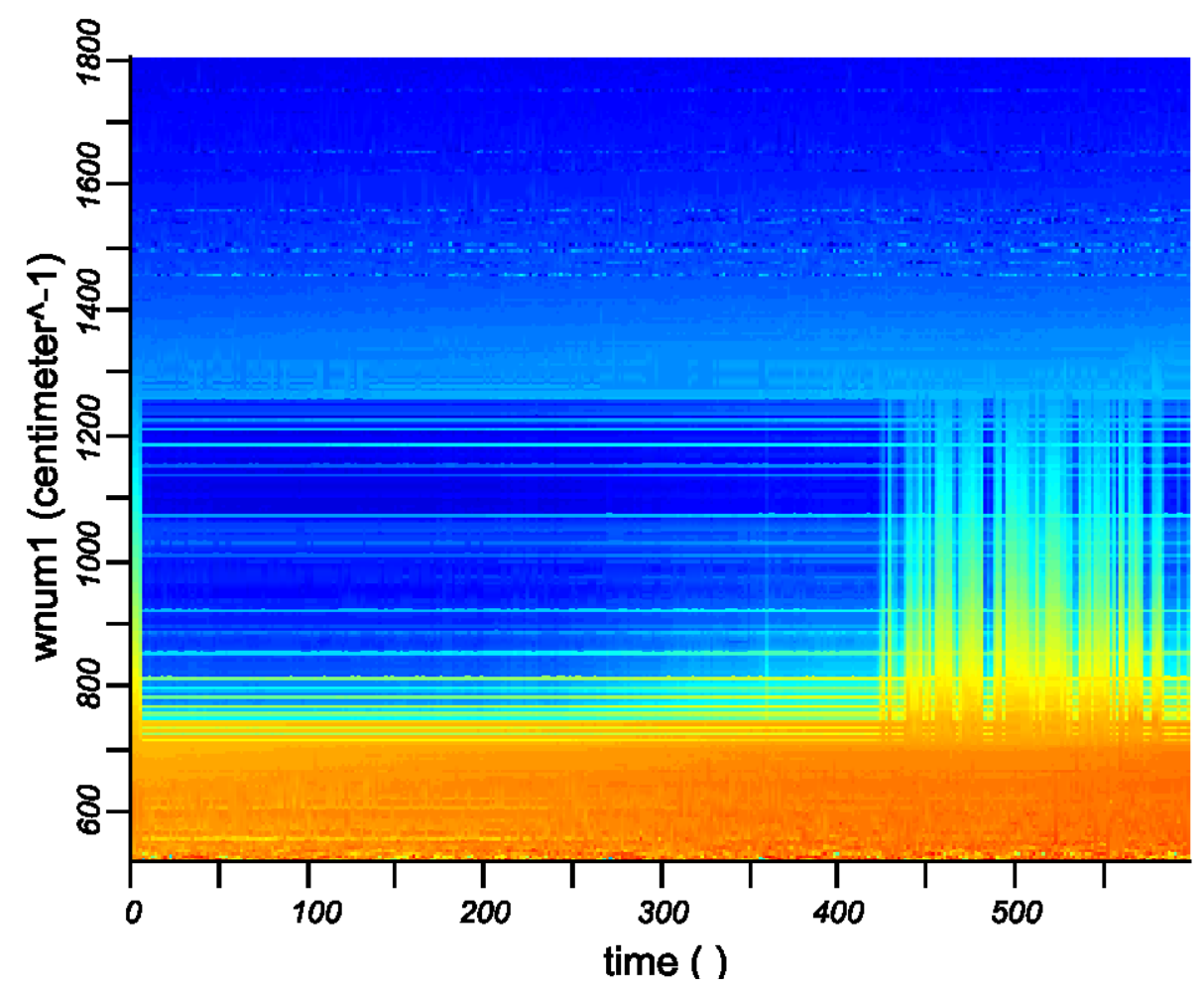

Figure 254. AERIbago mean radiance from 550 - 1800 wavenumbers.

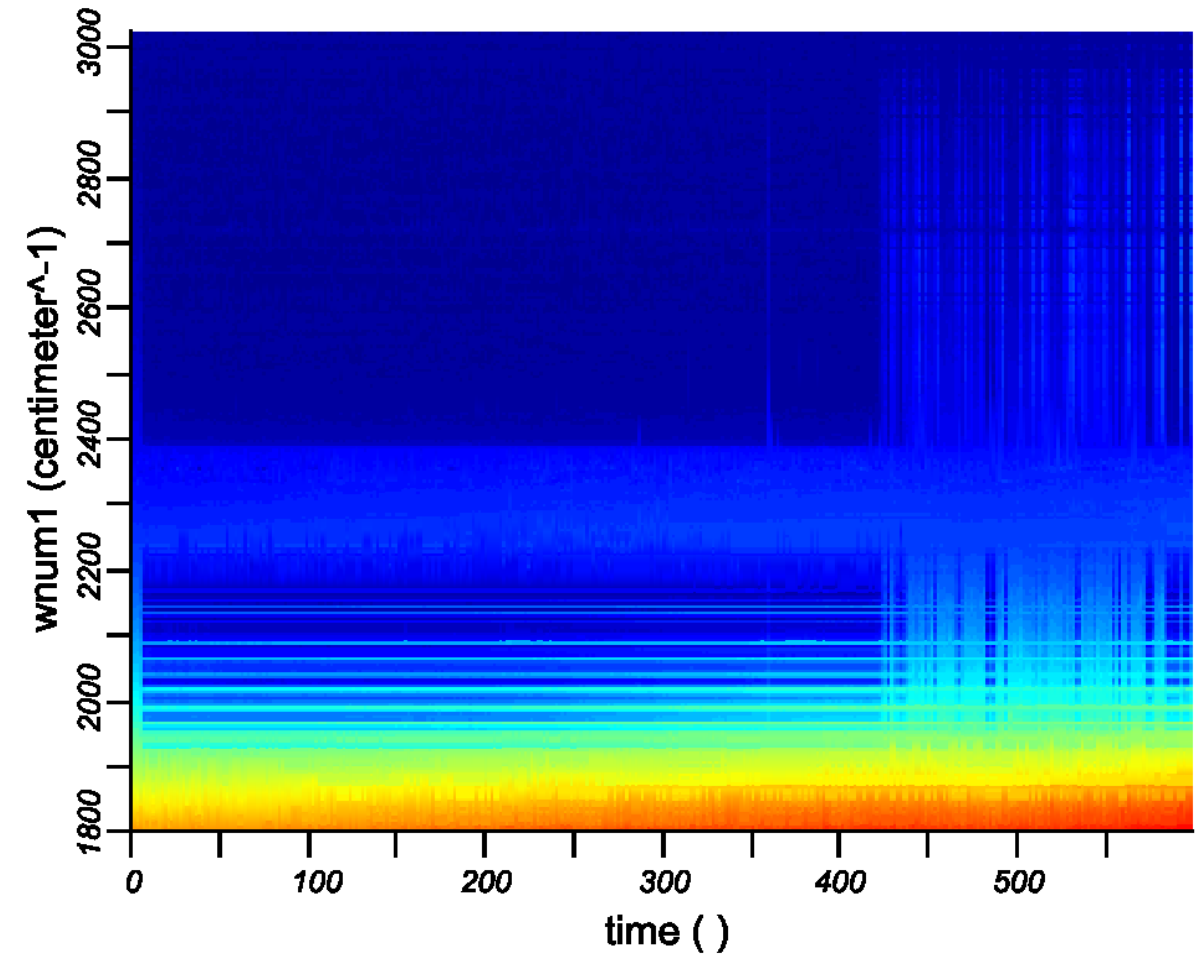

Figure 255. AERIbago mean radiance from 1800 - 3000 wavenumbers. 


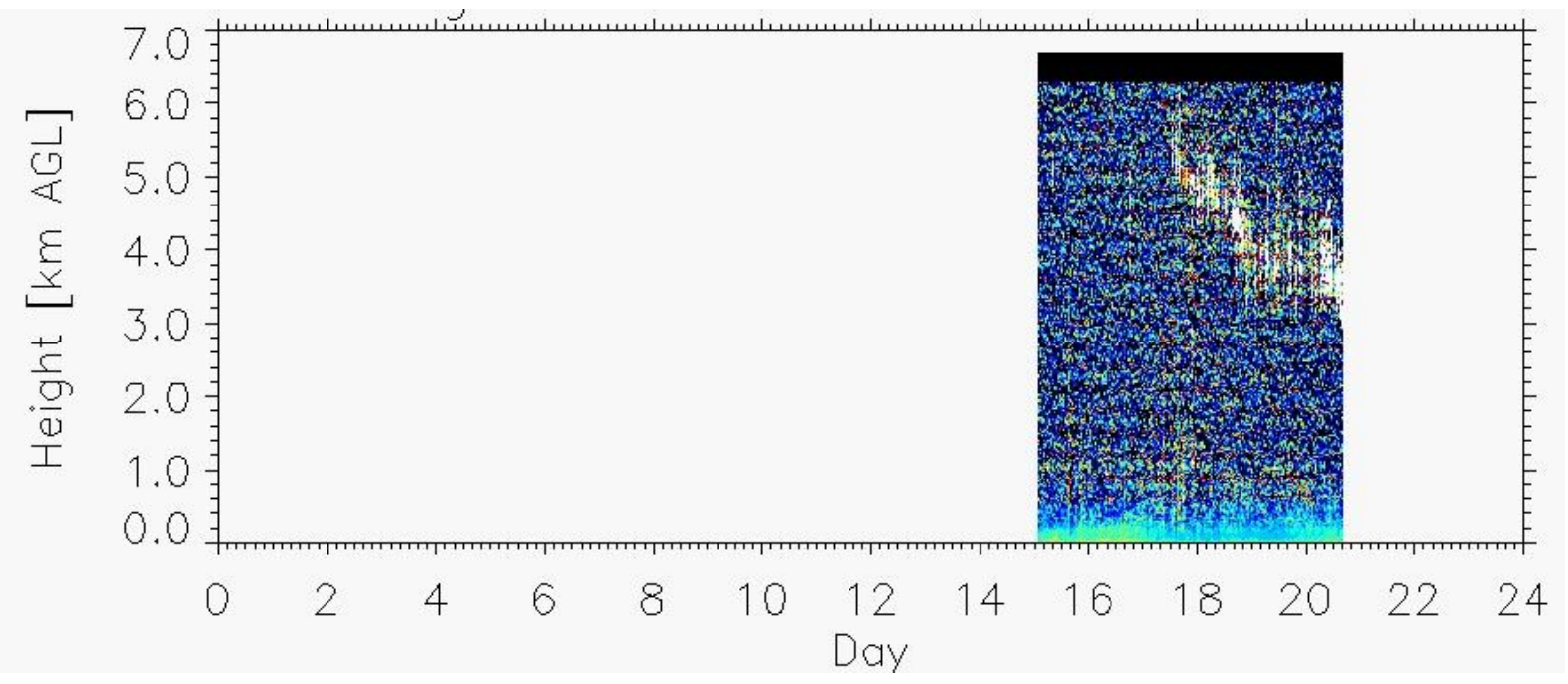

Figure 256. AERIbago Vaisala Backscatter, 060208.

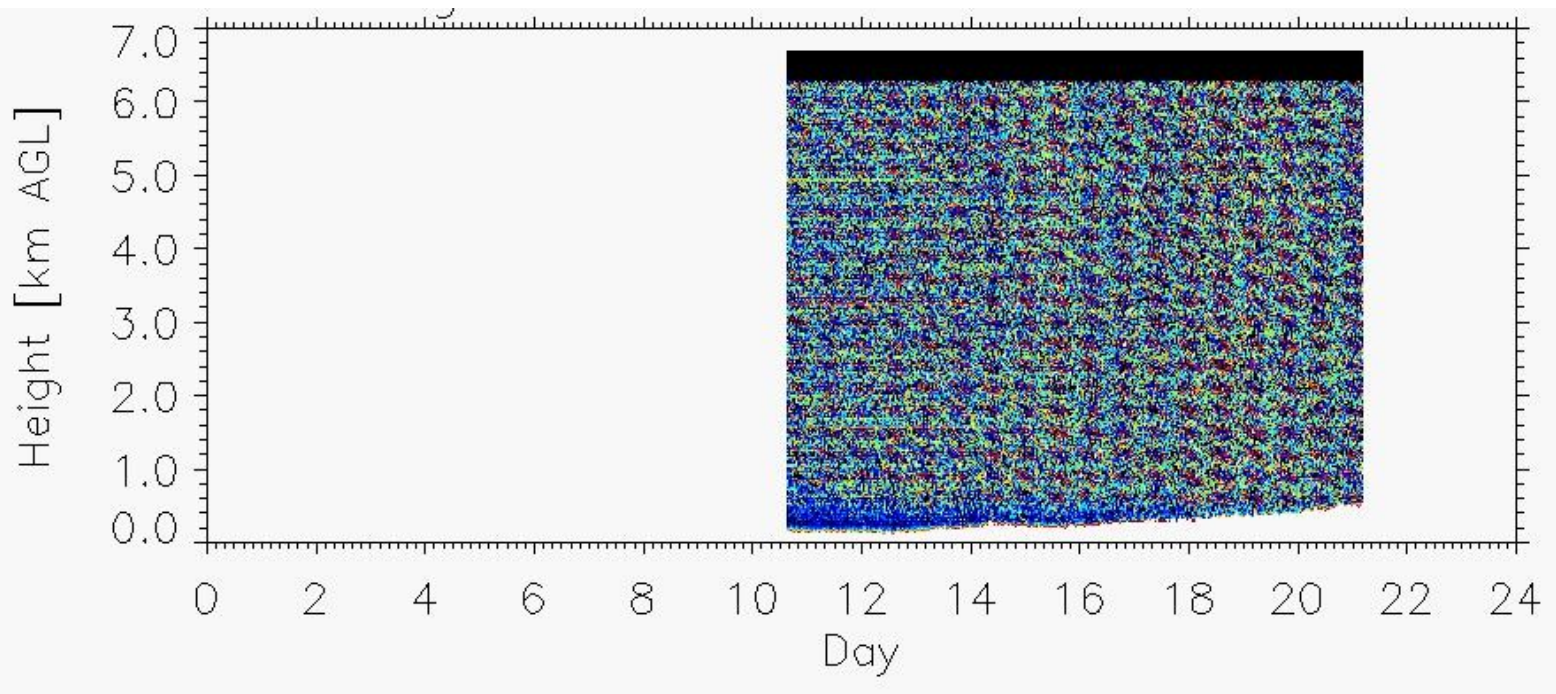

Figure 257. AERIbago Vaisala Backscatter, 060408. 


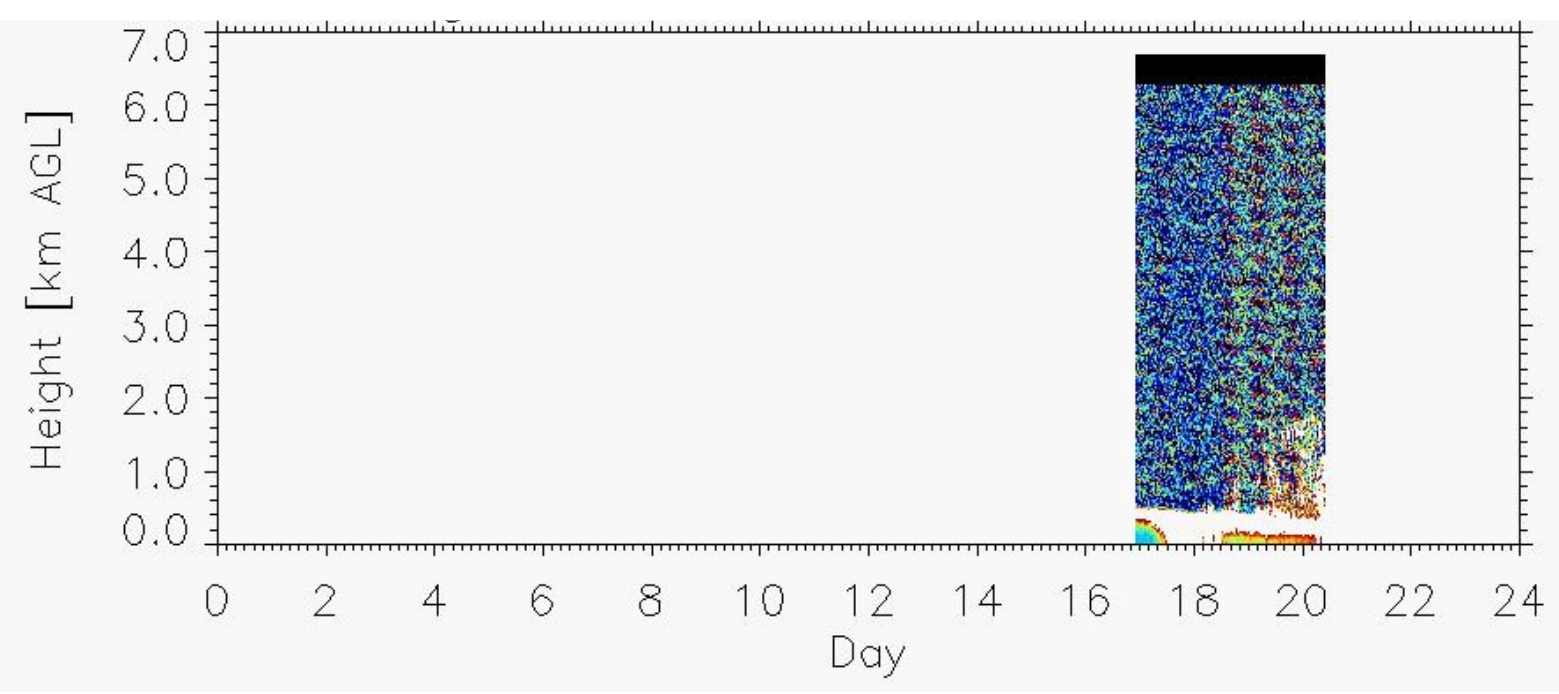

Figure 258. AERIbago Vaisala Backscatter, 060508.

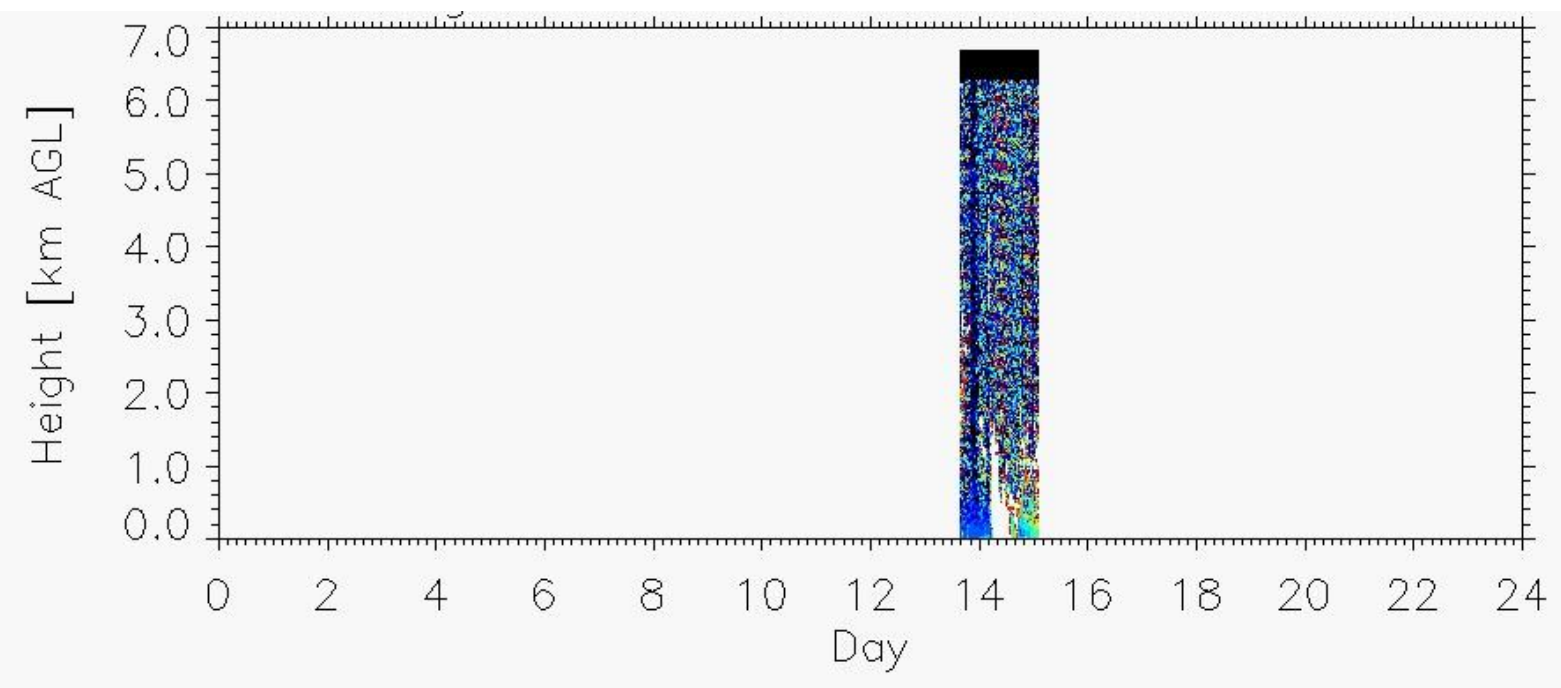

Figure 259. AERIbago Vaisala Backscatter, 060608. 


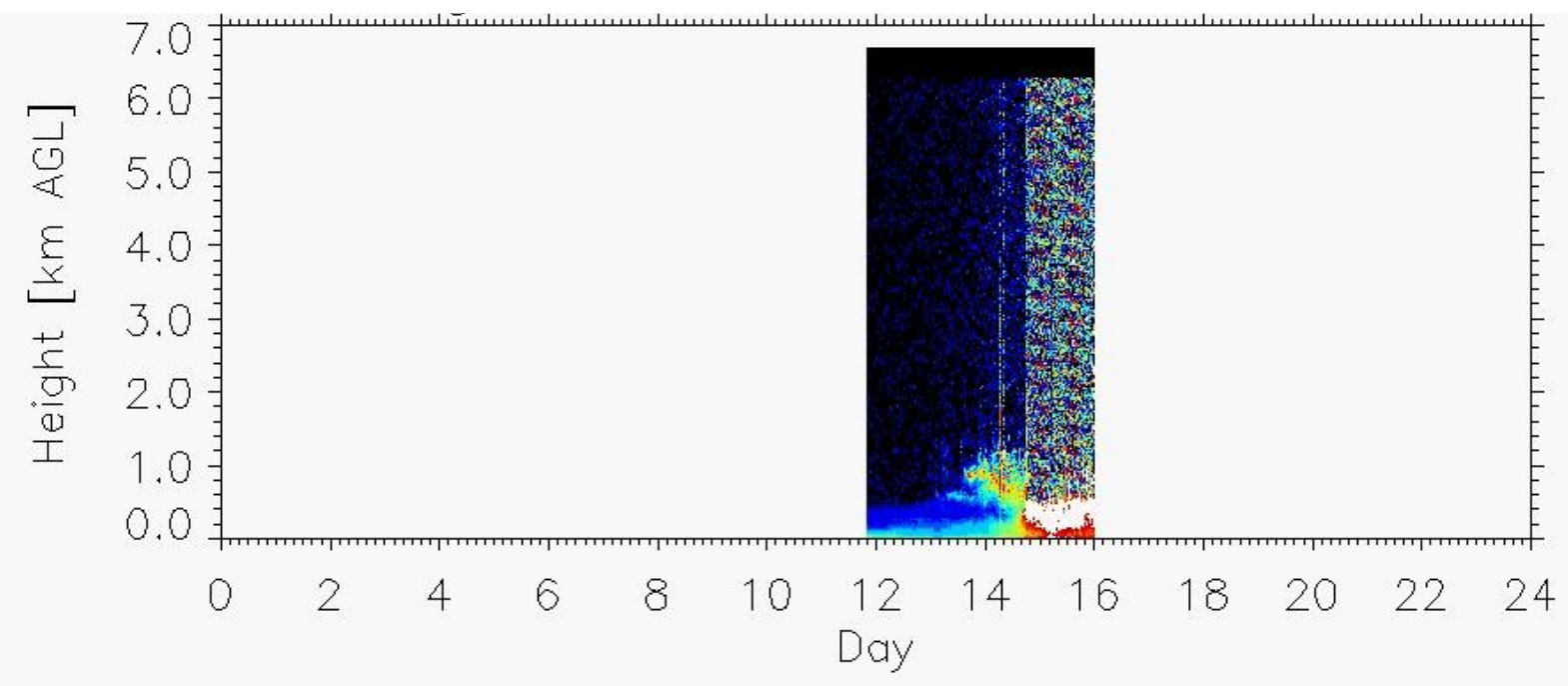

Figure 260. AERIbago Vaisala Backscatter, 060208 - 060708

Flight Schedule for June 7, 2008

\begin{tabular}{lrlrlr} 
Flight Arrivals & \multicolumn{1}{c}{ 6/7/2008 } & & & Actual Time \\
City & Time & Airline & Flight & Status & rwy21 \\
Chicago-O'Hare & $7: 25 \mathrm{AM}$ & American Eagle & 4072 & CRJ & $8: 38 \mathrm{AM}$ \\
Chicago-O'Hare & $8: 35 \mathrm{AM}$ & American Eagle & 3922 & CRJ & $8: 48 \mathrm{AM}$ \\
Chicago-O'Hare & $8: 49 \mathrm{AM}$ & United Express & 6028 & CRJ & rwy21 \\
Milwaukee & $9: 07 \mathrm{AM}$ & Midwest Connect & 2616 & $\mathrm{CRJ}$ & $9: 18 \mathrm{AM}$ \\
Detroit & $9: 29 \mathrm{AM}$ & Northwest & 837 & DC9 & $10: 13 \mathrm{AM}$ \\
Cleveland, OH & $10: 15 \mathrm{AM}$ & Continental Express & 8787 & DeHaviland DHC-8-200 "Dash 8" & $10: 53 \mathrm{AM}$ \\
St. Louis & $10: 40 \mathrm{AM}$ & American Connection & 5462 & CRJ
\end{tabular}

Valid flights in

yellow

clear blue sky, wind dir 180, 


\begin{tabular}{|c|c|c|c|c|c|}
\hline \multicolumn{5}{|c|}{ REPORT DOCUMENTATION PAGE } & $\begin{array}{c}\text { Form Approved } \\
\text { OMB No. 0704-0188 }\end{array}$ \\
\hline \multicolumn{6}{|c|}{$\begin{array}{l}\text { The public reporting burden for this collection of information is estimated to average } 1 \text { hour per response, including the time for reviewing instructions, searching existing data sources, } \\
\text { gathering and maintaining the data needed, and completing and reviewing the collection of information. Send comments regarding this burden estimate or any other aspect of this } \\
\text { collection of information, including suggestions for reducing this burden, to Department of Defense, Washington Headquarters services, Directorate for Information Operations and } \\
\text { Reports (0704-0188), } 1215 \text { Jefferson Davis Highway, Suite } 1204 \text {, Arlington, VA } 222202-4302 \text {. Respondents should be aware that notwithstanding any other provision of law, no person } \\
\text { shall be subject to any penalty for failing to comply with a collection of information if it does not display a currently valid OMB control number. } \\
\text { PLEASE DO NOT RETURN YOUR FORM TO THE ABOVE ADDRESS. }\end{array}$} \\
\hline \multirow{2}{*}{\multicolumn{2}{|c|}{$\begin{array}{l}\text { 1. REPORT DATE (DD-MM-YYYY) } \\
01-08-2010\end{array}$}} & \multirow{2}{*}{\multicolumn{3}{|c|}{$\begin{array}{l}\text { 2. REPORT TYPE } \\
\text { Technical Publication }\end{array}$}} & \multirow[t]{2}{*}{ 3. DATES COVERED (From - To) } \\
\hline & & Technic & & & \\
\hline \multirow{3}{*}{\multicolumn{5}{|c|}{$\begin{array}{l}\text { 4. TITLE AND SUBTITLE } \\
\text { Hazard Detection Analysis for a Forward-Looking Interferometer }\end{array}$}} & 5a. CONTRACT NUMBER \\
\hline & & & & & 5b. GRANT NUMBER \\
\hline & & & & & 5c. PROGRAM ELEMENT NUMBER \\
\hline \multirow{5}{*}{\multicolumn{5}{|c|}{$\begin{array}{l}\text { 6. AUTHOR(S) } \\
\text { West, Leanne; Gimmestad, Gary; Herkert, Ralph; Smith, William L.; } \\
\text { Kireev, Stanislav; Schaffner, Philip R.; Daniels, Taumi S.; Cornman, Larry } \\
\text { B.; Sharman, Robert; Weekley, Andrew; Perram, Glen; Gross, Kevin; } \\
\text { Smith, Greg; Feltz, Wayne; Taylor, Joe; Olson, Erik }\end{array}$}} & \multirow[t]{2}{*}{ 5d. PROJECT NUMBER } \\
\hline & & & & & \\
\hline & & & & & 5e. TASK NUMBER \\
\hline & & & & & \multirow{2}{*}{$\begin{array}{l}\text { 5f. WORK UNIT NUMBER } \\
\text { 609866.02.07.07.04 }\end{array}$} \\
\hline & & & & & \\
\hline \multirow{2}{*}{\multicolumn{5}{|c|}{$\begin{array}{l}\text { 7. PERFORMING ORGANIZATION NAME(S) AND ADDRESS(ES) } \\
\text { NASA Langley Research Center } \\
\text { Hampton, VA 23681-2199 }\end{array}$}} & \multirow[t]{2}{*}{\begin{tabular}{l|l} 
8. PERFORMING ORGANIZATION \\
REPORT NUMBER
\end{tabular}} \\
\hline & & & & & \\
\hline \multirow{4}{*}{\multicolumn{5}{|c|}{$\begin{array}{l}\text { 9. SPONSORING/MONITORING AGENCY NAME(S) AND ADDRESS(ES) } \\
\text { National Aeronautics and Space Administration } \\
\text { Washington, DC 20546-0001 }\end{array}$}} & 10. SPONSOR/MONITOR'S ACRONYM(S) \\
\hline & & & & & NASA \\
\hline & & & & & $\begin{array}{l}\text { 11. SPONSOR/MONITOR'S REPORT } \\
\text { NUMBER(S) }\end{array}$ \\
\hline & & & & & NASA/TP-2010-216845 \\
\hline \multicolumn{6}{|c|}{$\begin{array}{l}\text { 12. DISTRIBUTIONIAVAILABILITY STATEMENT } \\
\text { Unclassified - Unlimited } \\
\text { Subject Category } 03 \\
\text { Availability: NASA CASI (443) 757-5802 }\end{array}$} \\
\hline \multicolumn{6}{|c|}{ 13. SUPPLEMENTARY NOTES } \\
\hline \multicolumn{6}{|c|}{ 14. ABSTRACT } \\
\hline \multicolumn{6}{|c|}{$\begin{array}{l}\text { The Forward-Looking Interferometer (FLI) is a new instrument concept for obtaining the measurements required to alert flight crews to potential weather } \\
\text { hazards to safe flight. To meet the needs of the commercial fleet, such a sensor should address multiple hazards to warrant the costs of development, } \\
\text { certification, installation, training, and maintenance. The FLI concept is based on high-resolution Infrared Fourier Transform Spectrometry (FTS) technologies } \\
\text { that have been developed for satellite remote sensing. These technologies have also been applied to the detection of aerosols and gases for other purposes. The } \\
\text { FLI concept is being evaluated for its potential to address multiple hazards including clear air turbulence (CAT), volcanic ash, wake vortices, low slant range } \\
\text { visibility, dry wind shear, and icing during all phases of flight (takeoff, cruise, and landing). The research accomplished in this second phase of the FLI project } \\
\text { was in three major areas: further sensitivity studies to better understand the potential capabilities and requirements for an airborne FLI instrument, field } \\
\text { measurements that were conducted in an effort to provide empirical demonstrations of radiometric hazard detection, and theoretical work to support the } \\
\text { development of algorithms to determine the severity of detected hazards. }\end{array}$} \\
\hline \multicolumn{6}{|c|}{$\begin{array}{l}\text { 15. SUBJECT TERMS } \\
\text { Aviation; Safety; Detection; Interferometers; Fourier transformation; Spectrometry; Hazards; Weather }\end{array}$} \\
\hline \multicolumn{3}{|c|}{ 16. SECURITY CLASSIFICATION OF: } & 17. LIMITATION OF & 18. NUMBER & \multirow{2}{*}{$\begin{array}{l}\text { 19a. NAME OF RESPONSIBLE PERSON } \\
\text { STI Help Desk (email: help@sti.nasa.gov) }\end{array}$} \\
\hline \multirow[t]{2}{*}{ a. REPORT } & \multirow{2}{*}{\multicolumn{2}{|c|}{\begin{tabular}{l|l|} 
b. ABSTRACT & c. THIS PAGE
\end{tabular}}} & & $\begin{array}{l}\text { OF } \\
\text { PAGES }\end{array}$ & \\
\hline & & & & & 19b. TELEPHONE NUMBER (Include area code) \\
\hline $\mathrm{U}$ & $\mathrm{U}$ & $\mathrm{U}$ & UU & 260 & (443) 757-5802 \\
\hline
\end{tabular}

